Florida International University FIU Digital Commons

\title{
On-line Condition Monitoring, Fault Detection and Diagnosis in Electrical Machines and Power Electronic Converters
}

Alberto Berzoy

Florida International University, aberz001@fiu.edu

DOI: $10.25148 /$ etd.FIDC004096

Follow this and additional works at: https://digitalcommons.fiu.edu/etd

Part of the Electrical and Electronics Commons, and the Power and Energy Commons

\section{Recommended Citation}

Berzoy, Alberto, "On-line Condition Monitoring, Fault Detection and Diagnosis in Electrical Machines and Power Electronic Converters" (2018). FIU Electronic Theses and Dissertations. 3643.

https://digitalcommons.fiu.edu/etd/3643 


\section{FLORIDA INTERNATIONAL UNIVERSITY}

Miami, Florida

\section{ON-LINE CONDITION MONITORING, FAULT DETECTION AND DIAGNOSIS IN ELECTRICAL MACHINES AND POWER ELECTRONIC CONVERTERS}

A dissertation submitted in partial fulfillment of

the requirements for the degree of

DOCTOR OF PHILOSOPHY

in

ELECTRICAL ENGINEERING

by

Alberto Berzoy

2018 
To: Dean Dr. John Volakis

College of Engineering and Computing

This dissertation, written by Alberto Berzoy, and entitled On-line Condition Monitoring, Fault Detection and Diagnosis in Electrical Machines and Power Electronic Converters, having been approved in respect to style and intellectual contents, is referred to you for judgment.

We have read this dissertation and recommend its approval.

Sakhrat Khizroev

Bilal El-Zahab

Jean Andrian

Mark Roberts

Osama A. Mohammed, Major Professor

Date of Defense: March 22, 2018

The dissertation of Alberto Berzoy is approved.

Dean John Volakis

College of Engineering and Computing

Andrés G. Gil

Vice President for Research and Economic Development and Dean of the University Graduate School

Florida International University, 2018 
Copyright (c) 2018 by Alberto Berzoy

All rights reserved. 


\section{DEDICATION}

Dedicate to my wife Mrs. Neidegar Martinez for her love and support. To my parents Robinson Berzoy Aragon and Martha Llerena de Berzoy together with my sister Claudia Berzoy Llerena who has always encouraged and supported me during all of my educational life. 


\section{ACKNOWLEDGMENTS}

First, I would like to thank my major research advisor, Professor Osama Mohammed, whose guidance, support and expert technical advice have made all this work possible. Professor Mohammed has been an excellent and professional advisor to me. Working with him is a rewarding experience in many aspect of my life. His patience, expertise in managing and handling the research group is an eternal source of knowledge that helped me go through tough times with my projects. He provided support at all levels of research including technical guidance and discussions, laboratory facilities and financial support. His trust in my abilities allowed me to freely explore research ideas that eventually resulted in interesting and fulfilling work. I am deeply grateful for his guidance. I am also grateful for the chance he gave me to work at the world class facilities at the Energy Systems Research Laboratory. There, I found all the first-class equipment I needed to experimentally verify my results, and I developed professionally in an environment where work and engineering ethics are highly respected. The years I spent at the Energy Systems Research Laboratory, in FIU, have been the best rewarding years in my life.

I would like to thank the members of my committee, Dr. Mark Roberts, Dr. Jean H. Andrian, Dr. Sakhrat Khizroev and Dr. Bilal El-Zahab for the assistance they provided and their time in evaluating my dissertation and providing valuable feedback.

I am also grateful to Professor Dr. Jose Restrepo and Professor Dr. Jose Manuel Aller from Simon Bolivar University for their help with their profound knowledge on power electronics and electric machines. They have properly prepared me during my undergraduate and Master studies and research before joining FIU. Their serious attitude towards research and their interdisciplinary perspective helped me to better understand the 
phenomena of the inter-turn short-circuit fault of electric machines utilized in this dissertation.

I'd like to acknowledge the FIU university graduate school for awarding the Doctoral -Year Fellowship (DYF) to me in 2017. It significantly enhanced my ability to finish my doctoral research.

I'd like to acknowledge my doctoral student colleague, Ahmed A. S. Mohamed for his significant contribution and cooperation in my research and Hassan Hossam Hassan Eldeeb who put efforts in implementing and testing some of the fault detection techniques used in my dissertation. Also, I'm grateful for the help of undergraduate student interns who were involved in helping me test power electronic converters utilized in my research. Special acknowledgement to Professor Johnny Rengifo from Simon Bolívar University who teach me and contribute significantly in my best research publications.

In addition, I recognize that this research would not have been possible without the financial support from the Office of Naval Research (ONR) as well as other sources including the US Department of Energy brought to our research team through my major research advisor.

Lastly, I would like to dedicate this thesis to my wife Neidegar, my parents Robinson and Marthica, my sister Claudia and nephew Sebastian. It is their love and support throughout my entire life that makes me the person I am today. Here I wish them good health and happy living. 


\begin{abstract}
OF THE DISSERTATION
ON-LINE CONDITION MONITORING, FAULT DETECTION AND DIAGNOSIS IN

ELECTRICAL MACHINES AND POWER ELECTRONIC CONVERTERS
\end{abstract}

by

\author{
Alberto Berzoy \\ Florida International University, 2018 \\ Miami, Florida \\ Professor Osama A. Mohammed, Major Professor
}

The objective of this $\mathrm{PhD}$ research is to develop robust, and non-intrusive condition monitoring methods for induction motors fed by closed-loop inverters. The flexible energy forms synthesized by these connected power electronic converters greatly enhance the performance and expand the operating region of induction motors. They also significantly alter the fault behavior of these electric machines and complicate the fault detection and protection. The current state of the art in condition monitoring of power-converter-fed electric machines is underdeveloped as compared to the maturing condition monitoring techniques for grid-connected electric machines.

This dissertation first investigates the stator turn-to-turn fault modelling for induction motors (IM) fed by a grid directly. A novel and more meaningful model of the motor itself was developed and a comprehensive study of the closed-loop inverter drives was conducted. A direct torque control (DTC) method was selected for controlling IM's electromagnetic torque and stator flux-linkage amplitude in industrial applications. Additionally, a new driver based on DTC rules, predictive control theory and fuzzy logic inference system for the IM was developed. This novel controller improves the 
performance of the torque control on the IM as it reduces most of the disadvantages of the classical and predictive DTC drivers. An analytical investigation of the impacts of the stator inter-turn short-circuit of the machine in the controller and its reaction was performed. This research sets a based knowledge and clear foundations of the events happening inside the IM and internally in the DTC when the machine is damaged by a turn fault in the stator. This dissertation also develops a technique for the health monitoring of the induction machine under stator turn failure. The developed technique was based on the monitoring of the off-diagonal term of the sequence component impedance matrix. Its advantages are that it is independent of the IM parameters, it is immune to the sensors' errors, it requires a small learning stage, compared with $\mathrm{NN}$, and it is not intrusive, robust and online. The research developed in this dissertation represents a significant advance that can be utilized in fault detection and condition monitoring in industrial applications, transportation electrification as well as the utilization of renewable energy microgrids.

To conclude, this $\mathrm{PhD}$ research focuses on the development of condition monitoring techniques, modelling, and insightful analyses of a specific type of electric machine system. The fundamental ideas behind the proposed condition monitoring technique, model and analysis are quite universal and appeals to a much wider variety of electric machines connected to power electronic converters or drivers. To sum up, this $\mathrm{PhD}$ research has a broad beneficial impact on a wide spectrum of power-converter-fed electric machines and is thus of practical importance. 
CHAPTER

PAGE

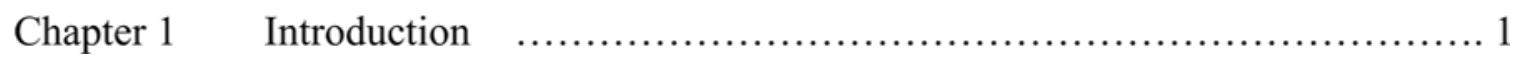

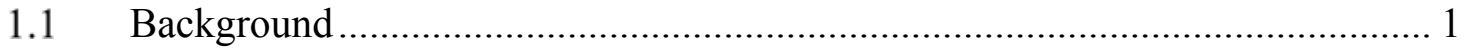

$1.2 \quad$ Problem Statement ................................................................................ 4

1.2.1 Condition Monitoring in Electric Machines ................................................. 4

1.2.2 Condition Monitoring and Performance Enhancement of the Power Electronics of the Drive Powertrain............................................................... 9

1.2.2.1 Performance Enhancement of Power Electronics .................................... 9

1.2.2.2 Condition Monitoring of the Power Electronics .................................... 10

$1.3 \quad$ Research Objective ................................................................................. 12

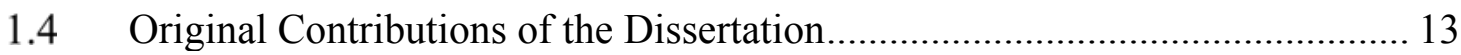

1.4.1 Condition Monitoring in Electric Machines .................................................... 13

1.4.2 Condition Monitoring and Performance Enhancement of the Power Electronics on the Energy Conversion Chain ................................................. 17

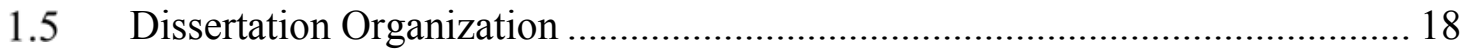

Chapter 2 Electrical Machine and Power Electronic Topology Selection: A

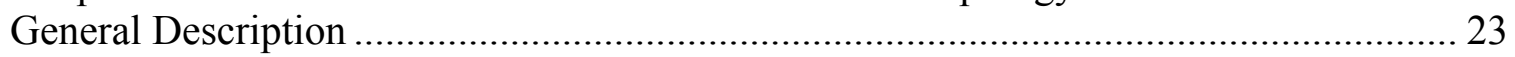

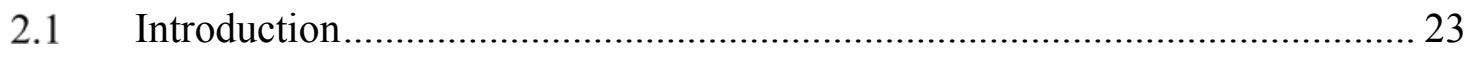

$2.2 \quad$ Electric Machine Selection ...................................................................... 23

2.2.1 Induction Machine Applications ................................................................. 24

2.2.2 Induction Machine Connections-Drives Classification ................................. 26

2.2.3 Selected Induction Machine Applications and Full Topologies ................... 30

2.2.3.1 High-performance Industrial Applications Energy Conversion Chain . 31

2.2.3.2 Transportation Electrification Applications (Hybrid and/or Electric Vehicles) Powertrain ....................................................................... 31

2.2.4 Induction Machine Failures Categorization.................................................... 35

$2.3 \quad$ Power Electronic Topology Selection ......................................................... 39

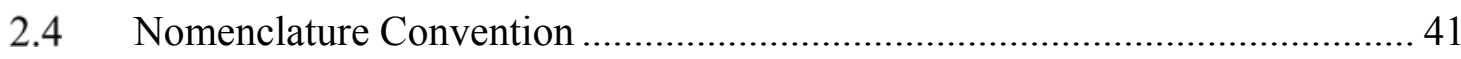

2.5 Reference Frame Theory and its Transformations......................................... 42

2.5.1 Space Vector Transformation ................................................................... 47

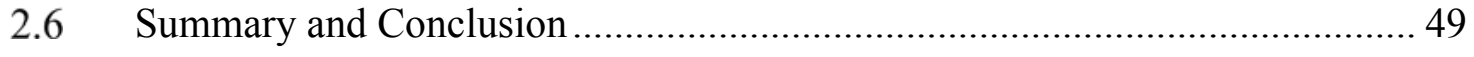

Chapter 3 Design and Control of Active Regenerative Rectifier in Transportation

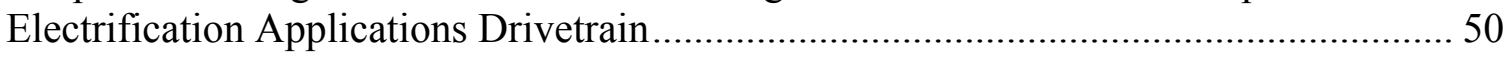

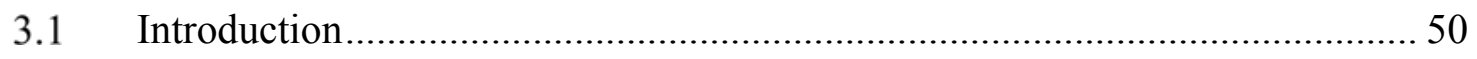


3.2 Active Regenerative Rectifier Modelling ………....................................... 54

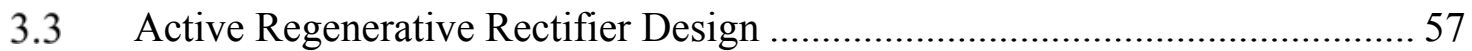

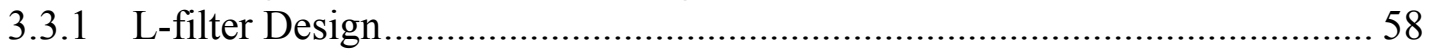

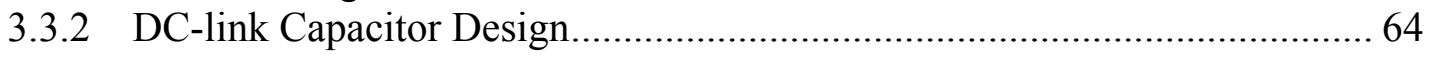

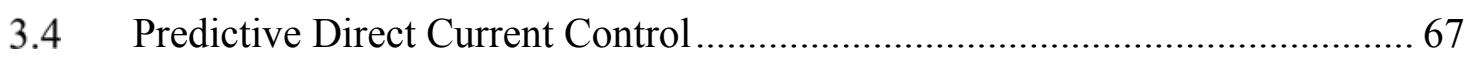

3.4.1 Problem Formulation: Predictive Direct Current Control ............................. 68

3.4.2 Predictive Direct Current Control .............................................................. 70

3.4.3 Best VSC Vector Selection - Direct Current Control (BVS-DCC).............. 71

3.4.4 Optimum VSC Vector's Computation - DCC (OVC-DCC) ……………..... 72

3.5 Performance Assessment of Different Modulation Strategies......................... 72

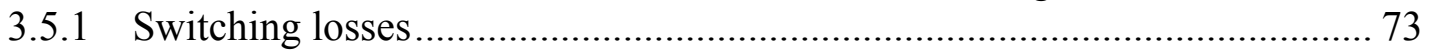

3.5.2 Current Distortion and Dynamic Response ……………............................. 74

3.6 Simulation and Experimental Results: Control .............................................. 74

3.6.1 OVS-DCC Simulation and Experimental Results ....................................... 75

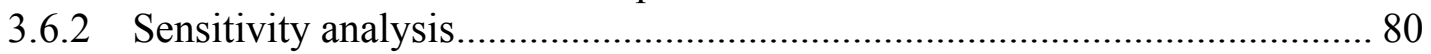

3.6.3 Pulse Width Modulation Strategies: Comparative Study .............................. 81

3.7 Simulation and Experimental Results: Design ……………………………....... 81

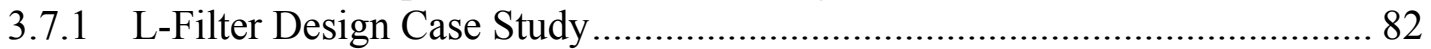

3.7.2 DC-link Capacitor Design Case Study …………………………............ 86

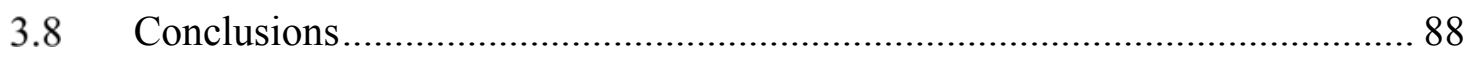

Chapter 4 Design of Power Converters in Transportation Electrification

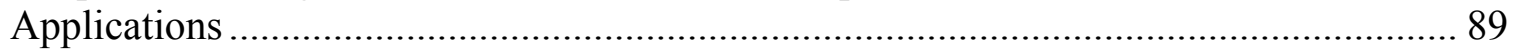

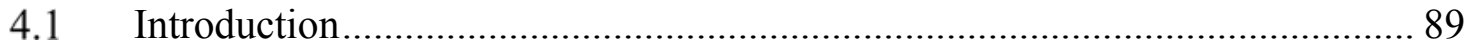

4.2 Optimizing Power Converter PCB Design for Lower EMI.............................. 89

4.3 Modeling of the DC-DC Converter ………………....................................... 91

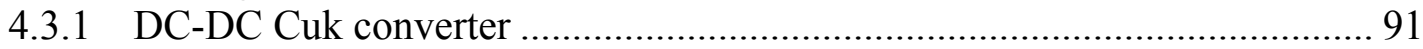

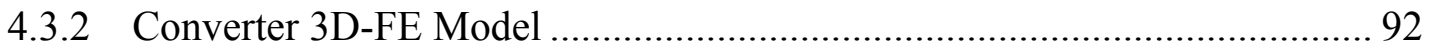

4.3.3 Electromagnetic Field Computation …………………………………...... 92

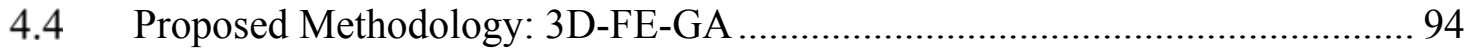

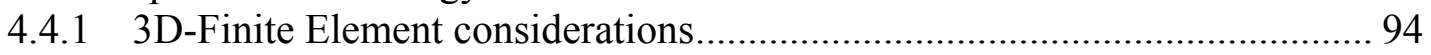

4.4.2 Genetic Algorithm Optimization Procedure ………………………............. 95

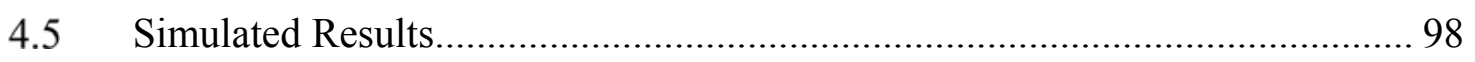

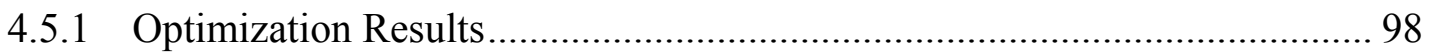

4.5.2 Optimal and Non-optimal Coils position.................................................. 100

4.5.3 Optimal and Non-optimal Capacitors position. ……….............................. 103

4.6 Experimental Results ............................................................................. 105

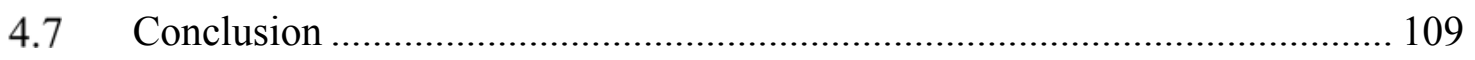


Chapter 5 Control of Induction Machine in the Drivetrain

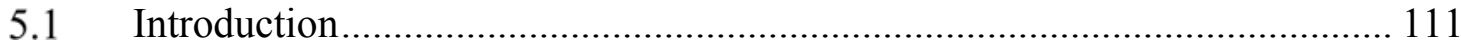

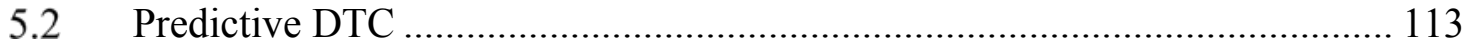

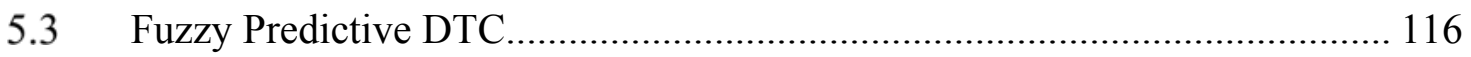

5.4 Performance Assessment of Different PWM Strategies ................................ 120

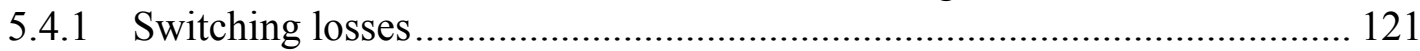

5.4.2 Current Distortion and Dynamic Response ................................................. 121

5.5 Simulation and Experimental Results .......................................................... 122

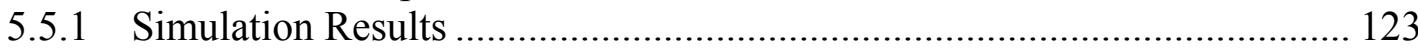

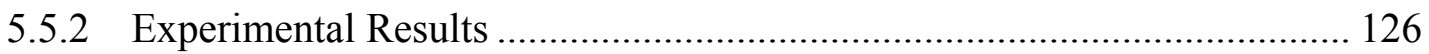

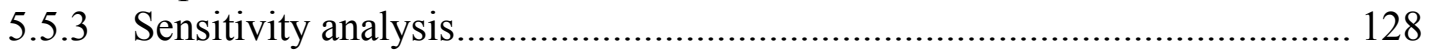

5.5.4 Pulse Width Modulation Strategies: Comparative Study ........................... 129

5.5.5 Simulation and Experimental Results for the Comparative Assessment between SVM and PWM ................................................................... 130

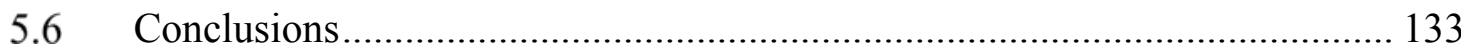

Chapter 6 Complex-Vector Transient and Steady-State Modelling of Induction Machines with Stator Winding Inter-turn Short-Circuit Fault........................................ 134

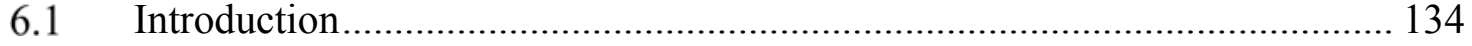

6.2 Modelling of IM with Stator ITSC Fault .................................................... 136

6.2.1 IM Equations in Primitive Coordinates .................................................... 137

6.2.1.1 Electromagnetic Torque Analysis ....................................................... 139

6.2.2 Asymmetric IM Model in Space-Vector Notation...................................... 140

6.3 State-Space Model of the Faulty IM ................................................................ 141

6.3.1 Flux-linkages State-Space Variable Model................................................. 142

6.3.2 Current State-Space Variable Model ......................................................... 144

6.4 Faulty IM: Complex Vector Equivalent Circuit ………………………....... 145

6.4.1 Steady State Analysis of Faulty IM ......................................................... 146

6.5 Simulation Results for Signatures Search...................................................... 147

6.5.1 Transient Comparative Results for Healthy and Faulty IM........................ 148

6.5.2 Steady State Results for Faulty IM ……………….................................. 153

6.5.2.1 Variation with Fault Severity Factor .................................................... 153

6.5.2.2 Variation with Faulty Resistance ...................................................... 154

6.5.2.3 Torque-Slip Characteristic ............................................................. 155

6.6 Conclusion and Future Research ............................................................... 157

Chapter 7 Proposed Dynamic Model of Induction Machines with Stator Winding

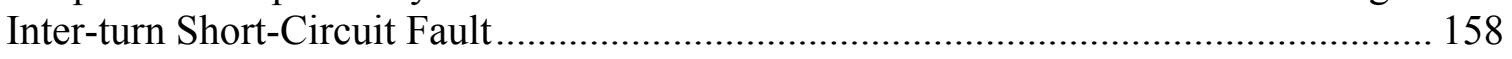

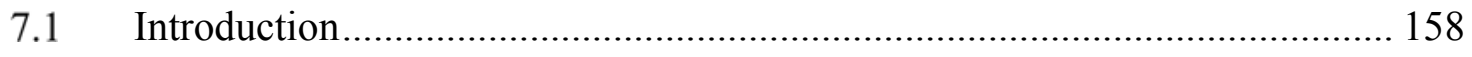




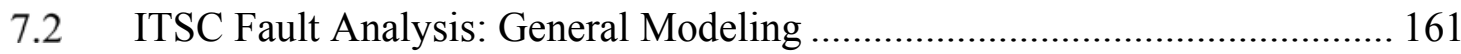

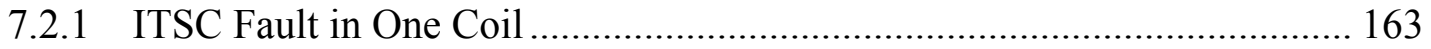

7.2.1.1 Parameter Determination for ITSC in One-Coil ................................ 165

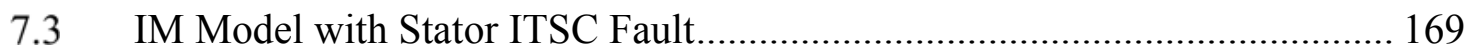

7.3.1 Parameter Determination for the phase with ITSC .................................... 170

7.3.2 IM Equations in Primitive Coordinates ..................................................... 173

7.3.3 IM Equations in Complex-Vector Notation................................................ 176

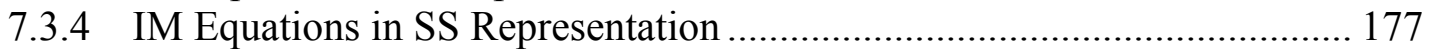

7.3.5 Equivalent Circuits: Faulty IM .............................................................. 178

7.3.6 Steady State Analysis: Symmetric Components EC................................... 180

7.4 Finite Element Model of Faulty IM ......................................................... 182

7.5 Simulation and Experimental Results for the IM model with ITSC............... 188

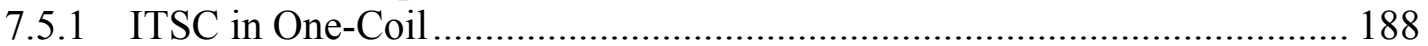

7.5.2 ITSC in Three-Phase IM: Case of Study ................................................... 194

7.5.2.1 Transient Regime of Faulty IM ....................................................... 196

7.5.2.2 Permanent Regime of Faulty IM ......................................................... 202

7.5.2.3 Comparative Analysis between Proposed Model and Linear Model of the Leakage Inductance...................................................................... 210

7.5.2.4 Electromagnetic Torque Discrepancy Study:....................................... 211

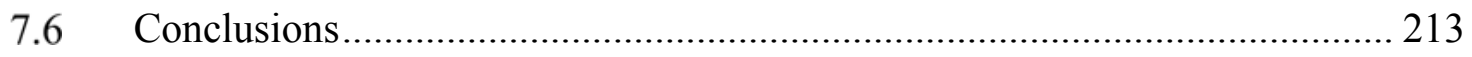

Chapter 8 Analysis of the Impact of Stator Inter-Turn Short-Circuit Faults on Induction Machines driven by Direct Torque Control................................................... 214

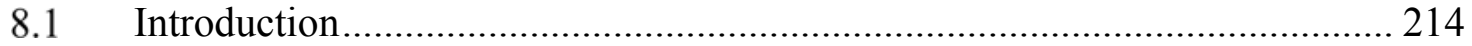

8.2 Analysis of the Complete Drive System ................................................... 215

8.2.1 Direct Torque Control Structure ……………......................................... 215

8.3 Influence of IM Stator Fault in the DTC ……........................................... 217

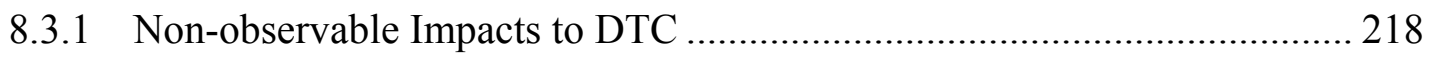

8.3.1.1 Problem of the Estimation Stage ..................................................... 218

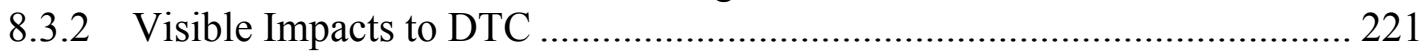

8.3.2.1 Effects on the Estimated Stator Flux-linkage .................................... 222

8.3.2.2 Effects on the Estimated Torque .......................................................... 223

$8.4 \quad$ Reaction of the DTC to the Asymmetric IM ……........................................ 224

8.4.1 DTC Compensation to the Visible Effects.................................................. 224

8.5 Robustness of the DTC to the Asymmetries................................................ 226

8.5.1.1 From the Standard Heuristic derivation of the DTC ............................. 226

8.5.1.2 From the point of view of non-linear control theory........................... 229

8.6 Simulation and Experimental Results .............................................................. 232

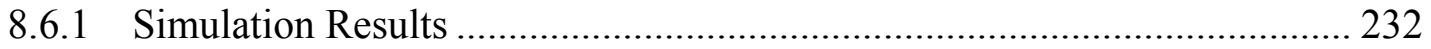

8.6.2 Experimental Results ............................................................................ 236

8.6.2.1 Transient Performance Examination of the Visible Effects ................. 237 
8.6.3 Steady State Inspection of the Visible Effects ............................................ 240

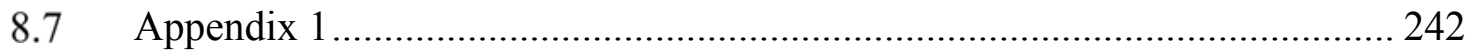

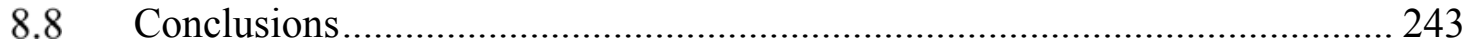

Chapter 9 Online Fault Detection of Inter-Turn Short Circuit in IM driven by DTC. 245

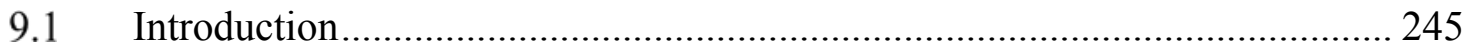

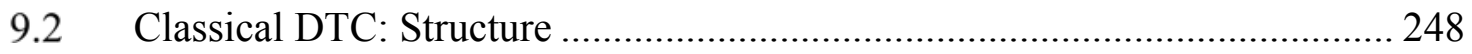

9.3 IM with Inter-Turn Fault: Steady-State Model .............................................. 250

9.4 Influence of IM Stator Fault in DTC ……………………………………..... 251

9.5 Fault Detection Technique ………………………………………………... 253

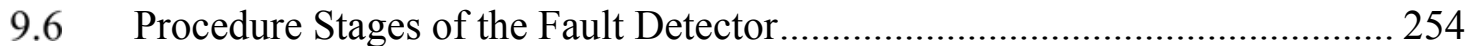

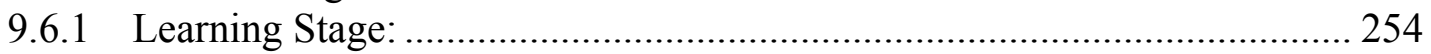

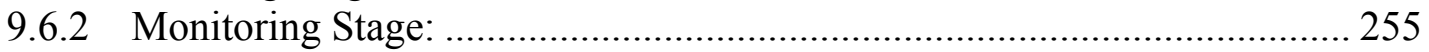

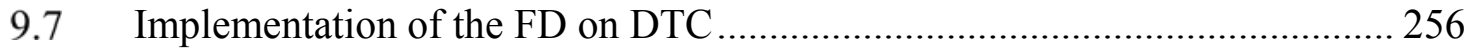

9.7.1 Computation of the Electric Frequency in DTC ........................................ 256

9.7.2 The Estimation of the Stator Voltages ..................................................... 257

9.7.3 Calculation of the Phase Angle of Phasors ............................................. 258

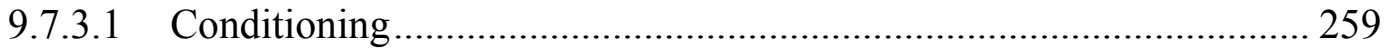

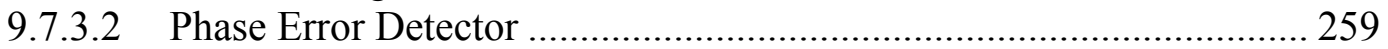

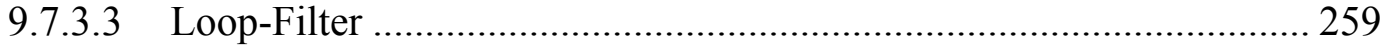

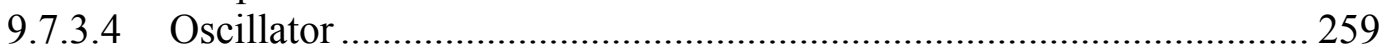

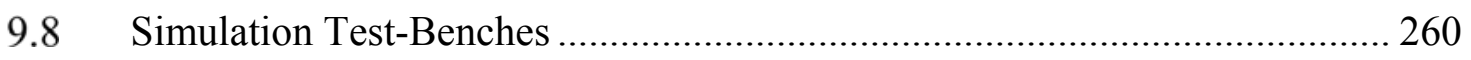

$9.9 \quad$ Simulation and Experimental Results …………........................................ 263

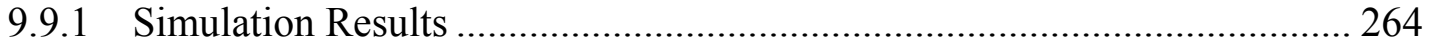

9.9.1.1 Transient Operation of the Faulty IM under DTC .............................. 264

9.9.1.2 Study of two Fault Severity Factors under DTC ................................. 266

9.9.1.3 The Fault Detection Demonstration..................................................... 269

9.9.2 Experimental Results ........................................................................ 272

9.9.2.1 Study of eight Fault Severity Factors under DTC for one operative

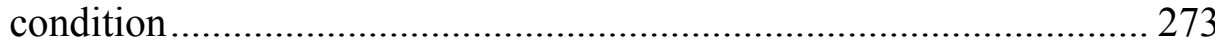

9.9.2.2 Fault Detection Corroboration ......................................................... 275

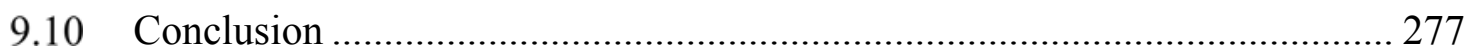

Chapter 10 Condition Monitoring in Power Electronic Devices: Breakdown Mechanism in Gallium Nitride MOSFETs ................................................................. 278

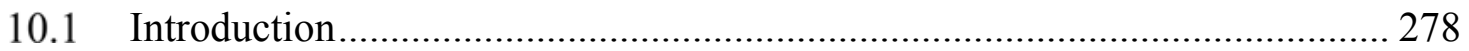

10.2 Breakdown Voltage Assessment of GaN HEMT Devices through Physics-

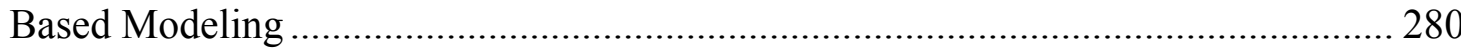


10.2.1 Electric Field by FEA of all the Cases...................................................... 283

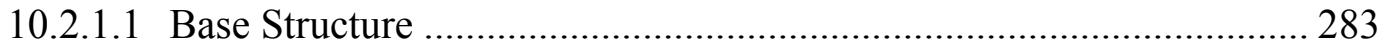

10.2.1.2 Changing the Passivation Layer ...................................................... 285

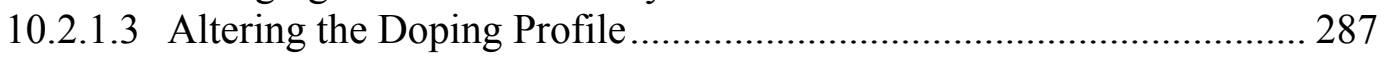

10.2.1.4 Replacing the Subtrate with Germanium ............................................ 289

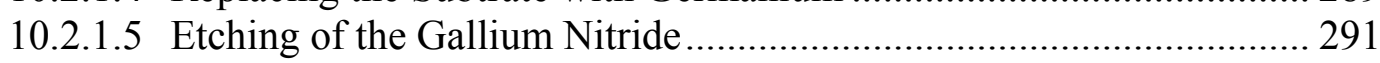

10.2.1.6 Applying Field Plate Contact ............................................................. 292

10.2.2 Comparative Results Analysis of GaN HEMT ......................................... 294

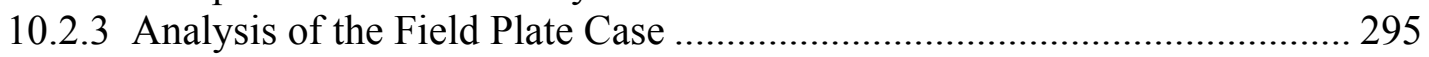

10.3 Field-Plate Length Variation on GaN Devices for BV and On-Resistance

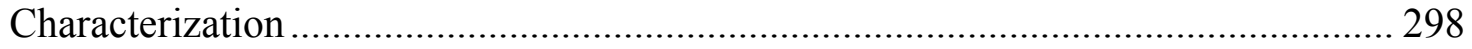

10.3.1 Analysis of FP Length on GaN Performance ……….............................. 300

10.3.2 Closer Analysis of Three Cases ............................................................... 302

10.4 Breakdown Voltage Improvement and Analysis of GaN HEMTs through

Field Plate Inclusion and Substrate Removal ................................................................ 303

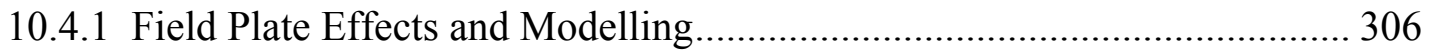

10.4.1.1 Field Plate Effects ………………………...................................... 306

10.4.1.2 Theoretical Model of HEMT with Field Plate ..................................... 308

10.4.2 Cases of Study and Simulation Results .................................................... 311

10.4.2.1 Varying Thickness of the Silicon Substrate ...................................... 312

10.4.3 Detailed Study of all the Cases ............................................................... 313

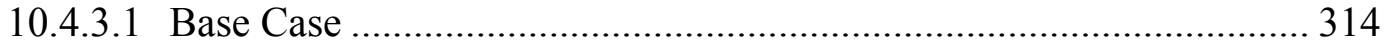

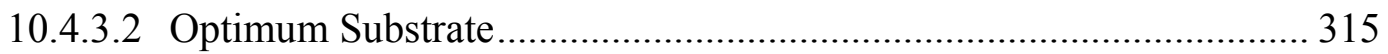

10.4.3.3 Source Field Plate 1........................................................................... 317

10.4.3.4 Source Field Plate 1 with SiN Insulator ............................................. 319

10.4.3.5 Source Field Plate 2 with SiN Insulator .............................................. 321

10.4.3.6 Source Field Plate 3 with SiN Insulator ............................................... 323

10.4.4 Comparative Results among all Studied Cases........................................... 325

10.4.5 Analysis of the Best Case with Field Plate ………………........................ 327

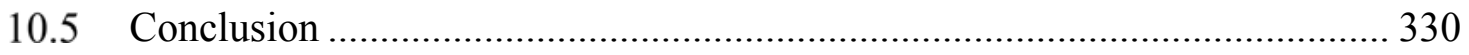

Chapter 11 Conclusions and Recommendations for Future Research .......................... 332

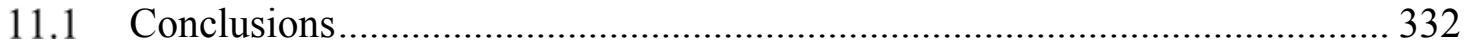

11.2 Recommendations for Future Research .................................................... 336

11.2.1 Recommendations for the Modelling of IM with ITSC.............................. 337

11.2.2 Recommendations for Online Condition Monitoring of IM........................ 339

11.2.3 Recommendations for the Condition Monitoring and Performance Enhancement of Power Electronic Stages .................................................... 341

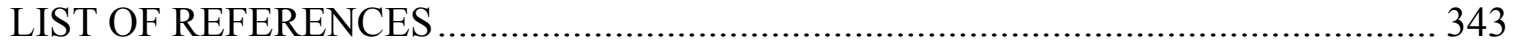

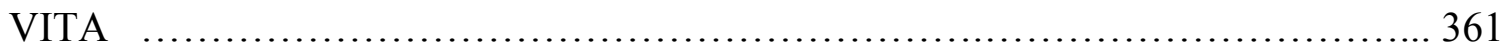


Table 1-1 Failure rate in the energy conversion chain...............................................11

Table 1-2 Failure rate of converter components ......................................................... 11

Table 2-1 Induction Machine Advantages and Drawbacks.........................................24

Table 2-2 Induction Machine connection-drives classification....................................2

Table 2-3 Classification of the Causes of Faults in Induction Machines ......................36

Table 2-4 Categorization of the Faults in an Induction Machine ……………...............37

Table 2-5 Fault Occurrence Possibility on Induction Motors.........................................38

Table 2-6 Variables Notation Convention. .............................................................. 41

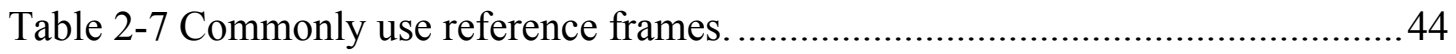

Table 3-1 Categorization of the Active Regenerative Rectifier's Controllers..............53

Table 3-2 Voltage Source Converter Voltage Space Vectors at the terminals [65]. ...55

Table 3-3 The Setup Parameters of ARR ………………………...............................

Table 3-4 Maximum MAE and RMSE for L-Filter variation........................................ 81

Table 3-5 Maximum Criteria Values for each Modulation Technique..........................81

Table 3-6 Comparative Results Desired Current Ripple ..............................................83

Table 3-7 Comparative Line Current Harmonics for $\mathrm{L}=0.012 \mathrm{H}$...............................86

Table 3-8 Comparative Results of DC-Link Voltage Ripple.......................................87

Table 4-1 Cases of Positioning for Inductors …………............................................95

Table 4-2 Cases of positioning for Capacitors.........................................................95

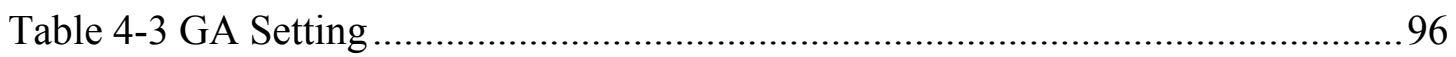




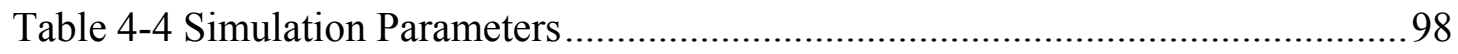

Table 4-5 Optimum Components Position ................................................................. 100

Table 4-6 Simulated and Measured Field at Sensor Center......................................... 107

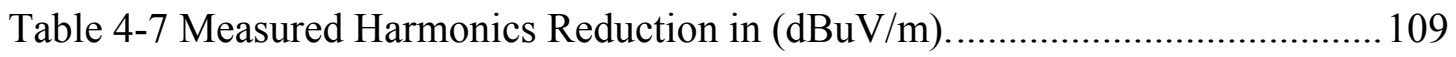

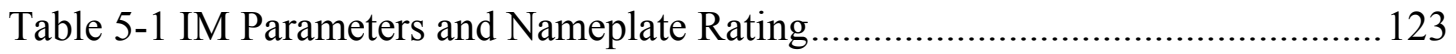

Table 5-2 Maximum Criteria Values for each Control Technique .............................. 124

Table 5-3 Simulation and Experimental Comparison among Torque, Flux and

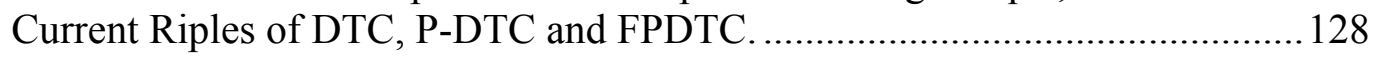

Table 5-4 Maximum MAE and RMSE of $R r, L l s, L l r$ and $L m$ for $50 \%$ variation

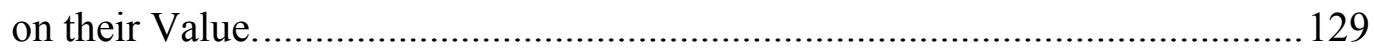

Table 5-5 Maximum Criteria Values for each Modulation Technique........................130

Table 5-6 Maximum Criteria Values for each Modulation Technique........................133

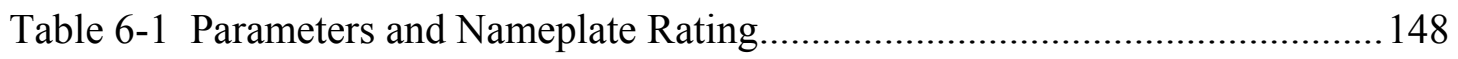

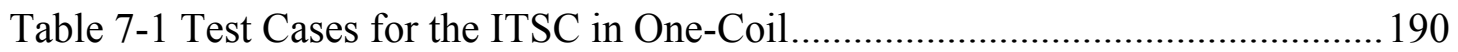

Table 7-2 Parameters for $\mu=0.33$ ITSC at $X 1-X 7$ for One Coil and Nameplate

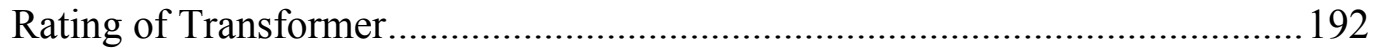

Table 7-3 Parameters and Nameplate Rating for the IM Weg..................................... 195

Table 7-4 ITSC Test Cases for the Faulty IM..............................................................196

Table 7-5 MAE and RMSE for Is $\alpha$, Is $\beta$, Ifault and $\Delta T e$ Sim. vs Exp. as a

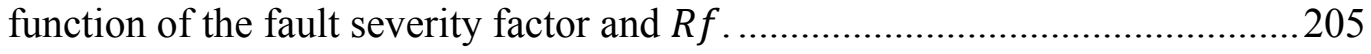

Table 7-6 MAE and RMSE for Is $\alpha$, Is $\beta$, If fault and $\Delta$ Te Sim. vs Exp...................209

Table 7-7 MAE and RMSE for Is $\alpha$, Is $\beta$, Ifault and $\Delta T e$ Sim. vs Exp.

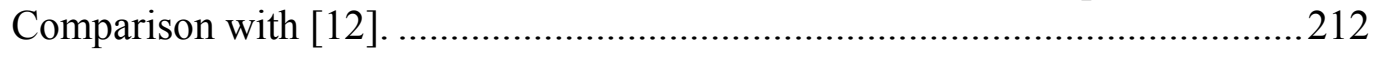

Table 8-1 Decision Look-up Table of DTC for IM ……………………….............216 
Table 8-2 Signal Waveforms and Description.......................................................220

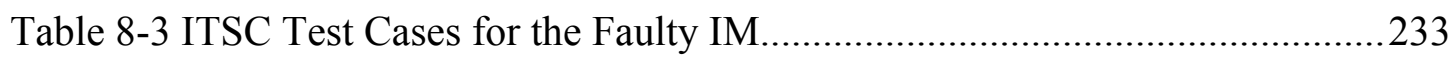

Table 9-1 Close-Loop Vector Controllers for IM and the References dealing with

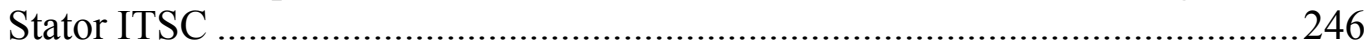

Table 9-2 Switching Look-Up Table for Classical DTC …………….......................247

Table 9-3 Parameters and Nameplate Rating for The IM Weg. ..................................264

Table 9-4 ITSC Test Cases for the Faulty IM...........................................................264

Table 9-5 Actual Torque and Flux MAE for the eight ITSC Test-Cases of Study at the eight Operational Points of the Faulty IM Driven by DTC ………................269

Table 9-6 Off-Diagonal Term of the Impedance Matrix, Mechanical Speed And Electrical Frequency for the 64 ITSC Test Cases of the Faulty IM Driven by DTC: From 0.6 to $1.2 \mathrm{Nm}$.

Table 9-7 Off-Diagonal Term of the Impedance Matrix, Mechanical Speed And Electrical Frequency for the 64 ITSC Test Cases of the Faulty IM Driven by DTC: From 1.2 to $2 \mathrm{Nm}$.

Table 9-8 Estimated Torque and Flux MAE for the eight ITSC Test-Cases for the one operational Point (Tref $=2 \mathrm{Nm}$ ) of the Faulty IM driven by DTC . 274

Table 9-9 Secuence Components and Off-Diagonal Term of the Impedance Matrix for the eight ITSC Test Cases of the Faulty IM driven by DTC at Tref $=2 \mathrm{Nm}$ 275

Table 10-1 GAN-FET Design Parameters and Cases of Study ................................282

Table 10-2 Doping Parameters for the GaN and AlGaN layers ...............................28

Table 10-3 BV and Maximum E Field for all Cases at 2 Heights ..............................2296

Table 10-4 GAN-FET Design Parameters and Cases of Study ……………..............299

Table 10-5 BV and Maximum E Field for all Cases at 2 Heights ...............................302

Table 10-6 Polarization Parameters for the Electrostatic Potential on AlGaN Devices. 309 
Table 10-7 GaN-FET Design Parameters and Cases of Study ...............................313

Table 10-8 GaNFET Design Parameters and Cases of Study.................................329 


\section{LIST OF FIGURES}

FIGURE

Figure 2.1 DOL or Grid-connected IM topology

Figure 2.2 Inverter-fed induction machine topology driven by open-loop control ....30

Figure 2.3 Inverter-fed induction machine topology driven by closed-loop control... 30

Figure 2.4 Complete and detailed schematic of the controlled induction machine drive for industry applications.

Figure 2.5 Complete and detailed schematic of the controlled IM drive for transportation electrification applications.

Figure 2.6 Complete and detailed diagram of the controlled active regenerative rectifier

Figure 2.7 Circuit schematic of the two-level three-phase voltage source inverter..... 40

Figure 2.8 Circuit schematic of the Cuk DC-DC converter

Figure 2.9 Transformation for stationary circuits portrayed by trigonometric relationships.

Figure 2.10 Complex Vector Plane for any stationary circuit variable referred to Stationary reference frame.

Figure 3.1 Complete topology of the controlled three-phase two-level active regenerative rectifier.

Figure 3.2 Geometrical Voltage Vector Plane for the Voltage Source Converter for different modulation strategies. 56

Figure 3.3 Current error calculation method. Grid line current ripple (top) and VSC terminal voltage (bottom).

Figure 3.4 Current error algebraic area summation. 60

Figure 3.5 Operation modes of the ARR and phasor diagram of the ARR. (a) $\mathrm{PF} \neq 1$ (b) $\mathrm{PF}=1$ 
Figure 3.6 Output regulation subspaces for the OVC-DCC.

Figure 3.7 The experimental laboratory test bench setup..................................... 75

Figure 3.8 Active and reactive power, and dc-link voltage of the ARR. (a)

Simulation and (b) Experimental results. 76

Figure 3.9 Control starting: grid line current, reference current and grid lineneutral voltage of phase $a$. (a) Simulation and (b) Experimental results.

Figure 3.10 Control mode transient from rectification to regeneration: grid line current, reference current and grid line voltage of phase $a$. (a) Simulation and

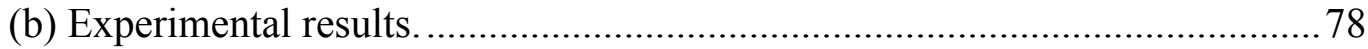

Figure 3.11 Current error or current line ripple of phase $a$. (a) Simulation and (b) Experimental results.

Figure 3.12 Simulated grid line current ripple and desired current ripple. (a) $\mathrm{L}=6$ $\mathrm{mH}$ (b) $\mathrm{L}=9 \mathrm{mH}$, (c) $\mathrm{L}=12 \mathrm{mH}$ and (d) $\mathrm{L}=15 \mathrm{mH}$.

Figure 3.13 Experimental results of line current ripple and desired current ripple.

(a) $\mathrm{L}=6 \mathrm{mH}$ (b) $\mathrm{L}=9 \mathrm{mH}$, (c) $\mathrm{L}=12 \mathrm{mH}$ and (d) $\mathrm{L}=15 \mathrm{mH}$.

Figure 3.14 (a) Current ripple, VSC voltage and their reconstruction in FS (b) Current ripple FS Coefficients (c) VSC voltage FS Coefficients.

Figure 3.15 Simulated results of line current spectrum. (a) $\mathrm{L}=9 \mathrm{mH}$ (b) $\mathrm{L}=12 \mathrm{mH}$, (c) $\mathrm{L}=15 \mathrm{mH}$ and (d) $\mathrm{L}=18 \mathrm{mH}$

Figure 3.16 Experimental results of line current spectrum. (a) $\mathrm{L}=9 \mathrm{mH}$ (b)

$\mathrm{L}=12 \mathrm{mH}$, (c) $\mathrm{L}=15 \mathrm{mH}$ and (d) $\mathrm{L}=18 \mathrm{mH}$.

Figure 3.17DC-link voltage ripple results. Experimental (a) $C=0.5 \mathrm{mF}$ (b) $C=1 \mathrm{mF}$, (c) $\mathrm{C}=2 \mathrm{mF}$ and simulation (d) $\mathrm{C}=0.5 \mathrm{mF}$, (e) $\mathrm{C}=1 \mathrm{mF}$, and (f) $\mathrm{C}=2 \mathrm{mF}$, 87

Figure 4.1 Circuit diagram of Cuk converter. . .92

Figure 4.2 3D-FE model of Cuk converter .93

Figure 4.3 GA and fitness function for the optimization. 97

Figure 4.4 Optimization results for the coil cases .99

Figure 4.5 Optimization results for the capacitor cases. 99 
Figure 4.6. Magnetic field for coils on the PCB (a) $|\mathrm{H}|$ non-optimum (b) $|\mathrm{H}|$ optimum 101

Figure 4.7 Magnitude of the magnetic field through a line in $\mathrm{x}$-direction and passing through the sensor center for optimal and non-optimal position.

Figure 4.8 Magnitude of the magnetic field through a line in $\mathrm{x}$-direction and passing through the sensor center at different heights.

Figure 4.9 Magnitude of the magnetic field through the sensor region in x-y plane. 103

Figure 4.10 Electric field for capacitors on the PCB (a) $|E|$ non-optimum (b) $|E|$ optimum 104

Figure 4.11 Magnitude of the electric field through a line in x-direction and passing through the sensor center for optimal and non-optimal position.

Figure 4.12 Magnitude of the electric field through the sensor region in x-y plane.. 105

Figure 4.13 Experimental Setup 106

Figure 4.14 Output voltage and inductors current for DC-DC converter. 106

Figure 4.15 Magnitude of the magnetic field through a line in x-direction and passing through the sensor center for optimal and non-optimal position.

Figure 4.16 Radiated Magnetic field from the experimental setup (a) In x direction (b) In y direction and (c) In z direction. 108

Figure 4.17 Radiated Magnetic field $(|\mathrm{H}|)$ from the experimental setup. 109

Figure 5.1 Torque and stator flux constants variation lines at the CVP. 116

Figure 5.2 Block diagram of the complete system. 118

Figure 5.3 Block diagram of the proposed fuzzy predictive DTC 118

Figure 5.4 Vector summation of Fuzzy out and correction

Figure 5.5 (a) Simulation results for comparison of the electromagnetic torque among DTC, P-DTC and FP-DTC. (b) Zoom of (a). 125

Figure 5.6 Simulation results of the stator flux locus and stator current comparison among the strategies. (a) DTC. (b) PDTC. (c) FP-DTC and (d) 
Figure 5.7 Experimental setup

Figure 5.8 Experimental comparison of the electromagnetic torque among DTC, P-DTC and FP-DTC.

Figure 5.9 Experimental comparison of the stator flux locus and de bus voltages

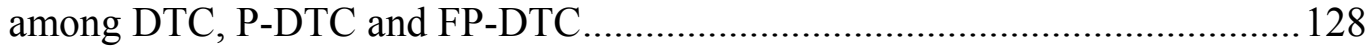

Figure 5.10 Simulation comparison of the electromagnetic torque of FP-DTC between two modulation techniques: PWM and SVM.

Figure 5.11 Simulation comparison of FP-DTC between two modulation techniques (a) stator flux magnitude PWM. (b) stator flux magnitude PWM and (c) phase a current

Figure 5.12 Experimental comparison of the electromagnetic torque of FP-DTC between two modulation techniques: PWM and SVM.

Figure 5.13 Experimental comparison of FP-DTC between two modulation techniques (a) stator flux magnitude PWM. (b) stator flux magnitude PWM (c) phase a current and (d) dc-link voltage

Figure 5.14 Experimental comparison the FFT for the stator current phase $a$ of FP-DTC between two modulation techniques: PWM and SVM.

Figure 6.1 Three-phase stator winding of an induction machine with inter-turn short-circuit in the phase a. (a) Physical Winding (b) Circuit diagram

Figure 6.2 (a) Complex vector equivalent circuit of Asymmetric IM (b) Faulty Circuit.

Figure 6.3 (a) Complex vector operational equivalent circuit of Asymmetric IM (b) Faulty Circuit.

Figure 6.4 Sequence-components equivalent circuit of Asymmetric IM (a) Positive Sequence (b) Negative Sequence (c) Faulty Circuit. 148

Figure 6.5 Real component stator current for healthy and faulty IM (a) is $\alpha$ (b) Zoom.

Figure 6.6 Imaginary component stator current for healthy and faulty IM (a) is $\beta$

(b) Zoomed is $\beta$. 
Figure 6.7 Electromagnetic torque for healthy and faulty IM (a) Te (b) First zoomed Te and (c) Second zoomed Te.

Figure 6.8 Rotor speed for healthy and faulty IM (a) $\omega r$ (b) Zoomed $\omega r$. 152

Figure 6.9 Vector components rotor current for healthy and faulty IM and faulty current (a) Zoomed $\operatorname{ir} \alpha$ (b) Zoomed $\operatorname{ir} \beta$ and (c) if.

Figure 6.10 (a) Fault current magnitude and electromagnetic torque ripple vs severity factor $\mu$. (b) Zoom.

Figure 6.11 (a) Stator current space-vector vs fault severity factor $\mu$ variation under load and no load conditions. (b) Zoom.

Figure 6.12 (a) Fault current magnitude and electromagnetic torque ripple vs severity factor $\mu$ (b) Stator current space-vector vs $\mu$ variation under load and no load conditions

Figure 6.13 (a) Stator component-currents (b) Equivalent admittances (c) Torque slip characteristic. 156

Figure 6.14 Electromagnetic torque slip characteristic (a) Input voltage vatiation and (b) Electric frequency variation.

Figure 7.1 ITSC types in magnetically coupled circuits (a) Single-phase Transformers. Three-phase transformer and EM connected in wye (b) high voltage connection (c) low voltage connection

Figure 7.2 ITSC fault in one-coil. (a) Physical winding (b) Circuit diagram ............ 164

Figure 7.3 Coil at no load condition (no ITSC fault) (a) Physical winding (b) Circuit diagram 165

Figure 7.4 Leakage factor inductance as a function of the fault severity factor 168

Figure 7.5 Equivalent circuit diagram for the ITSC in one coil or autotransformer . 168 Figure 7.6 Three-phase stator winding of an IM with ITSC in the phase " $a$ ". 170

Figure 7.7 (a) Complex-vector EC of an asymmetric IM (b) Faulty circuit. 179

Figure 7.8 (a) CV operational EC of an asymmetric IM (b) Faulty circuit. 180

Figure 7.9 Sequence-components EC of an asymmetric IM (a) Positive sequence 
(b) Negative sequence (c) Faulty circuit.

Figure 7.10 (a) Center taps in the phase " $a$ " of the IM for the ITSC creation. (b)

Phase " $a$ " single layer stator winding diagram

Figure 7.11 (a) IM internal geometry, slots, rotor bars and placement of the ITSC.

(b) 2D IM external geometry for the FEA. The unit's dimensions are millimeters (mm).

Figure 7.12 Phase " $a$ " circuit used in the FE co-simulation.

Figure 7.13 Four ITSC locations in the stator winding phase "a". Position $1(P 1)$ to 4 (P4): (a) speed (b) electromagnetic torque.

Figure 7.14 Four ITSC locations. Position $1(P 1)$ to $4(P 4)$ : (a) faulty current (b) zoom.

Figure 7.15 Four ITSC locations. Position $(P 1)$ to $(P 4)$ : (a) phase current and (b) zoom.

Figure 7.16 2D distribution of $B$ and flux lines for ITSC location $(P 1)$ at $\mathrm{t}=$ $0.117 \mathrm{sec}$

Figure 7.17 Experimental measures for the ferromagnetic core using Epstein frame (a) Hysteresis loops (b) Magnetization curve.

Figure 7.18 FEA simulation (a) Circuit diagram (b) Geometry and dimensions

Figure 7.19 (a) Experimental setup for ITSC in one-coil (b) Epstein frame.

Figure 7.20 ITSC cases of study in one coil, equivalent to the center taps from transformer.

Figure 7.21 Faulty current versus severity factor in the ITSC for one-coil case.

Figure 7.22 Simulation of the ITSC case of $\mu=0.33$ in one-coil. (a) current in the healthy winding, in the faulty winding and the faulty current in the shortcircuit. (b) Supply and faulty voltage.

Figure 7.23 Experimental measures of the ITSC case of $\mu=0.33$ in one-coil. (a) current in the healthy winding, in the faulty winding and the faulty current in the short-circuit. (b) Supply and faulty voltage.

Figure 7.24 Experimental setup for acquisition with a dSpace 1104. 196 
Figure 7.25 Real component of stator current for faulty IM (a) is $\alpha$ (b) Zoomed is $\alpha$.

Figure 7.26 Imaginary component stator current faulty IM (a) is $\beta$ (b) Zoomed is $\beta$.

Figure 7.27 Faulty leakage current (a) if and (b) Zoomed if ..................................198

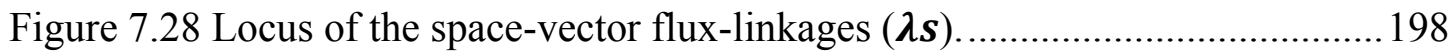

Figure 7.29 Rotor angular speed faulty IM (a) $\omega r$ (b) Zoomed $\omega r . . . \ldots \ldots \ldots \ldots \ldots \ldots \ldots \ldots . . . . . .199$

Figure 7.30 Electromagnetic torque in the faulty IM (a) Te (b) First zoom of Te

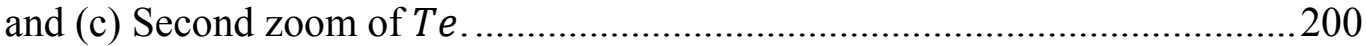

Figure 7.31 (a) Real component of the stator current for faulty IM (b) Imaginary component of the stator current faulty IM

Figure 7.32 Transient results faulty leakage current (a) if and (b) Zoomed if (c) Locus of the space-vector flux-linkages $(\lambda \boldsymbol{s})$.

Figure 7.33 (a) Rotor angular speed faulty IM ( $\omega r)$ (b) Electromagnetic torque faulty IM.

Figure 7.34 Simulation and experimental results for magnitude stator current space vector components (Is $\alpha, I s \beta)$ and If ault as a function of $\mu$. Using constant $\operatorname{Rcon}=3 \Omega$.

Figure 7.35 Simulation and experimental results for the magnitude stator current space vector components (Is $\alpha, I s \beta)$ and Ifault as a function of $R f$. Using constant $\operatorname{Rcon}=3 \Omega$

Figure 7.36 Real and imaginary component stator current space vector (Is $\alpha$, Is $\beta)$ and If ault as a function of $\mu$. Simulation and experimental results.

Figure 7.37 (a) Torque ripple vs $\mu$. Simulation and experimental results. Empirical impedance vs $\mu$ (b) Leakage faulty EC model considering the practical and empirical resistance Remp.

Figure 7.38 Real and imaginary component stator current space vector and Ifault as a function of the $R f$. Simulation and experimental results. 208

Figure 7.39 Electromagnetic torque ripple vs faulty resistance $R f$. Simulation and 
experimental results. Empirical impedance vs faulty resistance. 208

Figure 7.40 Real and imaginary component Stator current space vector and If ault as a function of $T L$. Simulation and experimental results.

Figure 7.41 Fig. 1............Electromagnetic torque ripple vs torque load. Simulation and experimental results. Empirical impedance vs torque load.

Figure 7.42 Experimental and two simulation results (proposed and linear leakage inductance) comparison using $\operatorname{Remp}=3 \Omega$. If ault as a function of $\mu$.

Figure 7.43 Experimental and two simulation results (proposed and linear leakage inductance) comparison using $\operatorname{Remp}=3 \Omega$. If ault as a function of $R f$........2212

Figure 7.44 Torque ripple comparison among experimental, simulation and FEA...213

Figure 8.1 Typical structure of a DTC with speed control of an induction motor with ITSC on phase " $a$ ".

Figure 8.2 Estimated and actual Stator Flux $\lambda \boldsymbol{s}$ at instant $t k$

Figure 8.3 Stator flux-linkage locus and VSI switching vectors. (a) Desired path

(b) real and estimated path.

Figure 8.4 (a) Real case of IVP in case of standard DTC with healthy IM and (b) real case of IVP case of standard DTC with faulty IM.

Figure 8.5 Experimental setup for acquisition with a dSpace 1104.

Figure 8.6 (a) Estimated and actual stator flux amplitude for the two cases of study (c2 and c4). (b) Error of flux estimation of both cases. Simulation Results.

Figure 8.7 (a) Estimated and actual electromagnetic torque the two cases of study (c2 and c4). (b) Error of torque estimation for both cases. Simulation Results. 235

Figure 8.8 (a) Fault Currents and (b) Mechanical speed for the two cases of study (case 1 and 2). Simulation Results. 236

Figure 8.9 Stator three-phase currents for the two cases of study. (a) case 1 and (b) case 2. Simulation Results 236

Figure 8.10 Estimated flux-linkage for the two cases of study (c2 and c7).

Experiemntal results. 
Figure 8.11 Estimated torque for the two cases of study (c2 and c7). Experiemntal results.

Figure 8.12 Stator three-phase currents for the two cases of study (case 2 and 7). Experimental Results. 238

Figure 8.13 Rotor mechanical speed for the two cases of study (case 2 and 7). Experimental Results.

Figure 8.14 Fault Current for the two cases of study (case 2 and 7). Experimental Results.

Figure 8.15 Fault Current and three-phase currents vs fault severity factor.

Figure 8.16 Mechanical speed and Estimated Torque ripple vs fault severity factor.

Figure 9.1 Typical structure of a DTC with speed control of an induction motor with ITSC on phase " $a$ ".

Figure 9.2 Estimated and actual Stator Flux $\lambda \boldsymbol{s}(t k)$

Figure 9.3 Sequence-components EC of Asymmetric IM (a) Positive Sequence (b) Negative Sequence (c) Faulty Circuit.

Figure 9.4 Fault Detection Technique (a) Learning Stage (b) Monitoring Stage......254

Figure 9.5 Implementation of the Fault Detection Technique for an inverter-fed IM under DTC. 256

Figure 9.6 Fault Detection Technique (a) Learning Stage (b) Monitoring Stage......260

Figure 9.7 Coded Simulation Test-bench programming levels, organization and filing.

Figure 9.8 (a) Simulink block of the damaged IM (b) Norton EC based Simulation Test-bench.

Figure 9.9 Estimated and actual Stator flux amplitude for the two cases of study (case 3 and 9). Simulation Results.

Figure 9.10 Estimated and actual Electromagnetic Torque the two cases of study (case 3 and 9). Simulation Results. 
Figure 9.11 Actual Electromagnetic Torque for the 8 machine operative points of the case 3. Simulation Results.

Figure 9.12 Actual Electromagnetic Torque for the 8 machine operative points of the case 9. Simulation Results.

Figure 9.13 Flux-linkages for the 8 machine operative points of the case 3.

Simulation Results.

Figure 9.14 Flux-linkages for the 8 machine operative points of the case 9 .

Simulation Results.

Figure 9.15 Locus of the Off-diagonal Term of the Sequence Component Impedance Matrix for the 64 cases of study. Simulation Results.

Figure 9.16 Simulation results of the proposed FD technique for the 64 cases of study. Fault detector versus number of experiments.

Figure 9.17 Estimated Electromagnetic Torque for the 8 cases of ITSC fault and the healthy IM at Tref $=2 \mathrm{Nm}$. Experimental results.

Figure 9.18 Locus of the Off-diagonal Term of the Sequence Component Impedance Matrix for the 64 cases of study. Experimental Results.

Figure 9.19 Experimental results of the proposed FD technique for the 8 fault testcases of study and the healthy IM at Tref $=2 \mathrm{Nm}$. Fault detector versus number of experiments.

Figure 10.1 Schematic layer structure (a) Base (b) Field Plate. 282

Figure 10.2 (a) True dimentions of the base case HEMT (b) Drain current versus Drain-source voltage characteristic of the base case HEMT.

Figure 10.3 Two dimentional electric field forth the base case HEMT. 284

Figure 10.4 (a) True dimentions of the case HEMT with SiN in the passivation (b) Drain current versus Drain-source voltage characteristic of the case HEMT with $\mathrm{SiN}$ in the passivation.

Figure 10.5 Two dimentional electric field forth the case HEMT with $\mathrm{SiN}$ in the passivation 286

Figure 10.6 Drain current versus Drain-source voltage characteristic of the case HEMT with SiN in the passivation. 
Figure 10.7 Two dimentional electric field forth the case HEMT with $\mathrm{SiN}$ in the

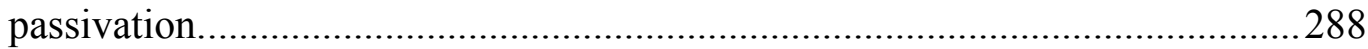

Figure 10.8 (a) True dimentions of the case HEMT with Germaium as substrate (b) Drain current versus Drain-source voltage characteristic of the case HEMT with Germaium as substrate.

Figure 10.9 Two dimentional electric field forth the case HEMT with Germaium as substrate. 290

Figure 10.10 (a) True dimentions of the case HEMT with etched substrate (b) Drain current versus Drain-source voltage characteristic of the case HEMT with etched substrate

Figure 10.11 Two dimentional electric field forth the case HEMT with etched substrate.

Figure 10.12 (a) True dimentions of the case HEMT with Field plate (b) Drain current versus Drain-source voltage characteristic of the case HEMT with Field plate.

Figure 10.13 Two dimentional electric field forth the case HEMT with Field plate.294

Figure 10.14 (a) BV curves for all cases of study (b) Drain, source and gate currents for the FP case.

Figure 10.15 E field distribution along $\mathrm{x}$ axis at (a) y_2 $=0.721 \mu \mathrm{m}$ (b) y_4 $=1.02$ $\mu \mathrm{m}$ 297

Figure 10.16 E field distribution along the $\mathrm{x}$ axis at $4 \mathrm{y}$ distances for the FP case. ..297

Figure 10.17 2D electric field distribution for the case of the FP 298

Figure 10.18 Schematic layer structure (a) Gate FP (G-FP) (b) Varying the G-FP. .300

Figure 10.19 Characteristic Curves for all Cases 301

Figure 10.20 Breakdown Voltage versus the Gate-FP Width. 301

Figure 10.21 $E f$ distribution along the $\mathrm{x}$ axis at $2 \mathrm{y}$ distances for the FP case.

Simulation performed at $V d s=853 \mathrm{~V}$. 303

Figure 10.22 2D $E f$ Distribution at $V d s=853 V$ (a) schematic (b) shortest $L F P=0.6 \mu \mathrm{m}(\mathrm{c})$ optimal $L F P=3.1 \mu \mathrm{m}(\mathrm{d})$ longest $L F P=5.1 \mu \mathrm{m}$. 304 
Figure 10.23 Cross-section of the HEMT with a Field Plate (FP-HEMT).

Figure 10.24 Illustration of the modelling (a) actual complex picture of the charges in the AlGaN layer (b) Simplified model of (a).

Figure 10.25 Schematic layer structure (a) Base (b) S-FP1 (c) S-FP2 and (d) GFP3.

Figure 10.26 BV against the Si substrate thickness for the etching reduction.

Figure 10.27 (a) True dimentions of the base case HEMT (b) Drain current versus Drain-source voltage characteristic of the base case HEMT.

Figure 10.28 Two dimentional electric field forth the base case HEMT.

Figure 10.29 (a) True dimentions of the HEMT with optimum substrate (b) Drain current versus Drain-source voltage characteristic of the HEMT optimum substrate.

Figure 10.30 Two dimentional electric field forth the HEMT optimum substrate....317

Figure 10.31 (a) True dimentions of the HEMT with Source FP1 (b) Drain current versus Drain-source voltage characteristic of the HEMT Source FP1.

Figure 10.32 Two dimentional electric field forth the HEMT Source FP1.

Figure 10.33 (a) True dimentions of the HEMT with Source FP1 and SiN insulator (b) Drain current versus Drain-source voltage characteristic of the HEMT Source FP1 and SiN insulator.

Figure 10.34 Two dimentional electric field forth the HEMT Source FP1 and SiN insulator.

Figure 10.35 (a) True dimentions of the HEMT with Source FP2 and SiN insulator (b) Drain current versus Drain-source voltage characteristic of the HEMT Source FP2 and SiN insulator.

Figure 10.36 Two dimentional electric field forth the HEMT Source FP2 and SiN insulator.

Figure 10.37 (a) True dimentions of the HEMT with Source FP3 and SiN insulator (b) Drain current versus Drain-source voltage characteristic of the HEMT Source FP3 and SiN insulator. 
Figure 10.38 Two dimentional electric field forth the HEMT Source FP3 and SiN

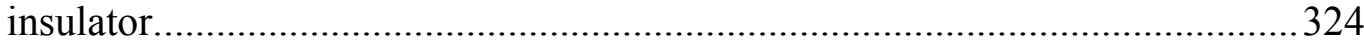

Figure 10.39 (a) BV curves for all cases (b) Drain, source and gate currents for the best Case of Si substrate thickness with FP1 and SiN

Figure 10.40 Locations of Electric Field Cross Sections............................................326

Figure 10.41 Electric field distribution along $\mathrm{x}$ axis for all cases (a) $\mathrm{y} 1=$ $0.535 \mu \mathrm{m}, \mathrm{b}$ y $2=0.721 \mu \mathrm{m} \quad$ (c) $\mathrm{y} 3=0.870 \mu \mathrm{m}$, (d) $\mathrm{y} 4=1.040 \mu \mathrm{m} . \ldots \ldots \ldots . .328$

Figure $10.42 \mathrm{E}$ field distribution along the $\mathrm{x}$ axis at $4 \mathrm{y}$ distances for the FP case. ...329

Figure 10.43 2D Solution for the FP1 with SiN Case. (a) Solid Material Model (b) 2D Electric Field Distribution Full View, (c) 2D Electric Field Zoom 330 


\section{LIST OF ACRONYMS}

\begin{tabular}{|l|l|}
\hline \multicolumn{1}{|c|}{ Acronym } & \\
\hline ADC & Analog to Digital Converter \\
\hline AC & Alternating Current \\
\hline ASD & Adjustable Speed Drive \\
\hline ARR & Active Regenerative Rectifier \\
\hline AlGaN & Aluminum Gallium Nitride \\
\hline ARF & Arbitrary Reference Frame \\
\hline BJT & Bipolar Junction Transistor \\
\hline BV & Breakdown Voltage \\
\hline CVP & Complex Vector Plane \\
\hline CV & Complex Vector \\
\hline CM & Common Mode \\
\hline CSI & Current Source Inverter \\
\hline CM & Condition Monitoring \\
\hline DC & Direct Current \\
\hline DM & Differential Mode \\
\hline DOL & Direct on-line \\
\hline DTC & Direct Torque Control \\
\hline DCC & Direct Current Control \\
\hline DPC & Direct Power Control \\
\hline DSC & Direct-Self Control \\
\hline DPLL & Electral Phase lock loop \\
\hline EMI & \\
\hline EV & \\
\hline
\end{tabular}




\begin{tabular}{|l|l|}
\hline EPRI & Electric Power Research Institute \\
\hline EM & Electric Machine \\
\hline EC & Equivalent Circuit \\
\hline EMF & Electromotive Force \\
\hline FEA & Finite Element Analysis \\
\hline FEM & Finite Element Model \\
\hline FOC & Field Oriented Control \\
\hline FLC & Fuzzy Logic Control \\
\hline FExA & Feature Extraction Approach \\
\hline FIS & Fuzzy Inference System \\
\hline FOM & Figure of Merit \\
\hline FP & Field Plate \\
\hline FP-DTC & Fuzzy Predictive- Direct Torque Control \\
\hline FD & Fault Detection \\
\hline FDI & Fault Detection and Identification \\
\hline FSMPC & Finite Set- Model Predictive Control \\
\hline FS & Fourier Series \\
\hline GA & Genetic Algorithms \\
\hline GaN & Gallium Nitride \\
\hline HEMT & High Electron Mobility Transistor \\
\hline HFET & Heterostructure Field-Effect Transistors \\
\hline HEV & Hybrid Electric Vehicle \\
\hline HB & Hysteresis Band \\
\hline HF & Induction Machine \\
\hline IM & Frequency \\
\hline
\end{tabular}




\begin{tabular}{|l|l|}
\hline IGBT & Insulated Gate Bipolar Transistor \\
\hline ITSC & Inter-turn Short-circuit \\
\hline KBA & Knowledge-based approach \\
\hline LF & Low Frequency \\
\hline LUT & Look-up Table \\
\hline MFOC & Magnetizing Field Oriented Control \\
\hline MRF & Multiple Reference Frame \\
\hline MOSFET & Metal Oxide Silicon Field Effect Transistor \\
\hline MAE & Mean Absolute Error \\
\hline MRA & Model Reference based Approach \\
\hline MMF & Magneto Motive Force \\
\hline RMSE & Root Mean Square Error \\
\hline MPPT & Maximum Power Point Tracking \\
\hline MF & Membership Function \\
\hline MPC & Model Predictive Control \\
\hline OVC & Optimum Voltage Vector Computation \\
\hline OCT & Open Circuit Test \\
\hline ODE & Ordinary Differential Equation \\
\hline P-DTC & Predictive- Direct Torque Control \\
\hline PI & Proportional Integral \\
\hline PCB & Printed Circuit Board \\
\hline PID & Proportional Integral Derivative \\
\hline PV & Photovoltaic \\
\hline PM & Permanent magnet \\
\hline PLL & PBM \\
\hline
\end{tabular}




\begin{tabular}{|l|l|}
\hline PFC & Power Factor Correction \\
\hline PWM & Pulse Width Modulation \\
\hline RFOC & Rotor-Field Oriented Control \\
\hline RK & Runge-Kutta \\
\hline SCIM & Squirrel-Cage Induction Machine \\
\hline SVM & Space Vector Modulation \\
\hline SV & Space Vector \\
\hline SC & Sequence Components \\
\hline SS & State Space \\
\hline SPWM & Sinusoidal Pulse Width Modulation \\
\hline SRF & Synchronous Reference Frame \\
\hline SiN & Silicon Nitride \\
\hline SiO2 & Silicon Dioxide \\
\hline SiC & Silicon Carbide \\
\hline SCT & Short Circuit Tests \\
\hline SHFETs & Single Heterojunction Field-Effect Transistors \\
\hline T-S & Takagi-Sugeno \\
\hline THD & Total Harmonic Distortion \\
\hline UPF & Unity Power Factor \\
\hline VAR & Voltage-Ampere Reactive \\
\hline VSI & Voltage Source Inverter \\
\hline VOC & Voltage Oriented Control \\
\hline VCT & Vector Control Techniques \\
\hline VF & Vonstant Volts-per-Hertz or volt-frequency control \\
\hline VGaN & \\
\hline VSC & Vertical GaN \\
\hline
\end{tabular}




\begin{tabular}{|l|l|}
\hline WRIG & Wound Rotor Induction Generator \\
\hline 3D-FE & Three dimension Finite Element \\
\hline 2DEG & Two-Dimensional Electron Gas \\
\hline
\end{tabular}




\section{Chapter 1 Introduction}

\subsection{Background}

Controlled ac motor drives are intelligent devices covering a wide range of diverse electro-mechanical apparatus and a wide scope of electric and electronic engineering skills. Today's ac motor drive power chain consists of five closely acting main parts: a dc source of energy, power electronic converter, an ac machine, motor control and control hardware, i.e. signal electronics. There is a vast variety of power electronic converters usable in controlled ac motor drives, however, they are comprehended in basically two or three main stages from mains to the load (electric power chain in industrial applications): ac-dc converter (rectifier), dc-dc converter (to boost or buck the voltage) and dc-ac converter (inverter). The number and type of stages in the power chain are highly dependable on the application. In the subsequent section, a brief background of each part is discussed.

The efficiency of electric power chains is of great importance for the electrification of the transport and industrial community. Different power rectifier topologies have various advantages and disadvantages, but, the active front-end rectifiers or active regenerative rectifiers (ARR) presents the best capabilities and performance. It have been widely used in multiple applications [1]. They have been recently employed in micro-grids and plugged-in electric vehicles where bidirectional power flow may be necessary [2]-[4]. They have the ability to control the dc-link voltage and to correct the power factor while reducing the current harmonic content. These advantages can be achieved during the two ARRs' modes: rectification and regeneration. Distortion factor and total harmonic distortion (THD) are directly proportional to each other, thus ARR can accomplish keeping 
the grid line currents in phase with the supply grid voltages whilst reducing their THD lower than 5\% (IEEE Std. 519-2014).

Nearly all applications are powered by some form of dc-dc converters for either battery charging, electronics power supply regulation or stepping up/down the required voltage needs. The most prevalent avenue for delivery of higher power levels to the retail market is in ac motor drives. It is the simplicity and cost-effectiveness of ac motors that make them commercially viable for higher power applications in the consumer market when compared to the dc motors. A typical ac motor drive requires an inverter which is supplied form a dc source. The dc source is not always in the same range of voltages as the ac machine drive. Thus, a dc-dc power electronic converter are needed. The use of power electronics has many advantages as we know, but they also suffer from some disadvantages as they inject considerable electromagnetic interference (EMI) [5] and harmonic content to both sides of its connections, its load side and its power source side. These harmonics may cause problems such as excess heating and more acoustic noise in the powertrain just to mention some problems. Therefore, attention is paid to procedures and methods for improving converter's efficiency and its EMI noise reduction: selecting the optimal topology, optimizing its design [6], using modern powerful switches in the power stage, improving the control system and enhancing the modulation techniques [7].

The development of the semiconductors and microelectronics has made the rapid development of the ac motor drives possible and with it, its condition monitoring as well. Power semiconductor switching devices play an important role in power electronics technologies because they are in charge of the electrical energy conversion systems. Since the birth of the first power transistor in 1947, power electronics technologies have 
developed in the world of silicon ( $\mathrm{Si}$ ) material [8]. Up until today, with more than 60 years of development, the Si power semiconductor devices fabrication process is very mature and can meet different power demands in electrical energy conversion. However, compared to $\mathrm{Si}$, wide band-gap (WBG) semiconductor materials have lots of intrinsic advantages which are very attractive for future power electronics applications. From the beginning of the 21st century, silicon carbide ( $\mathrm{SiC}$ ) [9] and gallium nitride ( $\mathrm{GaN}$ ) [10] power device fabrication has developed very fast. For the following decades, they have been and are good competitors to $\mathrm{Si}$. It has being estimated that by employing wide band gap (WBG) switches instead of conventional Si IGBTs, the efficiency of motor drives can be increased by 20 to $30 \%$ [11]. Highly efficient WBG devices make these power electronic converters highly adaptable for renovable energy originations. Although power electronic drives are widespread and are used in a variety of applications, they are prone to several failures. As [12] noted, the two components that are most likely to fail in switch-mode drives are electrolytic filtering capacitors and power transistors. Among the latter, the most typical power semiconductor failure are short-circuit and open-circuit faults. Although most of the work in the power converters fault detection (FD) addresses the open-circuit.

Semiconductors utilized in switching converters provide electronics processing capability of power, where intelligent devices such as microcontrollers and digital signal processors (DSP) provide power for the complex control algorithm [13]. DSP technology is enabling cost effective and energy efficient control system designs. The performance of a DSP architecture allows an intelligent approach to reduce the complete system costs of digital motion control applications using cheaper electrical motors, fewer sensors, and smaller sizes of EMI filters. It enables the system versatilly by the introduction of software 
and firmware algorithms that can make the system adaptable for many conditions including failures.

The final stage in the power chain and the most important of all, is the ac machine. The most famous ac machine is the three-phase squirrel-cage induction motor which is widely used in a variety of applications as a means to convert electric to mechanical power [14][20]. It is without a doubt the "workhorse" in industry applications due to its many advantages including high efficiency over a wide range of speeds, low cost, low maintenance, it is robust and sturdy (It can operate in a wide range of industrial conditions), its construction is simple and rugged (no brushes, slip rings or commutators), it does not require any complex circuit for starting. In addition, it is self-starting, it can be operated in hazardous environments and even under water, as it does not produce sparks, unlike dc motors and all this results in the motor running for many years with no cost and maintenance (durability).

\subsection{Problem Statement}

\subsubsection{Condition Monitoring in Electric Machines}

The most recognized electromechanical energy-conversion device in our modern civilization is the alternating current electric machinery. It has played essential roles in electric power generation, transportation, industrial processes, and residential applications. In many applications, they are subjected to environmental stresses, such as high ambient temperature, high moisture or dampness, corrosive environments, dusty medium, oil surrounding and water intrusion. These environmental stresses, combined with machines' internal stresses (electrical, mechanical, magnetic and thermal), could seed faults in electric machinery. These faults can evolve into disastrous machine failures if they are left 
undetected. This results in not only costly repairs, such as reconstructions or part replacement, but also significant financial losses because of unscheduled downtime in the production of industrial plants (idle time or off-line period) or safety hazards in transportation applications (electric vehicles).

The history of condition monitoring (CM), fault detection (FD), fault identification and diagnostics of electric machines is almost as old as electric machineries themselves. Originally, the electric machines were protected only against a misuse such as over current or over voltage [21]. The condition monitoring was periodic, with long intervals of time between inspections (months or semesters), and off-line (requiring machine disconnection and possibly dismantling). The maintenance and health monitoring of the machinery was executed only when it presents obvious malfunction problems. With the advent of semiconductor technology, many areas have been developed including sensors or transducers, digital-to-analog conversion, analog-to-digital conversion, data acquisition electronics, microprocessors, digital signal processors, field-programmable gate arrays, computation capability, etc. The advancements of semiconductor technology and signal processing techniques bring the possibility to study fault detection and identification of machinery in a more effective manner: the health monitoring can be performed while the electric machine is still in-service (on-line) and the time interval between inspections can be reduced to a point where it can be defined as continuous. The CM practitioners have made an effort to prove that the on-line and continuous diagnostics with real-time updates of the machine condition increases business profit through informed maintenance and service decisions. These capabilities enable the FD at their very inception, well before material machine failure evolution occurs [22]-[24]. The detection of incipient faults of 
electric machinery allows time for preventive maintenance to be scheduled, which improves the system reliability, availability, and maintainability.

To monitor the health of electric machines and thus detect and isolate a specific incipient faults, different types of sensors are added to them. These include search coils for stator or rotor faults [25], infrared sensors for corrupted electrical connection [26], thermal transducers, accelerometers for vibrations caused by bearing faults [27], magnetic velocity pickup [28], piezoelectric acoustic sensors, laser detectors, micro electro-mechanical sensors [28], etc. However, sensors and its associated installations and wirings are expensive, intrusive and difficult to implement for many applications. Therefore, only large high power and costly electric machines are equipped with some of these sensor-based CM schemes.

Recently, the research on the so-called sensorless CM of electric machines has gained a lot of interest. Instead of installing expensive special-purpose sensors for FD, existing hardware and transducers can provide health information of the machine condition that has traditionally not been exploited. For instance, in industrial plants, existing motor voltage and current measurements can be used for condition monitoring purposes. In motor drives, the existing current sensors and dc bus measurement can provide useful information about the wellbeing of the electric machines. Moreover, industrial plants and motor drives resources count with intelligent control devices (microprocessors or digital signal processors) that can offer processing capabilities for the fault detection computational burden. The actual advances on computational capabilities (high processor speed and large size of RAM) make these intelligent devices suitable and able of handling both control and diagnostic. 
Due to historic reasons, the majority of the FD methodologies are available for machines connected to directly to the mains or the so-called directly-on-line (DOL) electric machines. The emergent emphasis on energy efficiency and the inexpensive availability of power semiconductor devices cause more electric machines being now interconnected with various types of power electronic converters, some of which even have sophisticated control strategies. Whereas the malleable energy forms produced by these power electronic converters significantly enhance the performance of electric machines in an expanded operating region, they also have a substantial impact on fault behaviors of electric machines and introduce a greater complexity in terms of fault detection, fault identification, monitoring and protection. Typically, conventional FD methods already investigated for DOL electric machines cannot be applied to power-converter-fed machines anymore. This requires detailed analyses of the power converter effects on various faults of electric machines so that simple and reliable FD schemes can be created.

The plethora of diverse power electronic converters and electric machines available makes it impractical to study every possible combination. Additionally, the large number of fault types in an electric machinery make this situation even worse. One of the main goals of this $\mathrm{PhD}$ research focused on the condition monitoring of power-converter-fed three-phase squirrel-cage induction machine driven through a closed-loop controller.

Induction motors can be connected directly to the grid (grid-connected or DOL), or connected to power converters such as open-loop inverter drives or closed-loop inverter drives. This study mainly focuses on the condition monitoring of closed-loop inverter-fed induction machines. Closed-loop inverters are power electronic converters with current, torque, flux and/or speed feedback control capabilities. Closed-loop induction motor drives 
find many applications in traction system with high-performance motion control such as cranes, hoists, elevators, winding process, material handling, electric vehicles (EV), hybrid $\mathrm{EV}$, recent railway system, diesel-electric ships, and rolling steel mills for mention some of them. In this applications zero-speed torque capability and highly dynamic torque response are normally required. Compared to the DOL or open-loop inverter-fed electric machines, the machines fed by closed-loop inverters are generally connected to the most critical types of loads. Equipped with sensors (current, voltage and/or speed) and digital processors, the closed-loop inverter drives are also ideal platforms for implementing the sensorless health monitoring and FD schemes. In actual fact, however, closed-loop controlled machines are the least studied category in terms of FD, for two reasons: first because the closed-loop control dramatically changes the fault behaviors of the electric machines and complicates the fault analysis [29] and second due to historic reasons, closedloop controllers are newer than DOL and open-loop ones.

One of the weakest components in induction machines is the stator winding insulation which accounts for about 30 40\% of induction machine failures in industrial applications [21], [30]-[32]. Stator windings and their insulation system constitute the second potential source of failures since the various stresses that act on the motor cause gradual deterioration and aging of stator insulation. Short-circuit and open-circuit of stator windings, and magnetic core faults are the failure modes associated with the stator. Coil-to-coil, phaseto-phase and phase-to-ground short-circuit variants are consequences of insulation failure, often due to a combination of temperature rise, mechanical and electric stress. In closedloop induction motor drives, the large $d v / d t$ caused by the square voltage wave of the inverter will result in even higher electric stress on the induction machine, particularly on 
the first few turns in the terminal end of the stator winding [33]. Therefore, the condition monitoring of the stator insulation of inverter-fed induction machine is the primary focus of this work. The thesis will propose methods that not only detect solid stator insulation faults but understand the phenomena of the inter-turn short-circuit and its influence on the machine drive.

\subsubsection{Condition Monitoring and Performance Enhancement of the Power Electronics of the Drive Powertrain}

\subsubsection{Performance Enhancement of Power Electronics}

Since five decades, power electronics (PE) has witnessed a surprising growth due to the advancement made in the knowledge of devices, converter topologies and control. Static PE converters have gained popularity in energy conversion and their applications range from dc to ac and in many applications such as: variable speed-torque industrial drives, new architectures of autonomous electrical energy conversion systems, large electrical energy transport systems and low power mobile applications [8], for mention some of them.

Electric power represents about $40 \%$ of the total energy consumption. CPES and EPRI estimates that in few decades, PE devices will be supplying more than the $80 \%$ of consumers in the US. It is possible to save around $20 \%$ to $30 \%$ of electricity with proper use of a new generation of PE devices. Each percentage of improving converter efficiency has positive economic and environmental impacts. The biggest influence of $\mathrm{PE}$ are possible in the control of electric motors, lighting, electronic devices power supply, and renewable source generation. 
Some methods and procedures for PE converters efficiency improving are: optimal topology selection, design optimization, efficiency optimization by digital control and parallel connection of converters with the proper turn on/off strategy, appropriate frequency selection. As a consequence, the second focus of this dissertation is the improvement of the efficiency and performance of the all the stages in the energy conversion chain. The main PE converters to enhance would be the rectifier and the inverter design and control. Thus, for the rectifier this dissertation will propose a design and control strategies able to reduce the harmonic content in the grid currents as well as to reduce the reactive power consumption by achieving power factor correction (PFC). On the other hand, for the inverter that drives the IM, this dissertation will present control methodologies to advance the performance operation and reduce its drawbacks.

\subsubsection{Condition Monitoring of the Power Electronics}

The use of PE converters in the abovementioned applications has improved the quality and efficiency of the energy systems. However, we must ensure that such benefits are not gained at the expense of reliability, availability, and the safety of goods and people. Moreover, the equipment obtained through high investments must be preserved.

Table 1-1 and Table 1-2 indicate that more than $90 \%$ of failures in the energy conversion chain are due to the converter or the control circuits [8]. Apart from natural aging of the components, some of the extreme operational conditions, malfunctioning or harsh environments induce stresses on the system and its different components (electrical and thermal tensions on the semiconductors, and mechanical strains on moving parts). They lead to intermittent failures, which result in converter failure, and subsequently, the disruption of the power transfer. 
Table 1-1 Failure rate in the energy conversion chain

\begin{tabular}{|c|c|}
\hline Converter failure & $38 \%$ \\
\hline Control circuit failure & $53 \%$ \\
\hline Failure of external and auxiliary components & $9 \%$ \\
\hline
\end{tabular}

Among the converter components, the electrolytic capacitors and transistors are the elements that experience most of the failures.

Table 1-2 Failure rate of converter components

\begin{tabular}{|c|c|}
\hline Continuous bus capacitor & $60 \%$ \\
\hline Power transistors & $31 \%$ \\
\hline Diodes & $3 \%$ \\
\hline Inductive elements & $6 \%$ \\
\hline
\end{tabular}

The major difficulty in power electronics diagnosis is having a model that adequately represents the component or the converter, where there is sufficient availability of measurement-related information. Condition monitoring of the components, and therefore the converter, will depend on the obtainability of measurements, and the capacity to interpret them in order to analyze their behavior. The main obstacle lies in the limited number of sensors used, due to cost constraints and packaging problems.

Power transistor is the second cause of failure in power electronic converters and the converter failure is as well the second cause of failure in the energy conversion chain. The advent of wide bang gap power electronic devices, such as Silicon Carbide (SiC) or Gallium Nitride $(\mathrm{GaN})$ has demonstrated to be excellent devices for application in power 
electronics but also brings new challenges in the understanding of their failure mechanisms. Therefore, hurdles in the condition monitoring will arise and thus the final focus of this dissertation will be propose alternative design solutions that improves the robustness of the semiconductor devices as well as deeper understanding of the root cause of device failures.

\subsection{Research Objective}

Condition monitoring of energy conversion chain systems includes he health monitoring of the whole system including the ac electric machine and its power electronics stages. Condition monitoring of ac electric machines fed by power electronic converters, including is health monitoring, is of great importance to ensure the overall system reliability, availability and maintainability in a wide range of sensitive applications.

The objective of this $\mathrm{PhD}$ research is to develop simple, robust and non-intrusive condition monitoring method for the different stages of the energy conversion chain of electric drives, including power-converter-fed electric machines as a whole (closed-loop inverter-fed induction motors) and power converters.

The study proposes to undertake several important aspects of condition monitoring of this type of machine and its power electronic stages. A sequence of steps to achieve the final goal on health monitoring of inverter-fed IM is:

- Model the induction machine under the specific fault. The specific fault is stator turn-to-turn or inter-turn short-circuit. The model provides a mathematical tool for a machine under fault to be manipulated, understood, explained and investigated. It allows deep insights analyses of variables that are not accessible or cannot be measured. 
- Study the above-mentioned model of the damaged IM under the simplest connection: DOL. This investigation contributes to the behavior of the IM under the asymmetric condition. This objective will offer the possibilities of different diagnostics signatures available on the IM.

- Investigate the close-loop driving system for IM and the effect of the turn-toturn fault on the controller. Study the reactions of the controller to the fault.

- Propose and implement a simple, robust and non-intrusive condition monitoring method for the incipient detection and identification of stator inter-turn shortcircuits in IM driven by close-loop controllers.

The specifics goals for the condition monitoring and performance improvement of the power electronics stages are as follows:

- Propose and develop a design strategy of the active regenerative rectifier as a previous stage of dc power supply for the IM drive.

- Propose a control method for the active regenerative rectifier as a previous stage of de power supply for the IM drive.

- Present, develop and implement an enhanced control algorithm that solves the drawbacks of DTC as IM drive.

- Study and propose improvements to enhance the reliability and extension of the capabilities of wide band gap devices used in power electronic converter stages.

\subsection{Original Contributions of the Dissertation}

\subsubsection{Condition Monitoring in Electric Machines}

In this part of the dissertation the first main contribution was the developing of a unique and novel model of the three-phase squirrel-cage induction machine with stator inter-turn 
short circuit. For the development of this model, a previous study for the ITSC modelling in magnetically coupled circuits and its general parameter estimation was performed. The outcome of this study was a general theory of the ITSC that can be implemented and extended to any electric machine or any electro-mechanical device. This theory was developed for the simplest case possible: one coil with a unique winding. A more rigorous and meaningful approach to the faulty stator leakage inductances to construct a dynamic model for the three-phase squirrel-cage IM with ITSC was proposed, simulated, tested, compared and verified. This leakage inductance model has more accurate behavior with respect to the linear one, already pre-existing in the literature, keeping simplicity of the modelling.

The conception of this new proposed model of the anomalous IM was elicited by the antecedent study of a pre-existing faulty model of the IM [34] in which the behavior of leakage inductance is considered proportionally to the fault severity factor. This model was revised accurately, mathematically re-demonstrated and re-deduced for corroboration purposes. Subsequently, the model was completed and expressed in state-space (SS) representation for any formal further analysis and simulation. Additionally, it was simulated and compared with the healthy IM and experimental results. The benefits of this model in SS form lie in the characteristic that can be represented and separated in two modes: "Common Mode" (CM) and "Differential Mode" (DM). The common mode indicates the healthy behavior of the IM. The differential mode represents the faulty component of the ITSC. This decoupling is achieved because the model is based on the flux-linkages space-vector. 
As the main objective of this dissertation is to develop simple, robust and non-intrusive condition monitoring method for the inverter-fed IM driven by a closed-loop controller, a formal investigation on the different types of closed-loop drives was performed. With that in mind, the so-called direct torque control (DTC) was designated as the closed-loop drive, as this is the most common drive in industry applications, for the IM. However, DTC suffers from some particular drawbacks that are corrected with DTC modifications. Predictive DTC appears to be one of the most efficient strategies to overcome the DTC hurdles, but nevertheless other disadvantages emerged. In the light of this situation, an innovative and simple control strategy based on the integration of a Fuzzy Logic Controller (FLC) algorithm with minimum and linear membership function with a predictive methodology is developed. The advantages of both techniques are exploited to reduce the drawbacks and improve the performance of the direct torque controller. Simulations and experimental results show an improvement in the control performance of the torque, including ripple and steady state error reduction, and a satisfactory performance at the lowspeed region.

For simplicity purposes and as a start point, investigation of a three-phase squirrel-cage induction machine (IM) with inter-turn short-circuit (ITSC) fault driven by classical DTC was performed. The main output of this research was the understanding and analyzes of the impacts of the stator ITSC of an IM on the normal operation of DTC. The ITSC effects are twofold: DTC-observable and not observable. The former ones are visible in the DTC measured or estimated signals while the latter are hidden or invisible to the DTC operation. This categorization is exploited in the identification of the DTC reaction to the ITSC and in the computation of estimated errors. DTC reaction will be to compensate the observable 
effects of the ITSC until its limit, however its natural robustness stability will tolerate the invisible consequences of the ITSC.

With the learning of the effects and the reaction of the DTC controller to the stator ITSC fault plus a deep literature revision of fault detection techniques available in the literature it was simple to figure out which of the methodologies used in DOL IM were eligible for the inverter-fed IM driven by DTC. Ergo, the FD procedure presented in [35] was carefully chosen and adapted for its suitability to the DTC. The FD is based on the monitoring of the off-diagonal term of the Sequence Components (SCs) impedance matrix. Its advantages are that it is independent of the IM parameters, it is immune to the sensors' errors, it requires a small learning stage, compared with $\mathrm{NN}$, and it is not intrusive. The contribution lies in the adaptation of the technique to the DTC and in the developed solutions for the faced challenges in the signal processing implementation.

In summary the contributions achieved in this dissertation are:

- Developing of a new, general more meaningful model of the inter-turn short circuit on magnetically coupled devices.

- Implementing the general model of the inter-turn short circuit winding on the IM to develop a more advance and valid model of the IM under stator damage interturn short circuit.

- Deduction of the SS representation of the faulty model [34] and the discovery of its decoupling characteristics in the flux-linkage notation, which make it the simplest model in the literature and useful for FD in the incipient stage.

- Develop state-of-the-art driver that overcomes all the particular drawbacks of DTC for the healthy IM with the target of implementing the FD study on it. 
- Establish a deep understanding and analysis of the phenomena and influences of the stator ITSC of an IM on the DTC driver operation.

- Adapt and implement a FD technique based on the monitoring of the off-diagonal term of the sequence components impedance matrix

\subsubsection{Condition Monitoring and Performance Enhancement of the Power Electronics on the Energy Conversion Chain}

The contributions in this part of the dissertation are the developing a general and enhanced methodology for the design of the passive components of an active regenerative rectifier (ARR). For the L-filter, the design strategy developed is based on two techniques: the current area error in the time domain, considering the modulation strategy, and the highorder current harmonics in the frequency domain. The dc-link capacitor design procedure is based on the dc-link voltage ripple. Additionally, a non-linear deadbeat type predictive controller for the ARR was developed. This technique is based on the optimum voltage vector computation (OVC) of the voltage source converter (VSC) for direct current control (DCC), to provide fast dynamic, high-performance and parameter independency.

Regarding the condition monitoring on new semiconductor devices, the contributions are an extensive study to evaluate the breakdown mechanisms in gallium nitride $(\mathrm{GaN})$ high electron mobility transistors (HEMT). A comprehensive physics-based model of a common HEMT was developed providing the base comparison to conduct different material and geometric investigations. A theoretical analysis of the FP effects on the HEMT is carried out by modeling the electric fields inside the structure. The electric field model is deduced by solving the potential in the heterostructure, which should satisfy the Poisson equation. The developed comprehensive physics-based model of a common 
HEMT provided the base comparison to conduct two analyses: 1) a reduction of the silicon substrate and 2) the addition of different FPs. The electric field distribution across the source, gate, and drain is analyzed for each geometric case in order to determine the breakdown voltage origin as well as their I-V characteristic curves.

\subsection{Dissertation Organization}

This dissertation is organized as follows:

Chapter 2 will present the overview of the electrical machines with a special reference to three-phase squirrel-cage induction machines (SCIM), their benefits, drawbacks, classification, applications, starting techniques and failures. Based on their applications, the electric power drive-train is also studied from the point of view of power electronics theory. However, a prior selection of the power electronic topologies is performed. Thus, one of the purposes of this chapter is to illustrate, in an organized way, the topics covered in this book and their purposes. Here a perspective point of view from the general aspect to the concrete research developed during this dissertation will be given. Finally, we introduce all the necessary mathematical tools for the understanding of the analytical developments during this dissertation. Thus, a variable nomenclature convention is proposed and used throughout the book for any mathematical development. Additionally, a set of transformations, used in three-phase power systems to convert them from three components to two, is presented.

The following 3 chapters will provide a detailed overview of the applications and the different parts that constitute the powertrain for electric machine drives. Chapter 3 introduces the design and control of an active regenerative rectifier in the drivetrain. The applications are twofold: industry and transportation. This power electronic converter is 
typically a previous stage of the inverter drive for the electrical machine. It is utilized to convert the ac power into dc power, thus, dc voltage can be supplied with no reactive power consumption (power unity factor) and low current harmonic content in the mains. The design of dc-dc converters in the drivetrain is investigated in Chapter 4 to reduce the radiated electromagnetic interference (EMI). This chapter investigates the effect of components' placement on the radiated emissions of the printed circuit board (PCB) of dcde power converters. The main goal of this chapter is to find the best position and orientation for the passive components on the PCB to minimize the EMI at a specific location on the board. The optimum design for the location and orientation of the components on the PCB is achieved by Genetic Algorithms (GA) optimization. In Chapter 5 , the induction machine drive is selected, investigated and improved to enhance the drive performance in both applications: industrial and electric vehicle. In this chapter a torque controller that integrates the advantages of Fuzzy Logic Controller (FLC) and predictive control is proposed. The FLC helps in the reduction of the torque ripple with significant decrement of the parameters' dependency and limitation of the inrush current. The predictive control technique aims in the improvement of the performance at both starting condition and low-speed operation. In this approach, the FLC rules are based on a predictive DTC (P-DTC), where the main idea is to observe the non-perpendicular quadrants formed by the two lines of constant torque and constant stator flux magnitude drawn in the complex vector plane (CVP) of the voltage source inverter (VSI) or voltage $\alpha \beta$ plane.

Chapter 6 and Chapter 7 present the modelling of the IM with stator inter-turn shortcircuit. In Chapter 6, the simplest state-space (SS) representation for the IM with stator 
winding fault is derived following the model in [36], and a study seeking faulty signatures is performed. The benefits of this model lie in the characteristic that can be represented and separated in two modes: "Common Mode" (CM) and "Differential Mode" (DM). The common mode indicates the healthy behavior of the IM. The differential mode represents the faulty component of the ITSC. This decoupling is achieved because the model is based on the flux-linkages space-vector. In the search for fault signatures, the complex vector (CV), operational and the steady state equivalent circuits (ECs) for the faulty IM are developed.

A general study for the ITSC modelling in magnetically coupled circuits and its general parameter estimation is presented in detail in Chapter 7. The proposed analysis is based on the fact that the faulty winding can be modelled as a step-down autotransformer circuit and thus, fulfills the SS representation of a faulty magnetically coupled circuit. Also, general equations for estimating the faulty leakage inductances are presented. A case of ITSC study is presented: three-phase squirrel-cage induction machine. A more rigorous and meaningful approach to the faulty stator leakage inductances to construct a dynamic model for the three-phase squirrel-cage IM with ITSC is proposed. The faulty model is developed in detail and expressed in complex-vector (CV) notation. The SS representation is developed based on the flux-linkage space-vector. The CV operational and steady-state symmetric component equivalent circuits (ECs) are deduced from the faulty model for FDI purposes.

The Chapter 8 presents an investigation of a three-phase squirrel-cage induction machine (IM) with inter-turn short-circuit (ITSC) fault driven by Direct Torque Control (DTC). DTC is the most typical driver for controlling IM's electromagnetic torque and 
stator flux-linkage amplitude in industrial applications. Also, DTC has been classified as a non-linear hysteresis-based predictive controller. Two dynamic models in its state-space (SS) representation are used for the analysis. The first one uses the flux-linkages and the second one uses currents as state-variable. The advent of these models is to provide fluxlinkages and currents vectors information to analytically calculate the angle difference between the trajectories of constant torque and constant stator flux amplitude, expressed as a function of the $(\alpha \beta)$ inverter voltage components. The main goal in this article is to investigate the impacts of the stator ITSC of an IM on the normal operation of DTC. The ITSC effects are twofold: DTC-observable and not observable. The former ones are visible in the DTC measured or estimated signals while the latter are hidden or invisible to the DTC operation. This categorization is exploited in the identification of the DTC reaction to the ITSC and in the computation of estimated errors. DTC reaction will be to compensate the observable effects of the ITSC until its limit, however its natural robustness stability will tolerate the invisible consequences of the ITSC. Analytical development of the estimated mismatch are performed based on the mathematical models while the DTC reactions are analyzed based on its standard heuristic derivation and non-linear control theory. An easy-use circuit simulation block unit for the IM under ITSC was developed based on Norton's circuit. Its simplicity allows its use in educative environment and its versatility permits researchers not to concern on the complex mathematics behind the faulty IM. Simulation and experimental results are conducted for the dissymmetric IM driven by DTC to observe the limits of the controller and to corroborate their theoretical visible and not-visible impacts which in future research could be utilized for ITSC fault detection.

In Chapter 9, the FD procedure presented in [35], which was implemented for the DOL 
IM, is modified to be suited for an inverted-fed IM driven by classical DTC. The FD is based on the monitoring of the off-diagonal term of the Sequence Components (SCs) impedance matrix. Its advantages are that it is independent of the IM parameters, it is immune to the sensors' errors, it requires a small learning stage, compared with $\mathrm{NN}$, and it is not intrusive. Some challenges in the signal processing implementation are found for the afore-stated technique, however, its results are promising. Simulation and experimental results demonstrate that the technique is able to detect an ITSC fault on the onset (low fault severity factor).

Chapter 10 studies the mechanisms to increase the voltage breakdown on wide band gap semiconductor devices, mainly $\mathrm{GaN}$ based devices. For that purpose a model of the electric field was mathematically formulated when the device includes a field plate solution. A physics based-model of the GaN device was developed for its study and thus, investigate the breakdown voltage mechanism inside this type of devices.

The conclusions, contributions, and the recommendation for future work are summarized in Chapter 11. This chapter provides general discussion of the results and outcomes of this dissertation as well as presenting some suggestion and recommendations for further research and activities related to its topic. 


\section{Chapter 2 Electrical Machine and Power Electronic Topology Selection: A General Description}

\subsection{Introduction}

This chapter gives an overview of the electrical machines with a special reference to three-phase squirrel-cage induction machines (SCIM), their benefits, drawbacks, classification, applications, starting techniques and failures. Based on their applications, the energy conversion chain or electric power drive-train is also studied from the point of view of power electronics theory. However, a prior selection of the power electronic topologies is performed. Thus, one of the purposes of this chapter is to illustrate, in an organized way, the topics covered in this dissertation and their purposes. Here, a perspective point of view from the general aspect (overall) to the concrete research (specific) will be given. In this chapter, it is also introduced all the necessary mathematical tools for the analytical developments during this Phd. book. Thus, a nomenclature convention for the variables is proposed and used in this book. Additionally, a set of transformations, used in three-phase power system to convert it from three components to two, is presented.

\subsection{Electric Machine Selection}

Among the complete vast range of rotating electrical machines, three-phase SCIM are widely used in a variety of applications as a means to convert electric to mechanical power. It is without a doubt the "workhorse" in industry application due to its many advantages, as shown in Table 2-1. 
Table 2-1 Induction Machine Advantages and Drawbacks.

\begin{tabular}{|c|c|}
\hline Advantages & Drawbacks \\
\hline 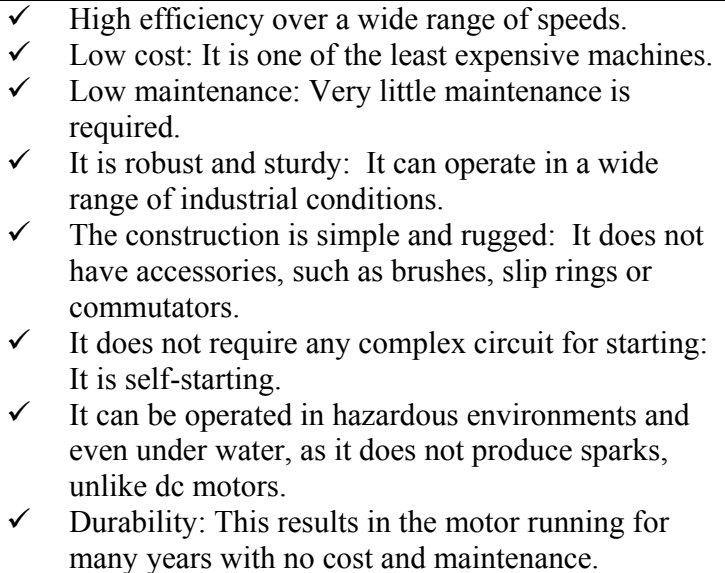 & 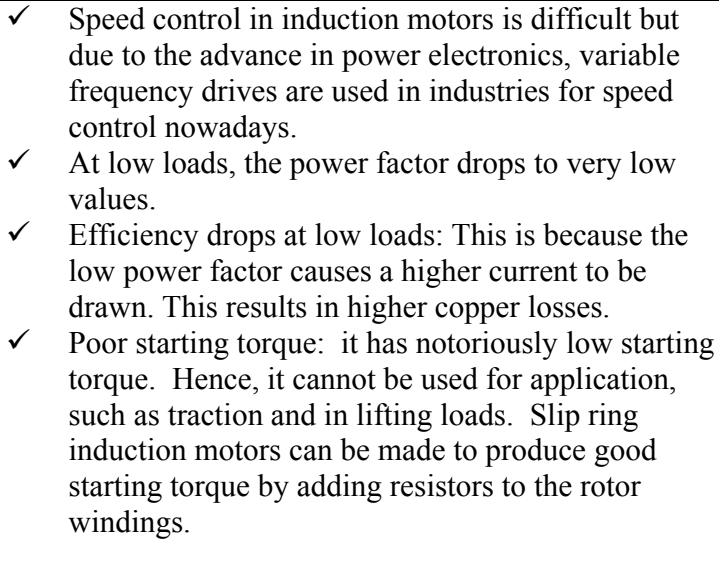 \\
\hline
\end{tabular}

Also, its drawbacks are in Table 2-1. Most of these drawbacks are fixed by closed-loop vector controllers, as it is studied in the next chapters. Close loop controllers increase the cost of the drive but keep a good cost-effective relationship for high-performance applications. In this sense, they are becoming more important every day due to the essential role that they are playing in our life development areas, such as: commercial, industrial, residential, transportation and military.

\subsubsection{Induction Machine Applications}

Many SCIM are available in the market to meet the demand of the several industrial applications and various starting and running condition requirements. They are classified according to the class and depending on it, they have different applications:

- Class A motors (normal starting torque, normal starting current, normal slip): The most popular class with applications such as fans, blowers, centrifugal pumps, compressors, conveyors, etc. 
- Class B motors (normal starting torque, low starting current, normal slip): The applications are basically the same as that of Class A motors: large fans, machine tools applications, machines for driving electric generators, centrifugal pumps, etc.

- Class C motors (high starting torque, low starting current, normal slip): Class C motors are generally the double squirrel cage type. They are used for crushers, compression pumps, reciprocating pumps, large refrigerators, textile machinery, wood working equipment, etc.

- Class D motors (high starting torque, low starting current, high slip): These motors are used where extremely high starting torque is required, e.g. bulldozers, shearing machines, foundry equipment, punch presses, stamping machines, metal drawing equipment, laundry equipment, die stamping machines, etc.

- Class E and F motors (low starting torque, normal starting current, low and normal slip): not many applications.

Another way to categorize the induction machine (IM) applications is depending of their required performance:

a) Low-performance applications: These are applications where the IM is connected directly to the mains (DOL), equally named grid-connected IM, or through an inverter (inverter-fed) driven by an open loop controller:

- fans

- pumps

- conveyors

- mixers

- centrifuges 
b) High-performance applications: These applications are inverted-fed IM managed by a close loop controller. In this case, the application requires high-performance motion control and traction system, such as:

- hoists

- elevators

- winding process

- material handling

- hybrid and electric vehicles

- the modern railway system

- diesel-electric ships

- rolling steel mills

The trend of the IM applications is moving towards the high-performance speed and torque response, where close loop controllers are essential for functioning. In this sense, this dissertation follows that trend and focuses the investigations in two specific applications:

- High-performance industrial applications.

- Transportation electrification applications, such as electric vehicles (EV), hybrid EV (HEV), etc.

\subsubsection{Induction Machine Connections-Drives Classification}

In all applications, the IM can be connected in two main ways, as aforementioned, which of course also defines the starter strategy (see Table 2-2). If the IM is attached to a transformer which is connected to the utility grid, then it is named DOL IM or "gridconnected IM." On the other hand, if the IM is started through a power electronic stage, 
then it is called "inverter-fed" [37]. Take into consideration that a modified IM can act as a generator. In this situation, the IM will be connected through a rectifier. This case it is not analyzed in this dissertation.

Table 2-2 Induction Machine connection-drives classification.

\begin{tabular}{|c|c|c|c|c|}
\hline \multicolumn{5}{|c|}{ IM Connection-Drives } \\
\hline \multicolumn{4}{|c|}{ Inverter-fed } & DOL \\
\hline \multicolumn{3}{|c|}{ Controlled Starter } & $\begin{array}{c}\text { Uncontrolled } \\
\text { Starter }\end{array}$ & $\begin{array}{c}\text { Uncontrolled } \\
\text { Starter }\end{array}$ \\
\hline \multicolumn{2}{|l|}{ Close Loop Control } & $\begin{array}{c}\text { Open Loop } \\
\text { Control }\end{array}$ & \multirow[t]{2}{*}{ No control } & \multirow[t]{2}{*}{ No control } \\
\hline Vector Control & Scalar Control & Scalar Control & & \\
\hline $\begin{array}{l}\checkmark \text { Control of IM with impressed } \\
\text { currents } \\
\checkmark \text { Stator Field Oriented Control (FOC) } \\
\checkmark \text { Rotor-Field Oriented Control (RFOC) } \\
\checkmark \text { Magnetizing Field Oriented Control } \\
\text { (MFOC) } \\
\checkmark \text { Indirect Rotor Field-Oriented Control } \\
\text { (IRFOC) } \\
\checkmark \text { Direct Torque Control (DTC) } \\
\checkmark \text { Direct-Self Control (DSC) } \\
\checkmark \text { Artificial-intelligence (AI) based } \\
\checkmark \text { vector controllers } \\
\checkmark \text { Predictive Direct Torque Control (P- } \\
\text { DTC) } \\
\checkmark \text { Any variation and combination of AI } \\
\text { and vector controllers } \\
\text { a) Fuzzy-DTC } \\
\text { b) Fuzzy-Predictive DTC (FP- } \\
\quad \text { DTC) }\end{array}$ & \begin{tabular}{|l}
$\checkmark$ Constant-Slip \\
current control \\
$\checkmark$ Scalar torque \\
control \\
$\checkmark$ Close loop scalar \\
$\quad$ speed control
\end{tabular} & $\begin{array}{l}\checkmark \text { Constant } \\
\text { Volts-per- } \\
\text { Hertz or volt- } \\
\text { frequency } \\
\text { (VF) control }\end{array}$ & $\begin{array}{l}\checkmark \text { Using } \\
\text { Modulatio } \\
\mathrm{n} \text { SPWM } \\
\text { or SVM. }\end{array}$ & $\begin{array}{l}\checkmark \text { Using a } \\
\text { transforme } \\
r \text { to supply } \\
\text { the ac } \\
\text { power } \\
\text { source. }\end{array}$ \\
\hline
\end{tabular}

Explanations of Table 2-2 are as follows:

a) DOL or Grid-connected: The IM is directly connected to the grid or any ac power source, as shown in Figure 2.1 In this situation, the IM is uncontrolled and it is started with full rated voltage and the currents can reach five times the rated values (inrush current). To limit this, starter like star-delta with an autotransformer is used. The autotransformer uses voltage steps of 40,60 and $75 \%$ of the rated voltage. The problem here is the reduction of the starting torque which is proportional to the square of the applied voltage [17], [18]. 
b) Inverter-fed: The IM is connected to a power electronic converter. The most common is a two-level three-phase voltage source inverter (VSI). There are two possibilities: uncontrolled and controlled starter:

i) Uncontrolled starter: No control. In this case the realization of a three-phase voltage source is performed by a fast switching squared waves achieved by means of the VSI. It does not require any sensor.

ii) Controlled starter which can be open loop and close loop:

(1) Open-loop controller: The IM machine is connected through a power electronic converter, typically VSI, as depicted in the block diagram of Figure 2.2. The control by preference in an open-loop system is Constant volts-per-hertz or volt-frequency (VF) control: It is a scalar controller and perhaps the simplest and least expensive control. This is a speed control strategy based on the torque-speed characteristic and in the phase-voltage equation of an IM [14], [18]. It requires a speed sensor but there is no feedback. It requires a modulation strategy.

(2) Closed-loop (CL) controller: The IM machine is connected through a power electronic converter, normally the same as in the open-loop controller but in this situation sensors and feedback connection is needed for the control purposes as in Figure 2.3. There are two main types:

(a) Scalar control: The CL control types are [38]:

(i) Constant-Slip current control which requires current phase feedback. It needs two sensors (current and speed), a speed proportional-integral (PI) controller and a modulation strategy. 
(ii) Scalar torque control: It is typical for winder drives, which are very common in the textile, paper, steel, plastic, or rubber manufacturing industries. It needs two sensors (current and speed).

(iii) Closed-loop scalar speed control: The speed is compared with the reference. The error is applied to a slip controller, usually a PI. The output of this control is added to the speed measurement to obtain the synchronous speed which goes to a voltage controller.

(b) Vector control: The most common vector controllers are as mentioned in Table 2-2. For more information, refer to [39], [40]. The vector controllers can be also categorized as quadrature control and slip control [41].

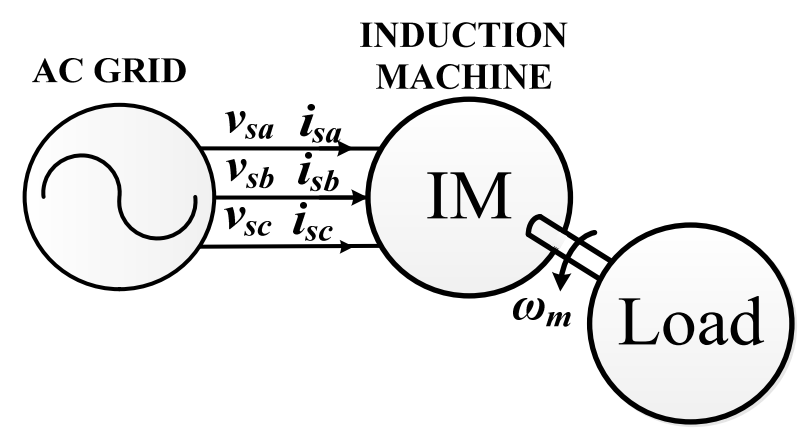

\begin{tabular}{|c|l|}
\hline Variables & \multicolumn{1}{c|}{ Meaning Variable } \\
\hline$v_{s x}$ where $x=\{a, b, c\}$ & $\begin{array}{l}\text { Per-phase grid or IM stator voltage. The subscript } s \text { comes from stator. It } \\
\text { could also be } g \text { from grid. The important element is the IM. The subscript } \\
x \text { refers to the phase. }\end{array}$ \\
\hline$i_{s x}$ where $x=\{a, b, c\}$ & Per-phase grid or IM stator current. Same as stator voltages. \\
\hline$\omega_{m}$ & $\begin{array}{l}\text { IM mechanical speed of the rotor. The subscript } m \text { comes from } \\
\text { mechanical. Also can be set as } r \text { from rotor. }\end{array}$ \\
\hline
\end{tabular}

Figure 2.1 DOL or Grid-connected IM topology 


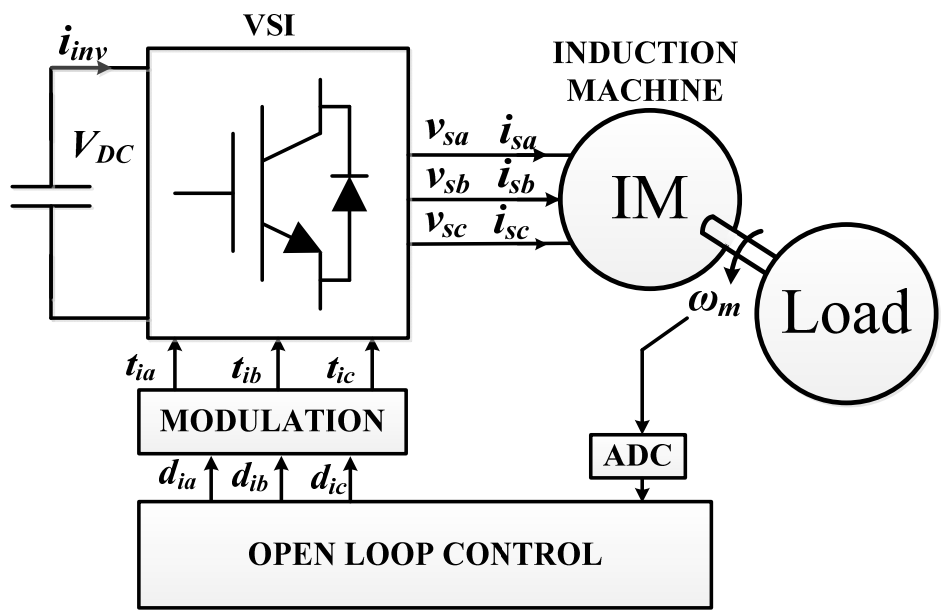

\begin{tabular}{|c|l|}
\hline Variables & \multicolumn{1}{c|}{ Meaning Variable } \\
\hline$V_{D C}$ & Dc link capacitor voltage. The subscript DC refers to the DC link. \\
\hline$i_{i n v}$ & Input current in the inverter. The subscript inv refers to the inverter. \\
\hline$t_{i x}$ where $x=\{a, b, c\}$ & $\begin{array}{l}\text { Square signal pulses, a time-dependent signal. The on-off commands for the } \\
\text { power semiconductor devices in this case, Insulate Gate Bipolar Transistors } \\
\text { (IGBT). These signals also originate their complementary } \bar{t}_{i x} \text { for the bottom } \\
\text { switching devices. The subscript } i \text { refers to inverter. }\end{array}$ \\
\hline$d_{i x}$ where $x=\{a, b, c\}$ & Duty cycle value as input of the modulation strategy. Same as $t_{i x}$. \\
\hline
\end{tabular}

Figure 2.2 Inverter-fed induction machine topology driven by open-loop control

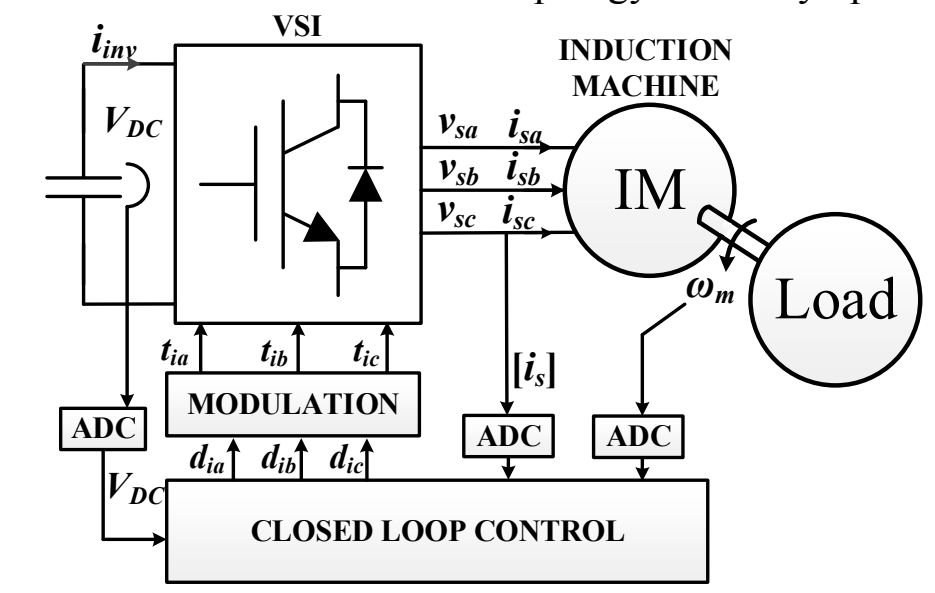

\begin{tabular}{|c|c|}
\hline Variables & Meaning Variable \\
\hline$\left[\boldsymbol{i}_{s}\right]=\left[i_{s a} i_{s b} i_{s c}\right]^{t}$ & Space-vector of the IM stator current. \\
\hline
\end{tabular}

Figure 2.3 Inverter-fed induction machine topology driven by closed-loop control

\subsubsection{Selected Induction Machine Applications and Full Topologies}

The selected application were mentioned before and here their complete topology it is presented in block diagrams as it is going to be studied over the dissertation. During this 
dissertation the topics of this complete topology will be studied separately as per simplicity approach to each of them.

\subsubsection{High-performance Industrial Applications Energy Conversion Chain}

In industrial applications, the connection of an IM is as stipulated in the previous subsection. In the past several decades, the price reduction of microprocessor, power semiconductor devices and their drives have allowed the cost-effective production of closeloop vector drivers. Therefore, the majority of recent IM applications are able to have highperformance motion and traction control, increasing the operative range of the IM and diminishing its drawbacks. Thus, in the case of the high-performance industrial application energy conversion chain we will refer to Figure 2.4, where four main components are remarked:

1. AC grid or source.

2. Controlled active regenerative rectifier (ARR): It is selected because of its bidirectional capabilities. With the ARR, it is possible to feed the machine drive and to perform power factor correction, current harmonic reduction and reactive power compensation.

3. Inverter motor drive with CL controller.

4. Mechanical Load.

\subsubsection{Transportation Electrification Applications (Hybrid and/or Electric} Vehicles) Powertrain

Figure 2.5 shows the detailed block diagram of an IM drive for an electric vehicle (EV). The EV is a slightly more complicated topology than the industrial applications, as it has two modes of operation: 
1. Driving or propulsion mode: In this case the battery is supplying the machine drive to move the $\mathrm{EV}$.

2. Charging mode: In this situation the EV is stand-still, unless it has dynamic wireless power transfer, and it is connected to the grid.

The two cases are depicted in Figure 2.5 and the topology is composed of:

1. AC grid.

2. Controlled ARR.

3. DC-DC converter: It can be bidirectional or unidirectional depending on the application and the capabilities towards the grid.

4. Inverter motor drive with CL controller.

5. Mechanical Load. 


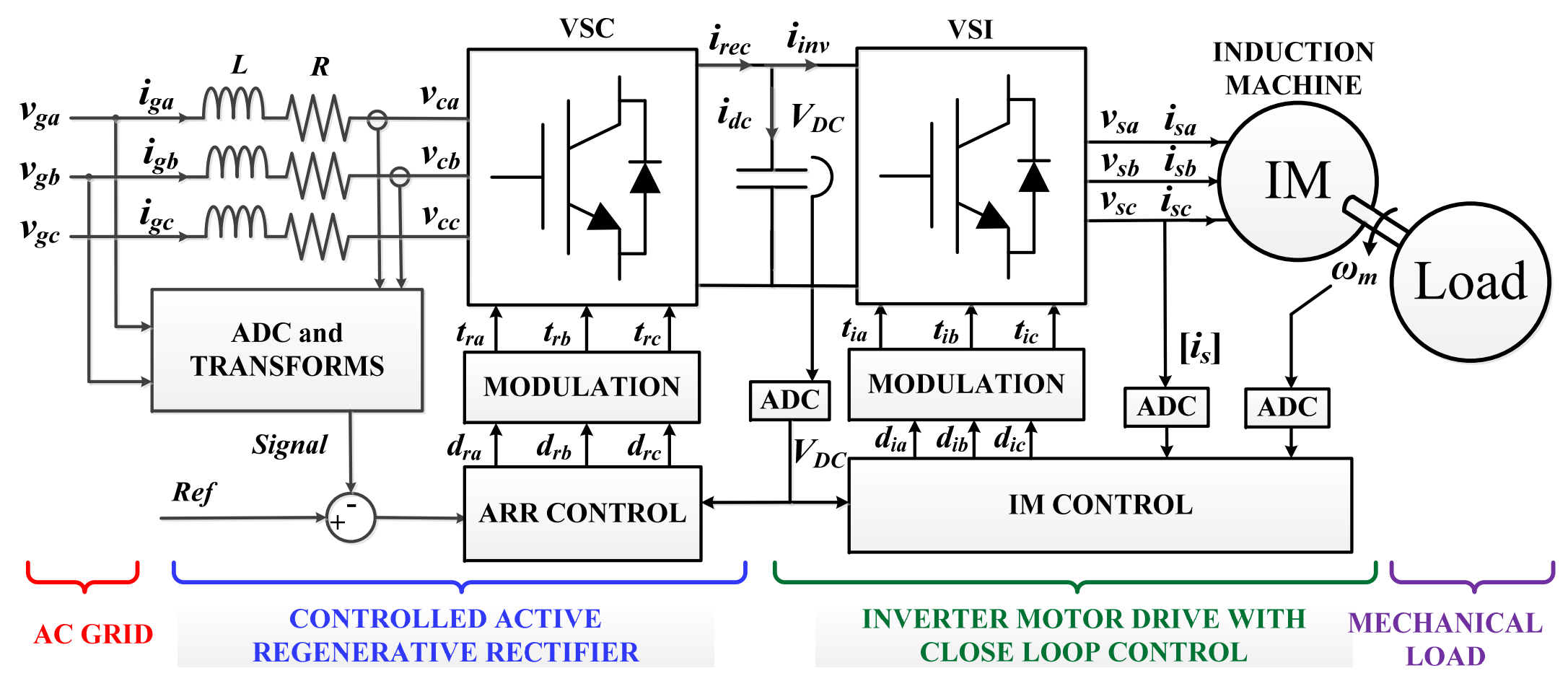

\begin{tabular}{|c|l|}
\hline Variables & \multicolumn{1}{c|}{ Meaning Variable } \\
\hline$v_{g x}$ where $x=\{a, b, c\}$ & Per-phase ac grid voltage. The subscript $g$ comes from grid. The subscript $x$ refers to the phase. \\
\hline$i_{g x}$ where $x=\{a, b, c\}$ & Per-phase ac grid current. Same as grid voltages. \\
\hline$v_{c x}$ where $x=\{a, b, c\}$ & Per-phase converter voltage. The subscript $c$ comes from converter. The subscript $x$ refers to the phase. \\
\hline$i_{r e c}$ & DC output current in the rectifier. The subscript $r e c$ refers to the rectifier. \\
\hline$t_{r x}$ where $x=\{a, b, c\}$ & $\begin{array}{l}\text { Square signal pulses, a time-dependent signal. The on-off commands for the power semiconductor devices in this case } \\
\text { Insulate Gate Bipolar Transistors (IGBT). These signals also originate their complementary } \bar{t}_{r x} \text { for the bottom switching } \\
\text { devices. The subscript } r \text { refers to rectifier. }\end{array}$ \\
\hline$d_{r x}$ where $x=\{a, b, c\}$ & Duty cycle value as input of the modulation strategy. The subscript $r$ refers to rectifier. \\
\hline
\end{tabular}

Figure 2.4 Complete and detailed schematic of the controlled induction machine drive for industry applications. 


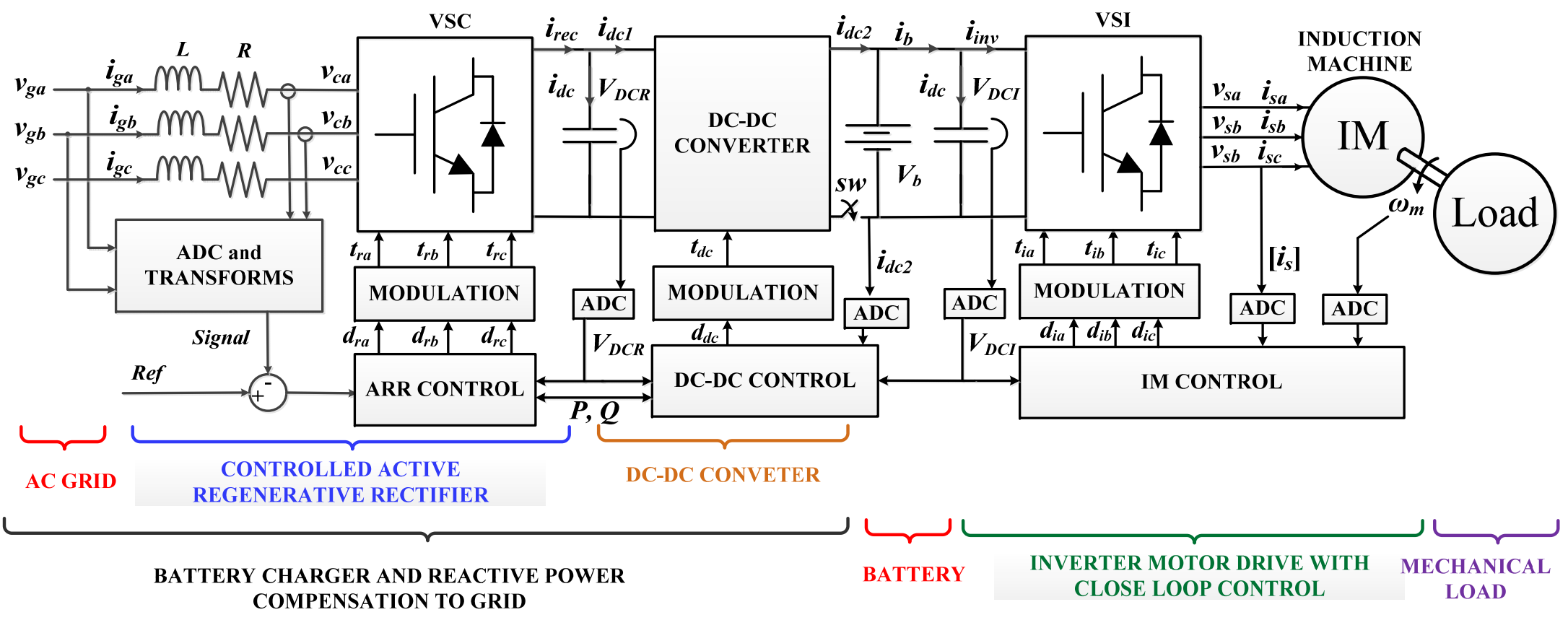

\begin{tabular}{|c|l|}
\hline Variables & \multicolumn{1}{c|}{ Meaning Variable } \\
\hline$V_{D C R}$ & DC link capacitor voltage in the rectifier. The subscript $D C$ refers to DC link and $R$ refers to rectifier. \\
\hline$V_{D C I}$ & DC link capacitor voltage in the inverter. The subscript $D C$ refers to DC link and R refers to inverter. \\
\hline$i_{d c 1}$ & Current in the terminal 1 of the dc-dc converter. The subscript $d c$ refers to the dc-dc converter and 1 refers to the terminal. \\
\hline$i_{d c 2}$ & Current in the terminal 2 of the dc-dc converter. Same as before. \\
\hline$V_{b}$ & Battery voltage. The subscript $b$ refers to the battery. \\
\hline$i_{b}$ & Battery current. In discharge mode. The subscript $b$ refers to the battery. \\
\hline$t_{d c}$ & Square signal pulses, a time dependent signal. The subscript $d c$ refers to dc-dc converter. \\
\hline$d_{d c}$ & Duty cycle value as input of the modulation strategy. The subscript $d c$ refers to dc-dc converter. \\
\hline$s w$ & Switch that change the mode of the dc-dc converter. \\
\hline
\end{tabular}

Figure 2.5 Complete and detailed schematic of the controlled IM drive for transportation electrification applications. 


\subsubsection{Induction Machine Failures Categorization}

The main goal of this dissertation is too study, develop and provide strategies to detect and identify faulty behavior on an induction machine drive. The main component in a machine drive is the electrical machine which in our situation is the SCIM. The identification of faults in an IM is a paramount topic in industry applications as IM is the workhorse motor. A failure of the IM may result in several consequences and in the worstcase scenario if an incipient fault is left undetected, it can develop into catastrophic failures. Some of the worst-case scenarios are:

$\checkmark$ Costly repairs if the fault reach a level where it is irreversible: damage to the stator winding or core.

$\checkmark$ Motor outage time and therefore significant financial loss because of unscheduled downtime in industrial plants.

$\checkmark$ Unexpected disaster that can cause safety hazards in transportation electrification applications

Electrical machines and drive systems often have several types of faults. These faults are caused by the combination of two main stresses: internal and external stresses [8], [23], [42]. The classification of the causes of faults in induction machines are shown in Table $2-3$. 
Table 2-3 Classification of the Causes of Faults in Induction Machines

\begin{tabular}{|c|c|c|c|}
\hline \multicolumn{4}{|c|}{ CAUSES OF FAULTS IN IM } \\
\hline \multicolumn{2}{|r|}{ External } & \multicolumn{2}{|r|}{ Internal } \\
\hline 莺 & $\begin{array}{ll}\checkmark & \text { Unbalanced power supply: voltage or current. } \\
\checkmark & \text { Voltage or current fluctuations: under and over voltage } \\
\checkmark & \text { and current. } \\
\checkmark & \text { Earth fault or grounding. } \\
\checkmark & \text { Noisy network. } \\
\checkmark & \text { Frequent starting } \\
\checkmark & \text { Frequent transients } \\
\checkmark & \text { Poor voltage regulation } \\
\checkmark & \text { Electrical construction defects on the windings, } \\
& \text { connectors, insulation or coating problems, wrong } \\
\checkmark & \text { winding tiding, etc. } \\
& \text { If inverter-fed: } \\
\text { (1) Failure on the power electronic components of the } \\
\text { drive. } \\
\text { (2) PWM inverter induce surges in the presence of } \\
\text { substantial long cable length between the motor and } \\
\text { its drive. }\end{array}$ & 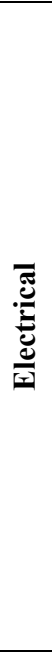 & $\begin{array}{l}\text { Electrical stresses in their stator and } \\
\text { rotor coils by: } \\
\checkmark \\
\checkmark \quad \text { Insulation degradation. } \\
\checkmark \quad \text { Aging of the winding. }\end{array}$ \\
\hline & $\begin{array}{ll}\checkmark & \text { Dynamic movement causes misalignment. } \\
\checkmark & \text { Accumulation of assembly may cause mass unbalance. } \\
\checkmark & \text { Tolerances may cause mass unbalance. } \\
\checkmark & \text { Non-homogeneous materials may cause mass unbalance. } \\
\checkmark & \text { Operational - uneven build-up of product causes mass } \\
& \text { unbalance. } \\
\checkmark & \text { Erosion of rotor material causes mass unbalance. } \\
\checkmark & \text { Assembly fault in general. } \\
\checkmark & \text { Frequently mechanical overload. } \\
\checkmark & \text { Oscillating load. } \\
\checkmark & \text { Improper load coupling or installation defects. } \\
\checkmark & \text { Gearbox failures. }\end{array}$ & 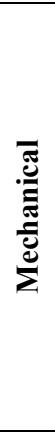 & $\begin{array}{ll}\checkmark & \text { Asymmetric constructions of the } \\
\text { air-gaps, rotor's cage, etc. } \\
\checkmark \quad \text { Coil and sheet steel motion in the } \\
\text { stator and/or rotor. } \\
\checkmark \quad \text { Foundation defect in its } \\
\text { construction. }\end{array}$ \\
\hline 莽 & $\begin{array}{l}\checkmark \text { Stalling } \\
\checkmark \text { Mechanical construction defects on machine. } \\
\checkmark \text { Human error at the installation, use or operation: } \\
\text { (1) Lack of appropriate standards and specifications. } \\
\text { (2) Poor tolerances and poor methods cause } \\
\text { misalignment. } \\
\text { (3) Good methods, but bad practices causes } \\
\text { misalignment. } \\
\text { (4) Lack of understanding of the precision process. } \\
\text { (5) Mis-diagnosis with unbalance or looseness } \\
\text { (6) Poor attention to assembly may cause mass } \\
\text { unbalance. } \\
\text { (7) Improper installation of the bearings. } \\
\text { (8) Inadequate lubrication of the bearings. } \\
\text { (9) Restricted ventilation }\end{array}$ & & $\checkmark \quad$ Failure of the cooling system. \\
\hline 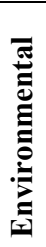 & $\begin{array}{ll}\checkmark & \text { High ambient temperatures } \\
\checkmark & \text { High moisture or damp. } \\
\checkmark & \text { Corrosive environments } \\
\checkmark & \text { Dusty and dirty medium } \\
\checkmark & \text { Oil surroundings } \\
\checkmark & \text { Water intrusion }\end{array}$ & 㞭 & $\begin{array}{l}\text { Magnetic stresses in the magnetic } \\
\text { circuits degradation and aging. }\end{array}$ \\
\hline
\end{tabular}


Table 2-4 Categorization of the Faults in an Induction Machine

\begin{tabular}{|c|c|c|}
\hline \multicolumn{3}{|c|}{ Internal Faults in IM } \\
\hline & Stator & Rotor \\
\hline 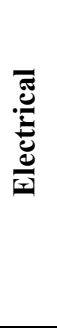 & $\begin{array}{l}\checkmark \text { Phase opening: broken turn of the coils for the IM } \\
\text { stator. } \\
\checkmark \text { Short-circuit on the winding: Typically, it is due } \\
\text { to problems in the winding insulation. When the } \\
\text { short-circuit is not in the full phase, it is called } \\
\text { inter-turn short-circuit and there are three types: } \\
\text { (1) within the same phase } \\
\text { (2) between two phases } \\
\text { (3) between one phase and the stator frame }\end{array}$ & $\begin{array}{l}\text { Phase opening } \\
\text { (1) Broken turn of the coils for wound rotor } \\
\text { machines } \\
\text { (2) Bar breakage for squirrel-cage machines } \\
\text { (3) Broken end-ring for squirrel-cage machines } \\
\checkmark \text { Short-circuit on the winding: It is the loss of the } \\
\text { insulation on the coils for wound rotor machines. }\end{array}$ \\
\hline 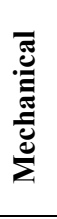 & \multicolumn{2}{|c|}{$\begin{array}{l}\checkmark \text { Bearing failure: it is not a significant source of vibration until quite advanced stages of failure are reached. } \\
\checkmark \text { Air-gap eccentricity: They cause unbalanced magnetic pull (UMP). This radial force is the main reason } \\
\text { for the noise in electrical machines. There are three categories: } \\
\text { (1) Dynamic } \\
\text { (2) Static } \\
\text { (3) Combined }\end{array}$} \\
\hline 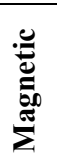 & \multicolumn{2}{|c|}{$\begin{array}{l}\checkmark \text { Demagnetization of the magnetic materials in the stator or rotor. } \\
\checkmark \text { Broken, cracked, or deteriorated magnetic material. }\end{array}$} \\
\hline \multicolumn{3}{|c|}{ External Faults in IM } \\
\hline 胥 & $\begin{array}{l}\checkmark \text { Phase opening: The opening is usually caused by } \\
\text { broken power line. } \\
\checkmark \text { Short-circuit on the winding: complete short circl } \\
\text { (1) between two phases } \\
\text { (2) between one phase and the ground } \\
\checkmark \text { Causes: they can be part of the external failures, } \\
\text { (1) Unsymmetrical mains supply. } \\
\text { (2) Voltage or current fluctuations: under anc } \\
\text { (3) Earth fault or grounding. } \\
\text { (4) Improper or abnormal connections of stat } \\
\text { (5) If inverter-fed: } \\
\text { (a) Failure on the power electronic compc } \\
\text { (b) PWM inverter induce surges in the pre } \\
\text { and its drive. }\end{array}$ & $\begin{array}{l}\text { bad fuse, bad connection, an open contactor or a } \\
\text { of the winding. It can be: } \\
\text { ch us: } \\
\text { ver voltage and current. } \\
\text { ents of the drive. } \\
\text { nce of substantial long cable length between the motor }\end{array}$ \\
\hline 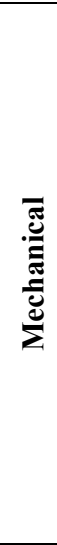 & $\begin{array}{l}\checkmark \text { Misalignment: It is universally recognized as the } \\
\checkmark \text { Mass unbalance: It is arguably the next most sign } \\
\checkmark \text { Bent shaft (akin to dynamic eccentricity): It can } \mathrm{r} \\
\text { serious damage to stator core and windings. } \\
\checkmark \text { Looseness: It may be considered as parts are not } \\
\checkmark \text { Blocked Rotor: the load or the mechanical coupli } \\
\checkmark \text { Fan Problems: damaged fan impellers. Ten to inc } \\
\checkmark \text { Resonance: It is a condition which occurs when a } \\
\text { speed) becomes coincident with, or close to, the } 1 \\
\text { appendage. This is a mix of internal and external } \\
\checkmark \text { Causes: they can be part of the external failures, } \\
\text { (1) Mechanical overload } \\
\text { (2) Underload } \\
\text { (3) Load failure } \\
\text { (4) Gearbox or coupling failure }\end{array}$ & $\begin{array}{l}\text { ading contributor to machinery failure. } \\
\text { icant contributor to premature machine failure. } \\
\text { ult in a rub between the rotor and stator, causing } \\
\text { ting tightly together. } \\
\text { is blocked or braked. } \\
\text { ase the temperature in the machine. } \\
\text { hachine forcing frequency (related to rotational } \\
\text { tural frequency of a machine component or } \\
\text { ult. } \\
\text { ch us: }\end{array}$ \\
\hline
\end{tabular}


Also, the faults in an electrical machine can be categorized into two groups: internal and external. In this dissertation, we concentrate on the internal faults, as the external ones are the same as their external causes and normally they are easy to detect, identify and sometimes to fix due to their external nature. In the case of internal failures, internal and external stresses exert an influence on the fault, which are not observable at the naked eye and therefore are more challenging to detect and identify.

The categorization of the faults in an electrical machine is in Table 2-4.

After observing the vast universe of causes and faults in an IM, it is necessary to determine the importance or the recurrence of these types of faults. Under Electric Power Research Institute (EPRI) sponsorship, a study was conducted by General Electric Company on the basis of the report of the motor manufacturer. As per their report, the main motor faults are presented in Table 2-5 [42].

Table 2-5 Fault Occurrence Possibility on Induction Motors.

\begin{tabular}{|c|c|c|c|c|}
\hline Studied by & Bearing Fault (\%) & Stator Fault (\%) & Rotor Fault (\%) & Others (\%) \\
\hline IEEE & 42 & 28 & 8 & 22 \\
\hline EPRI & 41 & 36 & 9 & 14 \\
\hline
\end{tabular}

Therefore, it can be seen that stator faults are the second most prone failures to happen and for these reason we have selected these faults for its study. Among the failures that can occur in the stator, we choose to work with incipient inter-turn short-circuits faults in one phase, for several reasons:

1) Inter-turn short circuits (ITSC) within the same phase are the most fundamental, common and probable among all the types of short-circuits that can develop in the stator winding. 
2) If they left undetected, they develop into phase-to-phase or phase-to-ground faults. Therefore, its study is priority.

3) In difference with other types of faults (Table 2-4) they are considered one of the most difficult failures to detect in its embryonic or incipient stage due to their subtle effects under this situation, mechanical load variations, power supply unbalances and manufacturing/construction imperfections within the machine itself.

\subsection{Power Electronic Topology Selection}

From the two cases of study in Figure 2.4 and Figure 2.5, it can be noticed that there are two power electronics topologies:

a) Active regenerative rectifier (in both cases, see Figure 2.6): The studied case is a bidirectional two-level three-phase active rectifier, also known as a synchronous rectifier (Figure 2.7). The next chapter will be dedicated to the following topics of the ARR:

i) Mathematical modelling.

ii) Design of the passive components.

iii) Control by means of non-linear predictive techniques.

b) DC-DC converter: It is investigated a Cuk converter, which has unidirectional capabilities (Figure 2.8). In this section the printed circuit board (PCB) design for electromagnetic interference reduction by means of Physics-Based Modelling (PBM) by Finite Element Analysis (FEA) is studied.

In this sense, a chapter per topology is dedicated with the complete contributions on these matters. 


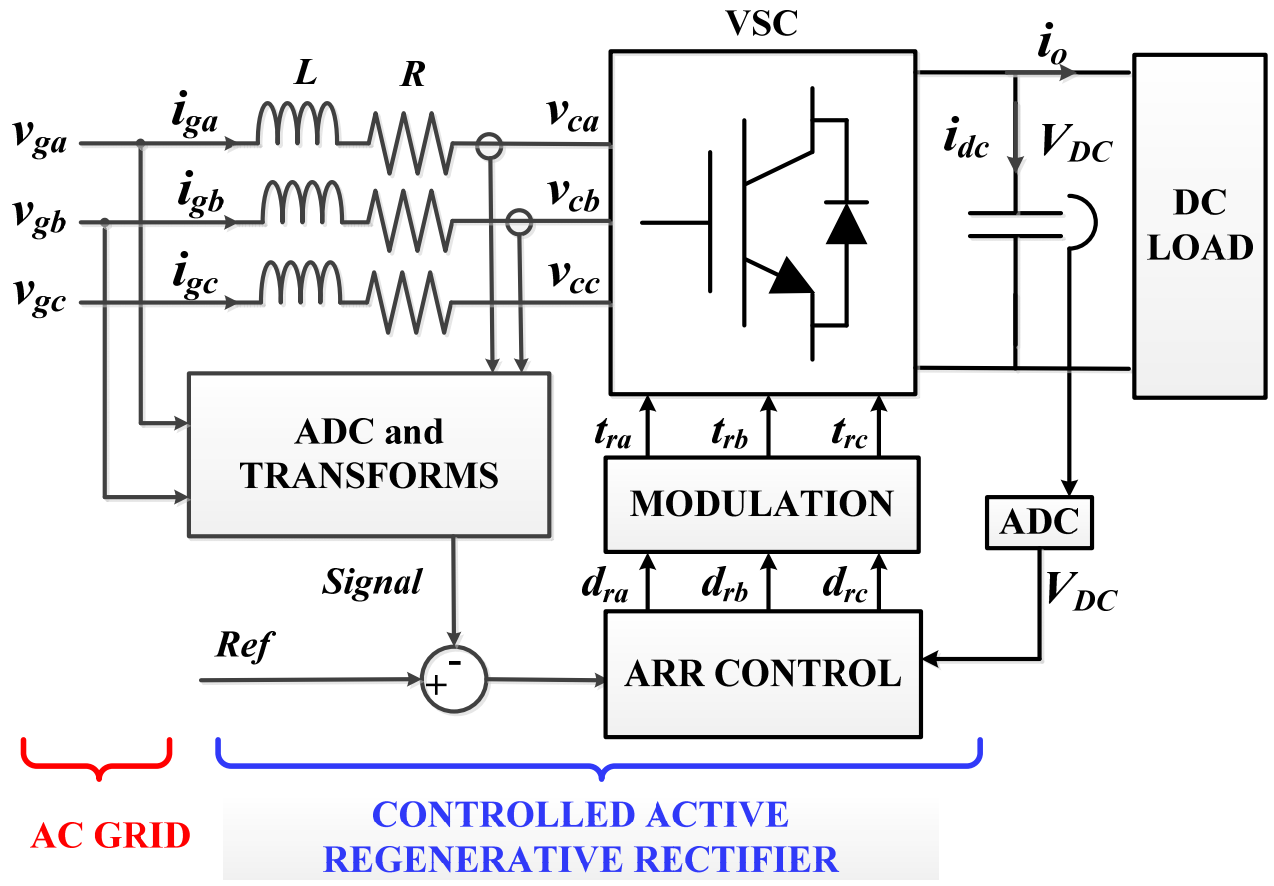

\begin{tabular}{|c|l|}
\hline Variables & \multicolumn{1}{c|}{ Meaning Variable } \\
\hline$i_{o}$ & Load current. \\
\hline Signal & Any signal that is measured and transform to any reference frame \\
\hline Ref & The reference signal for the control purposes. \\
\hline
\end{tabular}

Figure 2.6 Complete and detailed diagram of the controlled active regenerative rectifier

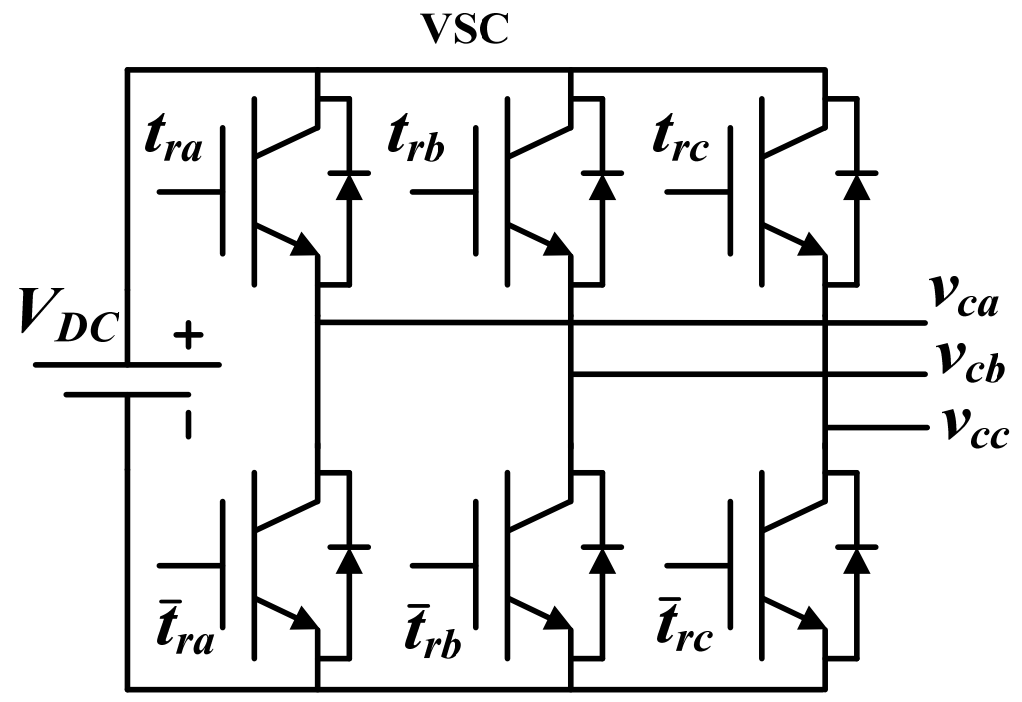

Figure 2.7 Circuit schematic of the two-level three-phase voltage source inverter. 


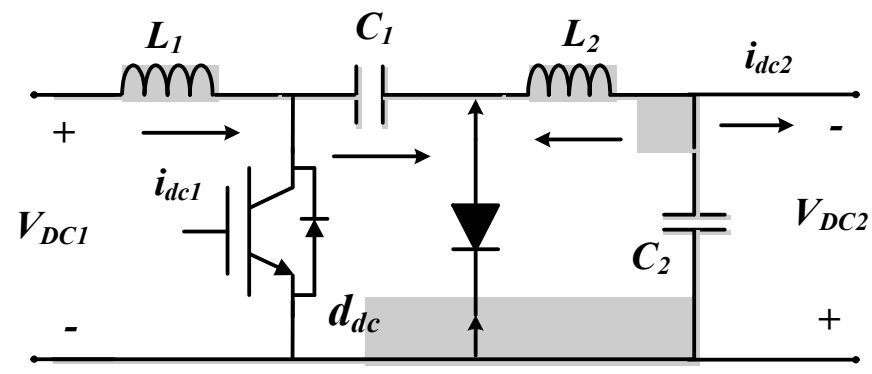

\begin{tabular}{|c|l|}
\hline Variables & \multicolumn{1}{c|}{ Meaning Variable } \\
\hline$i_{d c 1}$ & $\begin{array}{l}\text { Current in the terminal 1 of the dc-dc converter. The subscript } d c \text { refers to the dc-dc } \\
\text { converter and } 1 \text { refers to the terminal. }\end{array}$ \\
\hline$i_{d c 2}$ & Current in the terminal 2 of the dc-dc converter. Same as before. \\
\hline$V_{d c 1}$ & Voltage in the terminal 1 of the dc-dc converter. Same as before \\
\hline$V_{d c 2}$ & Voltage in the terminal 2 of the dc-dc converter. Same as before. \\
\hline
\end{tabular}

Figure 2.8 Circuit schematic of the Cuk DC-DC converter.

\subsection{Nomenclature Convention}

Throughout the dissertation we will follow a similar IEEE notation convention for the mathematical variables, as in Table 2-6.

Table 2-6 Variables Notation Convention.

\begin{tabular}{|c|c|c|c|}
\hline Type of variables & Variable case & Subscript case & Example \\
\hline Continues time dependent signal. & Lowercase italic & Lowercase italic & $v_{g x}(t)=v_{g x}$ \\
\hline $\begin{array}{l}\text { Continues time dependent signal and } \\
\text { small signal (similar as previous case) }\end{array}$ & Lowercase italic & $\begin{array}{l}\text { Lowercase italic } \\
+ \text { underscore } s s \\
\text { or ripple }\end{array}$ & $v_{d c_{-} s s}$ or $v_{d c_{-} r i p p l e}$ \\
\hline $\begin{array}{l}\text { DC signal component or average over a } \\
\text { period } T_{S} \text {. }\end{array}$ & Uppercase italic & Uppercase italic & $\begin{aligned} V_{D C} \text { or } V_{G X} & =\left\langle v_{g x}(t)\right\rangle_{T_{s}} \\
\left\langle v_{g x}(t)\right\rangle_{T_{s}} & =\frac{1}{T_{s}} \int_{t}^{t+T_{s}} v_{g x}(\tau) d \tau\end{aligned}$ \\
\hline $\begin{array}{l}\text { Peak amplitude of a time dependent } \\
\text { signal. In general scalar variables. } \\
\text { Typically the peak of a periodic signal. }\end{array}$ & Uppercase italic & Lowercase italic & $V_{g}$ or $\left|\bar{V}_{g}\right|$ \\
\hline $\begin{array}{l}\text { Peak or maximum transient value of a } \\
\text { signal different from its maximum } \\
\text { periodic value at permanent regime } \\
\text { (Design purposes) }\end{array}$ & Uppercase italic & $\begin{array}{l}\text { Lowercase italic } \\
+ \text { underscore } p k \\
\text { or } \max \end{array}$ & $V_{g_{\_} p k}$ or $V_{g_{-} \max }$ \\
\hline & & & $\tilde{V}_{g}$ \\
\hline $\begin{array}{l}\text { RMS value of a time dependent signal } \\
\text { or phasor. }\end{array}$ & $\begin{array}{l}\text { Uppercase italic } \\
\text { with virgulilla. }\end{array}$ & Lowercase italic & $\tilde{V}_{g}=\sqrt{\frac{1}{T_{s}} \int_{t}^{t+T_{s}} v_{g x}(\tau) d \tau}$ \\
\hline $\begin{array}{l}\text { Continuous total signal, sum of Time } \\
\text { dependent signal and dc component. } \\
\text { The time dependent can be small or } \\
\text { large signal. }\end{array}$ & Lowercase italic & Uppercase italic & $i_{D C}=I_{D C}+i_{d c}$ \\
\hline $\begin{array}{l}\text { State-Space vector o Space-Vector in } \\
\text { matrix form. }\end{array}$ & $\begin{array}{l}\text { Lowercase italic } \\
\text { bold with } \\
\text { brackets }\end{array}$ & $\begin{array}{l}\text { Lowercase italic } \\
\text { and bold }\end{array}$ & {$\left[\begin{array}{ll}\boldsymbol{v}_{g}\end{array}\right]=\left[\begin{array}{lll}v_{g a} & v_{g b} & v_{g c}\end{array}\right]^{t}$} \\
\hline
\end{tabular}




\begin{tabular}{|c|c|c|c|}
\hline Matrix for the SS model & $\begin{array}{l}\text { Uppercase italic } \\
\text { bold with } \\
\text { brackets }\end{array}$ & $\begin{array}{l}\text { Lowercase italic } \\
\text { and bold }\end{array}$ & {$\left[\boldsymbol{B}_{\lambda^{\prime}}\right]=\left[\begin{array}{lllll}1 & 0 & 0 & 0 & 0 \\
0 & 1 & 0 & 0 & 0\end{array}\right]^{t}$} \\
\hline $\begin{array}{l}\text { Space-Vector in complex vector } \\
\text { notation for any reference frame. In } \\
\text { general vector variables. They are time } \\
\text { dependent. These variables are } \\
\text { composed of different components, } \\
\text { typically } 2 .\end{array}$ & $\begin{array}{l}\text { Lowercase italic } \\
\text { bold. } \\
\text { Sometimes } \\
\text { reference } \\
\text { frames have } \\
\text { superscripts. }\end{array}$ & $\begin{array}{l}\text { Lowercase italic } \\
\text { and bold }\end{array}$ & $\begin{array}{c}\boldsymbol{v}_{\boldsymbol{g}}=v_{g \alpha}+j v_{g \beta} \\
\boldsymbol{v}_{\boldsymbol{g}}=\left|\boldsymbol{v}_{\boldsymbol{g}}\right| e^{j\left(\omega t+\theta_{g}\right)} \\
\quad \boldsymbol{v}_{\boldsymbol{g}}=\overline{\boldsymbol{v}}_{\boldsymbol{g}} e^{j \omega t} \\
v_{g \alpha}=V_{g \alpha} \cos \left(\omega t+\theta_{g \alpha}\right) \\
v_{g \beta}=V_{g \beta} \sin \left(\omega t+\theta_{g \beta}\right)\end{array}$ \\
\hline $\begin{array}{l}\text { Space-Phasor is the space-vector in } \\
\text { phasor notation, magnitude and angle. } \\
\text { They are still time dependent. This } \\
\text { Space-phasor can be related to the } \\
\text { Sequence components which are } \\
\text { phasor in frequency domain. }\end{array}$ & $\begin{array}{l}\text { Lowercase italic } \\
\text { bold with bar at } \\
\text { the top }\end{array}$ & $\begin{array}{l}\text { Lowercase italic } \\
\text { and bold }\end{array}$ & $\begin{aligned} \boldsymbol{v}_{\boldsymbol{g}} & =\left|\boldsymbol{v}_{\boldsymbol{g}}\right| e^{j \theta_{g}} e^{j \omega t} \\
\left|\boldsymbol{v}_{\boldsymbol{g}}\right| & =\sqrt{v_{g \alpha}{ }^{2}+v_{g \beta}{ }^{2}} \\
\theta_{g} & =\tan ^{-1}\left(\frac{v_{g \beta}}{v_{g \alpha}}\right)\end{aligned}$ \\
\hline $\begin{array}{l}\text { Discrete small signal or linear variation } \\
\text { first order approximation. }\end{array}$ & $\begin{array}{l}\text { Delta symbol }+ \\
\text { lowercase italic }\end{array}$ & Lowercase italic & $\Delta i_{g x(k)}$ \\
\hline Discrete time dependent signal & Lowercase italic & $\begin{array}{l}\text { Lowercase italic } \\
+\mathrm{k} \text { in } \\
\text { parenthesis }\end{array}$ & $v_{g x(k)}$ \\
\hline Discrete complex SV & $\begin{array}{l}\text { Lowercase italic } \\
\text { bold }\end{array}$ & $\begin{array}{l}\text { Lowercase italic } \\
+\mathrm{k} \text { in } \\
\text { parenthesis }\end{array}$ & $v_{g(k)}=v_{g \alpha(k)}+j v_{g \beta(k)}$ \\
\hline Estimated variable & $\begin{array}{l}\text { It has a hat. The } \\
\text { variable case } \\
\text { and type } \\
\text { depends on the } \\
\text { variable. }\end{array}$ & $\begin{array}{l}\text { The subscript } \\
\text { case and type } \\
\text { depends on the } \\
\text { variable. }\end{array}$ & $\widehat{\boldsymbol{v}}_{\boldsymbol{s}}$ is the estimated signal of $\boldsymbol{v}_{\boldsymbol{s}}$. \\
\hline $\begin{array}{l}\text { Phasor (frequency domain) it } \\
\text { represents a time domain, it is in steady } \\
\text { state. }\end{array}$ & $\begin{array}{l}\text { Uppercase italic } \\
\text { with bar at the } \\
\text { top }\end{array}$ & Lowercase italic & $\begin{array}{l}\bar{V}_{g}=\left|\bar{V}_{g}\right| e^{j \phi_{g}}=V_{g} \angle \phi_{g} \\
v_{g}=V_{g} \sin \left(\omega t+\phi_{g}\right)\end{array}$ \\
\hline Scalar potential. & $\begin{array}{l}\text { Lower or } \\
\text { Uppercase. }\end{array}$ & Lowercase & $\phi$ \\
\hline Vector potential or vector Field. & $\begin{array}{l}\text { Lower or } \\
\text { Uppercase with } \\
\text { arrow at the top. }\end{array}$ & Lowercase & $\overrightarrow{\mathrm{A}}$ \\
\hline
\end{tabular}

\subsection{Reference Frame Theory and its Transformations}

A brief summary of the history of the reference frame transformation [14] is as follows: Since 1920, some approaches to electrical machine analysis had been introducing change or replacing of variables for its study. R. H. Park introduced a transformation that eliminates all time varying inductances from the voltage equations of the synchronous machine. In the late 1930, H. C. Stanley employ a change of variable to eliminate the time varying inductances creating factious stationary windings. G. Kron demonstrate that referring the variables to a reference frame rotating in synchronism with the rotation 
magnetic field it can eliminate the position-time varying mutual inductances. This reference frame is referred as the synchronous reference frame (SRF). D. S. Brereton et al. also eliminates the time varying inductances by referring the stator variables to the reference frame in the rotor. This is the same as Park's Transformation. However, all this transformations are contained in one general transformation that eliminates the timevarying inductances referring the stator and rotor to a frame of reference that may rotate at any particular angular velocity or remain stationary: the arbitrary reference frame.

The transformation matrix for the arbitrary reference frame is [14]:

$\left[T_{a}\right]=k\left[\begin{array}{ccc|c}\cos \theta & \cos \left(\theta-\frac{2 \pi}{3}\right) & \cos \left(\theta+\frac{2 \pi}{3}\right) \\ \sin \theta & \sin \left(\theta-\frac{2 \pi}{3}\right) & \sin \left(\theta+\frac{2 \pi}{3}\right) \\ k_{3} & k_{3} & k_{3}\end{array}\right] \quad$ (2.1)
where $\omega=\frac{d \theta}{d t}$ is the angular velocity, $\theta$ is the angular displacement of the arbitrary reference frame and $k$ is a multiplicative factor for the matrix whilst $k_{3}$ is for the third row. In [14], the value of $k=\frac{2}{3}$ and $k_{3}=\frac{1}{2}$ is however they can be differently assigned. For this case, the inverse transformation is as:

$$
\left[T_{a}\right]^{-1}=\left[\begin{array}{ccc}
\cos \theta & \sin \theta & 1 \\
\cos \left(\theta-\frac{2 \pi}{3}\right) & \sin \left(\theta-\frac{2 \pi}{3}\right) & 1 \\
\cos \left(\theta+\frac{2 \pi}{3}\right) & \sin \left(\theta+\frac{2 \pi}{3}\right) & 1
\end{array}\right]
$$

This transformation with the previous values of $k$ and $k_{3}$ is power conservative or Hermitian. 
Now, the commonly used reference frames used in the analysis of electric machine and power system components are: arbitrary, stationary, rotor and synchronous reference frames. In the Table 2-7 the reference frames are given.

Notice from Figure 2.9 that this reference frame is has the quadrature axis pointing in the direction of the arbitrary reference frame and the direct axis is perpendicular with $-\frac{\pi}{2}$ rads. The axes $f_{s a}, f_{s b}$ and $f_{s c}$ are the primitive coordinate system in $a b c$ and the subscript $s$ refers to the stationary reference frame. Another, literature [43] has the direct axis pointing in the direction of the reference frame and the quadrature axis at $\frac{\pi}{2}$ rads. Thus, making reference to the names of the axes and easier to remember. However, this is matter of likes and it does not affect the results of the analysis.

Table 2-7 Commonly use reference frames.

\begin{tabular}{|c|c|c|c|}
\hline $\begin{array}{c}\text { Reference } \\
\text { Frame }\end{array}$ & Interpretation & \multicolumn{2}{|c|}{ Notation } \\
\cline { 3 - 4 } & $\begin{array}{c}\text { Stationary Circuit variables referred to the arbitrary } \\
\text { reference frame }\end{array}$ & $f_{q}, f_{d}, f_{0}$ & {$\left[T_{a}\right]$} \\
\hline$\omega=\omega_{s}=0$ & $\begin{array}{c}\text { Stationary Circuit variables referred to Stationary } \\
\text { reference frame }\end{array}$ & $f_{s q}, f_{s d}, f_{s 0}$ & {$\left[T_{s}\right]$} \\
\hline$\omega=\omega_{r}$ & $\begin{array}{c}\text { Stationary Circuit variables referred to rotor reference } \\
\text { frame }\end{array}$ & $f_{r q}, f_{r d}, f_{r 0}$ & {$\left[T_{r}\right]$} \\
\hline$\omega=\omega_{e}$ & $\begin{array}{c}\text { Stationary Circuit variables referred to synchronous } \\
\text { reference frame }\end{array}$ & $f_{e q}, f_{e d}, f_{e 0}$ & {$\left[T_{e}\right]$} \\
\hline
\end{tabular}

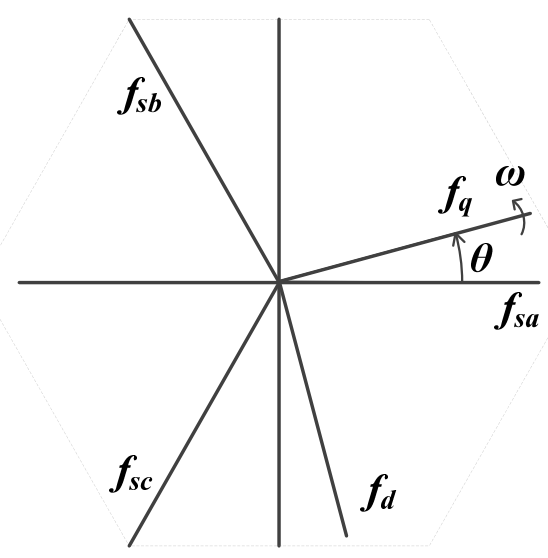

Figure 2.9 Transformation for stationary circuits portrayed by trigonometric relationships. 
The transformation of variables associated with the stationary circuits to a stationary reference frame was developed by E. Clarke [44] and it is the one used in the whole book for the ARR and the induction machine analysis. Nevertheless, some of the IM analysis we adopt the [14] nomenclature. Thus, taking $\theta=\theta_{s}=0$ and using the axes nomenclature as [43] and its value of $k=\sqrt{\frac{2}{3}}$, the stationary reference frame transformation can be shown as in (2.3). Using $k_{3}=\frac{1}{\sqrt{2}}$ the transformation is also power conservative.

$$
\left[T_{s}\right]=\sqrt{\frac{2}{3}}\left[\begin{array}{ccc}
1 & -\frac{1}{2} & -\frac{1}{2} \\
0 & \frac{\sqrt{3}}{2} & -\frac{\sqrt{3}}{2} \\
k_{3} & k_{3} & k_{3}
\end{array}\right]
$$

Its inverse can be written as:

$$
\left[T_{s}\right]^{-1}=\sqrt{\frac{2}{3}}\left[\begin{array}{ccc}
1 & 0 & \frac{1}{2 k_{3}} \\
-\frac{1}{2} & \frac{\sqrt{3}}{2} & \frac{1}{2 k_{3}} \\
-\frac{1}{2} & -\frac{\sqrt{3}}{2} & \frac{1}{2 k_{3}}
\end{array}\right]
$$

However, all the book analyses is considering three-phase star connections, then the transformation can be simplified to:

$$
\left[T_{s s}\right]=\sqrt{\frac{2}{3}}\left[\begin{array}{ccc}
1 & -\frac{1}{2} & -\frac{1}{2} \\
0 & \frac{\sqrt{3}}{2} & -\frac{\sqrt{3}}{2}
\end{array}\right] \quad\left[T_{s s}\right]^{-1}=\sqrt{\frac{2}{3}}\left[\begin{array}{cc}
1 & 0 \\
-\frac{1}{2} & \frac{\sqrt{3}}{2} \\
-\frac{1}{2} & -\frac{\sqrt{3}}{2}
\end{array}\right]
$$


The variables for this transformation will be defined as $f_{s \alpha}$ and $f_{s \beta}$ or just $f_{\alpha}$ and $f_{\beta}$ as only this reference frame will be used in the dissertation. This transformation is used in the ARR modelling, design and control analyses, and simulation and experimental results. Also, it is used in the modelling and control analysis, and simulation and experimental results of a symmetric IM. However, some different transformation approach is used in the modelling of the asymmetric or faulty IM.

For the asymmetric induction machine modelling and simulation, the transformation used is the one in [14] with the difference that the $q-d$ axes are set as in [43]. Thus, for the IM stator, the stationary reference frame transformation and its inverse is as (2.6). The reason to use this change of variables is to keep similar analysis to the one developed in [14] and in [34]. Transformations (2.5) and (2.6) are the same with different values of $k$. Thus, they share the same variable definition as well as same axes orientation (see Figure 2.10). In the case of the IM rotor the transformation is as in [14] substituting $\theta=\theta_{s}-\theta_{r}$ but $\theta_{s}=0$, therefore $\theta=-\theta_{r}$. The rotation transformation and its inverse are in (2.7) and its axes are shown in Figure 2.10. Notice that this transformation is Park and Clarke together and their variables are defined as $f_{r d}, f_{r q}$ and $f_{r 0}$.

$$
\left[T_{s}\right]=\frac{2}{3}\left[\begin{array}{ccc}
1 & -\frac{1}{2} & -\frac{1}{2} \\
0 & \frac{\sqrt{3}}{2} & -\frac{\sqrt{3}}{2} \\
\frac{1}{2} & \frac{1}{2} & \frac{1}{2}
\end{array}\right] \quad\left[T_{s}\right]^{-1}=\left[\begin{array}{ccc}
1 & 0 & 1 \\
-\frac{1}{2} & \frac{\sqrt{3}}{2} & 1 \\
-\frac{1}{2} & -\frac{\sqrt{3}}{2} & 1
\end{array}\right]
$$




\begin{tabular}{|c}
{$\left[T_{r}\right]=\frac{2}{3}\left[\begin{array}{ccc}\cos \theta_{r} & \cos \left(\theta_{r}-\frac{2 \pi}{3}\right) & \cos \left(\theta_{r}+\frac{2 \pi}{3}\right) \\
-\sin \theta_{r} & -\sin \left(\theta_{r}-\frac{2 \pi}{3}\right) & -\sin \left(\theta_{r}+\frac{2 \pi}{3}\right) \\
\frac{1}{2} & \frac{1}{2} & \frac{1}{2}\end{array}\right]$} \\
{$\left[T_{r}\right]^{-1}=\left[\begin{array}{ccc}\cos \theta_{r} & -\sin \theta_{r} & 1 \\
\cos \left(\theta_{r}-\frac{2 \pi}{3}\right) & -\sin \left(\theta_{r}-\frac{2 \pi}{3}\right) & 1 \\
\cos \left(\theta_{r}+\frac{2 \pi}{3}\right) & -\sin \left(\theta_{r}+\frac{2 \pi}{3}\right) & 1\end{array}\right]$}
\end{tabular} \mid

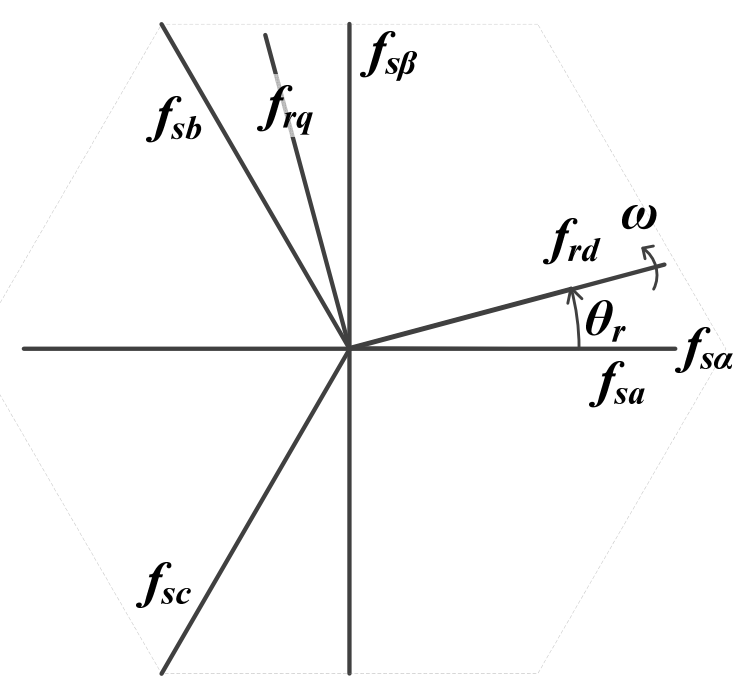

Figure 2.10 Complex Vector Plane for any stationary circuit variable referred to Stationary reference frame.

\subsubsection{Space Vector Transformation}

The model of the three-phase power systems including IM, normally define two type of matrices: symmetric and cyclic (see more in Chapter 4 for the case of IM). These matrices can be made diagonal and thus decoupled. It is demonstrated in [43] that the symmetric components transformation can achieved this by decoupling. The symmetric components transformation and its inverse are as in (2.8). 


$$
\begin{aligned}
& {\left[\begin{array}{l}
x_{z} \\
x_{p} \\
x_{n}
\end{array}\right]=\frac{1}{\sqrt{3}}\left[\begin{array}{ccc}
1 & 1 & 1 \\
1 & e^{j \frac{2 \pi}{3}} & e^{j \frac{4 \pi}{3}} \\
1 & e^{j \frac{4 \pi}{3}} & e^{j \frac{2 \pi}{3}}
\end{array}\right]\left[\begin{array}{l}
x_{a} \\
x_{b} \\
x_{c}
\end{array}\right]=\frac{1}{\sqrt{3}}\left[\begin{array}{ccc}
1 & 1 & 1 \\
1 & \alpha & \alpha^{2} \\
1 & \alpha^{2} & \alpha
\end{array}\right]\left[\begin{array}{l}
x_{a} \\
x_{b} \\
x_{c}
\end{array}\right]} \\
& {\left[\begin{array}{l}
x_{a} \\
x_{b} \\
x_{c}
\end{array}\right]=\frac{1}{\sqrt{3}}\left[\begin{array}{ccc}
1 & 1 & 1 \\
1 & e^{j \frac{4 \pi}{3}} & e^{j \frac{4 \pi}{3}} \\
1 & e^{j \frac{2 \pi}{3}} & e^{j \frac{4 \pi}{3}}
\end{array}\right]\left[\begin{array}{l}
x_{z} \\
x_{p} \\
x_{n}
\end{array}\right]=\frac{1}{\sqrt{3}}\left[\begin{array}{ccc}
1 & 1 & 1 \\
1 & \alpha^{2} & \alpha \\
1 & \alpha & \alpha^{2}
\end{array}\right]\left[\begin{array}{l}
x_{z} \\
x_{p} \\
x_{n}
\end{array}\right]}
\end{aligned}
$$

where $x_{z}, x_{p}$ and $x_{n}$ represent the zero, positive and negative sequence components for the variables $x=\{v, i, \lambda\}, \alpha=e^{j \frac{2 \pi}{3}}$.

This transformation can convert a coupled system in three independent systems. Zero sequence can only exist when the instantaneous summation of the variables is different from zero. Negative and positive sequence are similar and one is the conjugated of the other. Therefore, the symmetric three-phase power system can be represented only using the positive sequence component and this is denominated as Space Vectors (SV). To conserve the active power, the transformation should be as in (2.9). Following the variable nomenclature then we obtain:

$$
\boldsymbol{x}(\boldsymbol{t})=\sqrt{\frac{2}{3}\left[\begin{array}{lll}
1 & \alpha & \alpha^{2}
\end{array}\right]\left[\begin{array}{l}
x_{a} \\
x_{b} \\
x_{c}
\end{array}\right]}
$$

Notice that the SV transformation is exactly the same as (2.5) with the difference that (2.9) is in complex vector form (real and imaginary components) and (2.5) is in matrix form which has the same components as the one in the complex vector form.

For the asymmetric three-phase power system, the sequence components start having more sense and use. However, the application of symmetric components in three-phase power systems are in phasor notation. Thus, the system requires to be expressed in frequency domain (phase and magnitude) and after that, the implementation of the 
symmetric transformation as in (2.8). This is used in the modelling chapters of the IM with turn-fault as well as in the chapter of its condition monitoring.

\subsection{Summary and Conclusion}

From this chapter, it can be concluded and specified part of the objectives of this dissertation on the power electronic converter side, with no order or importance of apparition, are as follows:

- Active regenerative rectifier research: modelling, design and control.

- DC-DC converter research: PCB design for EMI reduction.

For the case of the condition monitoring in electrical machines, we have selected the three-phase squirrel-cage induction machine. Under this machine, the type of failure covered is the inter-turn short-circuit in one phase. The research in this dissertation englobes:

- Modelling of the IM with ITSC.

- Signature discovery for ITSC fault detection and identification on IM, under diverse conditions of the machine.

- Developing an online, robust, not intrusive fault detection scheme for inverterfed IM driven by close loop vector controllers. 


\section{Chapter 3 Design and Control of Active Regenerative Rectifier in Transportation Electrification Applications Drivetrain}

\subsection{Introduction}

Active front-end rectifiers or active regenerative rectifiers (ARR) have been widely used in multiple applications [1]. They are been recently employed in micro-grids and pluggedin electric vehicles where bidirectional power flow may be necessary [2]-[4]. They have the ability to control the dc-link voltage and to correct the power factor $(\mathrm{PF}=\mathrm{dpf} \times \mathrm{DF}$, where dpf is the displacement power factor and DF is the distortion factor) while reducing the current harmonic content. These advantages can be achieved during the two ARRs' modes: rectification and regeneration. DF and total harmonic distortion (THD) are directly proportional to each other, thus ARR can accomplish keeping the grid line currents in phase with the supply grid voltages whilst reducing their THD lower than 5\% (IEEE Std. 5192014). Therefore, plethora of investigations dealing with the ARRs' control are found in the literature [45]-[51], notwithstanding, not many papers are dedicated to the passive components' design [52]-[58] and even less research has attacked the design and control altogether [59]. Fundamentally, the ARR's design consists in the selection of the L-filter and dc-link capacitor under certain criteria. To the author's knowledge, there is not agreement for the most suitable ARR's design technique [52]-[57], [59]. The cause of this

controversy is the lack of a general and accurate formulation that can be implemented to different types of modulation techniques and to the diversity of power converters. Additionally, it has to be suitable to all abundant types of applications and finally also work for the different types of coupling filters (e.g. L and LCL). 
From an intensive literature revision, the ARR design methodologies can be categorized as: time domain [52], [56], [57] and frequency domain [56], [59]. Their development is in different domains, but under certain assumptions it is possible to conciliate them, as a first step, to find the general design methodology. Thus, this chapter presents a nonexclusive ARR design strategy that can be extended for different converter topologies, modulations, and applications, and under certain conditions can be implemented in converters with ancillary capabilities, such as: active power filters and reactive power compensators. The proposed design method determines a range of values for the L-filter and the dc-link capacitor, however, a proper selection of the ARR passive components is not sufficient to comply with the IEEE Std. 519-2014 and thus minimize current harmonic content and ripple. The design must be accompanied by the implementation of a control technique that is qualified to achieve the control goal (PF correction). The failure of either of these two procedures (design or control) causes the increase of the grid line current ripple and thus the no compliance of the stipulated IEEE standard. For the individual study and analysis of each procedure (design and control), it is assumed that the other is successfully granted. Nevertheless, this assumption bring errors generally attributed to the lack of considerations and practical imperfections, such as: acquisition errors, non-linearity of the transducers, non-linear phenomena of the passive components, losses on passive and active components, on and off delays of the switching devices, dead-time of the switches per inverter leg, temperature effects, modulation errors, digital-to-analog conversion errors, electromagnetic noise, etc. As a consequence, a significant increase of the actual current ripple can be accounted and this additional component can be defined as a delta current imperfection $\left(\Delta i_{\text {imperf }}\right)$. Therefore, the actual grid line current ripple $\left(\Delta i_{g}\right)$ is the 
summation of the desired ripple $\left(I_{r p}\right)$ and these non accounted ripple imperfections $\left(\Delta i_{\text {imperf }}\right)$ as: $\Delta i_{g}=I_{r p}+\Delta i_{\text {imperf }}$.

Clearly, there is a necessity of studying the design and control together, considering as much as possible the architecture of the controller and its modulation strategy. The optimal filter design technique will be the one that is able to consider all these imperfections in a simple and practical way, however, this is not an easy task. From the literature review and to the best of the author's knowledge, the majority of the design techniques only focus on physical characteristics of the current ripple plus the power electronic stage topology, but never include any further assumption of the modulation. In this chapter, the time domain L-filer design procedure contemplates a centered sinusoidal pulse width modulation (SPWM) technique, and this allows the results from the theoretical L-filter design (time domain) to match perfectly with the frequency domain results. The importance of considering the modulation strategy is due to the fact that the modulation highly affects the line current THD [7], and here is the main contribution of this research among others.

In Table 3-1, a categorization of ARR controllers is presented. This classification is a compendium of the previous survey articles [60]-[63]. In this table, two types of controllers were investigated and categorized thoroughly: linear and predictive controllers. Other research has also investigated and compared linear and predictive controllers [63], [64] concluding that predictive controllers have superior performance. Moreover, [51] and [32] compare two predictive controllers (deadbeat versus finite set model predictive control FSMPC), finding that the deadbeat type controller proposed in these articles surpasses the capabilities of the classical FSMPC. 
Thus, a non-linear deadbeat type predictive controller is proposed in this chapter. This technique is an extension of the work presented in [22], which is based on the optimum voltage vector computation (OVC) of the voltage source converter (VSC) for direct current control (DCC), to provide fast dynamic, high-performance and parameter independency. For purposes of the design technique development and control, a two-level three-phase voltage source converter (VSC) with centered SPWM is used. A sensibility analysis of the proposed controller is presented to study its robustness under parameter variations. Moreover, two modulations are studied and compared: SPWM and space vector modulation (SVM). Finally, the proposed design and control methodologies are verified by simulations and experimental tests in the ARR.

Table 3-1 Categorization of the Active Regenerative Rectifier's Controllers

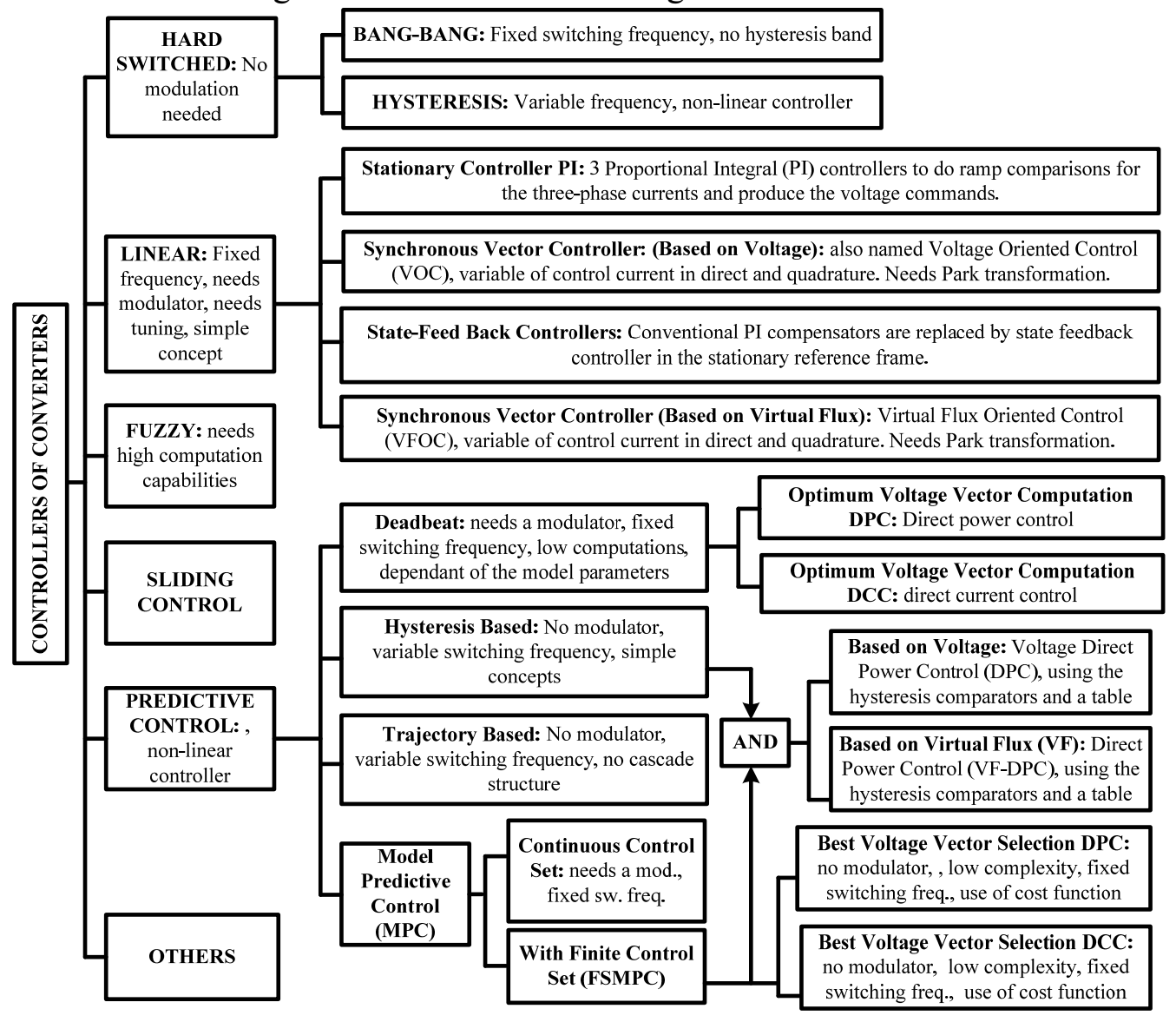




\subsection{Active Regenerative Rectifier Modelling}

The entire block diagram of the ARR is shown in Figure 3.1 and its state-space representation is given in (3.1).

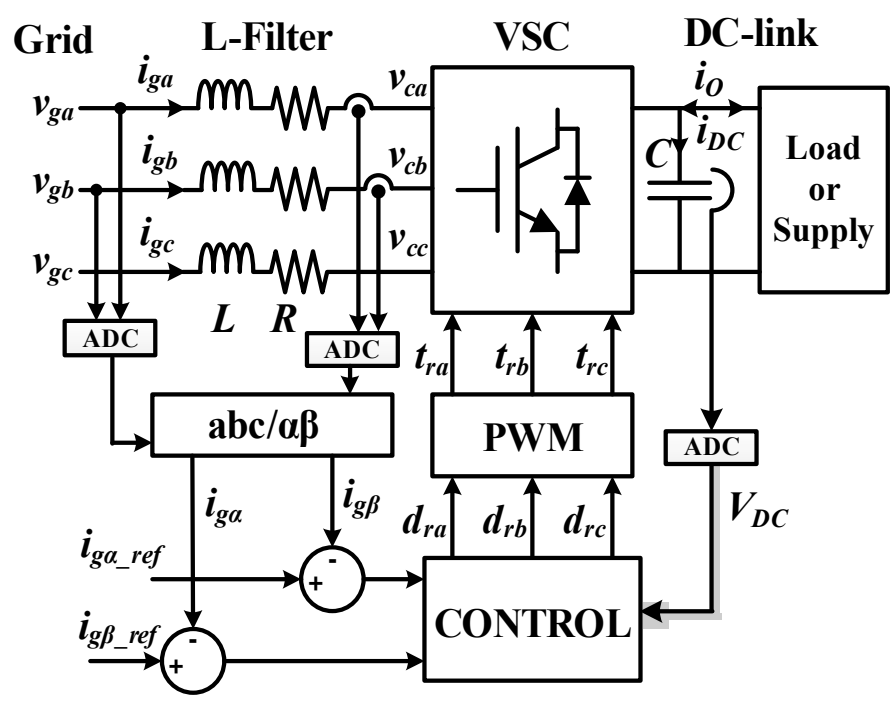

Figure 3.1 Complete topology of the controlled three-phase two-level active regenerative rectifier.

$$
\begin{gathered}
\frac{d\left[\boldsymbol{i}_{\boldsymbol{g}}\right]}{d t}=\frac{1}{L}\left(\left[\boldsymbol{v}_{\boldsymbol{g}}\right]-R\left[\boldsymbol{i}_{\boldsymbol{g}}\right]-\left[\boldsymbol{v}_{\boldsymbol{c}}\right]\right) \\
\frac{d v_{D C}}{d t}=\frac{1}{C}\left(\left[\boldsymbol{s}_{\boldsymbol{w}}\right]\left[\boldsymbol{i}_{\boldsymbol{g}}\right]^{t} \mp i_{O}\right)
\end{gathered}
$$

where $\left[\boldsymbol{v}_{g}\right]=\left[\begin{array}{lll}v_{g a} & v_{g b} & v_{g c}\end{array}\right]^{t}$ is the grid voltage source space-vector (SV), $\left[\boldsymbol{i}_{g}\right]=$ $\left[\begin{array}{llll}i_{g a} & i_{g b} & i_{g c}\end{array}\right]^{t}$ is the grid line current $\mathrm{SV}$ that is bi-directional (ac-to-dc and vice-versa), $\left[\boldsymbol{v}_{c}\right]=\left[\begin{array}{lll}v_{c a} & v_{c b} & v_{c c}\end{array}\right]^{t}$ is the converter voltage SV and $\left[\boldsymbol{s}_{w}\right]=\left[s_{w a} s_{w b} s_{w c}\right]^{t}$ is the switching function SV where $s_{w x}=\left\{\begin{array}{ll}0 & \text { off } \\ 1 & \text { on }\end{array}\right.$ models the VSC states $x=\{a, b, c\}$, the super index $t$ means transpose and $i_{O}$ is the total load-source current in the dc-link. The switching function can be also described by the normalized true inputs or outputs of the PWM block: $\left[\boldsymbol{d}_{\boldsymbol{i}}\right]=\left[\begin{array}{lll}d_{i a} & d_{i b} & d_{i c}\end{array}\right]^{t}$ and $\left[\boldsymbol{t}_{\boldsymbol{i}}\right]=\left[\begin{array}{lll}t_{i a} & t_{i b} & t_{i c}\end{array}\right]^{t}$, respectively. 
By applying one of the six non-zero vectors [65] of the VSC (Table 3-2) and neglecting losses in (3.1), the L-filter voltage $\left(v_{l x}\right)$ per-phase can be obtained as in (3.3). Notice that Table 3-2 is calculated by switching function representation of the converter as in (3.2) [7], [65] and Figure 3.2.

$$
\left[v_{c}\right]=\left[\begin{array}{l}
v_{c a} \\
v_{c b} \\
v_{c c}
\end{array}\right]=\frac{V_{D C}}{3}\left[\begin{array}{ccc}
2 & -1 & -1 \\
-1 & 2 & -1 \\
-1 & -1 & 2
\end{array}\right]\left[\begin{array}{l}
S_{w a} \\
s_{w b} \\
s_{w c}
\end{array}\right]
$$

Table 3-2 Voltage Source Converter Voltage Space Vectors at the terminals [65].

\begin{tabular}{|c|c|c|c|c|c|c|c|c|}
\hline \multirow[t]{2}{*}{$\begin{array}{l}\text { Complex } \\
\text { SV } \boldsymbol{v}_{\boldsymbol{c}}\end{array}$} & \multicolumn{3}{|c|}{$\begin{array}{l}\text { Switching Function } \\
\text { in Primitive } \\
\text { Coordinates }\end{array}$} & \multicolumn{3}{|c|}{$\boldsymbol{v}_{\boldsymbol{c}}$ in Primitive Coordinates } & \multicolumn{2}{|c|}{$\begin{array}{c}\boldsymbol{v}_{\boldsymbol{c}} \text { in } \alpha-\beta \\
\text { Reference Frame }\end{array}$} \\
\hline & $s_{w a}$ & $s_{w b}$ & $s_{w c}$ & $v_{c a}$ & $v_{c b}$ & $v_{c c}$ & $v_{c \alpha}$ & $v_{c \beta}$ \\
\hline$v_{c 0}$ & 0 & 0 & 0 & 0 & 0 & 0 & 0 & 0 \\
\hline$v_{c 1}$ & 0 & 0 & 1 & $-\frac{1}{3} V_{D C}$ & $-\frac{1}{3} V_{D C}$ & $\frac{2}{3} V_{D C}$ & $-\frac{1}{2} V_{D C}$ & $-\frac{\sqrt{3}}{2} V_{D C}$ \\
\hline$v_{c 2}$ & 0 & 1 & 0 & $-\frac{1}{3} V_{D C}$ & $\frac{2}{3} V_{D C}$ & $-\frac{1}{3} V_{D C}$ & $-\frac{1}{2} V_{D C}$ & $\frac{\sqrt{3}}{2} V_{D C}$ \\
\hline$v_{c 3}$ & 0 & 1 & 1 & $-\frac{2}{3} V_{D C}$ & $\frac{1}{3} V_{D C}$ & $\frac{1}{3} V_{D C}$ & $-V_{D C}$ & 0 \\
\hline$v_{c 4}$ & 1 & 0 & 0 & $\frac{2}{3} V_{D C}$ & $-\frac{1}{3} V_{D C}$ & $-\frac{1}{3} V_{D C}$ & $V_{D C}$ & 0 \\
\hline$v_{c 5}$ & 1 & 0 & 1 & $\frac{1}{3} V_{D C}$ & $-\frac{2}{3} V_{D C}$ & $\frac{1}{3} V_{D C}$ & $\frac{1}{2} V_{D C}$ & $-\frac{\sqrt{3}}{2} V_{D C}$ \\
\hline$v_{c 6}$ & 1 & 1 & 0 & $\frac{1}{3} V_{D C}$ & $\frac{1}{3} V_{D C}$ & $-\frac{2}{3} V_{D C}$ & $\frac{1}{2} V_{D C}$ & $\frac{\sqrt{3}}{2} V_{D C}$ \\
\hline$v_{c 7}$ & 1 & 1 & 1 & 0 & 0 & 0 & 0 & 0 \\
\hline
\end{tabular}




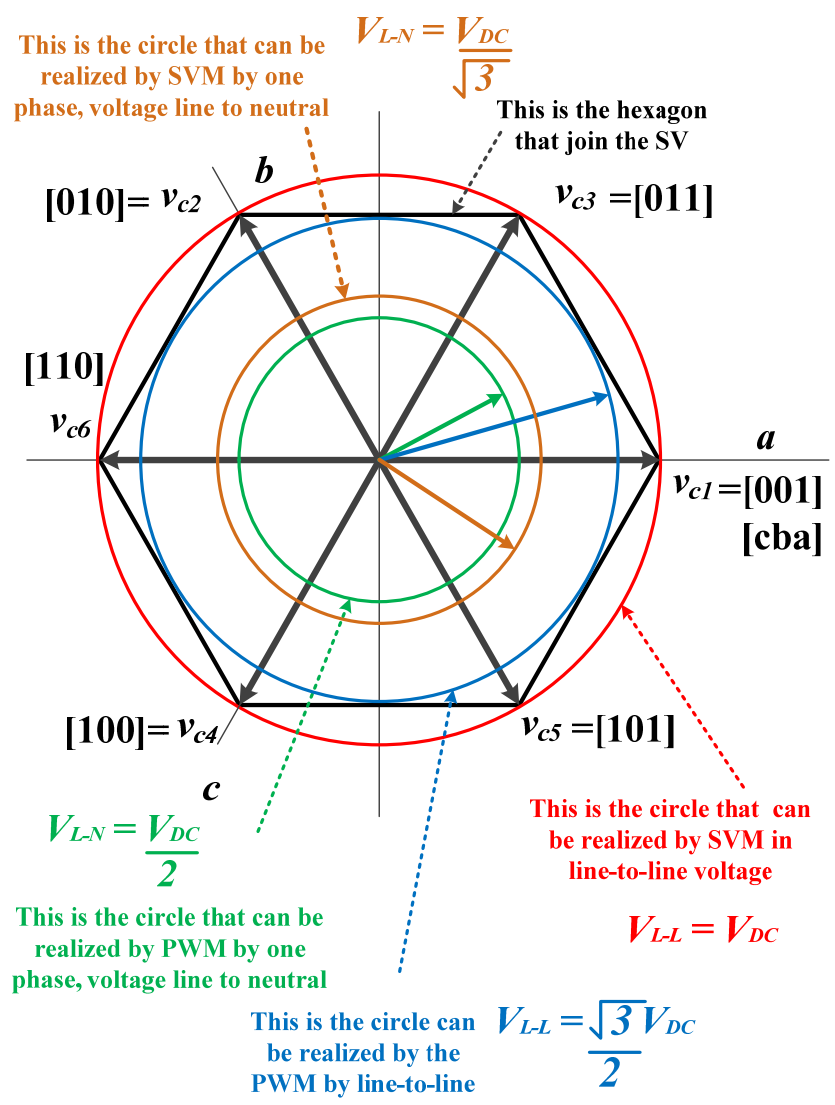

Figure 3.2 Geometrical Voltage Vector Plane for the Voltage Source Converter for different modulation strategies.

$$
v_{l x}= \pm \begin{cases}v_{g x}-\frac{m}{3} v_{D C} & \text { on state } \\ v_{g x}+\frac{m}{3} v_{D C} & \text { off state }\end{cases}
$$

where $m=\{0,-1,2\}$ depending on which of the eight possible switching states of the converter is applied, the \pm sign indicates rectification or regeneration mode respectively and $v_{D C}$ is the total de link capacitor voltage $\left(v_{D C}=V_{D C}+v_{d c_{-} s s}, v_{d c_{-} s s}\right.$ is the small signal). Based on the intrinsic voltage equation of the L-filter $v_{l}=L \frac{d i}{d t}$, assuming a high switching frequency $\left(f_{s}\right)$ with respect to the grid frequency $\left(f_{g}\right)$, then it can be approximated that the grid line current will vary in a linear fashion. In other words, a first 
order approximation to the ARR discrete model can be assumed. Selecting $m=2$, as the worst case, using the regeneration mode (negative sign), using centered-SPWM and doing a per phase analysis, then the current variation can be as in (3.4) at the discrete instant $k$.

$$
i_{g x(k)}= \begin{cases}-\frac{1}{L}\left(v_{g x(k)}-\frac{2}{3} v_{D C(k)}\right) d_{r x(k)} T_{s} & \text { on state } \\ -\frac{1}{L}\left(v_{g x(k)}+\frac{2}{3} v_{D C(k)}\right)\left(1-d_{r x(k)}\right) T_{s} & \text { off state }\end{cases}
$$

where $T_{s}=1 / 10000 \mathrm{sec}$ is the switching time period, $d_{r x(k)}$ is the rectifier duty cycle of the phase $x$. Due to the high switching frequency and assuming one sample acquisition per control period, at any instant of the discrete time $k$, the physical variables are observed, from the point of view of the controller, as constant values and we can get rid of the subscript $(k)$. Also, performing a per-phase analysis we can forget the phase subscript $x$, neglecting the small signal components and assuming the worst case at the peak of the sinusoidal grid voltage wave, then the equation (3.4) can be rewritten as:

$$
\Delta I= \begin{cases}-\frac{1}{L}\left(V_{g}-\frac{2}{3} V_{D C}\right) D T_{S} & \text { on state } \\ -\frac{1}{L}\left(V_{g}+\frac{2}{3} V_{D C}\right)(1-D) T_{S} & \text { off state }\end{cases}
$$

where $\Delta I$ is the grid line current variation per-phase, $V_{g}$ is the peak line-to-neutral amplitude of the grid voltage source per-phase, $V_{D C}$ is the dc component of dc-link voltage and $D$ is the duty cycle per-phase. All these variables and all the analyses are at the interesting instant of time $k$.

\subsection{Active Regenerative Rectifier Design}

The procedure for the ARR design follows [66] and requires selecting: dc-link voltage $\left(V_{D C}\right)$, desired dc-link voltage ripple $\left(V_{r p}\right)$, desired line current ripple $\left(I_{r p}\right)$, desired line 
current THD $\left(T H D_{\%}\right)$, ac grid peak voltage supply amplitude $\left(V_{g}\right)$ and line peak nominal current $\left(I_{g}\right)$ or nominal active power transfer $\left(P_{n}\right)$. Also, some assumptions are needed, such as: balanced sinusoidal grid voltage supply, unity PF operation (achieved by the controller), negligible associated internal coil resistance $(R)$ and higher switching frequency $\left(f_{s}\right)$ than the grid frequency $\left(f_{g}\right)$.

\subsubsection{L-filter Design}

The design purpose of the L-filter is to obtain short-term energy storage for the boosttype converter while keeping the switching noise away from the utility grid. Normally, the high-frequency switching noise in the line current is determined by the size of the inductors and the VSC control performance. Thus, assuming an acceptable control goal achievement, the procedure of the L-filter design is presented. The method is based on [57], where the current variation in one cycle of control is $\Delta I$ as in (3.5). The current area error $\left(S_{e}\right)$ and the current ripple are shown in Figure 3.3 and defined as in (3.6).

$$
\Delta I=\Delta I_{o n}+\Delta I_{o f f}=\Delta I_{o n}+2 \Delta I_{m}
$$

where $\Delta I_{o f f}=2 \Delta I_{m}$. Note that the variation of grid current in the "on-state" increases $\Delta I_{o n}$. Similarly, the variation in the "off-state" decreases $\Delta I_{o f f}$. The resultant current will be as (3.7).

$$
\Delta I=\Delta I_{o n}+2 \frac{\Delta I_{o f f}}{2}=\frac{1}{L}\left(V_{g}-\frac{2}{3} V_{D C}\right) D T_{S}+\frac{1}{L}\left(V_{g}+\frac{2}{3} V_{D C}\right)(1-D) T_{S}
$$


Thus,

$$
\Delta I=\frac{T_{S}}{2}\left[\frac{4}{3} V_{D C} D-\frac{2}{3} V_{D C}-V_{g}\right]
$$

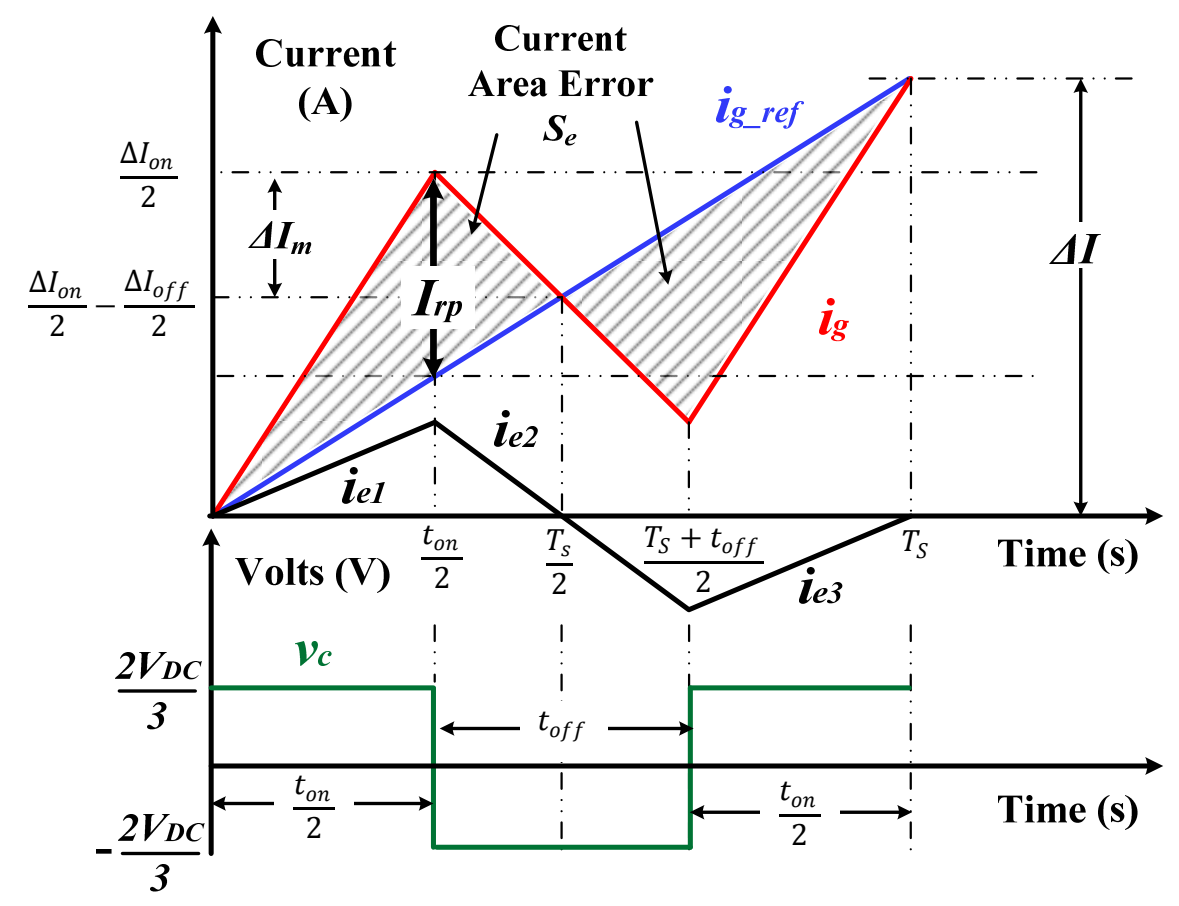

Figure 3.3 Current error calculation method. Grid line current ripple (top) and VSC terminal voltage (bottom).

The grid current error area between the reference and the actual current is the shadowed area as shown in Figure 3.4. This error area can be calculated as the algebraic summation of three triangles and one rectangle, as follows:

\begin{tabular}{|c|c|}
\hline$S_{e}=S_{1}+S_{2}-S_{3}-S_{4}$ & (3.9) \\
\hline$S_{e}=\frac{1}{2}\left(\frac{|\Delta I|}{2}+\left|\Delta I_{m}\right|\right) \frac{D T_{s}}{2}+\frac{1}{2}\left(\frac{|\Delta I|}{2}+\left|\Delta I_{m}\right|\right) \frac{(1-D) T_{s}}{2}-\frac{1}{2}(|\Delta I|) \frac{T_{s}}{2}-\frac{1}{2}\left(\left|\Delta I_{m}\right|\right) \frac{(1-D) T_{s}}{2}$ & (3.10) \\
\hline
\end{tabular}




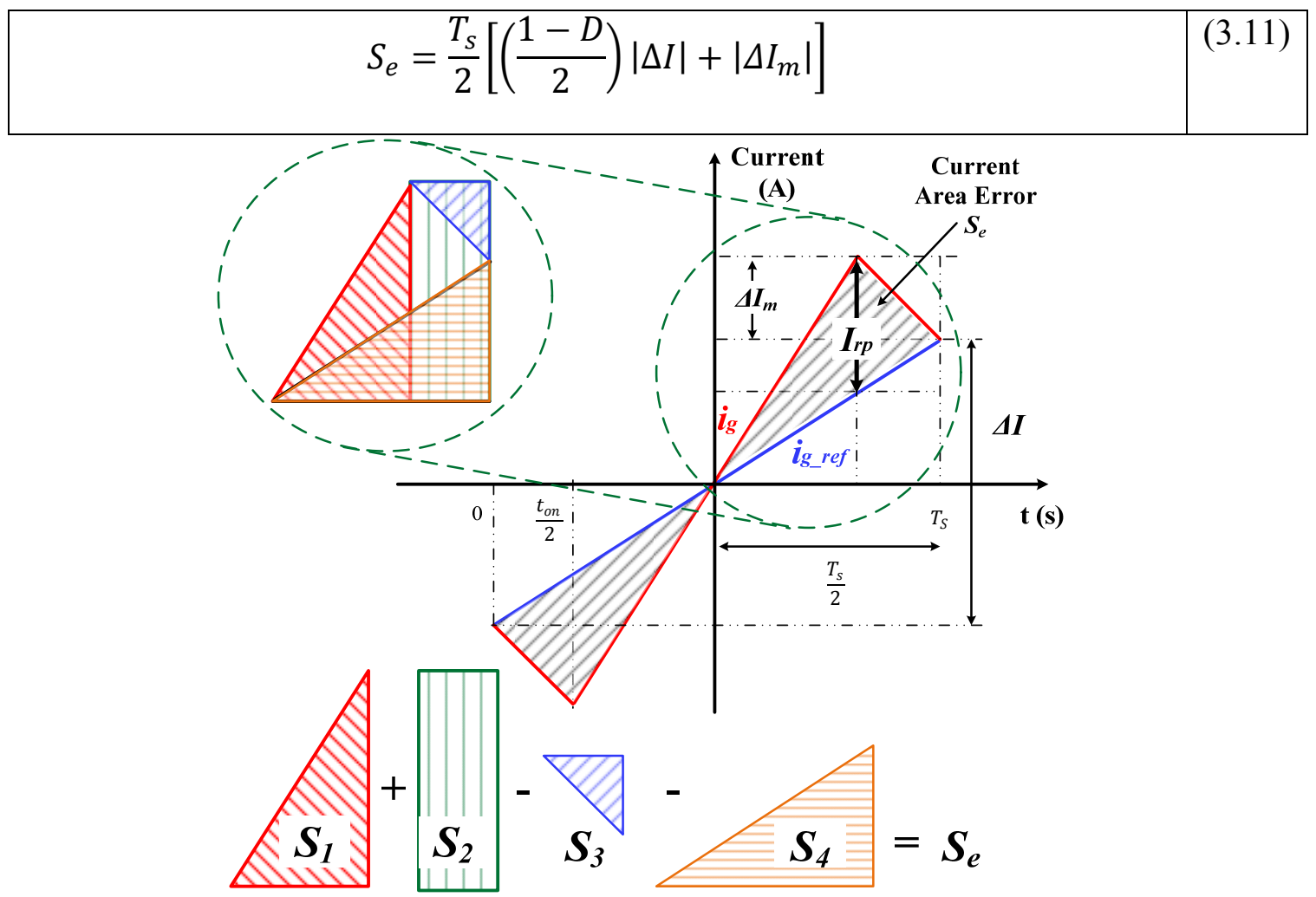

Figure 3.4 Current error algebraic area summation.

Inserting (3.8) and $\Delta I_{m}$ definition in (3.11):

$$
S_{e}=\frac{T_{s}^{2} V_{D C}}{3 L}\left[D-D^{2}\right]
$$

By calculating the derivative with respect to $D$, the duty cycle corresponding to the maximum area error can be found when the derivative equals zero.

$$
\frac{d S_{e}}{d t}=\frac{T_{s}{ }^{2} V_{D C}}{3 L}[1-2 D]=0
$$

By solving (3.13) the result is $D=0.5$. From Figure 3.3 the ripple is defined as:

$$
I_{r p}=\left(\frac{1-D}{2}\right)|\Delta I|+\left|\Delta I_{m}\right|
$$

Introducing (3.8) and $\Delta I_{m}$ definition in (3.14) and substituting $D=0.5$, the minimum limit value of the L-filter minimum value can be defined as in (3.14). 


$$
I_{r p}>\frac{T_{s} V_{D C}}{6 L} \quad \rightarrow \quad L_{\min }>\frac{V_{D C}}{6 f_{s} I_{r p}}
$$

By considering the fundamental components of all the ARR state-space vectors, in this case a phasor analysis is performed. The phasor diagram of an ARR is shown in Figure 3.5. The ARR can operate from the origin $(\mathbf{O})$ of the circle's center. In Figure 3.5(a) the ARR is at power factor different than 1 , it is consuming active $(p)$ and reactive power $(q)$. In Figure 3.5(b) the ARR is working at point $\mathrm{B}$, achieving unity power factor, therefore it is consuming only active power $(p)$ in rectification mode. When the ARR is operating at point $\mathrm{D}$, it is in regeneration mode $(\mathrm{PF}=-1)$.
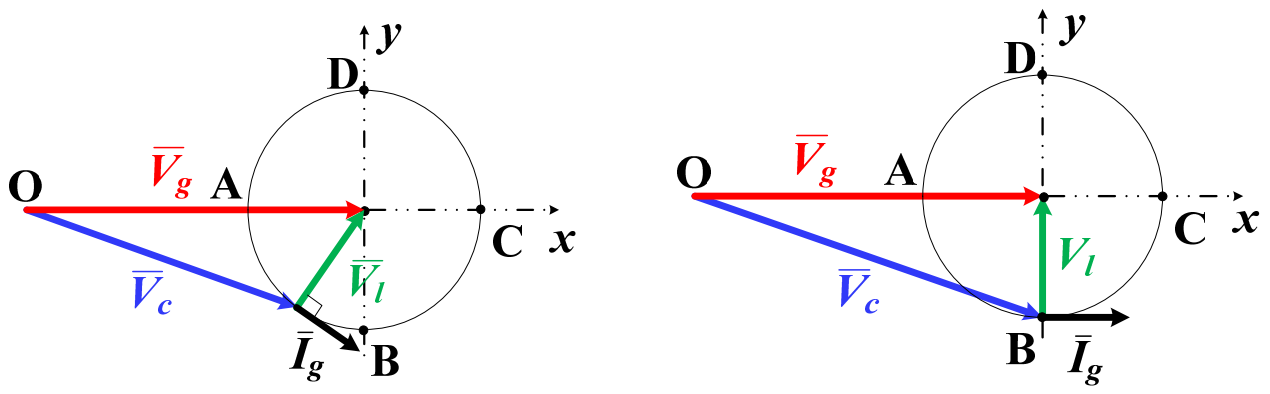

Figure 3.5 Operation modes of the ARR and phasor diagram of the ARR. (a) PF $\neq 1$ (b) $\mathrm{PF}=1$

In Figure 3.5(b), the voltage phasors are described, in amplitude and angle, as: $\bar{V}_{g}=$ $V_{g} \angle 0, \quad \bar{V}_{c}= \pm V_{c} \angle \cos ^{-1}\left(\frac{V_{g}}{V_{c}}\right)$ where $\tilde{V}_{c}=\frac{V_{c}}{\sqrt{2}}=\frac{V_{D C}}{\sqrt{2} M}$, and $M=\{\sqrt{3}, 2\}$ depending of modulation technique used $\{\mathrm{SVM}$ or $\mathrm{SPWM}\}$, using $\mathrm{SPWM} \tilde{V}_{c}=\frac{V_{D C}}{\sqrt{2} 2}$ and then $\bar{V}_{c}=$ $\pm \frac{V_{D C}}{2} \angle \cos ^{-1}\left(\frac{2 V_{g}}{V_{D C}}\right), \bar{V}_{l}=V_{l} \angle \frac{\pi}{2}$ and $V_{l}=\left|X_{L}\right| I_{g}=\omega_{g} L I_{g}$. Thus:

$$
V_{l}=\sqrt{V_{c}{ }^{2}-V_{g}^{2}}=\sqrt{\left(\frac{V_{D C}}{2}\right)^{2}-V_{g}{ }^{2}}
$$


From (3.16) it can be deduced that the maximum inductance drop voltage in both rectification and regeneration is as in (3.17).

$$
\omega_{g} L I_{g}<\sqrt{\frac{1}{4} V_{D C}{ }^{2}-V_{g}^{2}} \rightarrow L<\frac{\sqrt{V_{D C^{2}-4 V_{g}^{2}}}}{\omega_{g} I_{g}}
$$

Then finally, the optimal range of inductor design is:

$$
\frac{V_{D C}}{6 f_{S} I_{r p}}<L<\frac{\sqrt{V_{D C^{2}-4 V_{g}^{2}}}}{\omega_{g} I_{g}}
$$

On the other hand, a power flow stability analysis is considered following [67] and the maximum critic reactance for the voltage stability can be defined as in (3.19).

$$
X_{L}<\frac{V_{g}^{2}}{2 P_{n}} \quad \rightarrow \quad L_{\max }<\frac{V_{g}^{2}}{2 P_{n} \omega_{g}}
$$

where $P_{n}=\frac{V_{g} V_{c}}{X_{L}} \sin (\theta)$ is the nominal averaged active power, $\theta$ is the phase-shift between the supply voltage phasor and the converter voltage phasor. Noticing that $\frac{V_{g}{ }^{2}}{2 P \omega_{g}}<$ $\frac{\sqrt{V_{D C^{2}-4 V_{g}^{2}}}}{\omega_{g} I_{g}}$, the final design range for the L-filter is:

$$
L_{\min }=\frac{V_{D C}}{6 f_{s} I_{r p}}<L<\frac{V_{g}^{2}}{2 P \omega_{g}}=L_{\max }
$$

For completeness and to comply with the current distortion limits, for power systems rated from $120 \mathrm{~V}_{\mathrm{rms}}$ to $69 \mathrm{kV}_{\mathrm{rms}}$ on the IEEE std. 519-2014, a complementary frequency domain design procedure is developed. For this purpose, the line current is modelled, by its high-frequency harmonics, performing a Fourier Series (FS). Considering the aforementioned assumptions, any signal in a power system and specifically the grid line current in one phase can be divided into two signals: dc component $\left(I_{G}\right)$ and ac signal 
component. The ac component in this case is composed of the fundamental signal $\left(i_{g}\right)$ and a small signal $\left(i_{g_{-} s s}\right)$. Thus:

$$
i_{G}=I_{G}+i_{g}+i_{g_{-} s s}
$$

where $I_{G}=0$ for a balanced power system, $i_{g}=i_{g_{-} \text {ref }}$ as this is the pure frequency component signal at $f_{g}=60 \mathrm{~Hz}$, which is exactly the same as the current reference and $i_{g_{-} s}=i_{e}$ is the current error (current ripple) that can be seen as the small signal, which comes from the switching activity at $f_{s}=10 \mathrm{kHz}$ (Figure 3.3). Performing FS analysis, a general formula for FS coefficients can be obtained as in (3.22). The dc components and $a_{n}$ coefficients disappear as $i_{e}$ has odd symmetry.

$$
b_{n}=\frac{2\left[\int_{0}^{\frac{t_{o n}}{2}} i_{e 1} \mathrm{~s} 1 d t+\int_{\frac{t_{o n}}{2}}^{\frac{T_{S}+t_{o f f}}{2}} i_{e 2} \mathrm{~s} 1 d t+\int_{\frac{T_{S}+t_{o f f}}{2}}^{T_{S}} i_{e 3} \mathrm{~s} 1 d t\right]}{T_{s}}
$$

where $\mathrm{s} 1=\sin \left(n \omega_{s} t\right) d t, \omega_{s}=2 \pi f_{s}$ and $i_{e}$ is:

$$
i_{e}=\left\{\begin{array}{cc}
\frac{K_{G}}{D T_{s}} t & 0<t<\frac{t_{o n}}{2} \\
\frac{-K_{G}}{(1-D) T_{s}} t+\frac{K_{G}}{2(1-D)} & \frac{t_{o n}}{2}<t<\frac{T_{s}+t_{o f f}}{2} \\
\frac{K_{G}}{D T_{s}}\left(t-T_{s}\right) & \frac{T_{s}+t_{o f f}}{2}<t<T_{s} \\
K_{G}=\Delta I_{o n}(1-D)+\Delta I_{o f f} D
\end{array}\right.
$$

Substituting the current variations (3.5) in (3.23) and evaluating the integrals in (3.22), $i_{G}$ can be expressed as [66]:

$$
i_{G}=i_{g}+i_{e}=I_{g} \sin \left(\omega_{g} t\right)-\sum_{n=1}^{\infty} \frac{16 V_{D C}}{3 L f_{S}(2 \pi n)^{2}} \sin (\pi n D) \mathrm{s}_{1}
$$

where $n$ stands for the harmonic number and $\omega_{g}=2 \pi f_{g}=377 \mathrm{rad} / \mathrm{s}$. 
Therefore, the L-filter design is computed, considering that at high-frequencies the converter acts as a harmonic generator and the grid as a short-circuit [59], so the minimum value is calculated as in (3.25) [55], [59], [68]:

$$
L>\max \left(\frac{v_{c}(n)}{n \omega_{s} i_{e}(n)}\right) \quad \text { for } \mathrm{n}>2
$$

where $v_{c}(n)$ is the converter voltage at the terminal of the VSC (Figure 3.3) and its FS representation is given in (3.26).

$$
v_{c}=\frac{2}{3} V_{D C}(2 D-1)+\sum_{n=1}^{\infty} \frac{8 V_{D C}}{3 n \pi} \sin (\pi n D) \cos \left(n \omega_{s} t\right)
$$

\subsubsection{DC-link Capacitor Design}

The dc-link capacitor provides an intermediate energy storage that decouples the ac side from the dc side of the ARR. In the rectification mode, the dc side is connected to a load that consumes power and the variation on the dc-link depends only on the control. Nevertheless, in the regenerative mode the instantaneous difference in the active power $\left(\Delta P=P_{a c}-P_{d c}, P_{a c}\right.$ active power in the ac side and $P_{d c}$ power in the dc side) is stored in $\mathrm{C}$, which causes the dc-link voltage to vary. Hence, $\mathrm{C}$ is determined by the constraint on the maximum allowed dc-link voltage ripple $\left(V_{r p}\right)$.

The design of the dc-link capacitor is an extension for the procedure in [57] where the presented design is for an ARR in rectification mode with a resistive load in the dc side. In this chapter, a more general methodology for the ARR is proposed, where a load (dc motor, resistance, etc.), a power supply (dc generator, battery, photovoltaic panel, etc.) or both can be attached to dc-link. 
The capacitor's voltage-current relationship can be written as:

$$
v_{D C}\left(t_{f}\right)-v_{D C}\left(t_{i}\right)=\frac{1}{C} \int_{t_{i}}^{t_{f}} i_{D C}(t) d t
$$

where $t_{f}=t_{i}+T_{S}, t_{i}$ is the initial time and $i_{D C}(t)$ is the total capacitor current (Figure 3.1) given in (3.28).

$$
i_{D C}(t)= \begin{cases}{\left[\boldsymbol{s}_{w}\right]\left[\boldsymbol{i}_{g}\right]^{t}-i_{O}} & \text { rectification mode } \\ -\left[\boldsymbol{s}_{\boldsymbol{w}}\right]\left[\boldsymbol{i}_{\boldsymbol{g}}\right]^{t}+i_{O} & \text { regeneration mode }\end{cases}
$$

In the rectification mode, $i_{O}$ is the current towards the load and in the regeneration mode, it is the current from the dc source. By considering the switching function in one specific instant, then $\left[s_{w a} s_{w b} s_{w c}\right]=\left[\begin{array}{lll}1 & 0 & 0\end{array}\right]$ for the "on-state" and the complementary switches $\left[\begin{array}{lll}s_{w a} & s_{w b} s_{w c}\end{array}\right]=\left[\begin{array}{lll}0 & 1 & 1\end{array}\right]$ for the "off-state." Thus, (3.28) is expressed as:

$$
i_{D C}= \pm\left\{\begin{array}{lcl}
i_{g a}-i_{O} & t_{i}<t<t_{i}+d_{r x} T_{s} & \text { on } \\
-i_{g a}-i_{O} & t_{i}+d_{r x} T_{s}<t<t_{i}+T_{s} & \text { off }
\end{array}\right.
$$

where the \pm sign indicates rectification and regeneration mode. The analysis assumes the following:

- Neglecting the current ripple, the line current in the phase $a$ is $i_{g a}=I_{g} \sin \left(\omega_{g} t\right)$.

- The worst-case scenario for the duty cycle is $D=1$, which means that there is no SPWM.

- Constant $i_{O}=I_{O}+i_{o_{-} s s}$ means that $i_{o_{-} s s}=0$ for both modes, $I_{O}$ is the dc component of the load-source element connected to the dc-link.

- Steady-state condition exists.

- Neglecting the equivalent series resistance (ESR) and inductance (ESL) of the capacitor model. 
Analyzing (3.29), the maximum instantaneous capacitor current is at the minimum slope in the sinusoidal wave, which is at the peak $\left(\omega_{g} t=\pi / 2\right)$ and at no load. Therefore, $C_{\min }$ is defined at the previous condition. Thus, introducing (3.29) and solving (3.27) the design range is as in (3.30).

$C_{\min }=\frac{I_{g}}{f_{s} V_{r p}}<C<\frac{I_{g}+I_{o}}{f_{s} V_{r p}}=C_{\text {max }}$

where $V_{r p}=v_{D C}\left(t_{f}\right)-v_{D C}\left(t_{i}\right)=\Delta v_{D C}=v_{d c_{-} s s}$.

The other approach for the capacitor design is based on the balance of the active power in the ac side $\left(P_{a c}\right)$ and the dc power $\left(P_{d c}\right)$ [68], or the energy loss in the capacitor and active power [69]. Following a similar approach of [68] and using the "on-state" case in (3.29), the design can be as follows. The power balance is as (3.31).

$$
P_{a c}=\frac{3}{2} V_{g} I_{g}=3 \tilde{V}_{g} \tilde{I}_{g}=P_{d c}=V_{D C}\left(C \frac{d v_{D C}}{d t}-i_{O}\right)
$$

Linearization of (3.31) results in:

$$
C<\frac{3 V_{g} I_{g}+2 V_{D C} I_{o}}{2 \Delta v_{D C} V_{D C} f_{S}}=\frac{P_{a C}+P_{O}}{\Delta v_{D C} V_{D C} f_{S}}
$$

where $P_{O}$ is the power consumed (delivered) by the load (source) element connected to the dc-link.

However, by comparing (3.30) and (3.32), $\frac{I_{g}+I_{O}}{f_{s} V_{r p}}>\frac{3 V_{g} I_{g}+2 V_{D C} I_{o}}{2 V_{r p} V_{D C} f_{s}}$ as $\frac{3 V_{g}}{2 V_{D C}}<1$ because of the boost characteristic of the ARR. Thus, (3.33) represents the design range for the dclink capacitor.

$$
C_{\min }=\frac{I_{g}}{f_{s} V_{r p}}<C<\frac{3 V_{g} I_{g}+2 V_{D C} I_{o}}{2 V_{r p} V_{D C} f_{s}}=C_{\max }
$$




\subsection{Predictive Direct Current Control}

The switching noise on the grid line current depends on the L-filter design and ARR control. The consolidation of these two procedures results in the current ripple reduction to comply with the IEEE std. 519. For the control strategy, a proper design value of the Lfilter is considered. As aforementioned, a plethora of research is found about the ARR control [45]-[51] due to the ARR's characteristics, nevertheless, a niche area is the model predictive techniques [45], [61], [63], [65], [70], [71] by virtue of its benefits which make them suitable for power converters, such as:

1) The concepts are intuitive and easy to understand.

2) They can be applied to a variety of systems.

3) Constrains and nonlinearities can be easily included [63].

4) They do not require linear controllers as nested control loops can be incorporated in one loop [61].

5) They present very fast dynamic response as well as high-performance in the permanent regime (static behavior).

6) When compared with linear controllers like VOC, predictive types present their superiority [51], [60], [64], [65].

Predictive type controllers can be categorized as shown in Table 3-1, where direct power control (DPC) [46]-[48], [72] meets its family and it is explained in terms of non-linear control theory [73] as is in [45] using the output regulation subspaces (ORS). However, DPC requires the computation of the instantaneous active and reactive power. The control technique presented in [65], also known as optimum vector computation - direct current 
control (OVC-DCC), is a deadbeat type predictive controller, as shown in Table 3-1. However, OVC-DCC is a reduction of DPC where no power computation is required while keeping the control performance.

\subsubsection{Problem Formulation: Predictive Direct Current Control}

The state-space representation in (3.1) is transformed to the stationary reference frame by the Clarke transformation in (2.5). The representation in complex vector form is:

\begin{tabular}{|c|c|}
$\frac{d \boldsymbol{i}_{\boldsymbol{g}}}{d t}=\frac{1}{L}\left(\boldsymbol{v}_{\boldsymbol{g}}-R \boldsymbol{i}_{\boldsymbol{g}}-\boldsymbol{v}_{\boldsymbol{c}}\right)$ & \\
$\frac{d v_{D C}}{d t}=\frac{1}{C}\left(s_{w \alpha} i_{g \alpha}-s_{w \beta} i_{g \beta} \mp i_{O}\right)$
\end{tabular}

where $\boldsymbol{f}=f_{\alpha}+j f_{\beta}, \boldsymbol{f}=\left\{\boldsymbol{v}_{\boldsymbol{g}}, \boldsymbol{i}_{\boldsymbol{g}}, \boldsymbol{v}_{\boldsymbol{c}}, \boldsymbol{s}_{\boldsymbol{w}}\right\}, f_{\alpha}$ is the real component and $f_{\beta}$ is the imaginary component of the complex SV $\boldsymbol{f}$.

The control vector $\boldsymbol{v}_{\boldsymbol{c}}$, referred to the fixed frame, is the switching state of the VSC and form the input space of the control technique. The control objective is to design a switching sequence of $\boldsymbol{v}_{\boldsymbol{c}}$ to follow a desired current reference, achieving unity PF. The current tracking is direct instead of computing the instantaneous active and reactive powers as in DPC, which can be defined as in (3.35). This offers less computational effort and more accuracy.

$$
\begin{gathered}
p=\boldsymbol{v}_{\boldsymbol{g}} \cdot \boldsymbol{i}_{\boldsymbol{g}}=v_{g \alpha} i_{g \alpha}+v_{g \beta} i_{g \beta} \\
q=\boldsymbol{v}_{\boldsymbol{g}} \times \boldsymbol{i}_{\boldsymbol{g}}=v_{g \alpha} i_{g \beta}-v_{g \beta} i_{g \alpha}
\end{gathered}
$$

where $\boldsymbol{a} . \boldsymbol{b}=a_{\alpha} b_{\alpha}-b_{\beta} a_{\beta}$ is the dot product and $\boldsymbol{a} \times \boldsymbol{b}=a_{\alpha} b_{\beta}-b_{\alpha} a_{\beta}$ is the cross product of two vectors.

A mandatory theoretical condition for the generation of the ORS is that the selected outputs should be relative degree vector $\{1,1\}$ for the two outputs $\left\{y_{1}, y_{2}\right\}$. This ensures 
that the ORS will not be parallel and thus inducing a partition of the input space each perfectly characterized by the derivatives of $\left\{\dot{y}_{1}, \dot{y}_{2}\right\}$. Thus, also the controllability is guaranteed, since a particular output may be increased (decreased) by selecting a control vector above (below) the corresponding ORS, as shown in Figure 3.6.

The outputs for the OVC-DCC are defined as the magnitude and phase of the line current space-vector:

$$
\begin{gathered}
y_{1}=\left|\boldsymbol{i}_{\boldsymbol{g}}\right|=\sqrt{i_{g \alpha}{ }^{2}+i_{g \beta}{ }^{2}} \\
y_{2}=\angle \boldsymbol{i}_{\boldsymbol{g}}=\theta_{\boldsymbol{g}}=\tan ^{-1}\left(\frac{i_{g \beta}}{i_{g \alpha}}\right)
\end{gathered}
$$

Taking the time derivative of (3.36), using (3.34) and (3.35), we can write:

$$
\begin{gathered}
\frac{d y_{1}}{d t}=\frac{\boldsymbol{v}_{\boldsymbol{g}} \cdot \boldsymbol{i}_{\boldsymbol{g}}-R\left(i_{g \alpha}{ }^{2}+i_{g \beta}{ }^{2}\right)-\boldsymbol{v}_{\boldsymbol{c}} \cdot \boldsymbol{i}_{\boldsymbol{g}}}{\left|\boldsymbol{i}_{\boldsymbol{g}}\right| L}=\frac{p-p_{R}-p_{c}}{\left|\boldsymbol{i}_{\boldsymbol{g}}\right| L} \\
\frac{d y_{2}}{d t}=\frac{-\boldsymbol{v}_{\boldsymbol{g}} \times \boldsymbol{i}_{\boldsymbol{g}}+\boldsymbol{v}_{\boldsymbol{c}} \times \boldsymbol{i}_{\boldsymbol{g}}}{\left(i_{g \alpha}+i_{g \beta}\right) i_{g \alpha}}=\frac{-q+q_{c}}{\left(i_{g \alpha}+i_{g \beta}\right) i_{g \alpha}}
\end{gathered}
$$

where $p_{R}$ is the L-filter resistive losses, $p_{c}$ and $q_{c}$ are the active and reactive power of the VSC, respectively, at the ac terminals. From (3.37) it can be noticed that by controlling the magnitude and phase of the line current, the flow of the active and reactive power can be controlled in the same way as DPC. Nevertheless no power calculations are required.

Making $\left\{\dot{y}_{1}, \dot{y}_{2}\right\}=\{0,0\}$, two lines of constant variation of magnitude and angle of the current complex SV is written as a function of the input action of control $\left(\boldsymbol{v}_{\boldsymbol{c}}\right)$, as shown in Figure 3.6. These two lines define the ORS, which also determine the control actions for the different cases of variation of the two inputs:

- Both increase: $y_{1(k+1)}>y_{1(k)}$ and $y_{2(k+1)}>y_{2(k)}$.

- Both decrease: $y_{1(k+1)}<y_{1(k)}$ and $y_{2(k+1)}<y_{2(k)}$. 
- One increases and other decreases: $y_{1(k+1)}>y_{1(k)}$ and $y_{2(k+1)}<y_{2(k)}$.

- Vice versa: $y_{1(k+1)}<y_{1(k)}$ and $y_{2(k+1)}>y_{2(k)}$.

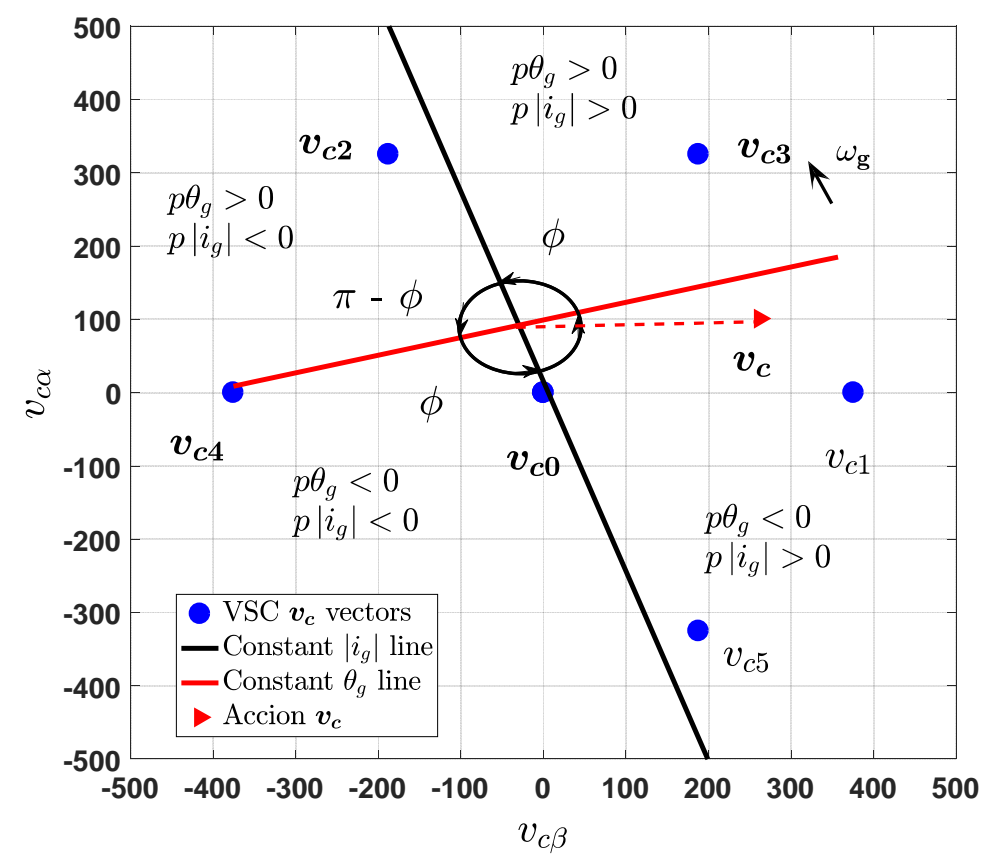

Figure 3.6 Output regulation subspaces for the OVC-DCC.

\subsubsection{Predictive Direct Current Control}

A discrete version of (3.34) is obtained by the following first order approximation:

$$
\Delta \boldsymbol{i}_{\boldsymbol{g}(\boldsymbol{k})}=\frac{T_{S}}{L}\left(\boldsymbol{v}_{\boldsymbol{g}(\boldsymbol{k})}-R \boldsymbol{i}_{\boldsymbol{g}(\boldsymbol{k})}-\boldsymbol{v}_{\boldsymbol{c}(\boldsymbol{k})}\right)
$$

where $\boldsymbol{f}_{(\boldsymbol{k})}$ represents the present sampled value of the complex SV $\boldsymbol{f}$. It is defined for every state-space vector:

$$
\boldsymbol{f}_{(\boldsymbol{k}+1)}=\boldsymbol{f}_{(\boldsymbol{k})}+\Delta \boldsymbol{f}_{(\boldsymbol{k})}
$$

Thus, using (3.38) and (3.39), in a lossless L-filter, the next line current value can be estimated depending on the seven VSC switching states (Table 3-2): 
\begin{tabular}{|c|c|}
$\boldsymbol{i}_{\boldsymbol{g}(\boldsymbol{k}+1)}=\boldsymbol{i}_{\boldsymbol{g}(\boldsymbol{k})}+\frac{T_{S}}{L}\left(\boldsymbol{v}_{\boldsymbol{g}(\boldsymbol{k})}-\boldsymbol{v}_{\boldsymbol{c}(\boldsymbol{k})}\right)$ & (3.40)
\end{tabular}

Defining the grid line current error as:

$$
e_{(k)}=i_{g_{-} r e f(k)}-i_{g(k)}
$$

where $\boldsymbol{i}_{\boldsymbol{g}_{-} \boldsymbol{r} \boldsymbol{f}(\boldsymbol{k})}=G \boldsymbol{v}_{\boldsymbol{g}(\boldsymbol{k})}, G$ is a scale factor used to build the grid current reference from the measured line voltage. For $G>0$, the ARR operates in rectification mode and for $G<$ 0 , the ARR operates in regeneration mode.

Assuming sinusoidal and balanced grid voltage supply, the ac voltage source complex $\mathrm{SV}$ at the next cycle of control can also be estimated as [51], [71]:

$$
\boldsymbol{v}_{\boldsymbol{g}(\boldsymbol{k}+\mathbf{1})}=\boldsymbol{v}_{\boldsymbol{g}(\boldsymbol{k})} e^{j \omega_{g} T_{s}}
$$

Then, using the current error definition, (3.40) and (3.42), the current error in the next cycle of control is obtained as:

$$
\boldsymbol{e}_{(\boldsymbol{k}+\mathbf{1})}=G \boldsymbol{v}_{\boldsymbol{g}(\boldsymbol{k})} e^{j \omega_{g} T_{S}}-\boldsymbol{i}_{\boldsymbol{g}(\boldsymbol{k})}+\frac{\boldsymbol{v}_{\boldsymbol{g}(\boldsymbol{k})}-\boldsymbol{v}_{\boldsymbol{c}(\boldsymbol{k})}}{L / T_{S}}
$$

\subsubsection{Best VSC Vector Selection - Direct Current Control (BVS-DCC)}

This control technique does not required modulation strategy and it is well-known as a Finite Set Model Predictive Control (FSMPC-DCC) as shown in Table 3-1. From (3.43), seven errors can be computed at every cycle of control depending on the seven switching states of the VSC (Table 3-2). Thus, the control law is the minimum of a cost quadratic function (3.44) that indicates which switching state SV is the best for the action of control. Notice that the SV current error is decomposed in its $\alpha-\beta$ components. 


$$
J_{(k+1) \_n}=k_{\alpha} e_{\alpha(k+1) \_n}{ }^{2}+k_{\beta} e_{\beta(k+1) \_n}{ }^{2}
$$

where the subscript $n=\{1,2 \ldots, 7\}$ indicates the switching state of the VSC (Table 3-2). The switching state $n$ that achieves the minimum error is the best selection and $k_{\alpha}$ and $k_{\beta}$ are the cost function weights set as 1 in this particular case.

\subsubsection{Optimum VSC Vector's Computation - DCC (OVC-DCC)}

Observing (3.43), it is deduced that the current error in the next cycle of control can be equal to zero, as a deadbeat type predictive controller:

$$
G \boldsymbol{v}_{\boldsymbol{g}(\boldsymbol{k})} e^{j \omega_{g} T_{S}}-\boldsymbol{i}_{\boldsymbol{g}(\boldsymbol{k})}+\frac{\boldsymbol{v}_{\boldsymbol{g}(\boldsymbol{k})-\boldsymbol{v}_{\boldsymbol{c}(\boldsymbol{k})}}}{\frac{L}{T_{S}}}=0
$$

From (3.45), it is found that the action of control, input space or $\boldsymbol{v}_{\boldsymbol{c}(\boldsymbol{k})}$ is:

$$
\boldsymbol{v}_{\boldsymbol{c}(\boldsymbol{k})}=\boldsymbol{v}_{\boldsymbol{g}(\boldsymbol{k})}-\frac{L}{T_{S}}\left(G \boldsymbol{v}_{\boldsymbol{g}(\boldsymbol{k})} e^{j \omega_{g} T_{S}}-\boldsymbol{i}_{\boldsymbol{g}(\boldsymbol{k})}\right)
$$

The selection of the modulation can be arbitrary, however, in this work, a classical centered-SPWM is selected. Assuming that the switching frequency is much higher than the electric grid frequency, then the exponential $e^{j \omega_{g} T_{s}}$ in the voltage estimation of (3.42) can be neglected. Therefore, $\boldsymbol{v}_{\boldsymbol{g}(\boldsymbol{k}+\mathbf{1})}=\boldsymbol{v}_{\boldsymbol{g}(\boldsymbol{k})}$, and thus, (3.46) be simplified to:

$$
\boldsymbol{v}_{\boldsymbol{c}(\boldsymbol{k})} \approx \boldsymbol{v}_{\boldsymbol{g}(\boldsymbol{k})}-\frac{L}{T_{S}}\left(G \boldsymbol{v}_{\boldsymbol{g}(\boldsymbol{k})}-\boldsymbol{i}_{\boldsymbol{g}(\boldsymbol{k})}\right)=\boldsymbol{v}_{\boldsymbol{g}(\boldsymbol{k})}-\frac{L}{T_{S}} \boldsymbol{e}_{(\boldsymbol{k})}
$$

\subsection{Performance Assessment of Different Modulation Strategies}

The goal of any modulation scheme is to achieve the desired voltage reference in the complex vector plane (Figure 3.6) with additional reduction of grid line current ripple, switching losses, common-mode voltages, electromagnetic interference, etc. In this sense, 
much research has been devoted towards the development of efficient ways of controlling the VSC with additional low computational burden in its implementation [74]. In general, the modulation strategies can be categorized in continuous and discontinuous PWM due to the different choices for the zero vector duty cycle subdivision [74]. In this work, we decided to compare two continues modulations: sinusoidal PWM (SPWM) and space vector modulation (SVM).

In this chapter, the behavior of the two modulation schemes are programmed using the generalized algorithm presented in [74]. A modulator is characterized by different performance parameters [75], i.e., generated current harmonics, maximum modulation index, switching frequency, switching losses and dynamic response. The performance criteria for the assessment, in this work, considers 3 parameters: switching losses, current distortion and dynamic response.

\subsubsection{Switching losses}

The switching losses evaluation is performed in the same way as [75], assuming that the VSC has switching losses that vary linearly with the amplitude of the current. Thus, the average value of the switching power losses $\left(P_{s w}\right)$ over a fundamental period is assessed in (3.48).

$$
P_{s w}=\frac{1}{2 \pi} \frac{1}{2}\left(V_{D C \cdot}\left(t_{o n}+t_{o f f}\right) \cdot f_{s} \cdot \int_{0}^{2 \pi}\left|I_{g}(\theta)\right| d \theta\right)
$$

where $t_{\text {on }}$ and $t_{\text {off }}$ are the respective turn-on and off intervals of the switching devices that can be found in its datasheet, $I_{g}$ is the output converter current and $|\cdot|$ is the absolute value function. 


\subsubsection{Current Distortion and Dynamic Response}

The current distortion is conducted by the computation of the total harmonic distortion (THD) of the grid line current. For the dynamic behavior, the mean absolute error (MAE) and root mean square error (RMSE) for grid line current in phase $a$ is computed. For this variable, the MAE and RMSE are calculated between the instantaneous value reference $\left(i_{g_{-} r e f(k)}\right)$ and the instantaneous current $\left(i_{g(k)}\right)$. As follows, the instantaneous errors are computed as (3.49) and MAE and RMSE as (3.50).

$$
\begin{gathered}
e_{i(k)}=\frac{i_{g_{-} r e f(k)}-i_{g(k)}}{\text { Pk }- \text { pkCurrent }} \\
M A E=\frac{1}{N} \sum_{k=1}^{N}\left|e_{i(k)}\right| \quad R M S E=\sqrt[2]{\frac{1}{N} \sum_{k=1}^{N} e_{i(k)}^{2}}
\end{gathered}
$$

\subsection{Simulation and Experimental Results: Control}

The simulations were performed in the MatLab environment under Simulink Simpower Systems, whereas the experiment implementation was tested in a prototype laboratory testbench, as shown in Figure 3.7. The ARR parameters used in the simulation and the experimental tests are indicated in Table 3-3.

The configuration of the ARR is shown in Figure 3.1, where the dc side is composed by a load resistance in parallel with a dc source. The dc source is built with a three-phase bridge diode rectifier supplied by an ac supply through a step-up transformer to reach lineline voltage equal to $346 \mathrm{~V}_{\text {rms. }}$. This voltage will set the dc-link to $490 \mathrm{~V}$ for purposes of bidirectional active power flow. The experimental platform consists of a dSpace 1104 for 
control, sensor board, measurement equipment, PC, VSC based on semikron IGBTs, dc and ac power supplies.

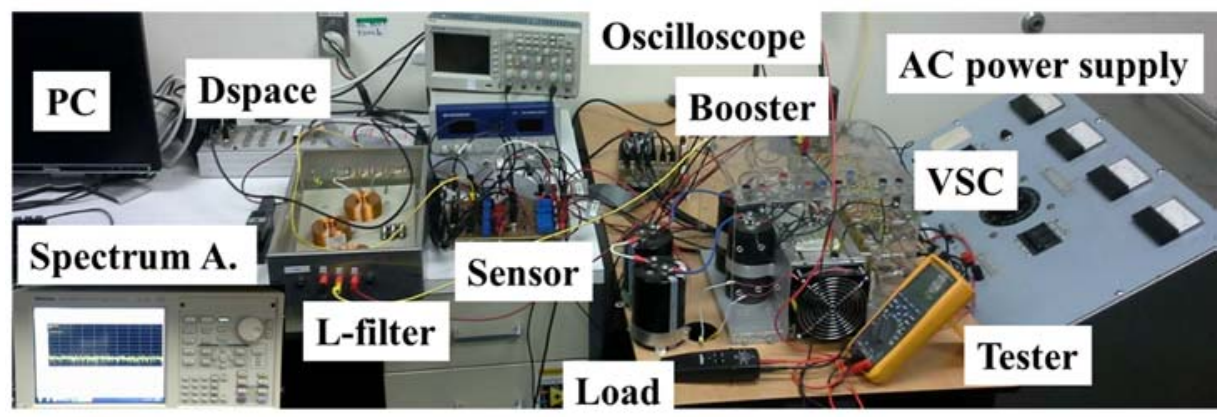

Figure 3.7 The experimental laboratory test bench setup.

\subsubsection{OVS-DCC Simulation and Experimental Results}

The predictive control used in this section is the optimum VSC's vector computation direct current control described in section IV-B. The simulation and experiment time is 6 sec. The simulation step time is $10^{-5} \mathrm{sec} .(100 \mathrm{kHz})$ whilst the acquisition frequency in the experiments is $20 \mathrm{kHz}$. In both types of results, a centered SPWM of $10 \mathrm{kHz}$ is used. The control algorithm is programmed in an embedded MatLab function to emulate a microprocessor with low-level programming language.

Table 3-3 The Setup Parameters of ARR

\begin{tabular}{|l|l|}
\hline Parameters & Value \\
\hline AC grid line-neutral voltage $\left(\tilde{V}_{g}\right)$ & $120 \mathrm{~V}_{\text {rms }}$ \\
\hline DC voltage supply $\left(V_{D C}\right)$ & $490 \mathrm{~V}$ \\
\hline Nominal grid line current $\left(I_{g}\right)$ & $\mathrm{G}=0.04$ of $\tilde{V}_{g}$ \\
\hline Switching frequency $\left(f_{s}\right)$ & $10 \mathrm{kHz}$ \\
\hline DC Load resistance $\left(R_{d c}\right)$ & $125 \Omega$ \\
\hline L-Filter $(L)$ & $12 \mathrm{mH}$ \\
\hline DC link capacitor $(C)$ & $1 \mathrm{mF}$ \\
\hline IGBT rating & $1200 \mathrm{~V}, 50 \mathrm{~A}$ \\
\hline
\end{tabular}




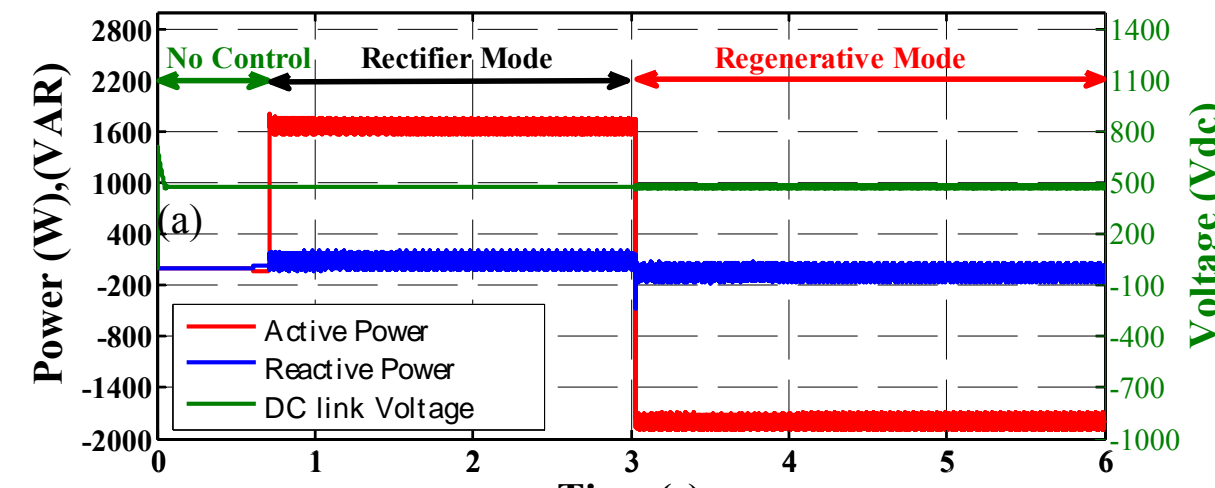

(a)

Time (s)

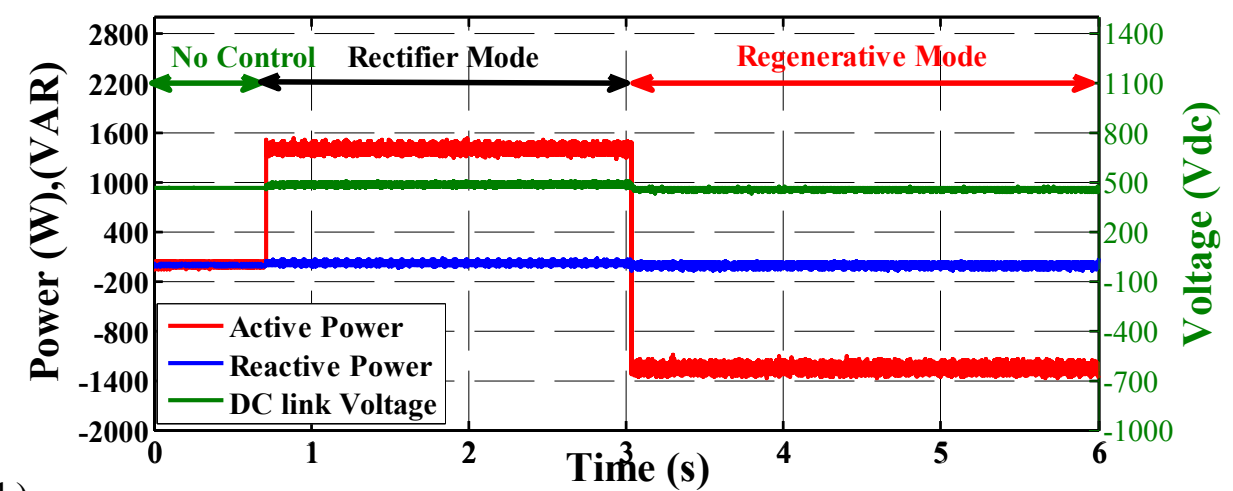

(b)

Figure 3.8 Active and reactive power, and dc-link voltage of the ARR. (a) Simulation and (b) Experimental results

From 0 to 0.715 secs. the ARR is operated at no control mode. After that, the ARR enters in rectification mode and then switches to regeneration mode at 3.03 secs. Figure 3.8 shows the three modes of operation. In Figure 3.8(a), during the no control mode, there is no active or reactive power flow. The dc load is being fed by the dc power supply. During the rectification mode, 1600 watts of active power flows from the ac to the dc side, keeping the reactive power at zero (unity PF operation). In the regeneration mode, 1600 watts flows from the dc to the ac side also maintaining the instantaneous reactive power in zero $(\mathrm{PF}=$ - 1). The same performance can be observed in the experimental results of Figure 3.8(b), however, the power flow is lower (1400 watts) due to the losses in the IGBTs and 
associated parasitic inductances, capacitors and resistances of wires, as well as ESR and ESL of capacitors.

Fast transient dynamic of the OVC-DCC can be observed from simulation and experimental results of Figure 3.8(a) and (b), respectively. In both cases, there is no power overshooting and almost instantaneous response at both transitions. For more detailed study, Figure 3.9 and Figure 3.10 show the grid line current for the two transition moments: control starting and control mode change. The figures present the actual grid line current, the reference current and the grid line-neutral voltage of the phase $a$, for the simulations and the experimental tests, respectively.

(a)
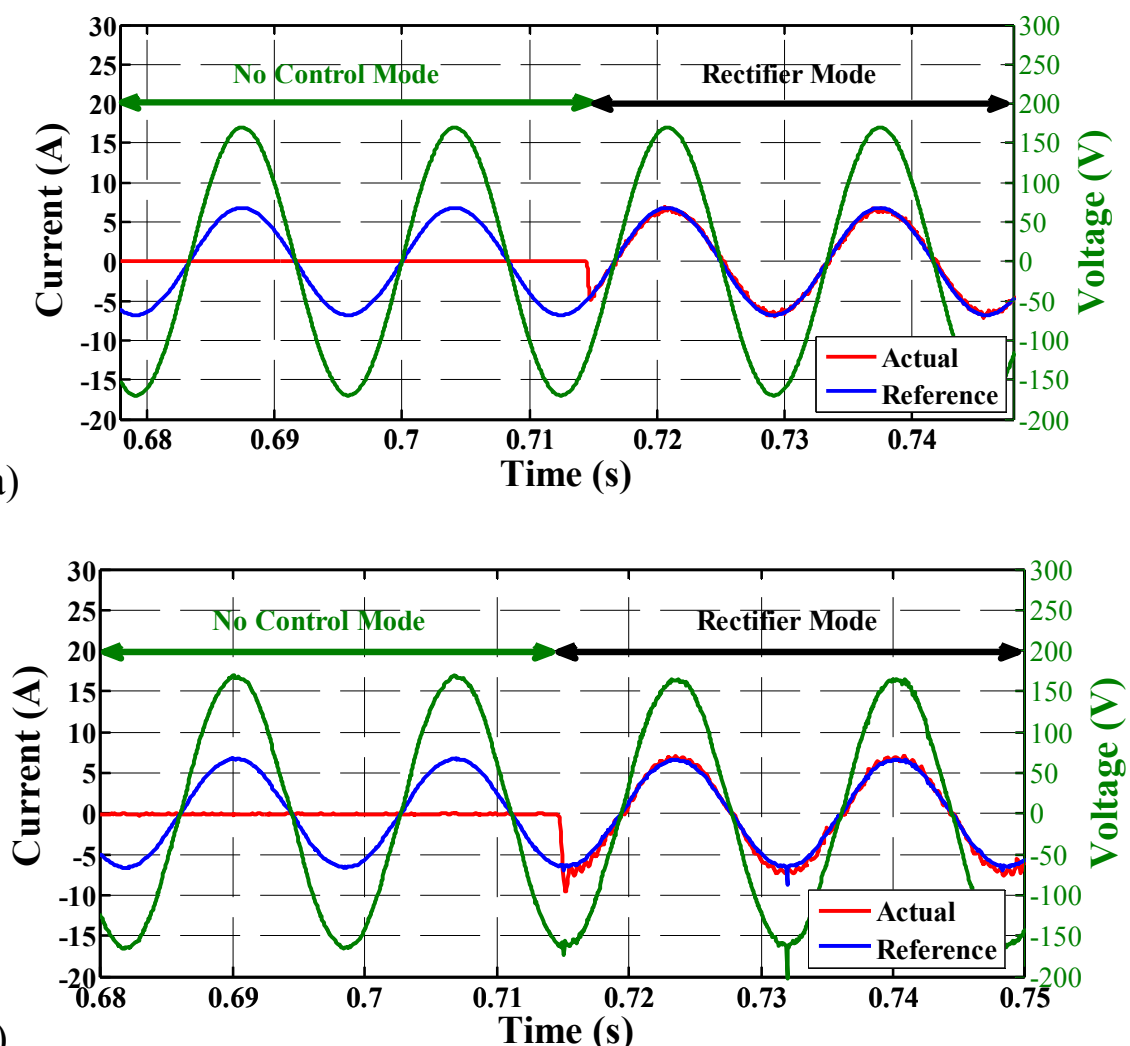

(b)

Time (s)

Figure 3.9 Control starting: grid line current, reference current and grid line-neutral voltage of phase $a$. (a) Simulation and (b) Experimental results. 


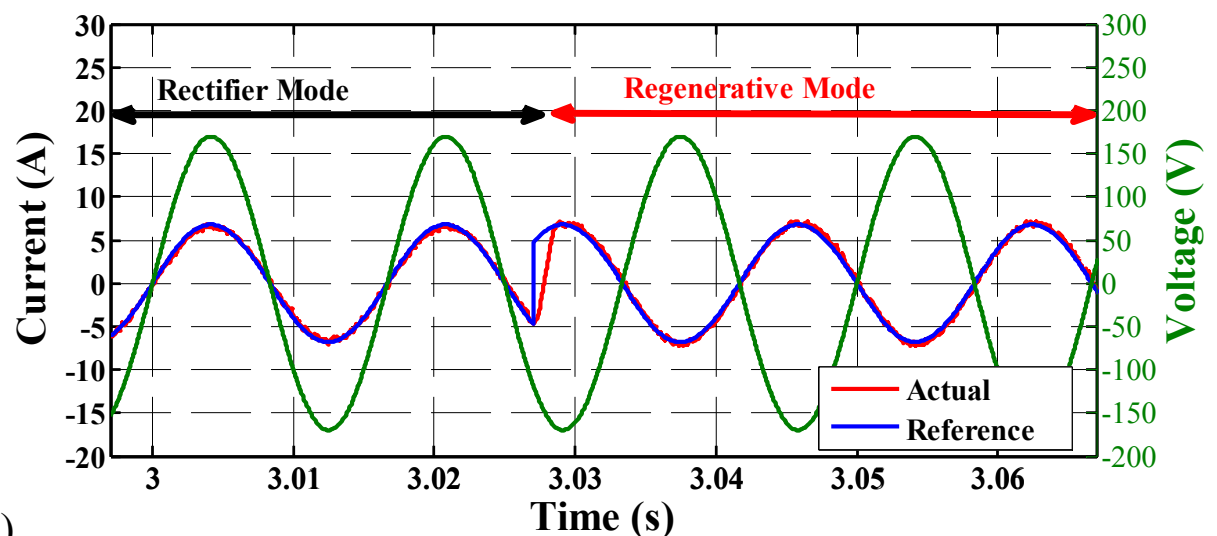

(a)

Time (s)

(b)

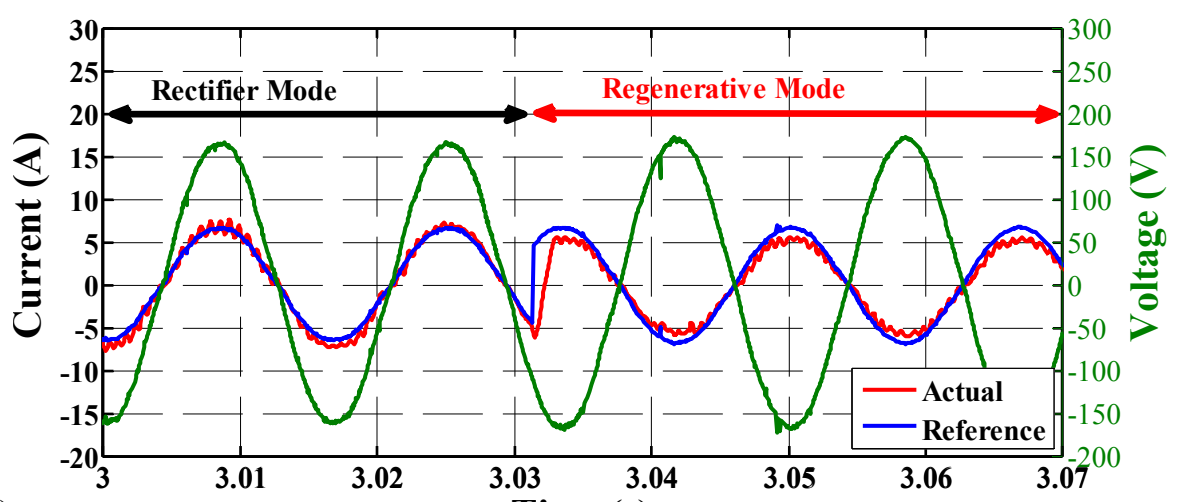

Time (s)

Figure 3.10 Control mode transient from rectification to regeneration: grid line current, reference current and grid line voltage of phase $a$. (a) Simulation and (b) Experimental results.

In Figure 3.9, previous to the control starting, the grid current is zero as the dc power supply voltage is higher than the ac peak line-to-line voltage, therefore the free-wheeling diodes of the IGBTs are inversed. Notice, once the control starts in rectification mode, the actual grid line current perfectly tracks the current reference $\left(i_{g a \_r e f}=G v_{g a}, G=0.04\right)$, achieving unity PF operation (in both simulation and experiment tests). In Figure 3.10, the control mode changes from rectification to regeneration. Before the transition, the current is in phase with the grid line-neutral voltage $(\mathrm{PF}=1)$ and after, the actual grid line current and current reference $\left(i_{L a \_r e f}=G v_{s a}, G=-0.04\right)$ are 180 degrees phase shifted $(\mathrm{PF}=-1)$. 
High correlation between simulation and experimental results can be observed, nevertheless, experimental results presents a slightly higher ripple. The quantification of the ripple is studied in the design results section.

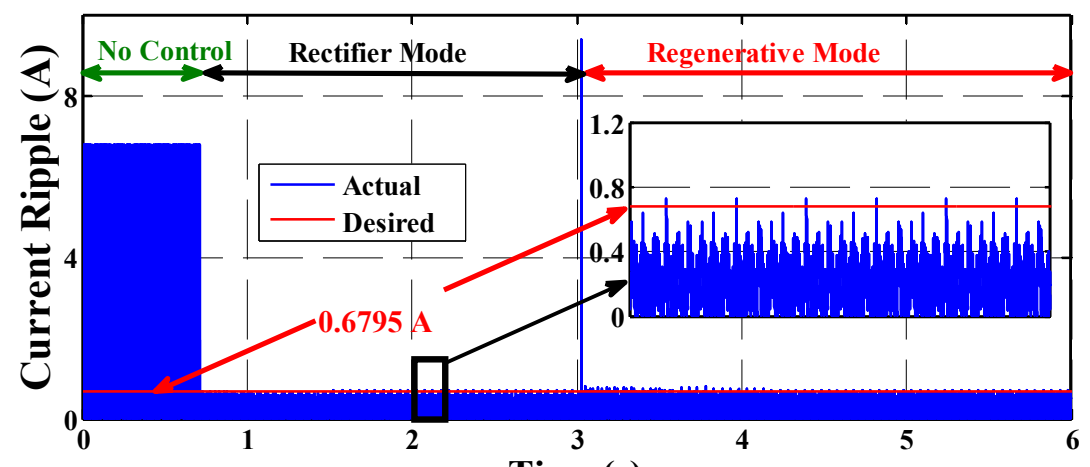

(a)

Time (s)

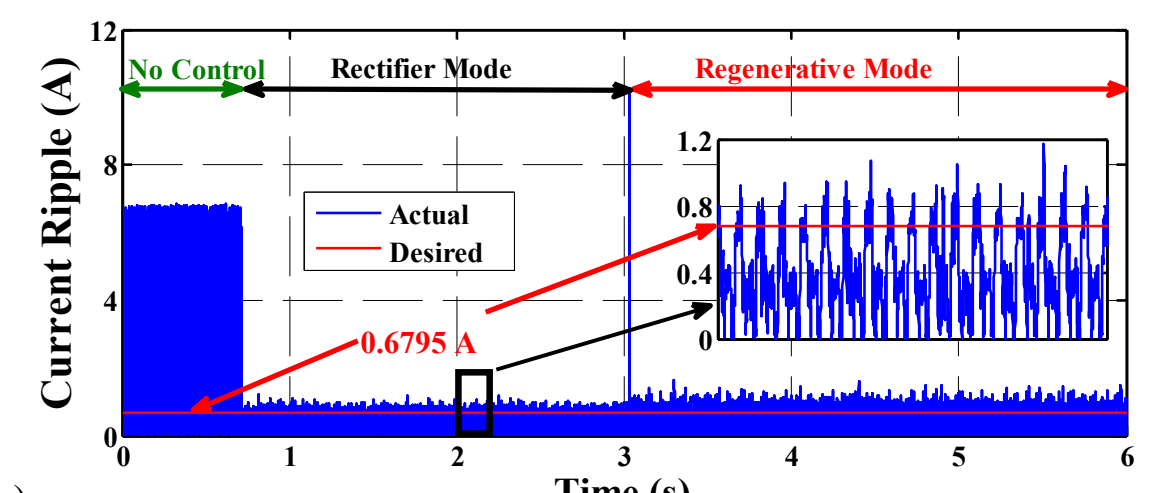

(b)

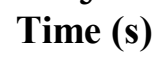

Figure 3.11 Current error or current line ripple of phase $a$. (a) Simulation and (b) Experimental results.

The actual grid line current ripple of the phase $a\left(\Delta i_{g a}\right)$ and the desired defined ripple $\left(I_{r p}\right)$ are presented in Figure 3.11, for the whole time of the simulation and experimental test. The grid line current ripple is defined in Figure 3.3 and it is computed as $\Delta i_{g a(k)}=$ $i_{g a \_r e f(k)}-i_{g a(k)}$ at every cycle of control $(10 \mathrm{kHz})$. The data acquisition, in the simulation, is $100 \mathrm{kHz}$, where 10 points decimation is programmed and synchronized with the SPWM. For the experimental results, 2 points decimation was performed. The desired 
current ripple is calculated from the design of minimum L-filter formula in (3.20). Thus, using the values in Table $3-3, I_{r p}=\frac{V_{D C}}{6 f_{s} L}=0.6795 A$. Notice that in the simulation (Figure 3.11(a)), the current ripple is $99.5 \%$ of the time below the desired value. Notwithstanding, in the experimental results (Figure 3.11(b)), the current ripple exceeds the design value due to the reasons mentioned in the introduction. Some of them are:

- The worst-case L-filter design is chosen as $L_{\text {min }}$, thus $\Delta i_{g a}$ will be maximum for the designed $I_{r p}$.

- The actual current ripple depends on the control algorithm, which is assumed in perfect operation for the design procedure.

- Errors of calibration in the acquisition and transducers nonlinearity affect the control performance.

- Parasitic components are not considered.

- VSC dead-time used in the experiments is not considered either in the simulation or in the theoretical analysis.

\subsubsection{Sensitivity analysis}

The proposed control strategy adjusts the action of control base on the ARR model. The main parameter in the ARR model is the L-filter. A sensitivity analysis is presented to evaluate the robustness of the proposed controller under uncertainty in this parameter. The inductance was changed over a range of $\pm 50 \%$. Each case was simulated using the same current profile of section VI-A. The MAE and RMSE among the instantaneous current reference in the phase $a$ are presented in Table 3-4. 
As it can be noted from Table 3-4, the MAE and RMSE are smaller than $6 \%$ and $12 \%$, respectively, indicating that the OVC-DCC is robust to the variation of the main parameter of the model. Similar MAE and RMSE are found for a correct value of the inductance.

Table 3-4 Maximum MAE and RMSE for L-Filter variation

\begin{tabular}{|c|c|c|c|c|c|c|c|c|}
\hline \multirow{2}{*}{$\begin{array}{c}\text { Real } \\
\text { Parameter }\end{array}$} & \multicolumn{2}{|c|}{$i_{g a}(\%)$} & \multirow{2}{*}{$\begin{array}{c}\text { Parameter } \\
\text { Changed } \\
\text { Increased } \\
(+50 \%)\end{array}$} & \multicolumn{2}{|c|}{$i_{g a}(\%)$} & \multirow{2}{*}{$\begin{array}{c}\text { Parameter } \\
\text { Changed } \\
\text { Decreased } \\
(-\mathbf{5 0 \% )}\end{array}$} & \multicolumn{2}{|c|}{$i_{g a}(\%)$} \\
\hline & MAE & RMSE & & MAE & RMSE & & MAE & RMSE \\
\hline$L=12 \mathrm{mH}$ & 5.21 & 11.37 & $L=6 \mathrm{mH}$ & 5.68 & 11.50 & $L=15 \mathrm{mH}$ & 5.70 & 11.78 \\
\hline
\end{tabular}

\subsubsection{Pulse Width Modulation Strategies: Comparative Study}

Two pulse width modulations, SPWM and SVM, are studied, simulated and compared as it is shown in Table 3-5. Three criteria are considered: switching losses $\left(P_{\text {loss }}\right)$, current distortion $\left(\right.$ THD $\left.I_{g}\right)$ and dynamic response (MAE and RMSE of $i_{g a}$ ).

Table 3-5 Maximum Criteria Values for each Modulation Technique

\begin{tabular}{|c|c|c|c|c|c|c|}
\hline \multirow{2}{*}{$\begin{array}{c}\text { Modulation } \\
\text { Technique }\end{array}$} & MAE $i_{g a}(\%)$ & \multirow{2}{*}{$R M S E i_{\text {ga }}(\%)$} & \multicolumn{3}{|c|}{$T H D i_{\text {ga }}(\%)$} & \multirow{2}{*}{$P_{\text {loss }}(W)$} \\
\cline { 4 - 6 } & & & Phase $a$ & Phase $b$ & Phase $c$ & \\
\hline PWM & 0.1148 & 0.2495 & 4.28 & 4 & 3.99 & 3.5917 \\
\hline SVM & 0.1064 & 0.2477 & 3.98 & 3.97 & 3.83 & 3.5644 \\
\hline
\end{tabular}

From Table 3-5, it can be observed that the SVM presents a slightly better performance in terms of less current error, current distortion, lower THD and lower losses. However, the difference is not significant.

\subsection{Simulation and Experimental Results: Design}

The ARR design consists on the selection of the L-filter and the dc-link capacitor. In this section, 4 designs for the L-filter and 3 designs for the dc-link capacitor are presented. 


\subsubsection{L-Filter Design Case Study}

In order to corroborate the L-filter design procedure, 4 cases have been chosen. The 4 cases are shown in Table 3-6, Figure 3.12 and Figure 3.13, for which four current ripples are calculated using the worst-case $\left(L_{\text {min }}\right)$ scenario in $(8), V_{D C}=400 \mathrm{~V}, I_{g}=0.03 V_{g}, \tilde{V}_{g}=$ $120 V_{r m s}$ and $f_{s}=10 \mathrm{kHz}$. Notice that $L_{\text {max }}$ is always the same, as it does not depend on the current ripple and it is selected for the stability issues.
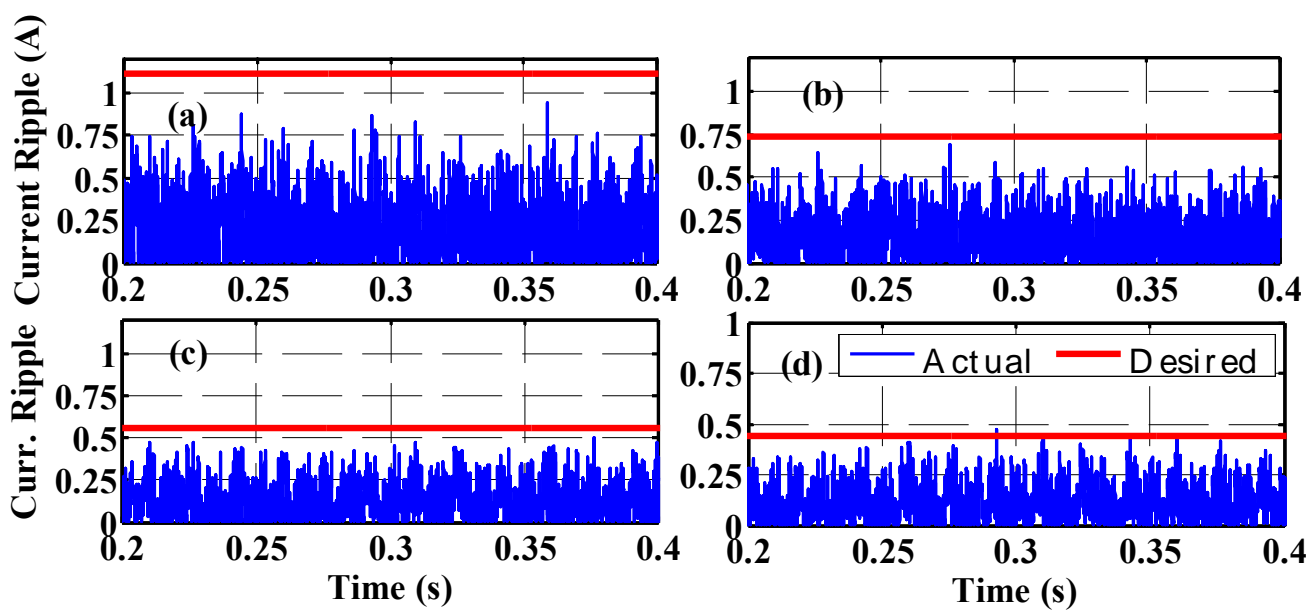

Figure 3.12 Simulated grid line current ripple and desired current ripple. (a) $\mathrm{L}=6 \mathrm{mH}$ (b) $\mathrm{L}=9 \mathrm{mH}$, (c) $\mathrm{L}=12 \mathrm{mH}$ and (d) $\mathrm{L}=15 \mathrm{mH}$.
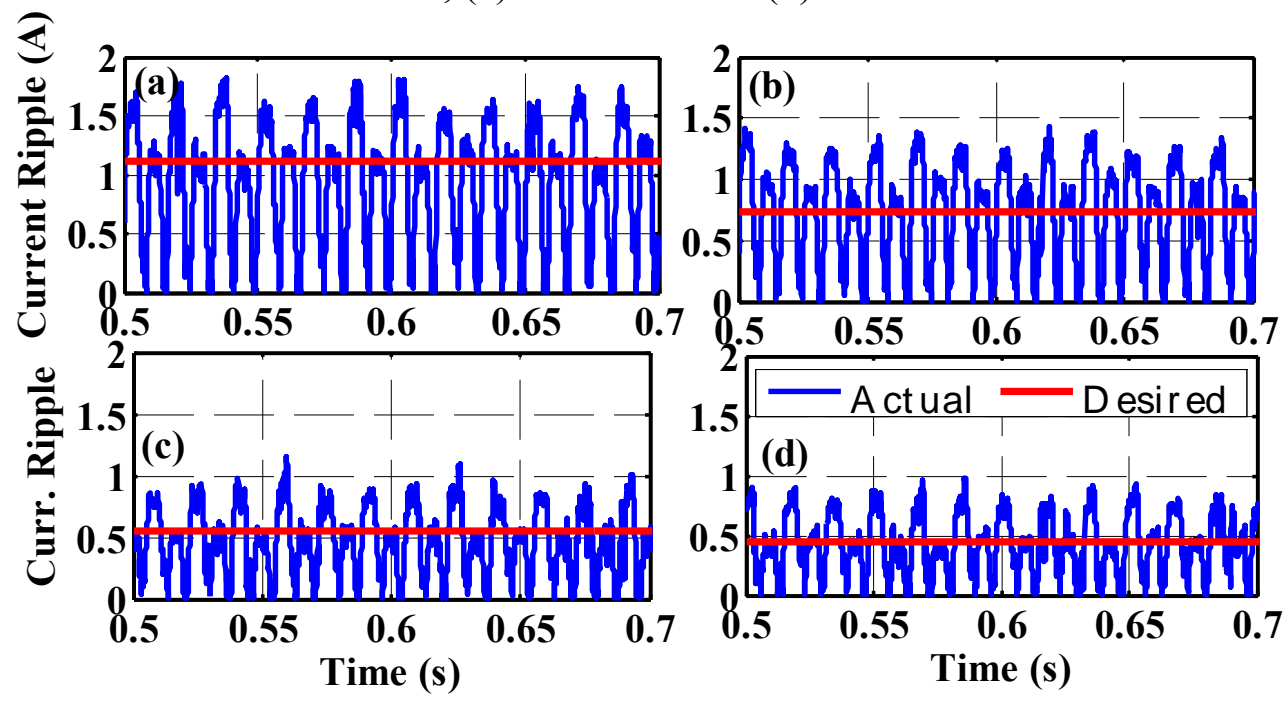

Figure 3.13 Experimental results of line current ripple and desired current ripple. (a) $L=6$ $\mathrm{mH}$ (b) $\mathrm{L}=9 \mathrm{mH}$, (c) $\mathrm{L}=12 \mathrm{mH}$ and (d) $\mathrm{L}=15 \mathrm{mH}$. 
In Figure 3.12 a zoom of the actual grid line current ripple from the simulation of the 4 L-filter design cases can be observed. In the 4 cases, the actual current ripple $\left(\Delta i_{g a}\right)$ is always below the desired current ripple $\left(I_{r p}\right)$. In Figure 3.13, the same analysis is presented for the experimental results. In this figure, the actual current ripple goes above the desired value due to the reasons discussed before. Table 3-6 summarizes the information shown in Figure 3.12 and Figure 3.13, where the average current ripple is computed. From these results, it can be concluded that the experimental current ripples are in average below the desired value of design.

Table 3-6 Comparative Results Desired Current Ripple

\begin{tabular}{|c|c|c|c|c|c|c|}
\hline \multicolumn{2}{|c|}{$L(m H)$} & $I_{r p}$ & \multicolumn{2}{c|}{$\Delta i_{g a}$ Simulation } & \multicolumn{2}{c|}{$\Delta i_{g a}$ Experimental } \\
\hline Min. & Max. & Desired & Max. & Mean & Max. & Mean \\
\hline 6 & 22 & 1.110 & 1.3037 & 0.2874 & 1.8601 & 0.9408 \\
\hline 9 & 22 & 0.740 & 0.7308 & 0.0224 & 1.431 & 0.7237 \\
\hline 12 & 22 & 0.555 & 0.5702 & 0.0181 & 1.159 & 0.4649 \\
\hline 15 & 22 & 0.444 & 0.5020 & 0.0153 & 0.9758 & 0.4284 \\
\hline
\end{tabular}

Finally, to verify the complementary frequency domain design procedure, the L-filter value equal to $12 \mathrm{mH}$ is chosen, with a desired current ripple of $0.555 \mathrm{~A}$. A MatLab script is written to compute the harmonic model of the current error as in (3.20) and the model of the VSC voltage as in (3.22).

Figure 3.14(a) demonstrates the FS modelling of the current ripple and the VSC converter waveform for $D=45 \%$. It is chosen $45 \%$ to show the apparition of the even harmonics. In a symmetric 50\% duty-cycle waveform, only odd harmonics appears. 


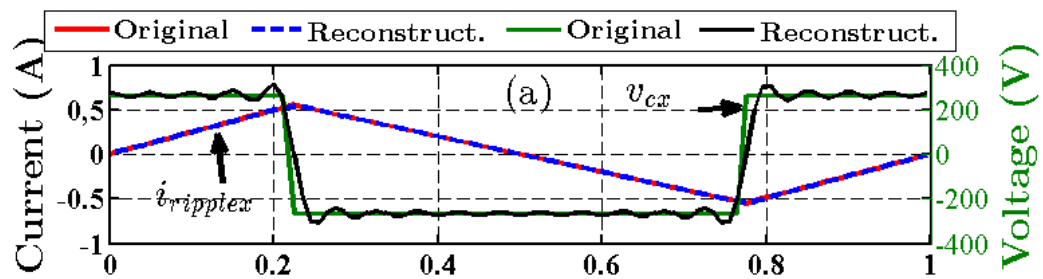

Time (s)

$\times 10^{-4}$
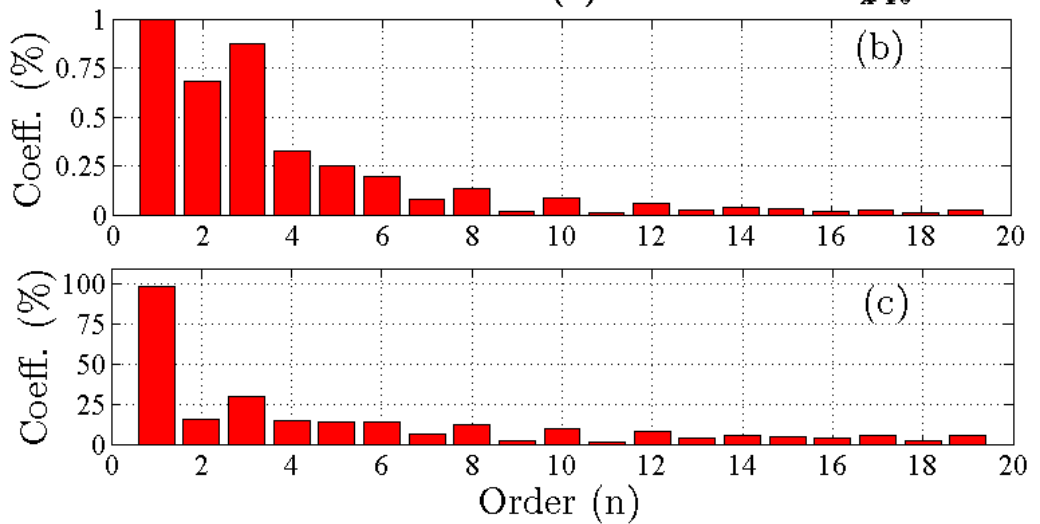

Figure 3.14 (a) Current ripple, VSC voltage and their reconstruction in FS (b) Current ripple FS Coefficients (c) VSC voltage FS Coefficients.
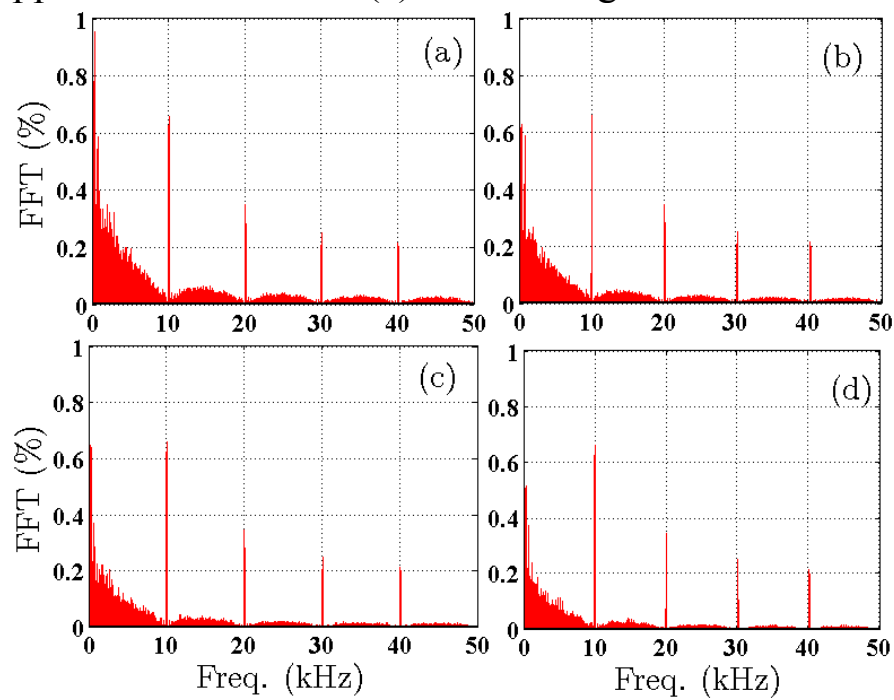

Figure 3.15 Simulated results of line current spectrum. (a) $\mathrm{L}=9 \mathrm{mH}$ (b) $\mathrm{L}=12 \mathrm{mH}$, (c) $\mathrm{L}=15 \mathrm{mH}$ and $(\mathrm{d}) \mathrm{L}=18 \mathrm{mH}$

In Figure 3.14(b) and (c) the FS coefficients of the current ripple and VSC converter are presented. In this FS, it is used 20 coefficients. From this analysis and using (3.21) it can be deduced that a minimum inductance of $12 \mathrm{mH}$ need to be designed for achieving the 
current ripple of $0.555 \mathrm{~A}$ (Figure 3.14(a)). Exactly the same value was found with the time domain analysis shown in Table 3-6, demonstrating thus the two methods are complementary.

The FFT (Matlab function) and spectrum analysis (from a Tektronix RSA 5103A Real Time Signal Analyzer) of the actual line current of the phase $a$ is presented in Figure 3.15 and Figure 3.16 for the simulation and the experimental tests respectively. In these figures the 4 inductance cases design are presented. It can be noticed that the second harmonic of $f_{S}$ appears as expected. Due to the acquisition resolution in the simulation $(100 \mathrm{kHz})$ the maximum frequency shown is until the fifth harmonic. Moreover, higher frequencies are more attenuated and they have very small effect.
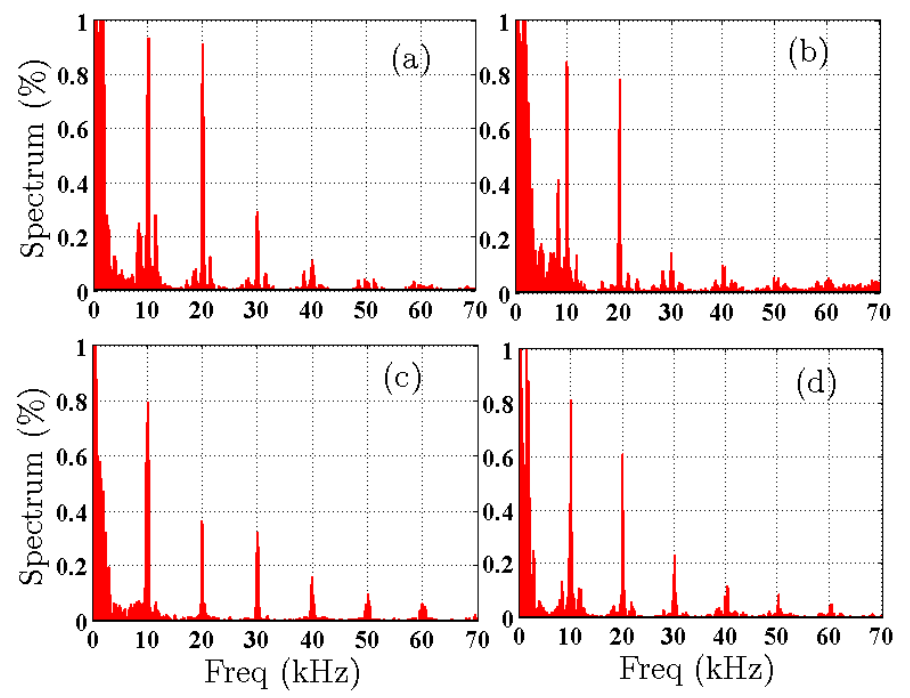

Figure 3.16 Experimental results of line current spectrum. (a) $\mathrm{L}=9 \mathrm{mH}$ (b) $\mathrm{L}=12 \mathrm{mH}$, (c) $\mathrm{L}=15 \mathrm{mH}$ and $(\mathrm{d}) \mathrm{L}=18 \mathrm{mH}$

A summary of the results in Figure 3.14(b), Figure 3.15 and Figure 3.16 for the case of $\mathrm{L}=12 \mathrm{mH}$ can be observed in Table 3-7. From this table a very good correlation between simulation and experimental results can be observed (as well as from Figure 3.15 
and Figure 3.16). The theoretical results has a huge discrepancy in the first harmonic, however the remaining harmonics are more correlated. This result difference comes from the assumptions considered in the frequency domain analysis, however, for practical issues it was demonstrated that the method is completely equivalent to the time domain for the inferior limit of the L-filter.

Table 3-7 Comparative Line Current Harmonics for $\mathrm{L}=0.012 \mathrm{H}$.

\begin{tabular}{|c|c|c|c|}
\hline Harm. order $f_{S}$ & Theoretical (\%) & Simulation (\%) & Experimental (\%) \\
\hline 1 & 8.72102 & 0.658 & 0.846 \\
\hline 2 & 0.682134 & 0.348 & 0.781 \\
\hline 3 & 0.874149 & 0.252 & 0.148 \\
\hline 4 & 0.324374 & 0.215 & 0.104 \\
\hline 5 & 0.249742 & 0.187 & 0.044 \\
\hline
\end{tabular}

\subsubsection{DC-link Capacitor Design Case Study}

Three cases of the $\mathrm{C}$ design are shown in Table 3-8, for which a synthesis procedure is performed. The values of the capacitors are selected as in the table. From (3.33), the maximum and minimum voltage ripples are calculated considering the same scenario as in the L-filter design. The maximum and minimum voltage ripples are in Table 3-8 and marked in Figure 3.17 with red and black lines. Figure 3.17 shows the dc-link voltage ripple for the 3 cases of capacitors. The left plots are experimental, whereas the right plots are simulation results. Also, Table 3-8 shows the summary of the results found in Figure 3.17. 
Experimentals
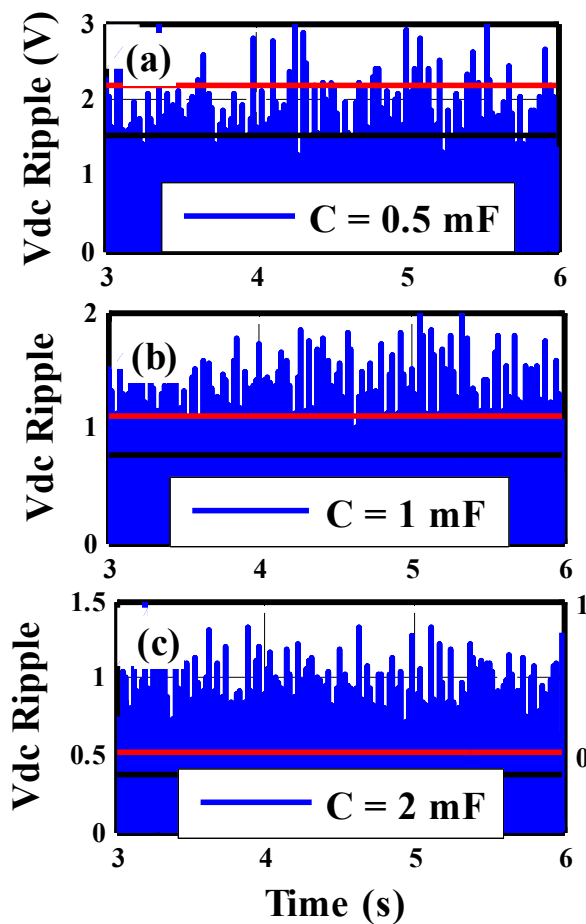

Simulations
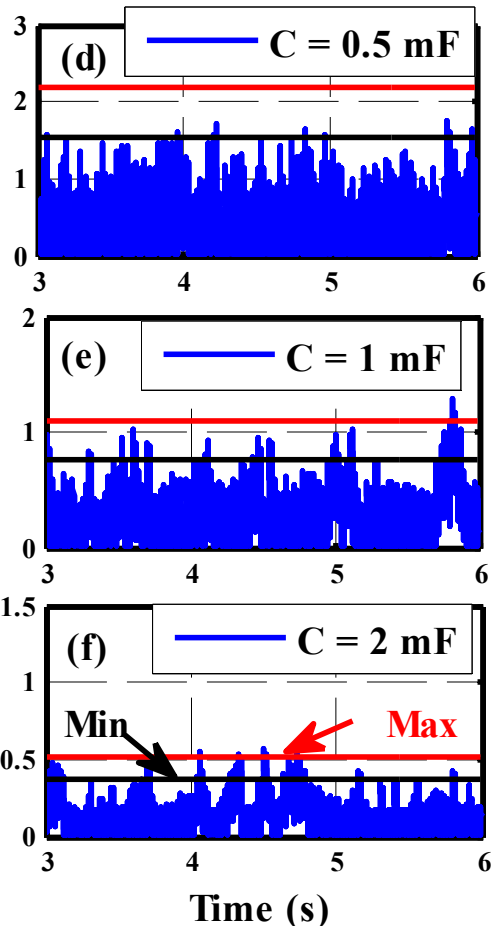

Figure 3.17DC-link voltage ripple results. Experimental (a) $C=0.5 \mathrm{mF}$ (b) $\mathrm{C}=1 \mathrm{mF}$, (c) $\mathrm{C}=2 \mathrm{mF}$ and simulation (d) $\mathrm{C}=0.5 \mathrm{mF}$, (e) $\mathrm{C}=1 \mathrm{mF}$, and (f) $\mathrm{C}=2 \mathrm{mF}$,

From Table 3-8, it can be noted that the simulations comply with the design procedure.

However, the voltage ripple from the experimental results is greater than the maximum limit value. This situation is explained by the assumptions in the design procedure:

1. Ideal capacitor by neglecting the ESR and equivalent series inductor (ESL).

2. The control is assumed to work perfectly.

Table 3-8 Comparative Results of DC-Link Voltage Ripple

\begin{tabular}{|c|c|c|c|c|}
\hline$C(\mathrm{mF})$ & \multicolumn{2}{|c|}{$V_{r p}(\mathrm{~V})$} & $\Delta v_{D C}$ Sim. $(\mathrm{V})$ & $\Delta v_{D C}$ Exp. $(\mathrm{V})$ \\
\hline Value & Min. & Max. & Value & Value \\
\hline 0.5 & 1.5300 & 2.1898 & 1.7694 & 3.5428 \\
\hline 1 & 0.7650 & 1.0949 & 1.2789 & 2.0389 \\
\hline 2 & 0.3643 & 0.5214 & 0.5725 & 1.5372 \\
\hline
\end{tabular}




\subsection{Conclusions}

A comprehensive study of the ARR is performed in this chapter, starting with an exhaustive literature review, classification and summarization of the control and design strategies. On top of that, the chapter proposes an improved and general design methodology for the passive components of the ARR. The novelty of the L-filter design strategy lies on the consideration of the modulation technique (centered-SPWM) in a simple manner to reach convergence with the current harmonic analysis in frequency domain. The dc-link capacitor design is based on the dc-link voltage ripple and power balancing. Both design strategies (L-filter and dc-link capacitor) were demonstrated and verified by means of simulation and experimental results. All these tests were performed under the ARR being controlled by a deadbeat-type predictive controller (OVC-DCC). Based on non-linear theory, a complete analysis of the OVC-DCC for a typical VSC is developed for the first time in the literature to the best of the author's knowledge. The dynamic performance and accuracy of the proposed controller was tested and corroborated also by means of simulations and experimental results where indices, such as MAE and RMSE, were computed. Two modulation strategies were compared (SPWM and SVM) under 3 criteria: dynamic performance, current THD and losses, finding that SVM performs better. A sensitivity study is performed to test the ARR parameter independency under diverse operating conditions. It is concluded that the OVC-DCC is highly robust to parameter uncertainty of $\pm 50 \%$. 


\section{Chapter 4 Design of Power Converters in Transportation Electrification Applications}

\subsection{Introduction}

As mentioned in the power electronic topologies selection of Chapter 2, we will discuss the design of a of dc-dc Cuk converter. This chapter investigates the effect of components' placement on the radiated emissions of the printed circuit board (PCB) of dc-dc power converters. The main goal of this chapter is to find the best position and orientation for the passive components on the PCB to minimize the electromagnetic interference (EMI) at a specific location on the board. The optimum design for the location and orientation of the components on the PCB is achieved by Genetic Algorithms (GA) optimization. The novelty of the design procedure lies on the fitness function programming, where an interface software platform is built through MatLab scripting to connect a 3D-Finite Element Analysis (FEA) and the GA. The FEA addresses the radiated EM calculation while the GA focuses on minimizing it. A comparison between the optimum and nonoptimum PCB designs were simulated and experimentally tested to verify the advantages of the proposed design technique. For this study, the Cuk converter is selected, as it presents a larger quantity of passive components (2 inductors and 2 capacitors).

\subsection{Optimizing Power Converter PCB Design for Lower EMI}

The wide use of high-frequency power electronic converters in high-power applications causes EMI. Electromagnetic compatibility is becoming a critical issue for power converters manufacturers, as the products should satisfy the national and the international standards, such as CISPR22, FCC part 15, EN 55022 and MIL-STD-464. Power converter designs should comply with the EMI standard limits. The switched operation of power 
electronic converters generates a high-frequency current and voltage ripples, which are mainly the source of the EMI problem.

EMI can propagate in the system by conduction or radiation. The conducted EMI travels through the circuit wiring while the radiated one grows by the inductive and capacitive coupling phenomena. The existence of inductive and capacitive components in the high-frequency power converter circuit spreads the electromagnetic waves in the medium. These waves are received and coupled, by other inductive and capacitive components in the circuit, with more or less degree depending on their relative position, dimension and function in the circuit.

Extensive attention is being given by researchers to mitigate the effect of conducted EMI. In [76] a methodology for EMI reduction based on using EMI filters is presented. Although these methods decrease the EMI, they add extra components to the system and increase the cost. Other research focuses on improving the modulation techniques of the controller to suppress EMI with emphasis on the conduction form [77]. These techniques need special EM sensors, higher data processing speed and memory, and complex control algorithms.

Recently, a plethora of studies are based on solving the EMI problem in the pre-design stage using numerical analysis to predict the radiated and conducted EMI behavior of the PCB [78]-[82]. References [78], [81] presented an optimized technique to locate the components on the PCB. In these works, very limited cases are considered, without applying optimization. In [83], far-field emissions analysis for the conducted and radiated EMI was presented. However, no near-field radiated analysis were performed. In [84], a method for placing the components, which have magnetic emissions, on a PCB is 
presented. The technique is based on calculating the magnetic coupling among these components to compute the set of minimum distance and rotation angle between them, and thus minimize the conducted EMI and PCB's volume. It is clear that less work has been done for the radiated EMI compared with the conducted EMI. The main reason is that the radiated EMI requires modeling of the physical geometry of the $\mathrm{PCB}$, as well as electric circuit analysis to compute the electromagnetic field distribution.

Currently, there is no software available to study the design of a PCB that helps to comply with the limit standards of radiated emission IEC61800-3. In this chapter, an optimization technique is proposed to define the components' position in a PCB of a highfrequency power converter reducing its stray fields to comply the standards in the predesign stage. The optimum design for the PCB is achieved by the combination of a quasistatic field analysis in a 3D-FE program and GA. The proposed technique is based on varying the distance and angle between passive components. These variations search for the cancelation of their radiated coupling effects at the point of interest on the PCB.

\subsection{Modeling of the DC-DC Converter}

\subsubsection{DC-DC Cuk converter}

DC-DC Cuk converter is commonly used in many control system applications. It provides an output voltage, which can be less or greater than the input voltage. The circuit diagram of Cuk converter is shown in Figure 4.1, which consists of a de voltage source, input inductor $\left(L_{l}\right)$, a power switch and a diode. Also, it usually has a filter capacitor $(C)$ and filter inductor $\left(L_{2}\right)$ to smooth the output. This topology is commonly used in water pump photovoltaic systems as a maximum power point tracker, where the EM susceptibility of the current transducer is a critical issue for the performance of the 
controller [85], [86]. In [87], this converter is used for MPPT purposes, as well as for driving the dc motor that actuates the water pump. The current sensor LA25-NP is used in this investigation as a sensitive device for studying the EMI effect.

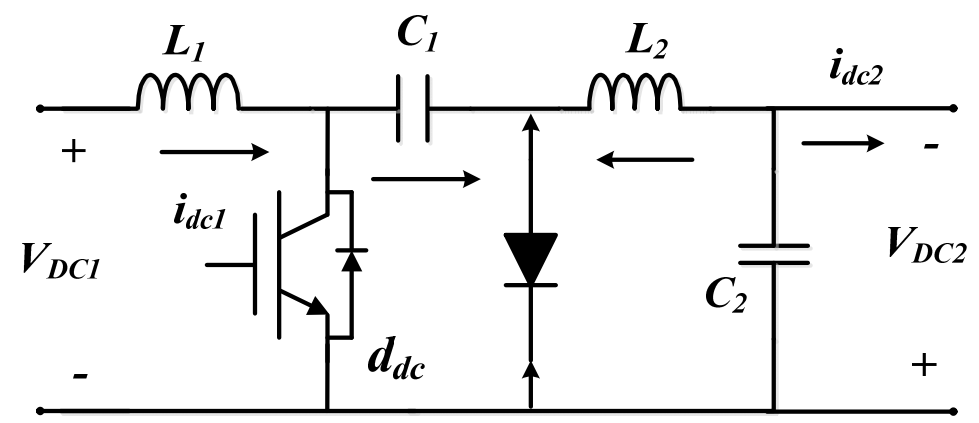

Figure 4.1 Circuit diagram of Cuk converter.

\subsubsection{Converter 3D-FE Model}

3D-FE analysis is a numerical tool, which provides an efficient and adequately accurate approach for physics-based modeling of the electric components. Thus, a 3D-FE model for Cuk converter is built to be used in this work. In this model, only the inductive and capacitive components are modeled numerically, as they have the most effect on radiated EMI propagation. The other components are considered as ideal and have no effect on the radiated EMI. Figure 4.2 shows the 3D-FE model of the dc-dc Cuk converter, which is used in this study.

\subsubsection{Electromagnetic Field Computation}

The electromagnetic field inside and outside the electrical components is governed by the following nonlinear partial differential equations:

$$
\nabla^{2} \phi+\frac{\partial}{\partial t}(\nabla \cdot \overrightarrow{\mathrm{A}})=\frac{\rho_{e}}{\varepsilon}
$$




$$
\nabla \times(v(\nabla \times \vec{A}))=\vec{J}_{e}+\sigma \frac{\partial \vec{A}}{\partial t}-\sigma(\nabla \phi)
$$

where $\phi$ is the electrostatic scalar potential, $\vec{A}$ is the magnetic vector potential (MVP), $\rho_{e}$ is the charge density $\left[\mathrm{C} / \mathrm{m}^{3}\right], \varepsilon$ is the permittivity of the medium $[\mathrm{F} / \mathrm{m}]$ and $J_{e}$ is the excitation current density vector $\left[\mathrm{A} / \mathrm{m}^{2}\right]$.

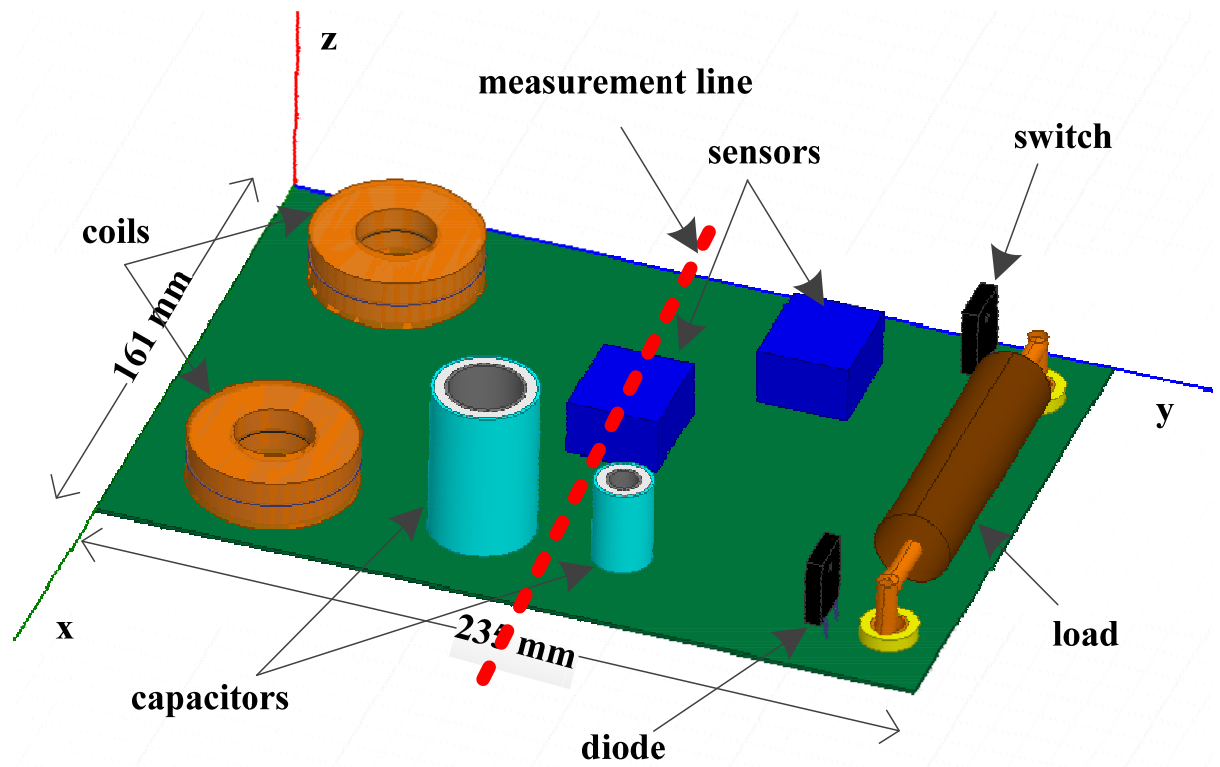

Figure 4.2 3D-FE model of Cuk converter

The excitation waveforms supplied to the FE model are calculated in the MatLab simulation. The second term on the RHS of (4.2) is used to analyze the Eddy currents effect resulted in the high-frequency switching operation $(40 \mathrm{kHz})$. A linear magnetization curve for the magnetic components was considered. The 3D-FE model utilizes the elemental equations for first-order tetrahedral elements, as shown in (4.3) [88]:

$$
\nabla \vec{A}_{i}(x, y, z)=\frac{1}{4}\left(\vec{A}_{1 i}+\vec{A}_{2 i}+\vec{A}_{3 i}+\vec{A}_{4 i}\right)
$$

where sub-indexes $1,2,3$ and 4 are the vertices of the tetrahedral element, $\vec{A}_{x i}$ is MVP of each vertices. This vector is used for calculation of the magnetic field $(\vec{H})$. Once the current 
distribution of the magnetic field is obtained, an accurate 3-D profile of the radiated magnetic field is calculated. The vector components of the magnetic field can be deduced from similar analysis of other tetrahedral elements oriented along the $\mathrm{x}, \mathrm{y}$ and $\mathrm{z}$-axes, which captures the 3-D electromagnetic solver. Then, the superposition principle is used to calculate the magnetic field at any arbitrary point $\mathrm{P}\left(x_{p}, y_{p}, z_{p}\right)$.

\subsection{Proposed Methodology: 3D-FE-GA}

This chapter proposes an effective methodology to find the optimum location and angle of the passive components in a PCB to cancel their radiated coupling effects at the point of interest. To achieve this purpose an interface platform is built based on the combination between GA and 3D-FE. The GA is applied using MatLab optimization toolbox while the 3D-FE is implemented in Magnet Infolityca software.

\subsubsection{D-Finite Element considerations}

The location of the device refers to its position on the PCB and the orientation is the axial rotation relative to the center of the device. In order to consider most of the possible positions and orientations, the optimization is divided into several cases. The proposed technique is based on five practical cases with different positions of the inductors and four cases for the capacitors. In these cases, the important parameters are:

1. The distance between the components (d).

2. The angle of orientation between the components $(\theta)$.

In all cases, one device is moved and the other is kept fixed. The different cases for coils are summarized in Table 4-1, and those for capacitors are shown in Table 4-2. In Table 4-1, the positive coil current means that the two coils have the same current direction, while the negative coil current means that they have opposite current directions. As a design 
constraint, the sensitive device (current sensor), is located in the center of the board, as shown in Figure 4.2.

Table 4-1 Cases of Positioning for Inductors

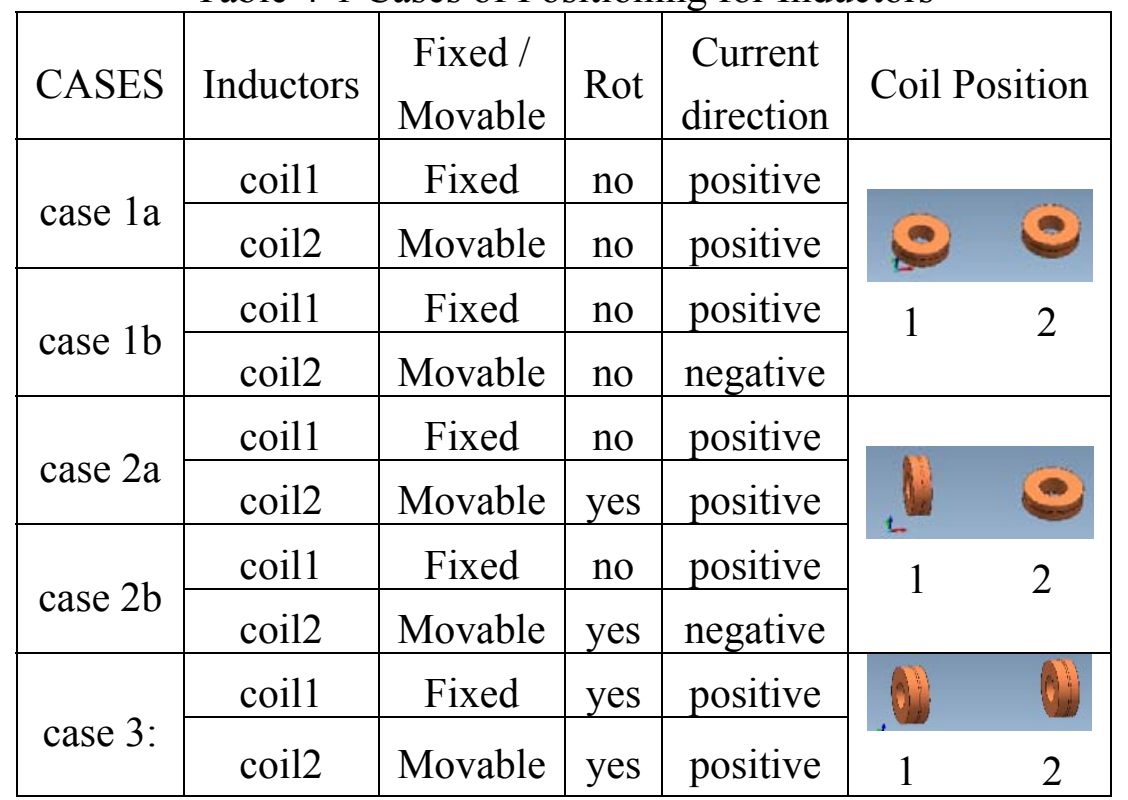

Table 4-2 Cases of positioning for Capacitors

\begin{tabular}{|c|c|c|c|c|}
\hline CASES & Capacitor & Fixed / Movable & Rotation & Capacitor Position \\
\hline \multirow{2}{*}{ CASE 1a } & Cap.1 & Fixed & no & \multirow{2}{*}{0} \\
\hline & Cap. 2 & Movable & no & \\
\hline \multirow{2}{*}{ CASE $1 b$} & Cap.1 & Movable & no & \multirow{2}{*}{2} \\
\hline & Cap. 2 & Fixed & no & \\
\hline \multirow{2}{*}{ CASE 2} & Cap.1 & Fixed & no & \multirow[t]{2}{*}{0} \\
\hline & Cap. 2 & Movable & yes & \\
\hline \multirow{2}{*}{ CASE 3} & Cap.1 & Fixed & no & 0 \\
\hline & Cap. 2 & Movable & yes & 1 \\
\hline
\end{tabular}

\subsubsection{Genetic Algorithm Optimization Procedure}

In our particular case, the genetic algorithm inputs (chromosomes) have the information for the variation of the distances $(\Delta x, \Delta y)$ and also the angle of rotation $(\Delta \theta)$. 
The setup for the genetic algorithm in one case of optimization, as an example, is shown in Table III.

Table 4-3 GA Setting

\begin{tabular}{|c|c|c|c|c|}
\hline \multicolumn{2}{|c|}{ Property } & Values & Property & Values \\
\hline \multirow{2}{*}{ Number of variables } & 3 & Mutation & Uniform $80 \%$ \\
\hline \multirow{3}{*}{ Bounds } & Lower & {$\left[\begin{array}{lll}0 & 0 & 0\end{array}\right]$} & Crossover & Single point \\
\cline { 2 - 5 } & Upper & {$\left[\begin{array}{llll}161 & 235 & 360\end{array}\right]$} & Generations & 10 \\
\hline \multicolumn{2}{|c|}{ Population } & 20 & - & - \\
\hline
\end{tabular}

The GA finds the optimum location and orientation of the passive components to get the minimum radiated magnetic and electric field at the sensitive device. The optimization process is achieved through two stages:

1. Coils optimization by Magnet (Infolytica) considering the magnetic field only. Once all the cases are optimized, the best case among the 5 coil cases is chosen. When the optimum coils' position become known, the second stage starts.

2. In this stage, the capacitors are optimized by Elecnet (Infolytica) considering the electric field only. In this stage, the 4 cases are optimized, and the best case is chosen.

The procedure and fitness function for the GA optimization is described by the flowchart in Figure 4.3 and explained as follows:

1: The GA generates random values for the variables $(\Delta x, \Delta y, \Delta \theta)$, and passes them to the MatLab script fitness function for placement and orientation of the movable component.

2: The MatLab script verifies if the movable component's position is overlapping with the other components on the PCB. If there is overlapping, then a linear penalization will be applied depending on the overlapped area. The penalization is as in (4.4). 


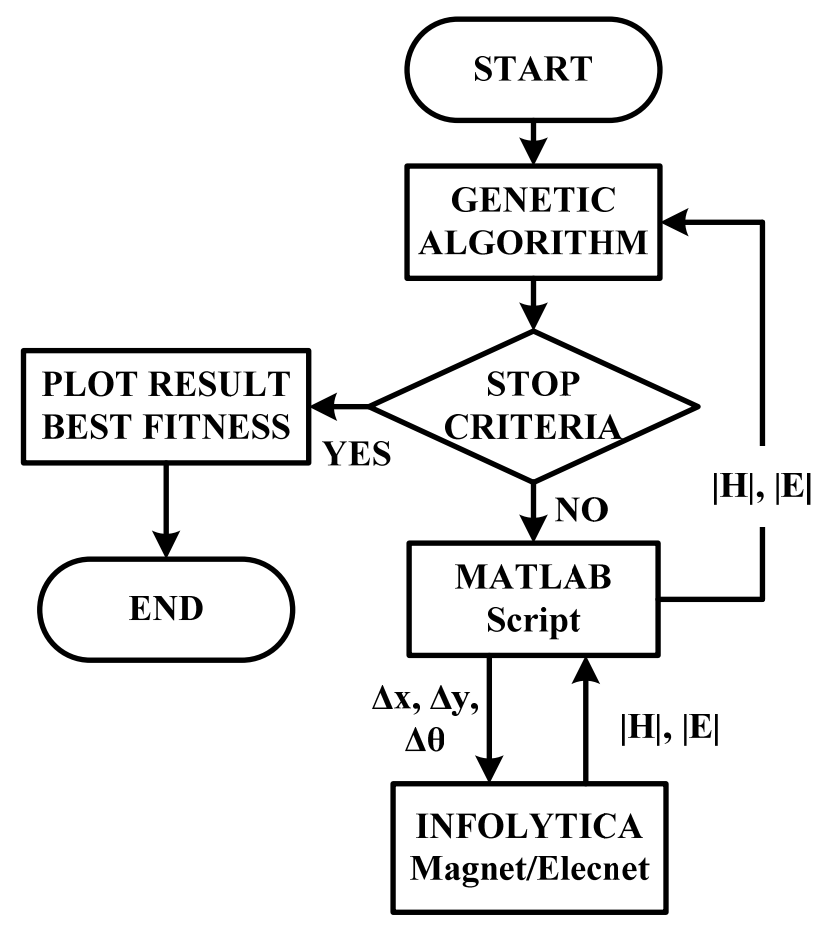

Figure 4.3 GA and fitness function for the optimization.

\begin{tabular}{|l|l|}
\hline $\begin{array}{l}\text { Cost }=|\vec{H}| \\
\text { If (overlapping = 1) }\end{array}$ & (4.4) \\
& $\quad|\vec{H}|=k *$ overlapped Area \\
else continue STEP 3 & \\
end & \\
\hline
\end{tabular}

where, " $k$ " is a constant value heuristically set in $20 \mathrm{~A} / \mathrm{m}$ for $|\vec{H}|$. The same procedure is carried out for the electric field.

3: If there is no overlapping, the MatLab script calls Infolytica program and passes random values of the variables $\Delta x, \Delta y$ and $\Delta \theta$.

4: The MatLab script summons the FE program to solve the model and passes the random values of the variables $\Delta x, \Delta y$ and $\Delta \theta$ to modify and shift the components in the FE model.

5: The converter model is analyzed in the 3D-FE software and the radiated magnetic $(|\vec{H}|)$ 
or electric field $(|\vec{E}|)$ is calculated at the line that crosses the center of the sensitive device, (the measurement line in Figure 4.2).

6: Once the 3D-FE analysis is finished, Infolytica passes the magnitude of the field $(|\vec{H}|$ or $|\vec{E}|)$ to the MatLab script.

7: The MatLab script returns the value of the field to the GA tool as the cost (4.3).

8: The GA tool internally processes the crossover, mutation and reproduction. It checks the stop criteria and if it is not reached, then it generates a new, random set of movement and rotation $(\Delta x, \Delta y, \Delta \theta)$, and the whole procedure is repeated again.

\subsection{Simulated Results}

The passive components in the PCB of the Cuk converter are modeled by FEA and simulated by the Infolytica software. The simulation conditions and the components' parameters are indicated in Table 4-4.

Table 4-4 Simulation Parameters

\begin{tabular}{|c|c|c|c|}
\hline Parameter & Value & Parameter & Value \\
\hline Frequency & $40 \mathrm{kHz}$ & Duty cycle & $50 \%$ \\
\hline Coil 1 inductance $\left(\mathrm{L}_{1}\right)$ & $1.35 \mathrm{mH}$ & Load resistance & $10 \Omega$ \\
\hline Coil 2 inductance $\left(\mathrm{L}_{2}\right)$ & $1.35 \mathrm{mH}$ & Input voltage & $38 \mathrm{~V}$ \\
\hline Capacitance $1\left(\mathrm{C}_{1}\right)$ & $470 \mathrm{uF}$ & Output voltage & $-33 \mathrm{~V}$ \\
\hline Load capacitance $(\mathrm{C})$ & $47 \mathrm{uF}$ & & \\
\hline
\end{tabular}

\subsubsection{Optimization Results}

The results of the proposed optimization technique is presented in this section. Figure 4.4 and Figure 4.5 indicate the best fitness values for each generation for all cases of the coils and capacitors, respectively. As it is clear from Figure 4.4, the best position for the 
coils is the case 1a, and for the capacitor is the case 1a also (see Table 4-1 and Table 4-2, respectively).

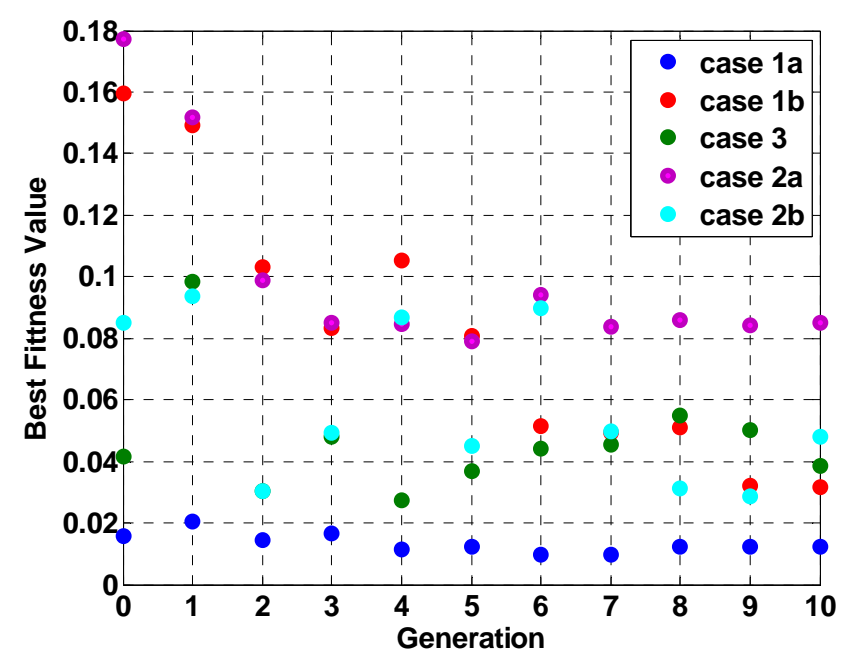

Figure 4.4 Optimization results for the coil cases.

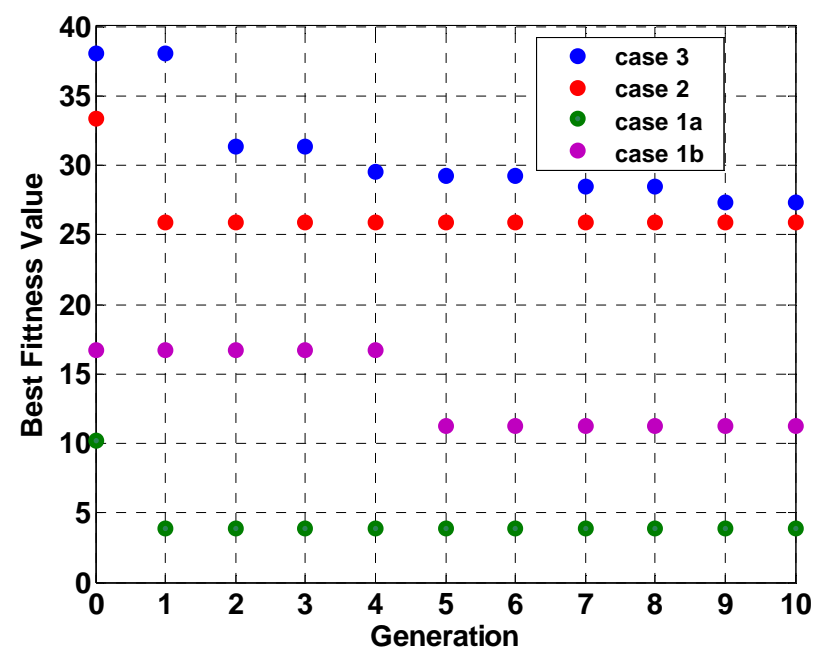

Figure 4.5 Optimization results for the capacitor cases.

The final positions of the passive components in the PCB, according to the optimization results, are summarized in Table 4-5. The computation time for each run of the simulation is estimated as 71 seconds. Each optimization case is 7100 seconds. Even, when this 
computation time appears to be large, the optimization is performed only one time in the pre-designed stage for the converter under design.

Table 4-5 Optimum Components Position

\begin{tabular}{|l|c|c|}
\hline \multirow{2}{*}{ COMPONENT } & \multicolumn{2}{c|}{ CENTER POSITION (X,Y) } \\
\cline { 2 - 3 } & Non-optimal & Optimal \\
\hline Coil1 (movable) & $(75,25)$ & $(27.0758,25)$ \\
\hline Coil2 (fixed) & $(135,25)$ & $(135,25)$ \\
\hline Capacitor1 (movable) & $(56,219)$ & $(17.038,219)$ \\
\hline Capacitor2 (fixed) & $(150,225)$ & $(150,225)$ \\
\hline
\end{tabular}

\subsubsection{Optimal and Non-optimal Coils position.}

Following the completion of the optimization steps, the passive components are rearranged on the PCB to match the optimum positions. A quasi-static field analysis using Maxwell software is applied for the original and the optimum arrangements. The magnetic field distribution in the $\mathrm{PCB}$ is shown in Fig. 6. The figure indicates that the field diminishes at the sensor region, as compared with the original arrangement.

The magnitude of the magnetic field is drawn along a line in the $\mathrm{x}$-direction and passes through the sensor's center (the measurement line Figure 4.2) for the original and optimum arrangement, as shown in Figure 4.7. The field magnitude at the sensor's center is reduced from $8.559 \times 10^{-5} \mathrm{AT} / \mathrm{m}$, for the non-optimum arrangement, to $4.211 \times 10^{-5} \mathrm{AT} / \mathrm{m}$, for the optimum arrangement. Figure 4.8 shows the magnetic field magnitude along the same line for the optimum arrangement at different heights (at bottom, middle and top of the sensor). The figure indicates that the proposed optimization topology is not affected significantly by the height variation. 

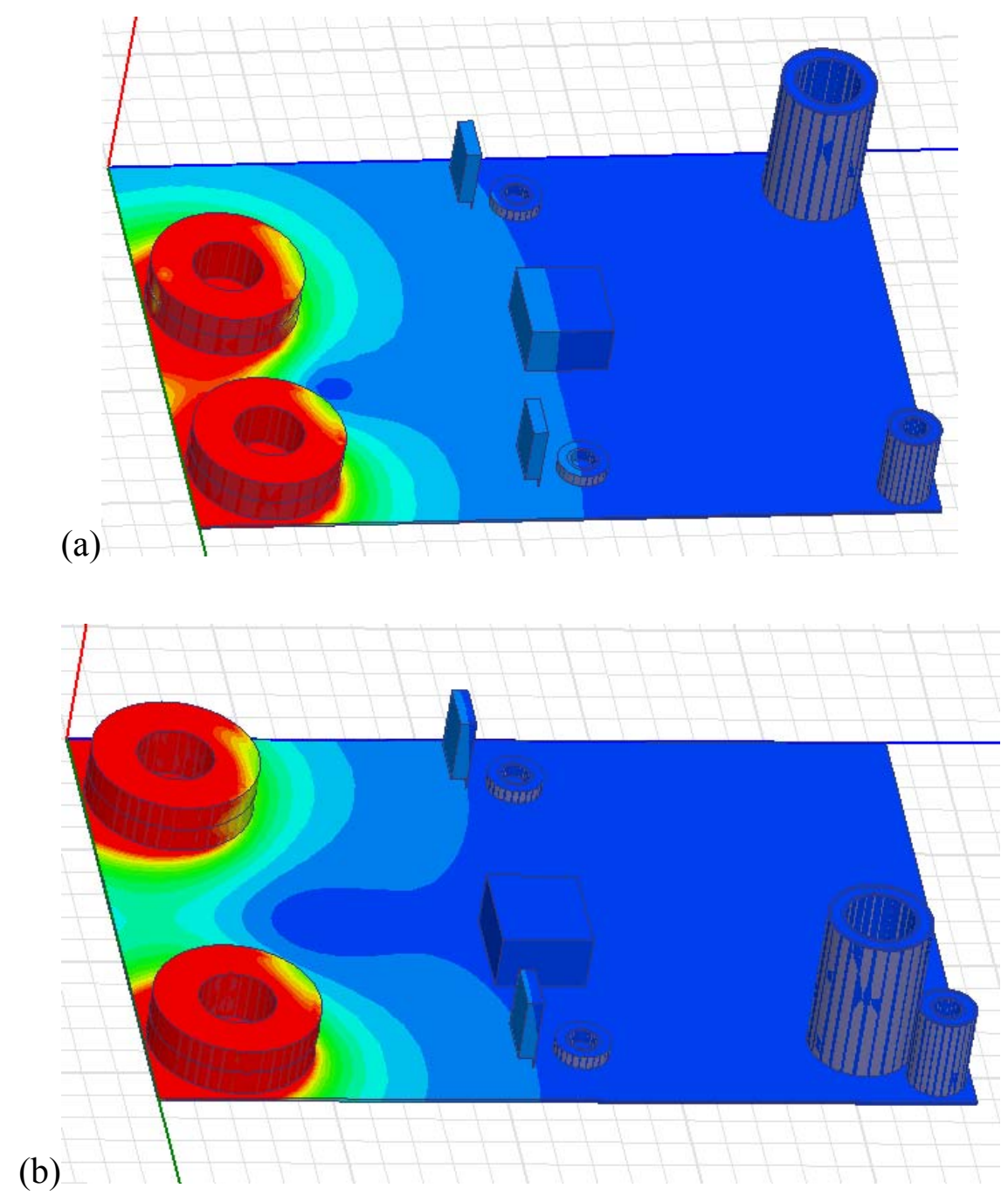

Figure 4.6. Magnetic field for coils on the PCB (a) $|\mathrm{H}|$ non-optimum (b) $|\mathrm{H}|$ optimum In order to study the magnetic field near the sensitive device, a 3-D plot of $|\vec{H}|$ versus variation on $x-y$ is shown in Figure 4.9. The figure indicates that the closer to the sensor, the less field magnitude is found. 


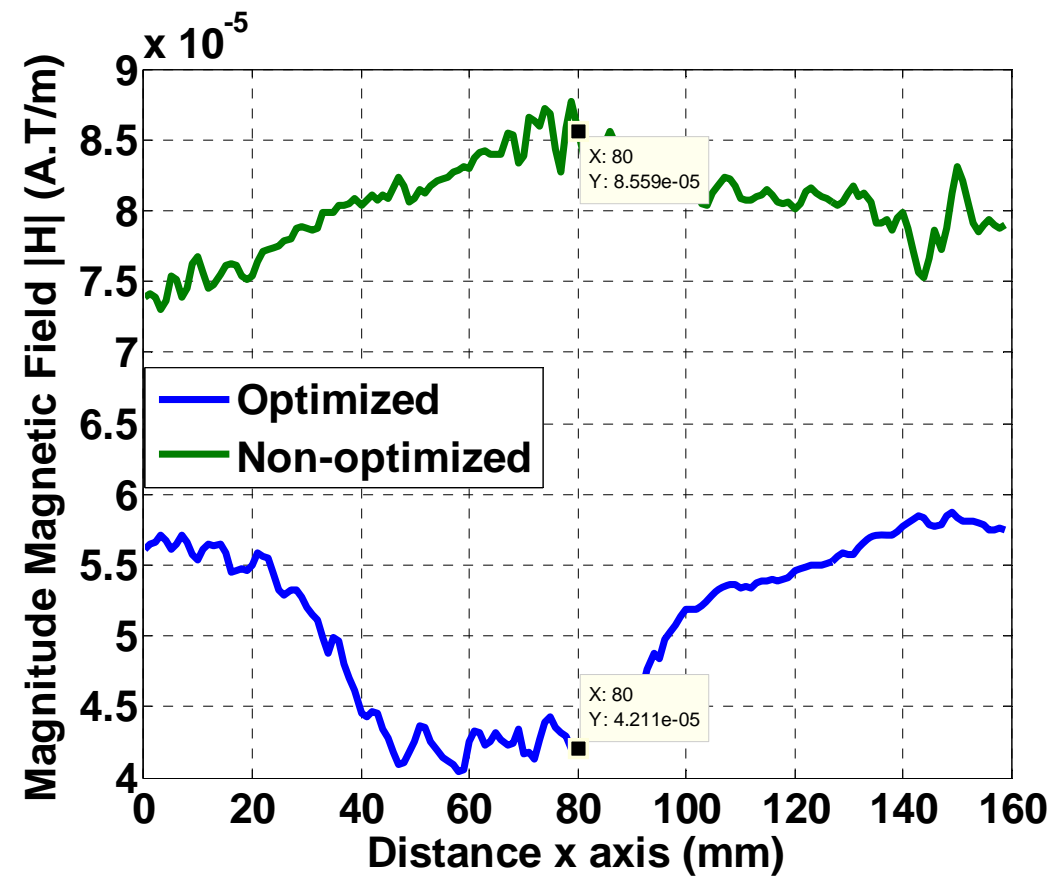

Figure 4.7 Magnitude of the magnetic field through a line in $\mathrm{x}$-direction and passing through the sensor center for optimal and non-optimal position.

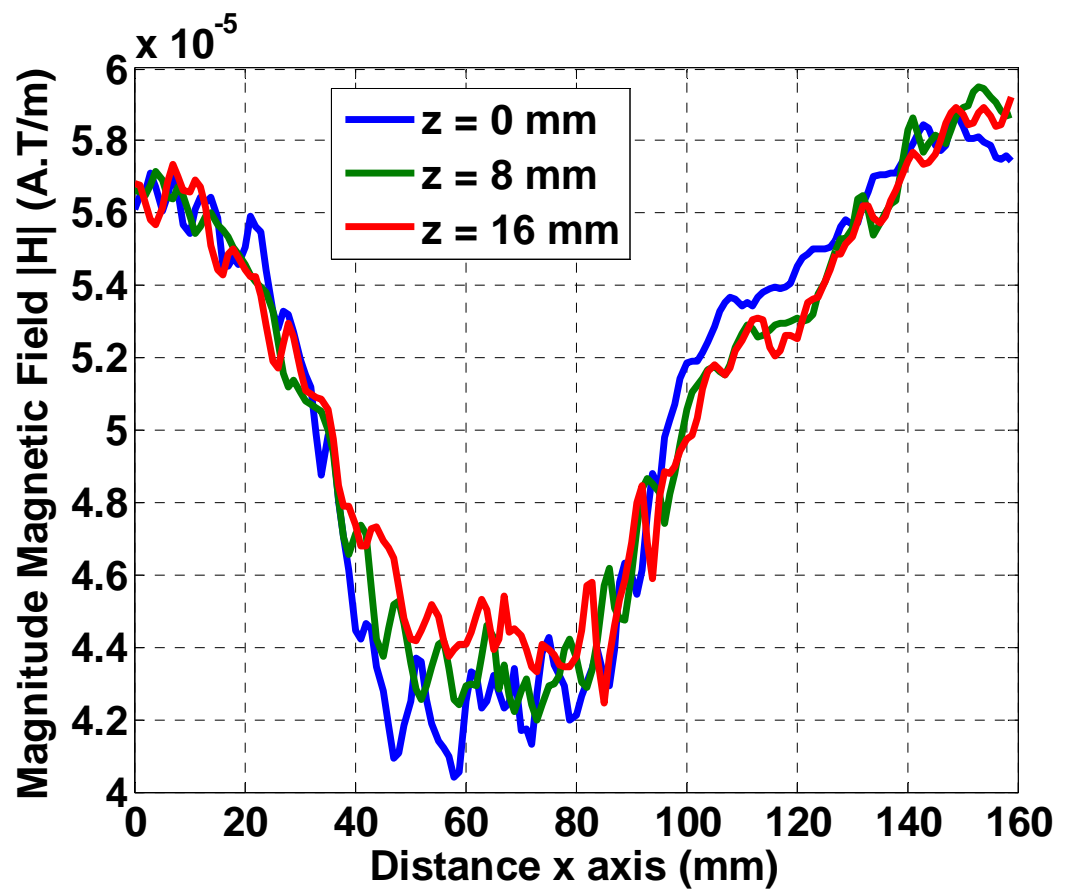

Figure 4.8 Magnitude of the magnetic field through a line in $\mathrm{x}$-direction and passing through the sensor center at different heights. 


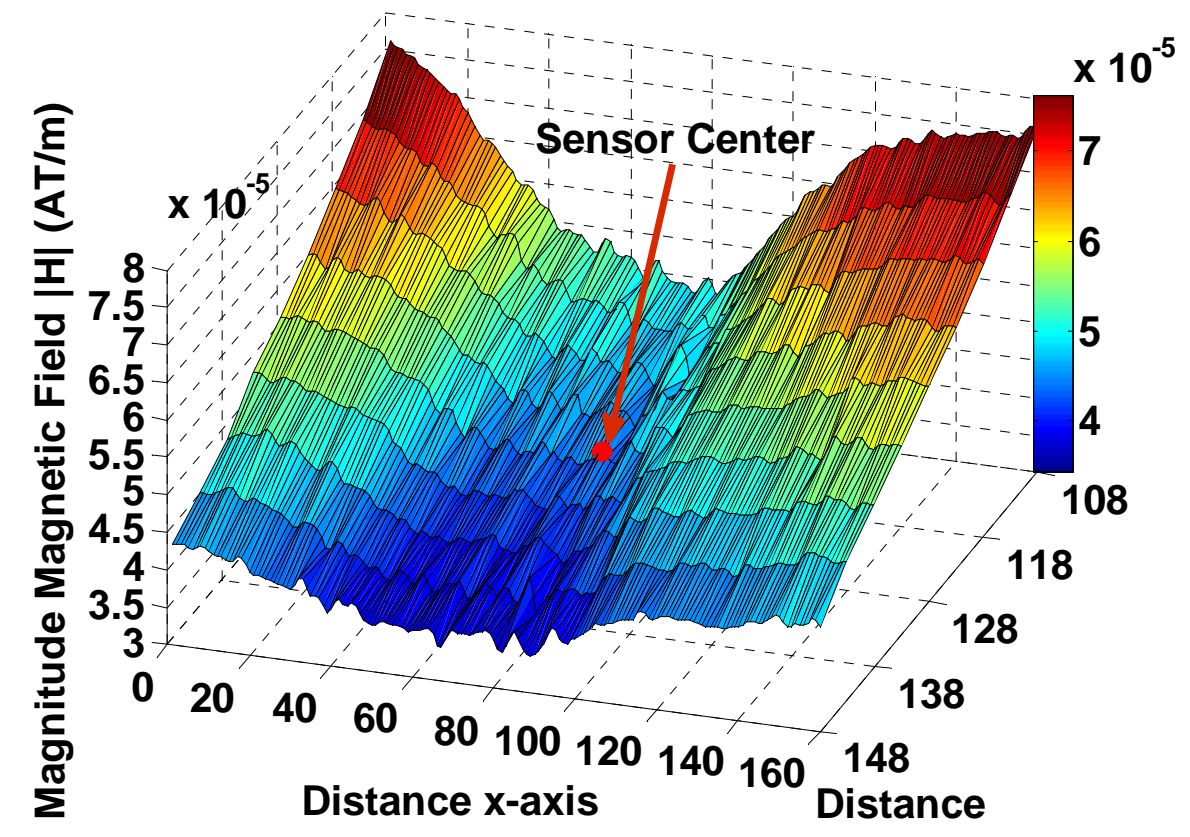

Figure 4.9 Magnitude of the magnetic field through the sensor region in x-y plane.

\subsubsection{Optimal and Non-optimal Capacitors position.}

Based on the optimization results, the capacitors are also rearranged to match the optimum positions. The non-optimum and optimum arrangement are solved for the magnitude of the electric field, as shown in Figure 4.10. The same abovementioned analyses for coils were also performed for the capacitors, and the results are indicated in Figure 4.11 and Figure 4.12. These figures show that the electric field decreases form 12.56 $\mathrm{V} / \mathrm{m}$ to $1.546 \mathrm{~V} / \mathrm{m}$ between the non-optimum and the optimum design, respectively, at the sensor's center. 
(a)

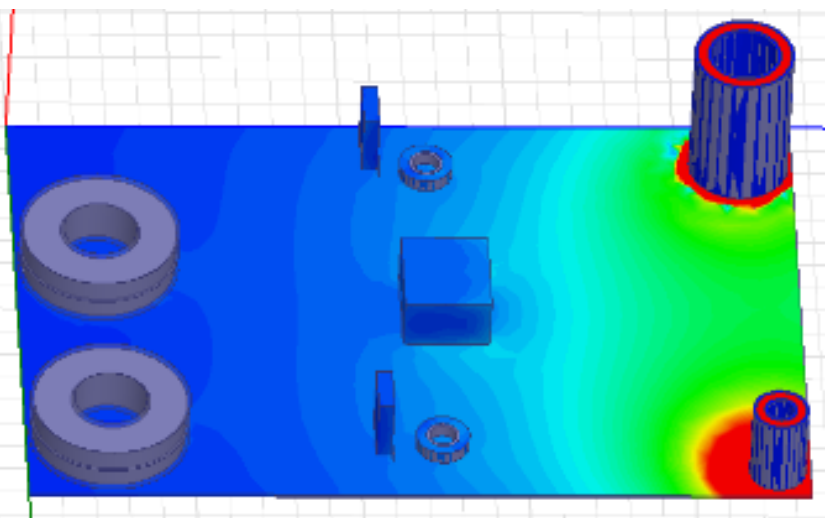

(b)

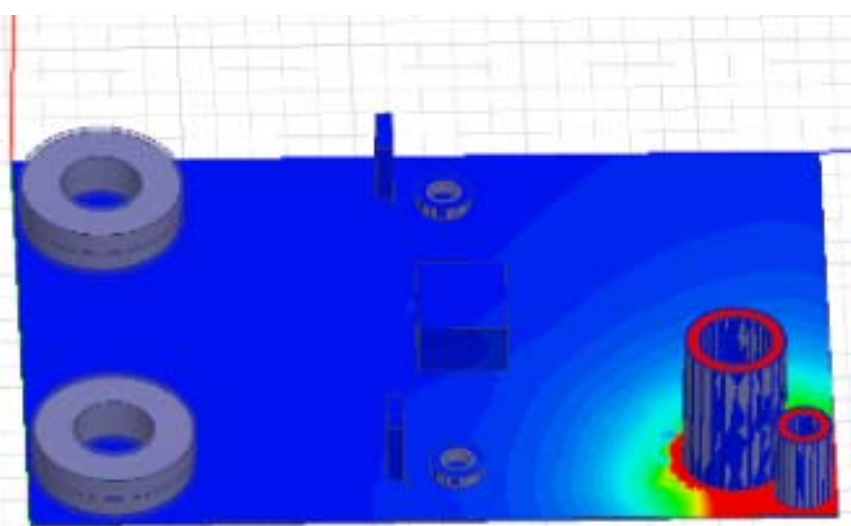

Figure 4.10 Electric field for capacitors on the PCB (a) $|\mathrm{E}|$ non-optimum (b) $|\mathrm{E}|$ optimum

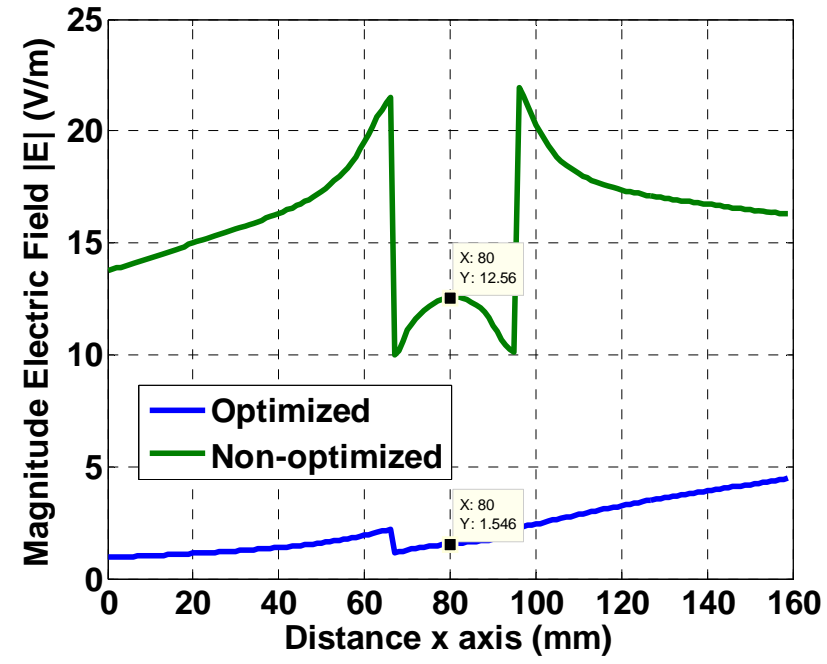

Figure 4.11 Magnitude of the electric field through a line in x-direction and passing through the sensor center for optimal and non-optimal position. 


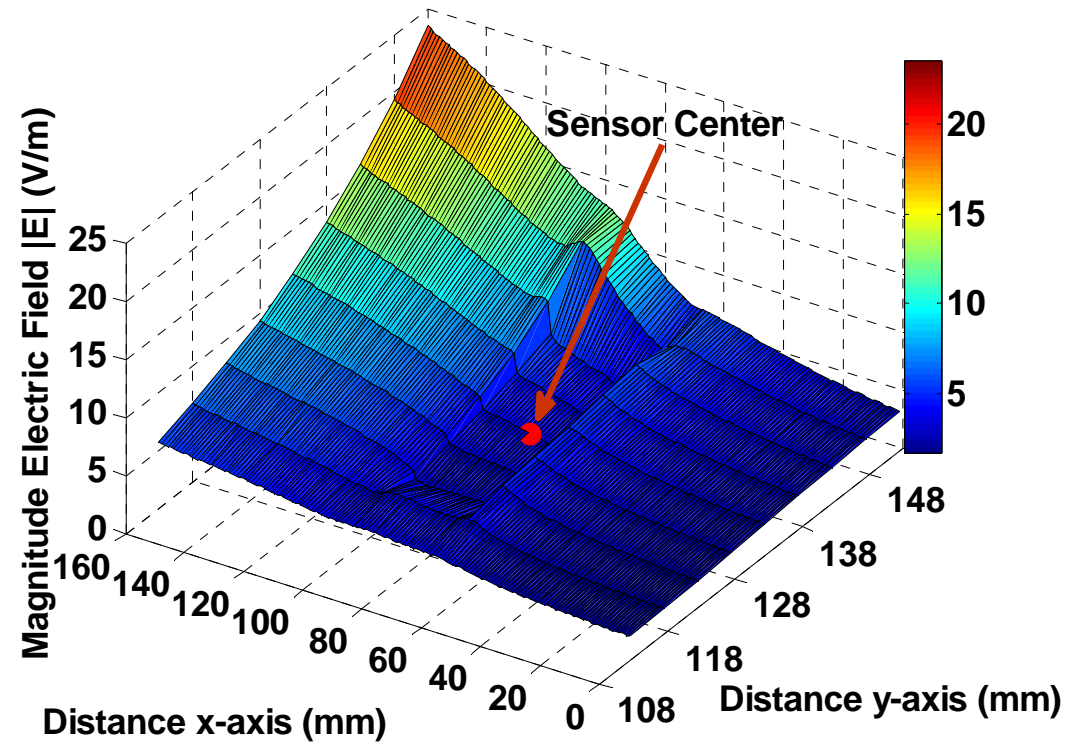

Figure 4.12 Magnitude of the electric field through the sensor region in x-y plane.

\subsection{Experimental Results}

In this section, the experimental results are presented to validate the proposed design technique for the proper location of passive components on the PCB for a dc-dc Cuk converter. Two experimental tests are performed; the first for the optimal position of coils and capacitors and the other for the non-optimum position that matches with the location on the simulated results. The dc-dc converter was designed and tested at the same conditions of the simulation, which are presented in Table 4-4. The experimental results are conducted in a Faraday chamber to isolate the circuit from the external interferences, as shown in Figure 4.13. The waveforms of the output voltage and the inductor currents of the Cuk converter are shown in Figure 4.14. The electromagnetic measurements are conducted using a Beehive Electronics 100C loop antenna, Beehive Electronics 150A Amplifier, and an EMC and Real time signal analyzer Tektronix RSA5103A. 


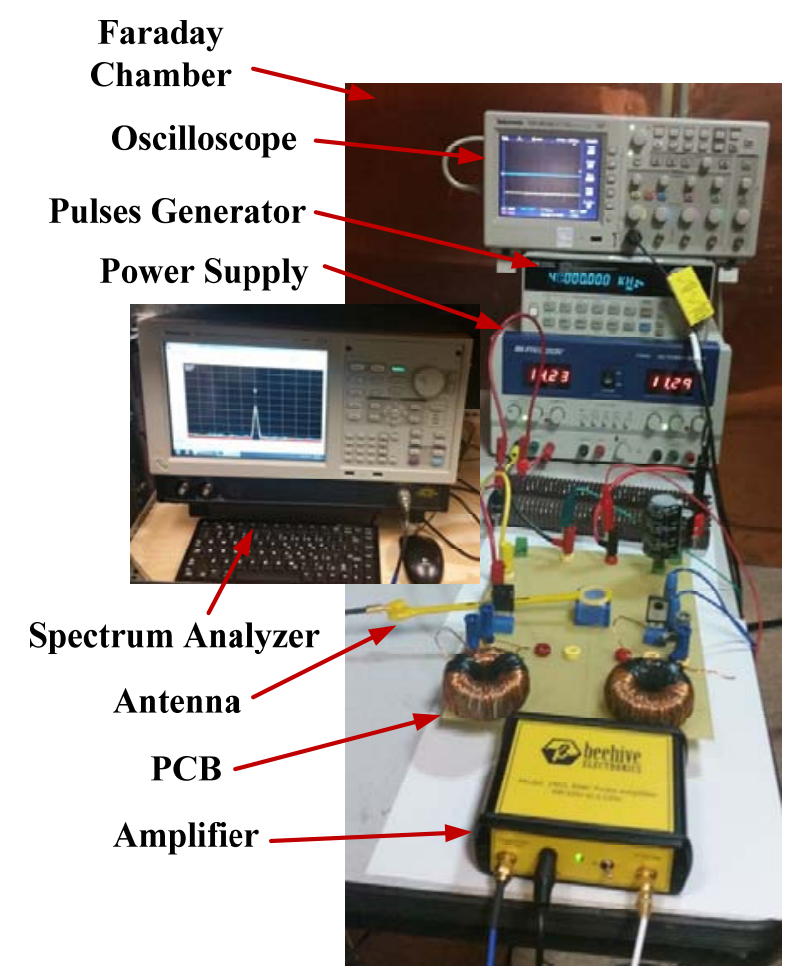

Figure 4.13 Experimental Setup

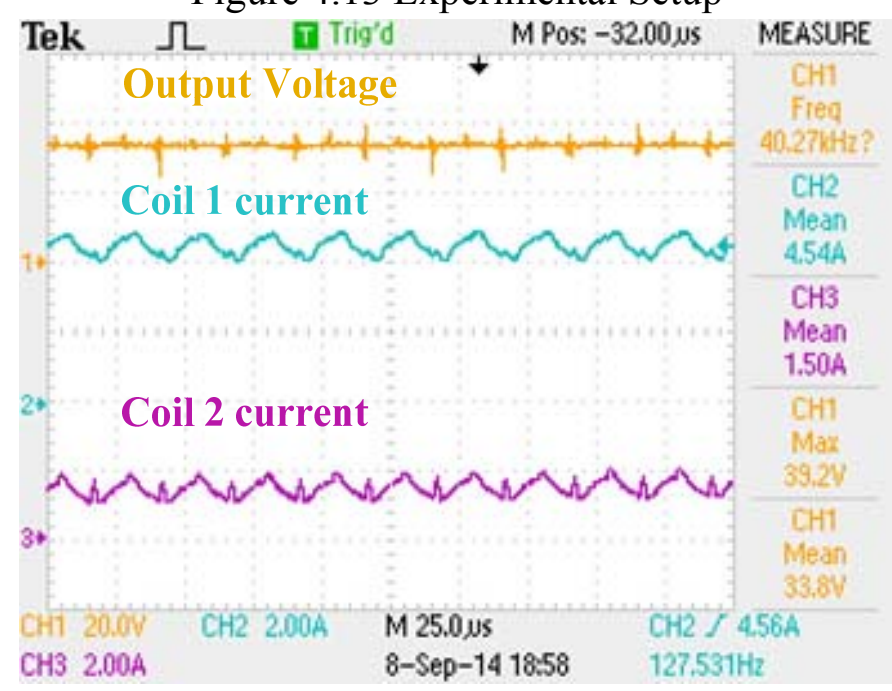

Figure 4.14 Output voltage and inductors current for DC-DC converter.

For comparison purposes, these measurements are performed for the optimum and nonoptimum arrangement. The magnitude of the magnetic field for the two cases is shown in Figure 4.15. In this figure, 9 measurements are depicted to indicate the variation of the 
magnetic field along the $x$-axis, passing through the center of the sensitive device, as shown in Figure 4.2. The figure also shows that the magnetic field interference is reduced from $7.891 \times 10^{-5}(\mathrm{~A} / \mathrm{m})$ to $4.542 \times 10^{-5}(\mathrm{~A} / \mathrm{m})$ at the center of the sensitive device, which means about $42.4 \%$ radiated EMI reduction with the proposed design. A comparison between the simulated and experimental results for the optimum and non-optimum arrangement are shown in Table 4-6.

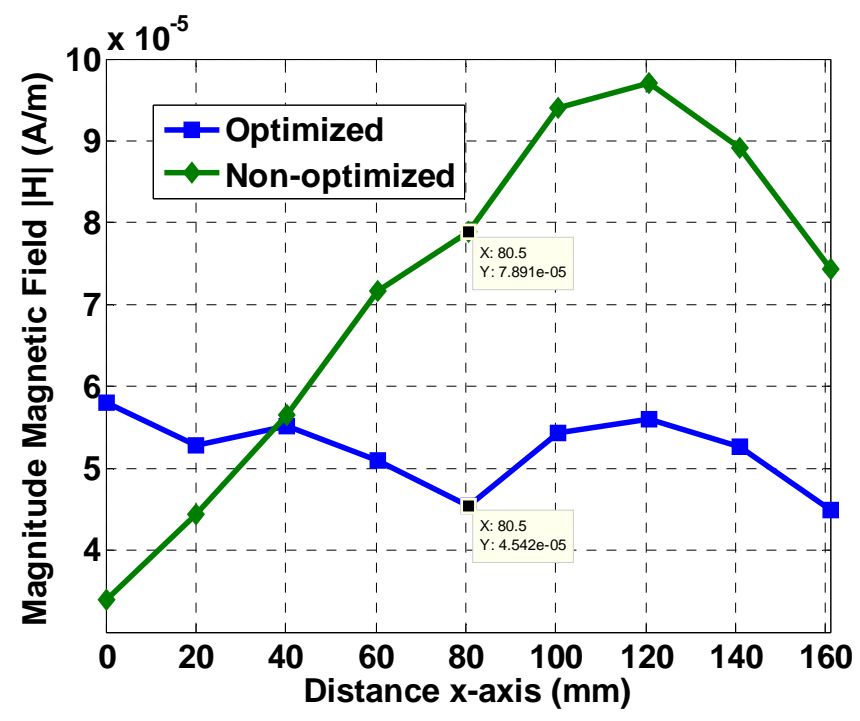

Figure 4.15 Magnitude of the magnetic field through a line in x-direction and passing through the sensor center for optimal and non-optimal position.

Table 4-6 Simulated and Measured Field at Sensor Center

\begin{tabular}{|l|c|c|c|}
\hline & $\begin{array}{l}|\mathbf{H}| \mathbf{O P T} \\
(\mathbf{A} / \mathbf{m})\end{array}$ & $\begin{array}{c}|\mathbf{H}| \text { NONOPT } \\
(\mathbf{A} / \mathbf{m})\end{array}$ & \%Reduction \\
\hline Simulated & $4.211 \times 10^{-5}$ & $8.559 \times 10^{-5}$ & 50.8 \\
\hline Experimental & $4.542 \times 10^{-5}$ & $7.891 \times 10^{-5}$ & 42.441 \\
\hline
\end{tabular}

The measurements of the magnetic field components were conducted by loop antenna in the three directions $\left(H_{x}, H_{y}\right.$ and $\left.H_{z}\right)$. For $H_{x}, H_{y}$ and $H_{z}$ component measurements, the antenna is oriented in the $\mathrm{xy}, \mathrm{xz}$ and yz plane, respectively. The magnetic field is detected in $\mathrm{dBuV} / \mathrm{m}$ and transformed to $\mathrm{A} / \mathrm{m}$. 
The three components of electromagnetic signature at the center of the sensitive device are shown in Figure 4.16. Inspecting this figure, it can be noticed that the optimized design exhibit lower radiated interference in the three directions and during the whole electromagnetic spectrum from $20 \mathrm{kHz}$ to $300 \mathrm{kHz}$ than the non-optimum one. It can also be observed that the magnetic field in the z-direction $(\mathrm{Hz})$ is almost the same for the optimum and the non-optimum positioning.

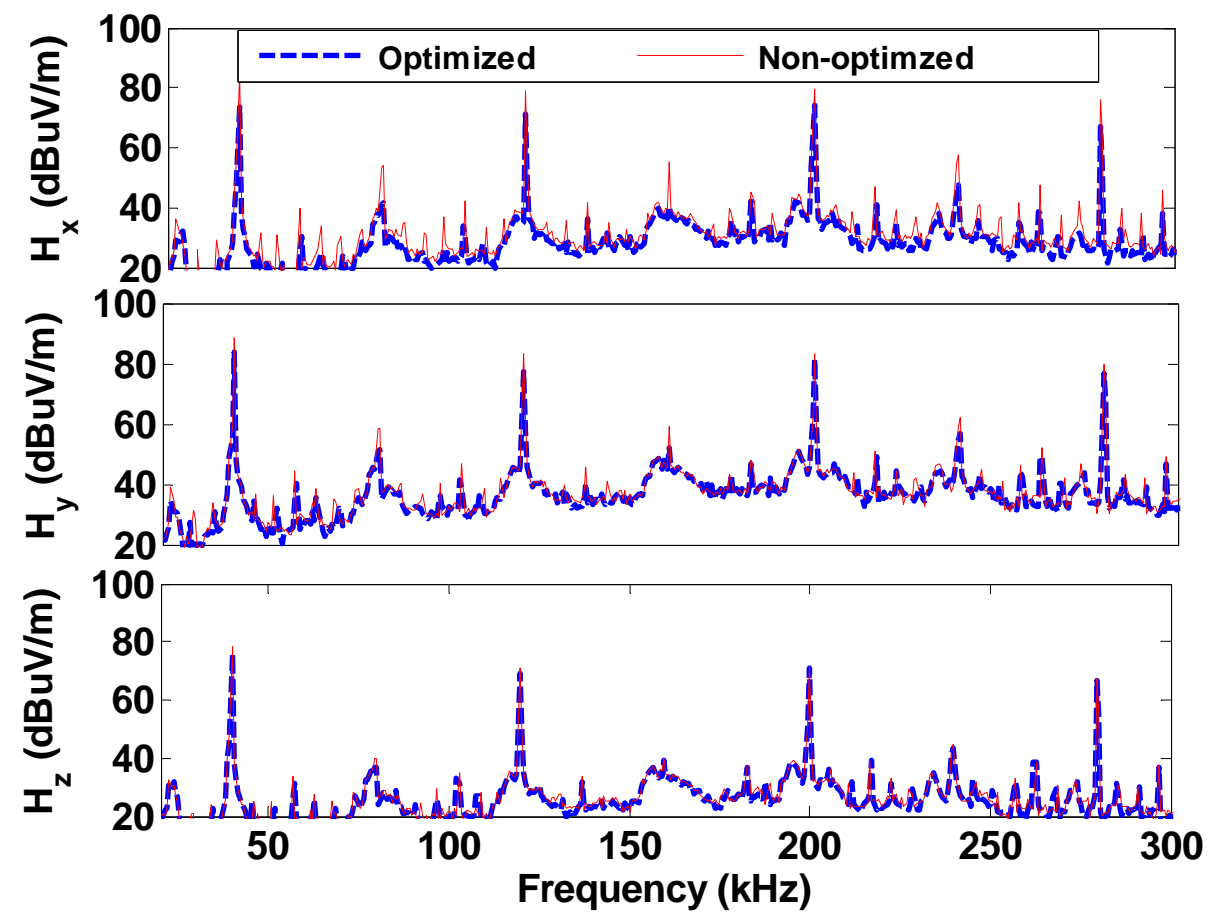

Figure 4.16 Radiated Magnetic field from the experimental setup (a) In x direction (b) In $\mathrm{y}$ direction and (c) In $\mathrm{z}$ direction.

The quasi-static simulation conducted by the FE procedure did not consider the time harmonic analysis in order to minimize timing and effort in the computation. However, it is clear that the reduction of the main harmonic component $(40 \mathrm{kHz})$ is accompanied by the reduction of other harmonics. This can be observed in Figure 4.17 and Table 4-7, where higher-level harmonics are shown. 


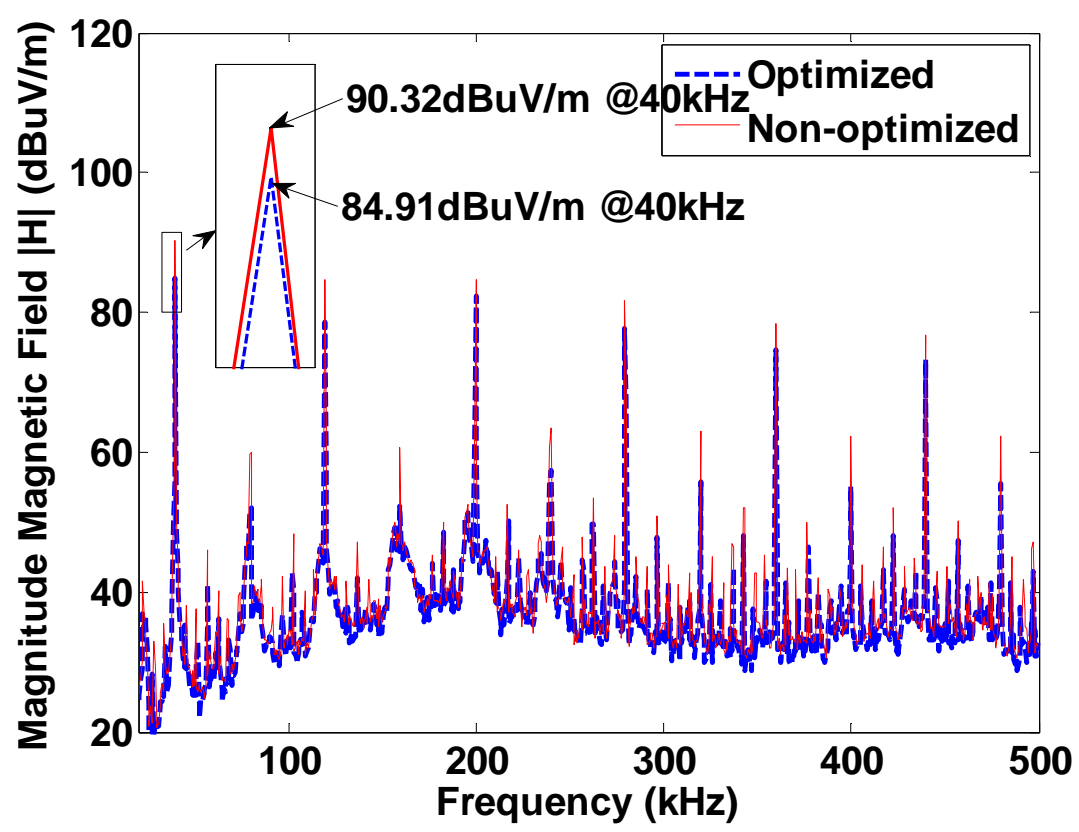

Figure 4.17 Radiated Magnetic field $(|\mathrm{H}|)$ from the experimental setup.

Table 4-7 Measured Harmonics Reduction in $(\mathrm{dBuV} / \mathrm{m})$.

\begin{tabular}{|l|l|l|l|l|l|l|l|}
\hline No & $|\mathbf{H}|_{\text {OPT }}$ & $|\mathbf{H}|_{\text {NOOPT }}$ & Red & No & $|\mathbf{H}|_{\text {OPT }}$ & $|\mathbf{H}|_{\text {NOOPT }}$ & Red \\
\hline 1 & 84.91 & 90.32 & 5.41 & 7 & 77.79 & 81.57 & 3.78 \\
\hline 2 & 51.99 & 59.95 & 7.96 & 8 & 55.8 & 62.92 & 7.12 \\
\hline 3 & 78.68 & 84.57 & 5.89 & 9 & 74.68 & 78.44 & 3.76 \\
\hline 4 & 52.36 & 60.71 & 8.35 & 10 & 55.09 & 62.39 & 7.3 \\
\hline 5 & 82.46 & 84.73 & 2.27 & 11 & 73.34 & 76.71 & 3.37 \\
\hline 6 & 57.49 & 63.58 & 6.09 & 12 & 55.5 & 62.3 & 6.8 \\
\hline
\end{tabular}

\subsection{Conclusion}

This chapter presents an optimization technique for the PCB design based on the arrangement of the passive components. A software platform was developed to perform the optimization using GA and 3D-FE. The proposed procedure for PCB design was verified by simulation and experimental results. The results show a significant reduction in the radiated electromagnetic field at a susceptible device in a PCB. Even when the 
optimization procedure is applied only for the sensor's center, the field reduction is extended for a wide region around the sensor. Moreover, the proposed technique not only reduces the fundamental field component, but also all the harmonic contents for the electromagnetic field. 


\section{Chapter 5 Control of Induction Machine in the Drivetrain}

\subsection{Introduction}

Induction machines are relevant in industrial applications and they are ideal for harsh environments due to their robustness, ruggedness, high efficiency, lower cost and maintenance. In the history of IM, there have been many types of controllers depending on the machine connections (see Table 2-2). One of the first controllers connects the IM directly to the mains or to scalar controllers, such as the Voltage-Frequency driver. The last one suffers from limitations at low speed and poor torque response [89]. The emergence of vector control techniques (VCT) moderately solves the IM control problems.

One of the most famous VCT is the Field Oriented Control (FOC) presented in [90]-[92]. This strategy grants control of the rotor flux and torque independently, acting over the stator current phase and quadrature components. Typically, the FOC design is simple but its performance depends on the knowledge of the IM parameters and the load variations [93]. Moreover, rotor flux observers are difficult to design when the IM's parameters vary with the frequency and temperature. Solutions for these problems were proposed in [94], [95].

More recent VCT are the Direct-Self Control (DSC) and Direct Torque Control (DTC) proposed in [96] and [97], respectively. The DSC is better suited for high-power and lowswitching frequency applications. The DTC is widely utilized and best suited for low and medium power and high switching frequency applications. DTC produces rapid torque response, while keeping the IM stator flux and torque decoupled. The main disadvantages of DTC are the large torque ripple, its high start-up current, variable switching frequency, 
and poor performance under overload and low speed operation. Plenty of research has been conducted to improve the DTC performance. DTC alterations are presented in [98], [99] for enhancing the starting condition and the low-speed operation. References [100]-[108] propose solutions to these drawbacks with predictive strategies, but they are parameter dependent. In [109], a DTC modification to reduce the torque ripple and enhance the response of the control under overload condition with the price of higher parameter sensitivity is presented. A modulated hysteresis DTC is proposed in [110] to achieve constant switching frequency and decrease the torque oscillations. In [111]-[116], fuzzy controllers are proposed to reduce torque ripple and limit the stator current.

In this chapter, a torque controller that integrates the advantages of Fuzzy Logic Controller (FLC) and predictive control (PC) is proposed. The FLC helps in the reduction of the torque ripple with significant decrement of the parameters' dependency and limitation of the inrush current. The PC technique aims in the improvement of the performance at both starting condition and low-speed operation. In this approach, the FLC rules are based on a predictive DTC (P-DTC), where the main idea is to observe the nonperpendicular quadrants formed by the two lines of constant torque and constant stator flux magnitude drawn in the complex vector plane (CVP) of the voltage source inverter (VSI) or voltage $\alpha \beta$ plane.

A comparative study is performed among the classical DTC, P-DTC and the proposed Fuzzy-Predictive DTC (FP-DTC), showing the advantages and disadvantages of every control under diverse torque profiles and different load conditions. An assessment analysis over eight different continues and discontinues modulation strategies is performed. A parameter sensitivity examination is conducted to corroborate the thesis of FP-DTC is 
robust to the parameter variations. Finally, experimental results are conducted, confirming all the expected performance and hypothesis.

\subsection{Predictive DTC}

The P-DTC objective is to control the electromagnetic torque and the magnitude of the stator flux-linkage space-vector (SV) independently. Thus, it is convenient to represent the IM dynamic state-space equations in terms of the stator current complex SV $\boldsymbol{i}_{\boldsymbol{s}}$ and stator flux-linkage complex SV $\lambda_{s}$, as in (5.1). The subscript $s$ comes from stator.

$$
\begin{gathered}
p \boldsymbol{i}_{\boldsymbol{s}}=\frac{\boldsymbol{v}_{\boldsymbol{s}}}{\hat{L}_{s}}-\left(\frac{L_{s}}{L_{r}} \frac{R_{r}}{\hat{L}_{s}}+\frac{R_{s}}{\hat{L}_{s}}-j P \omega_{m}\right) \boldsymbol{i}_{\boldsymbol{s}}+\left(\frac{R_{r}}{L_{r}}-j n_{p} \omega_{m}\right) \frac{\boldsymbol{\lambda}_{\boldsymbol{s}}}{\hat{L}_{s}} \\
p \boldsymbol{\lambda}_{\boldsymbol{s}}=\boldsymbol{v}_{\boldsymbol{s}}-R_{s} \boldsymbol{i}_{\boldsymbol{s}} \\
p \omega_{m}=\frac{1}{J}\left(T_{e}-T_{m}\right)
\end{gathered}
$$

where $R_{r}$ and $R_{s}$ are the resistances, and $L_{r}$ and $L_{s}$ are the inductances of the rotor and stator, respectively; $\hat{L}_{s}=L_{s}-\frac{L_{s r}^{2}}{L_{r}}, L_{s r}$ is the mutual inductance, $\omega_{m}$ is the mechanical speed, $T_{e}=n_{p} \lambda_{s} \times \boldsymbol{i}_{s}$ and $T_{m}$ are the electrical and mechanical load torque, respectively; $n_{p}$ is the number pole pairs, $J$ is the inertia of the machine and the stator SV $\boldsymbol{i}_{\boldsymbol{s}}, \boldsymbol{v}_{\boldsymbol{s}}$ and $\boldsymbol{\lambda}_{\boldsymbol{s}}$ are written in complex-vector notation referred to stationary $(\alpha \beta)$ reference frame, thusly $\boldsymbol{f}_{\boldsymbol{s}}=f_{\alpha s}+j f_{\beta s} ; \boldsymbol{f}_{\boldsymbol{s}}=\left\{\boldsymbol{i}_{\boldsymbol{s}}, \boldsymbol{v}_{\boldsymbol{s}}, \boldsymbol{\lambda}_{\boldsymbol{s}}\right\} ; p$ is the time-derivative operator. The transformation from the primitive coordinates $(a b c)$ to the stationary $(\alpha \beta)$ reference frame is Hermitian, or power conservative. The transformation used is in (2.5) or (2.9).

For the particular case of the IM and based on PC theory [73], it is possible to foresee the output error, $\boldsymbol{y}=\left[T_{e},\left|\boldsymbol{\lambda}_{s}\right|\right]$, in the next cycle of control $\left(t_{k+1}\right)$, as the output have a relative degree $\{1,1\}$, which is defined everywhere except at $\left|\lambda_{r}\right|=\mathbf{0}$ [117], [118]. This anticipation can be computed based on (5.2) and (5.3). 


\begin{tabular}{|c|c|}
\hline$p T_{e}=p\left(n_{p} \boldsymbol{\lambda}_{\boldsymbol{s}} \times \boldsymbol{i}_{s}\right)$ & (5.2) \\
$p\left|\boldsymbol{\lambda}_{s}\right|=\frac{2}{\left|\boldsymbol{\lambda}_{s}\right|} \boldsymbol{\lambda}_{s} \times j\left(\boldsymbol{v}_{\boldsymbol{s}}-R_{s} \boldsymbol{i}_{s}\right)$ & \\
\hline$p T_{e}=\left[\frac{\left(\boldsymbol{v}_{s}-j n_{p} \omega_{m} \boldsymbol{\lambda}_{s}\right)}{1 / n_{p}} \times\left(\boldsymbol{i}_{s}-\frac{\lambda_{s}}{\hat{L}_{s}}\right)-\left(\frac{L_{s} R_{r}+L_{r} R_{s}}{L_{r} \widehat{L}_{s}}\right) T_{e}\right]$ \\
$p\left|\boldsymbol{\lambda}_{s}\right|=\frac{2}{\left|\boldsymbol{\lambda}_{s}\right|} \boldsymbol{\lambda}_{\boldsymbol{s}} \times j\left(\boldsymbol{v}_{s}-R_{s} \boldsymbol{i}_{s}\right)$ & (5.3) \\
\hline
\end{tabular}

where $\boldsymbol{a} \times \boldsymbol{b}=a_{\alpha} b_{\beta}-b_{\alpha} a_{\beta}$ is the cross-product operation between two vectors.

The selection of the best VSI vector (input space $\boldsymbol{u}=\boldsymbol{v}_{\boldsymbol{s}}$ ) is achieved by the comparison of the values resulting from the cost function $(\psi)$ defined in (5.4), for the 7 inverter switching states or input control action (ICA) as in Table 3-2 and Figure 3.2.

\begin{tabular}{|l|l|}
\hline$\psi^{i}=k_{1}\left(\left\{p T_{e} T_{s}\right\}^{i}-\Delta T_{e}\right)^{2}+k_{2}\left(\left\{p\left|\lambda_{s}\right| T_{s}\right\}^{i}-\Delta\left|\lambda_{s}\right|\right)^{2}$ & (5.4) \\
\hline
\end{tabular}

where $\left(\Delta T_{e}, \Delta\left|\lambda_{s}\right|\right)$ are the actual output errors, $\left(\left\{p T_{e} T_{s}\right\}^{i},\left\{p\left|\lambda_{s}\right| T_{s}\right\}^{i}\right)$ are the predicted errors or variable incrementals, the superscript $i=\{1, \ldots, 7\}$ indicates the VSI states or ICA $v_{s i}=\left\{v_{s 1}, \ldots, v_{s 7}\right\}$, as shown in Figure 5.1 (blue dots) and Figure 3.2, e.g. for $i=1, v_{s 1}=$ $\left[\begin{array}{lll}v_{s a} & v_{s b} & v_{s c}\end{array}\right]=\left[\begin{array}{llll}2 V_{D C} / 3 & 0 & 0\end{array}\right]=v_{s \alpha}+j v_{s \beta}=\sqrt{2 / 3}\left(2 V_{D C} / 3\right)+j 0 ; \quad k_{1} \quad$ and $\quad k_{2} \quad$ are weighting constants. The index of the minimum among the 7 cost function values $\left[\psi^{1}, \ldots, \psi^{7}\right]$ determines the index of the ICA at the next instant $t_{k+1}$.

Setting both time derivatives in (5.3) equal to zero $\left(p T_{e}=0\right.$ and $\left.p\left|\lambda_{s}\right|=0\right)$, two linear voltage equations can be expressed as a function of the inverter voltages or input space $\left(v_{\alpha s}, v_{\beta s}\right)$ as in (5.5). Plotting these equations in the CVP for one cycle of control, they would be as shown in Figure 5.1. 
$v_{s \beta_{\lambda}}=R_{s} i_{s \beta}-\frac{\lambda_{s \alpha}}{\lambda_{s \beta}}\left(v_{s \alpha_{\lambda}}-R_{s} i_{s \alpha}\right)$
$v_{s \beta_{T}}=\frac{\left(\widehat{L}_{s} i_{s \beta}-\lambda_{s \beta}\right)}{\left(\hat{L}_{s} i_{s \alpha}-\lambda_{s \alpha}\right)}\left(v_{s \alpha_{T}}-\frac{\widehat{L}_{s} A}{\widehat{L}_{s} i_{s \beta}-\lambda_{s \beta}}\right)$

where $A=\frac{\left(\frac{L_{S}}{L_{r}} R_{r}+R_{s}\right) T_{e}}{n_{p} \hat{L}_{s}}-n_{p} \omega_{m}\left(\boldsymbol{\lambda}_{\boldsymbol{s}} \times j \boldsymbol{i}_{s}\right)+\frac{n_{p} \omega_{m}\left|\lambda_{s}\right|^{2}}{\hat{L}_{S}}$.

These two linear equations represent the constant torque variation $\left(v_{s \beta_{T}}\right)$ and constant magnitude of the stator flux vector variation $\left(v_{s \beta_{\lambda}}\right)$. The constant variation is due to their deduction that originates from the time derivative cancellation. Each plotted equation in the CVP divides the input space in two subspaces, where a perpendicular ICA to these lines will create the maximum change possible (at the instant) in the output variable. When the inverter ICA is over (under) one of the lines, then the trend is to increment (decrement) this variable. Generally, these two lines are not perpendicular between each other and they normally do not intersect in the CVP origin, hence four irregular quadrants (4 sub-spaces) are created in every cycle of control $\left(t_{k}\right)$. This irregularity generates an imbalanced number of input voltage vectors or ICA for each of the subspaces created. In classical DTC, it is assumed that these lines are perpendicular and in this way an even number of input voltage vectors is obtained, in each cycle of control, in each quadrant [117]. The four cases of increasing or decreasing the output errors $\Delta T_{e}=T_{r e f}-T_{e}$ and $\Delta\left|\lambda_{s}\right|=\lambda_{\text {ref }}-\left|\lambda_{s}\right|$ are as in (5.6). The angle in between these two lines is defined as $\phi$ (Figure 5.1).

$$
\begin{aligned}
& \Delta T_{e}>0 \text { and } \Delta\left|\lambda_{s}\right|>0 \\
& \Delta T_{e}>0 \text { and } \Delta\left|\lambda_{s}\right|>0 \\
& \Delta T_{e}>0 \text { and } \Delta\left|\lambda_{s}\right|>0 \\
& \Delta T_{e}>0 \text { and } \Delta\left|\lambda_{s}\right|>0
\end{aligned}
$$




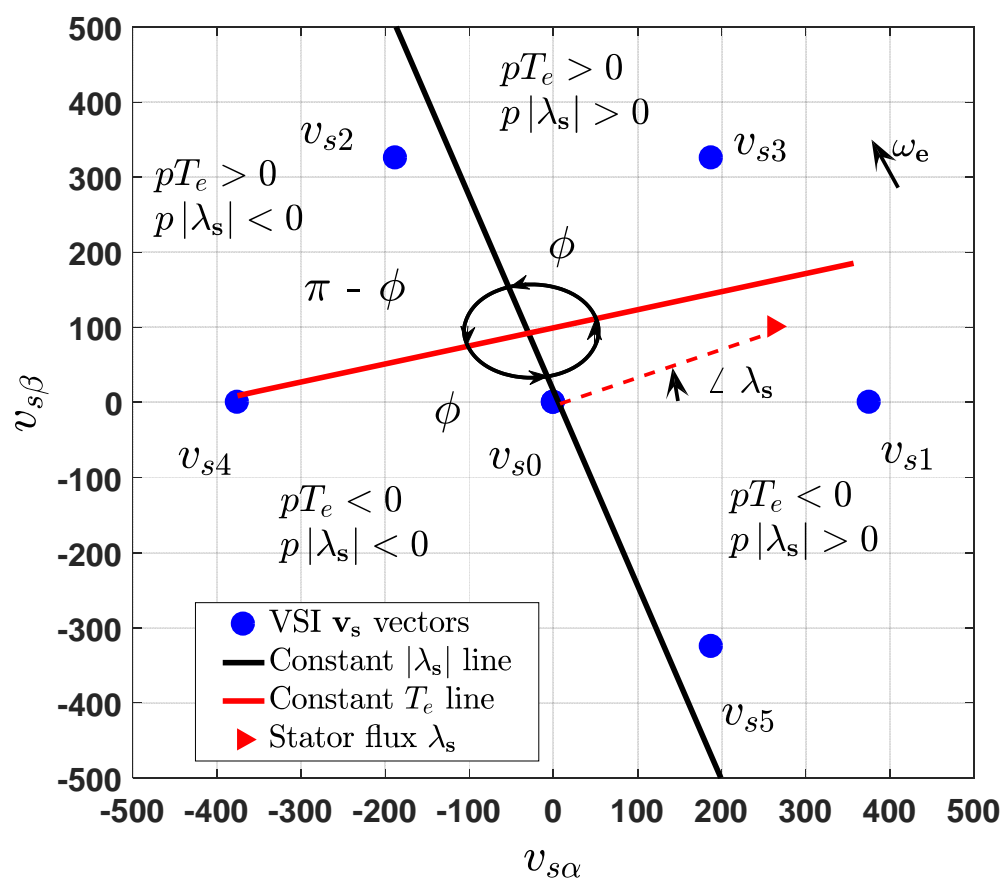

Figure 5.1 Torque and stator flux constants variation lines at the CVP.

\subsection{Fuzzy Predictive DTC}

An efficient solution for mitigating the issues of the classical DTC algorithm is to apply FLC as in [111]. The FL-based DTC provides an adaptive variation of the duty-cycle based on the switching table proposed by Noguchi [97], however, the rules are fixed, creating some drawbacks of classical DTC, such as poor performance at both starting and low-speed operation.

In the proposed FP-DTC, the contribution is the integration of FLC with a predictive control strategy, which allows not only adaptive variation, but also dynamic rules. The main limitation of FLC is the requirement of high-speed microprocessor ability with large memory size and the lack of the design strategies. Most FL system parameters are determined intuitively based on the designer and/or the operator experience [119]. To solve 
the first problem, the FLC system is designed by choosing the minimum number of linear membership functions (MF) for each input and using Takagi-Sugeno (T-S) Fuzzy Inference System (FIS) technique for calculating the control decision. The second problem is inherently solved, as the designed fuzzy rules are dynamic and depend on the defined angle $\phi$ (Figure 5.1). A block diagram for the complete system is depicted in Figure 5.2. In Figure 5.3, a detailed block diagram of the FIS is shown. The FIS is a zero-order T-S with two inputs $\left(\Delta T_{e}\right.$ and $\left.\Delta\left|\lambda_{s}\right|\right)$ and one output $\left(\boldsymbol{v}_{\boldsymbol{F u z z} y}=z e^{j \theta}\right)$. The universe of discourse of each input is described by three MF (N, Z and P). The fuzzy rules that define the relationship between the fuzzy inputs and output are chosen as a function of the angle $\phi$ (Figure 5.1). The matrix rule is the one presented in Figure 5.3. Each fuzzy rule has two parameters for the two outputs: magnitude ( $z$ ) and angle ( $\theta$ ); e.g. assume first case in (5.6), where $\Delta T_{e}>$ 0 and $\Delta\left|\lambda_{s}\right|>0$, thus $\mathrm{MF}_{T}=\mathrm{P}$ and $\mathrm{MF}_{\lambda}=\mathrm{P}$. The matrix rules indicate $z=L$ and $\theta=$ $\phi / 2$, so the maximum change in both torque and stator flux magnitude is achieved when $\theta=\phi / 2$. If the two lines of constant torque and flux are orthogonal and centered at the CVP, the rule would be $\theta=\pi / 2$ and the control will be the same as the one proposed in [111]. In this FLC, the rules are dynamic and varying at every period of control as a function of the angle $\phi$, which changes with the IM position and operative conditions. The angle $\phi$ is calculated from (5.5). 


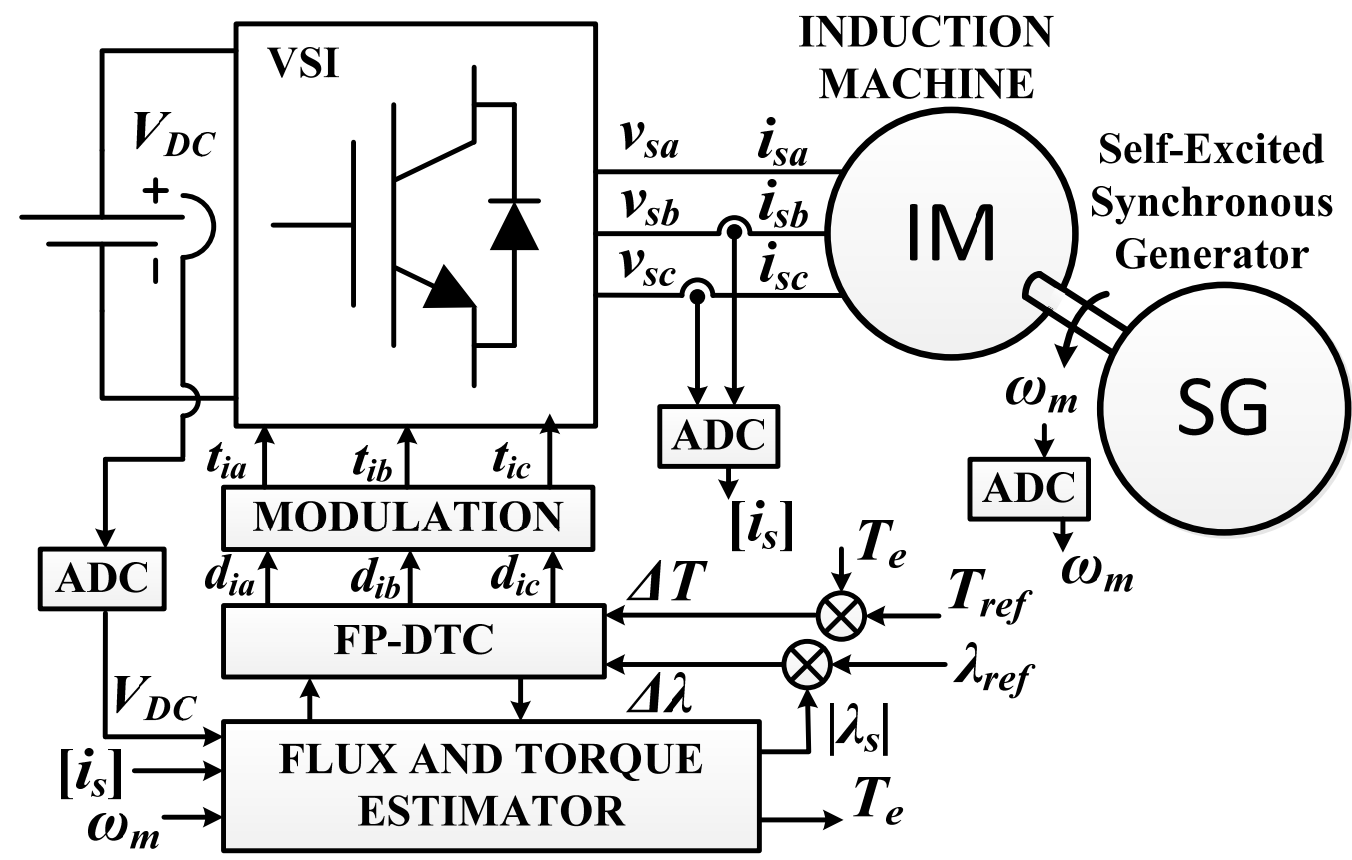

Figure 5.2 Block diagram of the complete system.

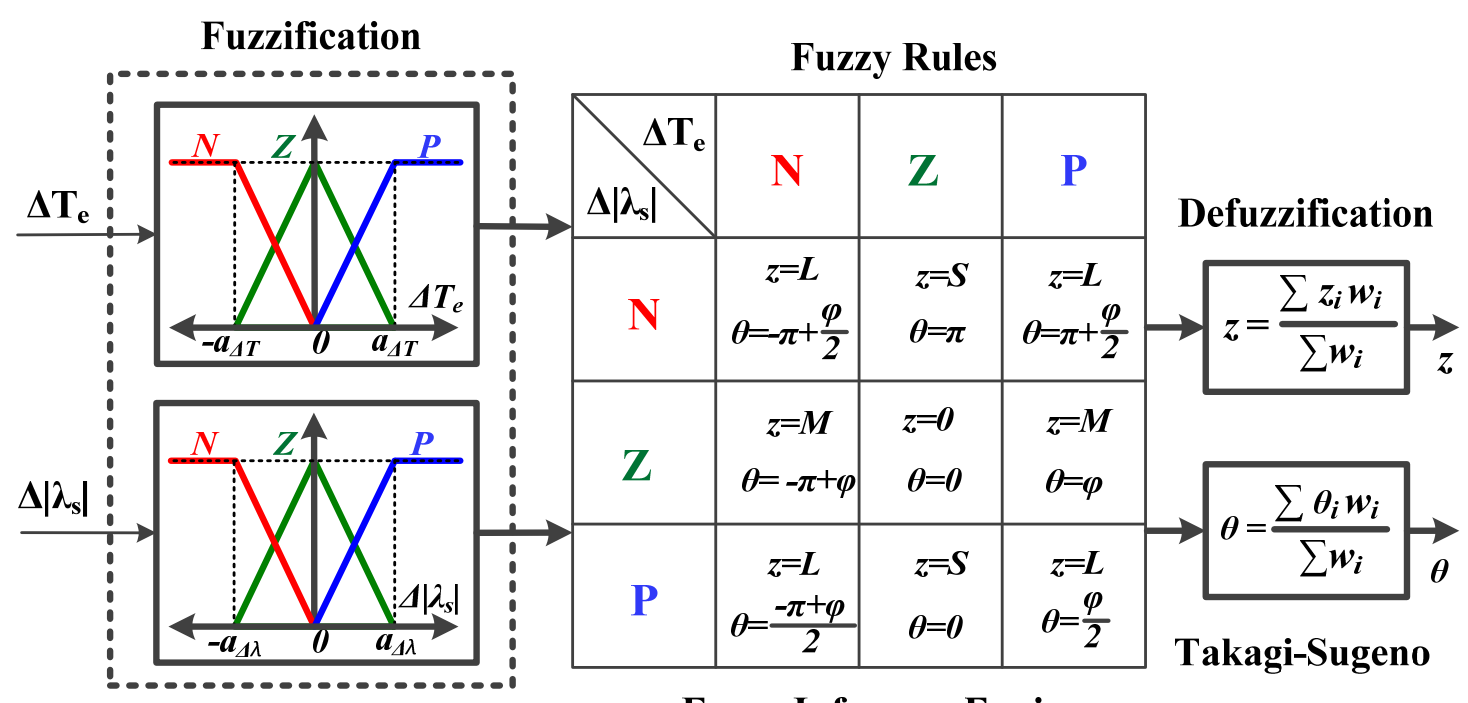

Fuzzy Inference Engine

Figure 5.3 Block diagram of the proposed fuzzy predictive DTC.

The FLC output ( $z$ and $\theta$ ) needs to be conditioned for the modulation technique, thus and so two conditioning methods can be implemented. Using (5.7) for mounting the fuzzy output over the flux-linkage SV or (5.8) that is a vector summation, as shown in Figure 5.4. 


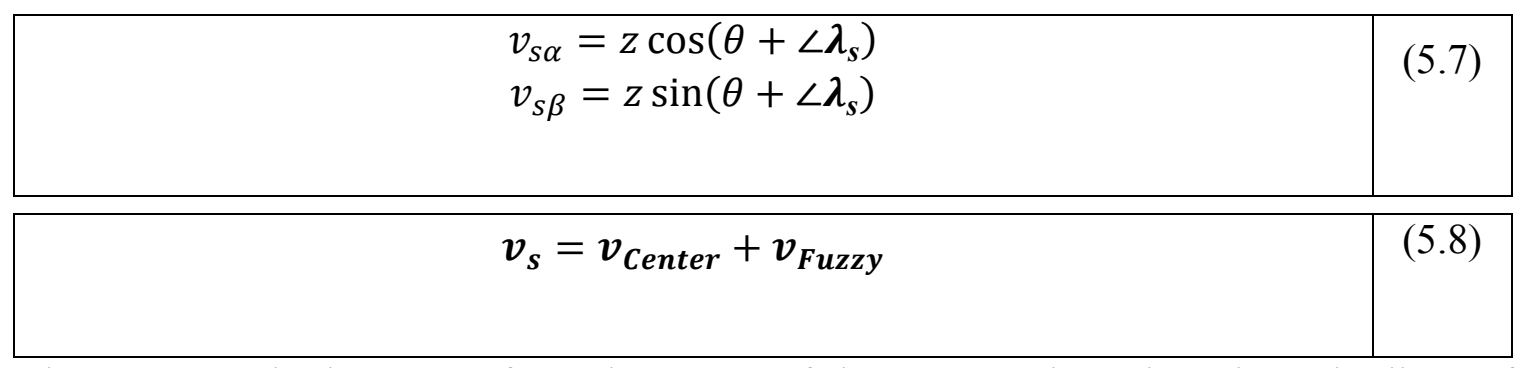

where $\boldsymbol{v}_{\text {Center }}$ is the vector from the center of the CVP to the point where the lines of constant torque and constant magnitude of flux cross. This vector can be realized by making the two voltage equations of (5.5) equal, in such a way $v_{s \beta_{\lambda}}=v_{s \beta_{T}}$. Therefore, the coordinates of this cross-point are:

\begin{tabular}{|c|c|}
\hline$v_{\text {Center } \beta}=\frac{R_{s}\left(i_{\beta}-\frac{\lambda_{\beta s}}{\hat{L}_{s}}\right) \lambda_{s} \times j i_{s}-A \lambda_{\beta s}}{\lambda_{s} \times j i_{s}-n_{p} \omega_{m} \frac{\left|\lambda_{s}\right|^{2}}{\widehat{L}_{s}}}$ & (5.9) \\
\hline$v_{\text {Center } \alpha}=R_{s} i_{\alpha s}-\frac{\lambda_{\beta s}}{\lambda_{\alpha s}}\left(v_{\beta s}-R_{s} i_{\beta s}\right)$ & (5.10) \\
\hline
\end{tabular}

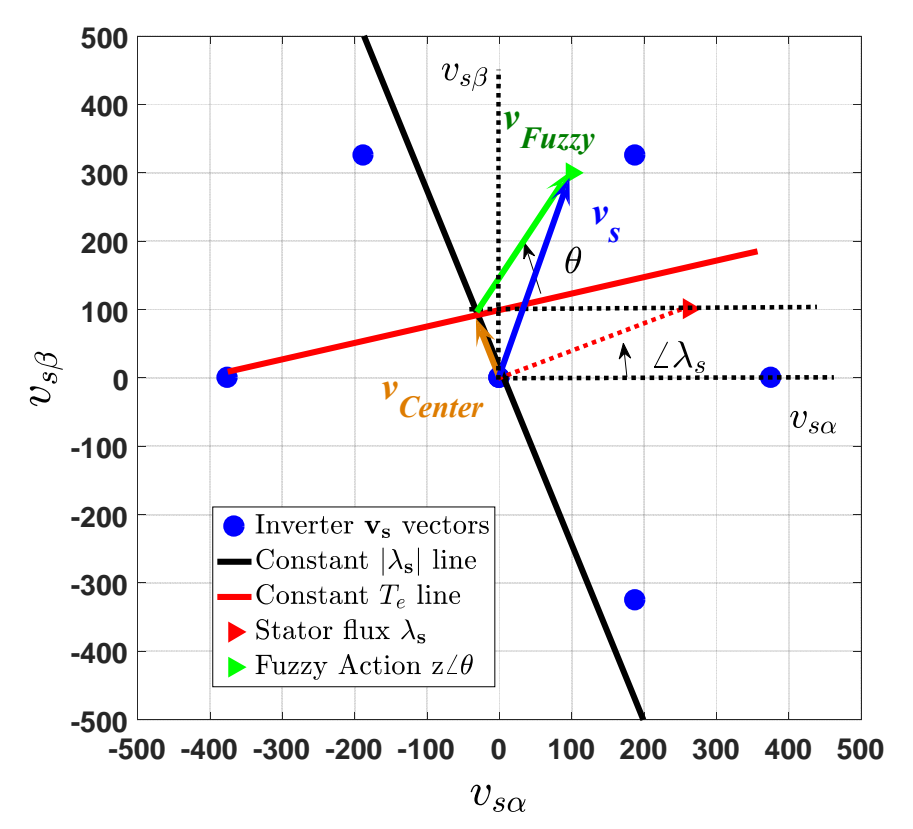

Figure 5.4 Vector summation of Fuzzy out and correction 
The computation in the first case is two trigonometric functions, and in the last case is just the calculation of two linear equations (constant torque and flux) and their tangents (lines slopes). The advantage of this proposed controller is that it corrects the control angle of the ICA compared with the classic table of DTC, which sometimes is erratic, causing the well-known big torque ripple and poor performance. Also, FLC decrease the parameter dependency of the predictive control.

\subsection{Performance Assessment of Different PWM Strategies}

The goal of any modulation scheme is to achieve the desired voltage reference in the CVP with additional reduction of stator current ripple, switching losses, common-mode voltages, electromagnetic interference, etc. In this sense, much research has been devoted towards the development of efficient ways of controlling the VSI with additional low computational burden in its implementation [74]. In general, the modulation strategies can be categorized in continuous and discontinuous PWM due to the different choices for the zero vector duty cycle subdivision [74], [120]. Despite the fact that there is an infinite number of possibilities to subdivide the zero vector duty cycle $\left(\delta_{z}\right)$, the performance and constraints of practical PWM in VSI drives reduce this to a finite number of viable choices [120]. The continuous modulations are sinusoidal PWM (SPWM) and space vector modulation (SVM) and the discontinuous are DPWM0, DPWM1, DPWM2, DPWM3, DPWMax and DPWMin, which have different clamp regions that aligns the reference voltage peak. DPWM1 is suitable for unity power factor, DPWM0 and DPWM2 are efficient for $30^{\circ}$ leading and lagging power factors, respectively and DPWM3 is a distortion-optimized modulation [75]. Deep analysis of these modulation strategies is found in [120] and their generalization is in [74]. 
In this chapter, the behavior of all these modulation schemes using the generalized algorithm presented in [74] is studied. The generalized algorithm is programmed and simulated. A modulator is characterized by different performance parameters [75], i.e., generated current harmonics, maximum modulation index, switching frequency, switching losses and dynamic response. The performance criteria for the assessment, in this work, considers 3 parameters: switching losses, current distortion and dynamic response.

\subsubsection{Switching losses}

The switching losses' evaluation is performed in the same way as [75], assuming that the VSI has switching losses that vary linearly with the amplitude of the current. Thus, the average value of the switching power losses $\left(P_{s w}\right)$ over a fundamental period is assessed in (5.11). This is the same way it was done in Chapter 3, section 3.5.1.

$$
P_{S w}=\frac{1}{2 \pi} \frac{1}{2}\left(V_{D C} \cdot\left(t_{o n}+t_{o f f}\right) \cdot f_{s} \cdot \int_{0}^{2 \pi}\left|I_{F}(\theta)\right| d \theta\right)
$$

where $V_{D C}$ is the dc-link voltage, $t_{o n}$ and $t_{o f f}$ are the respective turn-on and off intervals of the switching devices that can be found in its datasheet, $f_{s}$ is the switching frequency, $I_{F}$ is the output inverter current and $|\cdot|$ is the absolute value function.

\subsubsection{Current Distortion and Dynamic Response}

The current distortion is conducted by the computation of the total harmonic distortion (THD) of the stator current of the IM. For the dynamic behavior, the mean absolute error (MAE) and root mean square error (RMSE) for the electromagnetic torque and magnitude of the stator flux-linkage SV is computed. For these variables, the MAE and RMSE are calculated for the torque between the instantaneous torque reference $\left(T_{r e f(k)}\right)$ and the instantaneous electric torque $T_{e(k)}$, and for the flux-linkage between the magnitude of the 
flux-linkage reference $\left(\lambda_{\text {ref }(k)}\right)$ and magnitude of the flux-linkage $\left(\left|\lambda_{\boldsymbol{s}(\boldsymbol{k})}\right|\right)$. As follows, the instantaneous errors are computed as (5.12) and MAE and RMSE as (5.13). This is the same way it was done in Chapter 3, section 3.5.2.

\begin{tabular}{|c|c|c|}
\hline$e_{T(k)}=\frac{T_{r e f(k)}-T_{e(k)}}{\text { Rated Torque }} \quad e_{\lambda(k)}=\frac{\lambda_{\text {ref }(k)}-\left|\lambda_{\boldsymbol{s}(\boldsymbol{k})}\right|}{\lambda_{\text {ref }(k)}}$ & (5.12) \\
\hline$M A E=\frac{1}{N} \sum_{k=1}^{N}\left|e_{x(k)}\right| \quad R M S E=\sqrt[2]{\frac{1}{N} \sum_{k=1}^{N} e_{x(k)}^{2} \quad x=\{T, \lambda\}}$ & (5.13) \\
\hline
\end{tabular}

\subsection{Simulation and Experimental Results}

In order to compare the performance of the proposed FP-DTC, a simulation and experimental test comparison among classical DTC, P-DTC and proposed FP-DTC is carried out. The IM parameters used in this study are found by characterizing the induction motor at the starting condition under a step of ac voltage. The procedure followed for the parameter estimation is proposed in [121], [122]. The parameter estimation method adjusts the instantaneous input impedance motor during a start-up. For all the simulations and experimental tests, the machine is belt coupled with a self-excited synchronous generator for emulating the loading conditions. The parameter estimation was performed for the no load and loaded case to calculate the inertia of the load. Finally the model parameters are presented in Table 5-1 and these values are used in the simulations. The simulations are conducted in Simulink MatLab environment.

The simulation and experimental implementation were performed under the same conditions of dc-link capacitor voltage $V_{D C}=300 \mathrm{~V}$, switching frequency $f_{S}=10 \mathrm{kHz}$, 
current limitation $I_{\text {limit }}=30 / \sqrt{2} \mathrm{~A}_{\text {rms }}$. For purposes of accuracy and fairness comparison of the simulation and experimental results, the simulation step time was $10 \mu \mathrm{s}$ but the control algorithm operates at $0.1 \mathrm{~ms}$. The PWM in both simulation and experimental is at $10 \mathrm{kHz}$ and centered.

For purposes of control performance verification, a torque profile similar to the one found in electric vehicles' applications is implemented. Simulation and experimental tests are conducted for the 3 controllers. The torque profile starts with a step at $8 \mathrm{Nm}$, which is the rated torque from the name plate of the machine. Then, after 2 seconds, a down step of torque reaches the $4 \mathrm{Nm}$. Two seconds later, a ramp increases the profile until the nominal torque is reached again, keeping it for 2 more seconds, and a down ramp ends the profile in $5 \mathrm{Nm}$ (Figure 5.5(a) for simulation and Figure 5.8(a) for experimental). The flux reference in all the simulations and experiments is $\lambda_{\text {ref }}=0.33 \mathrm{~Wb}$.

Table 5-1 IM Parameters and Nameplate Rating

\begin{tabular}{|c|c|c|c|c|c|c|c|}
\hline Param. & Value & Param. & Value & Rating & Value & Rating & Value \\
\hline$L_{l s}$ & $3.08 \mathrm{mH}$ & $R_{S}$ & $1.506 \Omega$ & $P$ & $1.5 \mathrm{~kW}$ & $P F$ & 0.83 \\
\hline$L_{l r}$ & $3.46 \mathrm{mH}$ & $R_{r}$ & $0.6172 \Omega$ & $V$ & $208 \mathrm{~V}$ & Poles Pairs & 2 \\
\hline$L_{m}$ & $119.22 \mathrm{mH}$ & $J_{m}$ & $0.13 \mathrm{kgm}^{2}$ & $I$ & $5.9 \mathrm{~A}$ & $n_{r}$ & $1750 \mathrm{rpm}$ \\
\hline
\end{tabular}

\subsubsection{Simulation Results}

In Figure 5.5(a) the torque reference and the electromagnetic torque produced by the IM controlled by DTC, P-DTC and FP-DTC is presented. In this figure, it can be noticed that the dynamics of the three algorithms are very fast (well known for DTC), however, the ripple is not the same for the three techniques. In the Figure 5.5(b) a zoom of the 
electromagnetic torque for the three strategies are shown and it is clear that the classical DTC suffers two problems: steady state error and high torque ripple. On the other hand, the P-DTC corrects the steady state error and reduces the torque ripple, nonetheless, the FP-DTC is even better. The high torque ripple in DTC is produced by the selection of the non-optimum voltage vector, as is explained in [102], while FP-DTC chooses the control vector, using the constant torque and flux lines on the CVP, allowing to reduce the torque ripple. The locus of the stator flux vector for the DTC, P-DTC and FP-DTC are depicted in Figure 5.6(a) - (c). The three loci present a coincident response, although the locus of FP-DTC has less ripple. In Figure 5.6(d), a zoom for phase $a$ current for the three controllers is shown in steady state regime. It can be observed that the currents are sinusoidal and the current ripple of DTC is higher that P-DTC and FP-DTC, as well as the current ripple of P-DTC is higher that FP-DTC.

A comparison of the different control methods (DTC, P-DTC and FP-DTC) with respect to THD and power losses is presented, and it can be seen in Table 5-2. For the THD analysis, a preliminary FFT of the stator current is performed to calculate the fundamental component in the permanent regime. This fundamental frequency is the same for the 3 controllers at $42 \mathrm{~Hz}$. For the THD, 2 cycles of the signal are studied at 4.5 seconds.

Table 5-2 Maximum Criteria Values for each Control Technique

\begin{tabular}{|c|c|c|c|c|c|c|}
\hline Controllers & $M A E T_{e}(\%)$ & $R M S E T_{e}(\%)$ & $M A E\left|\lambda_{s}\right|(\%)$ & $R M S E\left|\lambda_{s}\right|(\%)$ & $P_{S w}(W)$ & $T H D I_{s}(\%)$ \\
\hline FPDTC & 3.23 & 6.31 & 0.75 & 2 & 0.0505 & 8.84 \\
\hline PDTC & 6.07 & 7.77 & 1.28 & 1.91 & 0.0442 & 18.81 \\
\hline DTC & 10.2 & 13.3 & 2 & 3.17 & 0.0457 & 25.76 \\
\hline
\end{tabular}



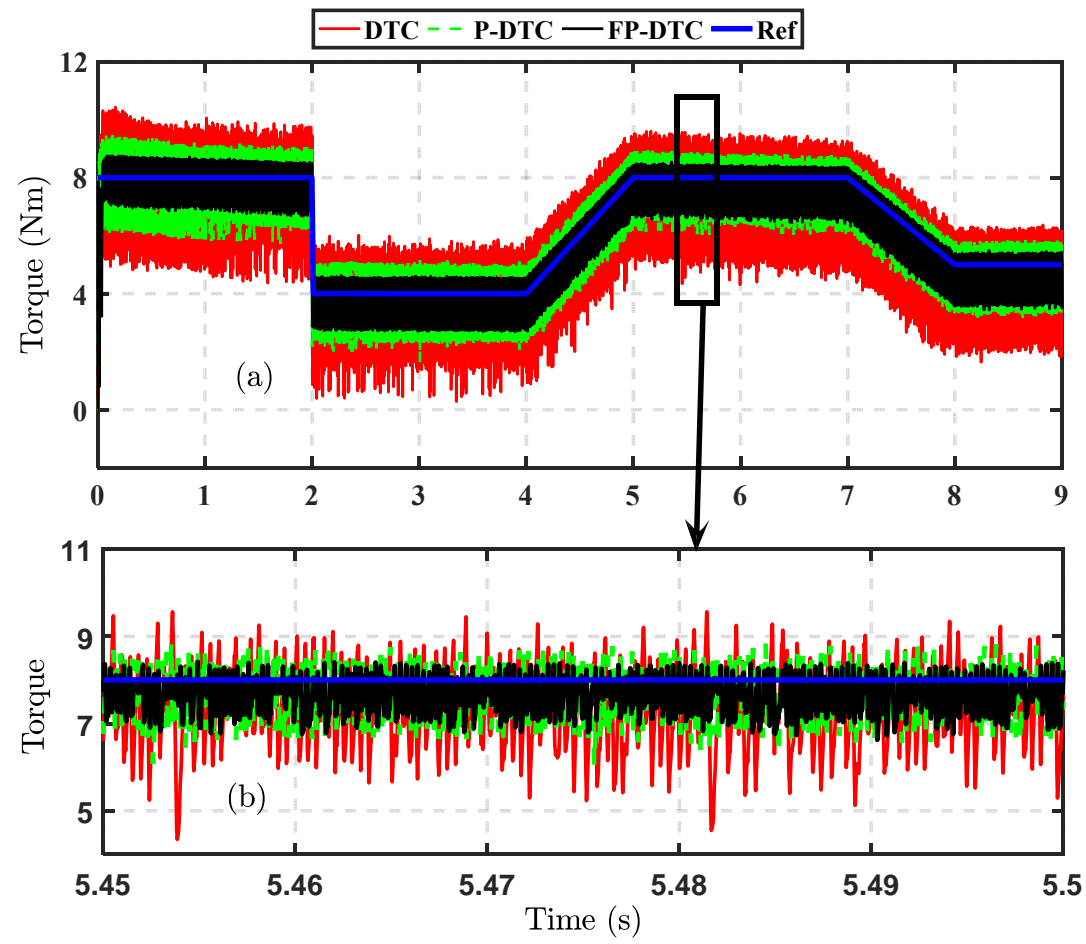

Figure 5.5 (a) Simulation results for comparison of the electromagnetic torque among DTC, P-DTC and FP-DTC. (b) Zoom of (a).
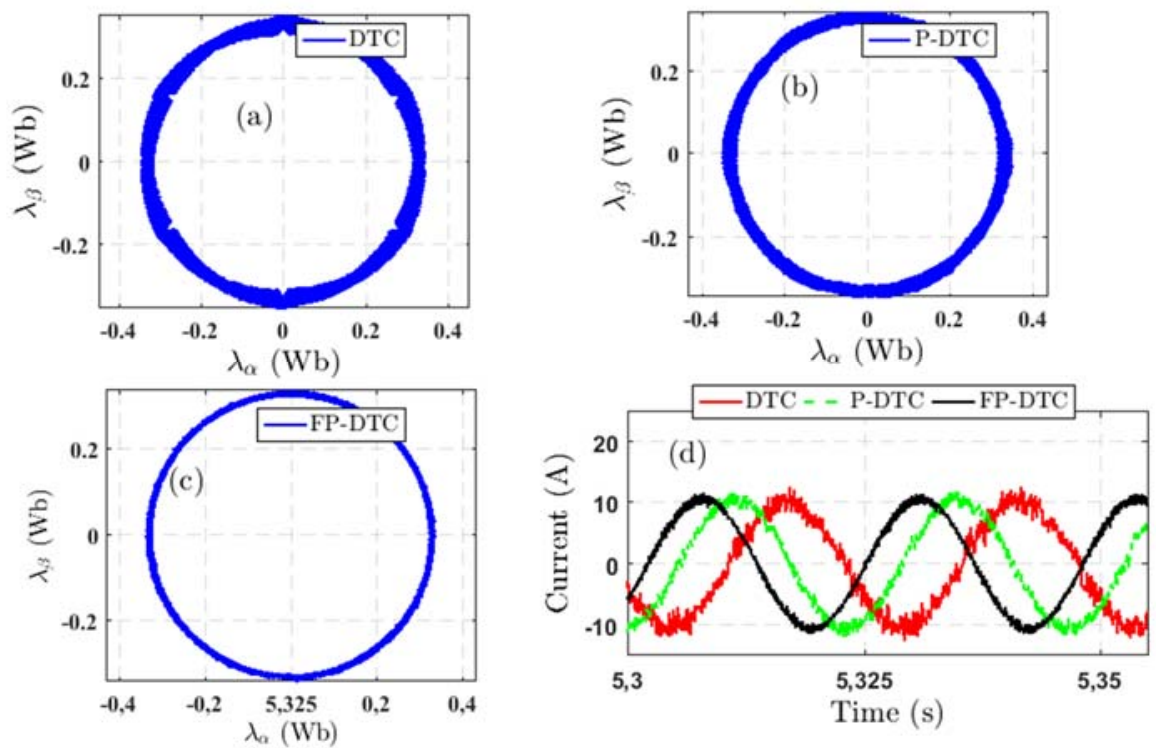

Figure 5.6 Simulation results of the stator flux locus and stator current comparison among the strategies. (a) DTC. (b) PDTC. (c) FP-DTC and (d) Stator currents. 


\subsubsection{Experimental Results}

In order to compare the performance of the proposed FP-DTC, an experimental comparison was accomplished. The Figure 5.7 presents the experimental test rig, which is composed by a PC, dSpace 1104, dc power supply for the control, booster for increase the pulses ( $0 \mathrm{~V}$ off $5 \mathrm{~V}$ on) from the dSpace to the IGBT drivers (-10 V off $15 \mathrm{~V}$ on), sensor board (LEM voltage and current transducers), oscilloscope Tecktronics, ac autotransformer, motor-generator set (motor WEG W21, generator Yanan SLG-164B) and voltage source inverter (VSI), which consist of Semikron IGBTs (1200 V-50 A), drivers and 2 electrolytic capacitors in series of $1200 \mu \mathrm{F}$ and $525 \mathrm{~V}$.

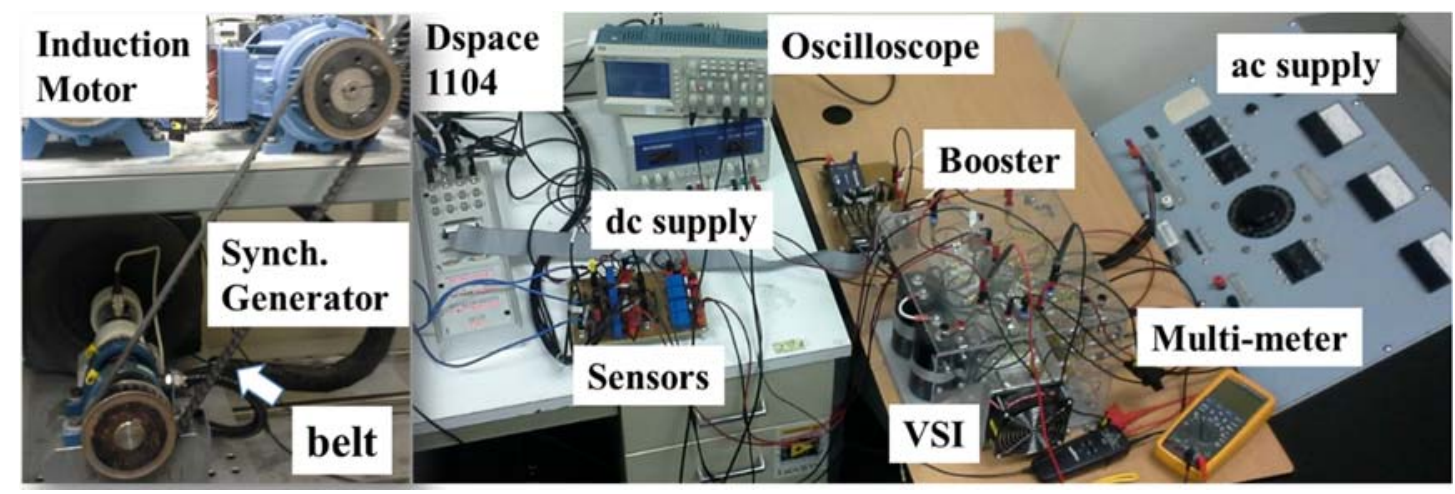

Figure 5.7 Experimental setup

The experimental results for the torque profile response can be observed in Figure 5.8(a).

Figure 5.8(b) presents a zoom of the response for a period of $0.05 \mathrm{~s}$. The experimental results are highly correlated with the simulation results presented in Figure 5.5(a) and (b). As it is expected, all the controllers have a fast dynamic response. Nonetheless, DTC and P-DTC have more torque ripple and steady state error than FP-DTC. 

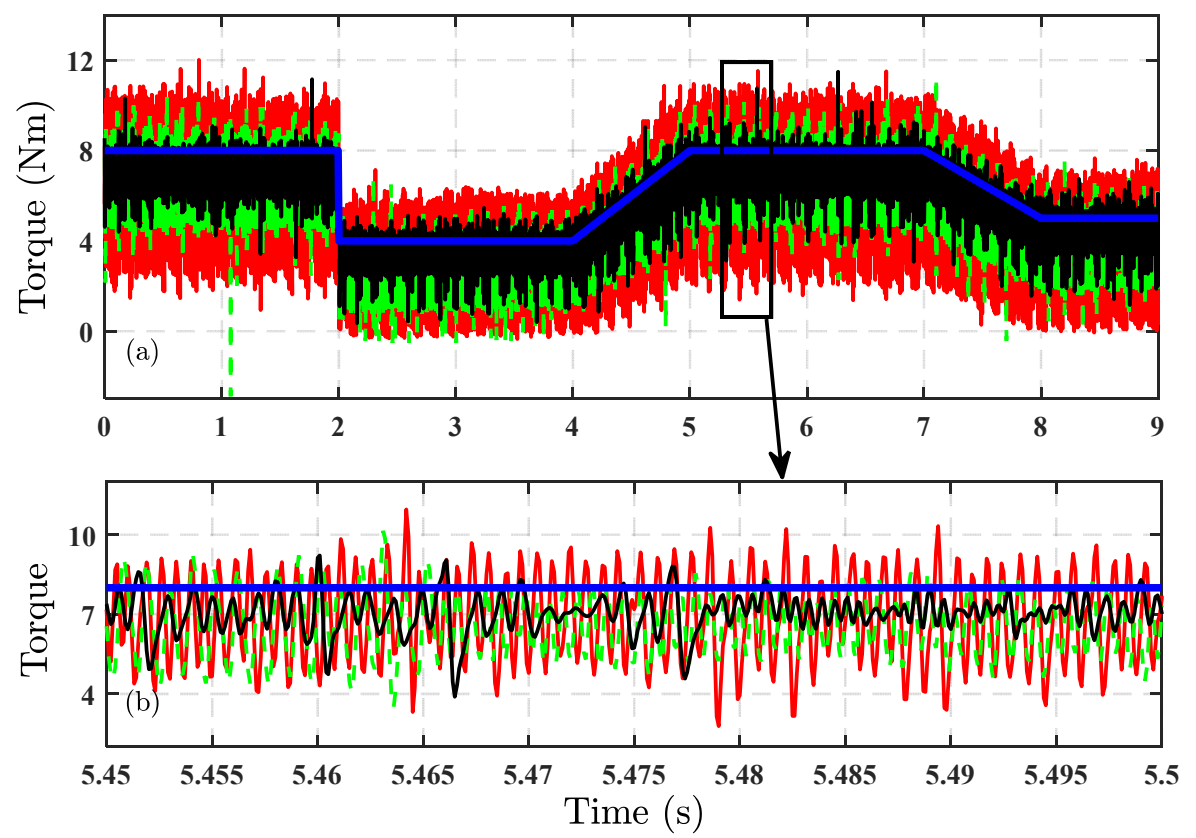

Figure 5.8 Experimental comparison of the electromagnetic torque among DTC, P-DTC and FP-DTC.

In Figure 5.9, the locus of the stator flux vector for the DTC, P-DTC and FP-DTC are presented. It can be seen, that the 3 loci present similar performance, as expected from the simulation, however, the FP-DTC demonstrates less ripple. In Figure 5.9(d), the dc-link voltage is presented, as it is not an ideal source like in the simulation. The effect on these variations affects the torque response of the experimental, resulting in higher torque ripple than simulations.

For final comparison among the three controllers and its correlation with the simulation results, a comparative results of the torque ripple, stator flux ripple and current ripple is presented in Table 5-3. 

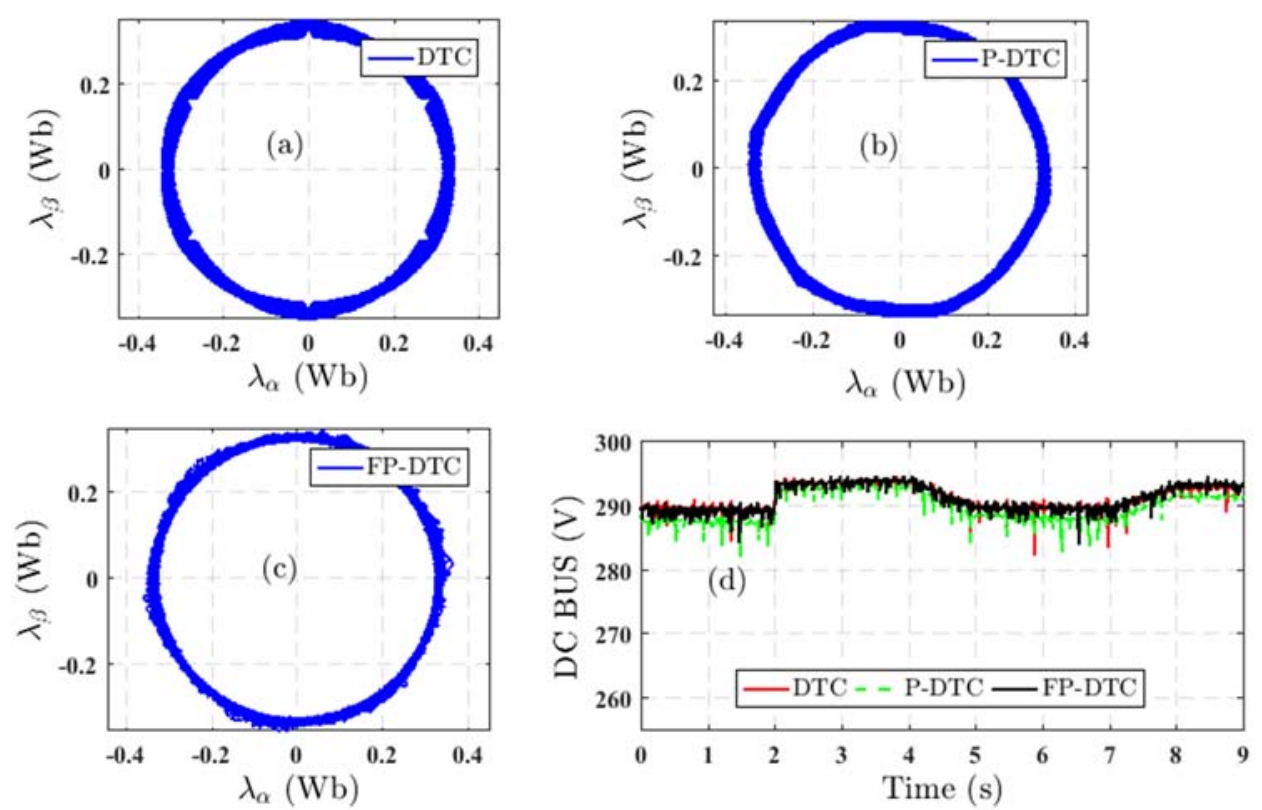

Figure 5.9 Experimental comparison of the stator flux locus and dc bus voltages among DTC, P-DTC and FP-DTC

Table 5-3 Simulation and Experimental Comparison among Torque, Flux and Current Riples of DTC, P-DTC and FPDTC.

\begin{tabular}{|c|c|c|c|c|c|c|}
\hline & \multicolumn{3}{|c|}{ Simulation Results } & \multicolumn{3}{c|}{ Experimental Results } \\
\hline & DTC & P-DTC & FP-DTC & DTC & P-DTC & FP-DTC \\
\hline $\begin{array}{c}\text { Torque Ripple } \\
\text { (Nm) }\end{array}$ & 4.5 & 2 & 1 & 6 & 2.5 & 1 \\
\hline Flux Ripple (Wb) & 0.05 & 0.035 & 0.02 & 0.05 & 0.05 & 0.04 \\
\hline Current Ripple (A) & 2 & 1.5 & 1 & 4 & 2 & 1 \\
\hline
\end{tabular}

\subsubsection{Sensitivity analysis}

The proposed control strategy adjusts the fuzzy rules based on the machine model. Thereby, to compute the FP-DTC algorithm, the machine's parameters are required. A sensitivity analysis is presented to evaluate the robustness of the proposed controller under uncertainty in the IM parameters. The rotor resistance, leakages and mutual inductances were changed over a range of $\pm 50 \%$. Each case was simulated using the same torque 
profile of section 5.5.1. The MAE between the instantaneous torque reference and the instantaneous electric torque, and between the magnitude of the stator flux reference and actual flux, are presented in Table 5-4. The RMSE is omitted for simplification purposes.

Table 5-4 Maximum MAE and RMSE of $R_{r}, L_{l s}, L_{l r}$ and $L_{m}$ for $50 \%$ variation on their Value.

\begin{tabular}{|c|c|c|c|c|c|c|c|c|}
\hline \multirow{2}{*}{ Parameters } & \multicolumn{2}{|c|}{ MAE } & \multirow{2}{*}{$\begin{array}{c}\text { Parameters } \\
\text { Change } \\
\text { Increase } \\
(+50 \%)\end{array}$} & \multicolumn{2}{|c|}{ MAE } & \multirow{2}{*}{\begin{tabular}{|c} 
Parameters \\
Change \\
Decrease \\
(-50\%)
\end{tabular}} & \multicolumn{2}{|c|}{ MAE } \\
\hline & $T_{e}(\%)$ & $\left|\lambda_{s}\right|(\%)$ & & $T_{e}(\%)$ & $\left|\lambda_{s}\right|(\%)$ & & $T_{e}(\%)$ & $\left|\lambda_{s}\right|(\%)$ \\
\hline$L_{l s}=3.08 \mathrm{mH}$ & \multirow{4}{*}{3.23} & \multirow{4}{*}{0.75} & $L_{l s}=4.62 \mathrm{mH}$ & 5.16 & 0.74 & $L_{l s}=1.54 \mathrm{mH}$ & 5.16 & 0.74 \\
\hline$L_{l r}=3.46 \mathrm{mH}$ & & & $L_{l r}=5.29 \mathrm{mH}$ & 5.05 & 0.75 & $L_{l r}=1.73 \mathrm{mH}$ & 503 & 0.67 \\
\hline$L_{m}=119.22 \mathrm{mH}$ & & & $L_{m}=178.83 \mathrm{mH}$ & 5.03 & 0.67 & $L_{m}=59.61 \mathrm{mH}$ & I 5.03 & 0.67 \\
\hline$R_{r}=0.6172 \Omega$ & & & $R_{r}=0.9258 \Omega$ & 5.25 & 0.78 & $R_{r}=0.3086 \Omega$ & 5.04 & 0.70 \\
\hline
\end{tabular}

As it can be noted from Table 5-4, the MAE is smaller than 6\%, indicating that the FPDTC is robust to the variation of the main parameters of the model. Also, it can be observed that the MAE for the flux is basically constant. Typically, the magnitude of the flux is a more stable variable than the electromagnetic torque.

\subsubsection{Pulse Width Modulation Strategies: Comparative Study}

In this section, 8 pulse width modulations (SPWM, SVM, DPWM0, DPWM1, DPWM2, DPWM3, DPWMax and DPWMin) were studied, simulated and compared as it is shown in Table 5-5. For this comparison assessment, 3 criteria are considered: switching losses $\left(P_{s w}\right)$, current distortion $\left(T H D I_{s}\right)$ and dynamic response (MAE and RMSE of $T_{e}$ and $\left|\lambda_{s}\right|$ ).

From Table 5-5, it can be observed that the SVM presents the best dynamic performance in terms of less torque error and the second best stator flux error. With respect to the current distortion, DPWMin has the lowest THD, nevertheless, SVM has the following lowest 
value. For the power switching losses, the criterion gives similar values for all the modulation techniques. DPWM3 presents the lower losses, per contra, SVM is not far from there. SVM is the fourth best technique. Therefore, in the combination of all the criteria, SVM is the one that presents the better behavior. In this fashion, SVM is chosen among the modulations for the real experimental comparison with the previous results.

Table 5-5 Maximum Criteria Values for each Modulation Technique

\begin{tabular}{|l|c|c|c|c|c|c|}
\hline & $\begin{array}{c}M A E T_{e} \\
(\%)\end{array}$ & $\begin{array}{c}R M S E T_{e} \\
(\%)\end{array}$ & $\begin{array}{c}M A E\left|\lambda_{s}\right| \\
(\%)\end{array}$ & $\begin{array}{c}R M S E\left|\lambda_{s}\right| \\
(\%)\end{array}$ & $\begin{array}{c}\text { THD } I_{s} \\
(\%)\end{array}$ & $\begin{array}{c}P_{S W} \\
(W)\end{array}$ \\
\hline PWM & 3.23 & 6.31 & 0.75 & 2.00 & 8.86 & 0.0505 \\
\hline SVM & 2.39 & 5.81 & 0.71 & 1.98 & 5.67 & 0.0509 \\
\hline DPWM0 & 3.69 & 6.69 & 0.71 & 1.87 & 6.78 & 0.0510 \\
\hline DPWM1 & 7.05 & 11.45 & 0.77 & 2.23 & 10.30 & 0.0531 \\
\hline DPWM2 & 3.80 & 6.83 & 0.72 & 2.15 & 7.25 & 0.0506 \\
\hline DPWM3 & 3.41 & 6.42 & 0.70 & 1.89 & 7.30 & 0.0502 \\
\hline DPWMax & 3.04 & 6.36 & 0.73 & 2.02 & 5.72 & 0.0516 \\
\hline DPWMin & 2.79 & 6.04 & 0.71 & 1.94 & 5.56 & 0.0512 \\
\hline
\end{tabular}

\subsubsection{Simulation and Experimental Results for the Comparative Assessment between SVM and PWM}

In this section, the experimental and simulation comparison results are presented between the selected modulation technique (SVM) and the original modulation used (PWM). The variables for the comparison are the same as used before: electromagnetic torque (Figure 5.10 and Figure 5.12, simulation and experimental results, respectively), locus of the stator flux linkage (Figure 5.11(a), (b) for simulation, Figure 5.13(a) and (b) for experimental results), stator phase $a$ current (Figure 5.11(c) and Figure 5.13(c), simulation and experimental results, respectively) and finally dc-link voltage (Figure 5.13(d) only experimental results). 
Figure 5.10 and Figure 5.12 introduced a similar profile as the one presented in Figure 5.5 and Figure 5.8. From these figures, not only the good torque dynamic of the FP-DTC, but also, the small torque ripple for both modulation techniques can be observed. In the zoom of these figures, for simulation and experiments results, it can be seen that the SVM presents less torque ripple than the PWM method.

From the comparison of the locus for both simulation and experimental results, it is difficult to observe any difference between the SVM and PWM techniques, however, Table 5-6 indicates better MAE and RMSE for the SVM modulation. From the simulation, the stator phase $a$ currents are perfectly sinusoidal waveforms for the 2 modulation strategies. The experimental results show some noise harmonic, which is similar for both techniques. Nevertheless, Figure 5.14 depicts the FFT for the stator current, in which it can be demonstrated that the FFT of the current phase $a$ for the PWM has more harmonic content than the SVM. Also, Table 5-6 indicates the numerical values that corroborate the results. Finally, the dc-link voltage is compared for the experimental results in Figure 5.13(d), where congruent behavior is found for the two modulations techniques.

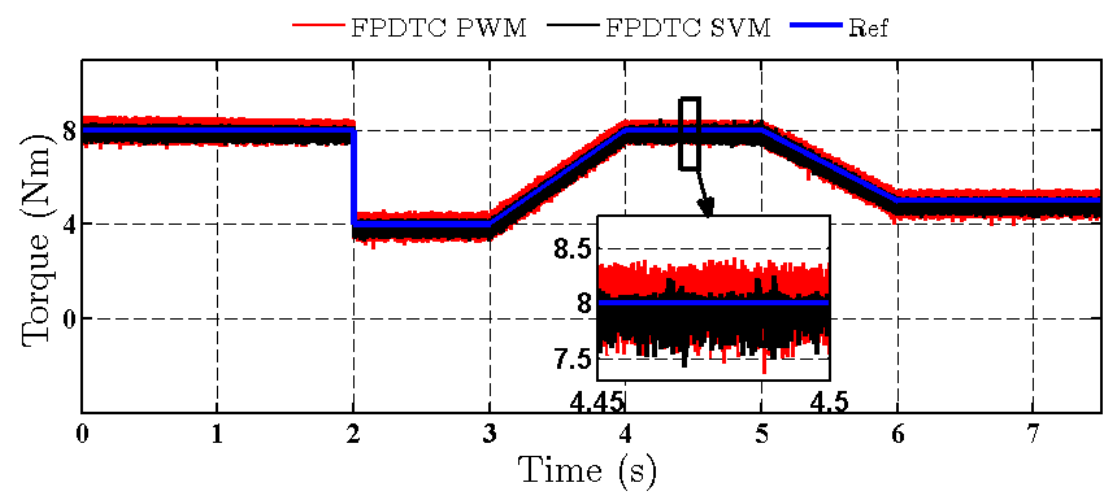

Figure 5.10 Simulation comparison of the electromagnetic torque of FP-DTC between two modulation techniques: PWM and SVM. 

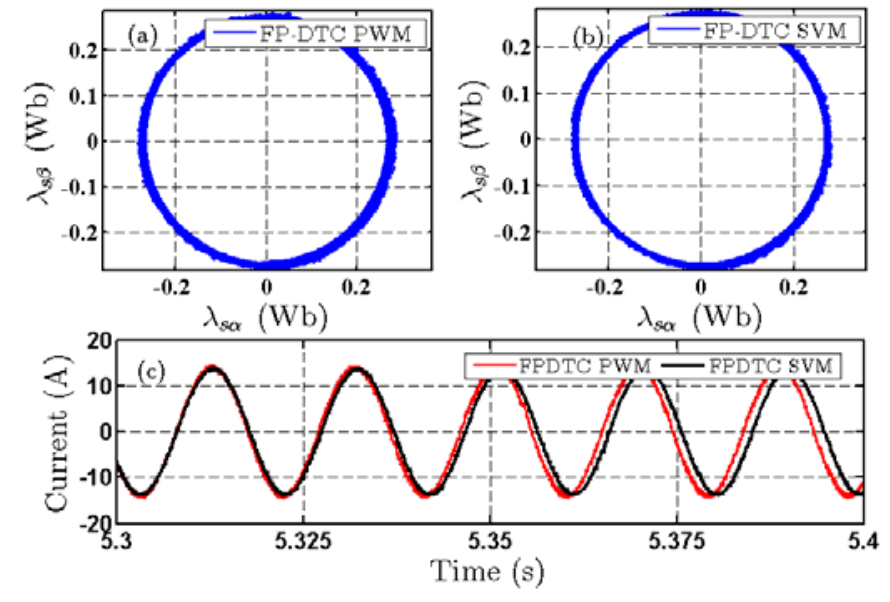

Figure 5.11 Simulation comparison of FP-DTC between two modulation techniques (a) stator flux magnitude PWM. (b) stator flux magnitude PWM and (c) phase a current

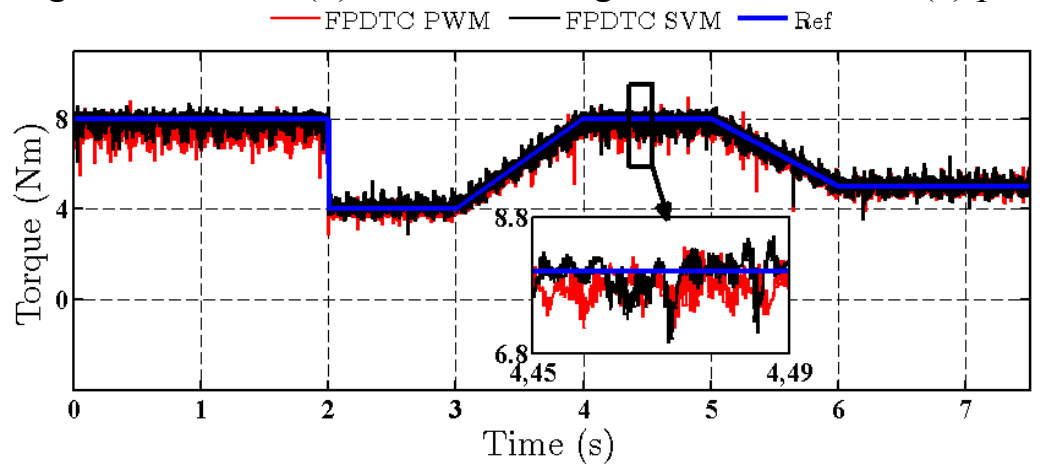

Figure 5.12 Experimental comparison of the electromagnetic torque of FP-DTC between two modulation techniques: PWM and SVM.
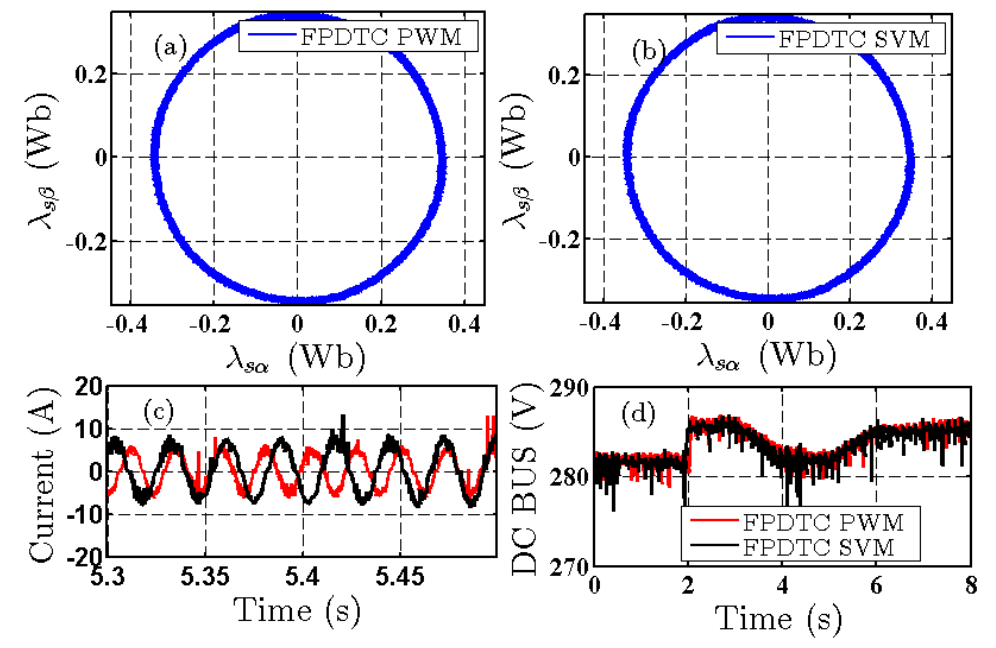

Figure 5.13 Experimental comparison of FP-DTC between two modulation techniques (a) stator flux magnitude PWM. (b) stator flux magnitude PWM (c) phase a current and (d) 


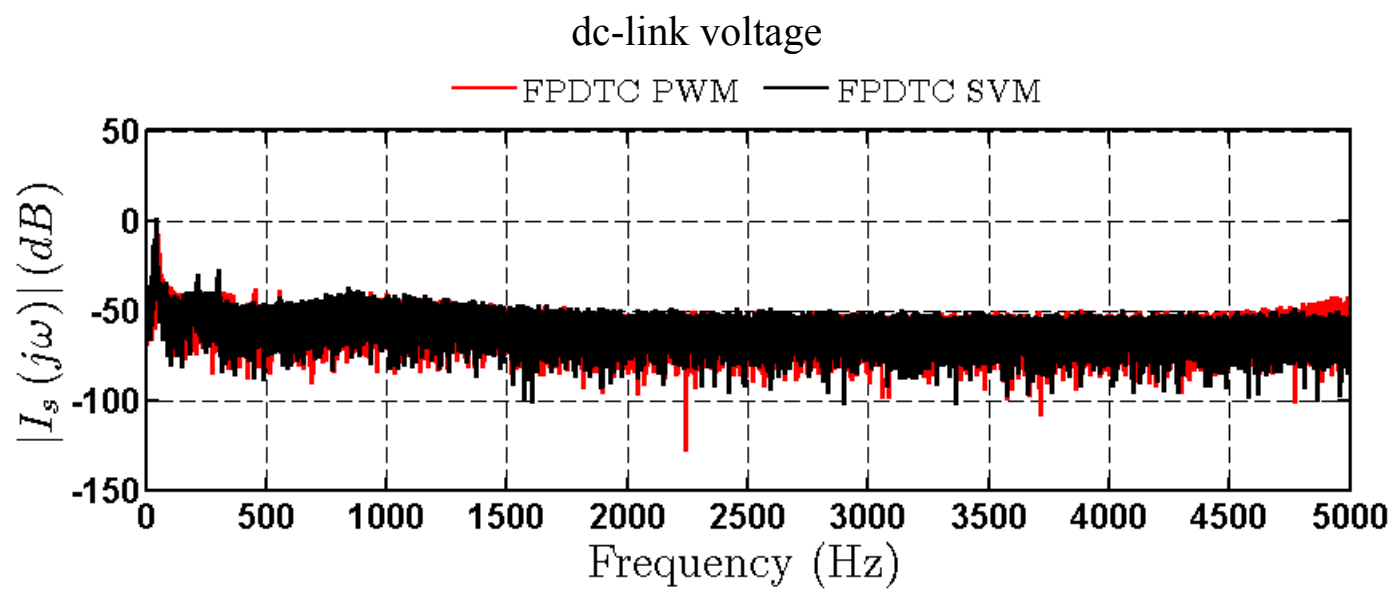

Figure 5.14 Experimental comparison the FFT for the stator current phase $a$ of FP-DTC between two modulation techniques: PWM and SVM.

Table 5-6 Maximum Criteria Values for each Modulation Technique

\begin{tabular}{|c|c|c|c|c|c|}
\hline & $M A E T_{e}(\%)$ & $R M S E T_{e}(\%)$ & $M A E\left|\lambda_{s}\right|(\%)$ & $R M S E\left|\lambda_{s}\right|(\%)$ & $T H D I_{s}(\%)$ \\
\hline PWM & 25.09 & 31.34 & 1.89 & 2.31 & 5.59 \\
\hline SVM & 19.62 & 25.40 & 1.94 & 2.10 & 4.98 \\
\hline
\end{tabular}

\subsection{Conclusions}

An innovative and simple control strategy based on the integration of a FLC algorithm with minimum and linear MF with a predictive methodology is presented. The advantages of both techniques are exploited to improve the drawbacks and performance of the direct torque controller. Simulations and experimental results show an improvement in the control performance of the torque, including ripple and steady state error reduction, and a satisfactory performance at the low-speed region. The proposed control was tested under $\pm 50 \%$ variation of the IM parameters, obtaining similar results and validating its low dependency. Different modulation strategies were studied, and it was found that SVM presents the best performance for the drive application under the proposed controller. 


\section{Chapter 6 Complex-Vector Transient and Steady-State Modelling of Induction Machines with Stator Winding Inter-turn Short-Circuit Fault}

\subsection{Introduction}

As it was mentioned in Chapter 2, three-phase induction motors (IM) are important in the industrial world due to their robustness, low cost, low maintenance and high performance. Driving IM with power electronics converters could be one of the causes of stator winding fault due to the fast switching frequency that results in large $\mathrm{dv} / \mathrm{dt}$. This, combined with environmental conditions and the machine's internal stresses, could lead to incipient stator faults. These faults can evolve into catastrophic failures if they are left undetected and they can cause financial losses due to costly repairs and downtime. Among the different types of IM's faults, the most recurrent is the winding inter-turn short-circuit in industrial applications [8].

This failure is generally the consequence of a combination of several conditions: aging, contamination, high temperatures, machine insulation class and large $\mathrm{dv} / \mathrm{dt}$ in the winding terminals. Basically, the fault starts with the degradation of the insulation characteristics of the materials, leading to the breakdown of the turn-to-turn insulation and thus to the incipient inter-turn short-circuit (ITSC) fault. Consequently, large current will flow due to the low impedance path, generating abnormal joule effect that may burn the neighboring turns' insulation [24].

The first step in the process of FDI of ITSC fault is the development of IM models regarding this situation. Several models for IM under stator fault have been proposed in [123]-[126]. The model presented in [124] is a method of harmonic analysis for computing 
the coupling impedances on the IM. The described models in [125]-[127] are derived by means of winding functions. In all these machine models, the IM design parameters are required, such as: the number of slots, coil pitch, number of turns per coil, number of rotor bars, stator and rotor conductor's distributions, etc. The models presented in [34], [36], [123] are based on mathematical transformations. In [123], a specific rotation transformation is applied to the rotor circuit in order to refer it to the stator " $a b c$ " coordinates. The inter-turn asymmetry of the three-phase winding is modeled by the reduction of $\mu$ percent (fault severity factor) of the turn's number $\left(N_{S x}\right)$ for the stator winding phase $x=\{a, b, c\}$, however, the short-circuit indeed is not modeled. In [34], [36] the stationary reference frame transformation is used. The advantage of this transformation is the order reduction of the ODE. The IM faulty model considers the reduction of the turns $(\mu$ percent) in the faulty winding phase, as well as the short-circuit analysis.

In this chapter, the simplest state-space (SS) representation for the IM with stator winding fault is derived following the model in [36], and a study seeking faulty signatures is performed. The benefits of this model lie in the characteristic that can be represented and separated in two modes: "Common Mode" (CM) and "Differential Mode" (DM). The common mode indicates the healthy behavior of the IM. The differential mode represents the faulty component of the ITSC. This decoupling is achieved because the model is based on the flux-linkages space-vector. In the search for fault signatures, the complex vector (CV), operational and the steady state equivalent circuits (ECs) for the faulty IM are developed. The SS representation and ECs are verified by means of simulation and compared with the healthy machine. The effect of the fault severity factor and fault 
resistance on different signatures is studied at different operation conditions. The torque pulsations and the stator current $\mathrm{CV}$ are computed to evaluate the faulty model.

\subsection{Modelling of IM with Stator ITSC Fault}

IM with stator winding ITSC on the phase " $a$ " is shown in Figure 6.1(a) where $N_{s a 2}$ represents the shorted turns and $N_{s a}=N_{s a 1}+N_{s a 2}$. Then the fault severity factor is defined as $\mu=\frac{N_{s a 2}}{N_{s a}}$, following the same notation as in [34], [36]. Figure 6.1(b) presents a circuit diagram of three-phase IM for the asymmetric stator winding, where two terms are highlighted: the transformer voltage and the generation terms, $e_{s a 1}=M \frac{d i_{s a 2}}{d t}, e_{s a 2}=$ $M \frac{d i_{s a 1}}{d t}$. For this analysis, it is assumed that the leakage inductance for the shorted turns is $\mu L_{l s}, R_{f}$ is fault impedance, $i_{f}$ is the faulty leakage current in the short-circuit path, $i_{s x}$ and $v_{s x}$ are the stator winding current and voltage for the phase $x$ where $x=\{a, b, c\}$.

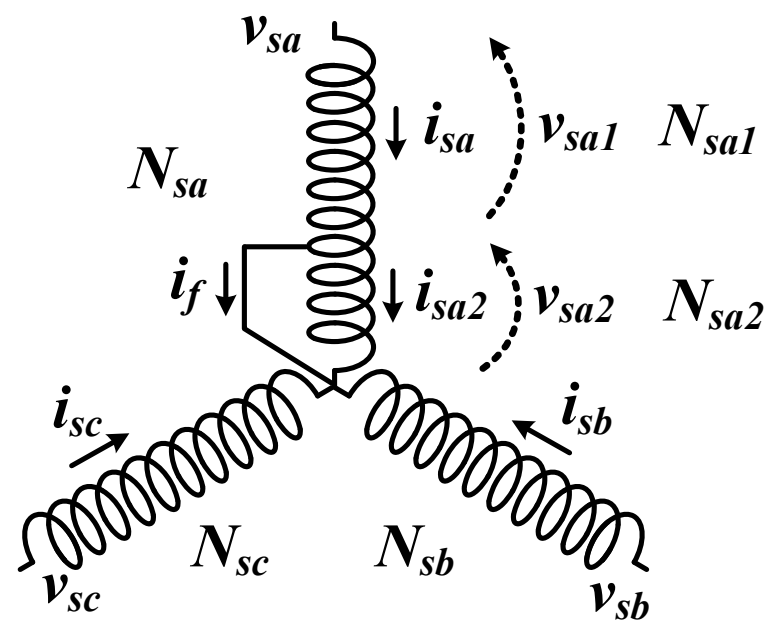

(a) Physical Winding 


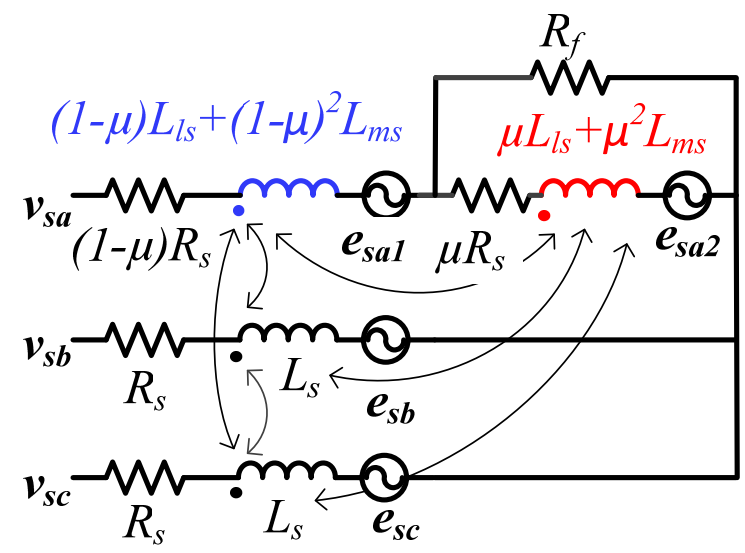

(b) Circuit diagram

Figure 6.1 Three-phase stator winding of an induction machine with inter-turn shortcircuit in the phase a. (a) Physical Winding (b) Circuit diagram

\subsubsection{IM Equations in Primitive Coordinates}

The stator and rotor voltage and flux linkages equations for the faulty machine in vector form, referred to the stator, can be expressed as [34]:

$\left[\begin{array}{c}{\left[\boldsymbol{v}^{\prime}{ }_{s}\right]} \\ {\left[\boldsymbol{v}_{\boldsymbol{r}}\right]}\end{array}\right]=\left[\begin{array}{cc}{\left[\boldsymbol{R}_{\boldsymbol{s}}^{\prime}\right]} & {[\mathbf{0}]} \\ {[\mathbf{0}]} & {\left[\boldsymbol{R}_{\boldsymbol{r}}\right]}\end{array}\right]\left[\begin{array}{c}{\left[\boldsymbol{i}_{\boldsymbol{s}}^{\prime}\right]} \\ {\left[\boldsymbol{i}_{\boldsymbol{r}}\right]}\end{array}\right]+p\left[\begin{array}{c}{\left[\boldsymbol{\lambda}^{\prime}{ }_{\boldsymbol{s}}\right]} \\ {\left[\boldsymbol{\lambda}_{\boldsymbol{r}}\right]}\end{array}\right]$
$\left[\begin{array}{c}{\left[\boldsymbol{\lambda}^{\prime}{ }_{\boldsymbol{s}}\right]} \\ {\left[\boldsymbol{\lambda}_{\boldsymbol{r}}\right]}\end{array}\right]=\left[\begin{array}{cc}{\left[\boldsymbol{L}^{\prime}{ }_{\boldsymbol{s s}}\right]} & {\left[\boldsymbol{L}^{\prime}{ }_{\boldsymbol{s r}}\right]} \\ {\left[\boldsymbol{L}_{\boldsymbol{r} \boldsymbol{s}}\right]^{t}} & {\left[\boldsymbol{L}_{\boldsymbol{r r}}\right]}\end{array}\right]\left[\begin{array}{c}{\left[\boldsymbol{i}^{\prime}{ }_{\boldsymbol{s}}\right]} \\ {\left[\boldsymbol{i}_{\boldsymbol{r}}\right]}\end{array}\right]$

where: $\left[\boldsymbol{v}^{\prime}{ }_{s}\right]=\left[\begin{array}{lllll}v_{s a 1} & v_{s a 2} & v_{s b} & v_{s c}\end{array}\right]^{t},\left[\boldsymbol{i}_{\boldsymbol{s}}^{\prime}\right]=\left[\begin{array}{llll}i_{s a} & i_{s a 2} & i_{s b} & i_{s c}\end{array}\right]^{t}$,

$\left[\begin{array}{lll}\boldsymbol{i}_{\boldsymbol{r}}\end{array}\right]=\left[\begin{array}{lll}i_{r a} & i_{r b} & i_{r c}\end{array}\right]^{t},\left[\lambda^{\prime}{ }_{s}\right]=\left[\begin{array}{llll}\lambda_{s a 1} & \lambda_{s a 2} & \lambda_{s b} & \lambda_{s c}\end{array}\right]^{t},\left[\begin{array}{ll}\lambda_{r}\end{array}\right]=\left[\begin{array}{lll}\lambda_{r a} & \lambda_{r b} & \lambda_{r c}\end{array}\right]^{t}$, $\left[\boldsymbol{R}_{\boldsymbol{s}}^{\prime}\right]=R_{s} \operatorname{diag}[(1-\mu) \quad \mu \quad 1 \quad 1],\left[\boldsymbol{R}_{\boldsymbol{r}}\right]=R_{r} \operatorname{diag}\left[\begin{array}{lll}1 & 1 & 1\end{array}\right]=R_{r}\left[\boldsymbol{I}_{3 \times 3}\right]$.

$\left[\boldsymbol{\lambda}^{\prime}{ }_{\boldsymbol{s}}\right]$ and $\left[\boldsymbol{\lambda}_{\boldsymbol{r}}\right]$ are the flux linkages vectors, $\left[\boldsymbol{R}_{\boldsymbol{s}}^{\prime}\right]$ and $\left[\boldsymbol{R}_{\boldsymbol{r}}\right]$ are the resistance matrices and $\left[\boldsymbol{L}^{\prime}{ }_{s \boldsymbol{s}}\right]=\left[\boldsymbol{L}^{\prime}{ }_{l \boldsymbol{s}}\right]+\left[\boldsymbol{L}^{\prime}{ }_{\boldsymbol{m} \boldsymbol{s}}\right]$ and $\left[\boldsymbol{L}_{\boldsymbol{r} r}\right]$ are the inductance matrices, all of them, for the stator and rotor respectively. $\left[\boldsymbol{L}^{\prime}{ }_{s r}\right]$ and $\left[\boldsymbol{L}_{r s}^{\prime}\right]^{t}$ are the mutual inductance matrices between stator and rotor. The symbol $p$ is the operator $\frac{d}{d t}$, the function diag[-] is the diagonal matrix, 
$\left[\boldsymbol{I}_{3 \times 3}\right]$ is the 3 by 3 identity matrix, the current $i_{s a 2}$ is defined in Figure $6.1\left(\right.$ a) as $i_{s a 2}=$ $i_{s a}-i_{f}$. The inductances matrices of (6.1) are:

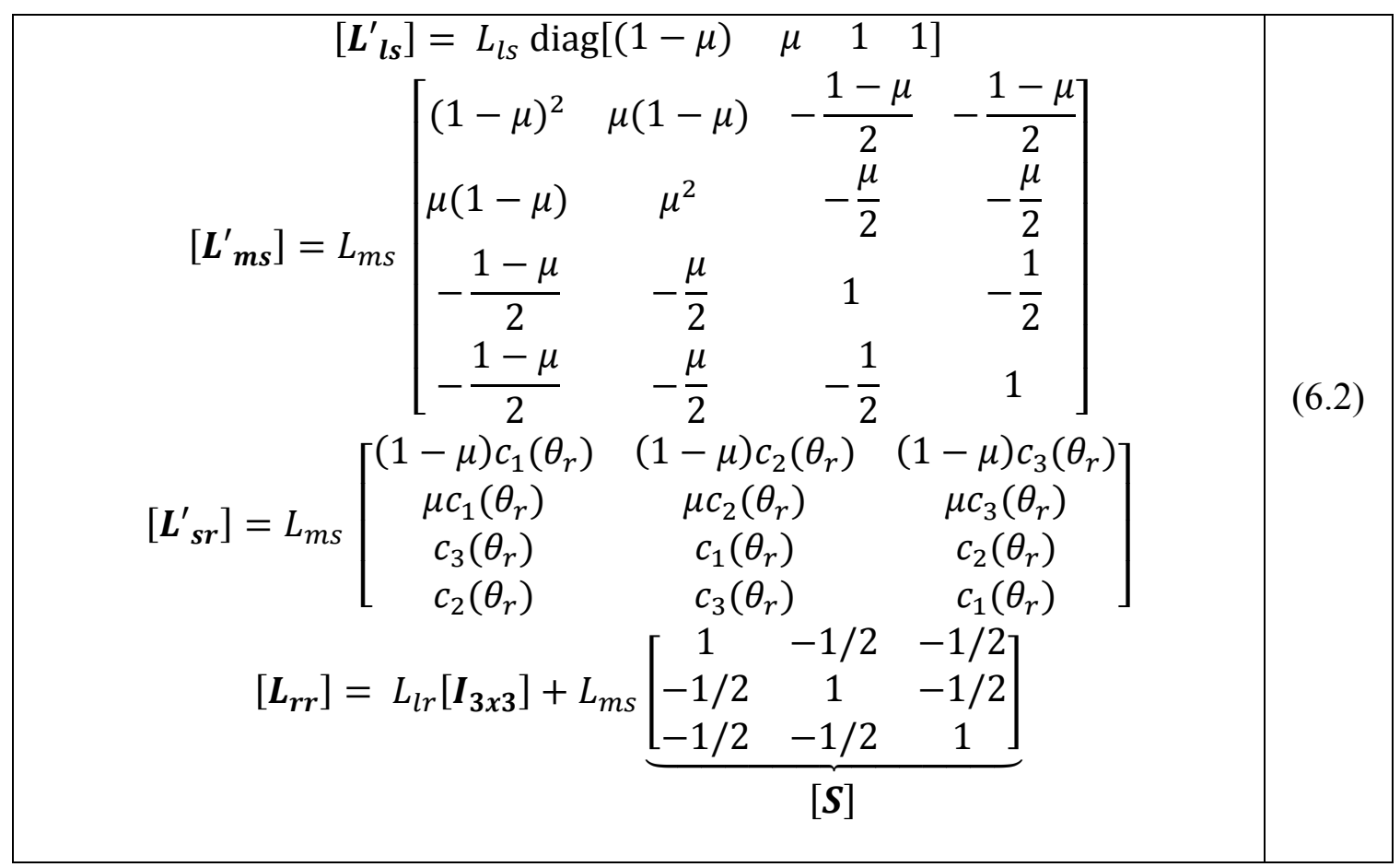

where in general $c_{1}(\zeta)=\cos \zeta, c_{2}(\zeta)=\cos \left(\zeta+\frac{2 \pi}{3}\right), c_{3}(\zeta)=\cos \left(\zeta-\frac{2 \pi}{3}\right), L_{l r}$ is the rotor leakage inductance and $[\boldsymbol{S}]$ is the symmetric matrix. The matrices and vectors $([\boldsymbol{f}]=$ $[\boldsymbol{R}],[\boldsymbol{L}],[\boldsymbol{v}],[\boldsymbol{i}]$ and $[\lambda])$ with the superscript prime $\left(\left[\boldsymbol{f}_{\boldsymbol{q}}{ }_{\boldsymbol{q}}\right]\right)$ represent the asymmetrical IM. The ones without the prime $\left(\left[\boldsymbol{f}_{\boldsymbol{q}}\right]\right)$ represent the symmetrical IM. The subscript $\boldsymbol{q}$ indicates the coordinate system for the stator $(\boldsymbol{q}=\boldsymbol{s})$ or rotor $(\boldsymbol{q}=\boldsymbol{r})$. Thus, the faulty machine equations can be rewritten in terms of the symmetrical IM matrices and vectors plus other terms that represent the asymmetrical part as follows: 


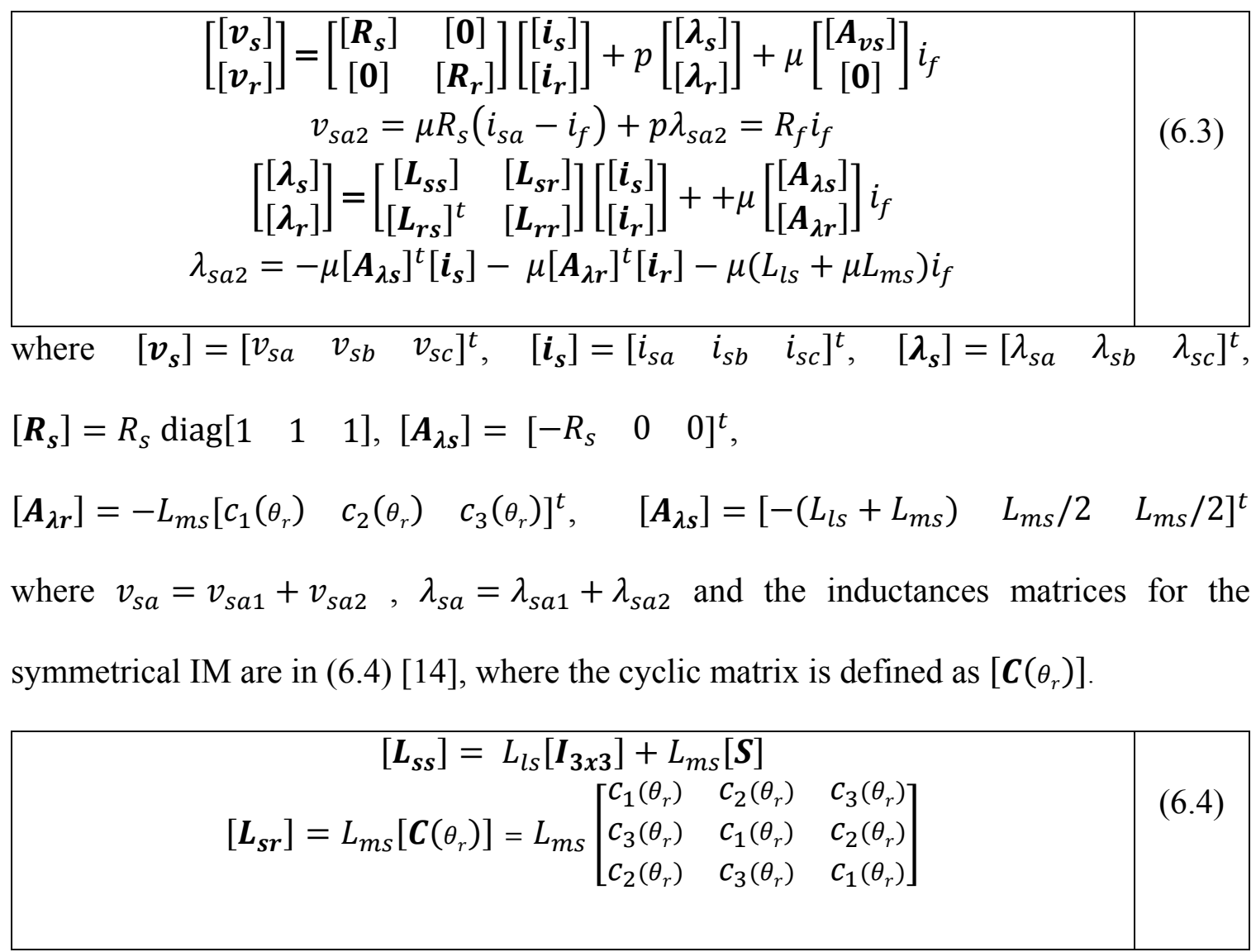

\subsubsection{Electromagnetic Torque Analysis}

The electromagnetic torque in terms of the primitive coordinates is [14]:

$$
T_{e}=\frac{P}{2}\left[\boldsymbol{i}_{\boldsymbol{s}}\right]^{t} \frac{\partial\left[\boldsymbol{L}_{\boldsymbol{s} \boldsymbol{r}}\right]}{\partial \theta_{r}}\left[\boldsymbol{i}_{\boldsymbol{r}}\right]
$$

Inserting the corresponding terms in (6.5), the torque equation can expressed as:

$$
T_{e}=\underbrace{\frac{P}{2}\left[\boldsymbol{i}_{\boldsymbol{s}}\right]^{t} \frac{\partial\left[\boldsymbol{L}_{\boldsymbol{s r}}\right]}{\partial \theta_{r}}\left[\boldsymbol{i}_{\boldsymbol{r}}\right]}_{T_{e m}}-\underbrace{\mu \frac{P}{2} L_{m s} i_{f}\left\{\frac{3}{2} i_{r a} s_{1}\left(\theta_{r}\right)+\frac{\sqrt{3}}{2}\left(i_{b r}-i_{c r}\right) c_{1}\left(\theta_{r}\right)\right\}}_{T_{e f}}
$$

where $s_{1}(\zeta)=\sin (\zeta)$. The equation (6.6) is expressed in terms of the torque equation for the symmetrical IM $\left(T_{e m}\right)$ and a faulty term $\left(T_{e f}\right)$ that will result in a double electric 
frequency oscillation, as indicated in many references [24], [124]. The double-line frequency oscillation comes from the multiplication of two sinusoidal waveforms with the same frequency $i_{f}=I_{f} \sin \left(\omega_{e} t+\delta_{1}\right)$ and $i_{x r}=I_{x r} \sin \left(\omega_{e} t+\delta_{2 x}\right)$, where the subscript $x$ indicates the rotor phases $(a, b, c)$ and $\omega_{e}$ is the stator electrical frequency, also called the synchronous frequency. Without losing accuracy, the frequency oscillation component in the torque can be deduced from the product of these two sinusoidal waveforms as:

\begin{tabular}{|l|l|}
\hline$T_{e f}=k i_{f} i_{r x}$ & $=k\left(\sin \left(\omega_{e} t+\delta_{1}\right)\right)\left(\sin \left(\omega_{e} t+\delta_{2 x}\right)\right)$ \\
$T_{e f}$ & $= \pm \frac{k}{2}\left\{1-\cos \left(2 \omega_{e} t+\delta_{3}\right)\right\}$
\end{tabular}

where $k$ is the magnitude of the torque ripple as a function of $\mu, \frac{P}{2}, L_{m}, I_{f}$ and $I_{x r}$ and $\delta_{z}$ for $z=\{1,2 x, 3\}$ are the phases of the signals $i_{f}, i_{r x}$ and $T_{e f}$, respectively.

\subsubsection{Asymmetric IM Model in Space-Vector Notation}

For simplification reasons, the voltage equations presented in (6.3) and (6.6) can be transformed from primitive coordinates to space-vector notation using the stationary reference frame transformation shown in Section 2.5.1 [14], which is known in the literature as Clarke [128] or Concordia [129] transformation. The transformed IM voltage equations are named to be in $(\alpha \beta)$ or stationary reference frame (SRF). Moreover, the model in this reference frame is also known as space-vector model and it can be derived from the positive sequence of the instantaneous symmetrical component transformation [130] proposed by Fortescue. Thus, the transformed voltage equations are: 
$\left[\begin{array}{c}\boldsymbol{v}_{s} \\ 0\end{array}\right]=\left[\begin{array}{cc}R_{s} & 0 \\ 0 & R_{r}\end{array}\right]\left[\begin{array}{l}\boldsymbol{i}_{s} \\ \boldsymbol{i}_{r}\end{array}\right]+\left[\begin{array}{cc}p & 0 \\ 0 & p-j \omega_{r}\end{array}\right]\left[\begin{array}{c}\lambda_{s} \\ \lambda_{r}\end{array}\right]-\left[\begin{array}{c}\frac{2 \mu R_{s}}{3} \\ 0\end{array}\right] i_{f}$
$v_{s a 2}=\mu R_{s}\left(i_{s \alpha}-i_{f}\right)+p \lambda_{s a 2}=R_{f} i_{f}$
$\left[\begin{array}{c}\lambda_{s} \\ \lambda_{r}\end{array}\right]=\left[\begin{array}{cc}L_{s} & L_{m} \\ L_{m} & L_{r}\end{array}\right]\left[\begin{array}{l}\boldsymbol{i}_{s} \\ \boldsymbol{i}_{r}\end{array}\right]-\frac{2}{3} \mu\left[\begin{array}{l}L_{s} \\ L_{m}\end{array}\right] i_{f}$
$\lambda_{s a 2}=-\mu L_{s} i_{s \alpha}-\mu\left(L_{l s}+\frac{2}{3} \mu L_{m}\right) i_{f}$

where: $\boldsymbol{f}_{\boldsymbol{q}}=f_{q \alpha}-j f_{q \beta}, \boldsymbol{f}=\{\boldsymbol{v}, \boldsymbol{i}, \boldsymbol{\lambda}\}, \boldsymbol{q}=\{\boldsymbol{s}, \boldsymbol{r}\}, L_{s}=L_{l s}+L_{m}$ and $L_{r}=L_{l r}+L_{m}$, $L_{m}=\frac{3}{2} L_{m s}$ and $P$ is the pair of poles.

The electromagnetic torque in $\mathrm{CV}$ notation is stated as:

\begin{tabular}{|l|l|}
\hline$T_{e}=\underbrace{\frac{3}{2} \frac{P}{2} L_{m}\left(i_{s \alpha} i_{r \beta}-i_{s \beta} i_{r \alpha}\right)}_{T_{e m}}+\underbrace{\mu \frac{P}{2} L_{m} i_{f} i_{r \beta}}_{T_{e f}}$ & (6.9) \\
\hline
\end{tabular}

\subsection{State-Space Model of the Faulty IM}

There are a plethora of state-space representations of IM that can be used for purposes of simulation in the continuous time domain. The three-phase IM can be modeled by using different state-space variables and keeping the stator voltages and the load torque as inputs, and the electromagnetic torque and the rotor angular velocity as outputs. The possible set of currents and flux linkages space-vectors are defined as follows: stator, rotor and magnetizing currents, and flux linkages. There are three types of models that can be done with these state-space variables [131]:

(1) Current state-space variable models

(2) Flux linkages state-space variable models

(3) Mixed currents-flux linkages state-space variable models 


\subsubsection{Flux-linkages State-Space Variable Model}

Define $\left[\lambda^{\prime}\right]=\left[\begin{array}{lllll}\lambda_{s \alpha} & \lambda_{s \beta} & \lambda_{r \alpha} & \lambda_{r \beta} & \lambda_{s a 2}\end{array}\right]^{t}$ to be the state-space variable. The flux linkages can be expressed as $\left[\boldsymbol{\lambda}^{\prime}\right]=\left[\boldsymbol{L}^{\prime}\right]\left[\boldsymbol{i}^{\prime}\right]$, where $\left[\boldsymbol{i}^{\prime}\right]=\left[\begin{array}{lllll}i_{s \alpha} & i_{s \beta} & i_{r \alpha} & i_{r \beta} & i_{f}\end{array}\right]^{t},[\boldsymbol{L}]$ comes from (6.1) and requires $\mu \neq 0$ to be a full-rank invertible matrix. $[\boldsymbol{L}]$ is presented in (6.10).

$\left[\boldsymbol{L}^{\prime}\right]=\left[\begin{array}{ccccc}L_{s} & 0 & L_{m} & 0 & -2 / 3 \mu L_{s} \\ 0 & L_{s} & 0 & L_{m} & 0 \\ L_{m} & 0 & L_{r} & 0 & -2 / 3 \mu L_{m} \\ 0 & L_{m} & 0 & L_{r} & 0 \\ \mu L_{s} & 0 & \mu L_{m} & 0 & -\mu\left(L_{l s}+2 / 3 L_{m}\right)\end{array}\right] \quad$ (6.10)

Then $\left[\boldsymbol{i}^{\prime}\right]=\left[\boldsymbol{L}^{\prime}\right]^{-1}\left[\boldsymbol{\lambda}^{\prime}\right]$, where $\left[\boldsymbol{L}^{\prime}\right]^{-1}=[\boldsymbol{N}]$ is as in (6.11).

$[N]=\left(\frac{1}{\Delta}\right)\left[\begin{array}{ccccc}N_{11} & 0 & L_{m} & 0 & N_{15} \\ 0 & -L_{r} & 0 & L_{m} & 0 \\ L_{m} & 0 & -L_{s} & 0 & 0 \\ 0 & L_{m} & 0 & -L_{s} & 0 \\ N_{51} & 0 & 0 & 0 & N_{55}\end{array}\right] \quad$ (6.11)

Also, $\Delta=L_{m}{ }^{2}-L_{s} L_{r}, N_{11}=-\frac{\mu L_{l s} L_{r}+2 / 3 \mu^{2} L_{m} L_{l r}}{L_{l s f}}, N_{15}=-\frac{2 / 3 \mu}{L_{l s f}}, N_{51}=\frac{\mu}{L_{l s f}}$

$N_{55}=-\frac{1}{L_{l s f}}$ and $L_{l s f}=\mu L_{l s}\left(1-\frac{2}{3} \mu\right)$

Thus, the simplest SS representation for a stator ITSC fault of IM, based on the authors' knowledge, is shown as:

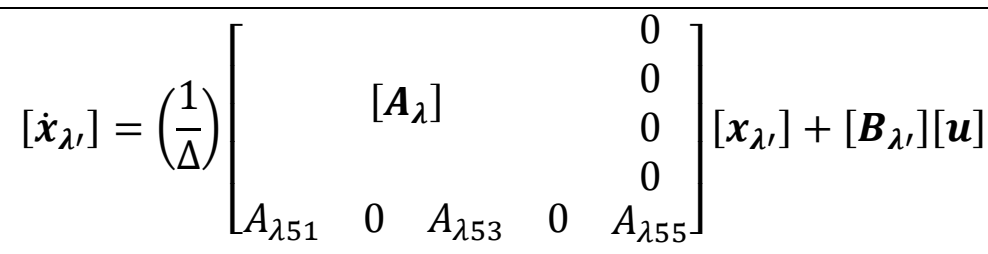


where $\left[\boldsymbol{A}_{\boldsymbol{\lambda}}\right]=\left[\begin{array}{cccc}R_{s} L_{r} & 0 & -R_{s} L_{m} & 0 \\ 0 & R_{s} L_{r} & 0 & -R_{s} L_{m} \\ -R_{r} L_{m} & 0 & R_{r} L_{s} & \omega_{r} \\ 0 & -R_{r} L_{m} & -\omega_{r} & R_{r} L_{s}\end{array}\right]$ is the matrix in flux-linkages SS

model for the healthy IM, $\left[\boldsymbol{x}_{\lambda}\right]=[\lambda], A_{\lambda 51}=\frac{\mu R_{f} \Delta-\mu R_{s} L_{m} L_{l r}}{L_{l s f}}, A_{\lambda 53}=-\mu R_{s} L_{m}$,

$A_{\lambda 55}=-\frac{\Delta\left(R_{f}+R_{s f}\right)}{L_{l s f}}, R_{s f}=\mu R_{s}\left(1-\frac{2}{3} \mu\right), L_{l s f}=\mu L_{l s}\left(1-\frac{2}{3} \mu\right),[\boldsymbol{u}]=\left[v_{\alpha s} v_{\beta s}\right]^{t}$ and

$\left[\boldsymbol{B}_{\lambda \prime}\right]=\left[\begin{array}{lllll}1 & 0 & 0 & 0 & 0 \\ 0 & 1 & 0 & 0 & 0\end{array}\right]^{t}$

The CM flux-linkages SS representation of the symmetric IM is in (6.13).

\begin{tabular}{|l|l|}
\hline$\left[\dot{x}_{\lambda}\right]=\left[\boldsymbol{A}_{\lambda}\right]\left[\boldsymbol{x}_{\lambda}\right]+\left[\boldsymbol{B}_{\lambda}\right][\boldsymbol{u}]$ & $(6.13)$ \\
\hline
\end{tabular}

where $\left[\boldsymbol{x}_{\lambda}\right]=[\lambda]=\left[\begin{array}{lllll}\lambda_{s \alpha} & \lambda_{s \beta} & \lambda_{r \alpha} & \lambda_{r \beta}\end{array}\right]^{t}$ is the healthy flux-linkage vector and $\left[\boldsymbol{B}_{\lambda}\right]=\left[\begin{array}{llll}1 & 0 & 0 & 0 \\ 0 & 1 & 0 & 0\end{array}\right]^{t}$

The DM is as (6.14).

$$
\dot{\lambda}_{s a 2}=A_{51} \lambda_{s \alpha}+A_{53} \lambda_{r \alpha}+A_{55} \lambda_{s a 2}
$$

The obvious deduction is that the faulty model of an IM is the healthy IM model (CM) plus an external circuit that represents the asymmetric part (DM). Many literature reviews [132], [133] have proposed this idea, however no mathematical foundations where demonstrated. However, the circuit separation is in the flux-linkage SS vector representation.

The load torque-speed characteristic defines the mechanical equation that complements the SS representation. This curve may vary widely from one application to the other, even though for simplicity, it is assumed that the load torque profile consists of the motor inertia 
$(J)$, an external load torque $\left(T_{L}\right)$ and friction $\left(k_{\text {loss }}\right)$. In this case, the mechanical equation following the second Newton's Law is:

$$
\frac{d \omega_{r}}{d t}=\frac{1}{\mathrm{~J}}\left(T_{e}-T_{L}-k_{\text {loss }} \omega_{r}\right)
$$

where $\left(\omega_{r}\right)$ is the mechanical speed.

\subsubsection{Current State-Space Variable Model}

From (6.8) and the properties of $[\boldsymbol{L}]$, the current derivatives can be deduced as in (6.16).

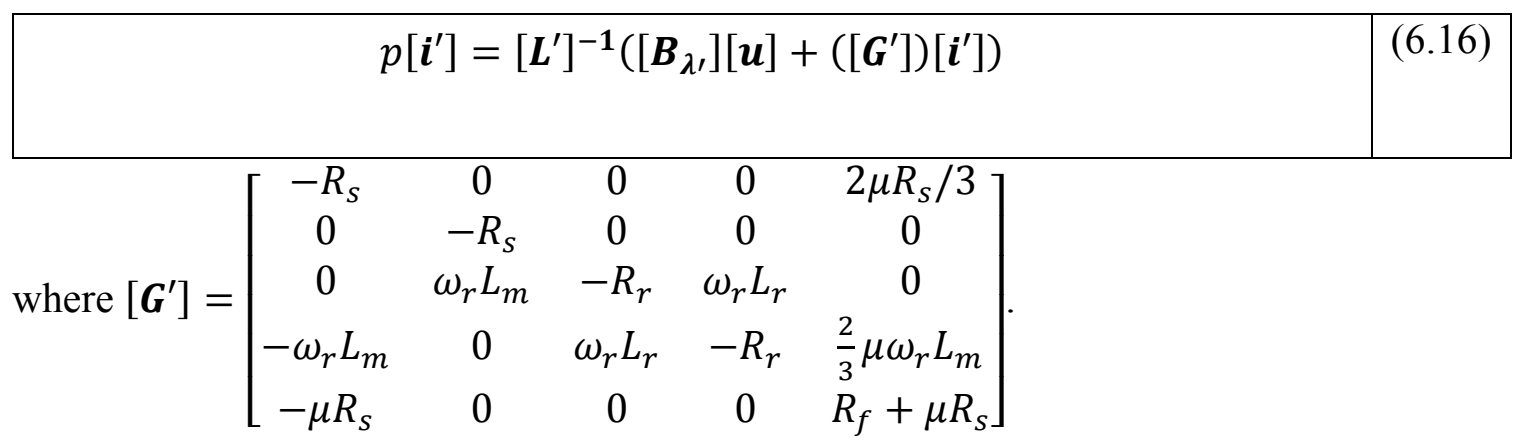

Solving, simplifying and rewriting (6.16), the current SS representation is obtained as in (6.17).

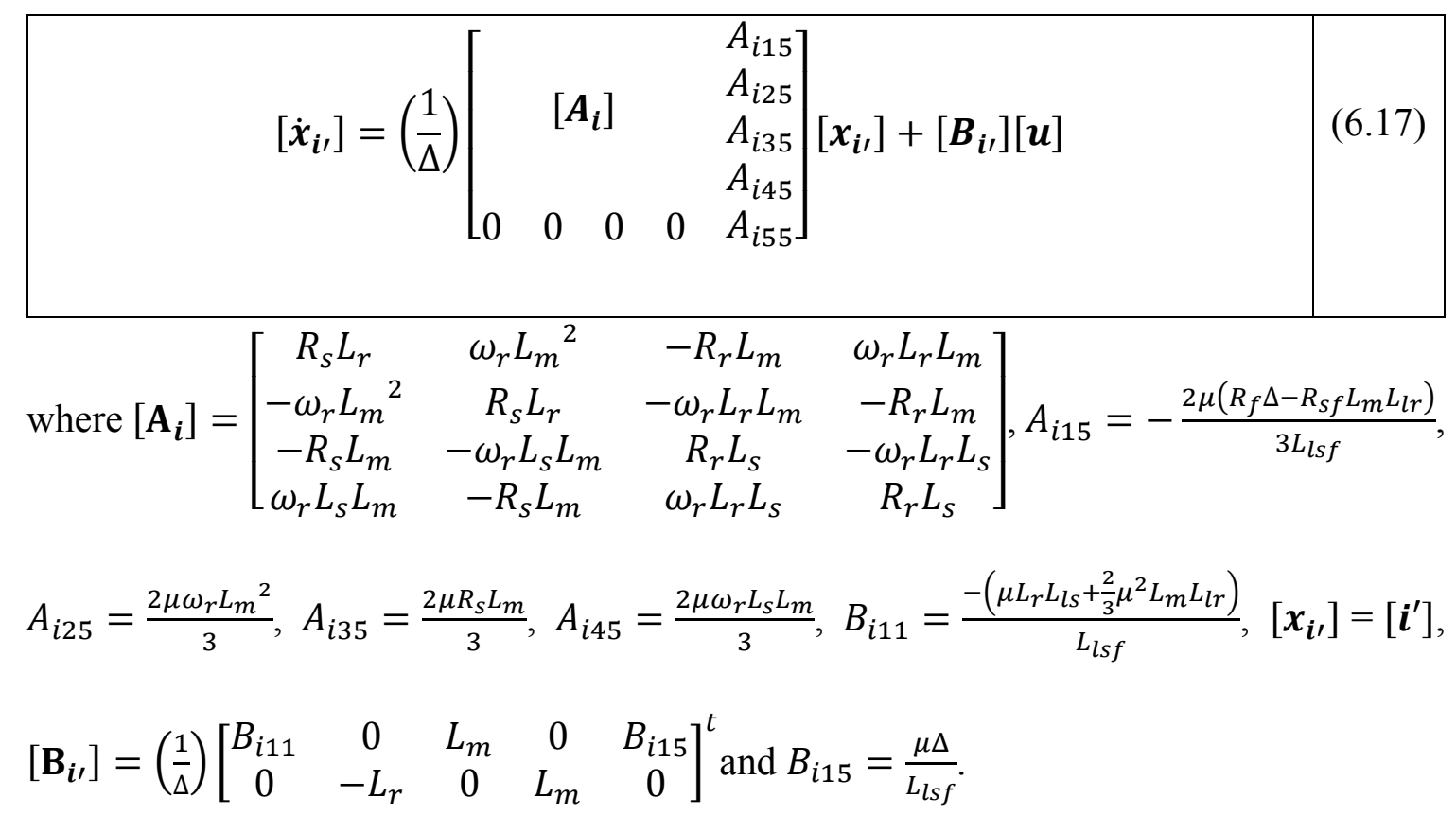




\subsection{Faulty IM: Complex Vector Equivalent Circuit}

The complex $\alpha \beta$-model for the faulty IM in (6.8) can be written as (6.18).

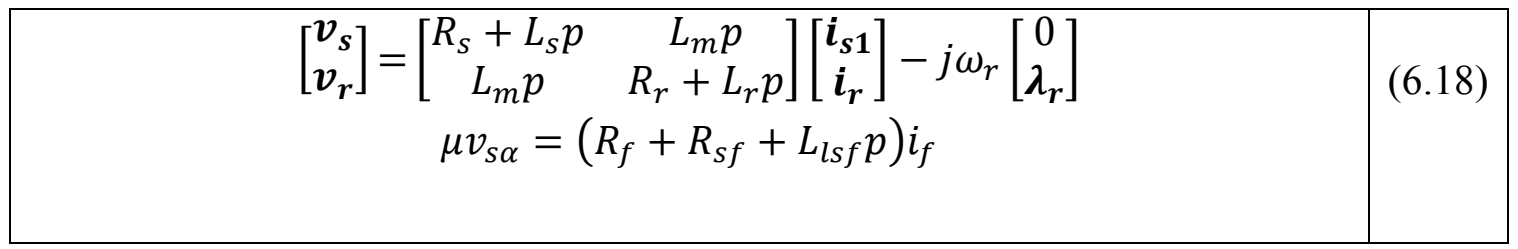

where $\boldsymbol{i}_{\boldsymbol{s} 1}=\boldsymbol{i}_{\boldsymbol{s}}-\frac{2}{3} \mu i_{f}$.

From (6.18), the complex EC model for the asymmetric IM can be plotted in Figure 6.2. Following the procedure in [40], an operational form of the EC can be represented in $\mathrm{CV}$ equations. This form is obtained by multiplying the rotor equation in (6.18) through $\frac{p}{p-j \omega_{r}}$.

Thus, the operational model can be stated as (6.19) and also represented by Figure 6.3.

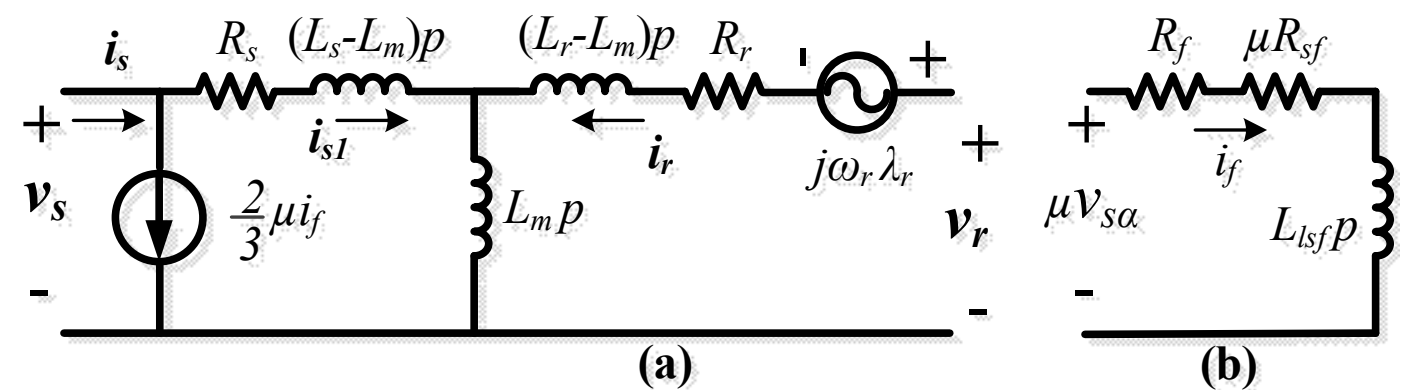

Figure 6.2 (a) Complex vector equivalent circuit of Asymmetric IM (b) Faulty Circuit.

$$
\left[\begin{array}{c}
\boldsymbol{v}_{\boldsymbol{s}} \\
0
\end{array}\right]=\left[\begin{array}{cc}
R_{s}+L_{s} p & L_{m} p \\
L_{m} p & \frac{R_{r} p}{p-j \omega_{r}}+L_{r} p
\end{array}\right]\left[\begin{array}{c}
\boldsymbol{i}_{\boldsymbol{s} \mathbf{1}} \\
\boldsymbol{i}_{\boldsymbol{r}}
\end{array}\right]
$$




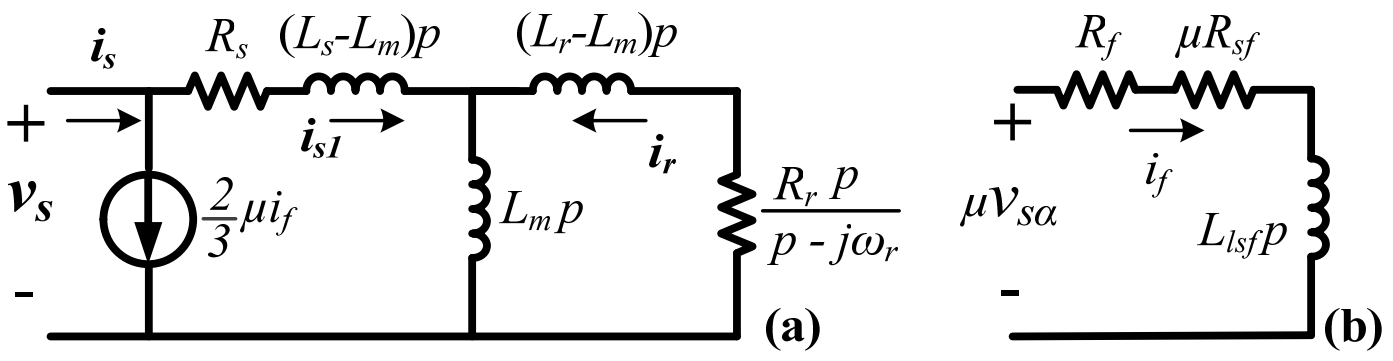

Figure 6.3 (a) Complex vector operational equivalent circuit of Asymmetric IM (b) Faulty Circuit.

\subsubsection{Steady State Analysis of Faulty IM}

Even when the dynamic model of the faulty machine includes all the necessary information for FDI, more insight analysis can be developed with operational and simple models in steady state. Many FDI techniques are based on monitoring the line, voltage and impedance sequence components of the asymmetric IM. Thus, steady state analysis is applied to (6.8) by following the procedure in [40].

In sinusoidal steady state, the complex variable $\boldsymbol{f}_{\boldsymbol{q}}$ can be expressed in terms of the positive $\left(\bar{F}_{q P}\right)$ and negative $\left(\bar{F}_{q N}\right)$ sequence component phasors as $(6.20)$ and the faulty current in Euler form as (6.21).

\begin{tabular}{|l|l|}
\hline $\boldsymbol{f}_{\boldsymbol{q}}=F_{q P} e^{j\left(\omega_{e} t\right)}+{F_{q N}}^{*} e^{-j\left(\omega_{e} t\right)}$ & (6.20) \\
\hline
\end{tabular}

where $\bar{F}_{q P}=F_{q P} e^{j\left(\phi_{F_{P}}\right)}, \bar{F}_{q N}{ }^{*}=F_{q N} e^{-j\left(\phi_{F_{N}}\right)}, \phi_{F_{P}}$ and $\phi_{F_{N}}$ are the positive and negative sequence-component phases of $\bar{F}_{q P}$ and $\bar{F}_{q N}$, respectively.

\begin{tabular}{|l|l|}
\hline$i_{f}=\bar{I}_{f P}+\bar{I}_{f N}=\frac{1}{2}\left(I_{f} e^{j\left(\omega_{e} t+\phi_{i_{f}}\right)}+I_{f} e^{-j\left(\omega_{e} t+\phi_{i_{f}}\right)}\right)$ & (6.21) \\
\hline
\end{tabular}

where $I_{f}$ and $\phi_{i_{f}}$ are the magnitude and phase of $i_{f}$, respectively. 
Applying (6.20) and (6.21) to (6.18), the steady state model as in (6.22) can be obtained. It can be shown that the steady state $v_{s \alpha}$ can be written as $\bar{V}_{s \alpha}=\bar{V}_{s P}+\bar{V}_{s N}$. s is the slip of the IM defined as $s=\frac{\omega_{e}-\omega_{r}}{\omega_{e}}, \bar{I}_{S P}=\bar{I}_{S P 1}-\frac{1}{3} \mu \bar{I}_{f}$ and $\bar{I}_{s N}=\bar{I}_{s N 1}-\frac{1}{3} \mu \bar{I}_{f}$.

Equation (6.22) can be represented in terms of the ECs for the faulty IM, as shown in Figure 6.4 [34]. The admittance matrix relating the sequence components of the IM voltages and currents can be obtained from (6.22) as (6.23).

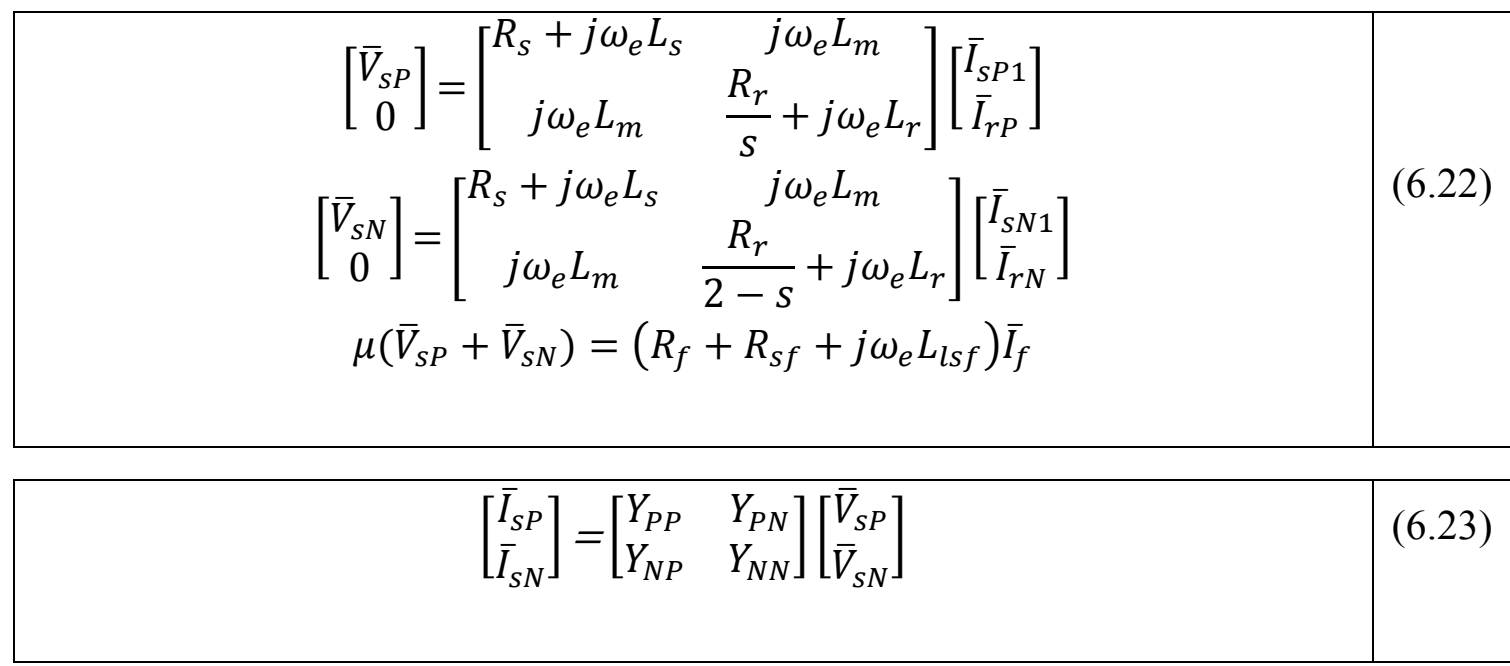

where $Y_{P N}=Y_{N P}=\frac{1 / 3 \mu^{2}}{R_{f}+\mu\left(R_{S}+j \omega_{e} L_{l s}\right)}, Y_{P P}=\frac{1}{R_{S}+j \omega_{e} L_{S}+\frac{\left(\omega_{e} L_{S}\right)^{2}}{\frac{R_{r}}{S}+j \omega_{e} L_{r}}}+Y_{P N}$,

$Y_{N N}=\frac{1}{R_{S}+j \omega_{e} L_{S}+\frac{\left(\omega_{e} L_{S}\right)^{2}}{\frac{R_{r}}{2-s}+j \omega_{e} L_{r}}}+Y_{P N}$

\subsection{Simulation Results for Signatures Search}

In the search for fault signatures, a comprehensive simulation of the transient and permanent regimens is performed. The simulation is performed for a three-phase IM with the model parameters and nameplate rating specifications presented in Table 6-1. 


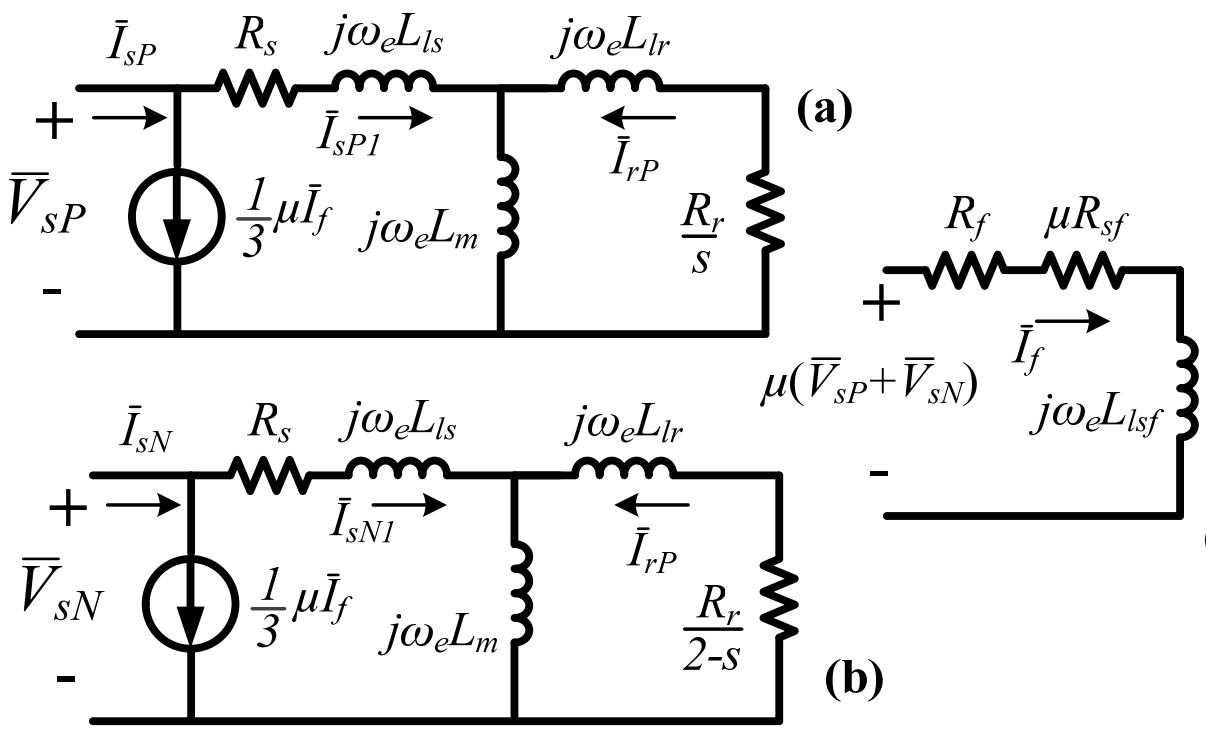

(c)

Figure 6.4 Sequence-components equivalent circuit of Asymmetric IM (a) Positive Sequence (b) Negative Sequence (c) Faulty Circuit.

Table 6-1 Parameters and Nameplate Rating

\begin{tabular}{|c|c|c|c|c|c|c|c|}
\hline Param. & Value & Param. & Value & Rating & Value & Rating & Value \\
\hline$L_{l s}$ & $5.4710^{-3} \mathrm{H}$ & $R_{s}$ & $1.889 \Omega$ & $P_{r}$ & $5.5 \mathrm{~kW}$ & $P F$ & 0.84 \\
\hline$L_{l r}$ & $6.0610^{-3} \mathrm{H}$ & $R_{r}$ & $1.719 \Omega$ & $V_{r}$ & $460 \mathrm{~V}$ & Poles & 2 \\
\hline$L_{m}$ & $0.212 \mathrm{H}$ & $J_{m}$ & $0.012 \mathrm{Kgm}^{2}$ & $I_{r}$ & $10.6 \mathrm{~A}$ & $n_{r}$ & $1750 \mathrm{rpm}$ \\
\hline
\end{tabular}

\subsubsection{Transient Comparative Results for Healthy and Faulty IM}

Figure 6.5 to Figure 6.9 present a comparison between the transient performance of the symmetrical IM (healthy) and the asymmetrical one (faulty) to reveal the differences in between and thus identify signatures for the FDI. A fault severity factor of $\mu=5 \%$ and a fault resistance of $R_{f}=0.01 \Omega$ is implanted. The machine starts with no load from stand still, and then a step of load of $20 \mathrm{Nm}$ at $0.3 \mathrm{sec}$, is applied. The entire time for the simulation is $0.6 \mathrm{sec}$ with integration step time of $0.0001 \mathrm{sec}$. The simulations are 
conducted by means of the MatLab code using the ode1 13 function. The electrical variables for this comparison are: stator current space-vector (Figure 6.5 and Figure 6.6), electromagnetic torque (Figure 6.7), the rotor speed (Figure 6.8) and rotor current spacevector (Figure 6.9). In Figure 6.9(c) the leakage faulty current is shown.

From Figure 6.5(a), Figure 6.6(a), Figure 6.7(a), Figure 6.8(a) and (b) and Figure 6.9(a), it can be observed that the behaviors of the faulty and healthy IM are similar during the transient and the steady state for no-load and loading condition. However, from the zoomed analysis of the steady-state near to the torque load step (from 0 to $20 \mathrm{Nm}$ ) the effects of the ITSC can be observed. Figure 6.5(b) shows that the real component of the faulty stator current vector has a substantial increase under load and no-load conditions, but for the imaginary component (Figure 6.6(b)) there is no difference under no-load, but it increases at the loading condition.

Figure 6.7(b) shows the electromagnetic torque at the steady state near to the load step $(t=0.3 \mathrm{sec})$. The double-line frequency oscillation can be noticed. This oscillation occurs under load condition. Under no load condition, the rotor current vector is almost zero and with very small friction torque (Figure 6.9(a) and (b)). The double-line frequency oscillation also appears at the starting of the IM (Figure 6.7(c)) due to the high inrush current to overcome the initial inertia, which corresponds to the same case of the loaded machine. 

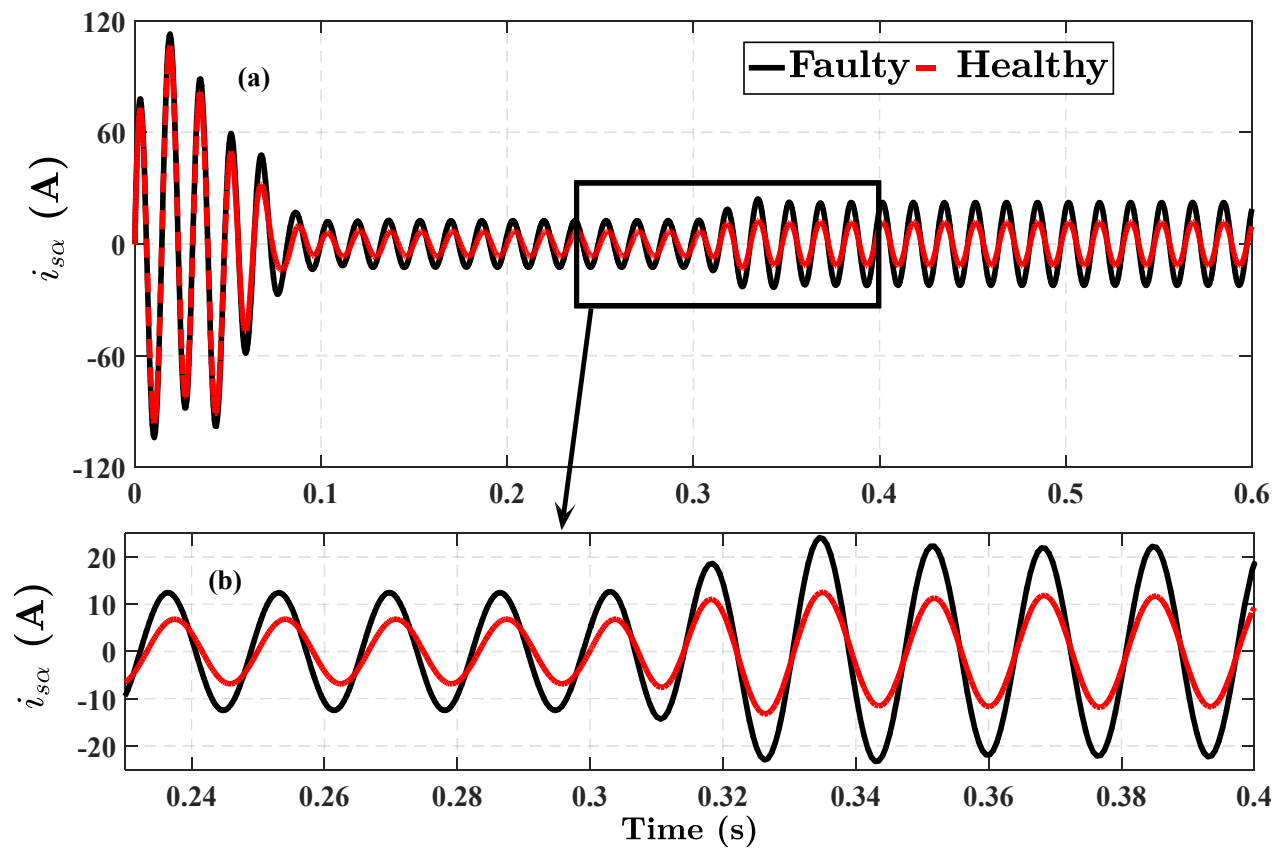

Figure 6.5 Real component stator current for healthy and faulty IM (a) $i_{s \alpha}$ (b) Zoom.
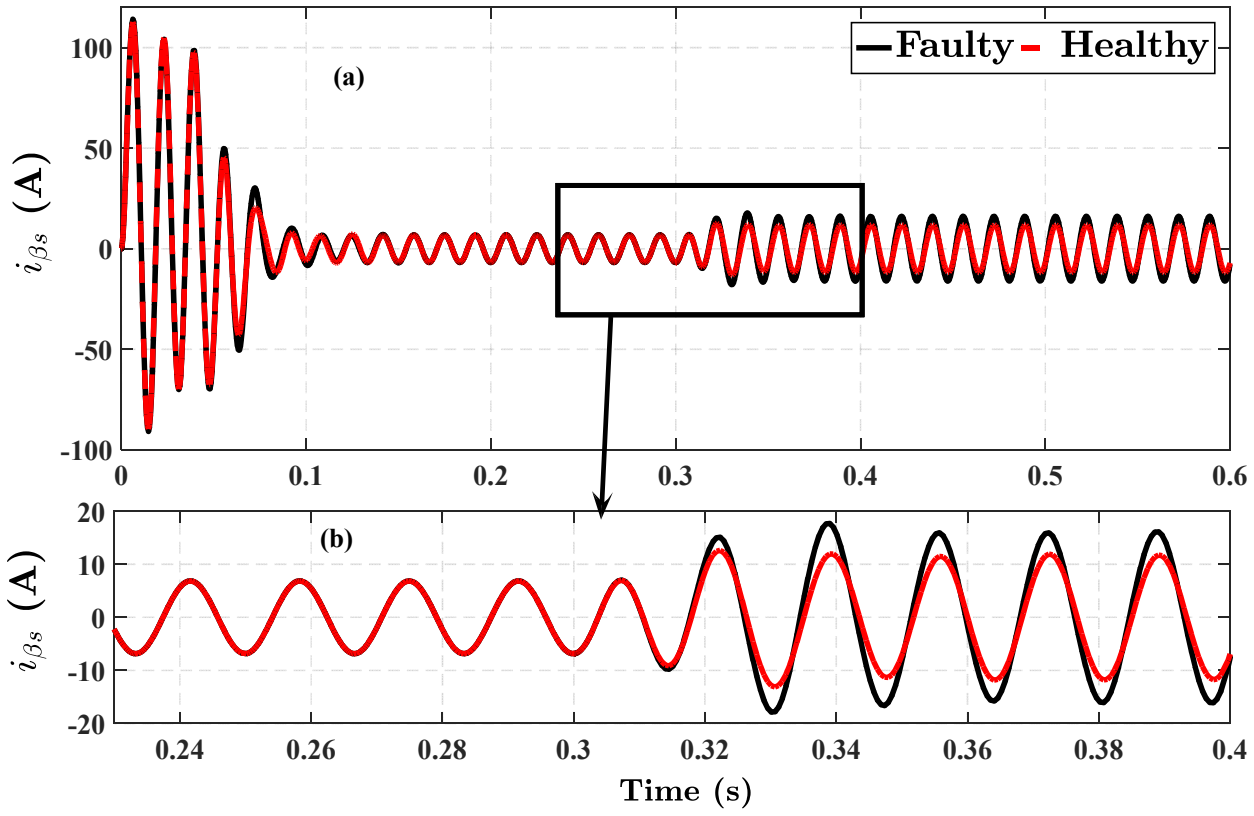

Figure 6.6 Imaginary component stator current for healthy and faulty IM (a) $i_{s \beta}$ (b) Zoomed $i_{s \beta}$. 
In Figure 6.8(b), the zoom for the rotor speed of the IM also presents the double-line frequency oscillation under load conditions, however, these oscillations are very small compared, in percentage, with the average speed.

The zoomed rotor current vector of the IM are presented in Figure 6.9(a) and (b), which indicate that under no load, the rotor currents of the symmetrical and asymmetrical IM are almost zero in steady state. However, under load conditions, the rotor currents in the faulty IM are increased in both real and imaginary components.
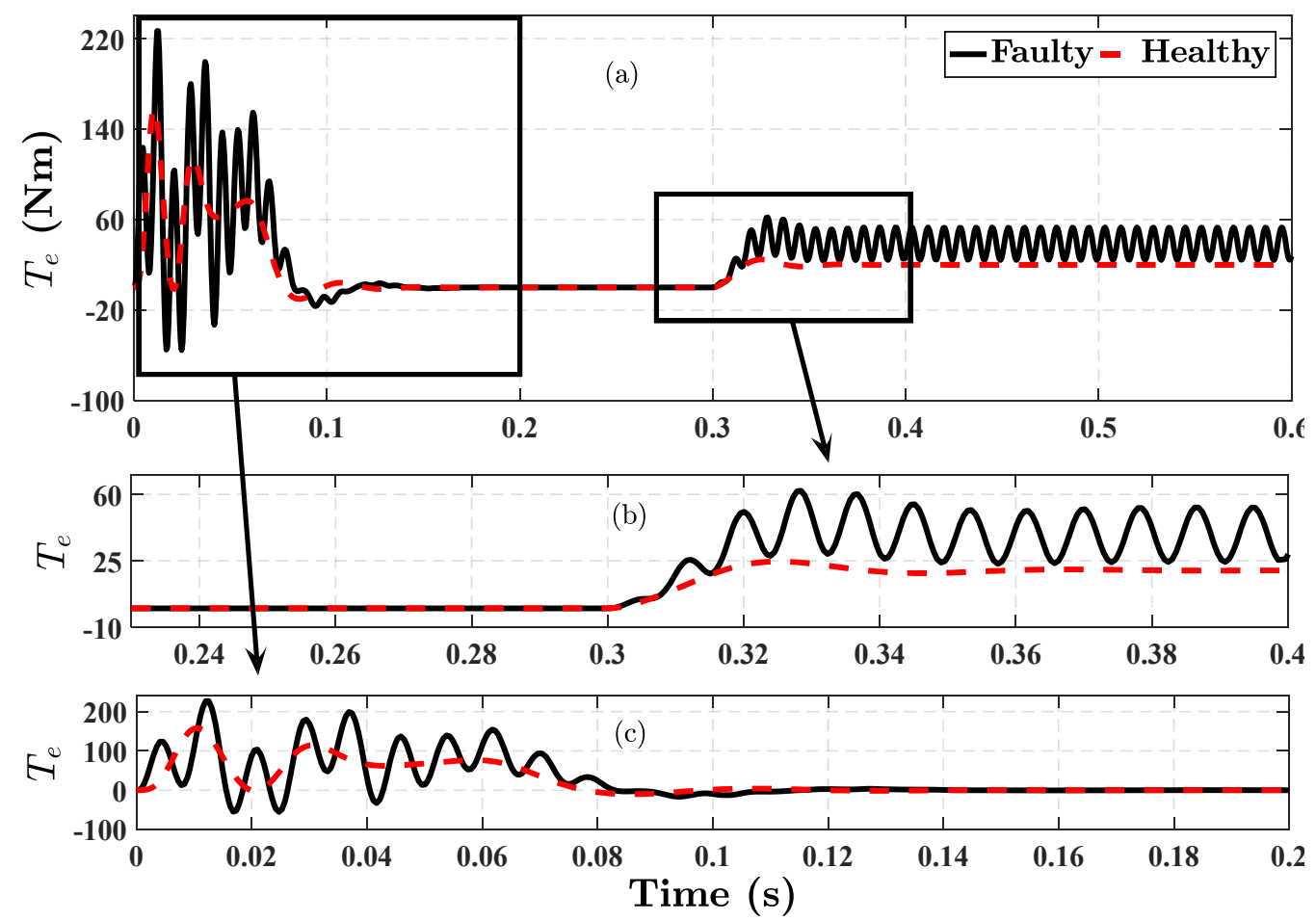

Figure 6.7 Electromagnetic torque for healthy and faulty IM (a) $T_{e}$ (b) First zoomed $T_{e}$ and (c) Second zoomed $T_{e}$. 

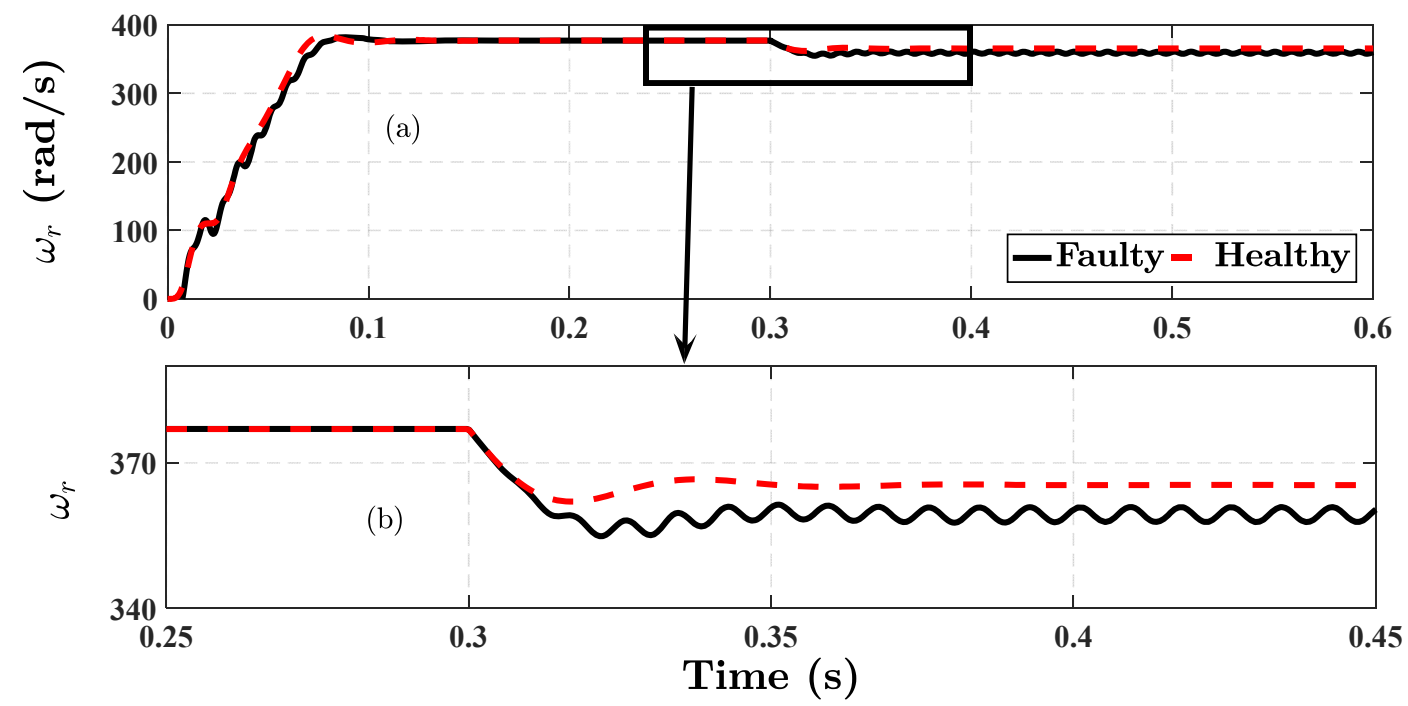

Figure 6.8 Rotor speed for healthy and faulty IM (a) $\omega_{r}$ (b) Zoomed $\omega_{r}$.
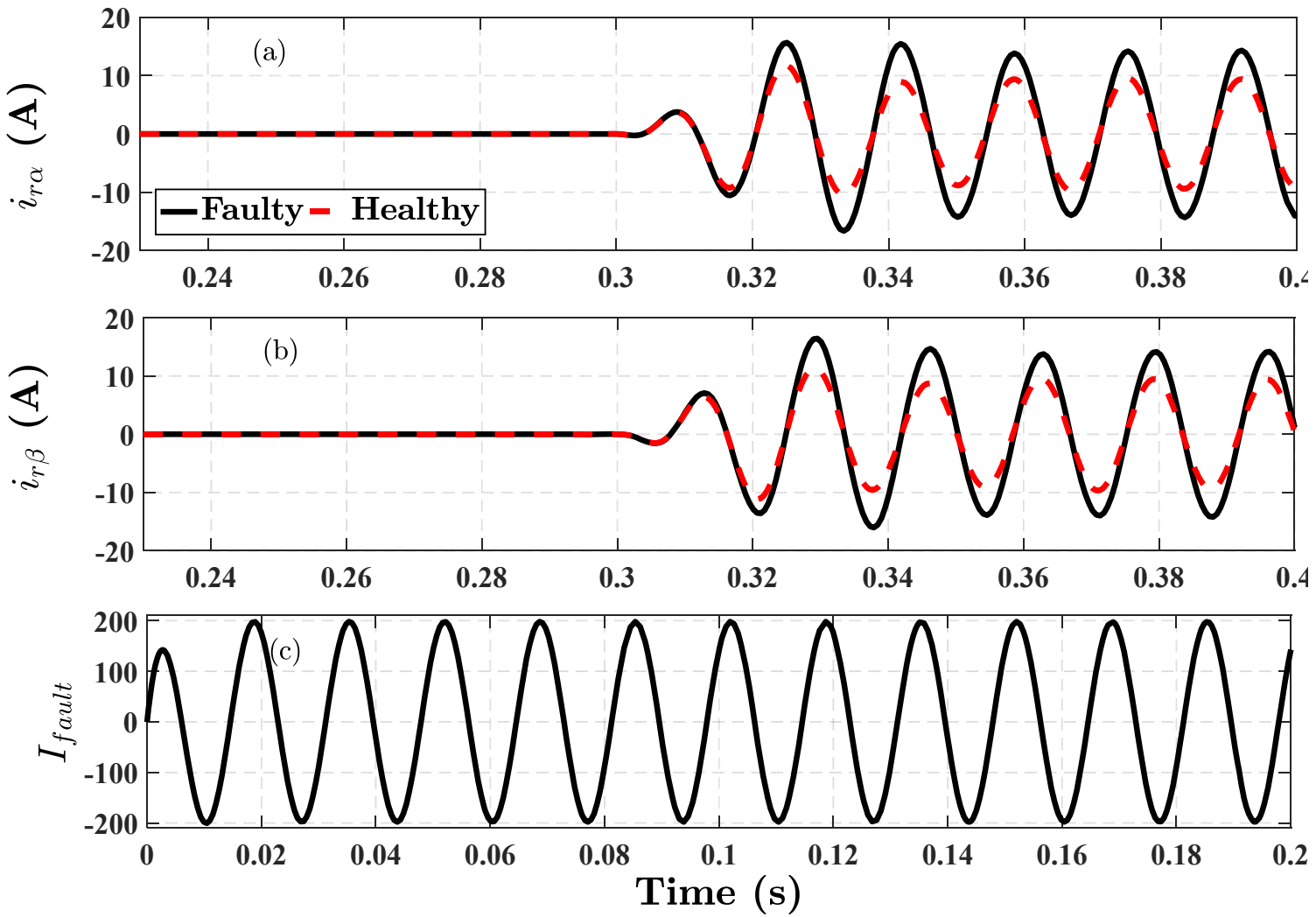

Figure 6.9 Vector components rotor current for healthy and faulty IM and faulty current (a) Zoomed $i_{r \alpha}$ (b) Zoomed $i_{r \beta}$ and (c) $i_{f}$. 


\subsubsection{Steady State Results for Faulty IM}

In this part, the steady state results are divided in three cases of study. The first and second cases investigate the magnitude variation of the leakage faulty current $\left(I_{f}\right)$, stator current space-vector $\left(I_{s \alpha}\right.$ and $\left.I_{s \beta}\right)$, and electromagnetic torque ripple $\left(\Delta T_{e}\right)$ as a function of:

(1) Fault severity factor $(\mu)$

(2) Faulty resistance $\left(R_{f}\right)$

The third case inspects the characteristic curve of the IM, torque versus slip under faulty condition and variations of supply voltage and frequency.

\subsubsection{Variation with Fault Severity Factor}

Figure 6.10 shows the variation of the leakage faulty current and the torque ripple as a function of $\mu$. Notice in Figure 6.10(a) that $I_{f}$ increases with the increment of $\mu$ and it shows a saturation behavior as $\mu$ becomes bigger. On the other hand, the double-line frequency oscillations of $T_{e}$ increases almost linearly with fault severity factor and $\Delta T_{e}$ gets worst as $\mu$ increases. From the zoom in Figure 6.10(b), the same behavior is noticed.

Figure 6.11 depicts the stator current space-vector $\left(I_{s \alpha}\right.$ and $\left.I_{s \beta}\right)$ as a function of $\mu$ for no-load and loading condition. It can be noticed three phenomena:

- For large $\mu$, the currents in both loading conditions are very similar.

- For small $\mu$ (Figure 6.11(b)) $I_{s \beta}$ does not change respect to $\mu$ in the no-load condition.

- During loading condition $I_{s \alpha}$ and $I_{s \beta}$ increase with the fault. 

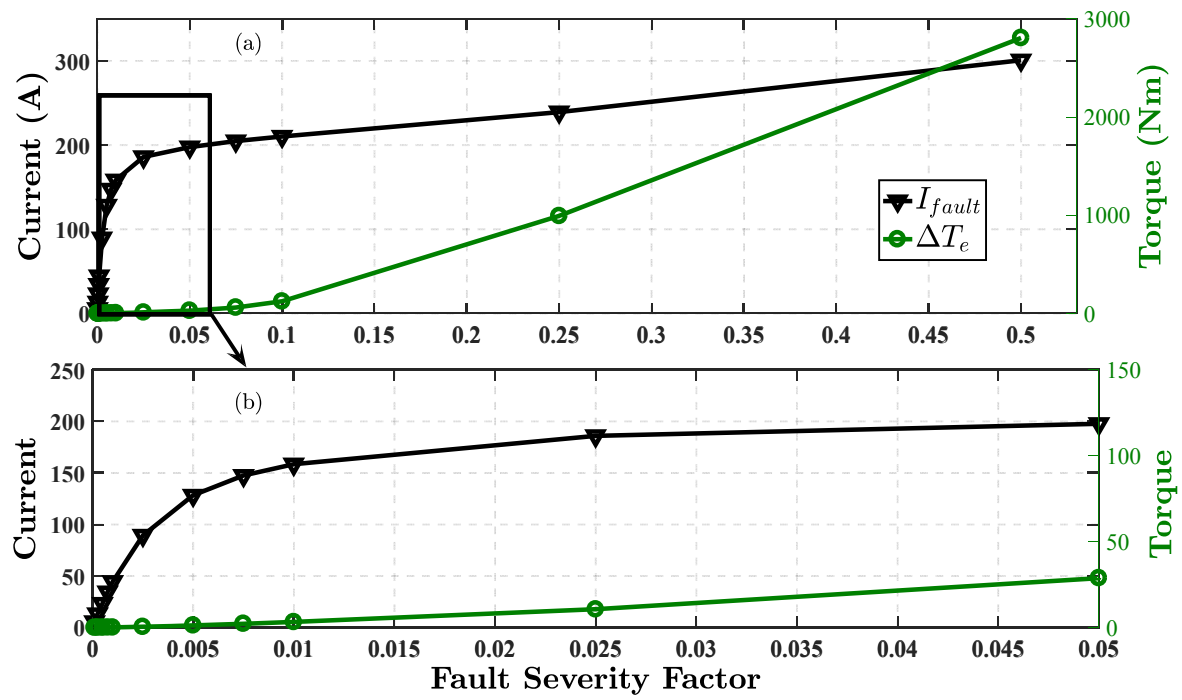

Figure 6.10 (a) Fault current magnitude and electromagnetic torque ripple vs severity factor $\mu$. (b) Zoom.

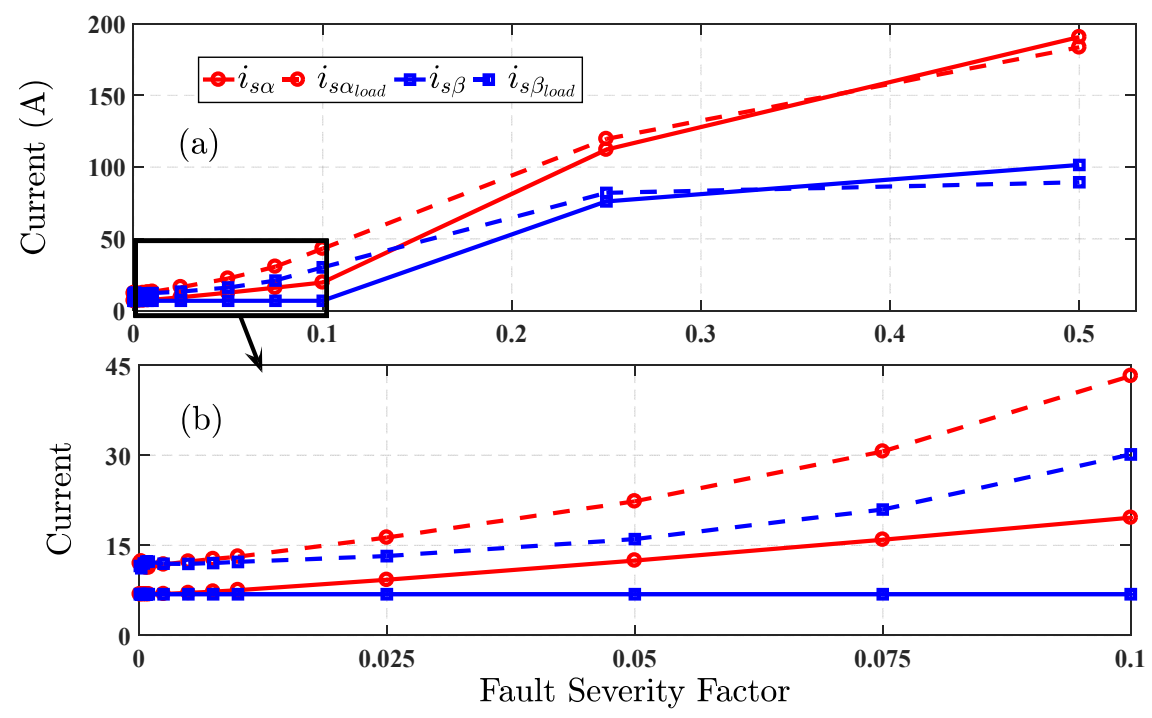

Figure 6.11 (a) Stator current space-vector vs fault severity factor $\mu$ variation under load and no load conditions. (b) Zoom.

\subsubsection{Variation with Faulty Resistance}

Figure 6.12(a) and (b) depict the variation of $I_{f}, \Delta T_{e}, I_{s \alpha}$ and $I_{s \beta}$ as a function of $R_{f}$. In

Figure $6.12, I_{f}, \Delta T_{e}$ and $I_{s \alpha}$ decrease exponentially as the faulty resistance increases.

Current $I_{s \beta}$ does not change respect to $R_{f}$ for no-load condition. 


\subsubsection{Torque-Slip Characteristic}

In this stage, the simulations are performed in MatLab scrip by solving (6.23). The results are verified by simulating the circuits in Figure 6.4 on Simulink. Also, the results correspond perfectly to the dynamic simulation presented before. The sequence components for the current and admittances as a function of slip are presented in Figure 6.13(a) and (b). The electromagnetic torque as a function of the slip is presented in Figure 6.13(c). It is interesting to notice that the negative sequence-components $\left(\bar{I}_{S N}\right.$ and $\left.Y_{P N}\right)$ in steady state almost have no effect respect with the slip variation (load variation), as shown partially in Figure 6.9. Moreover, the characteristic $T_{e}-s$ is also tested under variations of stator input voltage and electric frequency. The results of these changes are in Figure 6.14(a) and (b), respectively. The different voltage supplies are $V_{s 1}=460 \mathrm{~V}, V_{s 2}=360 \mathrm{~V}$ and $V_{s 3}=260 \mathrm{~V}$. The frequency variations are $\omega_{1}=377 \mathrm{rad} / \mathrm{s}, \omega_{2}=250 \mathrm{rad} / \mathrm{s}$ and $\omega_{3}=$ $150 \mathrm{rad} / \mathrm{s}$.

Observations: From these simulations it can be noticed:

(1) Here $\Delta T_{e}$ appears to be a good indicator of the ITSC fault when the IM is loaded.

(2) Effects on the negative sequence components (current and off-diagonal admittances $\left.Y_{P N}\right)$ show no variation with the slip. (Figure 6.13).

(3) The admittance off-diagonal terms of (6.23) looks to be a suitable tool for behavioral explanation of the torque ripple variation and fault current versus the turn fault severity factor shown in Figure 6.10. This admittance analysis is promising for the FDI. 

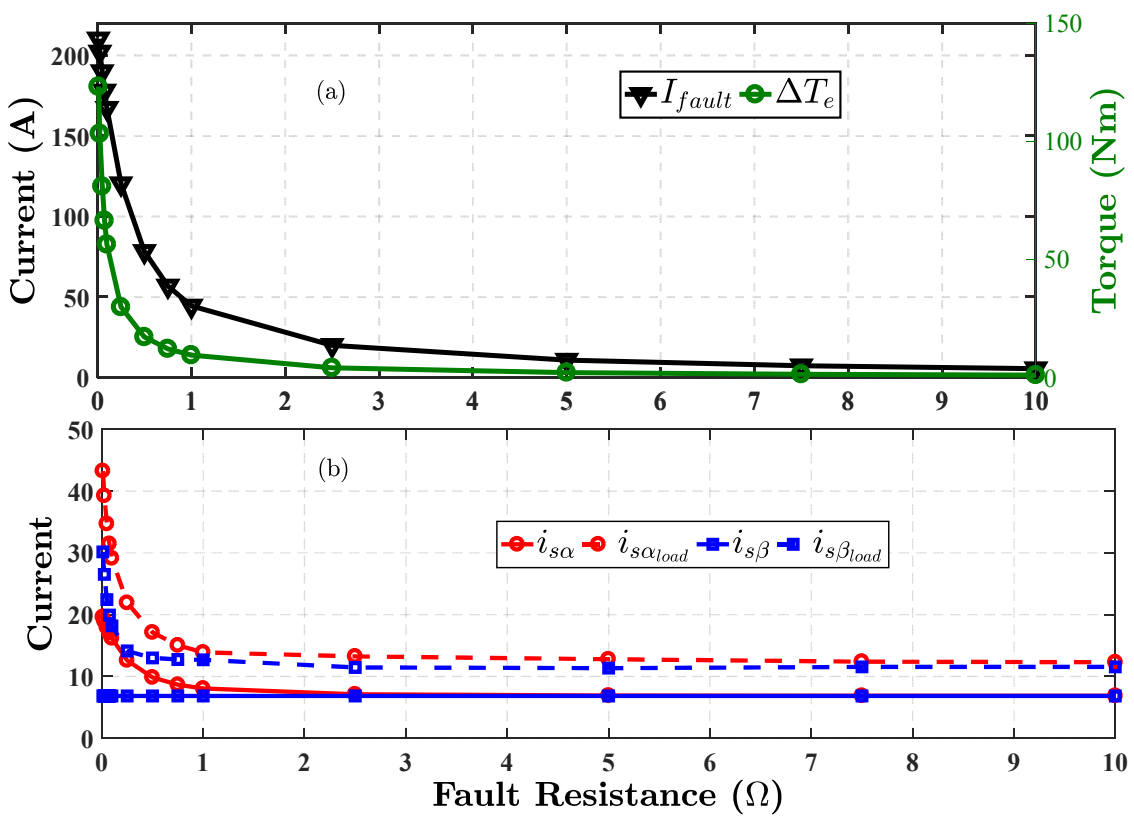

Figure 6.12 (a) Fault current magnitude and electromagnetic torque ripple vs severity factor $\mu$ (b) Stator current space-vector vs $\mu$ variation under load and no load conditions
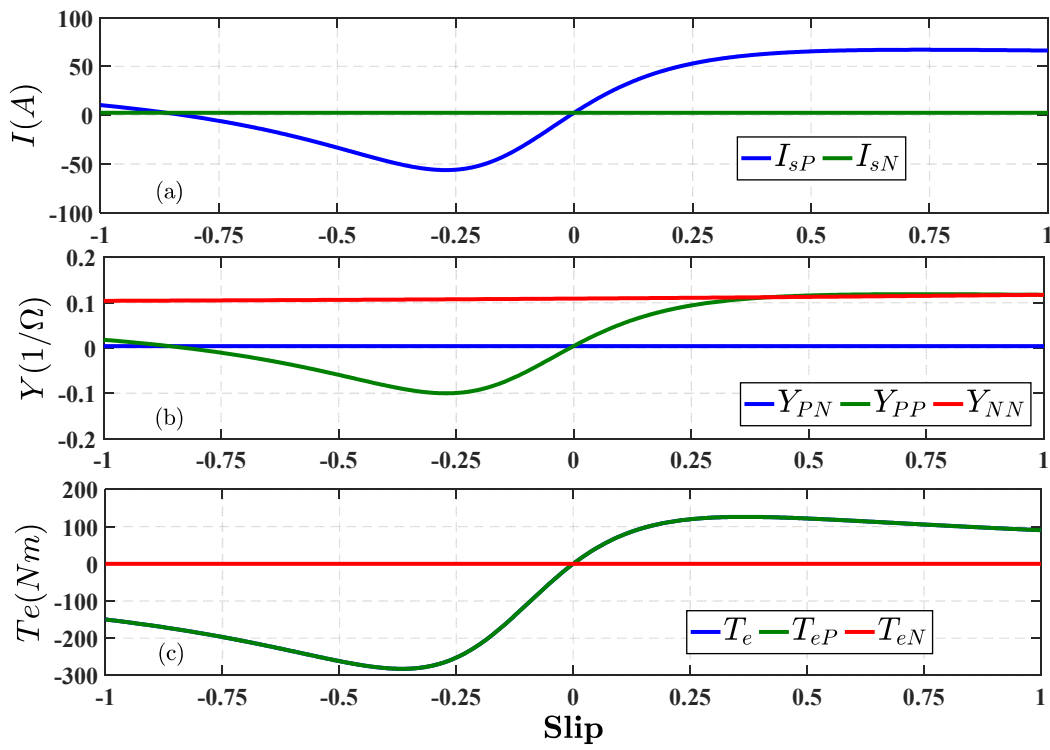

Figure 6.13 (a) Stator component-currents (b) Equivalent admittances (c) Torque slip characteristic. 

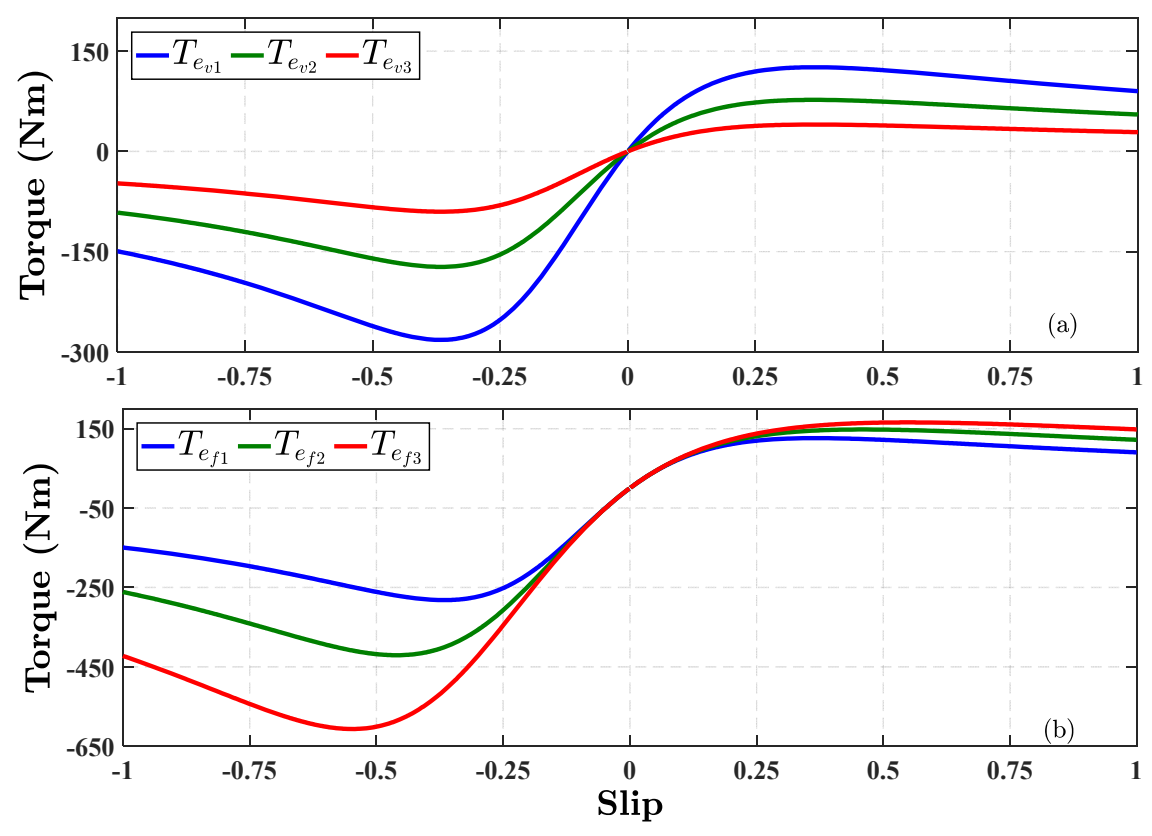

Figure 6.14 Electromagnetic torque slip characteristic (a) Input voltage vatiation and (b) Electric frequency variation.

\subsection{Conclusion and Future Research}

The simplest state-space model for the IM with stator winding inter-turn short-circuit fault on one phase is developed. It is demonstrated that this model can be separated in two modes: "common mode" and "differential mode," representing the healthy and faulty part of the IM. Complex vector and operational EC are derived to obtain the steady state component-sequence EC. Complete fault IM admittance deductions are developed and presented. Simulation for the transient and steady state behavior of the machine under fault condition are performed. Furthermore, SC analysis of the stator current and the torque ripple are studied to evaluate, compare and find usable signatures for FDI of stator windings faults in IM. Finally, torque slip characteristics are analyzed under voltage and frequency variation. 


\section{Chapter 7 Proposed Dynamic Model of Induction Machines with Stator Winding Inter- turn Short-Circuit Fault}

\subsection{Introduction}

The prompt fault detection and identification (FDI) of inter-turn short-circuits faults can avoid costly repairs, significant financial losses and safety problems. A survey of the most relevant literature reveals three general approaches to substantiate the FDI [129], [134]:

1. Feature extraction approach (FExA) [135], [136].

2. Feature extraction approach (MRA) [8], [137], [138].

3. Knowledge-based approach (KBA) [139], [140].

The accurate analytical modelling is mandatory for MRA and it may be helpful in FExA and KBA methods. In KBA, precise modelling can be used in neural network training procedures to avoid the difficulty, time consumption and danger of real experimentations.

Therefore, the need to develop a model of IM with ITSC fault is quite obvious and from this point of view, modeling is the initial stage of the FDI procedure. Different models for IM under stator faults have been proposed in [8], [124]-[127], [123], [34], [36]. The proposed model presented in [124] shows a technique for calculating the coupling IM faulty impedances based on harmonic analysis. The models in [125]-[127] derive the IM inductances using winding function approach, which introduces a great level of detail and accuracy but requires the full design parameters of the machine, such as: quantity of stator slots, coil pitch angle, number of turns per coil, number of rotor bars, stator and rotor conductor's distributions, etc. These parameters are typically unknown or difficult to find. The models in [129], [8], [123], [34], [36] based on mathematical transformations are 
simplified models compared to the full models in [125]-[127]. In [8], [129], the models are depicted in two modes: common (healthy IM) and differential (faulty IM) mode. This model works for FDI but the faulty circuit does not have a complete physical meaning. In [123], the ITSC is modeled by the reduction of $\mu$ percent (fault severity factor) of the number of turns per-phase, however, it does not model the faulty leakage circuit or the circulating current. In [34], [36] the stationary reference frame transformation is used. The IM faulty model considers the reduction of the turns in the faulty winding phase, as well as the short-circuit analysis for the faulty leakage current modeling. In [36], the state-space (SS) representation is presented in its simplest form and full transient and steady-state results were compared with the healthy IM in the search of signatures.

The main concern in any accurate SS modelling is its parameter estimation. The SS model of any magnetically coupled circuit basically depends on the leakage and magnetizing or self and mutual inductances. These parameters depend on the magnetomotive force (MMF) imposed by the winding configuration and the current in it. Magnetizing and mutual inductances, in general, are easy to estimate by means of magnetic circuits theory, winding functions [14], finite elements, etc. However, leakage inductance are highly dependent on the core and winding geometry, and type. Moreover, the difficulty of its estimation augments proportionally with the complexity of the geometry. In power transformers, leakage inductance calculation has been investigated in [141], [142] with a high degree of accuracy respect to the measurements. However, the ITSC fault can complicate the geometry of its computation, at a point where the magnetic field shape (MMF) gets much distorted and the hypotheses assumed for its calculation are not valid. To solve this issue, a correction factor based on experimental results is proposed in [143]. 
For rotating EM, particularly for induction machines (IM), the estimation of the leakage inductance is a complex procedure for the healthy machine, where the stator leakage inductance is the combination of distinct components: slot leakage, end-turn leakage, airgap or differential leakage (zig-zag and belt leakage). In such a way, the calculation of the faulty leakage inductances would be even more difficult. Previous research in faulty IM modelling [34], [36], [125], [127], [144] proposed excessive simplistic models for the leakage inductances. In [34], [36], the leakage is assumed linear dependent on the fault severity. In [144], it is proposed that the leakage is proportional to the number of turns squared which sounds to be better, however, the reluctance path is not considered. In [127], the leakage effect is not even considered.

The ITSC location can be affected in three different ways: the physical placement for a fix fault, the variation of the number of turns short-circuited and the phase position. The third case is just a rotation of 120 and/or 240 degrees studied in [145]. Therefore, the two first cases are the ones that are worth investigating. The first one is based on finite element analysis (FEA), to approach the following question: Does the location of an ITSC with fixed fault severity factor affect the damaged IM parameters? The second one is the complete full dynamic model in complex vector notation.

Thus, for the second part, in this chapter, a general study for the ITSC modelling in magnetically coupled circuits and its general parameter estimation is presented in detail. The proposed analysis is based on the fact that the faulty winding can be modelled as a step-down autotransformer circuit and thus, fulfills the SS representation of a faulty magnetically coupled circuit. Also, general equations for estimating the faulty leakage inductances are presented. A case of ITSC study is presented: three-phase squirrel-cage 
induction machine. A more rigorous and meaningful approach to the faulty stator leakage inductances to construct a dynamic model for the three-phase squirrel-cage IM with ITSC is proposed. The faulty model is developed in detail and expressed in complex-vector (CV) notation. The SS representation is developed based on the flux-linkage space-vector. The $\mathrm{CV}$ operational and steady-state symmetric component equivalent circuits (ECs) are deduced from the faulty model for FDI purposes. The SS representation and ECs are verified by means of simulation and compared with the experimental results. The effect of the fault severity factor $(\mu)$ and the faulty resistance $\left(R_{f}\right)$ is studied under different loading conditions.

\subsection{ITSC Fault Analysis: General Modeling}

Inter-turn short-circuits in single phase power transformers can be classified in two types [143]: turn-to-earth and turn-to-turn fault (Figure 7.1(a)). However, from the results of this chapter and by combining the two healthy parts of the windings in the turn-to-turn fault (over and under the fault), the categorization can be summarized in one case. For the case of three-phase transformers and rotating EM, the situation is different. ITSC can be categorized into three main types:

1. Turn-to-turn in one-phase (Figure 7.1(b1))

2. Phase-to-phase (Figure 7.1(b2))

3. Phase-to-core (Figure 7.1(b3))

Typically, the stator of IM has two windings per-phase that can be connected for high (Figure 7.1(b)) or low (Figure 7.1(c)) supply voltage. Thus, 3 different locations of onephase ITSC can be defined:

1. In the series connected windings (Figure 7.1(b1)) 
2. In one of the parallel connected windings (Figure 7.1(c1))

3. In between the parallel connected windings (Figure 7.1(c2))

In the case of phase-to-phase and phase-to-core, the ITSC depends on the machine connection (Figure 7.1(b2), (c3)).

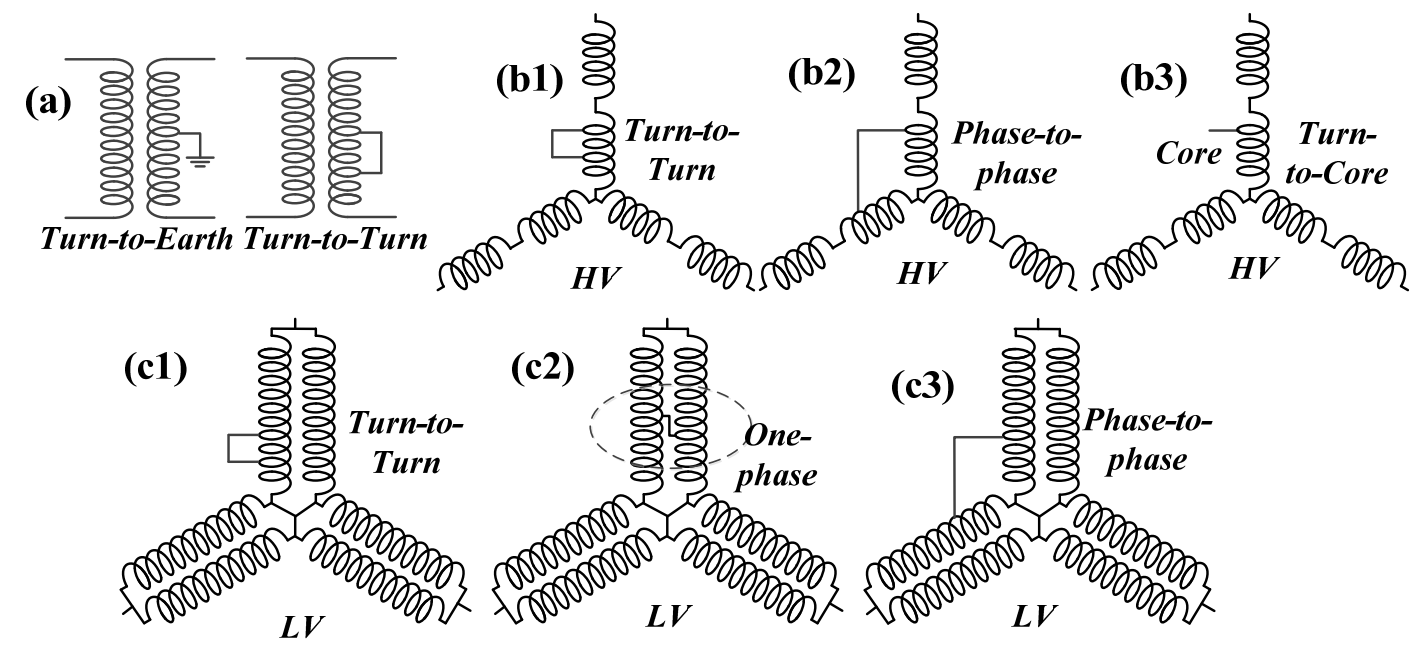

Figure 7.1 ITSC types in magnetically coupled circuits (a) Single-phase Transformers. Three-phase transformer and EM connected in wye (b) high voltage connection (c) low voltage connection.

Additionally, some difference in the modeling can be found when the three-phase system is wye or delta connected, however, the figures for the delta connection are omitted. Similar cases to the wye connection can happen. All these combinations are possible, nevertheless the most common fault is the one that occurs between adjacent turns in the same winding for the phase. Furthermore, the investigation conducted in [146] developed a model of the abnormal delta-connected IM under ITSC. They compared their model with the wye connection, discovering that the difference is just a rotation and magnitude transformation. Thus, in this chapter, ITSC in one winding has been studied. This analysis is applied to an IM as a case of study and it can be implemented for transformer and other 
types of three-phase ac machines. The general modelling principles and parameter estimation are deduced from the ITSC fault in one-coil in the next subsection.

\subsubsection{ITSC Fault in One Coil}

When an ITSC occurs in one coil, an accurate model to represent this circuit is done by means of a step-down autotransformer. In [147], the autotransformer model has been used to approach short-circuit studies in three-phase transformers.

The physical winding of the ITSC in one-coil, which is the same as a step-down autotransformer, is presented in Figure 7.2(a) where the number of turns of the healthy part is $N_{1}$ and the number of turns for the short-circuited part of the winding is $N_{2}$. The total number of coil turns is $N_{S}=N_{1}+N_{2}$. In Figure 7.2(b), the circuit diagram is also presented. According to Figure 7.2(a), two voltage equations can be written as in (7.1). In the figure, two terms of back electromotive force $e_{1}=M \frac{d i_{2}}{d t}$ and $e_{2}=M \frac{d i_{1}}{d t}$ can be noticed.

$$
\left[\begin{array}{l}
v_{1} \\
v_{2}
\end{array}\right]=\left[\begin{array}{cc}
R_{1} & 0 \\
0 & R_{2}
\end{array}\right]\left[\begin{array}{l}
i_{1} \\
i_{2}
\end{array}\right]+\left[\begin{array}{ll}
L_{1} & M \\
M & L_{2}
\end{array}\right] \frac{d}{d t}\left[\begin{array}{l}
i_{1} \\
i_{2}
\end{array}\right]
$$

where $L_{1}, L_{2}$ are the self-inductances and $R_{1}, R_{2}$ are the associated resistances of the windings and $M$ is the mutual inductance between coil 1 and 2, respectively. Additionally, three equations can be expressed: $v_{s}=v_{1}+v_{2}, i_{f}=i_{1}-i_{2}$ and $v_{2}=R_{f} i_{f}$, where $R_{f}$ is the resistance of the short-circuit path named faulty resistance. It is typically introduced in the experiments for avoiding high faulty currents. Combining these five equations, then the two voltage equations can be rewritten as in (7.2). 


$$
\left[\begin{array}{c}
v_{s} \\
0
\end{array}\right]=\left[\begin{array}{cc}
R_{h} & -R_{2} \\
R_{2} & -\left(R_{2}+R_{f}\right)
\end{array}\right]\left[\begin{array}{l}
i_{1} \\
i_{f}
\end{array}\right]+\left[\begin{array}{cc}
L_{h} & -\left(L_{2}+M\right) \\
L_{2}+M & -L_{2}
\end{array}\right] p\left[\begin{array}{l}
i_{1} \\
i_{f}
\end{array}\right]
$$

where $R_{h}$ and $L_{h}$ are the healthy resistance and inductance of the original coil, respectively.

The SS representation of the faulty one-coil or step-down autotransformer is stated in (7.3).

$$
p\left[\begin{array}{l}
i_{1} \\
i_{f}
\end{array}\right]=[\boldsymbol{L}]^{-\mathbf{1}}\left(\left[\begin{array}{c}
v_{s} \\
0
\end{array}\right]-\left[\begin{array}{cc}
R_{h} & -R_{2} \\
R_{2} & -\left(\mu R_{h}+R_{f}\right)
\end{array}\right]\left[\begin{array}{l}
i_{1} \\
i_{f}
\end{array}\right]\right)
$$

where $[\boldsymbol{L}]^{-\mathbf{1}}$ is the inverse inductance matrix that is required to be discovered by the parameter determination procedure in the next subsection.

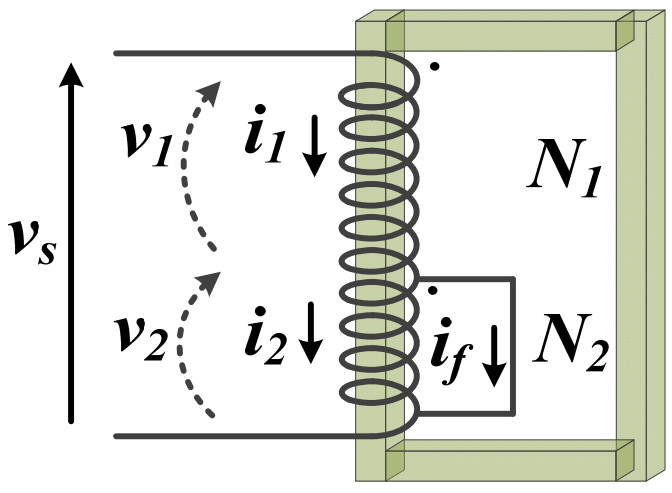

(a)

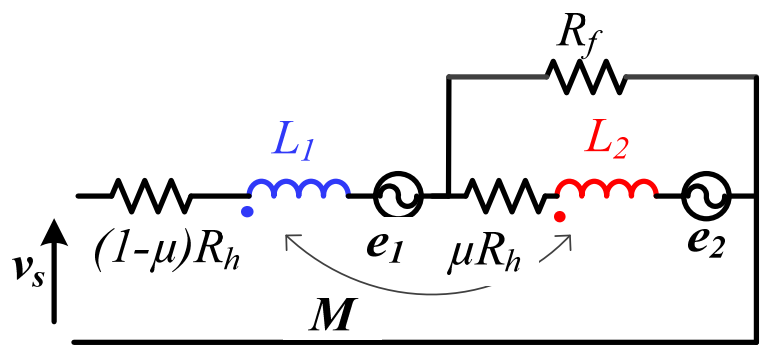

(b)

Figure 7.2 ITSC fault in one-coil. (a) Physical winding (b) Circuit diagram 


\subsubsection{Parameter Determination for ITSC in One-Coil}

The procedure to determine the faulty circuit parameters is based on three rules: consistency [143], proportionality [143] and magnetizing:

a) Consistency: let's assume that coil 1 and 2 from Figure 7.2(a) are in series, then the current $i_{1}$ flows through them (Figure 7.3(a)). The coil is at no load condition (no ITSC fault). Thus, the healthy inductance and resistance are as in (7.4).

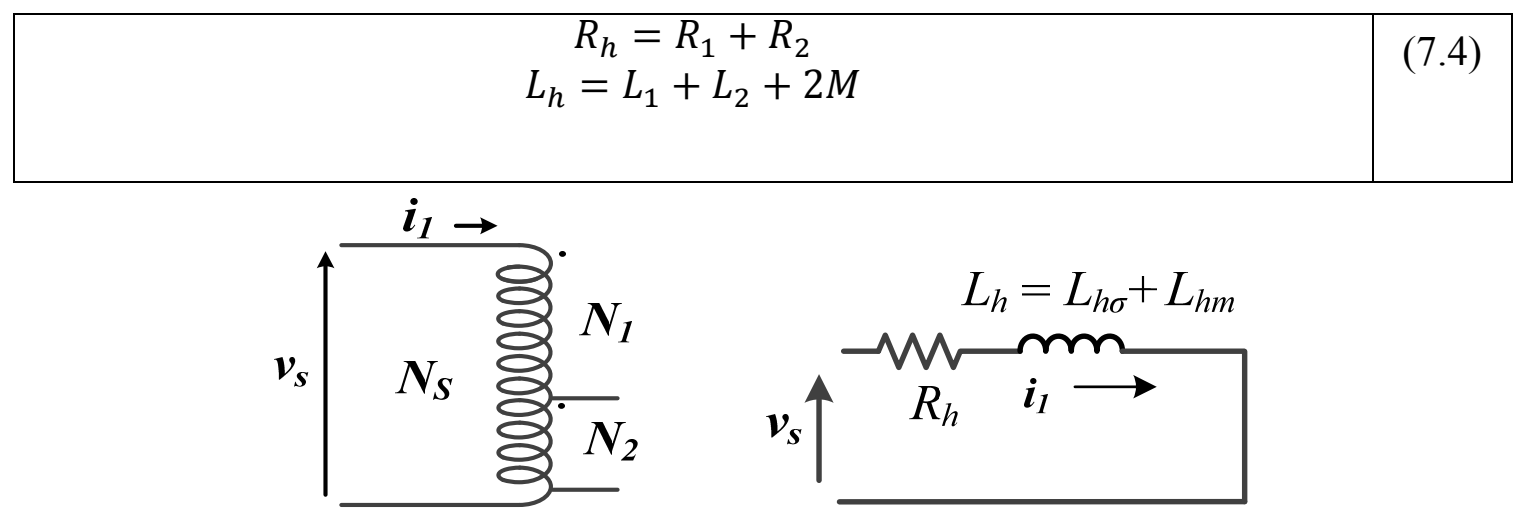

Figure 7.3 Coil at no load condition (no ITSC fault) (a) Physical winding (b) Circuit diagram

If a transformer is used for the testing of one coil case, the winding design of the primary and secondary winding, which generally have different cross sectional areas cables (different gauges), need to be considered.

b) Proportionality: the second rule that needs to be preserved is the turns' ratio:

$$
\frac{L_{1}}{L_{2}}=\left(\frac{N_{1}}{N_{2}}\right)^{2}=a^{2}
$$

where $a$ is the voltage ratio between coils 1 and 2. This rule is only true when the leakage inductances are neglected, however, it is an excellent approximation when leakage inductances are very small with respect to the magnetizing inductances. 
c) Magnetizing: it is used to find the magnetizing inductance. Based on the magnetically coupled circuit theory, the parameters of the autotransformer can be obtained as follows:

$$
\begin{aligned}
& L_{1}=L_{l 1}+L_{m 1}=\frac{N_{1}{ }^{2}}{\mathcal{R}_{l 1}}+\frac{N_{1}{ }^{2}}{\mathcal{R}_{m 1}} \\
& L_{2}=L_{l 2}+L_{m 2}=\frac{N_{2}{ }^{2}}{\mathcal{R}_{l 2}}+\frac{N_{2}{ }^{2}}{\mathcal{R}_{m 2}}
\end{aligned}
$$

where $L_{l 1}, L_{l 2}$ are the leakage inductances and $L_{m 1}$ and $L_{m 2}$ are the magnetizing inductances of the coil 1 and 2 , respectively, $\mathcal{R}_{l 1}$ and $\mathcal{R}_{l 2}$ are the leakage path reluctances for coil 1 and 2 , and $\mathcal{R}_{m 1}=\mathcal{R}_{m 2}=\mathcal{R}_{m}$ is the magnetizing path reluctance of the inductances.

By defining the healthy magnetizing inductance $L_{h m}=\frac{N^{2}}{\mathcal{R}_{m}}$, thus, (7.6) can be written as in (7.7) as a function of the fault severity factor $\mu=\frac{N_{2}}{N_{S}}$.

$$
\begin{gathered}
L_{1}=\frac{N_{1}{ }^{2}}{\mathcal{R}_{l 1}}+(1-\mu)^{2} L_{h m} \\
L_{2}=\frac{N_{2}{ }^{2}}{\mathcal{R}_{l 2}}+\mu^{2} L_{h m}
\end{gathered}
$$

The mutual inductance calculation is well-known as:

$$
M=\frac{N_{1} N_{2}}{\mathcal{R}_{m}}=\mu(1-\mu) L_{h m}
$$

Notice that the leakage inductance depends on the reluctance path formed by the fault, thus the assumptions in [34], [36], [144] are inaccurate. From (7.7), the leakage inductances are: 


$$
L_{l 1}=\frac{N_{1}^{2}}{\mathcal{R}_{l 1}}=\frac{(1-\mu)^{2} N^{2}}{\mathcal{R}_{\sigma 1}} \quad L_{l 2}=\frac{N_{2}{ }^{2}}{\mathcal{R}_{l 2}}=\frac{\mu^{2} N^{2}}{\mathcal{R}_{l 2}}
$$

From the consistency rule and the definition of healthy self-inductance:

$$
L_{h}=L_{l h}+L_{m h}=L_{1}+L_{2}+2 M
$$

where $L_{l h}$ is the leakage inductance of the healthy coil.

Thus, substituting (7.7), (7.8) and (7.9) into (7.10), the total leakage inductance can be deduced as a function of the faulty and healthy winding parts.

$$
L_{\sigma h}=L_{\sigma 1}+L_{\sigma 2}
$$

Notice that (7.11) is a logical equation, as typically the summation of the leakage effects in a winding is equal to the total leakage inductance. Using the proportionality rule:

$$
\mu^{2} L_{1}=(1-\mu)^{2} L_{2}
$$

Combining (7.7) with (7.12) and using (7.11), the leakage inductances can be determined as in (7.13).

$$
L_{l 1}=\frac{(1-\mu)^{2} L_{l h}}{1-2 \mu+2 \mu^{2}}=k_{l s 1} L_{l h} \quad L_{l 2}=\frac{\mu^{2} L_{l h}}{1-2 \mu+2 \mu^{2}}=k_{l s 2} L_{l h}
$$

where $k_{l s 1}=\frac{(1-\mu)^{2}}{1-2 \mu+2 \mu^{2}}$ and $k_{l s 2}=\frac{\mu^{2}}{1-2 \mu+2 \mu^{2}}$ are the leakage factors.

Finally, the $[\boldsymbol{L}]$ matrix is defined in (7.14).

$$
[\boldsymbol{L}]=\left[\begin{array}{cc}
L_{h} & -\left(L_{2}+\mu(1-\mu) L_{m}\right) \\
L_{2}+\mu(1-\mu) L_{m} & -\frac{\mu^{2} L_{l h}}{1-2 \mu+2 \mu^{2}}-\mu^{2} L_{m}
\end{array}\right]
$$


In Figure 7.4, the variation of the leakage inductances (leakage factor $k_{l s 2}$ ) from (7.13) as a function of the fault severity factor can be observed. For a very low severity factor, the proposed leakage factor $k_{l s 2}$ has similar behavior to the quadratic one, however $k_{l s 1}=$ $1-k_{l s 2}$ has poor correlation. It can be noticed that in some points, such as $\mu=[0,0.5,1]$, the linear and the proposed model match, however, the quadratic model only matches with the other two models at the ending points (no fault or full phase short-circuit).

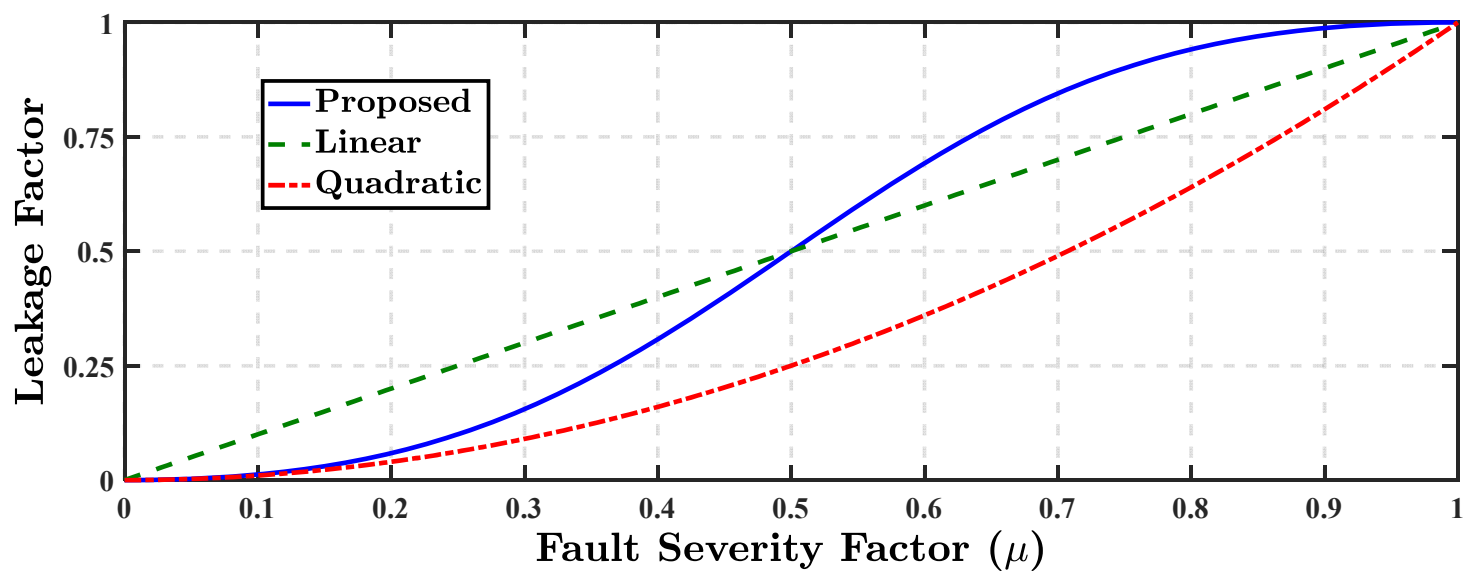

Figure 7.4 Leakage factor inductance as a function of the fault severity factor

The equivalent circuit model for the one-faulty-coil is presented in Figure 7.5 as a function of the self and mutual inductances, which is the same for the autotransformer.

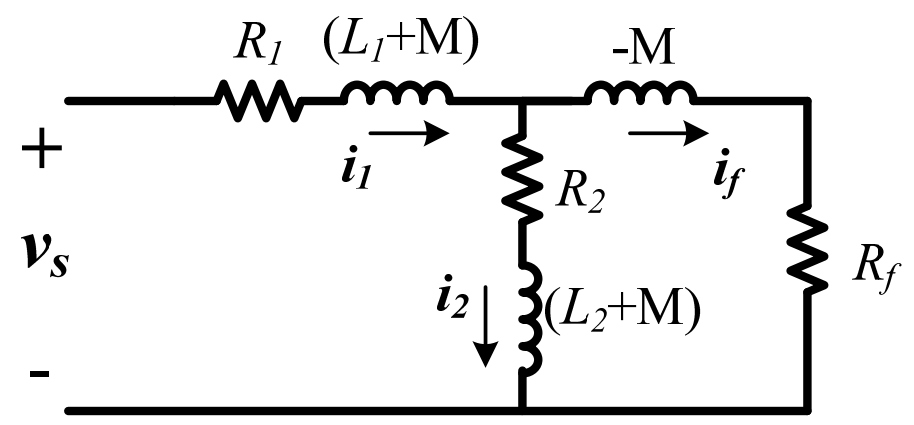

Figure 7.5 Equivalent circuit diagram for the ITSC in one coil or autotransformer 
The importance on modeling the ITSC fault as a step-down autotransformer is in the implications associated with the well-known case:

(1) If the turns ratio of the fault severity factor and current $i_{1}$ are known (e.g. by measurement), then the current $i_{2}$ and $i_{f}$ can be approximated by a simple calculation, as the step-down autotransformer $i_{2}=-\left(N_{1} / N_{2}\right) i_{1}$ and $i_{f}=i_{1}-i_{2}$.

(2) Reverse current phenomena of the autotransformer in the faulty winding part: this event occurs exactly at the moment of the short-circuit. Before the ITSC, the circuit of Figure 7.3 takes place and the current $i_{1}$ in the turns $N_{2}$ goes through all the winding. After the ITSC happens, Figure 7.2(a) takes place and the current in the faulty part is reversed in direction $-i_{2}$. This phenomenon occurs because the current in the faulty part needs to create a magnetic field that makes opposition to the field that is inducing this current in the $N_{2}$ turns (Lenz's Law).

(3) Notice that the current $i_{2}$ is still placed in the direction of the power supply for a simplified analysis and the polarity of the coils is additive, as it was demonstrated in (7.4), in the same way the dot rule is shown in Figure 7.2(a) and Figure 7.3(a).

\subsection{IM Model with Stator ITSC Fault}

A three-phase IM with stator winding ITSC in one phase, phase " $a$ ", is shown in Figure 7.6(a), where $N_{s a 2}$ represents the short-circuited turns and $N_{s a}=N_{s a 1}+N_{s a 2}$ is the total number of winding turns. The fault severity factor is defined as $\mu=\frac{N_{s a 2}}{N_{s a}}$. Figure 7.6(b) presents a circuit diagram of a three-phase IM stator for the failure, where two terms are highlighted: the transformer voltage (across the inductances) and the generation terms or back electromotive force (bemf), where $e_{s a 1}=M \frac{d i_{s a 2}}{d t}$ and $e_{s a 2}=M \frac{d i_{s a 1}}{d t}$ are the bemfs 
and $M$ is the mutual inductance. Notice the step-down auto-transformer configuration for phase " $a$ ". For this analysis, it is assumed that $R_{f}$ is the fault impedance that will determine the faulty leakage current $\left(i_{f}\right)$ magnitude in the short-circuit path, $i_{x s}$ and $v_{x s}$ are the stator winding current and voltage for the phase $x$, where $x=\{a, b, c\}$.

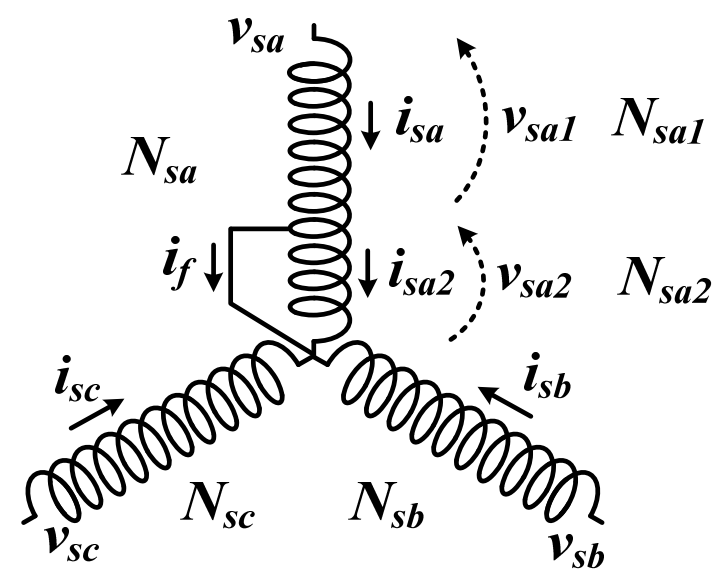

(a) Physical winding

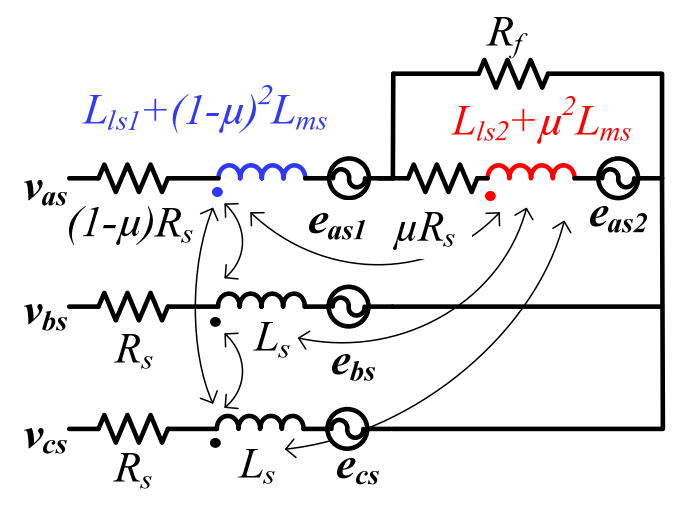

(b) Circuit diagram

Figure 7.6 Three-phase stator winding of an IM with ITSC in the phase " $a$ ".

\subsubsection{Parameter Determination for the phase with ITSC}

The procedure to determine the faulty parameters is based on the 3 rules abovementioned: 
a) Consistency: let's assume a healthy condition, therefore coil $s a_{1}$ and $s a_{2}$ from Figure 7.6(a) are in series and the same current $i_{s a}$ flows through them. Thus, the healthy inductance and resistance are:

$$
\begin{gathered}
R_{s a}=R_{s a 1}+R_{s a 2}=(1-\mu) R_{s a}+\mu R_{s a} \\
L_{a s}=L_{s a 1}+L_{s a 2}+2 M
\end{gathered}
$$

where $R_{s a 1}$ and $R_{s a 2}$ are the resistances and $L_{s a 1}$ and $L_{s a 2}$ are the self-inductances of $s a_{1}$ and $s a_{2}$ windings, respectively, $R_{s a}$ and $L_{s a}$ are the healthy resistance and self-inductance of the total winding of the phase " $a$," respectively.

b) Proportionality: the second rule that needs to be preserved is the turn's ratio:

$$
\frac{L_{s a 1}}{L_{s a 2}}=\left(\frac{N_{s a 1}}{N_{s a 2}}\right)^{2}=n^{2}
$$

where $n$ is the voltage ratio between $s a_{1}$ and $s a_{2}$ coils, $N_{s a 1}$ and $N_{s a 2}$ are the number of turns of coils $s a_{1}$ and $s a_{2}$, respectively.

c) Magnetizing: Based on the magnetically coupled circuit theory, the parameters of the IM are obtained as (7.17), where $L_{l s 1}, L_{l s 2}$ are the leakage inductances and $L_{m s 1}$ and $L_{m s 2}$ are the magnetizing inductances of the coils $s a_{1}$ and $s a_{2}$, respectively, $\mathcal{R}_{l 1}$ and $\mathcal{R}_{l 2}$ are the leakage reluctance path for coils $s a_{1}$ and $s a_{2}$, and $\mathcal{R}_{m 1}=$ $\mathcal{R}_{m 2}=\mathcal{R}_{m}$ is the magnetizing reluctance path of the inductances.

$$
\begin{aligned}
& L_{s a 1}=L_{l s 1}+L_{m s 1}=\frac{N_{s a 1}{ }^{2}}{\mathcal{R}_{l 1}}+\frac{N_{s a 1}{ }^{2}}{\mathcal{R}_{m 1}} \\
& L_{s a 2}=L_{l s 2}+L_{m s 2}=\frac{N_{s a 2}{ }^{2}}{\mathcal{R}_{l 2}}+\frac{N_{s a 2}{ }^{2}}{\mathcal{R}_{m 2}}
\end{aligned}
$$


By defining the healthy magnetizing inductance as $L_{m s}=\frac{N_{s a}{ }^{2}}{\mathcal{R}_{m}}$, then, (7.17) is written as in (7.18) as a function of $\mu$.

$$
L_{a s 1}=\frac{N_{a s 1}^{2}}{\mathcal{R}_{l 1}}+(1-\mu)^{2} L_{m s} \quad L_{a s 2}=\frac{N_{a s 2}^{2}}{\mathcal{R}_{l 2}}+\mu^{2} L_{m s}
$$

The mutual inductance calculation is well-known as:

$$
M=\frac{N_{s a 1} N_{s a 2}}{\mathcal{R}_{m}}=\mu(1-\mu) L_{m s}
$$

Note that the leakage inductances depend on the reluctance path formed by the fault as in (7.20). Hence, the assumptions in [34], [36], [127], [144] are inaccurate, as they consider $\mathcal{R}_{l 1}=\mathcal{R}_{l 2}=\mathcal{R}_{l}$, where $\mathcal{R}_{l}$ is the winding healthy reluctance path.

$$
L_{l s 1}=\frac{(1-\mu)^{2} N_{s a}^{2}}{\mathcal{R}_{l 1}} \quad L_{l s 2}=\frac{\mu^{2} N_{s a}^{2}}{\mathcal{R}_{l 2}}
$$

From the consistency rule, it can be written:

$$
L_{a s}=L_{l s}+L_{m s}=L_{s a 1}+L_{s a 2}+2 M
$$

where $L_{l s}$ is the leakage inductance of the healthy coil.

Substituting (7.18), (7.19) and (7.20) into (7.21):

$$
L_{l s}=L_{l s 1}+L_{l s 2}
$$

See that (7.22) is a meaningful equation, as typically the summation of the leakage effects in a winding is equal to the total leakage inductance. Using the proportionality rule:

$$
\mu^{2} L_{s a 1}=(1-\mu)^{2} L_{s a 2}
$$


Combining (7.18) with (7.23) and using (7.22), the leakage inductances are determined as:

$$
L_{l s 1}=\frac{(1-\mu)^{2} L_{l s}}{1-2 \mu+2 \mu^{2}}=k_{l s 1} L_{l s} \quad L_{l s 2}=\frac{\mu^{2} L_{l s}}{1-2 \mu+2 \mu^{2}}=k_{l s 2} L_{l s}
$$

where $k_{l s 1}$ and $k_{l s 2}$ are defined as the leakage factors.

From (7.20) and (7.24), it can be noticed that $\mathcal{R}_{l 1}=\mathcal{R}_{l 2}$ due to the approximation of the proportionality rule that holds when the magnetizing inductance is much greater than the leakage one. In this case, $\mathcal{R}_{l 1}=\mathcal{R}_{l 2}$, but they are different from $\mathcal{R}_{l}$, which is one step further in the physical representation of the IM with ITSC fault.

\subsubsection{IM Equations in Primitive Coordinates}

The asymmetric IM voltage equations for the stator and rotor are in vector form referred to the stator, and they are expressed as in (7.25) [34], [36]:

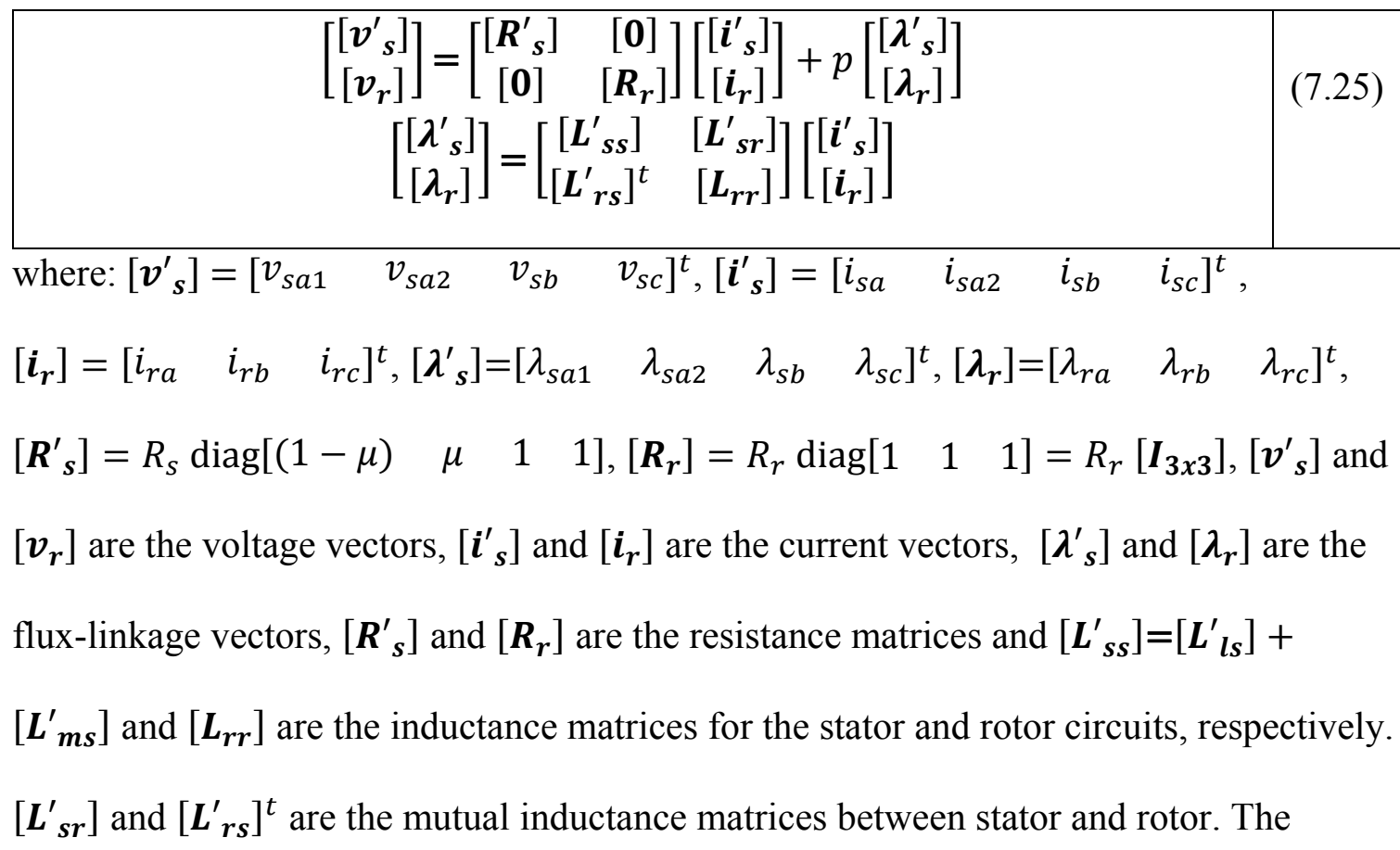


symbol $p$ is the derivative operator, the function $\operatorname{diag}[\cdot]$ is the diagonal matrix, the current $i_{s a 2}$ is defined in Figure 7.6(a) as $i_{s a 2}=i_{s a}-i_{f}$ and $\left[\boldsymbol{I}_{3 x 3}\right]$ is the identity matrix.

The inductance matrices $\left[\boldsymbol{L}_{\boldsymbol{l}}^{\prime}\right],\left[\boldsymbol{L}_{\boldsymbol{m} \boldsymbol{s}}^{\prime}\right],\left[\boldsymbol{L}_{\boldsymbol{s r}}^{\prime}\right]$ and $\left[\boldsymbol{L}_{\boldsymbol{r r}}\right]$ are in (7.26), where in general $c_{1}(\zeta)=\cos \zeta, c_{2}(\zeta)=\cos \left(\zeta+\frac{2 \pi}{3}\right), c_{3}(\zeta)=\cos \left(\zeta-\frac{2 \pi}{3}\right), L_{l r}$ is the rotor leakage inductance and $[\boldsymbol{S}]$ is the symmetric matrix. The superscript prime $\left(\left[\boldsymbol{f}_{\boldsymbol{q}}{ }_{\boldsymbol{q}}\right]\right)$ in matrices and vectors $([\boldsymbol{f}]=[\boldsymbol{R}],[\boldsymbol{L}],[\boldsymbol{v}],[\boldsymbol{i}]$ and $[\lambda])$ represent the asymmetrical IM. The absence of the prime $\left(\left[\boldsymbol{f}_{\boldsymbol{q}}\right]\right)$ represents the healthy IM. The subscript $\boldsymbol{q}=\{\boldsymbol{s}, \boldsymbol{r}\}$ indicates the stator and rotor coordinate reference frame, respectively.

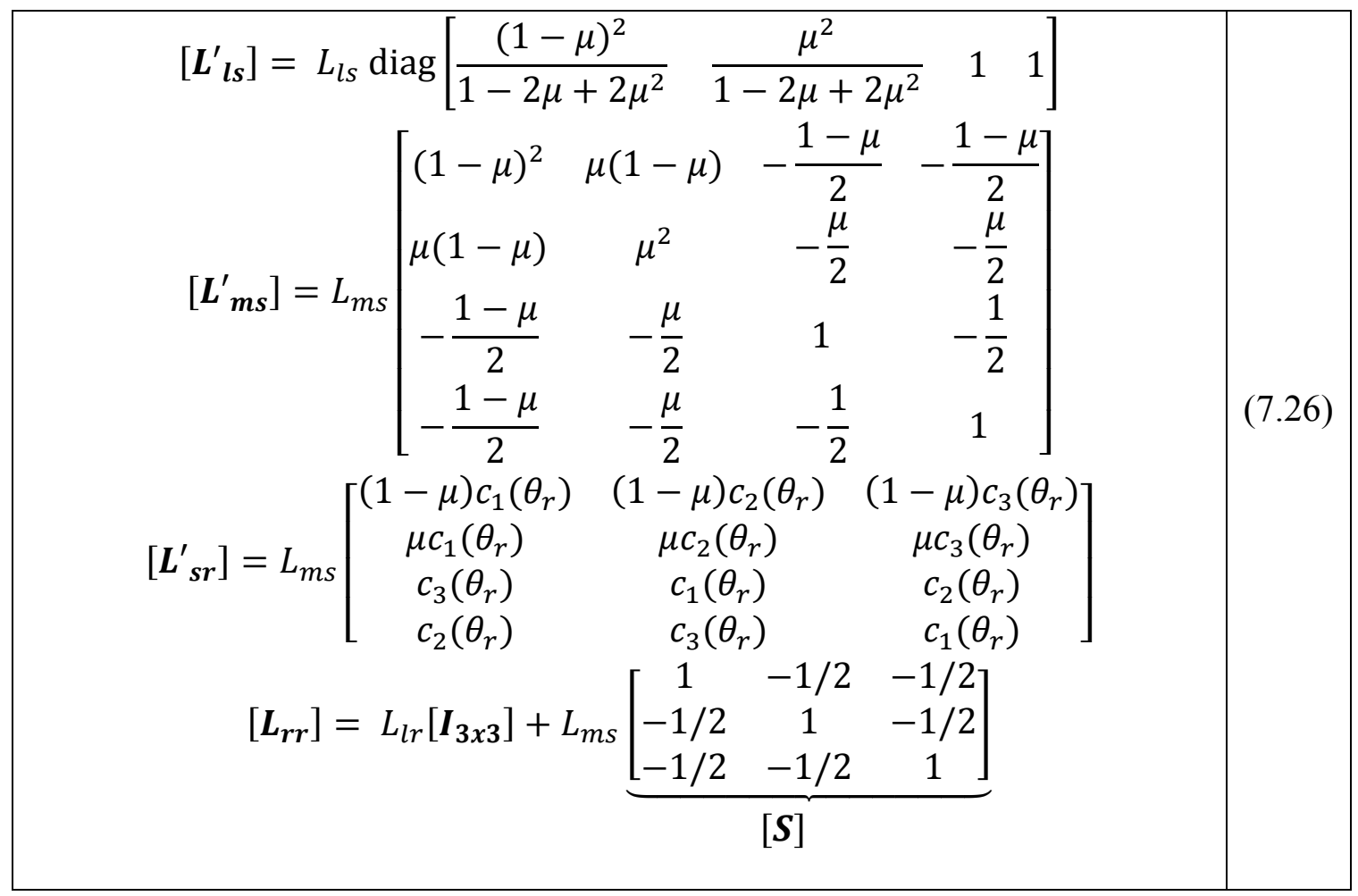

The asymmetric IM equations are written in terms of the healthy IM matrices and vectors plus the faulty terms as: 


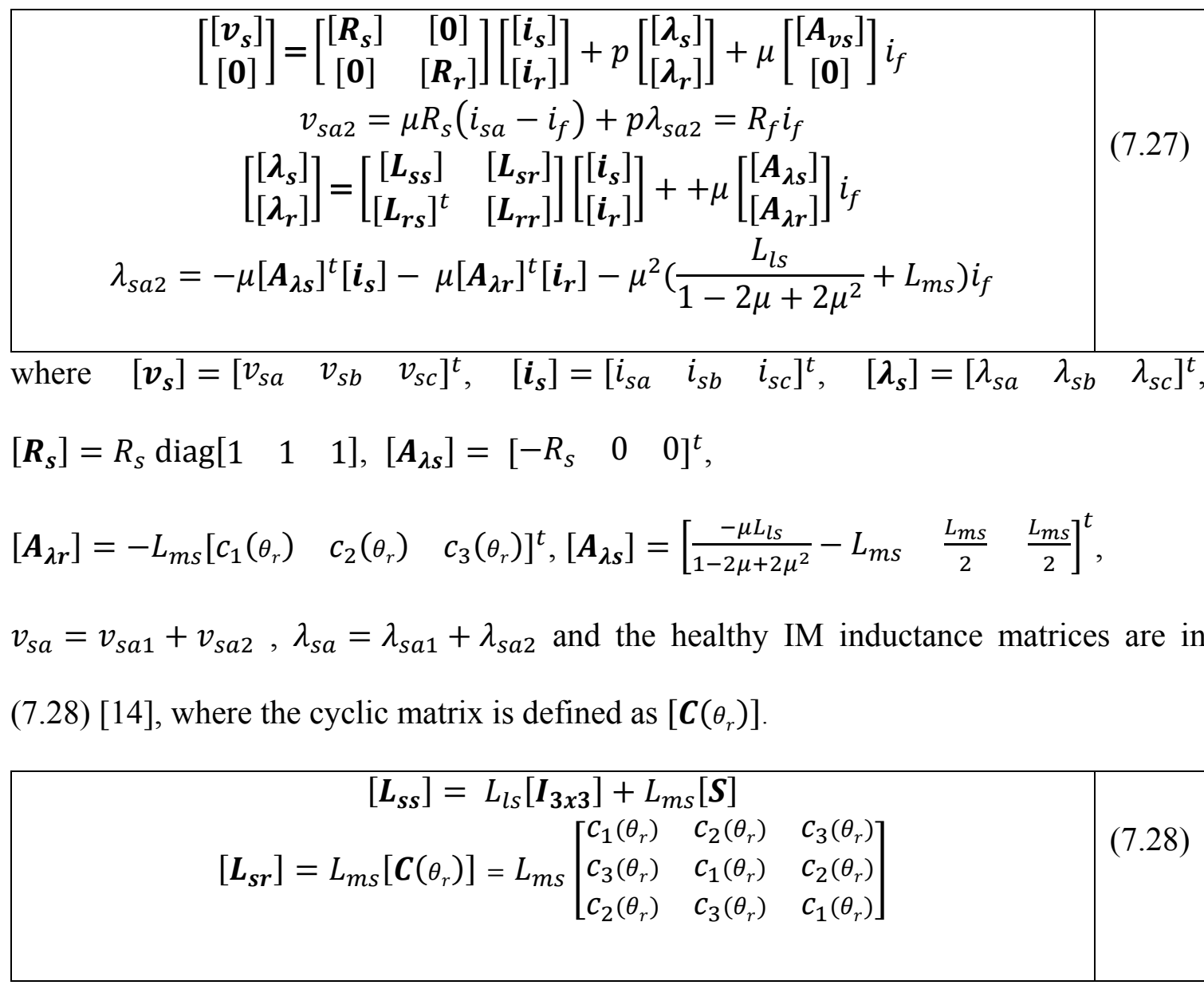

The torque for the faulty IM in terms of the primitive coordinates is as in (7.29).

\begin{tabular}{|l|l|}
\hline$T_{e}=\frac{P}{2}\left[\boldsymbol{i}^{\prime}{ }_{s}\right]^{t} \frac{\partial\left[\boldsymbol{L}^{\prime} \boldsymbol{s} \boldsymbol{r}\right]}{\partial \theta_{r}}\left[\boldsymbol{i}_{\boldsymbol{r}}\right]$ & (7.29) \\
\hline
\end{tabular}

Substituting $\left[\boldsymbol{L}_{\boldsymbol{s} \boldsymbol{r}}^{\prime}\right],\left[\boldsymbol{i}_{\boldsymbol{s}}^{\prime}\right]$ and $\left[\boldsymbol{i}_{\boldsymbol{r}}\right]$ in (7.29), the torque equation can be expressed in terms of the healthy IM torque equation $\left(T_{e m}\right)$ and a faulty term $\left(T_{e f}\right)$ that will result in a wave with the double frequency oscillation $\left(2 \omega_{s}\right)$ as in (7.30).

\begin{tabular}{|l|l|l|}
\hline$T_{e}=\underbrace{\frac{P}{2}\left[\boldsymbol{i}_{\boldsymbol{s}}\right]^{t} \frac{\partial\left[\boldsymbol{L}_{\boldsymbol{s} r}\right]}{\partial \theta_{r}}\left[\boldsymbol{i}_{\boldsymbol{r}}\right]}_{T_{e m}}-\underbrace{\frac{\mu P L_{m s}}{2}\left\{\frac{3}{2} i_{r a} s_{1}\left(\theta_{r}\right)+\frac{\sqrt{3}}{2}\left(i_{b r}-i_{c r}\right) c_{1}\left(\theta_{r}\right)\right\} i_{f}}_{T_{e f}} \quad$ (7.30) & \\
\hline
\end{tabular}

where $s_{1}(\zeta)=\sin \zeta$. 
This double-line frequency oscillation reported in [24], [124] comes in the mathematical representation from the multiplication of two sinusoidal waves that oscillate at the same frequency. In the physical world, it comes from the fact that when the ITSC happens the current in the fault winding $\left(i_{s a 2}\right)$ reverses its direction (opposite to $i_{s a}$ ), producing a counter rotating magnetic field (negative sequence), deforming the shape of the original MMF. All this as explained in the previous chapter.

\subsubsection{IM Equations in Complex-Vector Notation}

The voltage and torque equations in (7.27) and (7.30) are transformed from primitive coordinates to CV notation by utilizing the stationary reference frame transformation [14]. The transformed IM voltage equations are as in (7.31).

\begin{tabular}{|c|c|}
\hline 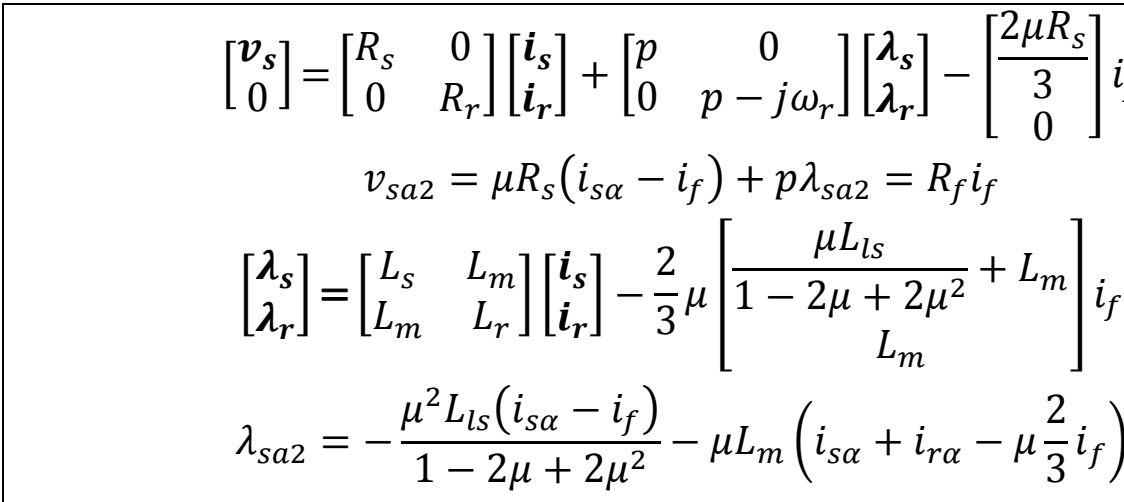 & $(7.31)$ \\
\hline $\begin{array}{c}\text { where } \boldsymbol{f}_{\boldsymbol{q}}=f_{q \alpha}-j f_{q \beta}, \boldsymbol{f}=\{\boldsymbol{v}, \boldsymbol{i}, \boldsymbol{\lambda}\}, \boldsymbol{q}=\{\boldsymbol{s}, \boldsymbol{r}\}, L_{s}=L_{l s}+L_{m}, L \\
L_{m}=\frac{3}{2} L_{m s}, \omega_{r} \text { is the mechanical speed and and } P \text { is the pair of po } \\
\text { The electromagnetic torque in complex-vector notation is: }\end{array}$ & \\
\hline$T_{e}=\underbrace{\frac{3}{2} \frac{P}{2} L_{m}\left(i_{s \alpha} i_{r \beta}-i_{s \beta} i_{r \alpha}\right)}_{T_{e m}}+\underbrace{\mu \frac{P}{2} L_{m} i_{f} i_{r \beta}}_{T_{e f}}$ & $(7.32)$ \\
\hline
\end{tabular}




\subsubsection{IM Equations in SS Representation}

The SS model for the IM can be represented in many different SS variables with the stator voltages and load torque as inputs, and the electromagnetic torque and the rotor angular speed as outputs. The flux-linkages are considered as the state-space variable $\left(\left[\lambda^{\prime}\right]=\left[\begin{array}{lllll}\lambda_{s \alpha} & \lambda_{s \beta} & \lambda_{r \alpha} & \lambda_{r \beta} & \lambda_{s a 2}\end{array}\right]^{t}\right)$, thus [ $\left.\lambda^{\prime}\right]$ is written in terms of the current SS vector as:

$$
\left[\lambda^{\prime}\right]=\left[\boldsymbol{L}^{\prime}\right]\left[\boldsymbol{i}^{\prime}\right]
$$

where $\left[\boldsymbol{i}^{\prime}\right]=\left[\begin{array}{lllll}i_{s \alpha} & i_{s \beta} & i_{r \alpha} & i_{r \beta} & i_{f}\end{array}\right]^{t},\left[\boldsymbol{L}^{\prime}\right]=[\boldsymbol{M}]$ comes from (7.31) and requires $\mu \neq$ 0 to be a full-rank invertible matrix.

$[\boldsymbol{M}]=\left[\begin{array}{ccccc}L_{s} & 0 & L_{m} & 0 & M_{15} \\ 0 & L_{s} & 0 & L_{m} & 0 \\ L_{m} & 0 & L_{r} & 0 & M_{35} \\ 0 & L_{m} & 0 & L_{r} & 0 \\ -3 / 2 M_{15} & 0 & -3 / 2 M_{35} & 0 & M_{55}\end{array}\right] \quad$ (7.34)

where $M_{15}=-\frac{2}{3} \mu\left(\frac{\mu L_{l s}}{1-2 \mu+2 \mu^{2}}+L_{m}\right), M_{35}=-\frac{2}{3} \mu L_{m}$ and $M_{55}=\frac{\mu^{2} L_{l s}}{1-2 \mu+2 \mu^{2}}+\mu^{2} \frac{2}{3} L_{m}$.

Then $\left[\boldsymbol{i}^{\prime}\right]=[\boldsymbol{M}]^{-1}\left[\boldsymbol{\lambda}^{\prime}\right]$, where $[\boldsymbol{M}]^{-1}=[\boldsymbol{N}]$ is as:

$[\boldsymbol{N}]=\left[\begin{array}{ccccc|c|}N_{11} & 0 & N_{13} & 0 & N_{15} \\ 0 & -L_{r} / \Delta & 0 & L_{m} / \Delta & 0 \\ N_{13} & 0 & N_{33} & 0 & N_{35} \\ 0 & L_{m} / \Delta & 0 & -L_{s} / \Delta & 0 \\ -3 / 2 N_{15} & 0 & -3 / 2 N_{35} & 0 & N_{55}\end{array}\right] \quad$ (7.35)

where: $\Delta=L_{m}^{2}-L_{s} L_{r}, N_{11}=\frac{-\left(3 L_{35}{ }^{2}+2 L_{r} L_{55}\right)}{D}, N_{13}=\frac{3 L_{15} L_{35}+2 L_{m} M_{55}}{D}$,

$$
\begin{aligned}
& N_{15}=\frac{2\left(L_{r} M_{15}-L_{m} M_{35}\right)}{D}, N_{33}=\frac{-\left(3 M_{15}{ }^{2} \mp 2 L_{s} M_{55}\right)}{D}, N_{35}=\frac{-2\left(L_{m} M_{15}-L_{S} M_{35}\right)}{D}, N_{55}=\frac{2 \Delta}{D} \text { and } \\
& D=6\left(\frac{M_{55} L_{m}^{2}}{3}+M_{15} M_{35} L_{m}-\frac{L_{r} M_{15}{ }^{2}}{2}-\frac{L_{s} M_{35}{ }^{2}}{2}-\frac{L_{r} L_{S} M_{55}}{3}\right) .
\end{aligned}
$$

The SS representation for the stator ITSC fault of an IM is: 


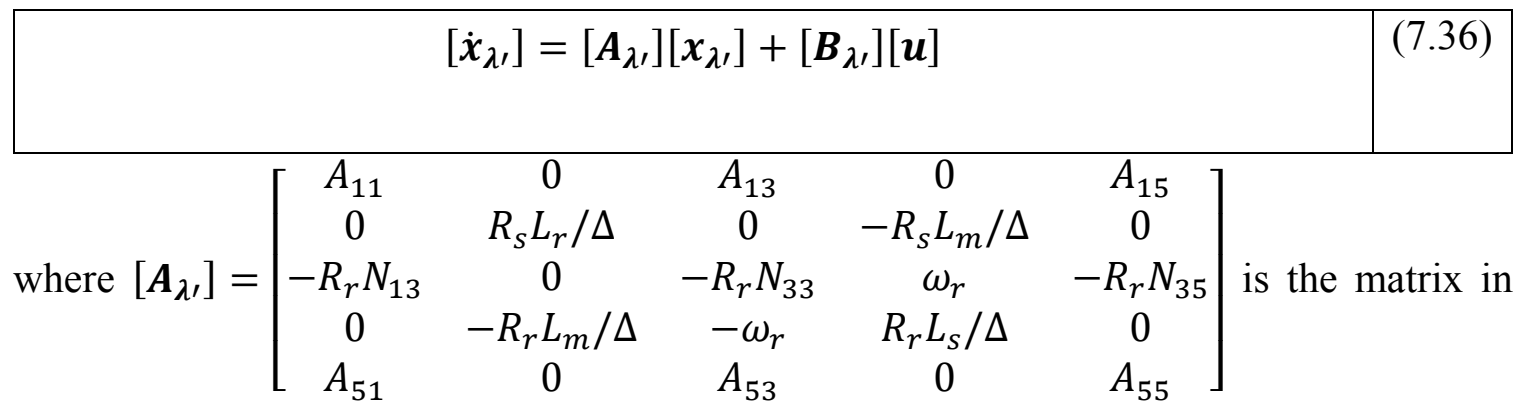

flux-linkages SS model, $A_{11}=-R_{S}\left(N_{11}+\mu N_{15}\right), A_{13}=-R_{S}\left(N_{13}+\mu N_{35}\right)$,

$$
\begin{aligned}
& A_{15}=R_{S}\left(-N_{15}+\mu \frac{2}{3} N_{55}\right), A_{51}=-\left(\frac{3}{2}\left(R_{f}+\mu R_{S}\right) N_{15}+\mu R_{S} N_{11}\right) \\
& A_{53}=-\left(\frac{3}{2}\left(R_{f}+\mu R_{S}\right) N_{35}+\mu R_{S} N_{13}\right) \text { and } A_{55}=\left(R_{f}+\mu R_{S}\right) N_{55}-\mu R_{S} N_{15}, \\
& {[\boldsymbol{u}]=\left[\begin{array}{c}
v_{s \alpha} \\
v_{s \beta}
\end{array}\right],\left[\boldsymbol{x}_{\lambda^{\prime}}\right]=\left[\boldsymbol{\lambda}^{\prime}\right] \text { and }\left[\boldsymbol{B}_{\lambda^{\prime}}\right]=\left[\begin{array}{lllll}
1 & 0 & 0 & 0 & 0 \\
0 & 1 & 0 & 0 & 0
\end{array}\right]^{t}}
\end{aligned}
$$

The load torque-speed characteristic defines the mechanical equation that complements the SS representation. This curve may vary widely from one application to the other, even though for simplicity, it is assumed that the load torque profile consists of the inertia of the motor $(J)$, an external load torque $\left(T_{L}\right)$ and friction $\left(k_{\text {loss }}\right)$. Thus, the mechanical equation is:

$$
\frac{d \omega_{r}}{d t}=\frac{1}{J}\left(T_{e}-T_{L}-k_{\text {loss }} \omega_{r}\right)
$$

\subsubsection{Equivalent Circuits: Faulty IM}

The $\mathrm{CV} \alpha \beta$-model for the faulty IM in (7.31) is written as (7.39) after approximating $M_{15}$, in (7.34), as (7.38). Notice that $M_{15}$ is one of the faulty terms out of five. This approximation accounts for the linear case of the leakage inductance factor in only the $M_{15}$ component. 


$$
M_{15}=-\frac{2}{3} \mu\left(\frac{\mu L_{l s}}{1-2 \mu+2 \mu^{2}}+L_{m}\right) \approx-\frac{2}{3} \mu L_{s}
$$

This approximation is always valid, whereas the leakage inductance is very small compared with the magnetizing inductance. The approximation effect is less than $2 \%$ error from the exact model.

$$
\begin{gathered}
{\left[\begin{array}{c}
\boldsymbol{v}_{\boldsymbol{s}} \\
\boldsymbol{v}_{\boldsymbol{r}}
\end{array}\right]=\left[\begin{array}{cc}
R_{s}+L_{s} p & L_{m} p \\
L_{m} p & R_{r}+L_{r} p
\end{array}\right]\left[\begin{array}{c}
\boldsymbol{i}_{\boldsymbol{s} \mathbf{1}} \\
\boldsymbol{i}_{\boldsymbol{r}}
\end{array}\right]-j \omega_{r}\left[\begin{array}{c}
0 \\
\lambda_{\boldsymbol{r}}
\end{array}\right]} \\
\mu v_{s \alpha}-j \omega_{s} L_{l s f \alpha} \mu i_{s \alpha}=\left(R_{f}+R_{s f}+L_{l s f} p\right) i_{f}
\end{gathered}
$$

where $\boldsymbol{i}_{\boldsymbol{s} \mathbf{1}}=\boldsymbol{i}_{\boldsymbol{s}}-\frac{2}{3} \mu i_{f}, R_{s f}=\mu\left(1-\frac{2}{3} \mu\right) R_{s}, L_{l s f}=\frac{\mu}{1-2 \mu+2 \mu^{2}}\left(1-\frac{2}{3} \mu\right) L_{l s}$ and $L_{l s f \alpha}=$ $\frac{1-3 \mu+2 \mu^{2}}{1-2 \mu+2 \mu^{2}} L_{l s} . R_{s f}$ and $L_{l s f}$ are the equivalent stator resistance and leakage inductance of the faulty winding determined by the fault severity factor, $L_{l s f \alpha}$ is a factor that accounts for the effect of the stator current in the faulty current, whereas the $R_{f}$ is the path resistance that creates the short-circuit.

From (7.39), the CV EC model for the defective IM is deduced in Figure 7.7. Following the procedure in [40], the operational EC is accomplished in (7.40) and shown in Figure 7.8 .

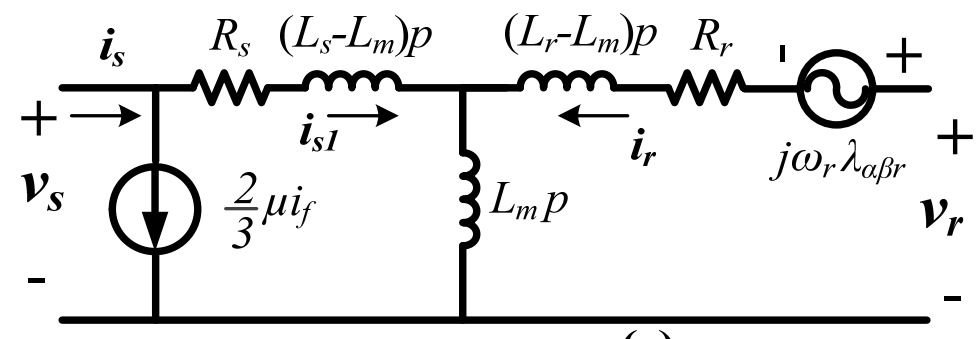

(a)

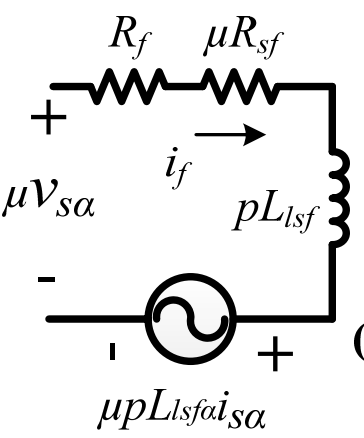

(b)

Figure 7.7 (a) Complex-vector EC of an asymmetric IM (b) Faulty circuit. 


$$
\left[\begin{array}{c}
\boldsymbol{v}_{s} \\
\mathbf{0}
\end{array}\right]=\left[\begin{array}{cc}
R_{s}+L_{s} p & L_{m} p \\
L_{m} p & \frac{R_{r} p}{p-j \omega_{r}}+L_{r} p
\end{array}\right]\left[\begin{array}{c}
\boldsymbol{i}_{\boldsymbol{s}} \\
\boldsymbol{i}_{r}
\end{array}\right]
$$

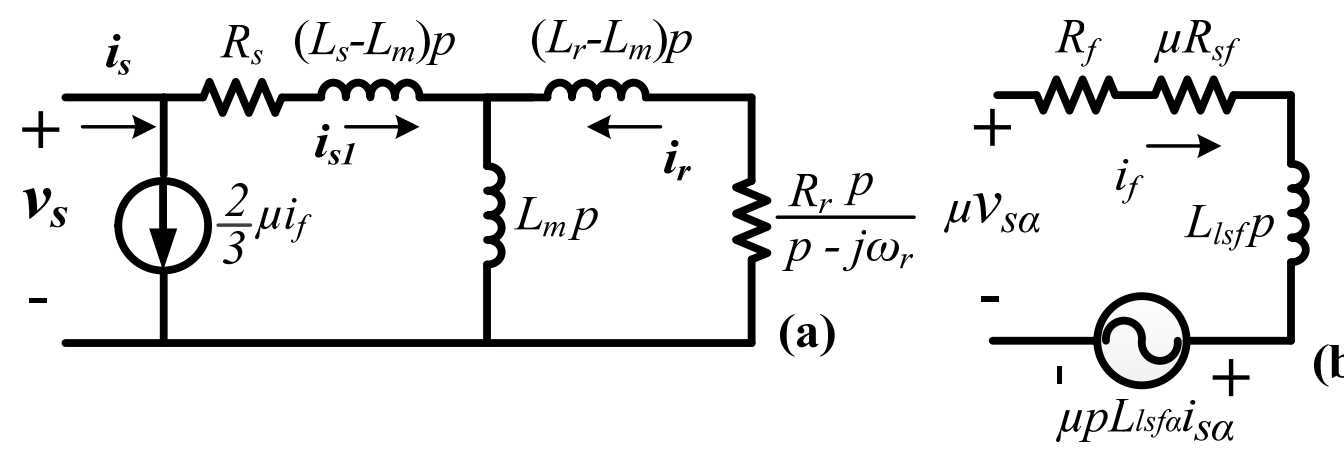

(b)

Figure 7.8 (a) CV operational EC of an asymmetric IM (b) Faulty circuit.

\subsubsection{Steady State Analysis: Symmetric Components EC}

The steady state analysis is applied to (7.31) by following the procedure in [40]. In the sinusoidal steady state regime, the $\mathrm{CV} \boldsymbol{f}_{\boldsymbol{q}}$ is expressed in terms of the positive $\left(\bar{F}_{q P}\right)$ and negative $\left(\bar{F}_{q N}\right)$ sequence component phasors as (7.41) and the faulty current in Euler form as in (7.42).

$$
\boldsymbol{f}_{\boldsymbol{q}}=F_{q P} e^{j\left(\omega_{e} t\right)}+F_{q N}{ }^{*} e^{-j\left(\omega_{e} t\right)}
$$

where $\bar{F}_{q P}=F_{q P} e^{j\left(\phi_{F_{P}}\right)}, \bar{F}_{q N}{ }^{*}=F_{q N} e^{-j\left(\phi_{F_{N}}\right)}, \phi_{F_{P}}$ and $\phi_{F_{N}}$ are the positive and negative sequence-component phases of $\bar{F}_{q P}$ and $\bar{F}_{q N}$, respectively, the superscript asterisk means the vector conjugate.

$$
i_{f}=\bar{I}_{f P}+\bar{I}_{f N}=\frac{1}{2}\left(I_{f} e^{j\left(\omega_{e} t+\phi_{i_{f}}\right)}+I_{f} e^{-j\left(\omega_{e} t+\phi_{i}\right)}\right)
$$

where $I_{f}$ and $\phi_{i_{f}}$ are the magnitude and phase of the faulty leakage current $i_{f}$, respectively. 
Substituting (7.41) and (7.42) into (7.40), the steady state model is obtained as in (7.43). It can be demonstrated that the steady state of the voltage and current real components $\left(v_{s \alpha}\right.$ and $i_{s \alpha}$ ) are written as $\bar{V}_{s \alpha}=\bar{V}_{s P}+\bar{V}_{s N}$ and $\bar{I}_{s \alpha}=\bar{I}_{S P}+\bar{I}_{s N}$.

$$
\begin{gathered}
{\left[\begin{array}{c}
\bar{V}_{s P} \\
0
\end{array}\right]=\left[\begin{array}{cc}
R_{s}+j \omega_{e} L_{s} & j \omega_{e} L_{m} \\
j \omega_{e} L_{m} & \frac{R_{r}}{s}+j \omega_{e} L_{r}
\end{array}\right]\left[\begin{array}{c}
\bar{I}_{s P 1} \\
\bar{I}_{r P}
\end{array}\right]} \\
{\left[\begin{array}{c}
\bar{V}_{s N} \\
0
\end{array}\right]=\left[\begin{array}{cc}
R_{s}+j \omega_{e} L_{s} & j \omega_{e} L_{m} \\
j \omega_{e} L_{m} & \frac{R_{r}}{2-s}+j \omega_{e} L_{r}
\end{array}\right]\left[\begin{array}{c}
\bar{I}_{s N 1} \\
\bar{I}_{r N}
\end{array}\right]} \\
\bar{I}_{f}=\frac{\mu\left(\bar{V}_{s P}+\bar{V}_{s N}\right)-j \omega_{e} L_{l s f \alpha} \mu\left(\bar{I}_{s P}+\bar{I}_{s N}\right)}{\left(R_{f}+R_{s f}+j \omega_{e} L_{l s f}\right)}
\end{gathered}
$$

where $s$ is the slip of the IM defined as $s=\frac{\omega_{e}-\omega_{r}}{\omega_{e}}, \bar{I}_{S P}=\bar{I}_{s P 1}-\frac{1}{3} \mu \bar{I}_{f}$ and $\bar{I}_{s N}=\bar{I}_{s N 1}-$ $\frac{1}{3} \mu \bar{I}_{f}$

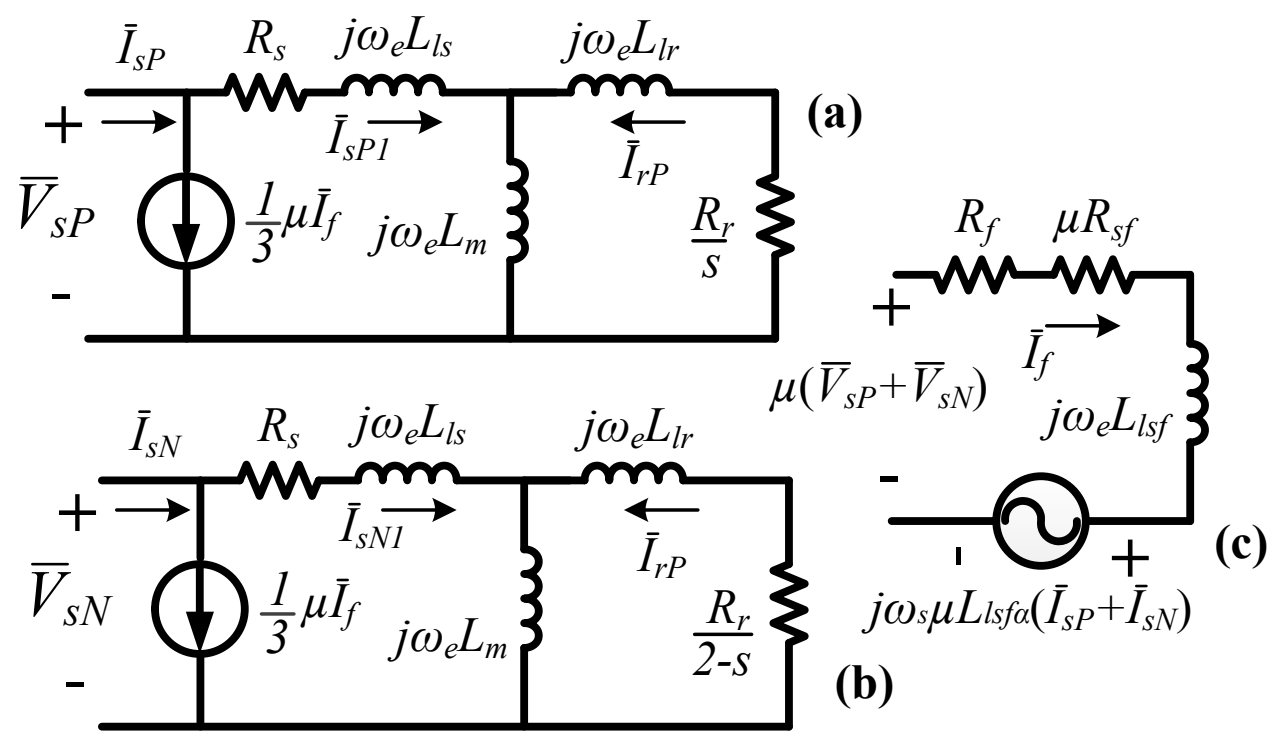

Figure 7.9 Sequence-components EC of an asymmetric IM (a) Positive sequence (b) Negative sequence (c) Faulty circuit. 
From the model in (7.43), the faulty IM EC is obtained, as in Figure 7.9. The admittance matrix for the sequence components of the IM are found from (7.43) as:

$$
\begin{aligned}
& \quad\left[\begin{array}{l}
\bar{I}_{S P} \\
\bar{I}_{S N}
\end{array}\right]=\left[\begin{array}{ll}
Y_{P P} & Y_{P N} \\
Y_{N P} & Y_{N N}
\end{array}\right]\left[\begin{array}{c}
\bar{V}_{S P} \\
\bar{V}_{S N}
\end{array}\right] \\
& \text { where } Y_{P N}=\frac{1 / 3 \mu^{2}}{R_{f}+\mu\left(R_{s f}+j \omega_{e} L_{l s f}\right)}, Y_{P P}=\frac{1}{R_{S}+j \omega_{e} L_{S}+\frac{\left(\omega_{e} L_{S}\right)^{2}}{\frac{R_{r}}{S}+j \omega_{e} L_{r}}}+Y_{P N}, Y_{N P}=Y_{P N} \text { and } \\
& Y_{N N}=\frac{1}{R_{S}+j \omega_{e} L_{S}+\frac{\left(\omega_{e} L_{S}\right)^{2}}{\frac{R_{r}}{2-s}+j \omega_{e} L_{r}}}+Y_{P N} .
\end{aligned}
$$

\subsection{Finite Element Model of Faulty IM}

The IM dealt with in this work has a stator winding, which is single layered, and each phase consists of two main coils $\left(U_{1}-U_{2}\right.$ and $U_{5}-U_{6}$ for the case of the phase " $a$ "). Each of these coils is divided into 3 sub-coils, as displayed in Figure 7.10(a), where $s_{1}, s_{12} \ldots s_{30}$ are the slot numbers, as in Figure 7.10(b). Each sub-coil has 85 turns, therefore the total number of turns per-phase is $N_{s a}=510$. For the purpose of creating the ITSC, the motor was dismantled and 8 center taps were performed in phase " $a$ " as depicted in Figure 7.10(a). The winding profile of the phase " $a$ " with one of the fault cases is shown in Figure 7.10(b). Four cases of the fault were performed with $\mu=42 / 510$ (8.2\%). The 4 cases were conducted in the following positions of the phase winding " $a$ ": $P_{1}: s_{1}-s_{12}, P_{2}: s_{2}-$ $s_{11}, P_{3}: s_{3}-s_{10}$ and $P_{4}: s_{19}-s_{30} . P_{y}$ indicates the position, where $y=\{1,2,3,4\}$. The fault resistance is imposed as $R_{f}=0.67 \Omega$. The complete geometry of the IM is depicted in Figure 7.11(a) and (b) with the dimensions in $\mathrm{mm}$. The stator consists of an iron core M19 USS with 36 slots numbered, as in Figure 7.10(b). The rotor bars are made of Aluminum $3.8 \mathrm{e} 7 \mathrm{Siemens} / \mathrm{m}$ and there are 44 of them. The air-gap is $0.31 \mathrm{~mm}$. 


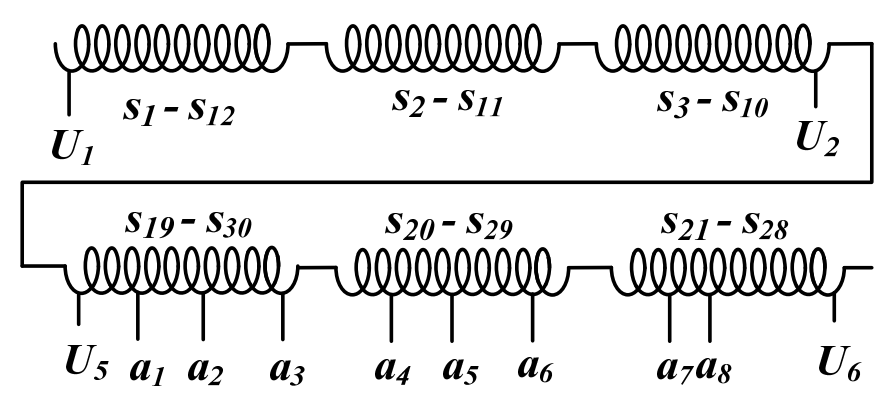

(a)

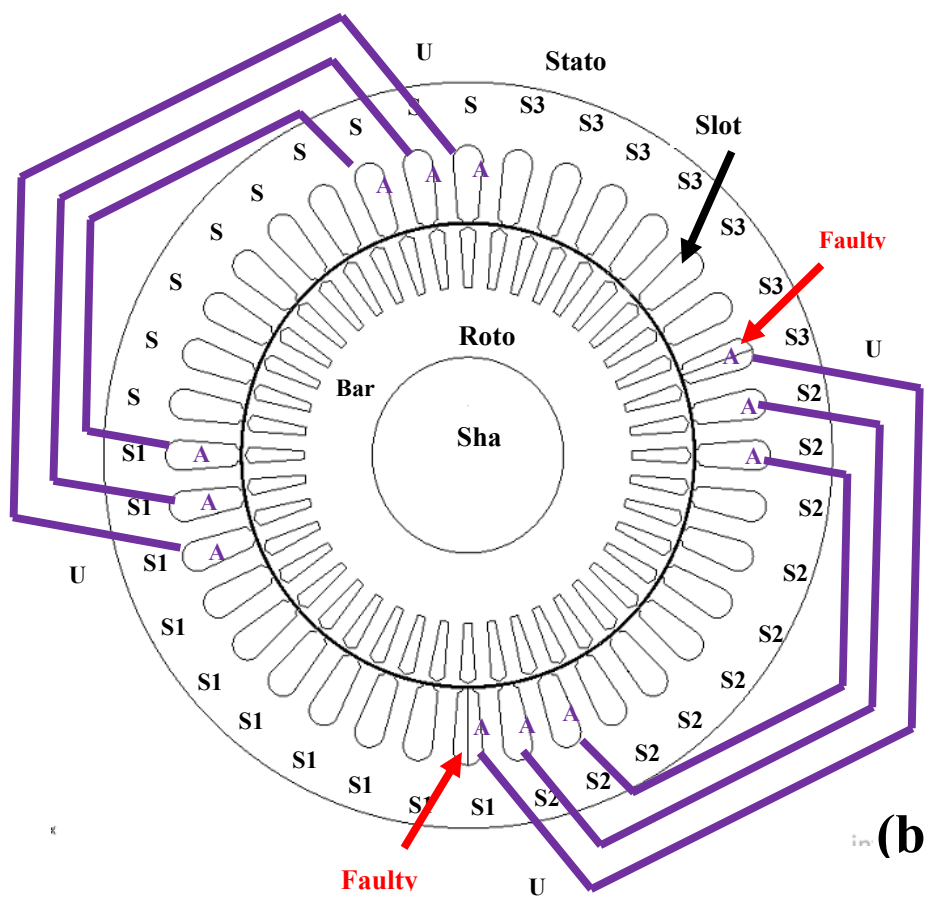

Figure 7.10 (a) Center taps in the phase " $a$ " of the IM for the ITSC creation. (b) Phase " $a$ " single layer stator winding diagram

The FEA is performed by strong coupling simulation between FE and circuit analysis. The FE is used to model the phase " $a$ " as the combination of 7 coils ( 6 healthy and 1 faulty windings) all connected in series to form the phase. The faulty circuit part is connected as a step-down autotransformer configuration, where $R_{f}$ is added, as in Figure 7.12.

In Figure 7.13, Figure 7.14, Figure 7.15 and Figure 7.16, the response of the IM for the 4 locations of the fault can be observed. Five IMs' variables are investigated: mechanical speed (Figure 7.13(a)), electromagnetic torque (Figure 7.13 (b)), faulty current (Figure 
7.14), the current in the winding (Figure 7.15), the 2D distribution of the magnetic field density's magnitude and flux lines (Figure 7.16). Notice that the electrical variables respond similarly with respect to the location of the fault. However, a zoom view of each variable indicates that the fault location is subtly affecting each of them. In Figure 7.13(a), a zoom of the speed reveals that in the transient state, still there is a small different acceleration for the 4 cases. The 4 cases reach to the rated speed $(187.87 \mathrm{rad} / \mathrm{s}=1794 \mathrm{rpm})$. The electromagnetic torque in Figure 7.13(b) is the one that has more instantaneous difference, however, in average all of them are close to zero (in steady state). The reason for this discrepancy is due to the MMF shape difference in the 4 cases. Distinct locations of the fault will create different MMFs with different harmonic components, but same general behavior. From the zoom of Figure 7.13(b), a double-line frequency oscillation can be noticed. This torque variation comes from the fact that when the ITSC happens, the current in the fault winding ( $i_{s a 2}$ from Figure 7.6(a)) reverses its direction (opposite to $i_{s a}$ ), producing a counter rotating magnetic field (negative sequence), deforming the shape of the original MMF and introducing a negative sequence component. Figure 7.14(b) and Figure 7.15(b) show the steady state currents in the fault and in the winding. Notice a small peak difference among all of them. 


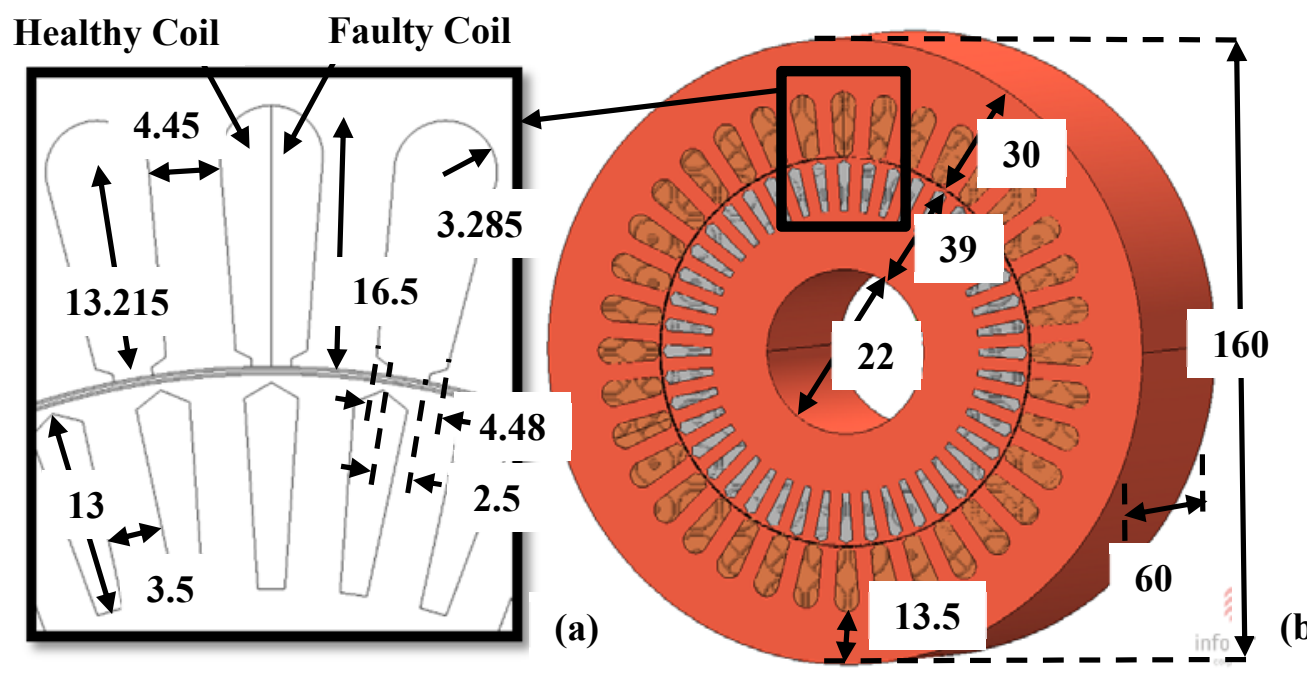

Figure 7.11 (a) IM internal geometry, slots, rotor bars and placement of the ITSC. (b) 2D IM external geometry for the FEA. The unit's dimensions are millimeters (mm).

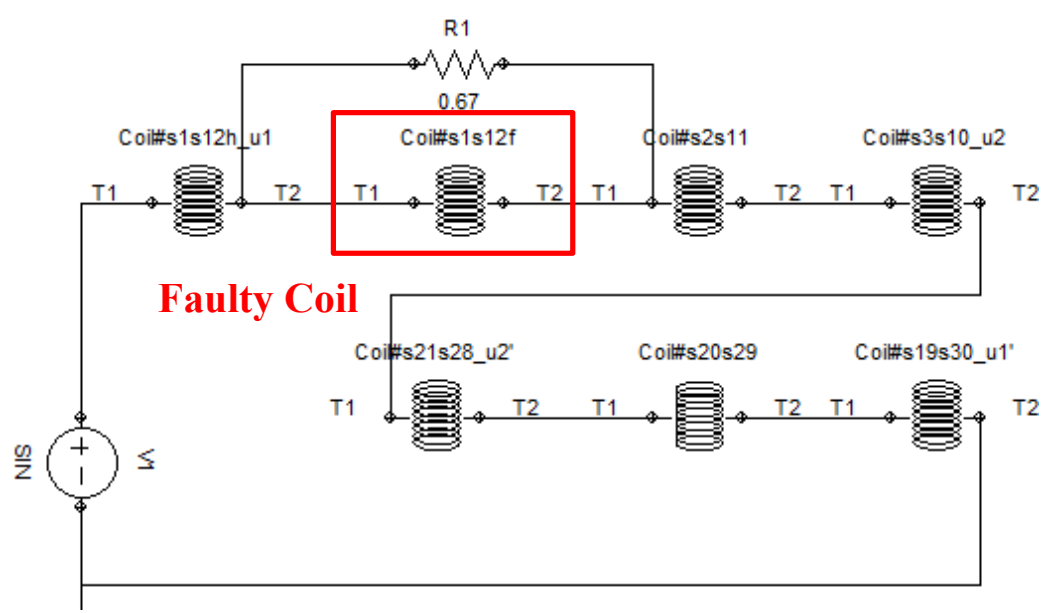

Figure 7.12 Phase " $a$ " circuit used in the FE co-simulation. 


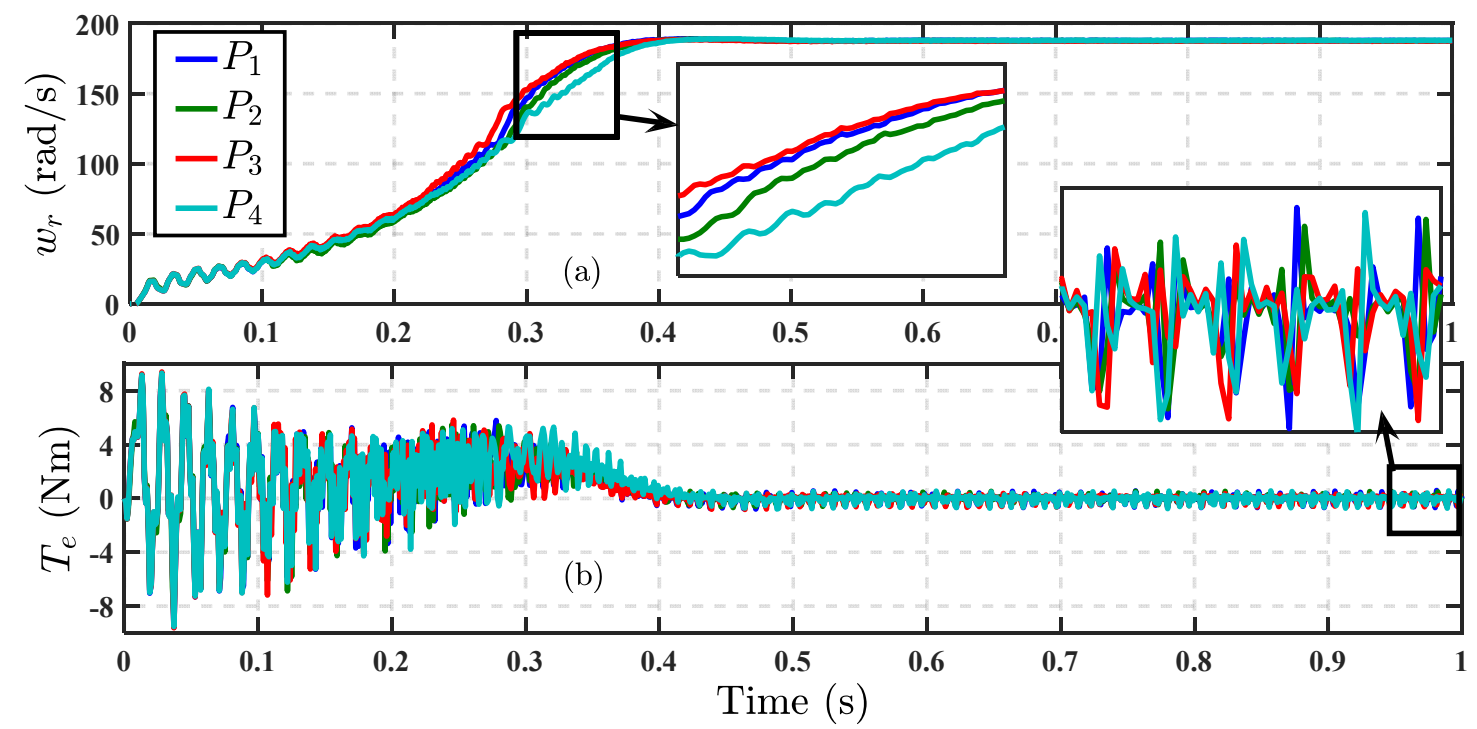

Figure 7.13 Four ITSC locations in the stator winding phase "a". Position $1\left(P_{1}\right)$ to $4\left(P_{4}\right)$ : (a) speed (b) electromagnetic torque.
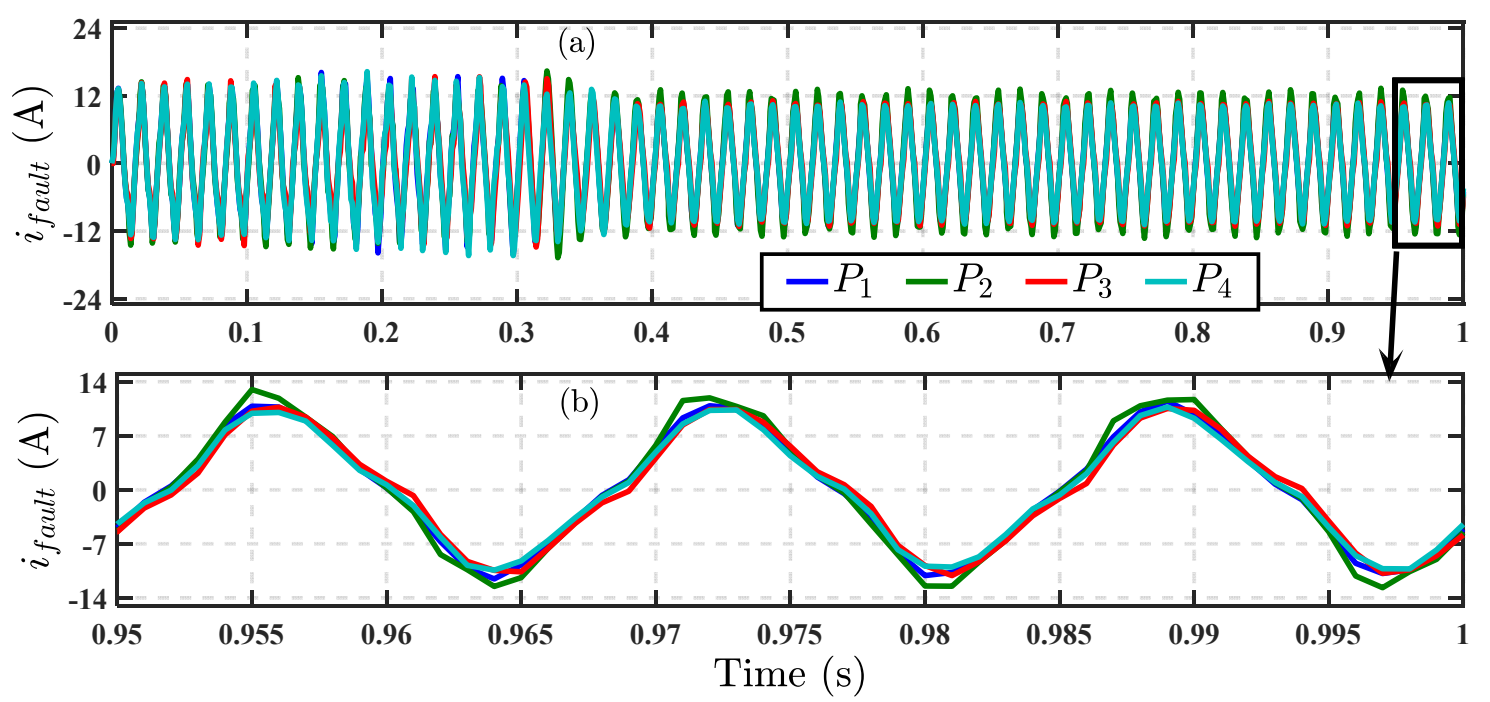

Figure 7.14 Four ITSC locations. Position $1\left(P_{1}\right)$ to $4\left(P_{4}\right)$ : (a) faulty current (b) zoom

From Figure 7.16, notice the 2D distribution for one case of study $\left(P_{1}\right)$. The other 3 cases are quite similar, which match with the previous results. It can be concluded, from these results, that the fault location impact on the IM electrical parameters, of the mathematical modeling, can be neglected. Therefore, in the next section a comparative analysis between 
the mathematical model (where the location of the ITSC is not in any specific position) and the experimental tests is presented.
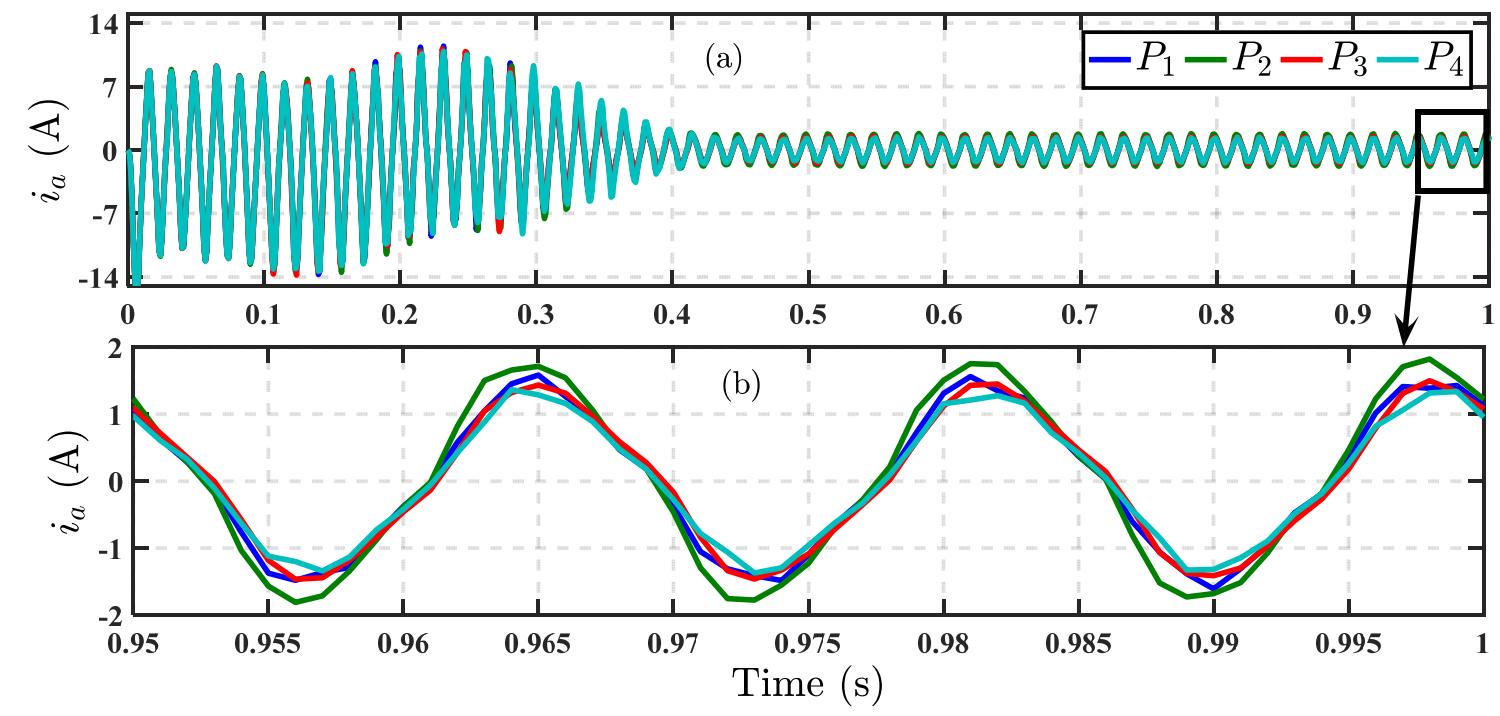

Figure 7.15 Four ITSC locations. Position $\left(P_{1}\right)$ to $\left(P_{4}\right)$ : (a) phase current and (b) zoom.

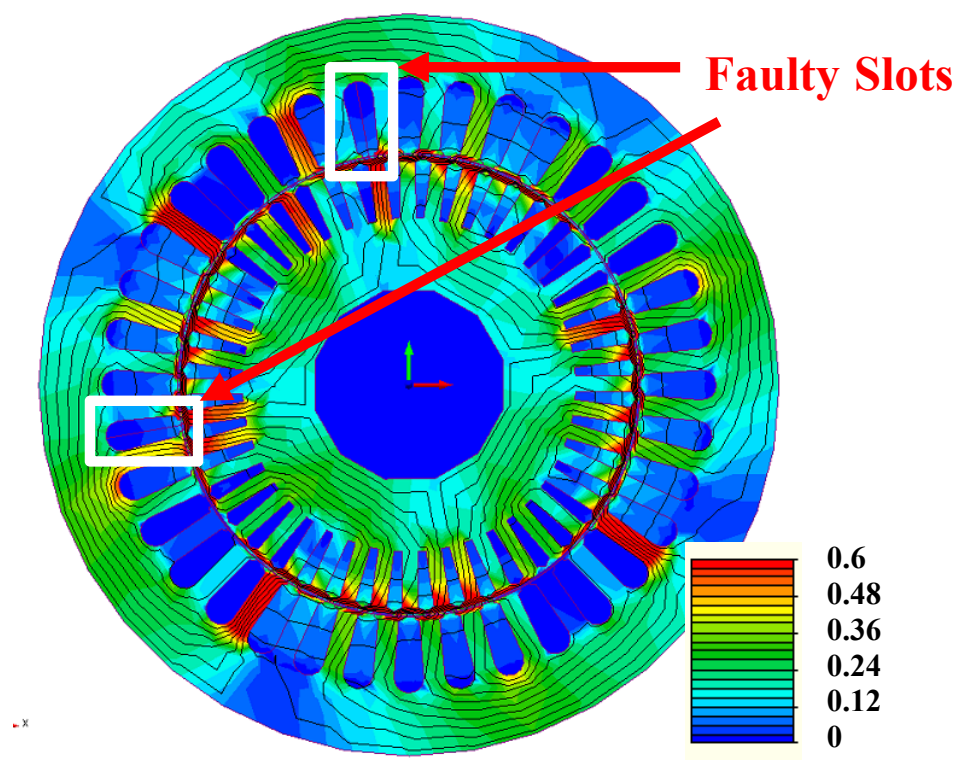

Figure 7.162D distribution of $|\vec{B}|$ and flux lines for ITSC location $\left(P_{1}\right)$ at $\mathrm{t}=0.117 \mathrm{sec}$. 


\subsection{Simulation and Experimental Results for the IM model with ITSC}

The simulation and experimental tests are divided in two sections: results for the general case of ITSC in one-coil and for the three-phase IM. The simulations for the two cases are performed in the MatLab environment.

\subsubsection{ITSC in One-Coil}

In this section, two simulation results are presented and compared: mathematical and physics-based model (PBM). A correct PBM requires an accurate device's geometry and material properties. In this sense, the magnetization $(\mathrm{B}-\mathrm{H})$ curve for the ferromagnetic core is required. Following the procedure presented in [148], the hysteresis loops for seven operative points are shown in Figure 7.17(a). Taking the maximum values of the magnetic flux density's magnitude $\left(B_{\max }\right)$ and the maximum magnitude of the of magnetic field intensity $\left(H_{\max }\right)$ for each hysteresis loop, the magnetization curve can be represented as in Figure 7.17(b). The experimental setup for the Epstein frame was built with $N_{1}=76$ and $N_{2}=14$ turns, as shown in Figure 7.19(b).

The PBM of the ITSC in one coil is based on FEA and performed in Infolytica Magnet software. The geometry and the dimensions of the transformer are presented in Figure 7.18(b). The magnetizing curve of the core is set as Figure 7.17(b). The conductivity of the cooper and the iron are corrected based on the comparison between experimental measurements and FE for the OCT and SCT when $\mu=0.33$. The core losses were fitted heuristically for the hysteresis and eddy phenomena with (7.45).

$$
P_{\text {loss }}=k_{h} f^{\alpha} B^{\beta}+k_{e}(s f B)^{2}
$$


where $P_{\text {loss }}$ is the total power loss in the core by the additive effects of hysteresis and eddy currents, $k_{h}$ and $k_{e}$ are the coefficients that depend on the geometry of the core for hysteresis and eddy currents, respectively, and sometimes used as fitting coefficients also, $\alpha$ and $\beta$ are the fitting coefficients, $f$ is the operative frequency, $B$ is the peak magnitude of the magnetic field density and $s$ is the lamination ratio thickness.
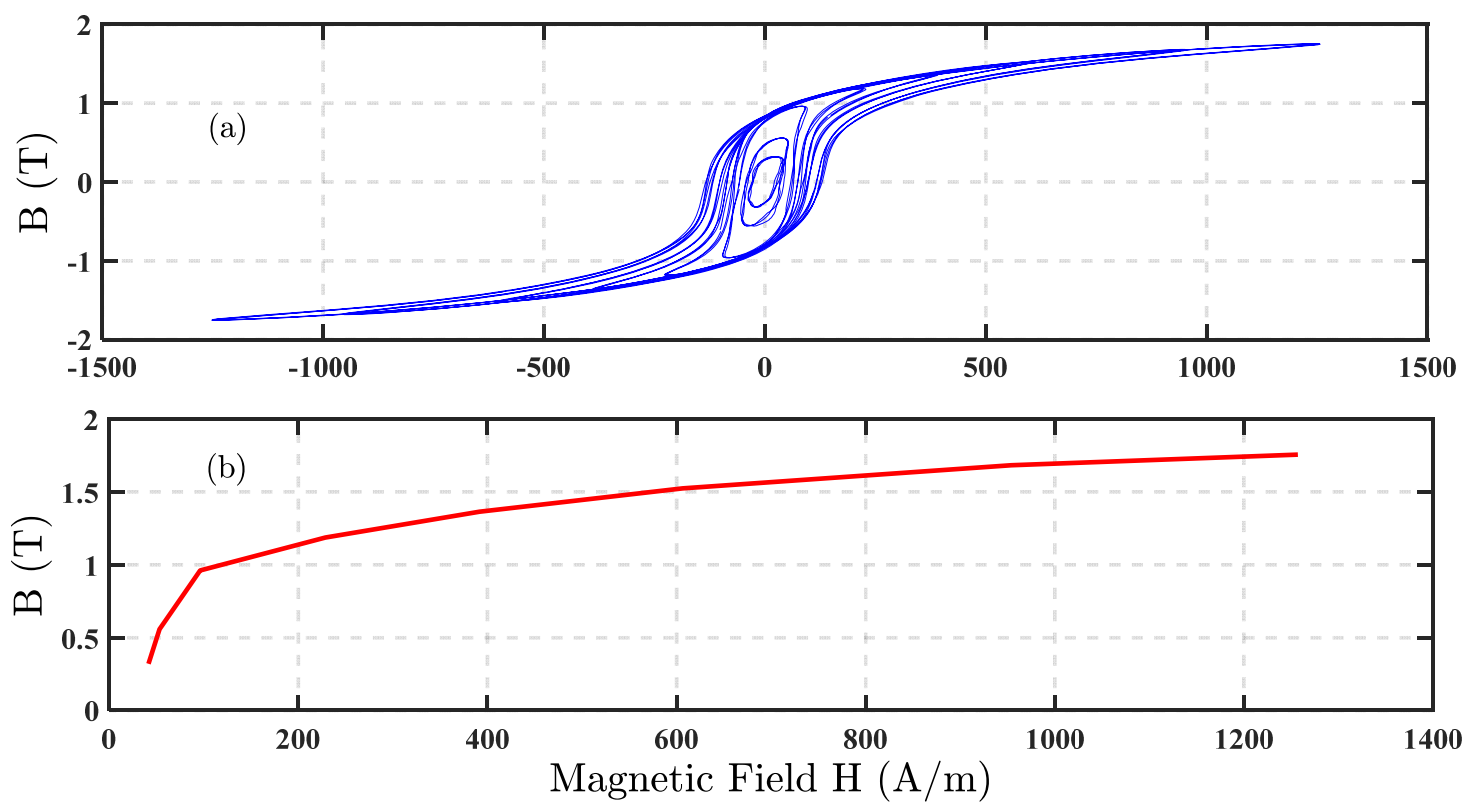

Figure 7.17 Experimental measures for the ferromagnetic core using Epstein frame (a) Hysteresis loops (b) Magnetization curve.

The FE simulation is performed by strong coupling simulation between FE and circuit analysis. The FE is used to model the ITSC as the combination of two coils (the healthy and faulty windings). The circuit part is where these two coils are connected as a stepdown autotransformer configuration and the supply voltage, and $R_{f}$ are added, as in Figure 7.18(a).

The tests are conducted by the setup shown in Figure 7.19(a). The device under test is a single-phase transformer connected as one-coil. During the fault, it behaves as a step- 
down autotransformer, as discussed before. The center taps of the transformer are used to create the ITSC cases of study, as shown in Figure 7.20 and Table 7-1. The transformer model T-100-3A is composed of three single-phase transformers with the connections and the side view of the transformer, as shown in Figure 7.19(a). The sensor board is based on LEM transducers and a dSpace 1104 is used for the data acquisition. A three-phase variac is used as an AC supply.

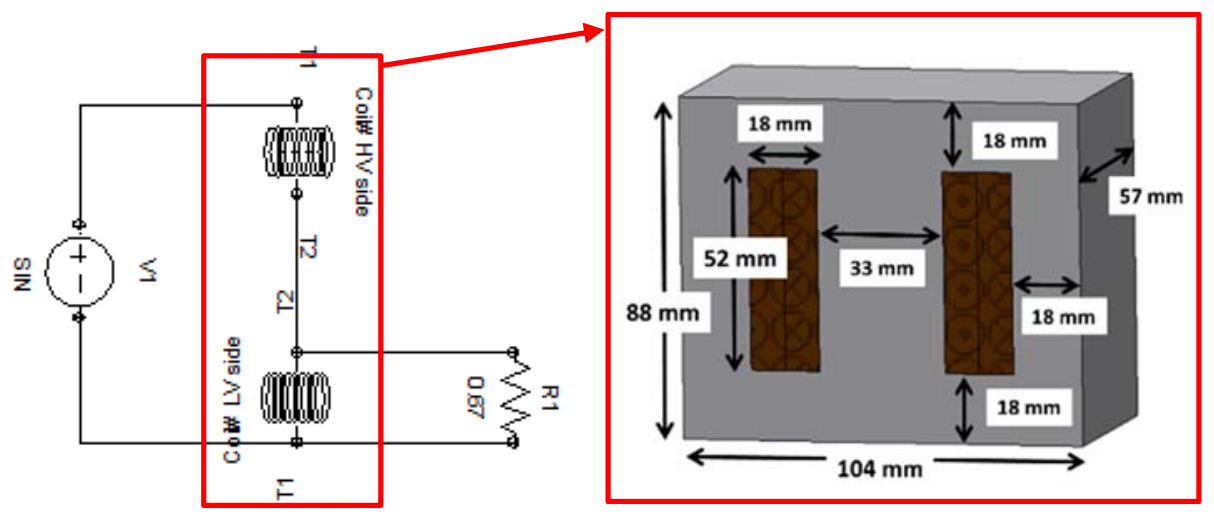

Figure 7.18 FEA simulation (a) Circuit diagram (b) Geometry and dimensions

Table 7-1 Test Cases for the ITSC in One-Coil

\begin{tabular}{|c|c|c|c|c|c|c|c|c|}
\hline ITSC & $\boldsymbol{N}_{\mathbf{2}}$ & $\boldsymbol{\mu}$ & $\mathbf{I T S C}$ & $\boldsymbol{N}_{\mathbf{2}}$ & $\boldsymbol{\mu}$ & ITSC & $\boldsymbol{N}_{\mathbf{2}}$ & $\boldsymbol{\mu}$ \\
\hline$X_{1}-X_{7}$ & 296 & 0.33 & $X_{3}-X_{6}$ & 196 & 0.216 & $X_{3}-X_{4}$ & 88 & 0.099 \\
\hline$X_{2}-X_{7}$ & 266 & 0.296 & $X_{1}-X_{4}$ & 148 & 0.167 & $X_{1}-X_{3}$ & 60 & 0.068 \\
\hline$X_{1}-X_{6}$ & 256 & 0.288 & $X_{2}-X_{4}$ & 118 & 0.133 & $X_{6}-X_{7}$ & 40 & 0.045 \\
\hline$X_{3}-X_{7}$ & 236 & 0.266 & $X_{4}-X_{6}$ & 108 & 0.122 & $X_{2}-X_{3}$ & 30 & 0.034 \\
\hline$X_{2}-X_{6}$ & 226 & 0.255 & & & & & & \\
\hline
\end{tabular}




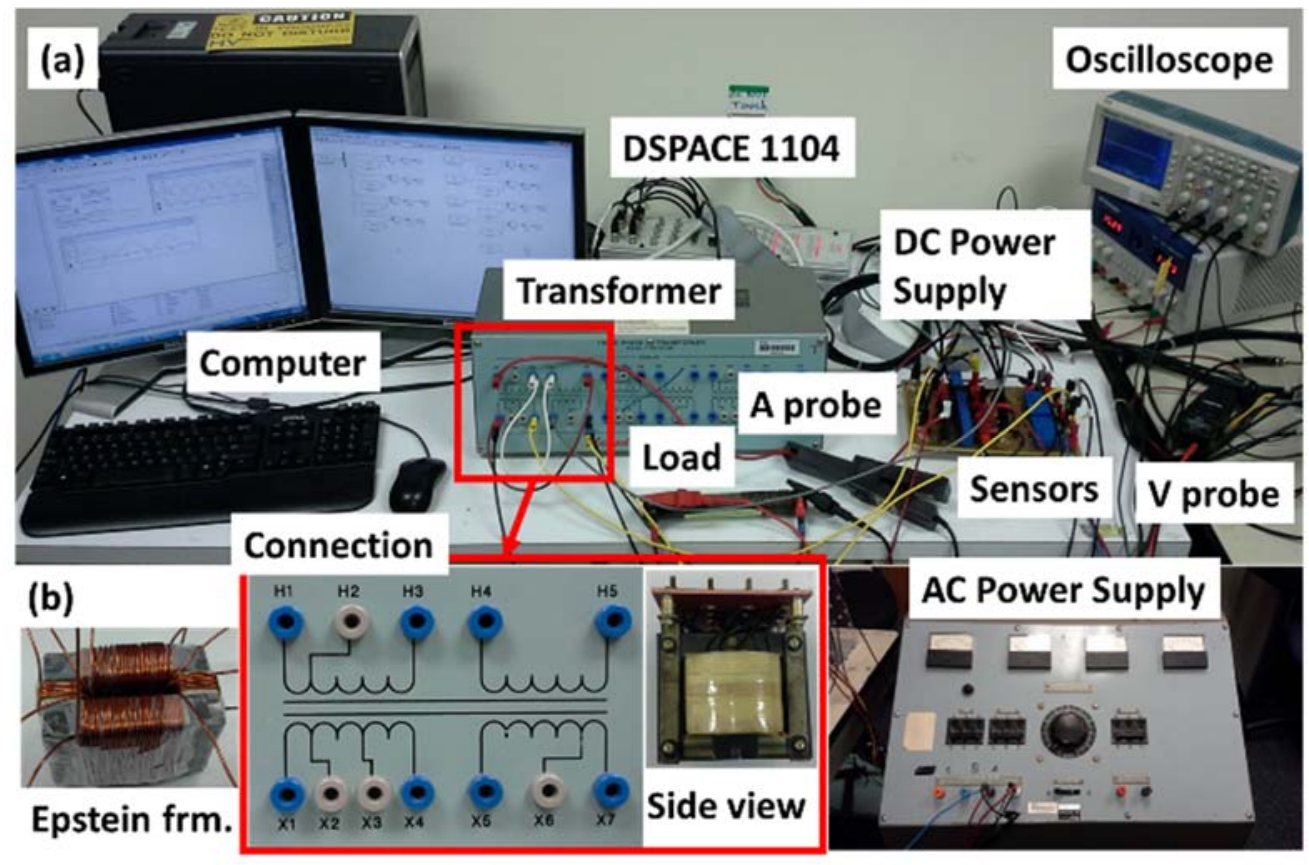

Figure 7.19 (a) Experimental setup for ITSC in one-coil (b) Epstein frame.

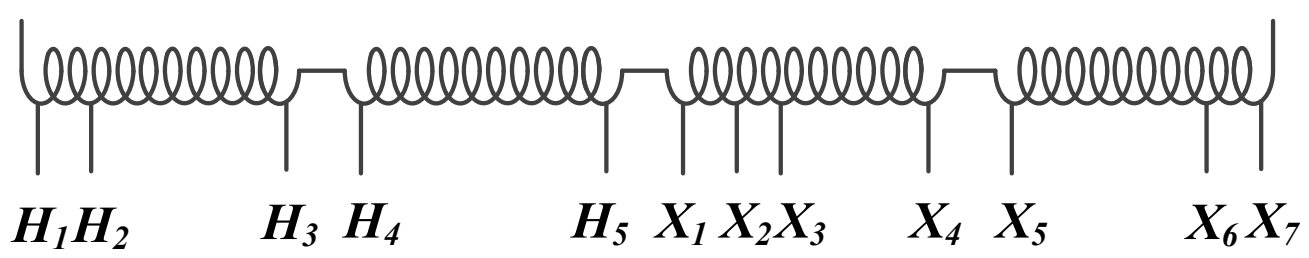

Figure 7.20 ITSC cases of study in one coil, equivalent to the center taps from transformer.

Figure 7.21 presents the faulty current $\left(i_{f}\right)$ as a function of the fault severity factor for the ITSC in one coil. Thirteen cases of ITSC are studied, as shown in Table 7-1. The simulation results are based on the circuit in Figure 7.5 and SS representation (7.3), the physics-based simulation is based on FEA and the experimental results are based on Figure 7.19(a). The input supply voltage is $12 \mathrm{~V}$ to avoid exceeding the nominal current of the transformer and to be in the linear region of the ferromagnetic core. The supply voltage 
was applied between $H_{1}-X_{7}$ terminals. The fault resistance is $R_{f}=0.67 \Omega$. The simulation requires the model parameters for the 13 fault cases. Initially, one case is selected and its parameters are calculated by means of open circuit (OCT) and short circuit tests (SCT). In Table 7-2, the parameters for the case of severity factor $\mu=0.33$, where the ITSC is performed between $X_{1}-X_{7}$ terminals, are presented. Thus, the healthy resistance and inductance are discovered, and using the equations in sub-section 7.2.1.1 the remaining parameters for the other 12 ITSC cases are determined.

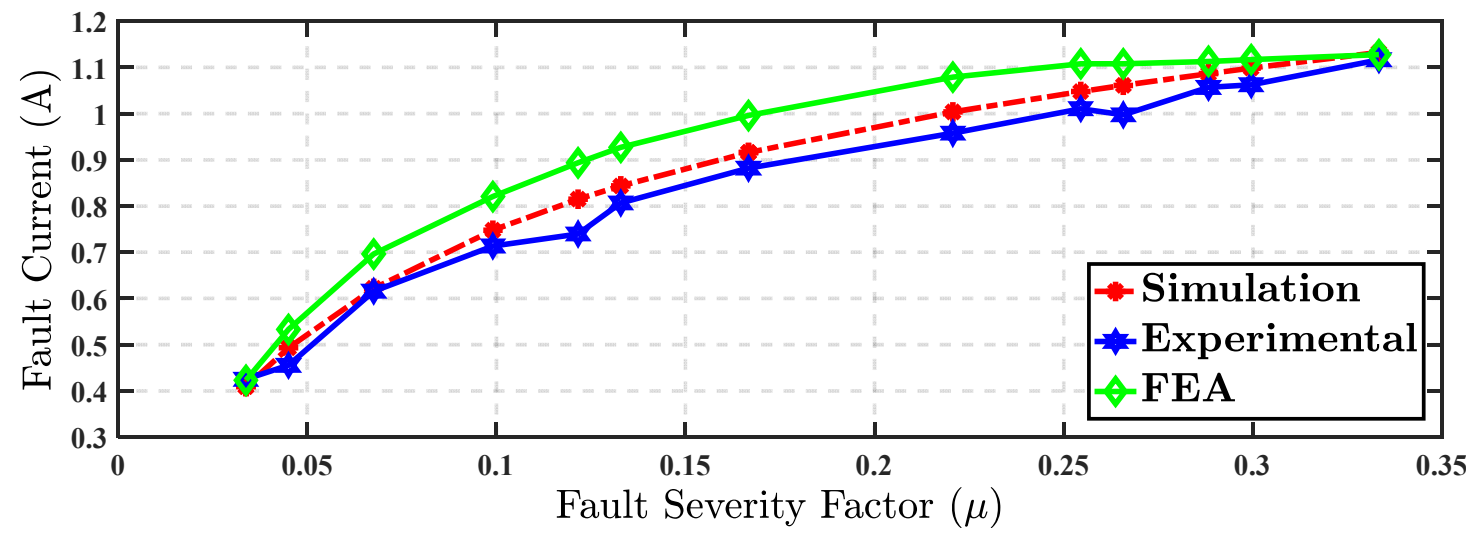

Figure 7.21 Faulty current versus severity factor in the ITSC for one-coil case.

Table 7-2 Parameters for $\mu=0.33$ ITSC at $X_{1}-X_{7}$ for One Coil and Nameplate Rating

\begin{tabular}{|c|c|c|c|c|c|}
\hline Par. & Value & Par. & Value & Rating & Value \\
\hline$L_{1}$ & $47.7544 \mathrm{H}$ & $L_{m 2}$ & $11.9375 \mathrm{H}$ & $S_{r}$ & $120 \mathrm{VA}$ \\
\hline$L_{2}$ & $11.9386 \mathrm{H}$ & $R_{1}$ & $12.8 \Omega$ & $V_{1 r}$ & $120 / 240 \mathrm{~V}$ \\
\hline$M$ & $23.8751 \mathrm{H}$ & $R_{2}$ & $3.2 \Omega$ & $V_{2 r}$ & $60 / 120 \mathrm{~V}$ \\
\hline$L_{l 1}$ & $4.2310^{-3} \mathrm{H}$ & $R_{h}$ & $16 \Omega$ & $I_{r}$ & $1 \mathrm{~A}$ \\
\hline$L_{l 2}$ & $1.0510^{-3} \mathrm{H}$ & $L_{h}$ & $5.2810^{-3} \mathrm{H}$ & $N_{\text {total }}$ & 888 \\
\hline$L_{m 1}$ & $47.7502 \mathrm{H}$ & $L_{m}$ & $106.3716 \mathrm{H}$ & $R_{m}$ & $13.8 \mathrm{k} \Omega$ \\
\hline
\end{tabular}


From Figure 7.21, it can be seen that the simulation and the experimental results are correlated. The shape of both curves have the same behavior, however, the FEA results shows some deviations with respect to the experimental ones. These deviations are the effect of the difficulty to obtain accurate information about the properties of the core material. Even when the magnetization curve was obtained, the core losses were fitted heuristically. Also additional excess core losses were not considered.
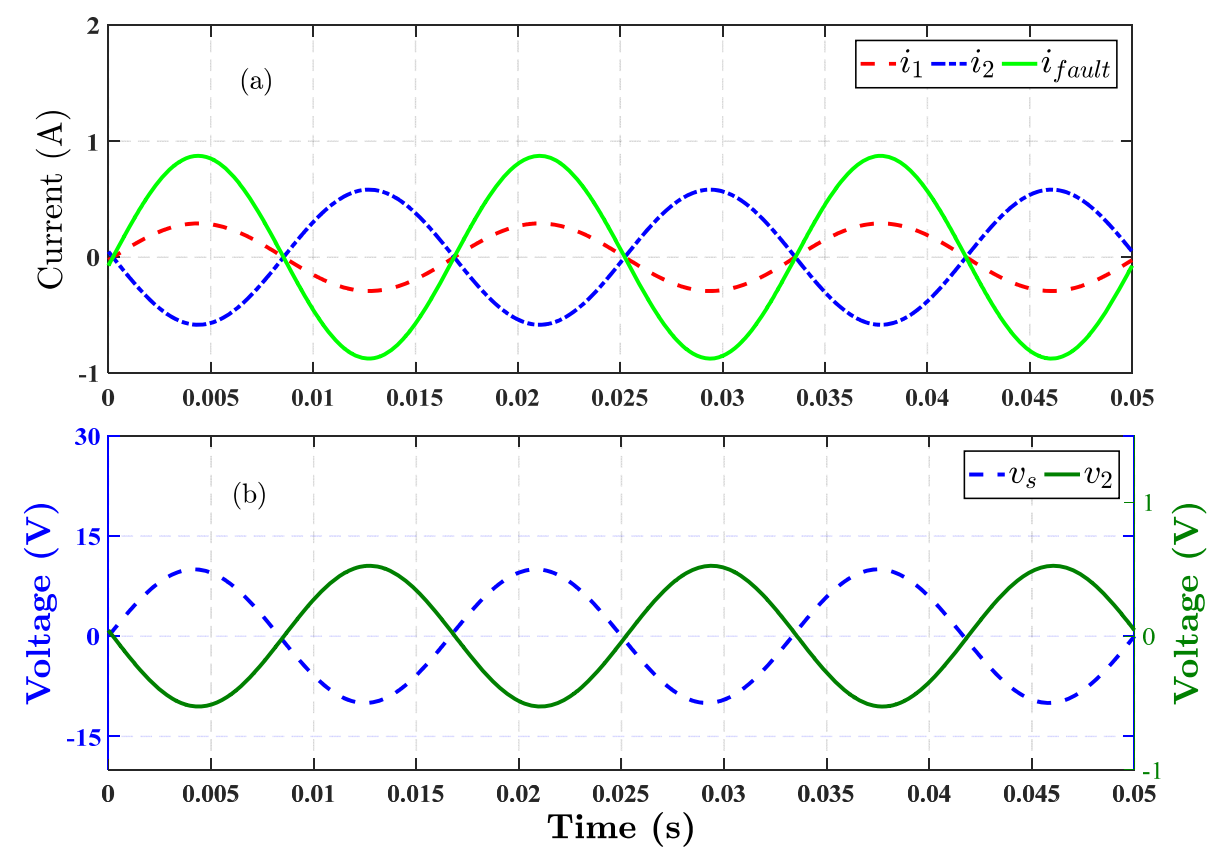

Figure 7.22 Simulation of the ITSC case of $\mu=0.33$ in one-coil. (a) current in the healthy winding, in the faulty winding and the faulty current in the short-circuit. (b) Supply and faulty voltage.

Figure 7.22 and Figure 7.23 present the simulation and experimental results of the ITSC case for a fault severity of $\mu=0.33$ in one-coil case. In Figure 7.22(a) and Figure 7.23(a), the current in the healthy part of the winding $\left(i_{1}\right)$, the current in the faulty part of the winding $\left(i_{2}\right)$ and the faulty current in the short-circuit path can be observed. The current in 
the faulty part of the winding $\left(i_{2}\right)$ was measured, as indicated in Figure 7.2 and Figure 7.5. Notice that this current is $180^{\circ}$ phase-shift with respect to the current in the healthy winding $\left(i_{1}\right)$. Figure 7.22(b) and Figure 7.23(b) show the supply voltage and the induced voltage $\left(v_{2}\right)$ in the faulty winding.
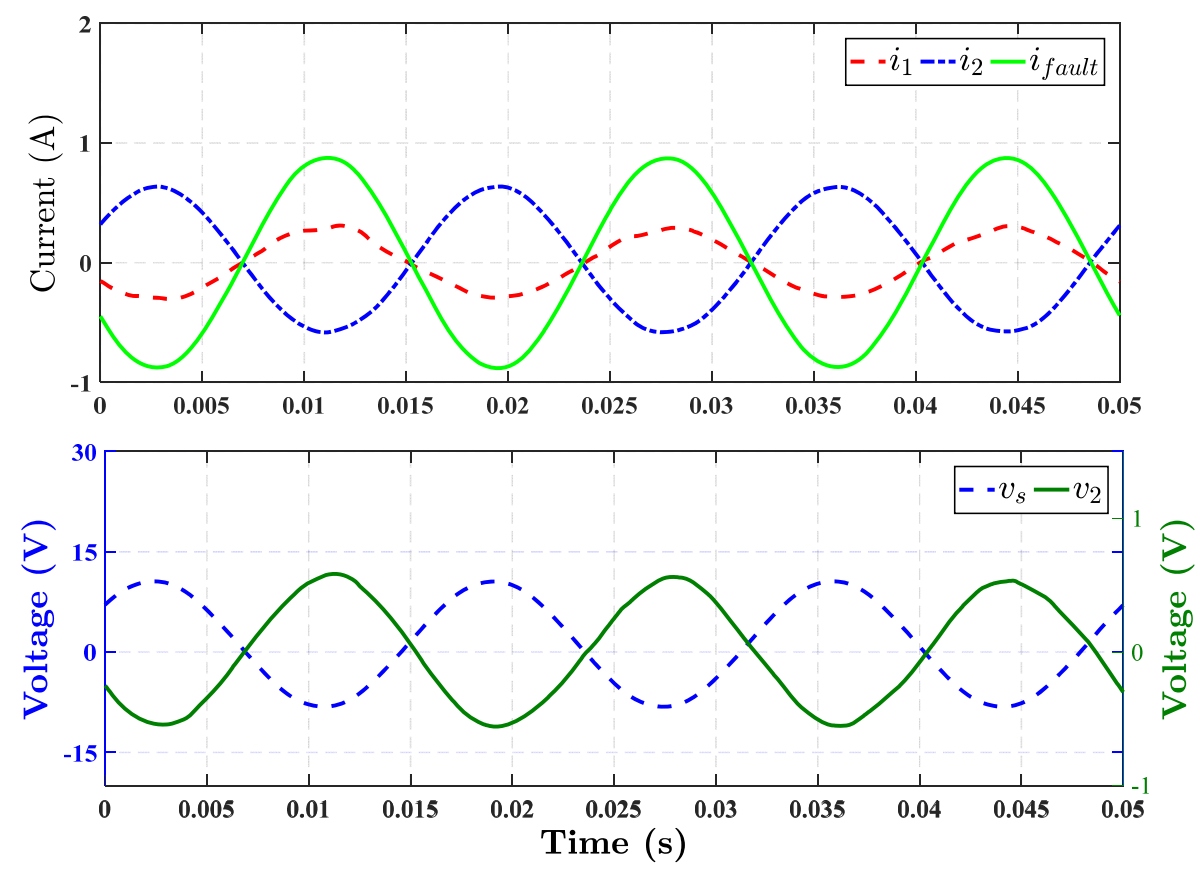

Figure 7.23 Experimental measures of the ITSC case of $\mu=0.33$ in one-coil. (a) current in the healthy winding, in the faulty winding and the faulty current in the short-circuit. (b) Supply and faulty voltage.

\subsubsection{ITSC in Three-Phase IM: Case of Study}

The simulation and experimental test are performed for a 1 HP IM Weg. The nameplate ratings of the motor are shown in Table 7-3. The IM parameters were estimated using the IM transient response at no load condition with an optimization technique over the vector impedance of the IM following the procedure in [122]. The IM parameters are exposed in Table 7-3. 
Table 7-3 Parameters and Nameplate Rating for the IM Weg.

\begin{tabular}{cc|cc|cc|cc}
\hline \hline Param. & Value & Param. & Value & Rating & Value & Rating & Value \\
\hline$L_{l s}$ & $32.110^{-3} \mathrm{H}$ & $R_{S}$ & $9.292 \Omega$ & $P_{r}$ & $746 \mathrm{~W}$ & $P F$ & 0.8 \\
$L_{l r}$ & $37.010^{-3} \mathrm{H}$ & $R_{r}$ & $7.231 \Omega$ & $V_{r}$ & $460 \mathrm{~V}$ & Poles & 2 \\
$L_{m}$ & $0.895 \mathrm{H}$ & $J_{m}$ & $0.053 \mathrm{Kgm}^{2}$ & $I_{r}$ & $1.47 \mathrm{~A}$ & $n_{r}$ & $1730 \mathrm{rpm}$ \\
\hline \hline
\end{tabular}

The IM stator winding is single layered and each phase consists of two main coils $\left(U_{1}-\right.$ $U_{2}$ and $U_{5}-U_{6}$ for the case of the phase "a"). Each of these coils is divided into 3 subcoils, as displayed in Figure 7.10(a), where the variables $s_{1}, s_{12} \ldots s_{30}$ represent the slot numbers, as in Figure 7.10(b). Each sub-coil has 85 turns, therefore the total number of turns per-phase is $N_{a s}=510$. For the purpose of creating the ITSC, the motor was dismantled and 8 center taps were performed in the IM phase " $a$," as depicted in Figure 7.10(a). Sixteen cases of ITSC were studied and tested. They are shown in Table 7-4.

For the experimental test, a platform exhibited in Figure 7.24 is used. The IM shaft is mechanically coupled to a dc permanent magnet (PM) machine of $250 \mathrm{~W}$ and $42 \mathrm{~V}$ dc. The dc PM machine is acting as a generator with variable load connected to the armature to emulate the mechanical loading conditions. The sensor board is based on LEM voltage and current transducers and a dSpace 1104 is used for the data acquisition. A three-phase variac is used as ac supply. The motor angular speed is measured by an encoder of $1000 \mathrm{ppr}$ coupled to the PM machine. The simulation and experimental results are divided into two parts: transient and permanent regime. 


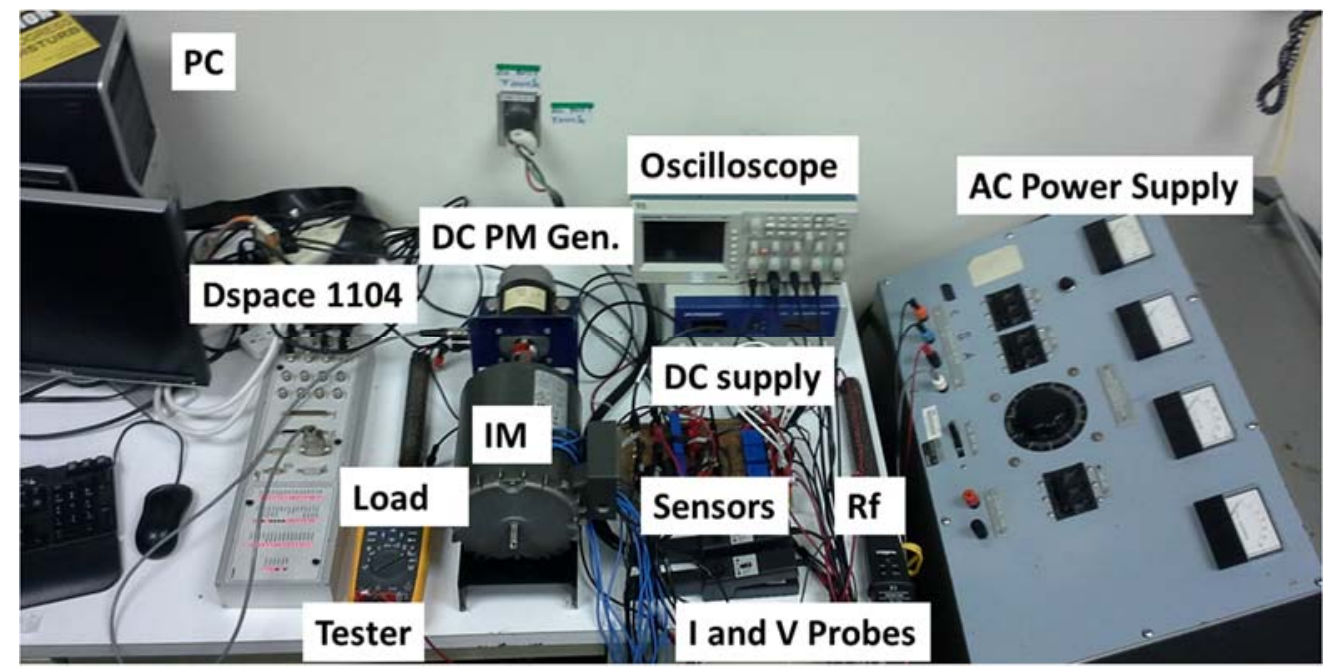

Figure 7.24 Experimental setup for acquisition with a dSpace 1104.

Table 7-4 ITSC Test Cases for the Faulty IM

\begin{tabular}{ccc|ccc|ccc}
\hline \hline ITSC & $\boldsymbol{N}_{\boldsymbol{a s} \mathbf{2}}$ & $\boldsymbol{\mu ( \% )}$ & ITSC & $\boldsymbol{N}_{\boldsymbol{a s} \mathbf{2}}$ & $\boldsymbol{\mu ( \% )}$ & ITSC & $\boldsymbol{N}_{\boldsymbol{a s} \mathbf{2}}$ & $\boldsymbol{\mu ( \% )}$ \\
\hline$a_{1}-a_{2}$ & 14 & 2.8 & $U_{5}-a_{4}$ & 85 & 16.1 & $a_{2}-a_{8}$ & 174 & 34.3 \\
$U_{5}-a_{2}$ & 19 & 3.7 & $a_{2}-a_{6}$ & 96 & 18.3 & $U_{5}-a_{7}$ & 187 & 36.7 \\
$a_{1}-a_{3}$ & 27 & 5.4 & $a_{1}-a_{6}$ & 108 & 21.1 & $U_{5}-a_{8}$ & 194 & 38.2 \\
$U_{5}-a_{3}$ & 32 & 6.4 & $U_{5}-a_{6}$ & 113 & 22.2 & $U_{5}-U_{1}$ & 255 & 50 \\
$a_{3}-a_{5}$ & 50 & 9.8 & $a_{3}-a_{7}$ & 152 & 29.9 & & & \\
$a_{2}-a_{4}$ & 64 & 12.3 & $a_{2}-a_{7}$ & 167 & 32.8 & & & \\
\hline \hline
\end{tabular}

\subsubsection{Transient Regime of Faulty IM}

A comparative analysis for the transient performance of the faulty IM between simulation and experimental results is presented. The study includes the IM starting at no load and loaded condition ( $1 \mathrm{Nm}$ ), from stand still until reaching the maximum speed with $120 V_{r m s}$ (balanced three-phase voltage line-to-neutral) and rated frequency. The investigation is performed at a voltage supply lower than half of the nominal voltage to 
avoid IM overheating and being damaged. The entire time of the test is $1.5 \mathrm{sec}$, however, for purposes of better plotting, $0.7 \mathrm{sec}$ is shown in some curves. The simulation integration step time is $0.1 \mathrm{msec}$ and the experimental acquisition frequency is $5 \mathrm{kHz}$.

\subsection{No Load Condition:}

A fault severity factor of $3.78 \%$ and fault resistance of $1 \Omega$ are implanted. The electrical variables for this comparison are: stator current space-vector (Figure 7.25 and Figure 7.26), faulty leakage current (Figure 7.27), locus of the stator flux-linkage space-vector (Figure 7.28), the rotor angular speed (Figure 7.29) and electromagnetic torque (Figure 7.30).

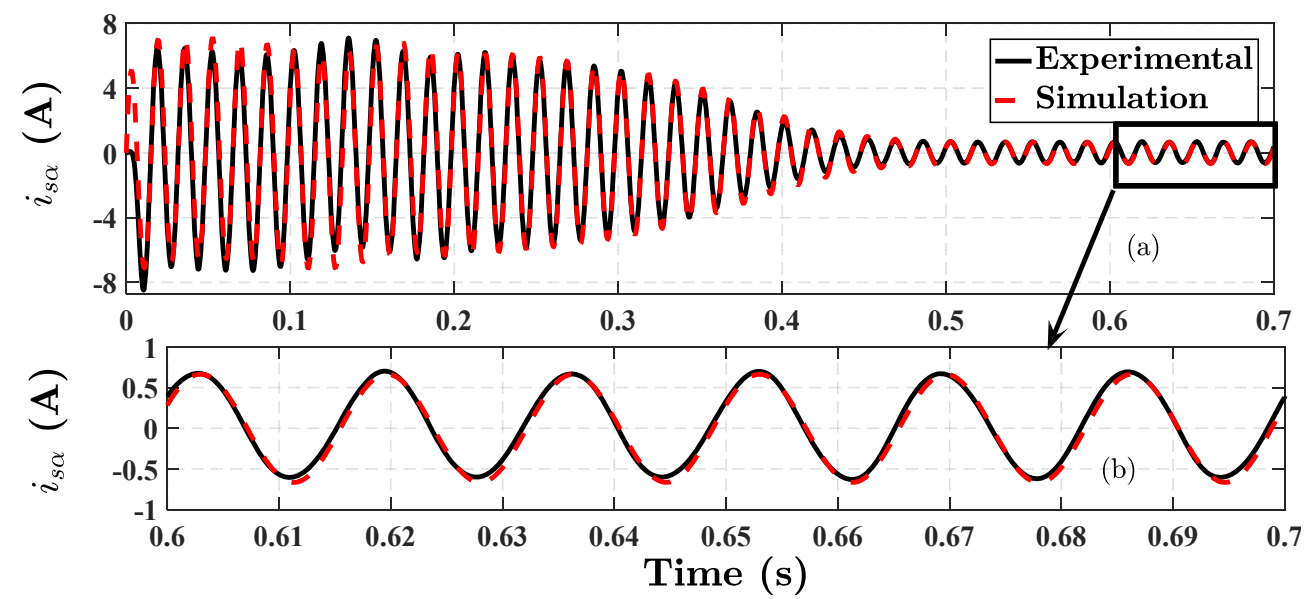

Figure 7.25 Real component of stator current for faulty IM (a) $i_{s \alpha}$ (b) Zoomed $i_{s \alpha}$.

From Figure 7.25(a) to Figure 7.30(a), the simulation and experimental results are fully correlated during the transient and the steady state time. From the zoomed analysis of the steady state in Figure 7.25(b) to Figure 7.27(b) and Figure 7.29(c), high correlation and matching can be observed. Figure 7.27(a) shows the transient analysis for the faulty leakage current, which presents a slight difference with the experimental results at the starting, although, the steady state demonstrates good agreement. From Figure 7.28, the locus of $\lambda_{s}$ shows high correlation. 


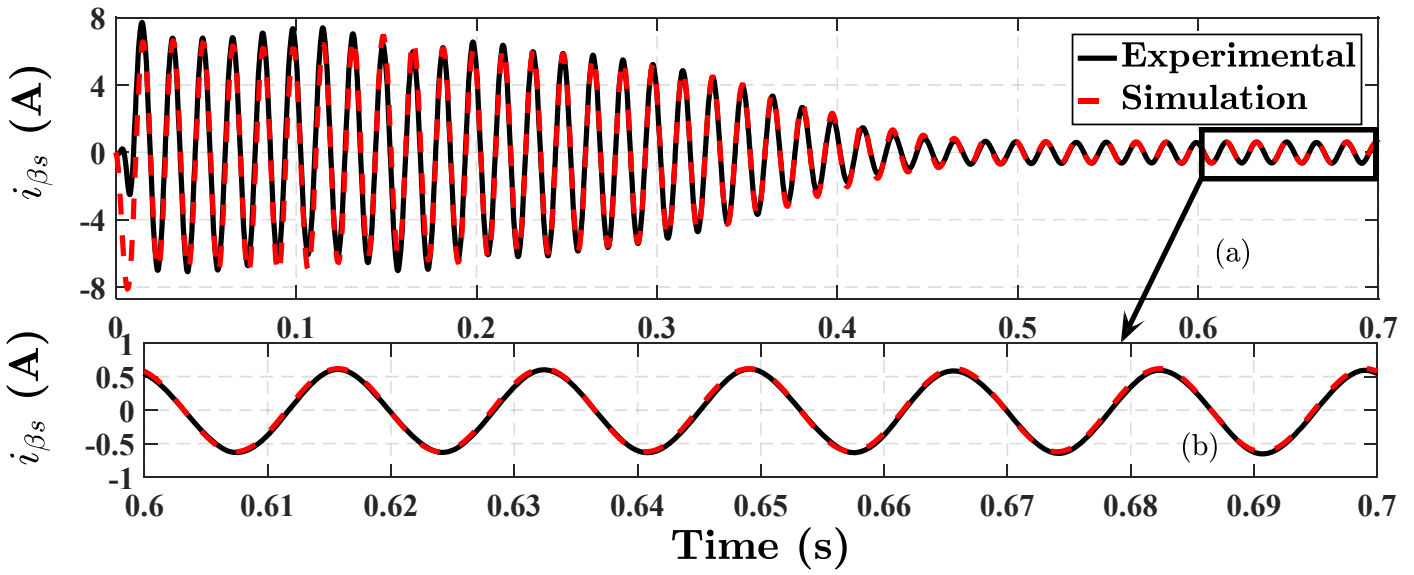

Figure 7.26 Imaginary component stator current faulty IM (a) $i_{s \beta}$ (b) Zoomed $i_{s \beta}$.

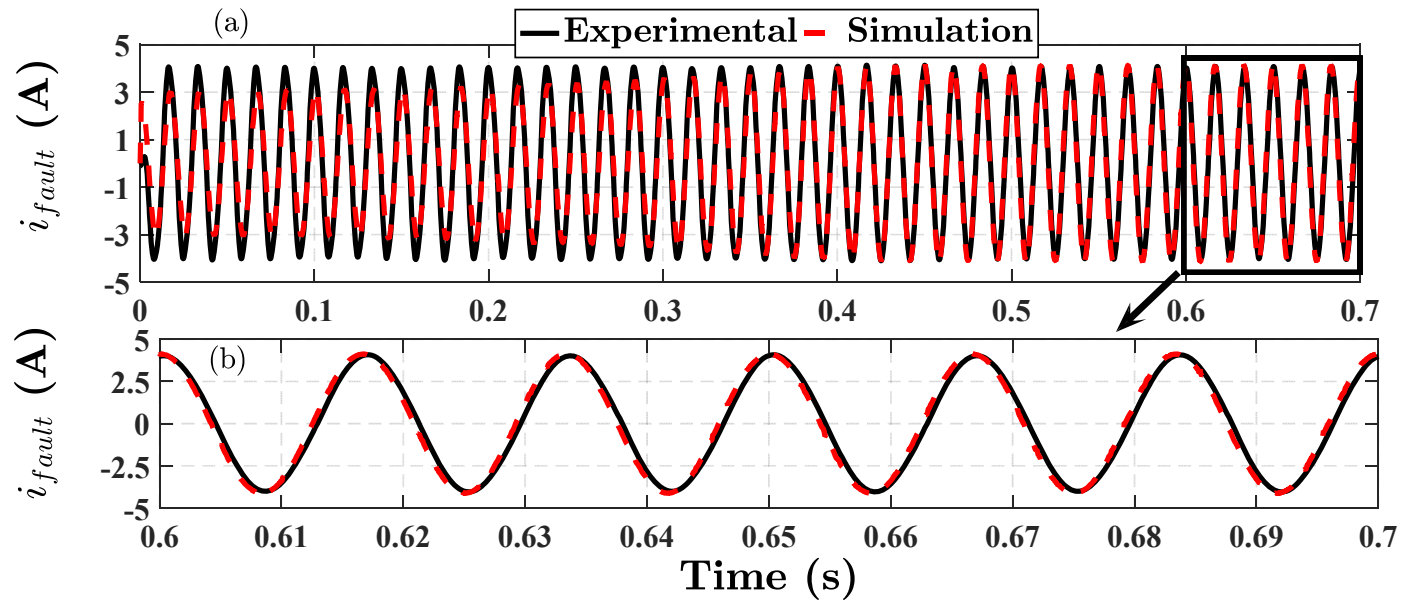

Figure 7.27 Faulty leakage current (a) $i_{f}$ and (b) Zoomed $i_{f}$.

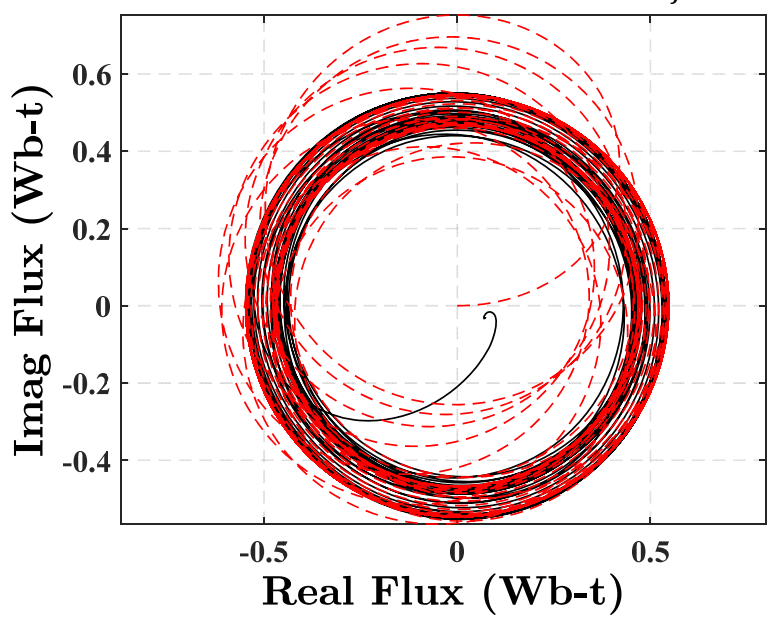

- Experimental

- - Simulation

Figure 7.28 Locus of the space-vector flux-linkages $\left(\lambda_{s}\right)$. 


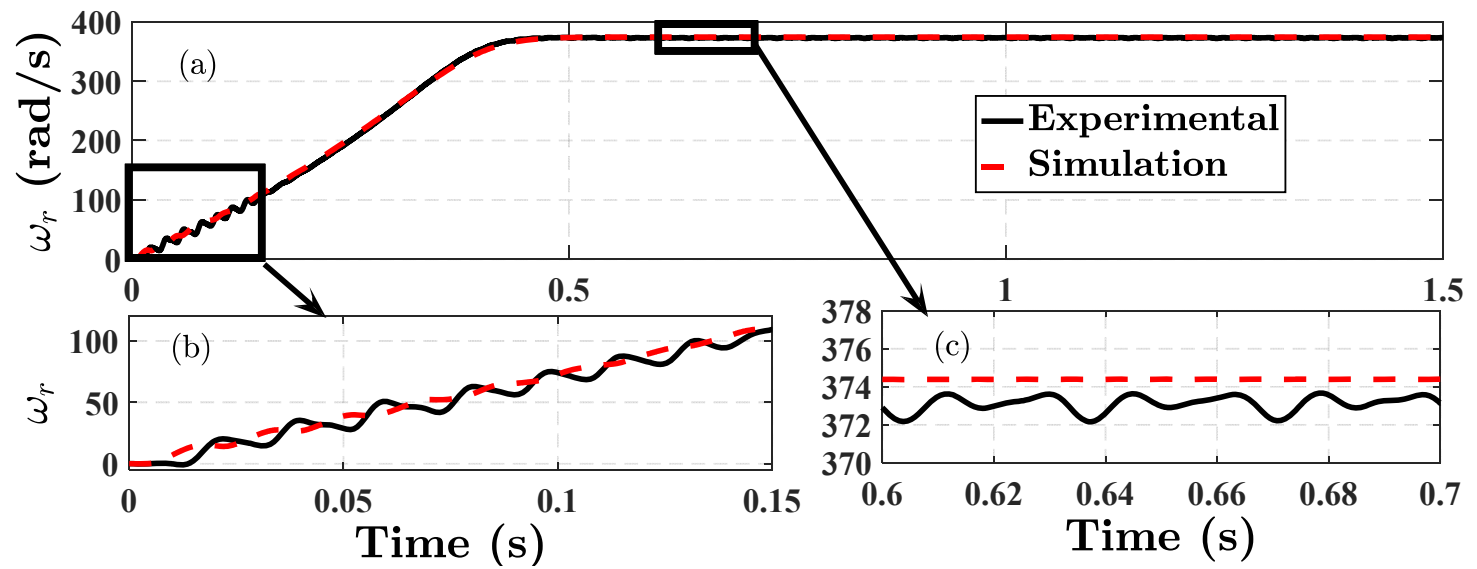

Figure 7.29 Rotor angular speed faulty IM (a) $\omega_{r}$ (b) Zoomed $\omega_{r}$.

In Figure 7.30(b) and (c), the zoom of the electromagnetic torque displays some discrepancy that is typically acceptable for the following reasons:

1. First, the torque ripple is being estimated and not measured in the experimental test.

2. Second, this variable depends on the shape, geometry and position of the rotor bars in the IM, which are not considered in the proposed model.

3. Third, the input voltage source in the experimental test presents small unbalanced behavior $\left(v_{s a}=168.2 \mathrm{~V}, v_{s b}=173.4 \mathrm{~V}\right.$ and $\left.v_{s c}=167.3 \mathrm{~V}\right)$.

4. On the other hand, the simulations are performed with a balanced three-phase power source, which makes the comparison of the electromagnetic torque unfair.

5. Finally, typically, a real IM has small asymmetries inherent to its construction. Some of the asymmetries are static eccentricity, different values of stator resistances per-phase, deformation and defects in the stator windings, etc [149].

In Figure $7.30(\mathrm{c})$, the double line frequency predicted in the theoretical analysis (7.30) and (7.32) appears in both simulation and experimental results. 


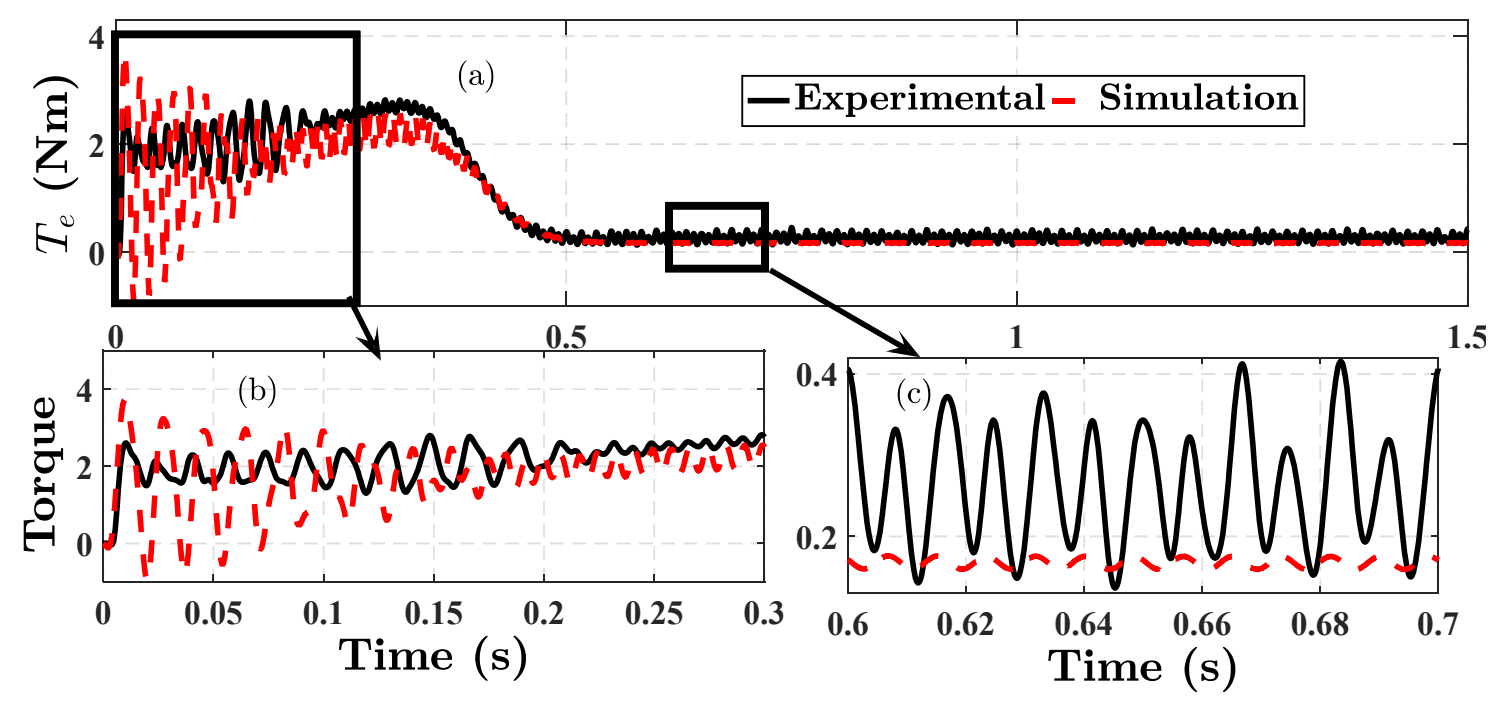

Figure 7.30 Electromagnetic torque in the faulty IM (a) $T_{e}$ (b) First zoom of $T_{e}$ and (c) Second zoom of $T_{e}$.

\subsection{Loading Condition:}

Transient test comparison analysis for the faulty IM between simulation and experimental results at the loaded condition are shown in Figure 7.31, Figure 7.32 and Figure 7.33. A fault severity factor of $3.78 \%$, a fault resistance of $2.5 \Omega$ and a mechanical load of $1 \mathrm{Nm}$ are implanted. The electrical variables for this comparison are: stator current space-vector (Figure 7.31), faulty leakage current (Figure 7.32(a) and (b)), locus of the stator flux-linkage space-vector (Figure 7.32(c)), the rotor angular speed (Figure 7.33(a)) and electromagnetic torque (Figure 7.33(b)). From these figures, it can be noted that the model behaves accurately at the loading condition. However, there is a small mismatch at the transient of the speed and electromagnetic torque. The steady state response is correlated for both variables. 


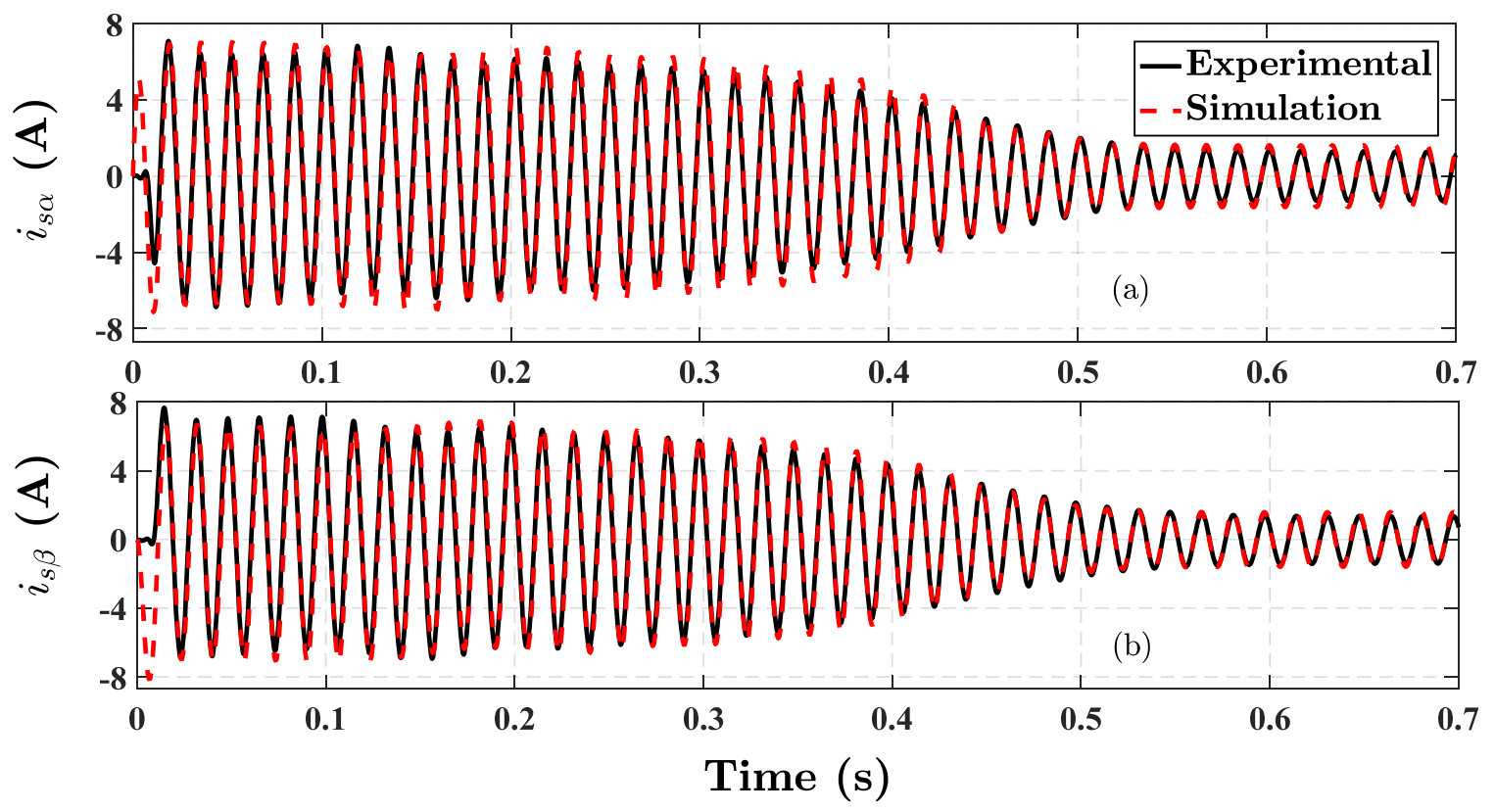

Figure 7.31 (a) Real component of the stator current for faulty IM (b) Imaginary component of the stator current faulty IM
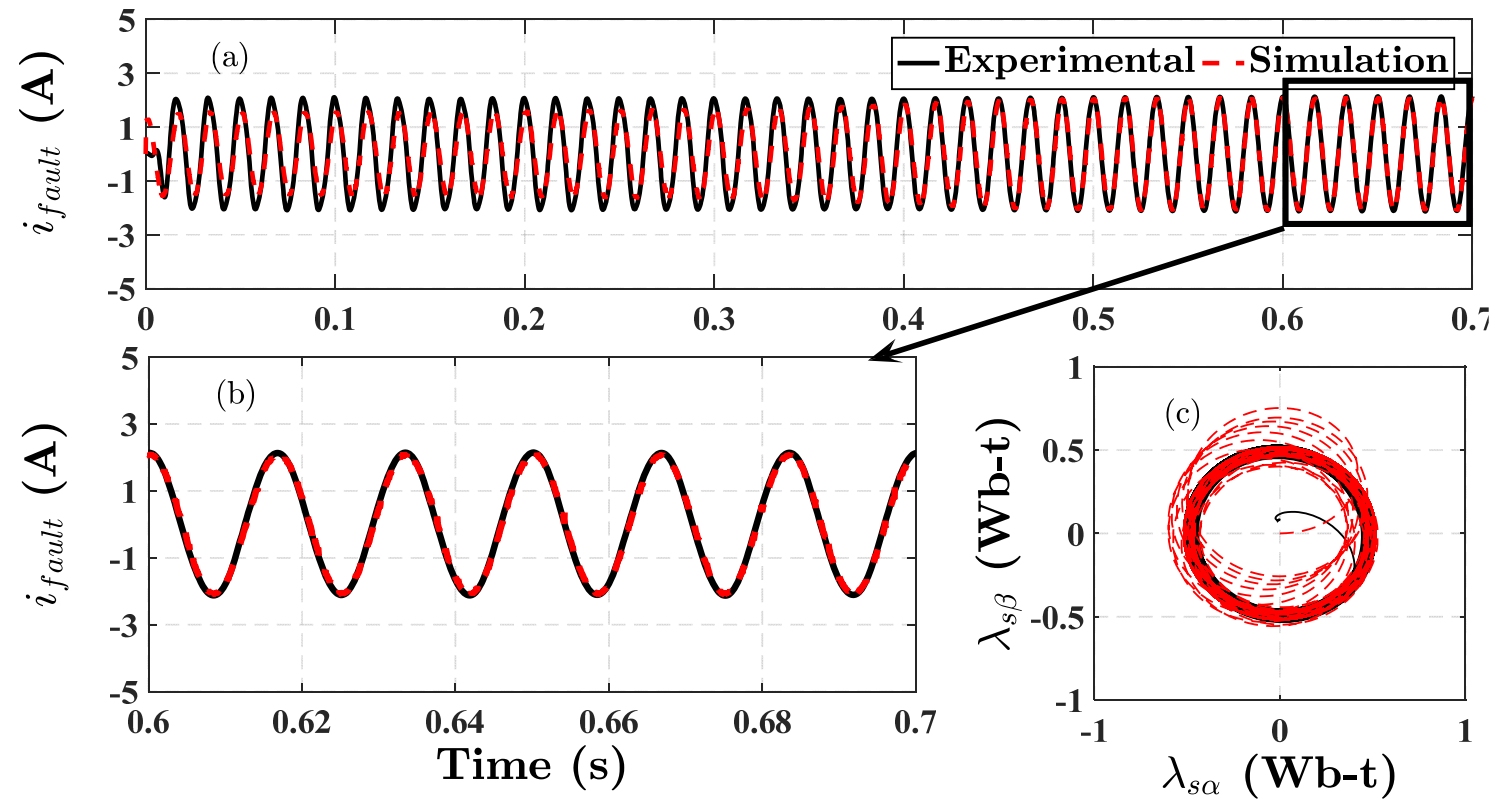

Figure 7.32 Transient results faulty leakage current (a) $i_{f}$ and (b) Zoomed $i_{f}$ (c) Locus of the space-vector flux-linkages $\left(\boldsymbol{\lambda}_{\boldsymbol{s}}\right)$. 

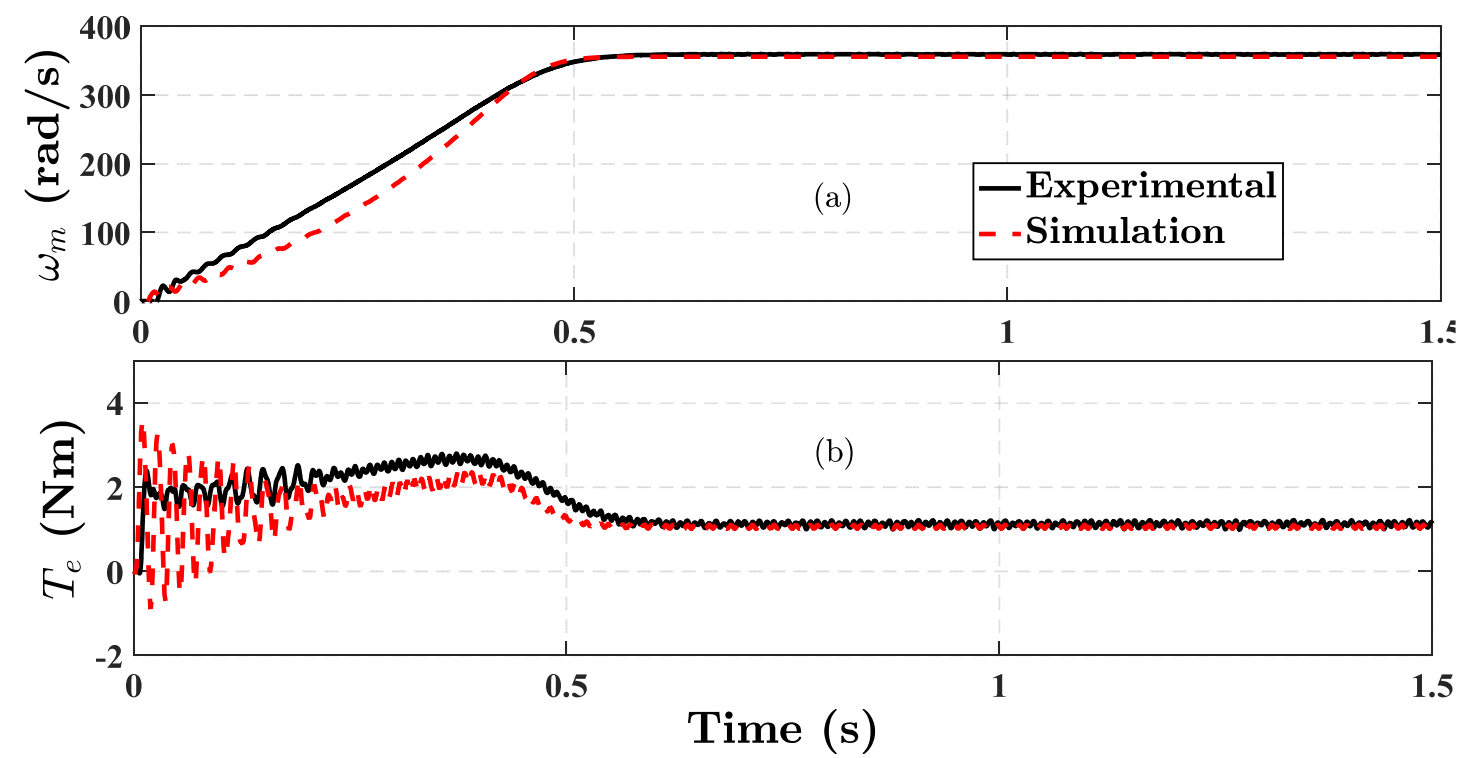

Figure 7.33 (a) Rotor angular speed faulty IM $\left(\omega_{r}\right)$ (b) Electromagnetic torque faulty IM.

\subsubsection{Permanent Regime of Faulty IM}

In this section, the variation of the faulty leakage current $\left(i_{f}=i_{\text {fault }}\right)$, stator current space-vector and electromagnetic torque ripple as a function of: the severity factor $(\mu)$, faulty resistance $\left(R_{f}\right)$, and the torque load $\left(T_{L}\right)$ are investigated.

\subsection{Variation with Fault Severity Factor:}

The fault severity factor range is shown in Table $7-4$ from 0.0282 to 0.5 . The faulty resistance is set as $10 \Omega$ for safety purposes, thus achieving $\mu=50 \%$ and avoiding permanent IM damage. In the simulation results, an empirical value of resistance is introduced in the faulty circuit model. This resistance $\left(R_{c o n}\right)$ models the extra wire introduced to perform the center taps and their soldering connection (junction). Research [8] has also included this practical resistance in its modelling. For our simulation, $R_{c o n}=$ $3 \Omega$. From Figure 7.34, it can be perceived that the model has a high correlation with the experimental results until $\mu=32.8 \%$, which means good accuracy for the first 12 cases of 
ITSC. The proposed model has some limitations for high $\mu$ from 32.8 to $50 \%$. Also, the correlation of the real and imaginary components of $\boldsymbol{i}_{\boldsymbol{s}}$ is good for the entire $\mu$ range. Table 7-5 presents the mean absolute error (MAE) and root mean square error (RMSE) between the experimental and simulated variables $\left(I_{s \alpha}, I_{s \beta}, I_{\text {fault }}\right.$ and $\left.\Delta T_{e}\right)$ as a function of $\mu$. These calculations are based on (7.46).

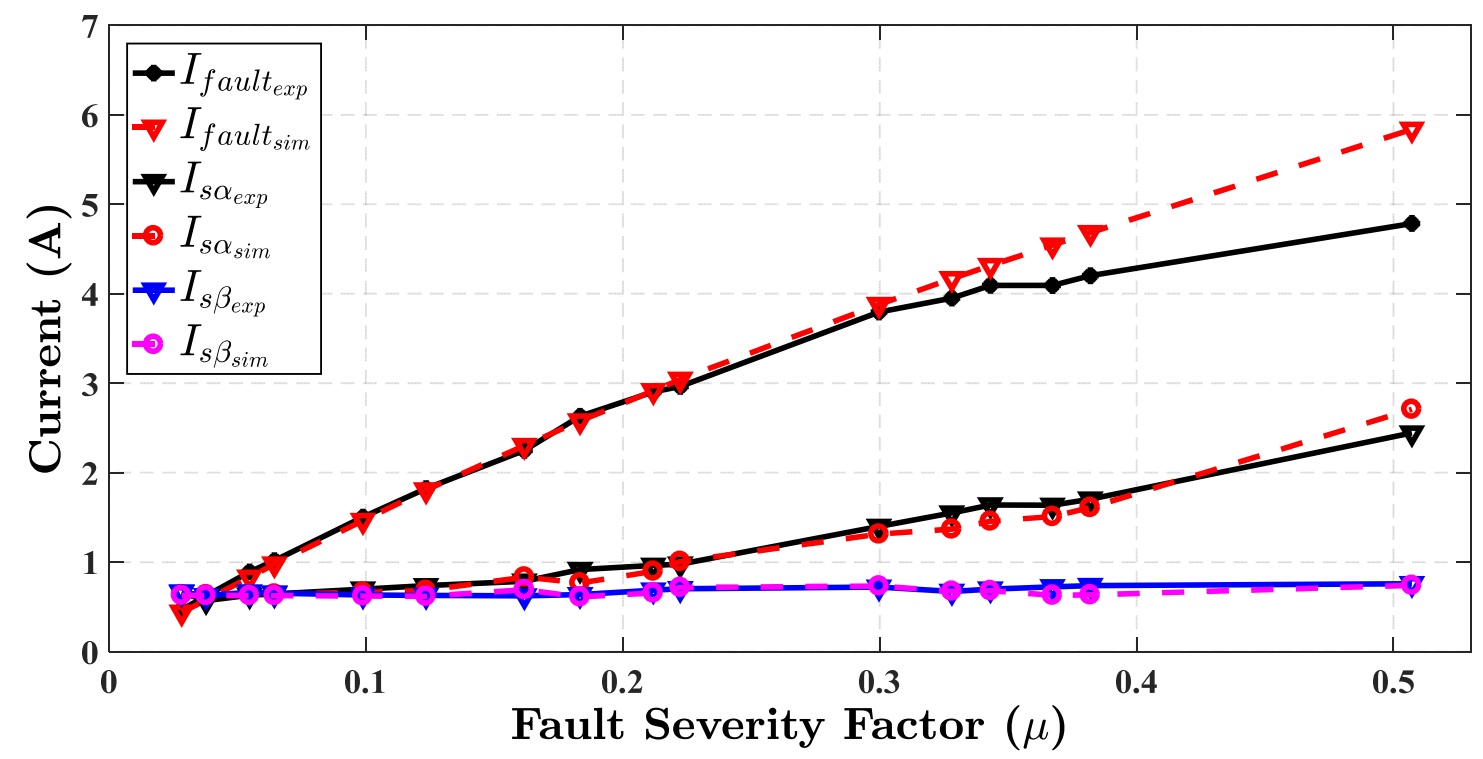

Figure 7.34 Simulation and experimental results for magnitude stator current space vector components $\left(I_{s \alpha}, I_{s \beta}\right)$ and $I_{\text {fault }}$ as a function of $\mu$. Using constant $R_{c o n}=3 \Omega$.

$z_{k}=\frac{z_{\exp (k)}-z_{\operatorname{sim}(k)}}{z_{\exp (k)}}$
$M A E=\frac{1}{N} \sum_{k=1}^{N}\left|z_{k}\right| \quad R M S E=\sqrt{\frac{1}{N} \sum_{k=1}^{N} z_{k}^{2}}$

From Table 7-5, it can be realized that the MAE and RMSE decrease considerably for $I_{\text {fault }}$ and decrement for $I_{s \alpha}$ and $I_{s \beta}$ in the first 12 fault severity factor cases compared to the complete set of 16 cases. 


\subsection{Variation with Faulty Resistance:}

The variation of the faulty resistance is, 13 cases, from 0 to $10 \Omega$. Figure 7.35 delineates the same variables, as before, as a function of $R_{f}$. The study is conducted for $\mu=3.78 \%$ at no load condition and $R_{\text {con }}=3 \Omega$. Table 7-5 exposes MAE and RMSE for the same variables as a function of $R_{f}$. Notice that the model has a very good accuracy for $I_{f a u l t}$, when $R_{f}$ is in the range from 2 to $10 \Omega$. On the other hand, it displays some limitations once $R_{f}$ is below $2 \Omega$. Also, the same behavior can be observed, where the components of $\boldsymbol{i}_{\boldsymbol{s}}$ do not present a considerable error change between all the 13 cases and the last 9 fault resistances.

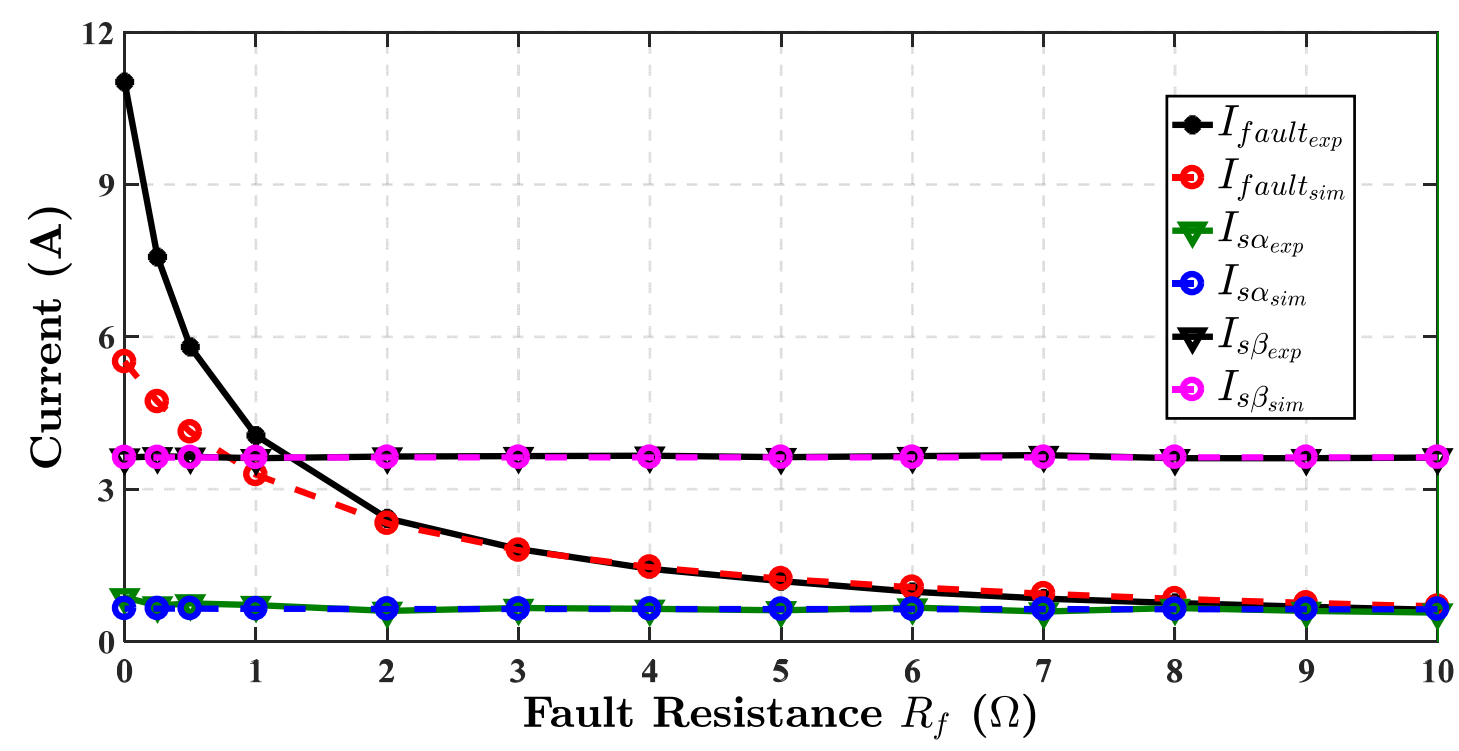

Figure 7.35 Simulation and experimental results for the magnitude stator current space vector components $\left(I_{s \alpha}, I_{s \beta}\right)$ and $I_{\text {fault }}$ as a function of $R_{f}$. Using constant $R_{\text {con }}=3 \Omega$

The proposed model behaves accurately in a certain range of the faulty conditions, however, it presents some limitations once they are too severe. These limitations are observed in the faulty leakage current, as shown in Figure 7.34, Figure 7.35 and Table 7-5. Different behavior is realized in $\boldsymbol{i}_{\boldsymbol{s}}$, which keeps a good correlation for the complete set of 
experiments. Thus, a second empirical resistance value is added in series with the fault $\left(R_{f}\right)$ and connection $\left(R_{c o n}\right)$ resistances to account for this mismatch, which has a non-linear behavior $\left(R_{\text {non_linear }}\right)$. Therefore, the final added resistance $R_{\text {emp }}$ (Figure $\left.7.37(\mathrm{~b})\right)$ is as (7.47).

Table 7-5 MAE and RMSE for $I_{s \alpha}, I_{s \beta}, I_{\text {fault }}$ and $\Delta T_{e}$ Sim. vs Exp. as a function of the fault severity factor and $R_{f}$.

\begin{tabular}{c|cc|cc|cc|cc}
\hline & \multicolumn{4}{|c|}{ Fault Severity Factor } & \multicolumn{4}{c}{ Fault Resistance } \\
\cline { 2 - 9 } & \multicolumn{1}{|c|}{16 ITSC cases } & \multicolumn{2}{|c|}{ First 12 cases } & \multicolumn{2}{c}{13 cases of $R_{f}$} & \multicolumn{2}{c}{ Last 9 cases } \\
\cline { 2 - 9 } & $M A E$ & $R M S E$ & $M A E$ & $R M S E$ & $M A E$ & $R M S E$ & $M A E$ & $R M S E$ \\
\hline$I_{\text {fault }}$ & 5.79 & 7.81 & 3.56 & 4.19 & 15.3 & 20.95 & 7.15 & 8.17 \\
$I_{s \alpha}$ & 5.30 & 7.02 & 4.57 & 5.58 & 6.41 & 8.31 & 5.38 & 6.39 \\
$I_{s \beta}$ & 7.65 & 9.51 & 6.34 & 7.87 & 3.05 & 3.64 & 3.47 & 4.03 \\
$\Delta T_{e}$ & 79.4 & 80.9 & 84.7 & 85.61 & 96.8 & 96.8 & 96.5 & 96.5 \\
\hline \hline
\end{tabular}

$$
R_{\text {emp }}=R_{\text {con }}+R_{\text {non_linear }}
$$

The purpose of $R_{e m p}$ is to demonstrate that the asymmetric model can reproduce the real behavior of the real damaged IM for different cases of $\mu, R_{f}$ and $T_{L}$. The next subsections demonstrate this. This will allow the model to be used in different techniques of condition monitoring, such as: parameter estimation and observers-based MRA.

\subsection{Variation with Fault Severity Factor, variable $\boldsymbol{R}_{e m p}$ :}

Figure 7.36 shows $\boldsymbol{i}_{s}, I_{\text {fault }}$ and $\Delta T_{e}$ as a function of $\mu$. A good agreement between the simulation and experimental results (Table 7-6) can be noted. Observe that $I_{\text {fault }}$ increases with $\mu$, as well as the real component of $\boldsymbol{i}_{\boldsymbol{s}}$. The faulty current depicts a small non-linear shape due to the leakage inductance dependency, as shown in (7.43). The imaginary component of $\boldsymbol{i}_{\boldsymbol{s}}$ does not vary with respect to $\mu$. Figure 7.37(a) displays $\Delta T_{e}$ and $R_{e m p}$ as 
a function of $\mu$. Notice that the torque ripple increases almost linearly with respect to $\mu$ for both experimental and simulation results. A large error between them can be viewed (Table 7-6). The reasons for this error were previously discussed in the transient analysis and are summarized as:

a) $T_{e}$ is estimated.

b) $T_{e}$ depends on the shape, geometry and position of the rotor bars in the IM, which are not contemplated in the proposed model.

c) Experimental input voltage source presents small unbalances.

d) A real IM has small asymmetries inherent to its construction.

Observe that $R_{e m p}$ is $3 \Omega$ for 8 cases of ITSC, for reasonable $\mu$ from 0.0282 to 0.1835 .

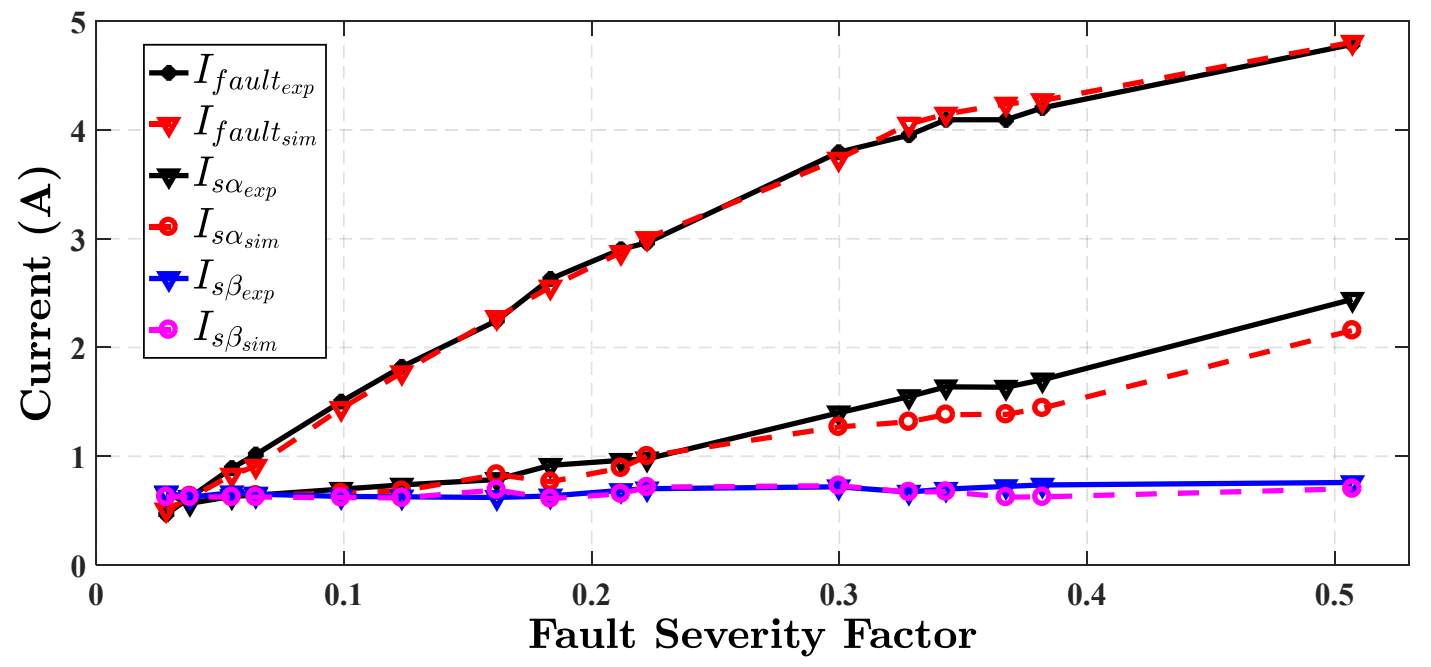

Figure 7.36 Real and imaginary component stator current space vector $\left(I_{s \alpha}, I_{s \beta}\right)$ and $I_{\text {fault }}$ as a function of $\mu$. Simulation and experimental results. 

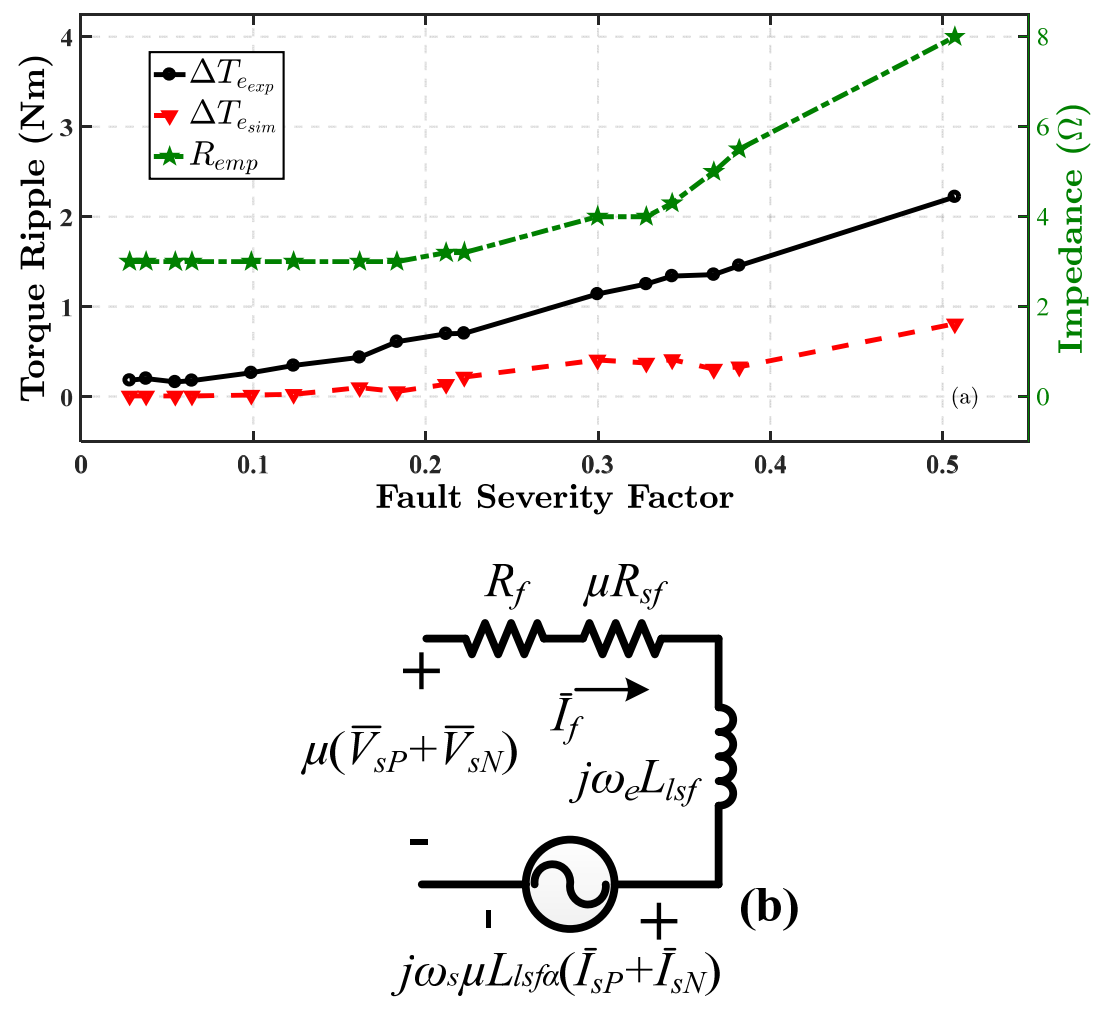

Figure 7.37 (a) Torque ripple vs $\mu$. Simulation and experimental results. Empirical impedance vs $\mu$ (b) Leakage faulty EC model considering the practical and empirical resistance $R_{e m p}$.

\subsection{Variation with Fault Resistance, variable $\boldsymbol{R}_{e m p}$ :}

From Figure 7.38, a perfect agreement between simulation and experimental results can be seen (Table 7-6) among $\boldsymbol{i}_{s}, I_{\text {fault }}$ and $\Delta T_{e}$ as a function of $R_{f} . I_{\text {fault }}$ decreases exponentially with $R_{f}$, as it can be seen from (7.43). Re-writing (7.43) as (7.48) the hyperbolic dependency is clearly observed.

$$
I_{f}=\frac{k_{1}}{\left(R_{f}+k_{2}\right)}
$$

where $k_{1}$ and $k_{2}$ are the remaining terms of $I_{f}$ that do not depend on $R_{f}$. The real and imaginary components of $\boldsymbol{i}_{\boldsymbol{s}}$ do not vary with respect to $R_{f}$. For avoiding the plot 
overlapping of $I_{s \alpha}$ and $I_{s \beta}$, the imaginary component is shown as $I_{s \beta}+3$. Notice in Figure 7.39 that $\Delta T_{e}$ is almost constant for both experimental and simulation results. Still, there is a large error between them (Table 7-6). Observe that for 9 cases of $R_{f}, R_{e m p}$ is $3 \Omega$.

\subsection{Variation with Load Torque:}

This study is conducted for $\mu=3.78 \%$ with a $T_{L}$ distribution from 0.3 to $0.9 \mathrm{Nm}, R_{f}=$ $1 \Omega$. Figure 7.40 and Figure 7.41 depict the variation of the same variables of previous cases as a function of $T_{L}$.

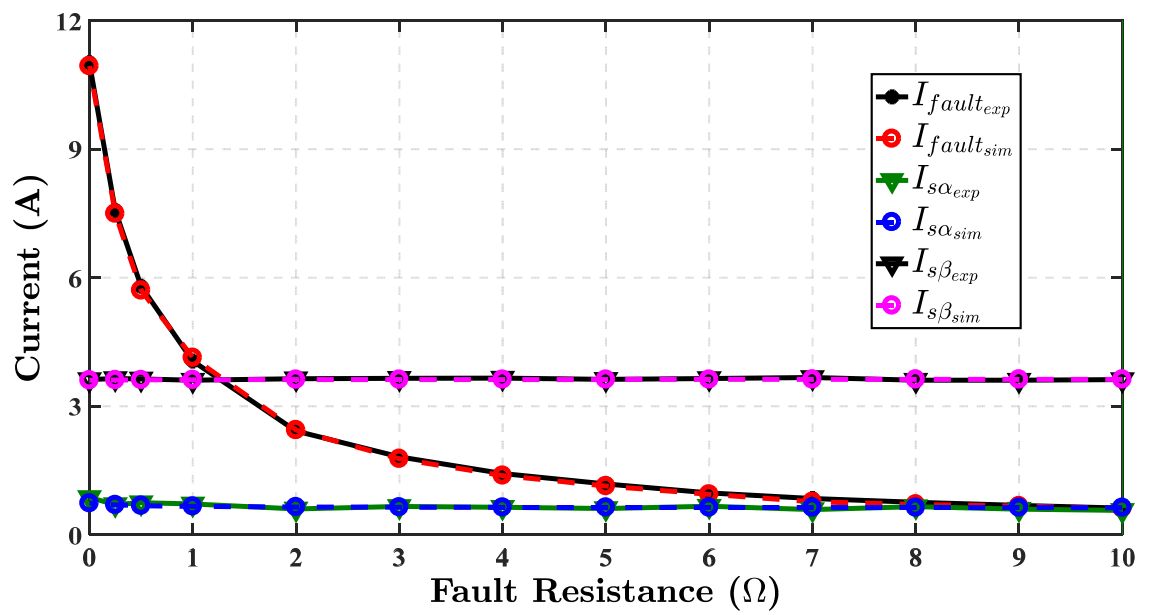

Figure 7.38 Real and imaginary component stator current space vector and $I_{\text {fault }}$ as a function of the $R_{f}$. Simulation and experimental results.

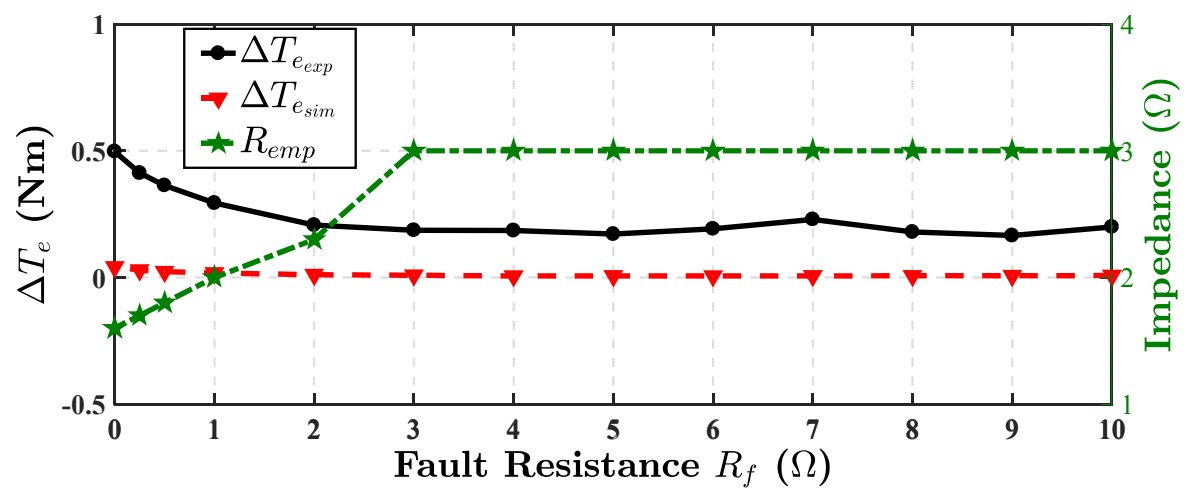

Figure 7.39 Electromagnetic torque ripple vs faulty resistance $R_{f}$. Simulation and experimental results. Empirical impedance vs faulty resistance. 
Table 7-6 MAE and RMSE for $I_{s \alpha}, I_{s \beta}, I_{\text {fault }}$ and $\Delta T_{e}$ Sim. vs Exp.

\begin{tabular}{c|cc|cc|cc}
\hline \multirow{2}{*}{} & \multicolumn{2}{|c|}{ Severity Factor } & \multicolumn{2}{|c|}{ Fault Resistance } & \multicolumn{2}{c}{ Load } \\
\cline { 2 - 7 } & $M A E$ & $R M S E$ & $M A E$ & $R M S E$ & $M A E$ & $R M S E$ \\
\hline$I_{\text {fault }}$ & 3.2 & 4.3 & 2.9 & 3.7 & 2.1 & 2.2 \\
$I_{s \alpha}$ & 8.9 & 10.4 & 6.2 & 7.4 & 16.5 & 16.9 \\
$I_{s \beta}$ & 5.1 & 6.7 & 3.3 & 3.7 & 9.0 & 9.5 \\
$\Delta T_{e}$ & 81.8 & 82.7 & 95.2 & 95.2 & 73.0 & 73.7 \\
\hline \hline
\end{tabular}

Figure 7.40 describes that $I_{\text {fault }}$ is almost constant and $R_{e m p}$ is constant as a function of the $T_{L}$, which means that the loading condition is not a factor affecting $I_{\text {fault }}$. This is also noted in the faulty EC in Figure 7.9 and Figure 7.37(b), where the dependency on $I_{\text {fault }}$ with the slip is introduced by the factor $j \omega_{s} L_{l s f \alpha} \mu\left(\bar{I}_{S P}+\bar{I}_{s N}\right)$ in (7.43), which is small. Investigations in [34], [36] have obtained the same results. Notice that the simulation and experimental results are correlated (Table 7-6). For avoiding the plot overlapping, $I_{s \beta}$ is shown as $I_{s \beta}+0.5$.

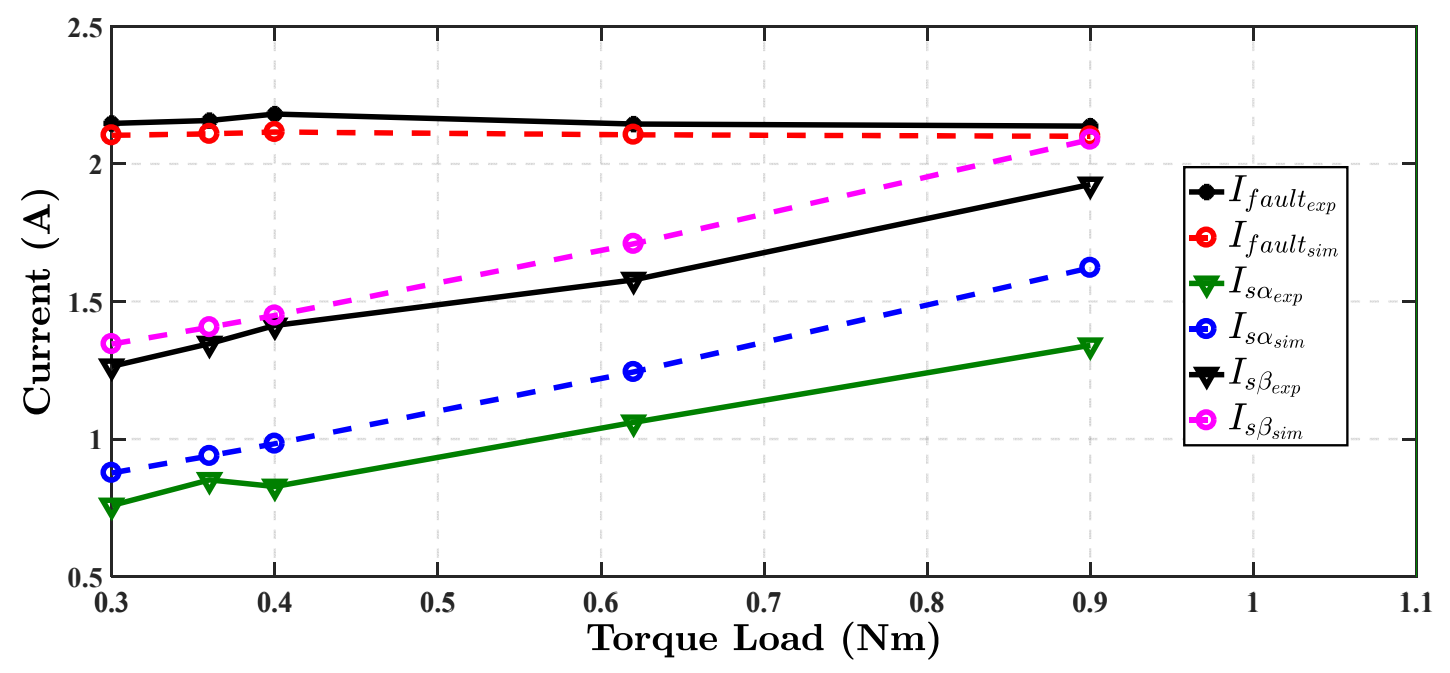

Figure 7.40 Real and imaginary component Stator current space vector and $I_{\text {fault }}$ as a function of $T_{L}$. Simulation and experimental results. 


\subsubsection{Comparative Analysis between Proposed Model and Linear Model of the \\ Leakage Inductance.}

The model presented in [34] has been programmed and simulated in the same way and under the same conditions of the proposed model. A full comparison of all the cases for the permanent regime analysis is performed. This comparative analysis studies the difference among the experimental and the two simulations (proposed and linear leakage inductance [34]) for the real and imaginary components of $\boldsymbol{i}_{\boldsymbol{s}}$ and $I_{\text {fault }}$ as a function of $\mu$ and $R_{f}$. Figure 7.42 and Figure 7.43 show the comparison of both models using $R_{e m p}$ set in $3 \Omega$ for fairness. Figure 7.42 demonstrates $I_{\text {fault }}$ varying as a function of $\mu$ and Figure 7.43 delineates $I_{\text {fault }}$ varying as a function of $R_{f}$ for $\mu=3.78 \%$.

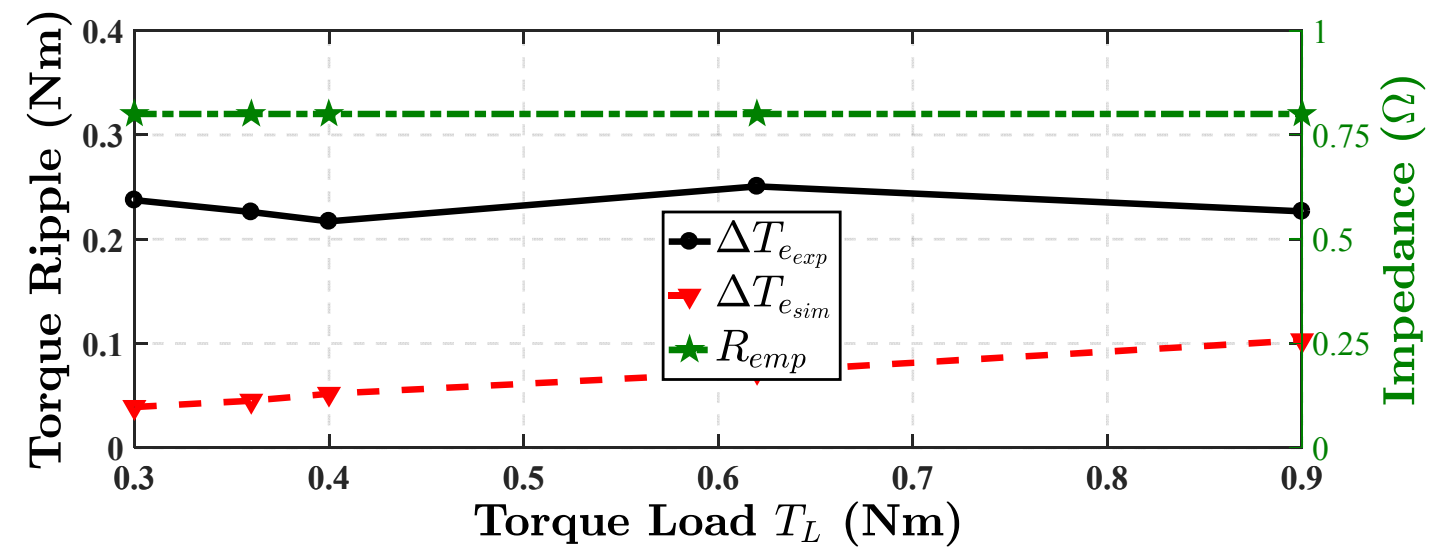

Figure 7.41 Fig. 1. Electromagnetic torque ripple vs torque load. Simulation and experimental results. Empirical impedance vs torque load.

Table 7-7 reveals the numerical values for the MAE and RMSE for the two comparative studies. From this table and Figure 7.42, it is perceived that the proposed model is more accurate than the linear model presented in [34] for the variation of $\mu$. The proposed model 
has better correlation in $I_{\text {fault }}$, mainly where it is noticed that for large $\mu$ the linear model of the leakage inductance [34] diverges more than the proposed model (these cases are where the leakage inductance become significant). Also, the real and imaginary components of $\boldsymbol{i}_{\boldsymbol{s}}$ exhibit a slightly better MAE and RMSE for the proposed model compared to the linear one. However, the torque ripple exposes a quite similar mismatch. For the studied cases of fault resistance variation, it is perceived from Figure 7.43 and Table 7-7 that both models performed similarly due to the small percentage of fault ( $\mu=$ 3.78\%), where the leakage inductance can be neglected.

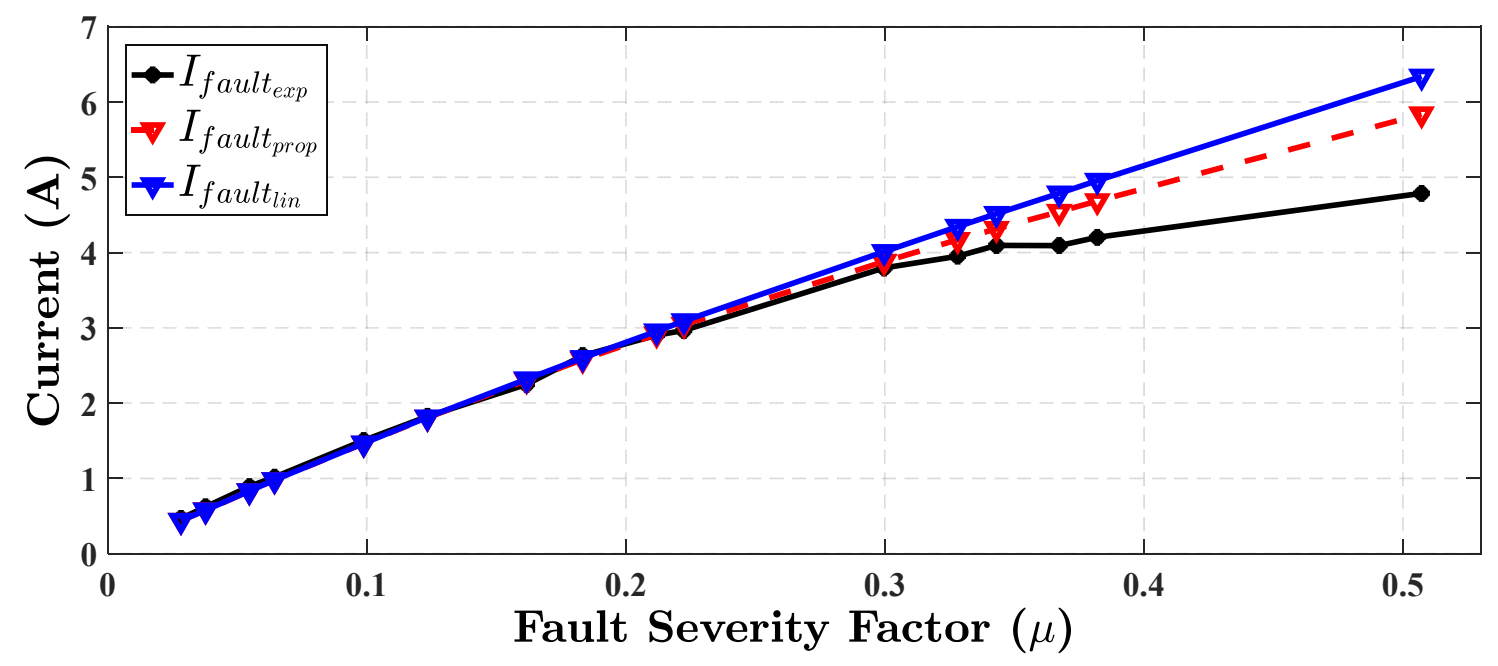

Figure 7.42 Experimental and two simulation results (proposed and linear leakage inductance) comparison using $R_{e m p}=3 \Omega$. $I_{\text {fault }}$ as a function of $\mu$.

\subsubsection{Electromagnetic Torque Discrepancy Study}

For purposes of studying the $\Delta T_{e}$ discrepancy and corroborating the reasons of its mismatch, a finite elements analysis (FEA) study was performed for the faulty IM with the worst case of $\mu(50 \%)$. The $\Delta T_{e}$ was computed and compared with the experimental and simulation results in Figure 7.44. As it can be noted, the FEA torque ripple is $1.82 \mathrm{Nm}$, which is more than 2 times the one from the proposed model $(0.83 \mathrm{Nm})$. Notwithstanding, 
it is still less than the experimental one $(2.22 \mathrm{Nm})$, as no construction asymmetries were included. The imbalance of the voltage source was contemplated.

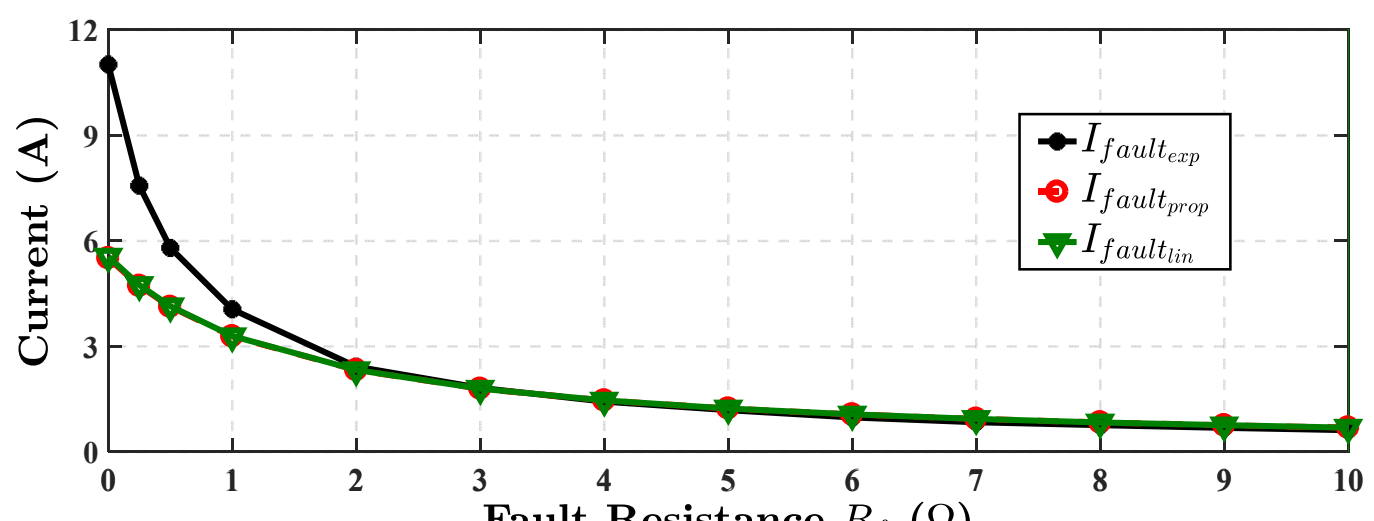

Figure 7.43 Experimental and two simulation results (proposed and linear leakage inductance) comparison using $R_{e m p}=3 \Omega$. $I_{\text {fault }}$ as a function of $R_{f}$.

Table 7-7 MAE and RMSE for $I_{s \alpha}, I_{s \beta}, I_{\text {fault }}$ and $\Delta T_{e}$ Sim. vs Exp. Comparison with [12].

\begin{tabular}{c|cc|cc|cc|cc}
\hline \multirow{2}{*}{} & \multicolumn{3}{|c|}{ Fault Severity Factor } & \multicolumn{4}{c}{ Fault Resistance } \\
\cline { 2 - 9 } & \multicolumn{2}{|c|}{ Proposed } & \multicolumn{2}{|c|}{$[34]$ Model } & \multicolumn{2}{c}{ Proposed } & \multicolumn{2}{|c}{ [34]Model } \\
\cline { 2 - 9 } & $M A E$ & $R M S E$ & $M A E$ & $R M S E$ & $M A E$ & $R M S E$ & $M A E$ & $R M S E$ \\
\hline$I_{\text {fault }}$ & 5.79 & 7.81 & 8.16 & 11.43 & 15.3 & 20.95 & 15.2 & 20.78 \\
$I_{S \alpha}$ & 5.30 & 7.01 & 5.70 & 7.31 & 6.41 & 8.31 & 6.41 & 8.31 \\
$I_{S \beta}$ & 7.65 & 9.51 & 7.95 & 9.81 & 3.05 & 3.64 & 3.05 & 3.64 \\
$\Delta T_{e}$ & 79.4 & 80.9 & 77.3 & 78.78 & 96.8 & 96.38 & 96.8 & 96.39 \\
\hline \hline
\end{tabular}




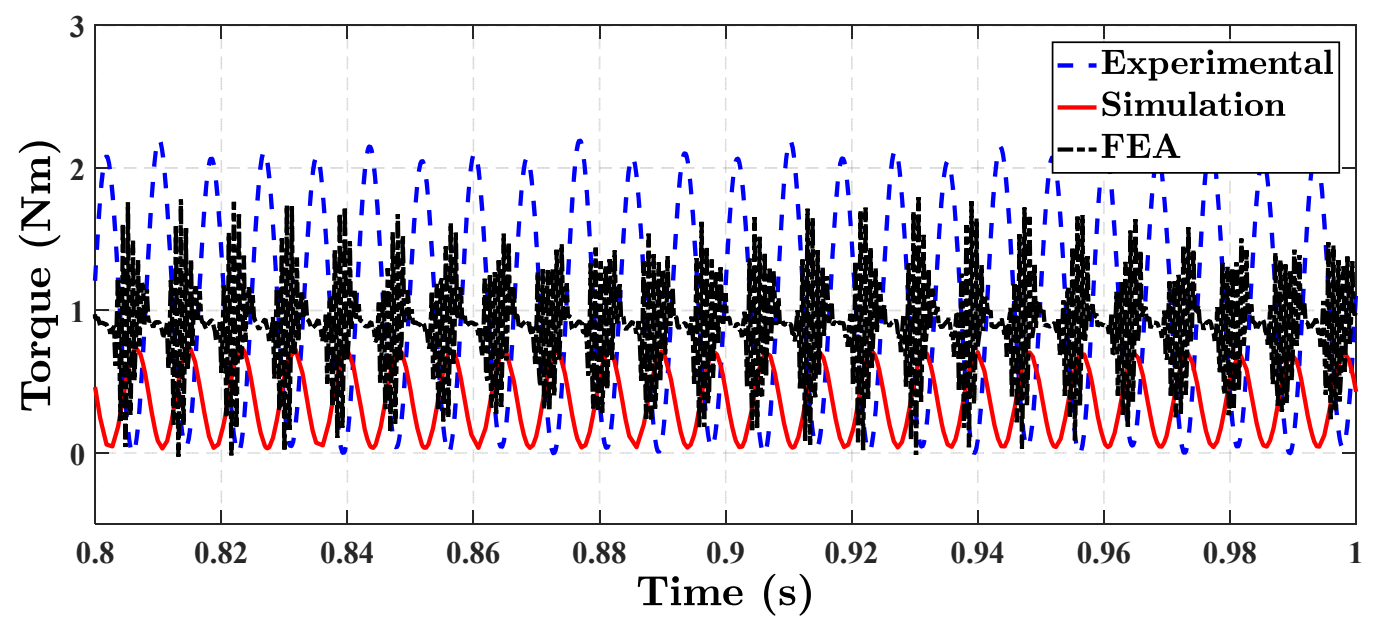

Figure 7.44 Torque ripple comparison among experimental, simulation and FEA.

\subsection{Conclusions}

Inter-turn short-circuits in the stator winding of a three-phase IM was fully theoretically analyzed, studied, modelled, simulated, experimentally tested and compared. This work proposes an alternative faulty leakage inductance approach with additional physical meaning compared with the ones presented in the literature. This leakage inductance model has more accurate behavior with respect to the linear one, keeping simplicity of the modelling. The faulty model is completely deduced and verified by comparing simulation with experimental tests under different load conditions, regimes and variation of the fault severity factor and faulty resistance. These two variables define the magnitude of the faulty leakage current and thus the damaging of the IM. From the results, it can be concluded that the faulty model is accurate and behaves similarly to the real asymmetric IM. Also, the chapter collects a series of EC that are useful for future FDI techniques, however, they are more utilizable for improving some MRA techniques, such as: off-line impedance signature, parameter estimation and observer-based techniques. 


\section{Chapter 8 Analysis of the Impact of Stator Inter-Turn Short-Circuit Faults on Induction Machines driven by Direct Torque Control}

\subsection{Introduction}

Stator inter-turn short-circuit (ITSC) faults in three-phase induction machines (IM) represent about 40 percent of machine failures in industrial applications [8]. This situation tends to worsen when motors are fed from a voltage source inverter (VSI), as there are higher stresses imposed in the inter-turn isolation. This augmented stress is caused by the steep voltage waveform produced by the fast switching solid-state devices. In general, inverter-fed controllers can be roughly classified as scalar and vector controllers. Field oriented control (FOC) and direct torque control (DTC) are the most common vectorcontrol drives for IM. The ITSC fault detection (FD) is a very well-researched topic for grid-connected or direct on-line (DOL) IM, however, little research can be found in literature dealing with the asymmetric IM under vector controllers. References [29], [150][155] studied the faulty IM driven by FOC, whereas [153], [156], [157] investigate the failure under DTC. Literature [29], [150], [151] employ a feature extraction approach (FExA). In [150], a statistical analysis calculating the mean average of the VSI outputs is exploited, while in [29], [151] the spectrum stator current analysis is applied as a FD technique. Additionally, [29], [153] propose internal signals of the Direct FOC for FD purposes. Neural networks (NN) are used in [151], [154]. In [151], a negative sequence faulty voltage signal is proposed as the output of the NN for the FD, while in [154], a fault progression indicator of a stator phase evaluates the ITSC asymmetry. An impedance identification approach is presented in [155] to locate and detect the fault. In both 
references [156] and [157], two techniques are exhibited, one is FExA-based, taking advantage of stator current spectrum and the second one (preferred by the authors of [156]) is based on a model reference approach (MRA), where a fault severity signature is derived from the mathematical model of the damaged IM. The characteristic analysis of the components on the internal control signals is studied in [153] for a DTC driver.

In this chapter, a detailed analysis of DTC in an IM under ITSC is investigated. For this purpose, a mathematical analysis of the non-linear predictive theory of the faulty IM for a DTC [117], [158] is presented. Several models for IM under stator fault have been proposed in [34], [159]. For simplicity, the model in [34] is implemented and from it two state-space (SS) models are developed. Simulation and experimental results of the impaired IM driven by DTC are displayed to corroborate the aforementioned analysis.

\subsection{Analysis of the Complete Drive System}

\subsubsection{Direct Torque Control Structure}

The DTC objective is to directly control electromagnetic torque $\left(T_{e}\right)$ and the stator fluxlinkage amplitude $\left(\left|\lambda_{s}\right|\right)$. The DTC algorithm in our work is performed at fixed switching frequency $\left(f_{s}\right)[112]$ and it has a PWM, which is not required. The DTC comprises the following stages (Figure 8.1):

Acquisition: two current and one voltage sensors measure the motor currents space-vector $\left(\boldsymbol{i}_{s}\right)$ and the dc-link level $\left(V_{D C}\right)$, respectively.

Transformation: the signals in primitive coordinates are transformed into $(\alpha \beta)$ complex vectors in the stationary frame.

Voltage motor reconstruction: with $V_{D C}$ and the switching control sequence at $t_{k-1}$, the VSI output $\left(\widehat{\boldsymbol{v}}_{\boldsymbol{s}}\right)$ can be deduced. 
Estimation: The stator flux-linkage $\left(\hat{\lambda}_{\boldsymbol{s}}\right)$ is estimated using $\widehat{\boldsymbol{v}}_{\boldsymbol{s}}, \boldsymbol{i}_{\boldsymbol{s}}$ and the stator resistance $\left(R_{s}\right)$. The electromagnetic torque $\left(\widehat{T}_{e}\right)$ is estimated with $\hat{\lambda}_{\boldsymbol{s}}$ and $\boldsymbol{i}_{\boldsymbol{s}}$. The formulas for the basic observers are in subsection 8.3.1.1.

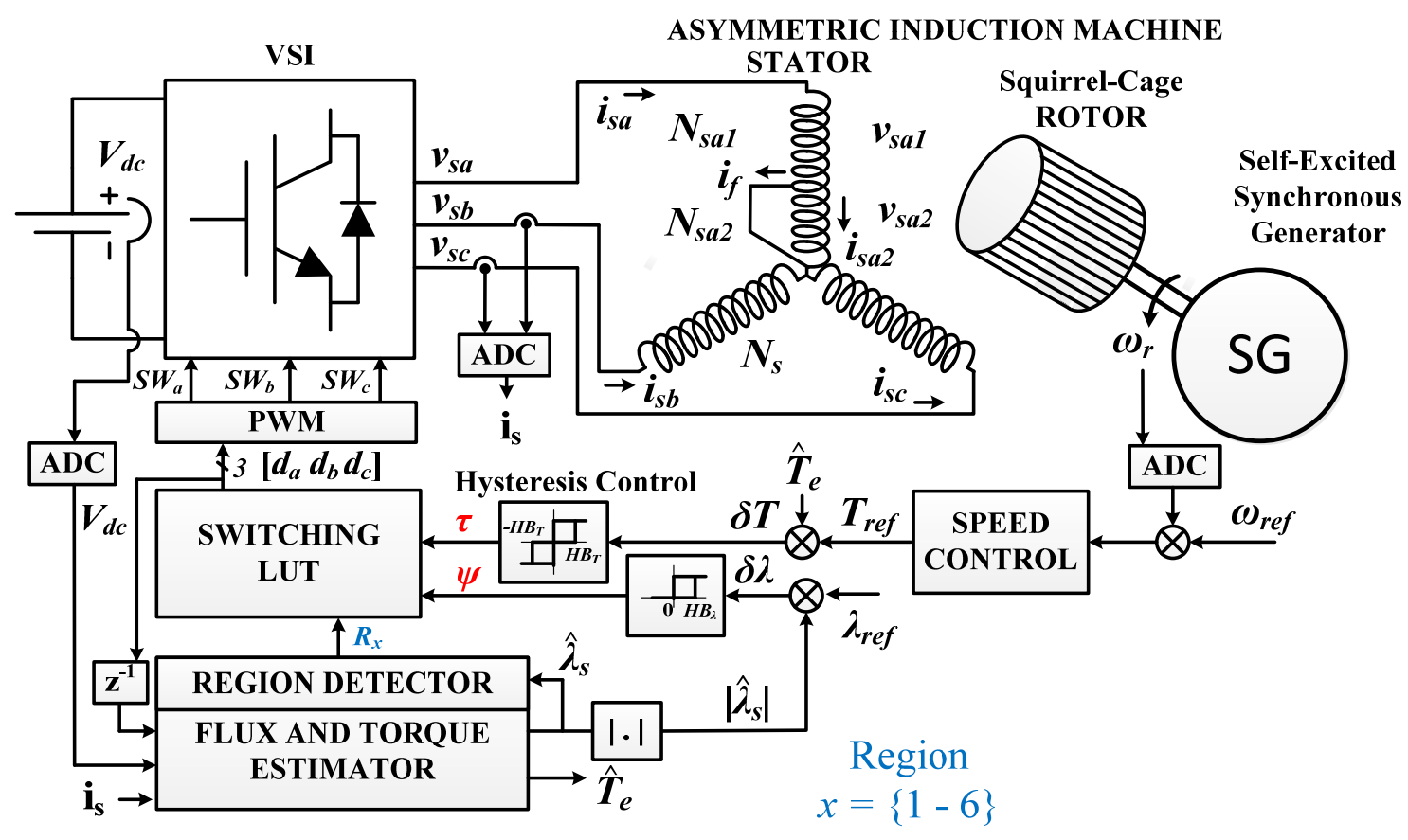

Figure 8.1 Typical structure of a DTC with speed control of an induction motor with ITSC on phase " $a$ ".

Table 8-1 Decision Look-up Table of DTC for IM

\begin{tabular}{|c|c|c|c|c|c|c|c|c|}
\hline$\tau, \psi$ & $\downarrow \downarrow$ & 0 & $\uparrow \downarrow$ & 0 & $\downarrow \uparrow$ & 0 & $\uparrow \uparrow$ & 0 \\
\hline$S_{1}$ & $\boldsymbol{v}_{\boldsymbol{s} 4}$ & 0 & $\boldsymbol{v}_{\boldsymbol{s} 2}$ & 0 & $\boldsymbol{v}_{\boldsymbol{s} 5}$ & 0 & $\boldsymbol{v}_{\boldsymbol{s} 3}$ & 0 \\
\hline$S_{2}$ & $\boldsymbol{v}_{\boldsymbol{s} 5}$ & 0 & $\boldsymbol{v}_{\boldsymbol{s} 6}$ & 0 & $\boldsymbol{v}_{\boldsymbol{s} 1}$ & 0 & $\boldsymbol{v}_{\boldsymbol{s} 2}$ & 0 \\
\hline$S_{3}$ & $\boldsymbol{v}_{\boldsymbol{s} 1}$ & 0 & $\boldsymbol{v}_{\boldsymbol{s} 4}$ & 0 & $\boldsymbol{v}_{\boldsymbol{s} 3}$ & 0 & $\boldsymbol{v}_{\boldsymbol{s} 6}$ & 0 \\
\hline$S_{4}$ & $\boldsymbol{v}_{\boldsymbol{s} 3}$ & 0 & $\boldsymbol{v}_{\boldsymbol{s} 5}$ & 0 & $\boldsymbol{v}_{\boldsymbol{s} 2}$ & 0 & $\boldsymbol{v}_{\boldsymbol{s} 4}$ & 0 \\
\hline$S_{5}$ & $\boldsymbol{v}_{\boldsymbol{s} \mathbf{2}}$ & 0 & $\boldsymbol{v}_{\boldsymbol{s} 1}$ & 0 & $\boldsymbol{v}_{\boldsymbol{s} 6}$ & 0 & $\boldsymbol{v}_{\boldsymbol{s 5}}$ & 0 \\
\hline$S_{6}$ & $\boldsymbol{v}_{\boldsymbol{s} \mathbf{6}}$ & 0 & $\boldsymbol{v}_{\boldsymbol{s} \mathbf{3}}$ & 0 & $\boldsymbol{v}_{\boldsymbol{s} 4}$ & 0 & $\boldsymbol{v}_{\boldsymbol{s} 1}$ & 0 \\
\hline
\end{tabular}

Hysteresis Control: two and three-level hysteresis controllers for stator flux amplitude and torque, respectively. The role of these controllers is to maintain the levels of the two 
variables within a narrow hysteresis bands $\left(H B_{\lambda}\right.$ and $\left.H B_{T}\right)$ around the references $\left(T_{r e f}\right.$ and $\left.\lambda_{\text {ref }}\right)$. The input of these controllers are errors $\delta T_{e}=T_{r e f}-T_{e}$ and $\delta\left|\lambda_{s}\right|=\lambda_{\text {ref }}-\left|\lambda_{s}\right|$. The output of the two hysteresis are $\tau$ and $\psi$ signals (see table in Figure 8.1).
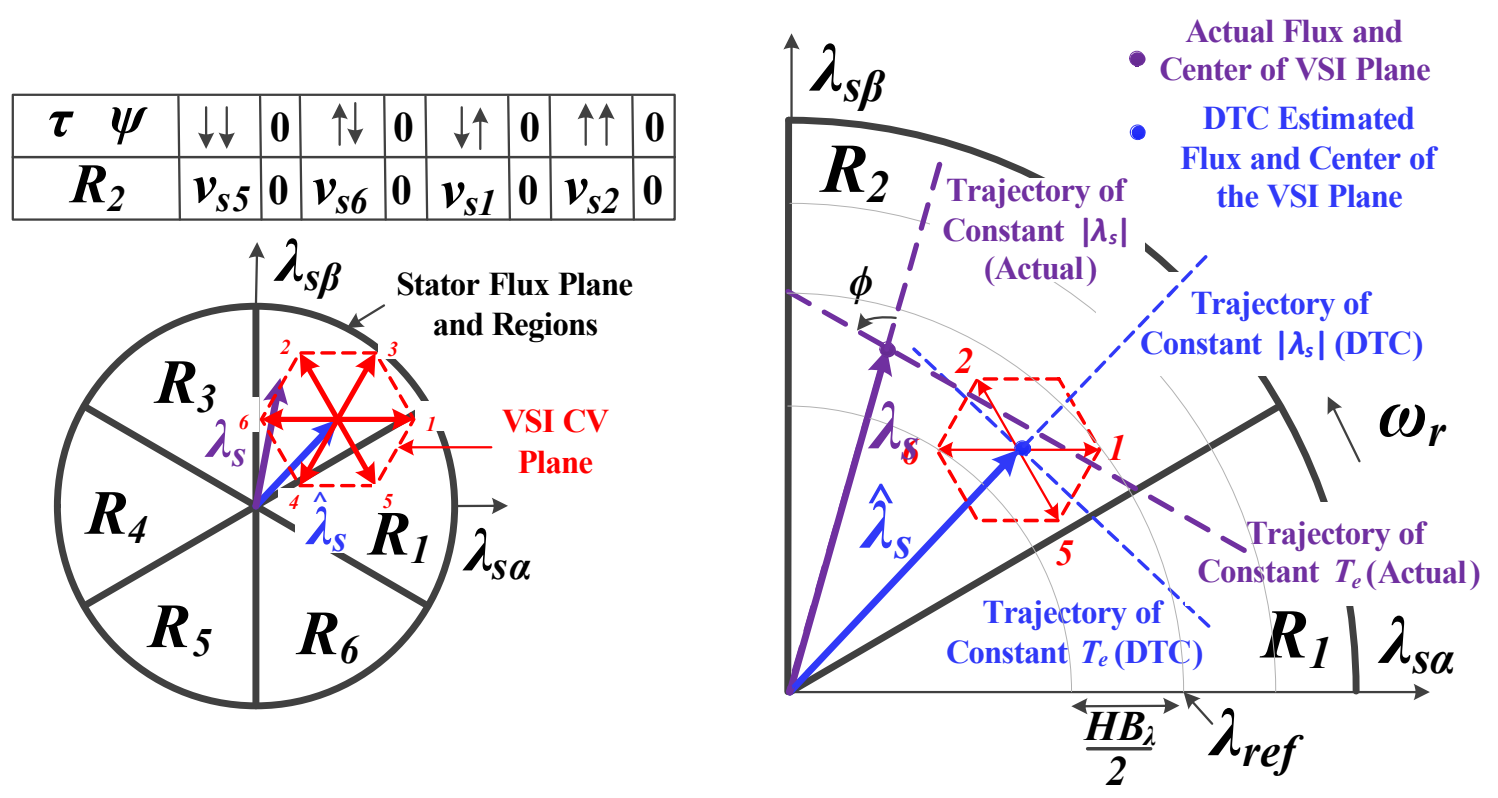

Figure 8.2 Estimated and actual Stator Flux $\lambda_{s}$ at instant $t_{k}$

Region Detection: the location of the stator flux-linkage space vector in the complex plane will indicate one of the 6 regions (see Table 8-1 and top of Figure 8.2) in which the fluxlinkage complex plane is divided $\left(R_{x}\right.$ where $\left.x=\{1-6\}\right)$.

VSI switch state selection: with $(\tau, \psi)$ in conjunction with $\left(R_{x}\right)$ the output signals to the gates of the VSI can be generated from the DTC look-up table (LUT), as in Table 8-1.

\subsection{Influence of IM Stator Fault in the DTC}

The effects of the IM stator ITSC on the DTC are a set of unbalances in its variables (current, fluxes, torque and speed) that can be categorized as: non-observable and invisible. 


\subsubsection{Non-observable Impacts to DTC}

These impacts are invisible effects of the abnormal IM over the DTC. These physical phenomena remain hidden from the DTC as the information to compute them is not available, such as the faulty circulating current, the severity of the fault and/or fault resistant. They create a series of consequences analyzed below.

\subsubsection{Problem of the Estimation Stage}

When there is no fault $(\mu=0)$ the estimated stator flux-linkage $\left(\hat{\lambda}_{s}\right)$ and electromagnetic torque $\left(\widehat{T}_{e}\right)$ are the true values:

$$
\begin{gathered}
\hat{\lambda}_{s}=\int\left(\boldsymbol{v}_{\boldsymbol{s}}-R_{s} \boldsymbol{i}_{\boldsymbol{s}}\right) d t \\
\hat{T}_{e}=\frac{3}{2} \frac{P}{2} \frac{L_{m}}{L_{s}}\left(i_{s \beta} \hat{\lambda}_{s \alpha}-i_{s \alpha} \hat{\lambda}_{s \beta}\right)
\end{gathered}
$$

\subsection{Stator Flux Amplitude Estimation Problem}

At the ITSC onset, if the degree of fault is small and the short circuit is not bold $\left(R_{f}>\right.$ 0), the DTC continues its apparent normal operation. In this situation, the estimated flux and electromagnetic torque can only be evaluated with (8.1) as no information is provided about the faulty quantities. Typically, in DTC, the initial estimation for the stator flux is as in (8.1), however, the actual stator flux is as in (8.2).

$$
\begin{gathered}
\lambda_{s \alpha}=\int\left(v_{s \alpha_{\lambda}}-R_{s} i_{s \alpha}-\frac{2}{3} \mu R_{s} i_{f}\right) d t \\
\lambda_{s \beta}=\int\left(v_{s \beta_{\lambda}}-R_{s} i_{s \beta}\right) d t
\end{gathered}
$$


As a product of the actual flux, a stator flux amplitude estimation error is obtained $\left(e_{\left|\lambda_{s}\right|}=\left|\hat{\lambda}_{s}\right|-\left|\lambda_{s}\right|\right)$. Using the Taylor series of $\sqrt{x}$, we can re-write an approximated version of $e_{\left|\lambda_{s}\right|}$ (see Appendix 1) as in (8.3).

$$
e_{\left|\lambda_{s}\right|} \approx \frac{e_{\left|\lambda_{s}\right|^{2}}}{4}
$$

where $e_{\left|\lambda_{s}\right|^{2}}$ is define as $e_{\left|\lambda_{s}\right|^{2}}=\left|\hat{\lambda}_{s}\right|^{2}-\left|\lambda_{s}\right|^{2}$.

$$
e_{\left|\lambda_{s}\right|^{2}}=\left(\hat{\lambda}_{s \alpha}{ }^{2}+\hat{\lambda}_{s \beta}{ }^{2}\right)-\left(\lambda_{s \alpha}{ }^{2}+\lambda_{s \beta}{ }^{2}\right)
$$

where $\lambda_{s \alpha}=L_{s} i_{\alpha s}+L_{m} i_{\alpha r}-2 / 3 \mu L_{s} i_{f}$ comes from (6.8), $\hat{\lambda}_{s \alpha}=L_{s} i_{\alpha s}+L_{m} i_{\alpha r}$ and $\hat{\lambda}_{s \beta}=\lambda_{s \beta}$. Thus:

$$
e_{\left|\lambda_{s}\right|}=\left(\hat{\lambda}_{s \alpha}-\frac{2 \mu L_{s} i_{f}}{3}\right) \frac{\mu L_{s} i_{f}}{3}
$$

Thus, the estimated flux-linkage magnitude error depends on the circulating fault current squared, the degree of the fault squared and the real flux-linkage.

\subsection{Torque Estimation Problem}

From the DTC point of view, after the stator flux estimation is performed, the torque is observed as in (8.1). Comparing it with the actual electromagnetic torque:

$$
e_{T_{e}}=\widehat{T}_{e}-T_{e}=\widehat{T}_{e}-T_{e m}-T_{e f}
$$

Introducing the actual stator real flux component and $T_{e f}$ in (8.6), the estimated torque error is as (8.7).

$$
e_{T_{e}}=\frac{3}{2} \frac{P}{2} \frac{L_{m}}{L_{s}} i_{s \beta} e_{\lambda_{s \alpha}}-\mu \frac{P}{2} L_{m} i_{f} i_{r \beta}
$$


where the stator real flux component estimation error is $e_{\lambda_{s \alpha}}=\hat{\lambda}_{s \alpha}-\lambda_{s \alpha}=2 / 3 \mu L_{s} i_{f}$. Thus:

$$
e_{T_{e}}=\mu \frac{P}{2} L_{m} i_{f}\left(i_{s \beta}+i_{r \beta}\right)
$$

The two new error indexes $e_{\left|\lambda_{s}\right|}$ and $e_{T_{e}}$ and faulty term of the torque $\left(T_{e f}\right)$, result from the multiplication of two sinusoidal signals at the same electric frequency $\left(\omega_{e}\right)$ with different phase shift $\left(\phi_{x}\right)$, where $x$ depends on the variable selected. Let assume only the fundamental component of the motor supplies currents. Thus, they are as shown in Table 8-2.

Therefore, $T_{e f}, e_{\left|\lambda_{s}\right|}$ and $e_{T_{e}}$ will have a similar shape as:

$$
\begin{gathered}
i_{f} i_{g x}=K \sin \left(\omega_{e} t+\phi_{f}\right) \sin \left(\omega_{e} t+\phi_{g x}\right) \\
i_{f} i_{g x}=K_{1}+K_{2} \cos \left(2 \omega_{e} t+\phi_{1}\right)
\end{gathered}
$$

From (8.9) it can be noticed two errors: static error which is constant and introduces an offset and a dynamic error at twice the electric frequency of the supply voltages $\left(2 \omega_{e}\right)$. Thus, torque and flux-linkage errors will be as in Error! Reference source not found..

Table 8-2 Signal Waveforms and Description

\begin{tabular}{|c|l|c|}
\hline Index & \multicolumn{1}{|c|}{ Signal name and variables } & Sinusoidal form \\
\hline$e_{T_{e}}$ & Ceneric Signal: $i_{g x}$ & $i_{g x}=I_{g x} \sin \left(\omega_{e} t+\phi_{g x}\right)$ \\
\hline$e_{T_{e}}$ & $\begin{array}{l}\text { Summation of the Imaginary component of the rotor } \\
\text { current and stator current: } i_{g 1}=i_{s \beta}+i_{r \beta}\end{array}$ & $i_{f}=I_{f} \sin \left(\omega_{e} t+\phi_{f}\right)$ \\
\hline$e_{\left|\lambda_{s}\right|}$ & $\begin{array}{l}\text { Summation of the real component of the stator flux- } \\
\text { linkage and faulty current: } i_{g 2}=\hat{\lambda}_{s \alpha}+2 / 3 \mu L_{s} i_{f}\end{array}$ & $i_{g 2}=I_{g 2} \sin \left(\omega_{e} t+\phi_{g 1}\right)$ \\
\hline$T_{e_{-} f}$ & \begin{tabular}{l} 
Imaginary component of the rotor current: $i_{g 3}=i_{r \beta}$ \\
\hline
\end{tabular} & $i_{g 3}=I_{g 3} \sin \left(\omega_{e} t+\phi_{g 3}\right)$ \\
\hline
\end{tabular}




$$
\begin{gathered}
e_{T_{e}}=T_{\text {sta_inv }}+\underbrace{T_{2} \cos \left(2 \omega_{e} t+\phi_{T_{1}}\right)}_{T_{\text {dyn_inv }}} \\
e_{\left|\lambda_{s}\right|}=\lambda_{\text {sta_inv }}+\underbrace{\lambda_{2} \cos \left(2 \omega_{e} t+\phi_{\lambda_{1}}\right)}_{\lambda_{\text {dyn_inv }}}
\end{gathered}
$$

where $T_{\text {sta_inv }}$ and $\lambda_{\text {sta_inv }}$ are the static errors for the torque and flux-linkage, respectively,

due to the invisible effects. $T_{\text {dyn_inv }}$ and $\lambda_{\text {dyn_inv }}$ are the dynamic errors for the torque and flux, respectively, due to the invisible effects. The subscripts inv stands for the invisible effects of the ITSC on the DTC.

\subsubsection{Visible Impacts to DTC}

These effects can be observed by the controller. They are noticeable in the measured and estimated variables during the DTC operation. These effects are less intense than the non-observable ones, unless the fault is too severe, case in which the DTC will become unstable. The visible impacts are completely related with the DTC reaction as they are linked by the closed-loop control. However, its separation has been performed for understanding purposes. In the next section, the reaction of the DTC to the ITSC is analyzed starting with the visible effects and finalizing with the tolerance to the nonobservable ones.

The previous sub-section for the non-observable effects is valid only for the fundamental component of the motor supply voltage as no other components were considered. In DTC the action of the inverter commutation will introduce additional components in the motor supply voltages and currents, which must be taken into account. Therefore, it is important to predict the behavior of the two hysteresis controllers, sector detection and VSI state selection when the fault emerges. This problem is not easy to deal with in analytical terms 
due to the nature of the control and its sequential algorithm. Even though, an attempt to improve the analysis on [156] is presented.

Let's assume the IM is healthy and the DTC is running with a constant load and a fixed torque and flux-linkage amplitude references. In the fault onset, the first effect is the appearance of a negative sequence component (SC) and an additional quantity in the positive SC of the motor supply currents. The space-phasor of $\boldsymbol{i}_{\boldsymbol{s}}$ can be expressed as:

$$
\boldsymbol{i}_{\boldsymbol{s}}=\left|\bar{I}_{S P}\right| e^{j\left(\omega_{e} t+\phi_{i_{S P}}\right)}+\left|\bar{I}_{S N}\right| e^{-j\left(\omega_{e} t+\phi_{i_{S N}}\right)}
$$

where $\left|\bar{I}_{S P}\right|$ and $\left|\bar{I}_{S N}\right|$ are the amplitude of the positive and negative SC of the stator currents respectively. They are defined as in (6.23).

When the machine is healthy and fed with balanced voltages, then negative SC of the voltage $\bar{V}_{S N}=0, Y_{P N}=0, \bar{I}_{S N}=0$ and $\bar{I}_{S P}=Y_{P} \bar{V}_{S P}$. When the fault arises, $Y_{P N} \neq 0$ and assuming that the fault severity factor is small and DTC keeps balanced voltages, then $\bar{V}_{S N}=0$. Thus, $\bar{I}_{S P}=Y_{P P} \bar{V}_{S P}$, giving an extra amount in the positive SC $\Delta \bar{I}_{S P}=Y_{N P} \bar{V}_{S P}$ and the negative SC appears as $\bar{I}_{S N}=Y_{N P} \bar{V}_{S P}=\Delta \bar{I}_{S P}$. In case of a severe fault, the DTC voltages will be unbalanced and subsequently an increase in the positive and negative SC will happen.

\subsubsection{Effects on the Estimated Stator Flux-linkage}

Neglecting the stator resistance, the flux controller has no reason to react to this new condition. In that case, it will impose a sinusoidal flux at the fundamental supply electrical frequency $f_{e}$. The estimation of the flux before the DTC reaction will be: 


$$
\hat{\lambda}_{s}=\int v_{s} d t=\lambda_{r e f} e^{j\left(\omega_{e} t+\phi_{\lambda_{s}}\right)}
$$

Nonetheless, once the fault evolves and if the stator resistance is not negligible, the effect of $R_{S} \boldsymbol{i}_{\boldsymbol{s}}$ is significant. Thus, the flux-linkage will perceive some decrease in the positive SC and the appearance of a small negative SC. Then, the $\hat{\lambda}_{s}$ in space-phasor notation is as follows:

$$
\hat{\lambda}_{s}=\left|\bar{\psi}_{S P}\right| e^{j\left(\omega_{e} t+\phi_{\lambda_{S P}}\right)}+\left|\bar{\psi}_{s N}\right| e^{-j\left(\omega_{e} t+\phi_{\lambda_{S N}}\right)}
$$

\subsubsection{Effects on the Estimated Torque}

The estimated torque is given by (8.1) or equivalently by:

$$
\widehat{T}_{e}=\frac{3}{2} \frac{P}{2} \frac{L_{m}}{L_{s}} \operatorname{Im}\left\{\hat{\lambda}_{s}^{*} \boldsymbol{i}_{s}\right\}
$$

Introducing (8.11) and (8.13) in (8.14), $\widehat{T}_{e}$ is re-written as:

$$
\widehat{T}_{e}=\frac{3}{2} \frac{P}{2} \frac{L_{m}}{L_{s}}\left(T_{\text {const }}+T_{\text {dyn_vis }}\right)
$$

where $T_{\text {const }}$ and $T_{\text {dyn_vis }}$ are as in (8.16) and (8.17).

$$
\begin{gathered}
T_{\text {const }}=T_{\text {mech }}-T_{\text {sta_vis }}=\frac{\left|\bar{\psi}_{S P}\right|\left|\bar{I}_{S P}\right| \sin (\vartheta)-\left|\bar{\psi}_{S N}\right|\left|\bar{I}_{S N}\right| \sin (\varsigma)}{\frac{4 L_{S}}{3 P L_{m}}} \\
T_{\text {dyn_vis }}=\frac{\left|\bar{\psi}_{S P}\right|\left|\bar{I}_{S N}\right| \sin \left(2 \omega_{e} t+\zeta\right)-\left|\bar{\psi}_{s N}\right|\left|\bar{I}_{S P}\right| \sin \left(2 \omega_{e} t+\gamma\right)}{\frac{4 L_{S}}{3 P L_{m}}}
\end{gathered}
$$

where $\vartheta, \varsigma, \zeta$ and $\gamma$ are the angle difference between the respective positive and negative SC of the $\boldsymbol{i}_{s}$ and $\hat{\boldsymbol{\lambda}}_{\boldsymbol{s}}$. 
Three terms appear: (1) an electromechanical torque doing work $\left(T_{\text {mech }}\right),(2)$ a static error ( $\left.T_{\text {sta_vis }}\right)$ and (3) a dynamic error at double electric frequency $\left(T_{\text {dyn_vis }}\right)$. The subscripts vis stands for the visible effects of the ITSC on the DTC.

The total impacts on electromagnetic torque have two non-observable components in the actual torque described by $e_{T_{e}}$ : a static error $\left(T_{\text {sta_inv }}\right)$ and a dynamic error $\left(T_{\text {dyn_inv }}\right)$ at twice the supply voltage frequency $\left(2 \omega_{e}\right)$. Additionally, from (8.15) two visible components appears: a constant term $\left(T_{\text {sta_vis }}\right)$ and a double frequency component $\left(T_{\text {dyn_vis }}\right)$. Thus the total oscillating torque component is:

$$
T_{\text {osc }}=T_{\text {dyn_inv }}+T_{\text {dyn_vis }}
$$

\subsection{Reaction of the DTC to the Asymmetric IM}

The reaction of the DTC to the stator turn fault is twofold: DTC will compensate the visible impacts of the ITSC and at the same time it will tolerate the non-observable disturbances.

\subsubsection{DTC Compensation to the Visible Effects}

It is known that the torque and flux controllers force the estimated errors to lie within the hysteresis bands around the references $\left(T_{r e f}\right.$ and $\left.\lambda_{r e f}\right)$. So, the estimated flux follows the reference no matter the condition and the controller cancels any flux's negative SC. This flux's negative SC was originated by the stator resistance in the flux estimation. Thus, the control system has to introduce a negative flux SC equal to $\lambda_{s_{-} \text {comp }}=$ $-\left|\bar{\psi}_{s N}\right| e^{-j\left(\omega_{e} t+\phi_{\lambda_{S N}}\right)}$ and the estimated flux will follow the flux reference as (8.19) and $\left|\bar{\psi}_{s P}\right|=\lambda_{\text {ref }}$ 


$$
\hat{\lambda}_{s}=\lambda_{r e f} e^{j\left(\omega_{e} t+\phi_{\lambda_{S P}}\right)}
$$

On the other hand, the estimated torque has to track the reference in every situation as well. The control eliminates any visible torque oscillations ( $\left.T_{\text {dyn_vis }}\right)$ as shown in (8.17). Consequently, the control system must introduce a torque component equal to $T_{\text {dyn_comp }}=-T_{\text {dyn_vis }}$. By the analysis of $(8.14)$ it is concluded that [156]:

1) The introduction of a negative $\mathrm{SC}$ in the stator flux and consequently in the motor supply voltages.

2) The introduction of a positive $\mathrm{SC}$ in the stator flux linkage at a frequency of $3 f_{e}$. Before analyzing these two possibilities, let's study the consequences of the flux cancellation. When the controller removes the negative $\mathrm{SC}$ in the flux by injecting $\lambda_{s_{-} \text {comp }}$, part of the torque oscillations will also disappear. In this case the second term in (8.17) written as $\left|\bar{\psi}_{s N}\right|\left|\bar{I}_{S P}\right| \sin \left(2 \omega_{e} t+\gamma\right)$ will no longer exist. The torque visible dynamic errors that the controller needs to compensate is $T_{\text {dyn_comp }}=-T_{\text {dyn_vis }}:$

$$
T_{\text {dyn_vis }}=\frac{3}{2} \frac{P}{2} \frac{L_{m}}{L_{s}}\left|\bar{\psi}_{s P}\right|\left|\bar{I}_{S N}\right| \sin \left(2 \omega_{e} t+\zeta\right)
$$

Cruz et al. [156] explains, and corroborated here, that the maximum allowed amplitude of the negative SC needed in the stator flux to compensate the torque oscillations is:

$$
\left|\bar{\psi}_{s N}\right| \approx \frac{H B_{\lambda}}{2 \lambda_{\text {ref }}}\left|\bar{\psi}_{s P}\right|
$$

From (8.21), there is not too much room for introducing a flux and voltage's negative $\mathrm{SC}$ as this is limited by hysteresis band of the flux controller. So, the system has to find an alternative to compensate the torque oscillations. 
The other possibility is that the controller produces a variation of the angle difference between the stator and the rotor flux $\left(\phi_{\lambda_{s}}-\phi_{\lambda_{r}}\right)$ at the same frequency of $2 f_{e}$. This is equivalent to consider a stator flux linkage as:

$$
\hat{\lambda}_{s}=\lambda_{r e f} e^{j\left(\omega_{e} t+\phi_{\lambda_{S P}}+a \cos \left(2 \omega_{e} t+\xi\right)\right)}
$$

A flux-linkage like this will inject third harmonic components in the flux, however, smaller fifth and seventh harmonics are included as well. This injection of third harmonics can be done without major restrictions.

In this work, we agreed with the research [156] in disregarding option 1 and the hypothesis of a third harmonic injection in the stator flux, however, it is not clear why the control is able to modify the angle difference $\left(\phi_{\lambda_{s}}-\phi_{\lambda_{r}}\right)$. An alternative explanation would be the injection of zero SC in the supplied voltage instead of controlling $\phi_{\lambda_{s}}-\phi_{\lambda_{r}}$. In DTC there is not neutral connection in the VSI, this virtual center can be freely moved by injecting zero SC in the voltages. This zero SC can develop a third order harmonic in the voltage and thus in the flux-linkage with no restriction as stipulated by [156].

\subsection{Robustness of the DTC to the Asymmetries}

It is well-known that DTC allows certain amount of errors and approximations, such as: measurements and acquisition problems, estimation errors and uncertainty in the stator resistance parameter $\left(R_{S}\right)$. The DTC tolerance is analyzed in to parts: (1) from the standard heuristic derivation of the DTC and (2) from point of view of non-linear control theory.

\subsubsection{From the Standard Heuristic derivation of the DTC}

In a healthy IM, these uncertainties, errors and approximation are overcome due to the DTC robustness, which is based on its finite-set control action and the no requirement of 
exact flux position. DTC only needs the correct sector in which it lies inside the locus in the flux-linkage plane (Figure 8.2). Each DTC sector can be qualitatively divided as: safe and critical regions. The safe region lies in the vicinity of the sector's center or when $\lambda_{\boldsymbol{s}}$ is far from the sector's borders. The critical region is close to the sectors' limits and it is where DTC tends to select an incorrect control action. When $\lambda_{s}$ lies in this critical region, small measurement or estimation error could lead to a wrong sector identification. So, the selection of a control action will incur in higher deviation from the torque and flux-linkage magnitude references.

Assume that at the instant of control $k$, the torque demand $(\tau)$ is required to increase (acceleration action - torque is lower than the reference) and $\boldsymbol{\lambda}_{\boldsymbol{s}}$ (in purple) is as shown in Figure 8.3 . Let's also assume that $\lambda_{s}$ lies in the sector 2 (at point $P_{0}$ ) in the vicinity of the critical region. For errors of estimation, $\hat{\lambda}_{s}$ (in blue) is evaluated in sector 1 (at point $P_{0 \prime}$ ). In Figure 8.3 three flux paths are presented: (1) desired path (red straight lines in Figure 8.3(a)) is from $P_{0} \rightarrow P_{1 \prime \prime} \rightarrow P_{2 \prime \prime} \rightarrow P_{3 \prime \prime}$. In this track $\lambda_{s}$ follows the reference. (2) The real path (purple lines in Figure 8.3(b)) is from $P_{0} \rightarrow P_{1} \rightarrow P_{2} \rightarrow P_{3}$. In this path $\lambda_{s}$ follows the reference with an offset and (3) Estimated path (blue lines in Figure 8.3(b)) from $P_{0} \rightarrow$ $P_{1} \rightarrow P_{2 \prime} \rightarrow P_{3 \prime}$. In this path $\hat{\lambda}_{s}$ follows the reference with some small errors that are corrected once it is in the same sector as $\lambda_{s}$ (safe region). The detailed sequence of events and VSI control selection action is as follows:

1. Initial state at instant of control $t_{k}: \lambda_{s}$ is in sector 2 and $\hat{\lambda}_{s}$ is in sector 1 .

2. As the magnitude of $\hat{\lambda}_{s}$ is greater than $\lambda_{\text {ref }}$ then the flux demand $\psi$ is set to decrease. From Table 8-1: as $\lambda_{s}$ is in sector 2, the action that decreases flux and increases torque 
demand is $\boldsymbol{v}_{\boldsymbol{s} \mathbf{6}}$. As $\hat{\boldsymbol{\lambda}}_{\boldsymbol{s}}$ is in sector 1 , the action that decreases flux and increases torque demand is $\boldsymbol{v}_{\mathbf{s} \mathbf{2}}$. The correct action that decreases flux and increases torque demand is $v_{s 6}$ for $\lambda_{s}$.

3. Thus: $\hat{\lambda}_{s}$ pass from $P_{0}$, to point $P_{1}$. This results in an erratic control action as $\hat{\lambda}_{s}$ is in the wrong sector compared to the actual $\lambda_{s} . \lambda_{s}$ pass from $P_{0}$ to point $P_{1}$. In reality $\lambda_{s}$ is increased from $P_{0}$ toward the point $P_{1}$ and now the flux-linkage in control instant $\left(t_{k+1}\right)$ is larger than in control instant $\left(t_{k}\right) \cdot \lambda_{s}$ should pass from $P_{0}$ to point $P_{1 \prime \prime}$ (desired path Figure 8.3(a)).

4. At control instant $\left(t_{k+1}\right), \hat{\lambda}_{s}$ is still greater than the reference but now is in sector 2 (safe region).

5. Thus, control action vector $\boldsymbol{v}_{s 6}$ is selected: $\hat{\lambda}_{s}$ pass from $P_{1}$, to point $P_{21}, \lambda_{s}$ pass from $P_{1}$ to point $P_{2}$. For the ideal case, the control action at $t_{k+1}$, should be $\boldsymbol{v}_{s 2}$, thus $\lambda_{s}$ pass from $P_{1 \prime \prime}$ to point $P_{2 \prime \prime}$.

6. The path of $\hat{\lambda}_{s}$ and $\lambda_{s}$ continues in the same way choosing $\boldsymbol{v}_{\boldsymbol{s} \mathbf{2}}$ and $\boldsymbol{v}_{\boldsymbol{s} \mathbf{6}}$. See Figure 8.3(b).

Even when the control makes some wrong decisions, once $\hat{\lambda}_{s}$ pass from sector 1 to sector 2 (safe region) the action of control $\boldsymbol{v}_{\boldsymbol{s} 6}$ is selected partially correcting the control path. The drawback is that a pseudo reference $\lambda_{\text {ref }}+\Delta \lambda_{\text {offset }}$ is followed instead of $\lambda_{\text {ref }}$. Figure 8.3(b) has been exaggerated for the explanation purposes, the offset will be small when compared with $\lambda_{\text {ref }}$ as $f_{s}$ is high. Thus, the control will keep operation close to the desired operative point. 

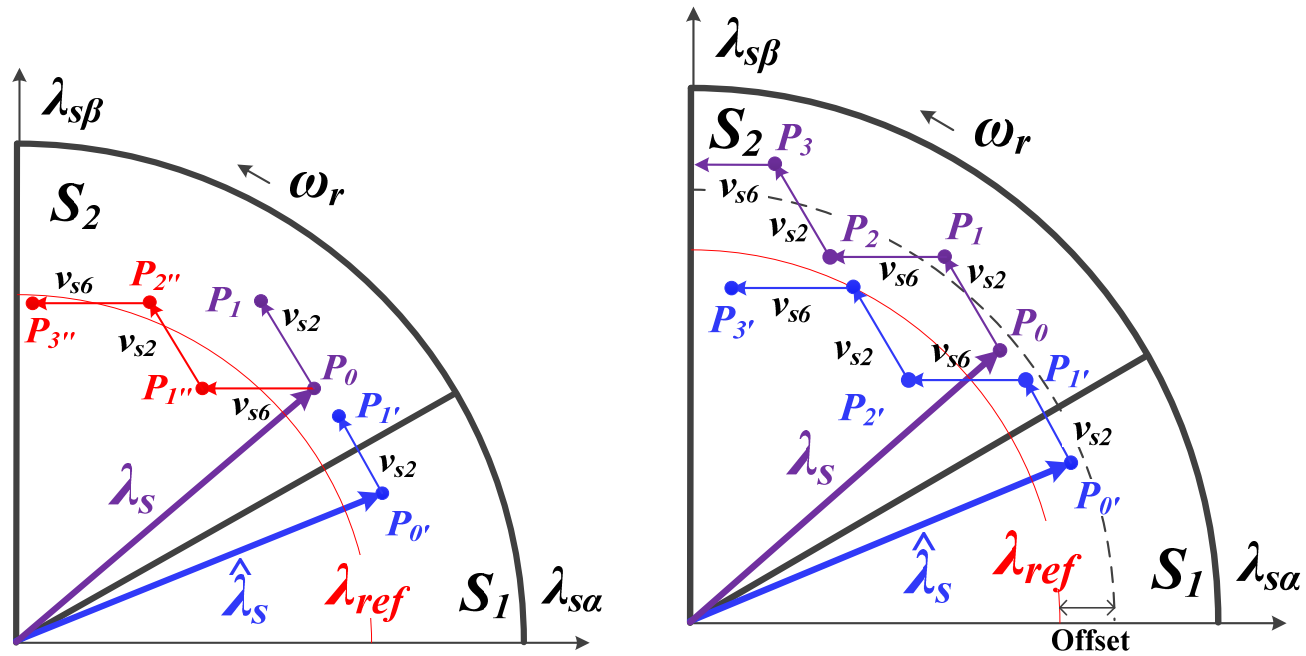

Figure 8.3 Stator flux-linkage locus and VSI switching vectors. (a) Desired path (b) real and estimated path.

\subsubsection{From the point of view of non-linear control theory}

DTC is categorized as hysteresis-based predictive control (PC) [63] and it can be explained using its foundations. Based on PC theory [73] and for the particular case of the damaged IM, it is possible to anticipate the error of the output, $\boldsymbol{y}=\left[T_{e},\left|\boldsymbol{\lambda}_{\boldsymbol{s}}\right|\right]$, in the next cycle of control $\left(t_{k+1}\right)$, as the output has a relative degree $\{1,1\}$, which is defined everywhere except at $\left|\lambda_{\boldsymbol{r}}\right|=\mathbf{0}[117]$, [118]. This prediction can be computed based on the time derivatives of the two outputs along the dynamics. Thus, using the two SS models presented in (6.12) and (6.17) the trajectories of constant torque and flux amplitude are obtained as in (8.23).

$$
\begin{aligned}
& p T_{e}=p\left(\frac{P}{2} \lambda_{s} \times i_{s}+\mu \frac{P}{2} L_{m} i_{f} i_{r \beta}\right)=0 \\
& p\left|\lambda_{s}\right|=\frac{1}{\left|\lambda_{s}\right|}\left(\lambda_{s \alpha} p \lambda_{s \alpha}+\lambda_{s \beta} p \lambda_{s \beta}\right)=0
\end{aligned}
$$

Writing (6.17) in more compact form: 


$$
\left[\dot{\boldsymbol{x}}_{\boldsymbol{i}}\right]=\left[\begin{array}{l}
{\left[A_{i 1}\right]} \\
{\left[A_{i 2}\right]} \\
{\left[A_{i 3}\right]} \\
{\left[A_{i 4}\right]} \\
{\left[A_{i 5}\right]}
\end{array}\right]\left[\boldsymbol{x}_{\boldsymbol{i}}\right]+\left[\begin{array}{l}
{\left[B_{i 1}\right]} \\
{\left[B_{i 2}\right]} \\
{\left[B_{i 3}\right]} \\
{\left[B_{i 4}\right]} \\
{\left[B_{i 5}\right]}
\end{array}\right][\boldsymbol{u}]
$$

where $\left[A_{i n}\right]$ is the $\mathrm{n}$ row of the matrix $\left[\mathbf{A}_{\boldsymbol{i}}\right],\left[B_{i \mathbf{n}}\right]$ is the $\mathrm{n}$ row of the matrix $\left[\mathbf{B}_{\boldsymbol{i}}\right]$. Thus, each variable of $\left[\dot{\boldsymbol{x}}_{\boldsymbol{i}}\right]$ is as $(8.25)$.

$$
\dot{x}_{i \mathrm{n}}=\left[A_{i \mathrm{n}}\right]\left[\boldsymbol{x}_{\boldsymbol{i}}\right]+\left[B_{\text {in }}\right][\boldsymbol{u}]
$$

where $\mathrm{n}$ can go from 1 to 5 .

The two trajectories that correspond to the constant torque variation $v_{s \beta_{T}}\left(v_{s \alpha_{T}}\right)$ and constant amplitude of the stator flux-linkage variation $v_{s \beta_{\lambda}}\left(v_{s \alpha_{\lambda}}\right)$ are as in (8.26).

$v_{s \beta_{T}}=\frac{K_{\alpha_{T}} v_{s \alpha_{T}}-K_{T}}{K_{\beta_{T}}}$
$v_{s \beta_{\lambda}}=R_{s} i_{s \beta}-\frac{\lambda_{s \alpha}}{\lambda_{s \beta}}\left(v_{s \alpha_{\lambda}}-R_{s} i_{s \alpha}-\frac{2}{3} \mu R_{s} i_{f}\right)$

Under the assumption of a healthy IM, the inverter voltage plane (IVP), which is the red-dashed hexagon, is located at the estimated stator flux-linkage $\left(\hat{\lambda}_{s}=\lambda_{s}\right)$ as shown in Figure 8.4(a). Figure 8.4(a) shown another set of trajectories: the actual ones in purple. Each trajectory divide the IVP space in two subspaces. As shown in Figure 8.4(a), generally these two trajectories are not perpendicular $(\phi \neq \pi / 2)$ and they do not intersect in the origin of the IVP, thus four irregular quadrants are created in every control cycle. This irregularity can generate an unbalanced number of input VSI vectors for each of the 4 subspaces. In classical DTC it is assumed that these trajectories are perpendicular $(\phi=$ 
$\pi / 2$ ) and their origin lies on $\hat{\lambda}_{s}$ (see blue trajectories in Figure 8.4(a)). This means that $\left|\lambda_{s}\right|$ and $T_{e}$ are decoupled and even number of inputs are in each quadrant.
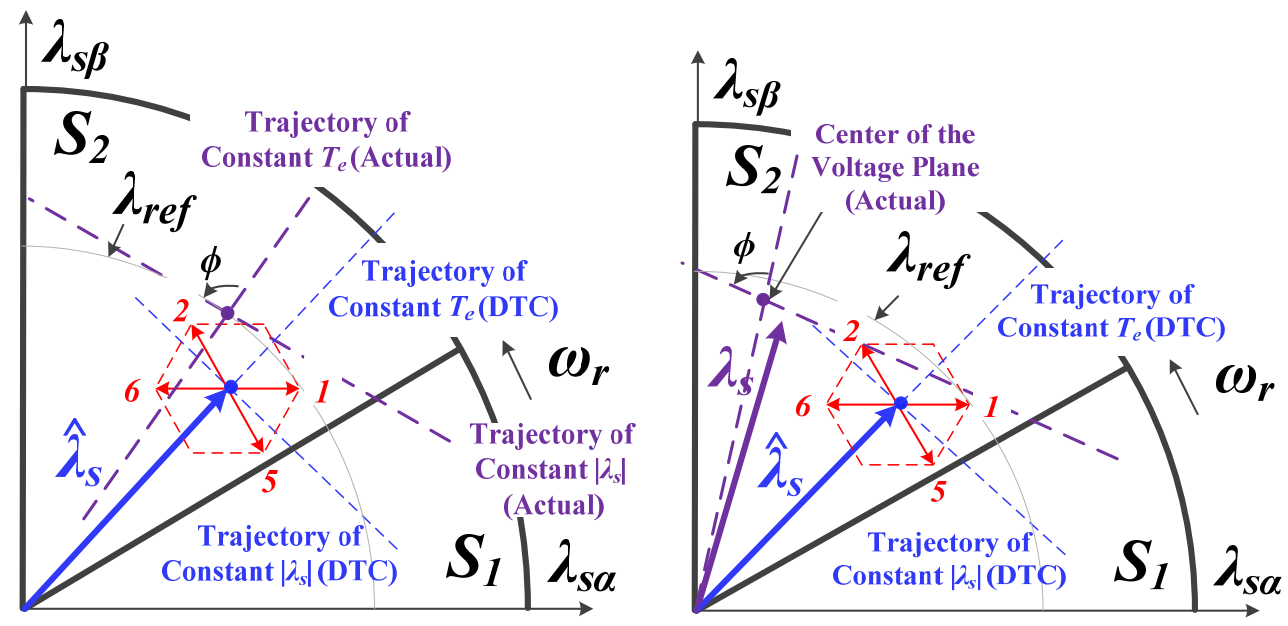

Figure 8.4 (a) Real case of IVP in case of standard DTC with healthy IM and (b) real case of IVP case of standard DTC with faulty IM.

In the fault onset the estimated stator flux-linkage is different from the actual (see Figure 8.4(b)) and from (8.26) it is clear that the both trajectories of constant $T_{e}$ and $\left|\lambda_{s}\right|$ are affected by $i_{f}$. One time segment is plotted in Figure 8.4(b) where $\lambda_{s}$ (purple) is depicted against the $\hat{\lambda}_{s}$ (blue). In this figure the plot indicates the real situation where the DTC keeps operation under the estimated value $\hat{\lambda}_{s}$ and thus the IVP origin is placed on it. However, $\lambda_{s}$ and the actual IVP lies in other places. Notice that the constant trajectory of $\lambda_{s}$ is not perpendicular to the $\lambda_{s}$ forming an angle $\phi<\pi / 2$. The constant trajectory of $T_{e}$ is as well neither perpendicular to the constant trajectory of $\lambda_{\mathrm{s}}$ nor parallel to $\lambda_{s}$.

An analytical comparison of the actual trajectories versus the DTC trajectories for the both cases of healthy and faulty IM is complex. Therefore, simulations using (8.26) are required to aim this analysis. To conclude this section, it has been stablished that the normal 
erratic behavior of DTC becomes even worse at the appearance of ITSC. However, in the

previous subsection it was explained that while $\hat{\lambda}_{\boldsymbol{s}}$ lies in the same sector as $\lambda_{\boldsymbol{s}}$ the control can be hold $\left|\lambda_{s}\right|$ to the reference.

\subsection{Simulation and Experimental Results}

The simulation and experimental test are performed for a 1 HP IM Weg. Table 7-3 shows the nameplate ratings of the motor and the IM parameters. Figure 8.5 shows the experimental test platform. The IM shaft is mechanically coupled to a $250 \mathrm{~W}$ and $42 \mathrm{~V}$ dc permanent magnet (PM) machine. The dc PM machine is acting as a generator with variable load connected to the armature to emulate the mechanical loading conditions. The sensor board is based on LEM voltage and current transducers and a dSpace 1104 board is used for the control. The motor angular speed is measured with a 1000 ppr coupled to the PM machine. For all simulation and experimental tests, the IM is driven by DTC with $T_{\text {ref }}=2 \mathrm{Nm}$ and $\lambda_{\text {ref }}=0.56 \mathrm{~Wb}$. The start-up is from stand-still until reaching the maximum speed with $V_{D C}=300 \mathrm{~V}$ in the dc-link of the VSI. The IM has enough load to achieve the reference torque with $R_{f}=1 \Omega$. Seven cases of ITSC are studied between simulations and experimental results and they are shown in Table 8-3. The first case (c1) is the healthy IM not shown in Table 8-3.

\subsubsection{Simulation Results}

For the simulation results, two cases of ITSC are shown: case 2 (c2) has a $\mu=2.68 \%$ and case 4 (c4) $\mu=12.01 \%$ (see Table 8-3). The transient (start-up) and steady-state performance are shown. The entire time for the test is $2 \mathrm{sec}$, however, for purposes of better plotting, 0.2 sec. is shown in some curves. The simulation integration step time is $10^{-5} \mathrm{sec}$. 
Table 8-3 ITSC Test Cases for the Faulty IM.

\begin{tabular}{cccc|cccc}
\hline \hline ITSC & $\boldsymbol{N}_{\text {as } 2}$ & $\boldsymbol{\mu ( \% )}$ & Cases & ITSC & $\boldsymbol{N}_{\boldsymbol{a s} 2}$ & $\boldsymbol{\mu ( \% )}$ & Cases \\
\hline$a_{1}-a_{2}$ & 14 & 2.68 & $\mathrm{C} 2$ & $a_{1}-a_{6}$ & 108 & 20.9 & $\mathrm{C} 5$ \\
$a_{1}-a_{3}$ & 27 & 5.55 & $\mathrm{C} 3$ & $a_{2}-a_{7}$ & 167 & 31.39 & $\mathrm{C} 6$ \\
$a_{2}-a_{4}$ & 64 & 12.01 & $\mathrm{C} 4$ & $U_{5}-a_{8}$ & 194 & 38.2 & $\mathrm{C} 7$ \\
\hline
\end{tabular}

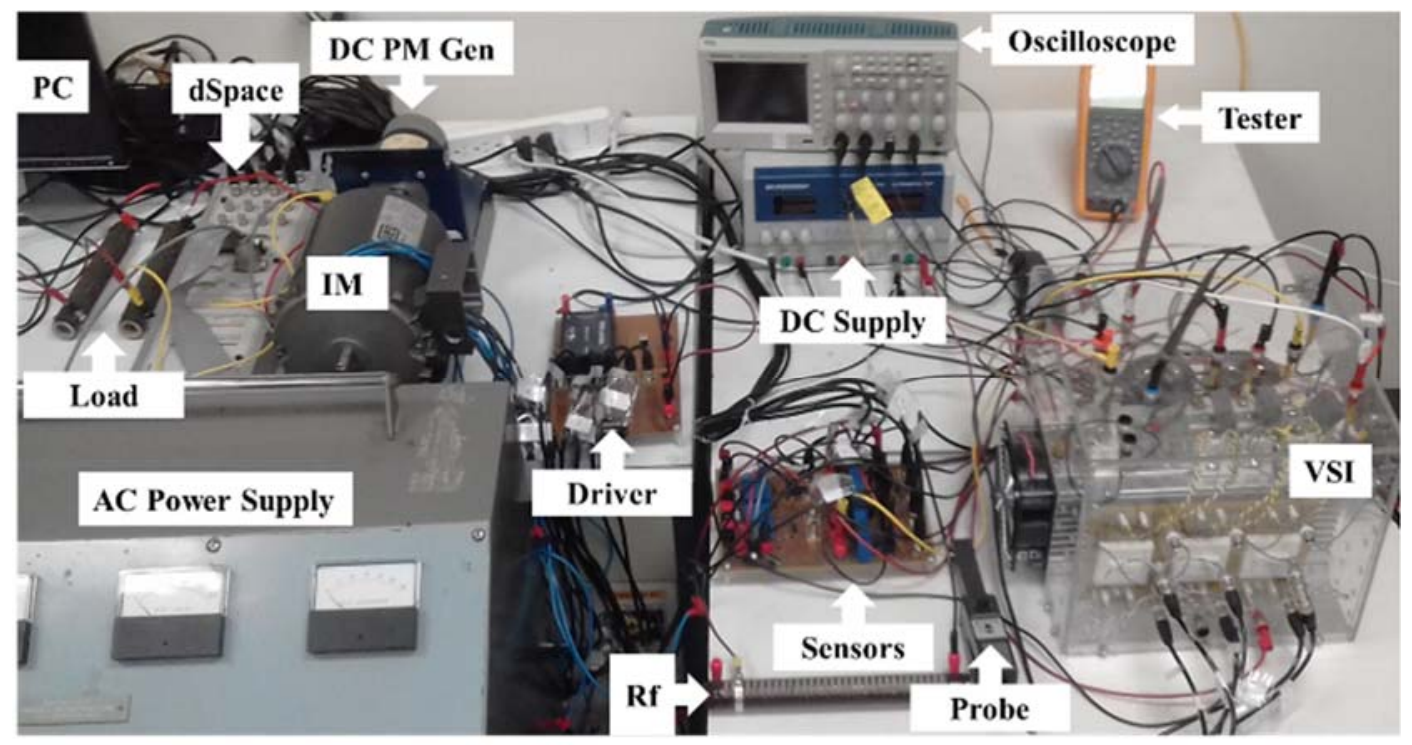

Figure 8.5 Experimental setup for acquisition with a dSpace 1104.

The simulation comprises the comparison of the estimated and actual variables $\left(\widehat{T}_{e}\right.$ and $\left|\hat{\lambda}_{s}\right|$ versus $T_{e}$ and $\left.\left|\lambda_{s}\right|\right)$ for the two cases of study. Figure 8.6(a) presents the amplitudes of the stator fluxes $\left(\left|\hat{\lambda}_{s}\right|\right.$ and $\left.\left|\lambda_{s}\right|\right)$ and the $\lambda_{\text {ref }}$ in red, where a zoom over a period of the signal shows the following: (1) both estimated fluxes for the cases c2 and c4 are overlapped with the actual flux $\mathrm{c} 2$ and tracking $\lambda_{\text {ref }}$. This means that the control system is compensating the visible effects on the estimated flux in both cases. For $\mathrm{c} 2$, the invisible impacts are not strong as the fault severity is low and thus $\left|\lambda_{\boldsymbol{s}}\right|$ is similar to the estimated ones. (2) In the 
case of $\mathrm{c} 4$, there is a significant difference between actual (black and dashed) and estimated flux (orange). In this case the dynamic error at the double frequency can be clearly observed in the $\left|\lambda_{s}\right|$. This oscillation is a non-observable impact, as the DTC depicts $\left|\hat{\lambda}_{s}\right|$ (orange).

Figure 8.6(b) illustrates the new index of the flux error $\left(e_{\left|\lambda_{s}\right|}\right)$ between the estimated and actual stator flux-linkage amplitude. Same observations as in Figure 8.6(a). The flux error in the case $\mathrm{c} 2$ indicates no dynamic error appearance while in the case $\mathrm{c} 4$ a double frequency term appears as predicted in Section 8.3.

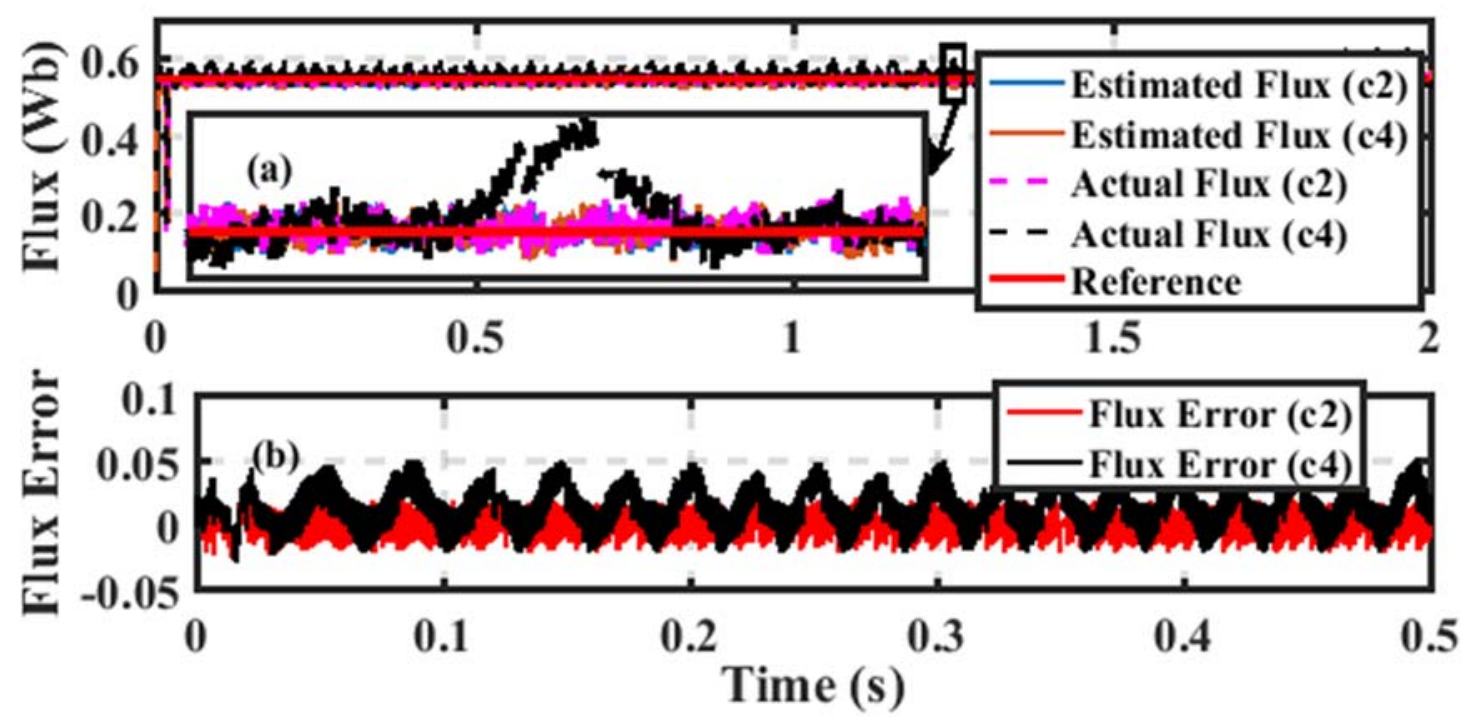

Figure 8.6 (a) Estimated and actual stator flux amplitude for the two cases of study (c2 and c4). (b) Error of flux estimation of both cases. Simulation Results.

Figure 8.9(a) shows torque (estimated and actual) comparison for the two cases of study. The estimation problem can be seen, where $\widehat{T}_{e}$ for $\mathrm{c} 2$ and $\mathrm{c} 4$ track the reference together with $T_{e}$ in $\mathrm{c} 2$ whilst $T_{e}$ in $\mathrm{c} 4$ does not. Similar situation as in Figure 8.6(a). In the same context, Figure 8.9(b) illustrates the new index of torque error $\left(e_{T_{e}}\right)$ between $\widehat{T}_{e}$ and $T_{e}$. The torque error in the case $\mathrm{c} 2$ indicates no dynamic component generation while in the 
case $\mathrm{c} 4$ a $2 f_{e}$ term shows up as predicted in section III.

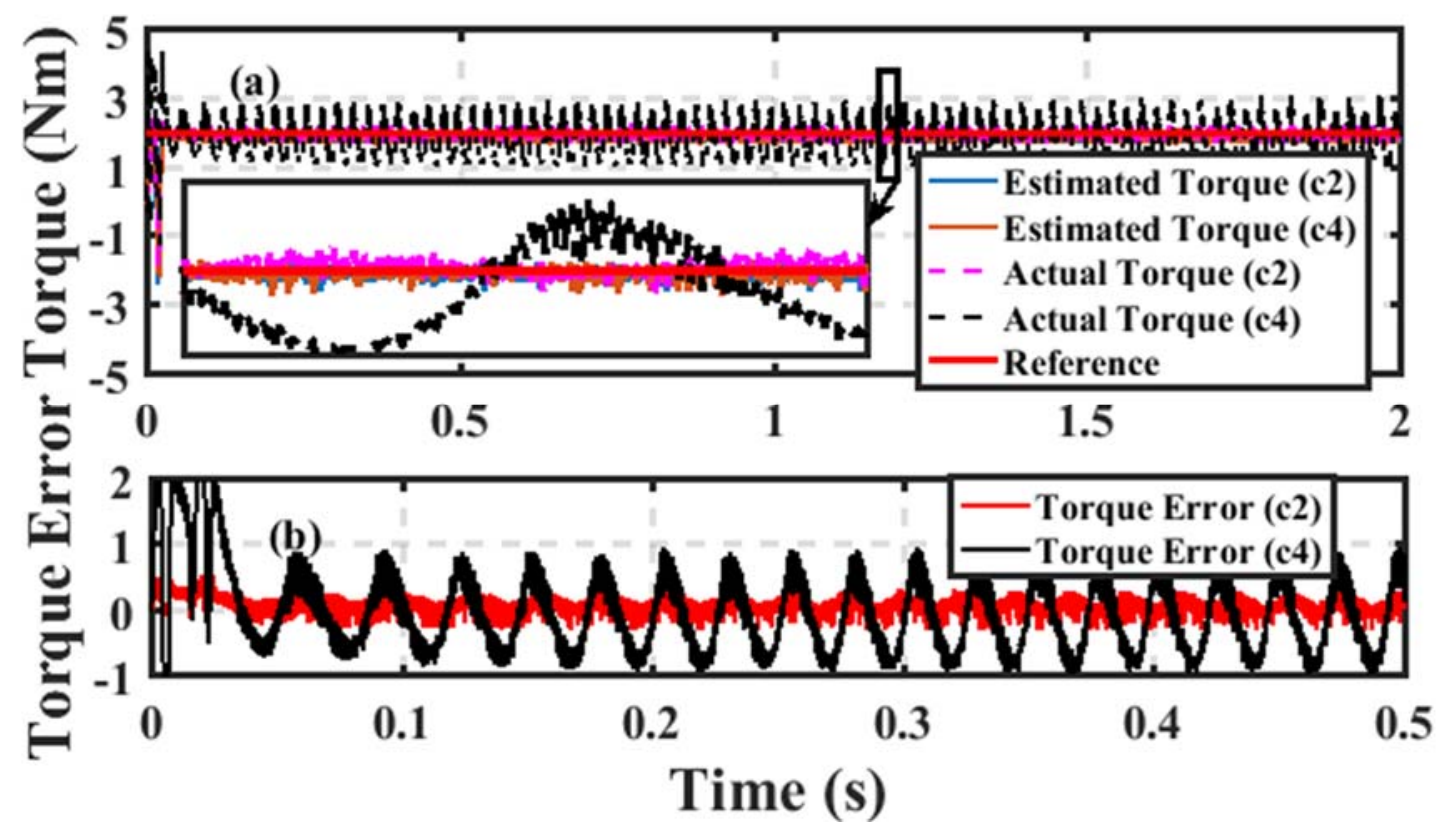

Figure 8.7 (a) Estimated and actual electromagnetic torque the two cases of study (c2 and c4). (b) Error of torque estimation for both cases. Simulation Results.

The leakage fault current increases with $\mu$, as it is shown in Figure 8.8, and expected from the literature. The mechanical speed is inversely proportional to $\mu$. The fault circuit consumes power, and to balance the power equation the speed varies inversely proportional to the power consumption whilst keeping the torque constant. Notice also the doublefrequency noise for $\mathrm{c} 4$.

In Figure 8.9, the three-phase currents indicate that for $\mathrm{c} 4$ there is an unbalanced situation that can be explained by the hypothesis mentioned in Section 8.4. 


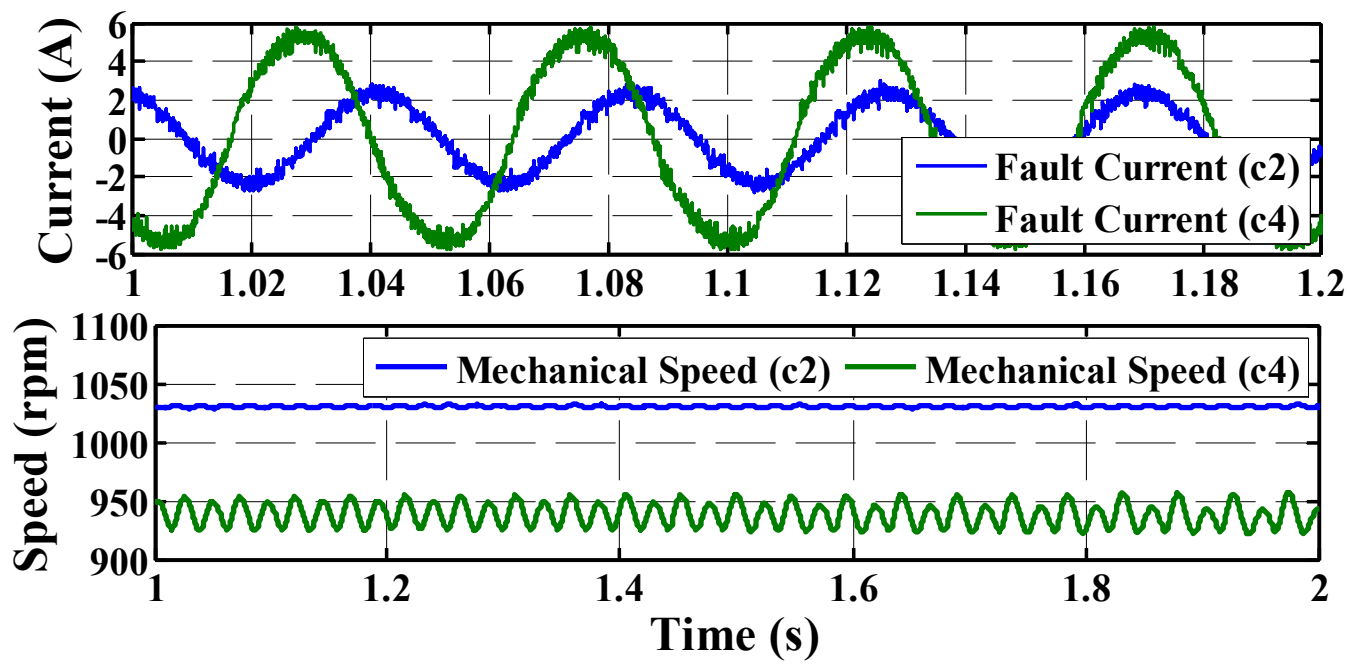

Figure 8.8 (a) Fault Currents and (b) Mechanical speed for the two cases of study (case 1 and 2). Simulation Results.

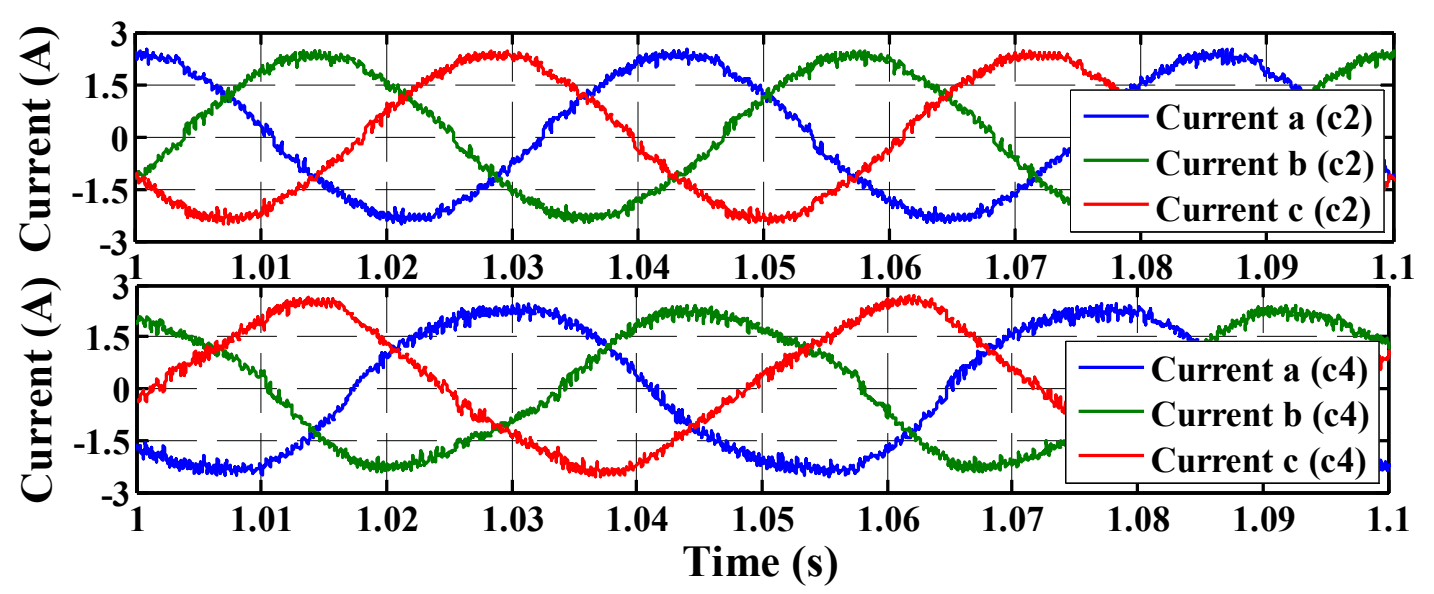

Figure 8.9 Stator three-phase currents for the two cases of study. (a) case 1 and (b) case 2. Simulation Results.

\subsubsection{Experimental Results}

Two analyses are performed: transient and steady state. The dynamic inspection considers two faulty cases: case 2 (c2) and case 7 (c7) (see Table 8-3). The steady state examination studies all 7 cases of ITSC. The first case is the healthy IM and the second and fourth were previously simulated. The acquisition and control frequency is $10 \mathrm{kHz}$. 
The time scoped for the results is 8 seconds. These results present the study of the visible effects.

\subsubsection{Transient Performance Examination of the Visible Effects}

For the transient examination the two most extreme cases are selected to demonstrate the visible effect of the DTC on the control estimated variables under small and severe ITSC. From the point of view of DTC, the estimated flux (Figure 8.10) and torque (Figure 8.11) are clearly not affected by the ITSC, however, from the zoom of both figures it can be noticed that at the seveth second, the control suffers a instability in the control for $\mathrm{c} 7$. In the estimated flux in Figure 8.10 a notch spike appears and in the estimated torque an small increase on the average value is noticed. Nevertheless, during the remainig time, the estimated variables still are virtually controlled and tracking the reference.

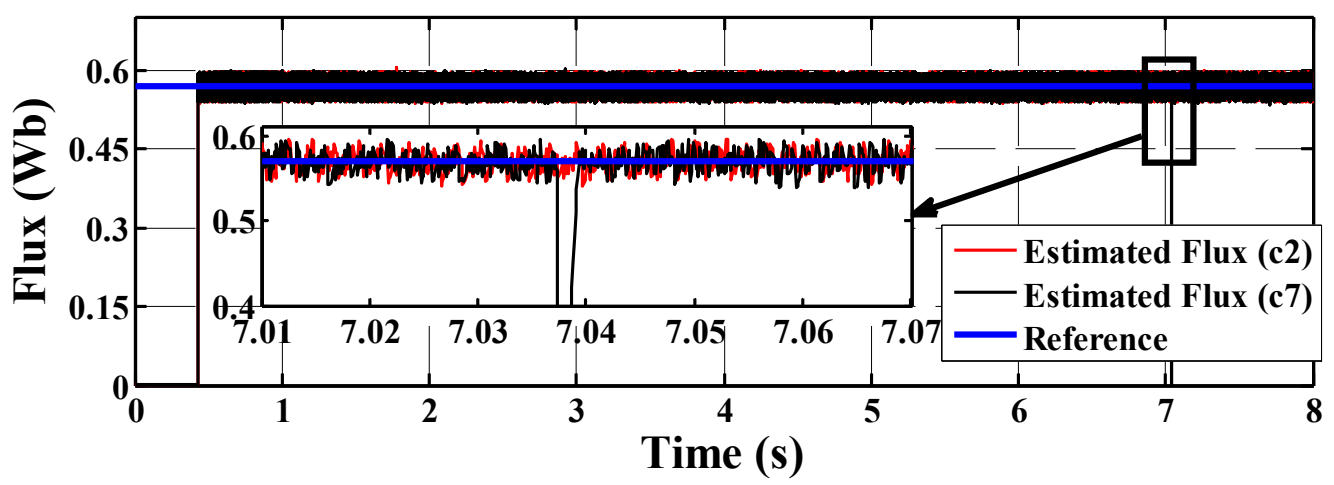

Figure 8.10 Estimated flux-linkage for the two cases of study (c2 and c7). Experiemntal results. 


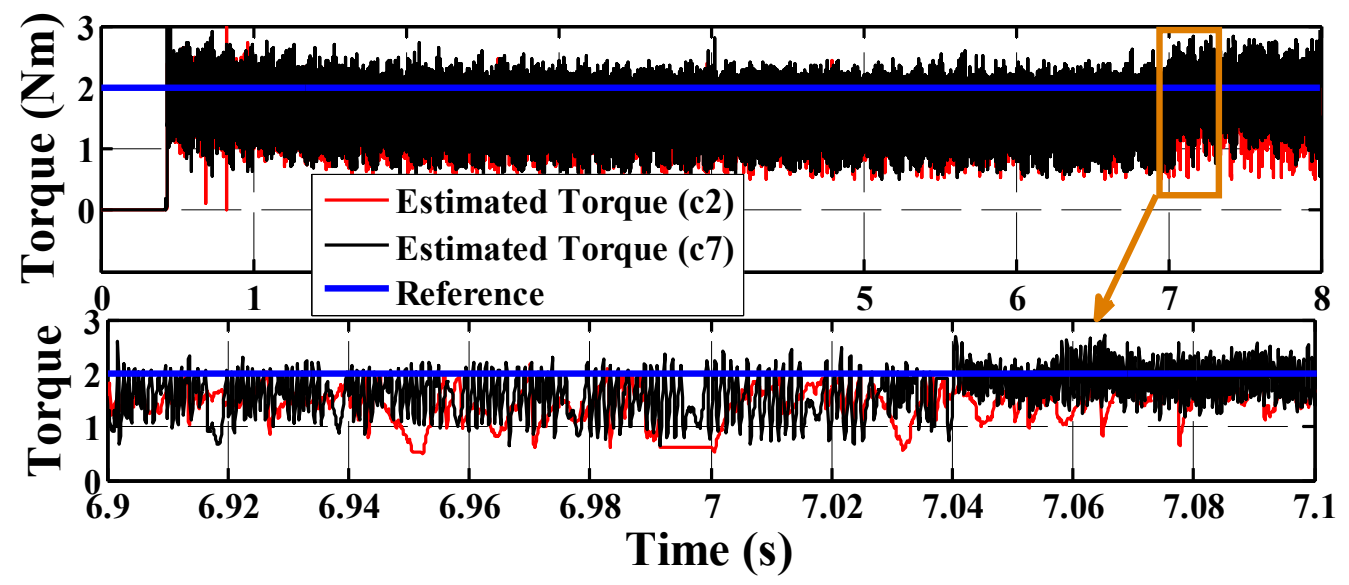

Figure 8.11 Estimated torque for the two cases of study (c2 and c7). Experiemntal results.

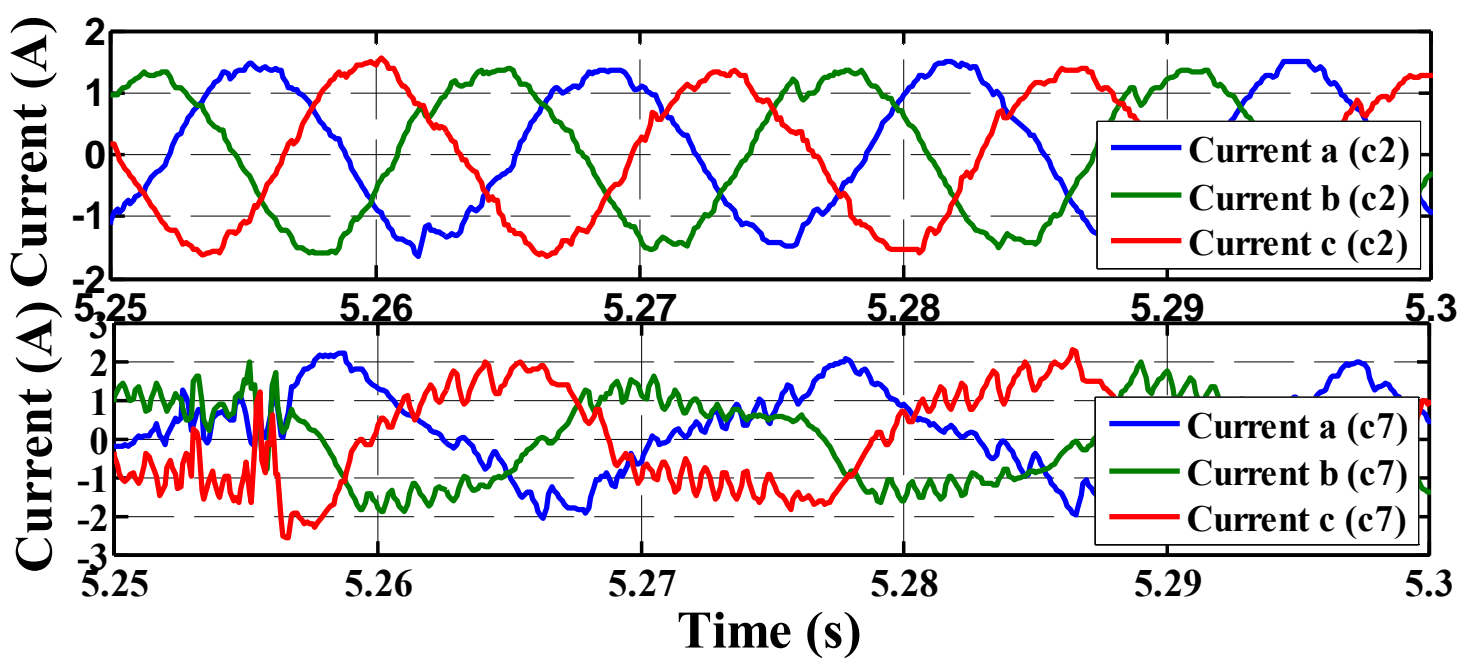

Figure 8.12 Stator three-phase currents for the two cases of study (case 2 and 7). Experimental Results. 


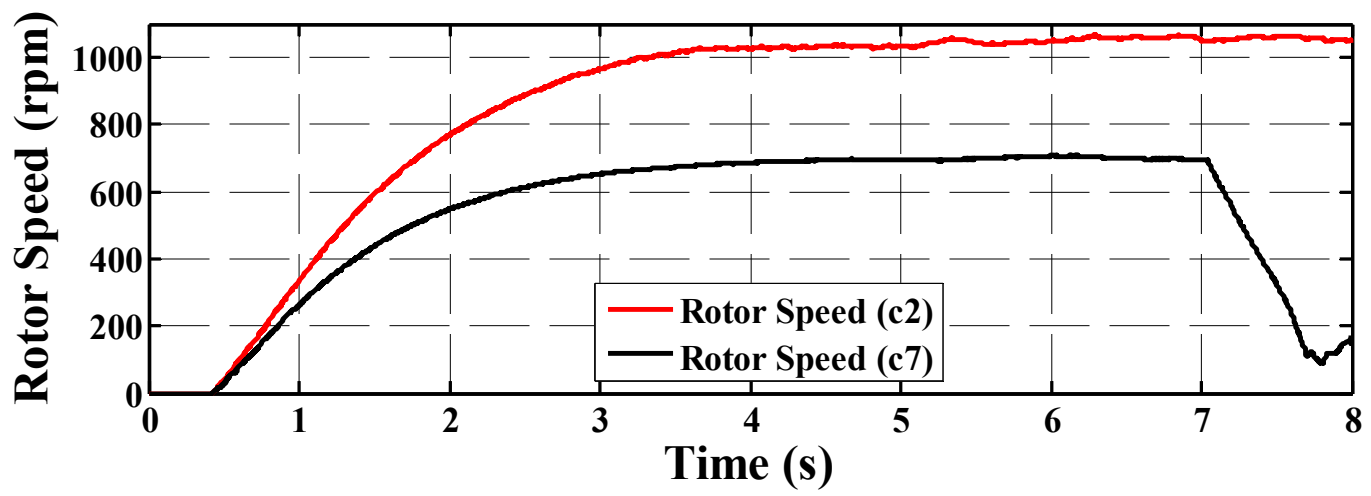

Figure 8.13 Rotor mechanical speed for the two cases of study (case 2 and 7). Experimental Results.

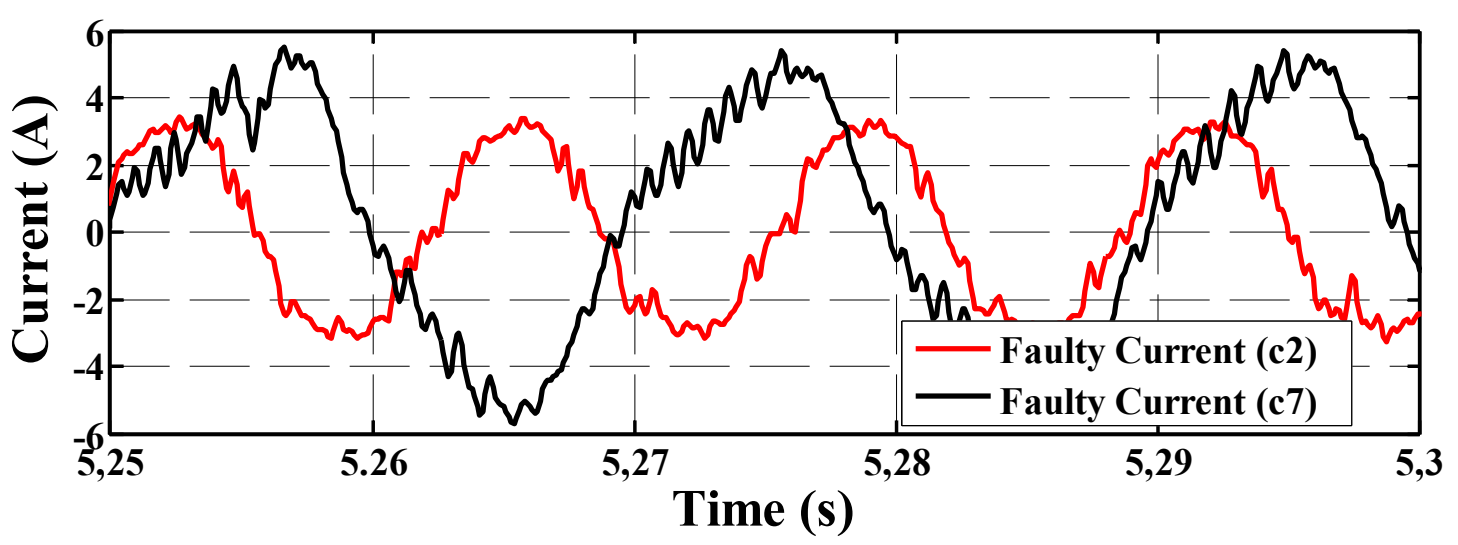

Figure 8.14 Fault Current for the two cases of study (case 2 and 7). Experimental Results.

Deeper analysis is performed observing the phase currents (Figure 8.12), mechanical speed (Figure 8.13) and circulating faulty current (Figure 8.14). The phase currents are plotted instants before the instability point and an obvious unbalance behavior and harmonic content is depicted for $\mathrm{c} 7$. This distortions comes as a compensation effect of the visible effects on the DTC (Section 8.3). The mechanical speed corroborated the loss of the control stability accordingly with the flux and torque variations at time $7 \mathrm{sec}$. 


\subsubsection{Steady State Inspection of the Visible Effects}

Steady state results are presented for the following variables: $I_{\text {fault }}$ (Figure 8.15), phase currents (Figure 8.15), $\omega_{r}$ (Figure 8.16) and $\Delta T_{e}$ (Figure 8.16). An interesting behavior of the fault current against $\mu$ can be seen in Figure 8.15. $I_{\text {fault }}$ raises from healthy until $\mathrm{c} 3$ at $\mu=5.55 \%$ (stage I) and then remains approximately constant until c6 at $\mu=31.39 \%$ (stage II) and then start decreasing (stage III). This characteristic requires further analysis to be explained, however, it can be analyzed by the hypothesis stated in Section 8.3 and 8.4:

$\checkmark$ In the stage I the DTC reacts to the visible effects compensating them. For a low fault severity factor, it is easy for the DTC to force the estimated variables to follow the reference in the same fashion as the healthy IM.

$\checkmark$ In stage II, the fault severity factor increases. With this increment, the currents become more unbalanced (see phase $a$ ) and the visible effects are greater. In this situation, DTC is able to compensate these visible effects managing to keep them in a constant manner as the fault severity factor increases. The constant fashion is attributed to the constant circulating faulty current versus the severity of the fault.

$\checkmark$ In the stage III the unbalance in the currents is excessive creating bigger differences between the estimated values and the actual ones. Thus, some small instabilities appears as shown in Figure 8.10, Figure 8.11 and Figure 8.13 for the case 7.

Another indication of the compensation of the visible effects is in the torque ripple of the estimated torque plotted Figure 8.16. In here it can be noted that the torque ripple almost remain constant with the increase of the fault. However, a subtle increment indicates the inability to the controller to compensate larger effects. Additionally in Figure 8.16, the 
rotational speed is recorded with the increment of the fault severity observing a loading effect and a drastic reduction of the speed.

The non-observable effects stablished in section 8.3 cannot be identified in the experimental results unless certain special sensors are acquired and installed: two internal search coils to sense the real and imaginary components of the stator flux-linkage and a force meter. Additionally, the voltage sensed in the two search coils requires to be highly processed to finally compute $\left|\lambda_{s}\right|$.

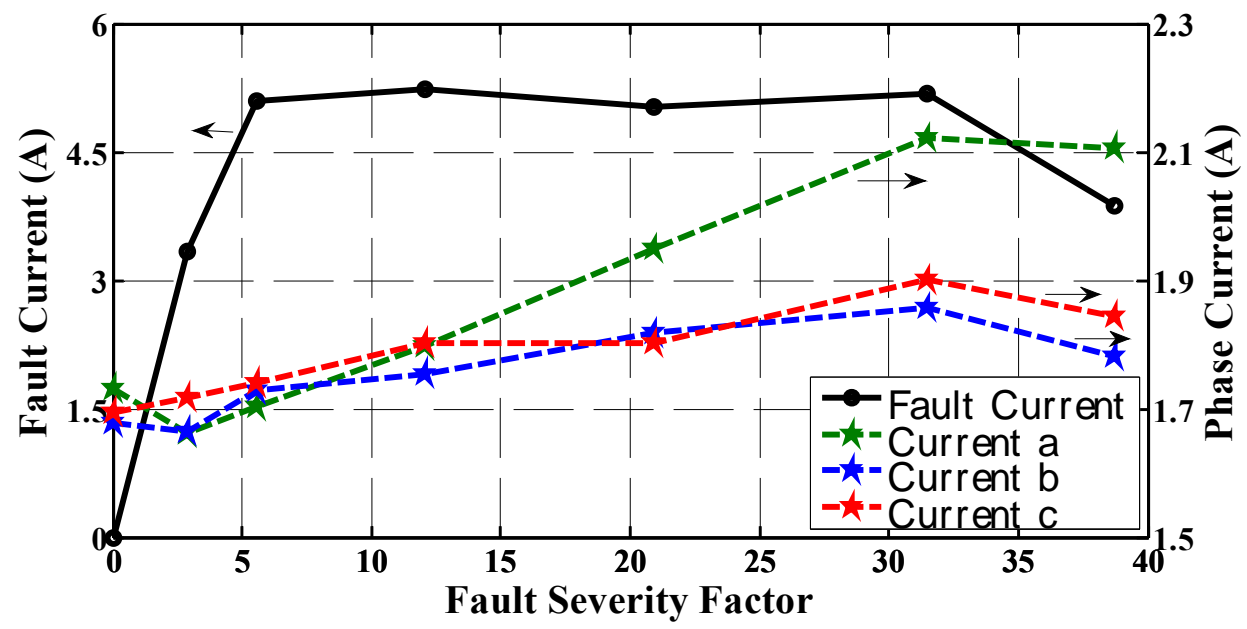

Figure 8.15 Fault Current and three-phase currents vs fault severity factor.

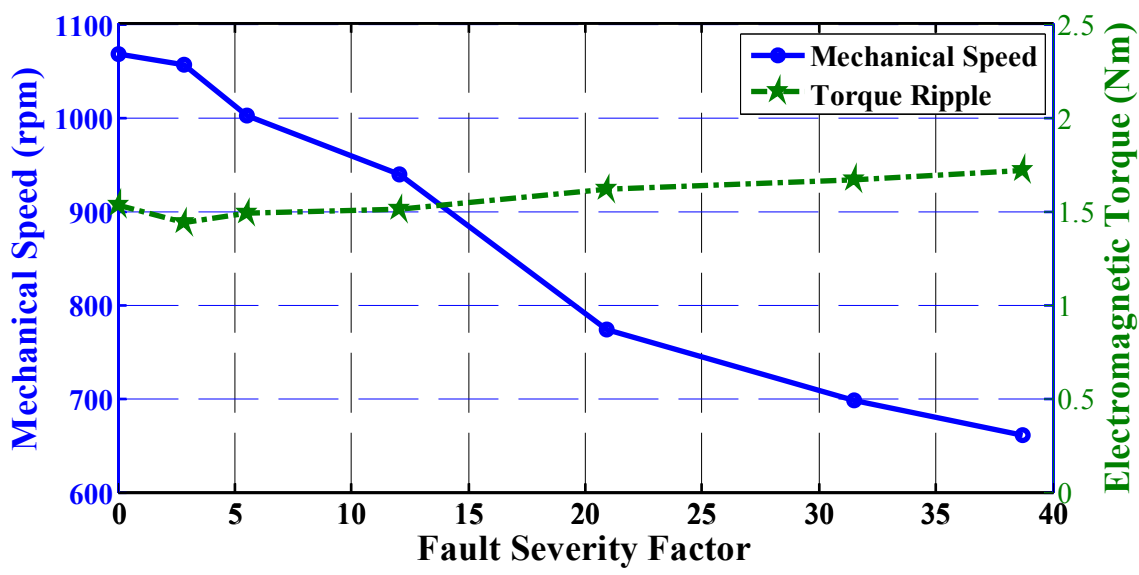

Figure 8.16 Mechanical speed and Estimated Torque ripple vs fault severity factor. 


\subsection{Appendix 1}

Using the Taylor series of the square root of $x$ in (8.27), we can re-write an approximated version of $e_{\left|\lambda_{s}\right|}$.

$$
\sqrt{x}=\sum_{n=0}^{\infty} \frac{(-1)^{n}\left((-1+x)^{n}\left(-\frac{1}{2}\right)_{n}\right)}{n !}
$$

where $n$ ! is the factorial of the variable $n$, and $(.)_{n}$ is the Pochhammer function $(y)_{n}=$ $y(y+1) \ldots(y+n-1)$. Thus, truncating the Taylor series of $\sqrt{x}$ to the third element and introducing the stator flux-linkage amplitude, it can be obtained:

$$
\begin{aligned}
& e_{\left|\lambda_{s}\right|}=\left|\hat{\lambda}_{s}\right|-\left|\lambda_{s}\right|=\sqrt{\hat{\lambda}_{s \alpha}{ }^{2}+\hat{\lambda}_{s \beta}{ }^{2}}-\sqrt{\lambda_{s \alpha}{ }^{2}+\lambda_{s \beta}{ }^{2}} \\
& e_{\left|\lambda_{s}\right|}=\frac{\left|\hat{\lambda}_{s}\right|^{2}-\left|\lambda_{s}\right|^{2}}{2}-\frac{\left(\left|\hat{\lambda}_{s}\right|^{2}-1\right)^{2}-\left(\left|\lambda_{s}\right|^{2}-1\right)^{2}}{8} \\
& e_{\left|\lambda_{s}\right|}=\frac{\left|\hat{\lambda}_{s}\right|^{2}-\left|\lambda_{s}\right|^{2}}{4}-\frac{\left|\hat{\lambda}_{s}\right|^{4}-\left|\lambda_{s}\right|^{4}}{8}=\frac{e_{\left|\lambda_{s}\right|^{2}}}{4}-\frac{e_{\left|\lambda_{s}\right|^{4}}}{8}
\end{aligned}
$$

The first term of (8.30) is defined as in (8.31).

$$
\frac{e_{\left|\lambda_{s}\right|^{2}}}{4}=\frac{\left|\hat{\lambda}_{s}\right|^{2}-\left|\lambda_{s}\right|^{2}}{4}=\frac{\left\{\left(\hat{\lambda}_{s \alpha}{ }^{2}+\hat{\lambda}_{s \beta}{ }^{2}\right)-\left(\lambda_{s \alpha}{ }^{2}+\lambda_{s \beta}{ }^{2}\right)\right\}}{4}
$$

where $\lambda_{s \alpha}=L_{s} i_{\alpha s}+L_{m} i_{\alpha r}-2 / 3 \mu L_{s} i_{f}$ comes from (6.8), $\hat{\lambda}_{s \alpha}=L_{s} i_{\alpha s}+L_{m} i_{\alpha r}$ and $\hat{\lambda}_{s \beta}=\lambda_{s \beta}$. Thus:

$$
\frac{e_{\left|\lambda_{s}\right|^{2}}}{4}=\frac{\hat{\lambda}_{s \alpha}\left(\mu L_{s} i_{f}\right)}{3}-\frac{2\left(\mu L_{s} i_{f}\right)^{2}}{9}=\left(\hat{\lambda}_{s \alpha}-\frac{2 \mu L_{s} i_{f}}{3}\right) \frac{\mu L_{s} i_{f}}{3}
$$

The second term of (8.30) is: 
$\frac{e_{\left|\lambda_{s}\right|^{4}}}{8}=\frac{\left|\hat{\lambda}_{s}\right|^{4}-\left|\lambda_{s}\right|^{4}}{8}=\frac{\left\{\left(\hat{\lambda}_{s \alpha}{ }^{4}-\lambda_{s \alpha}{ }^{4}\right)+2 \hat{\lambda}_{s \beta}{ }^{2}\left(\hat{\lambda}_{s \alpha}{ }^{2}-\lambda_{s \alpha}{ }^{2}\right)\right\}}{8}$

Which can be further developed to: $\frac{e_{\left|\lambda_{S}\right|^{4}}}{8}=\frac{e_{\text {terms }}{ }^{4}}{8}+\frac{\widehat{\lambda}_{s \beta}{ }^{2} e_{\left|\lambda_{S}\right|^{2}}}{4}$

$$
\frac{e_{\mathrm{terms}}{ }^{4}}{8}=\frac{\frac{8 \hat{\lambda}_{s \alpha}{ }^{3}\left(\mu L_{s} i_{f}\right)}{3}-\frac{2 \hat{\lambda}_{s \alpha}{ }^{2}\left(\mu L_{s} i_{f}\right)^{2}}{3}+\frac{32 \hat{\lambda}_{s \alpha}\left(\mu L_{s} i_{f}\right)^{3}}{27}-\frac{32\left(\mu L_{s} i_{f}\right)^{4}}{81}}{8}
$$

Then:

$$
e_{\left|\lambda_{s}\right|}=\frac{e_{\left|\lambda_{s}\right|^{2}}}{4}+\left(\frac{e_{\mathrm{terms}}}{8}+\frac{\hat{\lambda}_{s \beta}^{2} e_{\left|\lambda_{s}\right|^{2}}}{4}\right)
$$

The new index $e_{\left|\lambda_{s}\right|}$ can be approximated neglecting the terms elevated to the power of 4 as the fluxes are normally values lower than 1 . Then, these terms will be smaller than terms elevated to the power of 2 .

$$
e_{\left|\lambda_{s}\right|} \approx \frac{e_{\left|\lambda_{s}\right|^{2}}}{4}=\left(\hat{\lambda}_{s \alpha}-\frac{2 \mu L_{s} i_{f}}{3}\right) \frac{\mu L_{s} i_{f}}{3}
$$

\subsection{Conclusions}

Turn faults in the stator winding of an inverter-fed IM driven by DTC was theoretically analyzed, studied, simulated, experimentally tested and compared. This paper enhances and develops novel explanations of the ITSC influence to the DTC. It is found that these effects are twofold: visible and invisible. Also, it is discovered that DTC reacts to the visible effects by compensation until its collapse. It also reacts tolerating the impacts that cannot detect. All these discoveries are based on the development of a current SS model of the faulty IM, which is presented in the literature for the first time. Also, these findings are attributed to the study of the DTC under its standard heuristic derivation and predictive 
control theory. Closed-form formulas for the estimated errors were developed and they can be utilized in future condition monitoring techniques. The simulation and experimental results verified the theoretical findings. 


\section{Chapter 9 Online Fault Detection of Inter-Turn Short Circuit in IM driven by DTC}

\subsection{Introduction}

Early fault detection (FD) of incipient stator winding faults in induction machines (IM) driven by vector controllers is becoming critical in industry applications. Online condition monitoring of those types requires sensible techniques to detect embryonic stator asymmetries. Inter-turn short circuit (ITSC) faults indeed are considered one of the most crucial and difficult failures to detect and identify. The problem is more challenging when the IM is driven by a closed-loop controller, as the last one hides the signatures, typically, available in the flawed IM. A plethora of research has been devoted to this type of fault, when the IM is connected directly to the mains (direct on-line - DOL) [24], however, despite its seriousness and considering that nowadays the majority of the IM are inverterfed, few attempts exists currently in the literature to address this problem [29], [150]-[153], [155]-[157], [160]-[162]. Among all the IM vector controllers, Direct Torque Control (DTC) is the most famous, nevertheless, it has been less researched compared to Field Oriented Control (FOC) in the case of stator winding turn fault IM. A comparative analysis of the amount and type of research per vector control in IM is summarized in Table 9-1. Reference [156] presented two techniques to address that problem. The first one monitors the third order harmonic component of the supply currents $\left(\boldsymbol{i}_{\boldsymbol{s}}\right)$, based on the fact that it increases significantly in the case of fault. The second method is based on the multiple reference frame theory (MRF). 
Table 9-1 Close-Loop Vector Controllers for IM and the References dealing with Stator ITSC

\begin{tabular}{|c|c|c|}
\hline Closed-Loop Vector Control & $\begin{array}{l}\text { Amount } \\
\text { research }\end{array}$ & References FDI \\
\hline Control with Impressed Currents & 1 & [163] \\
\hline Stator Field Oriented Control & 7 & $\begin{array}{c}{[29],[150]-[153],[155],} \\
{[161]}\end{array}$ \\
\hline Rotor-Field Oriented Control & 0 & - \\
\hline Magnetizing Field Oriented Control & 0 & - \\
\hline $\begin{array}{l}\text { Indirect Rotor Field-Oriented } \\
\text { Control }\end{array}$ & 0 & - \\
\hline Direct Torque Control & 3 & {$[156],[157],[162]$} \\
\hline Direct-Self Control & 0 & - \\
\hline Total & 11 & \\
\hline
\end{tabular}

The same authors illustrated in [157] the implementation of the later techniques using a digital signal processor. A Neural Network (NN) was used in [162] to detect the ITSC fault severity and adapt the classical DTC operation, accordingly. The proposed methodology is based on the fact that the ITSC will increase the magnitude of the third order harmonic in the stator current, while the proposed fault-tolerant system re-estimates and reconfigures the stator resistance.

From the performed deep literature review, only these 3 investigations are found studying the detection of stator faults in IM driven by classical DTC. Reference [162] uses $\mathrm{NN}$ that requires a huge amount of experimental data for the learning, training and testing stages. Furthermore, it uses the third harmonic component of $\boldsymbol{i}_{\boldsymbol{s}}$, in the same way as the first approach of [156] and [157]. These last two references are from the same authors using frequency domain ( $3^{r d}$ harmonic of $\boldsymbol{i}_{\boldsymbol{s}}$ ) and the MRF theory approach. Therefore, it can be concluded that fundamentally, only two techniques have been researched. Investigation 
[153] uses the internal signals of the controllers to detect the ITSC fault for FOC and DTC. The DTC, dealt with, uses 2 proportional-integral controllers (for torque and magnitude of the flux), instead of the very well-known DTC look-up table (LUT), as shown in Table II [98].

It is obvious that this field has not been fully researched and there is a large necessity to address this problem, as the majority of the IM on the industrial, commercial, military and domestic fields are being controlled by classical DTC or derivatives of it, such as predictive DTC [106], fuzzy-DTC [113] fuzzy-predictive-DTC [164], etc. Consequently, in this chapter the FD procedure presented in [35], which was implemented for the DOL IM, is modified to be suited for an inverted-fed IM driven by classical DTC. The FD is based on the monitoring of the off-diagonal term of the Sequence Components (SCs) impedance matrix. Its advantages are that it is independent of the IM parameters, it is immune to the sensors' errors, it requires a small learning stage, compared with $\mathrm{NN}$, and it is not intrusive. Some challenges in the signal processing implementation are found for the afore-stated technique, however, its results are promising. Simulation and experimental results demonstrate that the technique is able to detect an ITSC fault on the onset (low fault severity factor).

Table 9-2 Switching Look-Up Table for Classical DTC

\begin{tabular}{|c|c|c|c|c|}
\hline$\tau, \psi$ & $\downarrow \downarrow$ & $\uparrow \downarrow$ & $\downarrow \uparrow$ & $\uparrow \uparrow$ \\
\hline$R_{1}$ & $\boldsymbol{v}_{s 4}$ & $\boldsymbol{v}_{s 2}$ & $\boldsymbol{v}_{s 5}$ & $\boldsymbol{v}_{s 3}$ \\
\hline$R_{2}$ & $\boldsymbol{v}_{s 5}$ & $\boldsymbol{v}_{s 6}$ & $\boldsymbol{v}_{s 1}$ & $\boldsymbol{v}_{s 2}$ \\
\hline$R_{3}$ & $\boldsymbol{v}_{s 1}$ & $\boldsymbol{v}_{s 4}$ & $\boldsymbol{v}_{s 3}$ & $\boldsymbol{v}_{s 6}$ \\
\hline$R_{4}$ & $\boldsymbol{v}_{s 3}$ & $\boldsymbol{v}_{s 5}$ & $\boldsymbol{v}_{s 2}$ & $\boldsymbol{v}_{s 4}$ \\
\hline$R_{5}$ & $\boldsymbol{v}_{s 2}$ & $\boldsymbol{v}_{s 1}$ & $\boldsymbol{v}_{s 6}$ & $\boldsymbol{v}_{s 5}$ \\
\hline$R_{6}$ & $\boldsymbol{v}_{s 6}$ & $\boldsymbol{v}_{s 3}$ & $\boldsymbol{v}_{s 4}$ & $\boldsymbol{v}_{s 1}$ \\
\hline
\end{tabular}




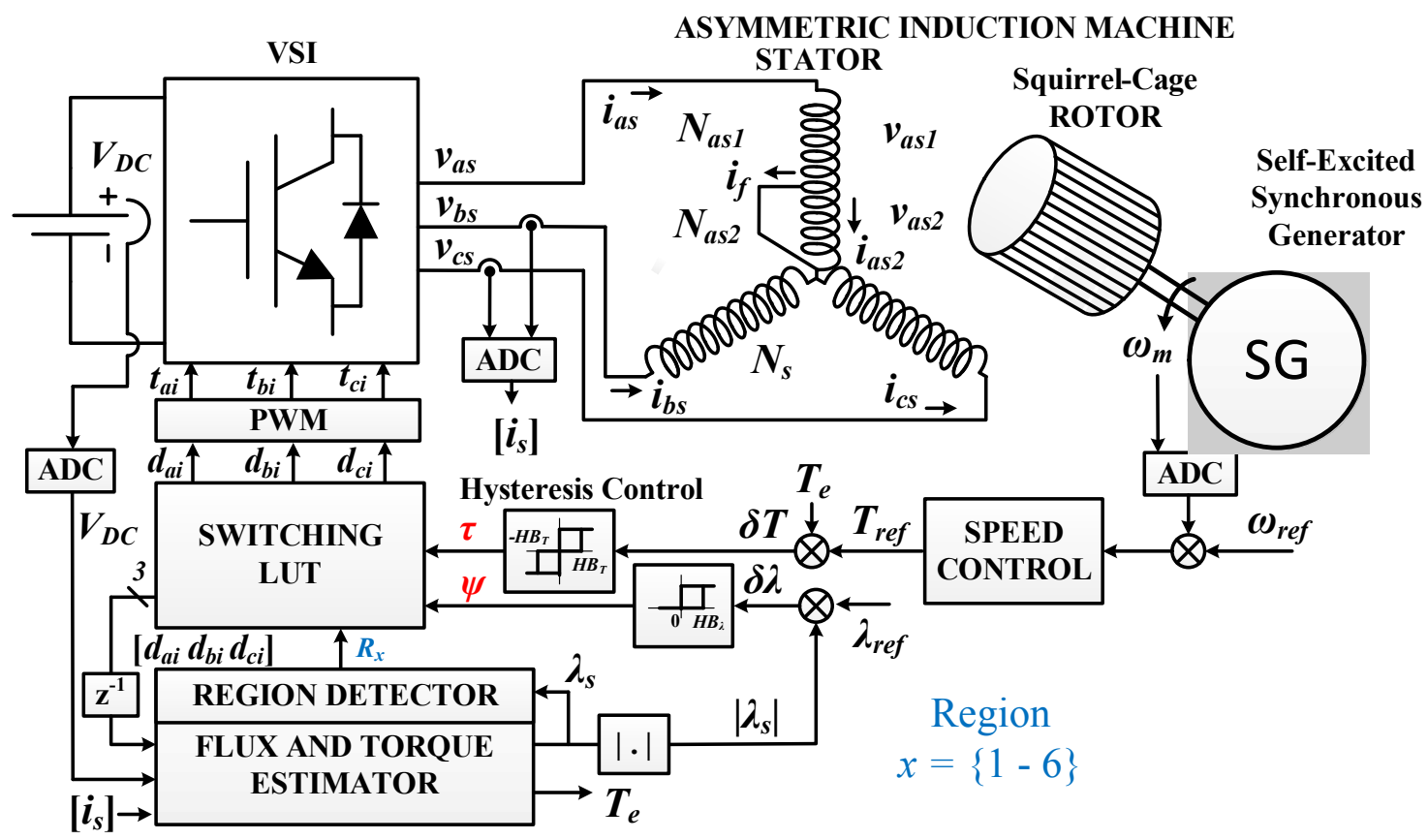

Figure 9.1 Typical structure of a DTC with speed control of an induction motor with ITSC on phase " $a$ ".

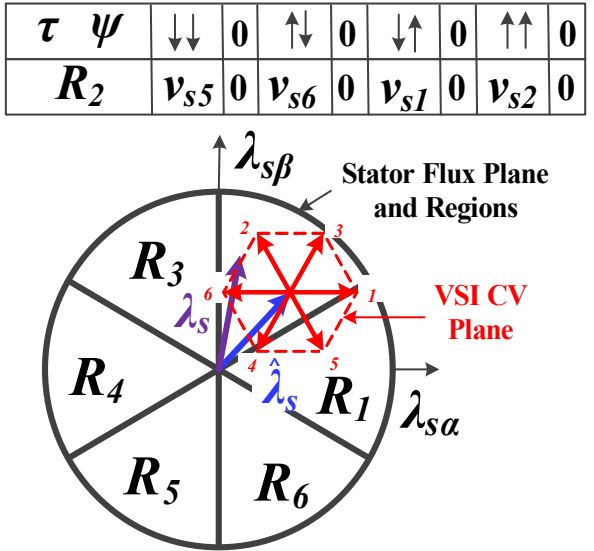

Figure 9.2 Estimated and actual Stator Flux $\lambda_{s}\left(t_{k}\right)$

\subsection{Classical DTC: Structure}

The classical DTC objective is to directly control electromagnetic torque $\left(T_{e}\right)$ and the stator flux-linkage amplitude $\left(\left|\lambda_{s}\right|\right)$. The DTC algorithm in our work is performed at fixed switching frequency $\left(f_{s}\right)$ and it uses a PWM for the FD. The DTC comprises the following stages (Figure 9.1): 
Acquisition: two current and one voltage sensors measure the motor stator current vector $\left(\left[\begin{array}{lll}\boldsymbol{i}_{\boldsymbol{s}}\end{array}\right]=\left[\begin{array}{lll}i_{s a} & i_{s b} & i_{s c}\end{array}\right]^{t}\right)$ and the dc-link level $\left(V_{D C}\right)$, respectively.

Transformation: the signals in primitive coordinates are transformed into $(\alpha \beta)$ complex space-vectors (SV) in the stationary frame.

$\checkmark$ Stator Voltage Estimation: with $V_{D C}$ and the switching control sequence $\left(\left[\boldsymbol{d}_{\boldsymbol{i}}\right]=\right.$ $\left.\left[\begin{array}{lll}d_{i a} & d_{i b} & d_{i c}\end{array}\right]^{t}\right)$ at the previous cycle of control $\left(t_{k-1}\right)$, where $k$ is the time sample index, the output voltage SV of the voltage source inverter (VSI) can be deduced $\left(\widehat{\boldsymbol{v}}_{\boldsymbol{s}}\right)$.

$\checkmark \quad$ Stator Flux Estimation: the stator flux-linkage SV $\left(\hat{\lambda}_{s}\right)$ is estimated using estimated stator voltage $\mathrm{SV}$, current $\mathrm{SV}\left(\boldsymbol{i}_{\boldsymbol{s}}\right)$ and the stator resistance $\left(R_{S}\right)$. The estimated electromagnetic torque $\left(\widehat{T}_{e}\right)$ is computed with $\hat{\boldsymbol{\lambda}}_{\boldsymbol{s}}$ and $\boldsymbol{i}_{\boldsymbol{s}}$. The estimation equations are in [98], [164].

Hysteresis Control: uses two and three-level hysteresis controllers, for the stator fluxlinkage amplitude and the torque, respectively. The function of these controllers is to maintain the levels of the two variables within a narrow hysteresis bands $\left(H B_{\lambda}\right.$ and $\left.H B_{T}\right)$ around the references $\left(T_{r e f}\right.$ and $\left.\lambda_{\text {ref }}\right)$. The input of these controllers are the torque and flux-linkage amplitude errors $\delta T_{e}=T_{r e f}-T_{e}$ and $\delta\left|\lambda_{\mathrm{s}}\right|=\lambda_{\text {ref }}-\left|\lambda_{\mathrm{s}}\right|$. The output of the two hysteresis ( $\tau, \psi$ signals) results in 4 possible control actions: (1) the two variables increase, (2) the two variables decrease, (3) one increases and the other decreases and (4) vice-versa.

$\checkmark$ Region Detection: the location of the stator flux SV in the complex plane will indicate one of the 6 regions (see Table 9-2 and table at top of Figure 9.2) in which the flux-linkage complex plane is divided $\left(R_{x}\right.$ where $\left.x=\{1-6\}\right)$. See Figure 9.2.

$\checkmark \quad$ VSI switch state selection: with $(\tau, \psi)$ in conjunction with $\left(R_{x}\right)$, the output signals to the gates of the VSI can be generated from the DTC look-up table (LUT), as in Table II. 


\subsection{IM with Inter-Turn Fault: Steady-State Model}

ITSC on one of the phases (i.e. phase " $a$ ") of an IM is shown in the Figure 9.1. Following [34], [36], [165], $N_{s a 2}$ and $N_{s}$ are the numbers of shorted turns and number of turns perphase, respectively. While $\mu=N_{s a 2} / N_{s}$ is the fault severity factor, $R_{f}$ and $i_{f}$ are the fault impedance and the circulating current in the short-circuit path, respectively. From [165], the steady state model and equivalent circuits (EC) in SC are presented in (9.1) and (9.2), and Fig. 3. In (9.1) and (9.2), $\bar{V}_{S P}, \bar{V}_{S N}, \bar{I}_{S P}$ and $\bar{I}_{S N}$ are the positive and negative SC of the steady-state stator voltage and current, respectively.

$$
\begin{gathered}
{\left[\begin{array}{c}
\bar{V}_{s P} \\
0
\end{array}\right]=\left[\begin{array}{cc}
R_{s}+j \omega_{e} L_{s} & j \omega_{e} L_{m} \\
j \omega_{e} L_{m} & \frac{R_{r}}{s}+j \omega_{e} L_{r}
\end{array}\right]\left[\begin{array}{c}
\bar{I}_{s P 1} \\
\bar{I}_{r P}
\end{array}\right]} \\
{\left[\begin{array}{c}
\bar{V}_{s N} \\
0
\end{array}\right]=\left[\begin{array}{cc}
R_{s}+j \omega_{e} L_{s} & j \omega_{e} L_{m} \\
j \omega_{e} L_{m} & \frac{R_{r}}{2-s}+j \omega_{e} L_{r}
\end{array}\right]\left[\begin{array}{c}
\bar{I}_{s N 1} \\
\bar{I}_{r N}
\end{array}\right]} \\
\mu\left(\bar{V}_{s P}+\bar{V}_{s N}\right)=\left(R_{f}+R_{s f}+j \omega_{e} L_{l s f}\right) \bar{I}_{f}
\end{gathered}
$$

$$
\left[\begin{array}{l}
\bar{I}_{S P} \\
\bar{I}_{S N}
\end{array}\right]=\left[\begin{array}{ll}
Y_{P P} & Y_{P N} \\
Y_{N P} & Y_{N N}
\end{array}\right]\left[\begin{array}{c}
\bar{V}_{S P} \\
\bar{V}_{S N}
\end{array}\right]
$$

where $\omega_{e}$ is the electrical frequency, $L_{s}$ and $L_{r}$ are the self-inductances of the stator and rotor, respectively, $L_{m}$ is the magnetizing inductance, $L_{l s}$ is the leakage inductance of the stator, $s=\frac{\omega_{e}-\omega_{r}}{\omega_{e}}$ is the slip, $\omega_{r}$ is the mechanical speed in the shaft, $Y_{P N}=\frac{1 / 3 \mu^{2}}{R_{f}+\mu\left(R_{S}+j \omega_{e} L_{l s}\right)}$, $Y_{P P}=\frac{1}{R_{S}+j \omega_{S} L_{S}+\frac{\left(\omega_{e} L_{S}\right)^{2}}{\frac{R r}{S}+j \omega_{S} L_{r}}}+Y_{P N}, Y_{N N}=\frac{1}{R_{S}+j \omega_{e} L_{S}+\frac{\left(\omega_{e} L_{S}\right)^{2}}{\frac{R_{r}}{2-s}+j \omega_{e} L_{r}}}+Y_{P N}, Y_{P N}=Y_{N P}$, and $L_{l s f}=$ $\mu L_{l s}\left(1-\frac{2}{3} \mu\right)$ 


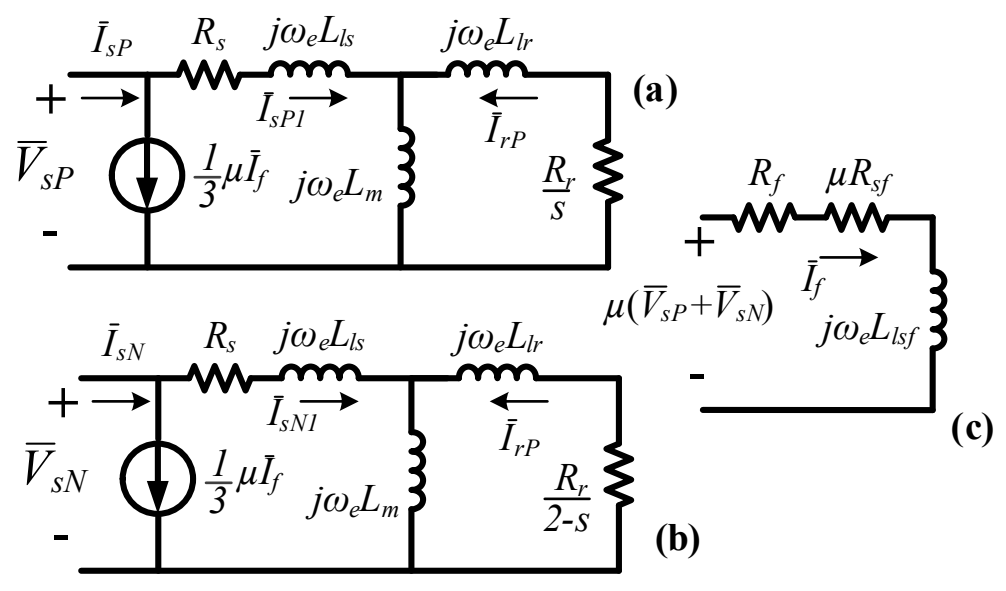

Figure 9.3 Sequence-components EC of Asymmetric IM (a) Positive Sequence (b) Negative Sequence (c) Faulty Circuit.

\subsection{Influence of IM Stator Fault in DTC}

When there is no fault $(\mu=0)$, the estimated stator flux-linkage SV $\left(\hat{\lambda}_{s}\right)$ and the estimated electromagnetic torque $\left(\widehat{T}_{e}\right)$ are the true values. However, on the fault onset, if the degree of fault is small and the short circuit is not bold $\left(R_{f}>0\right)$, the DTC continues its apparent normal operation. In this situation, the estimated flux and electromagnetic torque is evaluated as if the IM would be healthy and no information is provided about the faulty quantities (see Figure 9.3(c) and the current sources in Figure 9.3(a) and (b)). The flux and torque estimation would be erroneous and they will differ from the actual values. The only correct information gathered by the DTC is the stator currents and voltages, however, it has been proven and found in the literature and by their own simulations and experimentations that the controller keeps its normal operation under a certain range of fault severity fault. Reference [156] explains that in order to keep the DTC operation, the control has to compensate for these error components in the electromagnetic torque and amplitude of the flux. Two options can be possible: (a) the introduction of negative SC in 
the flux-linkage (and consequently in the voltages) and (b) the injection of a positive sequence component in the stator flux-linkage at the frequency of $3 f_{s}$. It is concluded that there is not too much room for introducing a negative sequence voltage, as this is limited by $H B_{\lambda}$. The second possibility (preferred by the authors of [156]) is the introduction of third harmonic component in the flux-linkage and by consequence in the stator currents and voltages by the torque hysteresis controller to compensate the double frequency torque component. All this without major restrictions.

The first option requires an unbalanced behavior of the VSI, which is not achieved easily in the case of the DTC. It requires uneven selection, per electric cycle, of the VSI states, that depend on the flux-linkage position and signals $\tau$ and $\psi$. In this work, this option is discarded, following the results from [156], as well. The second option has a more physical meaning, however, by a different explanation that is the injection of a zero sequence voltage component instead of a positive sequence component in the stator flux. The zero sequence component is introduced by the no neutral connection of the wye center in the inverter-fed IM. An alternative third explanation to the DTC reaction is that the controller is not compensating, yet tolerating the asymmetries due to its robustness.

In conclusion, DTC is not able to observe the stator anomalies. Consequently, many signatures in the stator current, torque, speed, etc, are hidden. However, the off-diagonal term of the SC impedance matrix signature is revealed. Indifferent of the DTC operation and its reaction to the ITSC, the FD technique is able to discriminate the abnormal behavior of the IM as the technique is based on the impedance change, which is a ratio between SC voltages and currents, as shown in the next section. 


\subsection{Fault Detection Technique}

The SC voltage equation of an IM can be expressed as in (9.3) [35], where the subscripts $p$ and $n$ represent the positive, negative SCs of the stator voltage and current phasors. As the analysis will be only considering the measured variables (stator), the following analysis will drop the subscript $s$, referred to the stator. Furthermore, as the IM is wye-connected, the zero sequence component does not need to be considered.

$$
\left[\begin{array}{l}
\bar{V}_{p} \\
\bar{V}_{n}
\end{array}\right]=\left[\begin{array}{ll}
Z_{p p} & Z_{p n} \\
Z_{n p} & Z_{n n}
\end{array}\right]\left[\begin{array}{c}
\bar{I}_{p} \\
\bar{I}_{n}
\end{array}\right]
$$

For a symmetrically constructed machine, the off-diagonal terms of the impedance matrix are zero. Moreover, the SCs are independent from each other. Nevertheless, in a real IM, imperfections in the motor structure cause asymmetry and therefore the offdiagonal SCs terms exist. These off-diagonal impedance terms are slip dependent. From (9.3), the negative sequence voltage can be written as (9.4).

$$
\bar{V}_{n}=Z_{n p} \bar{I}_{p}+Z_{n n} \bar{I}_{n}
$$

Assuming $Z_{n p}=Z_{p n}$, the focus of the fault detection is based on the computation of one of these two off-diagonal terms of the SC impedance matrix. In the fault onset, the value of $Z_{n p}$ varies, compared with the healthy value of the off-diagonal term $\left(Z_{n p h}\right)$, as the motor becomes unsymmetrical differently. A stator fault, such as ITSC can be detected by continuous monitoring of $Z_{n p}$. The monitoring is based on the deviation between $Z_{n p}$ and $Z_{n p h}$. The FD indicator is as in (9.5).

$$
\Delta Z_{n p}=Z_{n p}-Z_{n p h}
$$

Calculation of $Z_{n p}$ at any given slip condition requires two data points, since (4) 
comprehends two unknowns $\left(Z_{n p}\right.$ and $\left.Z_{n n}\right)$. Assuming 2 linearly independent sets of data at the same slip condition, $Z_{n p}$ can be calculated as (9.6).

$\left[\begin{array}{l}Z_{n p} \\ Z_{n n}\end{array}\right]=\left[\begin{array}{ll}\bar{I}_{p 1} & \bar{I}_{n 1} \\ \bar{I}_{p 2} & \bar{I}_{n 2}\end{array}\right]\left[\begin{array}{c}\bar{V}_{n 1} \\ \bar{V}_{n 2}\end{array}\right]$
$Z_{n p}=\frac{\bar{I}_{n 2} \bar{V}_{n 1}-\bar{I}_{n 1} \bar{V}_{n 2}}{\bar{I}_{p 1} \bar{I}_{n 2}-\bar{I}_{p 2} \bar{I}_{n 1}}$

The data-set is defined as $S_{x}=\left\{\bar{I}_{p x}, \bar{V}_{n x}, \bar{I}_{n x}\right\}$, where $x=\{1,2\}$ represents the first and second data-set of measurements, which are obtained at different unbalanced supply voltage to avoid singular matrix in (9.6). It is noteworthy to mention that sensor errors would give non-zero $Z_{n p}$, however this does not affect the technique, as the indicator works with the change of $Z_{n p}$.

\subsection{Procedure Stages of the Fault Detector}

The implementation technique consists of two stages: learning and monitoring, as shown in Figure 9.4. Z-LUT stands for the impedance look-up table to avoid confusion with DTC LUT.

Learning Stage

Measure
$S_{b}, S_{u}$$\rightarrow \begin{gathered}\text { Calculate } \\ Z_{n p h}\end{gathered} \rightarrow \begin{gathered}\text { Store in Z-LUT } \\ S_{u, Z_{n p h}}\end{gathered}$

Monitoring Stage

\begin{tabular}{|c|c|c|c|}
\hline $\begin{array}{c}\text { Measure } \\
S_{m}\end{array}$ & $\begin{array}{c}\text { Calculate } \\
Z_{n p}\end{array}$ & $\begin{array}{c}\text { Search Z-LUT } \\
S_{u}, Z_{n p h}\end{array}$ & $\begin{array}{c}\text { Calculate } \\
\qquad \Delta Z_{n p}\end{array}$ \\
\hline
\end{tabular}

Figure 9.4 Fault Detection Technique (a) Learning Stage (b) Monitoring Stage.

\subsubsection{Learning Stage:}

The calculation of $Z_{n p}$ requires two data points, defined before $\left(S_{x}\right)$ at different unbalanced supply voltage. The data-set 1 is for the balanced voltage condition $\left(S_{1}=S_{b}\right)$ 
and data-set 2 is for unbalanced supply voltage $\left(S_{2}=S_{u}\right)$. These two data-sets are defined per-slip value. For this purpose, two procedures can be performed: (a) The DTC output voltage is unbalanced by means of the use of the modulation strategy. Using the pulse width modulation, the output in one phase can be set to a lower value than $100 \%$ of the dutycycle. For instance: $\left[\begin{array}{lll}\boldsymbol{d}_{\boldsymbol{i}}\end{array}\right]=\left[\begin{array}{lll}d_{i a} & d_{i b} & 0.8 * d_{i c}\end{array}\right]^{t}$, where $d_{i x}$ only takes values of 0 and 1 (see Figure 9.5). (b) Inclusion of a physical resistance $\left(R_{u}\right)$ between the VSI and the IM, as in Figure 9.5.

To obtain different slip conditions, the torque reference of the DTC is changed assuming that the mechanical load in the IM is rigorous to follow any reference and keeping the flux amplitude constant. With those data, the Z-LUT $Z_{n p h}-$ slip is elucidated and stored together with $S_{u}-$ slip. This technique was originally designed for a DOL IM. However, the technique has been adapted to inverter-fed IM in this research. For the latter, the IM does not behave similarly to the DOL IM. When the machine is controlled by DTC, the voltage and frequency in the stator change to comply with the torque and flux references. In the DOL machine, the voltage and frequency are kept fixed and the mechanical loading makes the slip changes.

\subsubsection{Monitoring Stage:}

Once all the data is stored in Z-LUT from the learning stage, the $Z_{n p}$ value is continuously evaluated with 2 new data-sets: $S_{1}=S_{m}$ that is the actual measurements during normal operation and the stored unbalanced data $S_{2}=S_{u}$ at the slip condition measured in the learning stage. Each $S_{u}$ is paired with a $Z_{n p h}$, which is used by the fault detector, as in (9.5). 


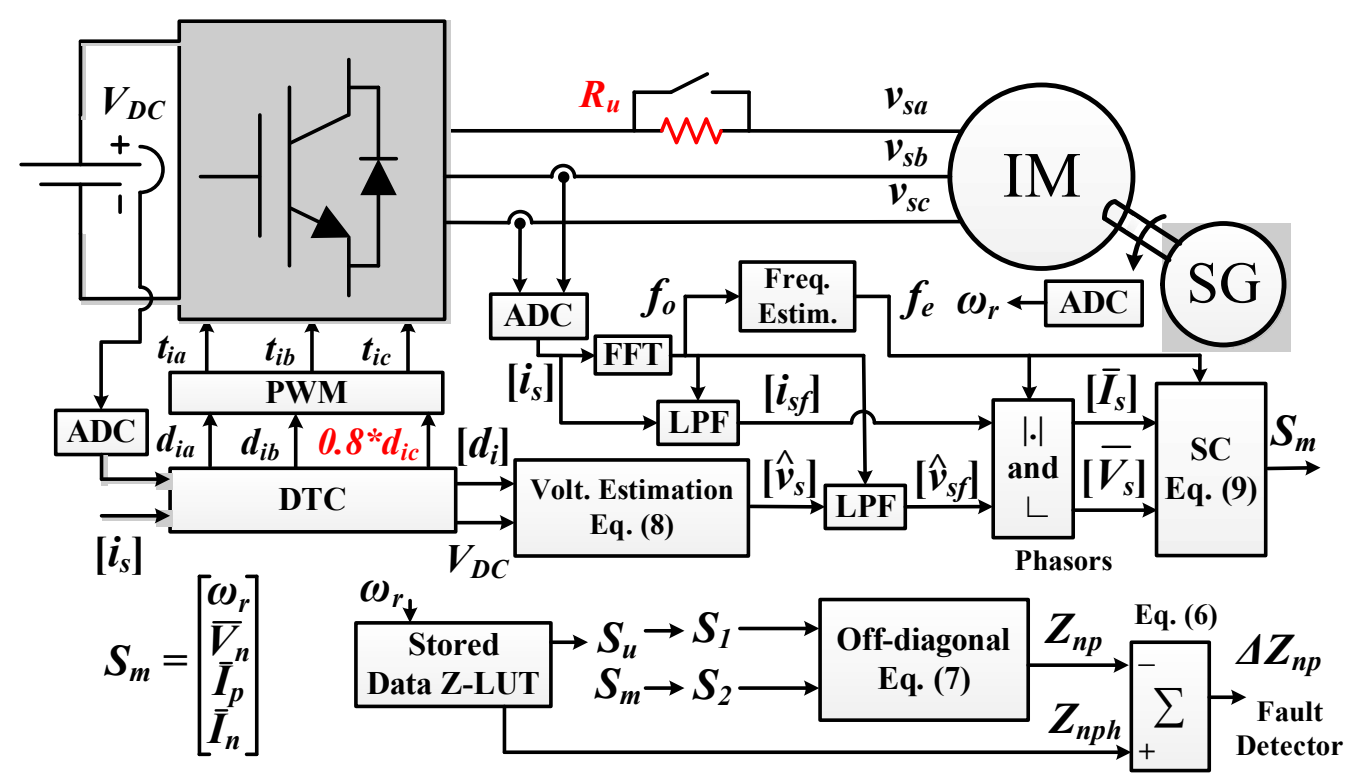

Figure 9.5 Implementation of the Fault Detection Technique for an inverter-fed IM under DTC.

\subsection{Implementation of the FD on DTC}

For the implementation of the fault detection technique, some signal processing hurdles were overcome: (a) the computation of the electric frequency, which varies with the operational point in DTC, (2) the estimation of the stator voltages and (c) the calculation of the phase-angle of the voltage and current phasors.

Prior to the computation of these signals, a buffer stage of enough data points is required to be programmed. The buffer needs to be filled cyclically for the continuous monitoring.

\subsubsection{Computation of the Electric Frequency in DTC}

The first challenge, for the adaptation of the FD technique to the DTC, is to compute the $\mathrm{SC}$ for which the fundamental stator electric frequency $\left(f_{e}\right)$ is required. It is well-known that DTC varies this frequency to achieve its control goal. There are many techniques to estimate the frequency: a phase lock loop (PLL) is proposed in [166], a Discrete Fourier Transform (DFT) has been widely adopted due its inherent harmonic rejection [167], 
however it presents accuracy problems. Other techniques like Kalman filters [168], least square mean methods [169], Newton type methods [170] and wavelet transforms [171] are proposed. Nevertheless, the convergence speed and bulk processing are the main problems in real-time applications. Soft computing techniques, such as neural networks [172] and genetic algorithms [173], have been utilized for frequency estimation, however, these techniques require a trade-off between accuracy and computational complexity. In this chapter, the frequency estimation proposed in [174] is used.

A small adaptation to the technique is performed as the method requires the nominal frequency $\left(f_{o}\right)$, which is obtained by the Fast Fourier Transform (FFT), as shown in Figure 9.5. The first step is to implement a Fast Fourier Transform (FFT) to the stator currents. The current FFT will discover the nominal frequency. With this information, a filter stage in the stator voltage and currents is conducted. A digital second-order low-pass filter is designed with a natural frequency from the FFT and damping ration of 0.707 (see Figure 9.5). With $f_{o}$, then the estimator is programmed to obtain $f_{e}$.

\subsubsection{The Estimation of the Stator Voltages}

The second complication faced was the absence of the stator voltages in the DTC. The DTC does not require the stator voltages in the primitive coordinate frame and they are square waves that come from the output of the VSI. Therefore, measuring these voltages would require high sampled acquisition frequency. The DTC in question here is working at fixed switching frequency of $10 \mathrm{kHz}$, thus, an acquisition of at least $100 \mathrm{kHz}$ would give some resolution. To avoid additional cost, the voltages were estimated in a similar way DTC does for the calculation of the stator flux-linkages. However, typically, DTC estimation is performed in the complex-vector notation and we required the voltages in 
primitive coordinates. Using the information of the sequence switching of DTC $\left(\left[\boldsymbol{d}_{\boldsymbol{i}}\right]\right)$ and the dc-link voltage $\left(V_{D C}\right)$, the line-to-neutral voltage can be deduced as in (9.7). In Figure 9.5 the procedure is depicted.

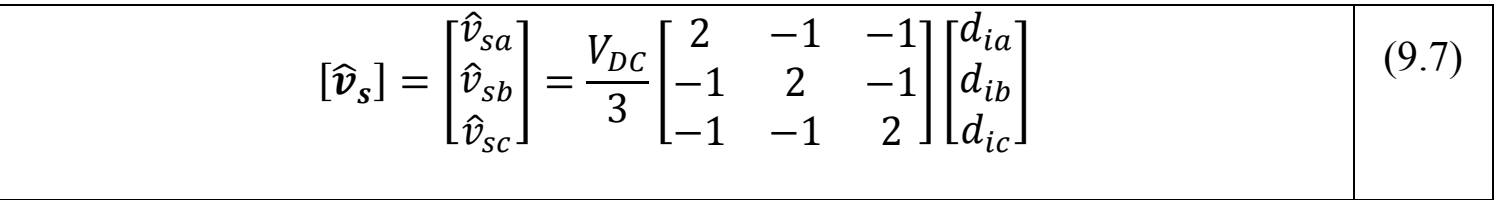

where $d_{i x}=\left\{\begin{array}{ll}0 & \text { off } \\ 1 & \text { on }\end{array}\right.$.

With, the electric frequency and the filtered signals (current and voltage), the sequence components of the voltage and currents are calculated as in (9.8) [175].

$$
\left[\begin{array}{l}
x_{z} \\
x_{p} \\
x_{n}
\end{array}\right]=\frac{1}{\sqrt{3}}\left[\begin{array}{ccc}
1 & 1 & 1 \\
1 & \alpha & \alpha^{2} \\
1 & \alpha^{2} & \alpha
\end{array}\right]\left[\begin{array}{l}
x_{a} \\
x_{b} \\
x_{c}
\end{array}\right]
$$

where $\alpha=e^{j \frac{2 \pi}{3}}$ and $x=\{v, i\}$.

\subsubsection{Calculation of the Phase Angle of Phasors}

Although, the symmetric components' transformation is a straightforward procedure, before it, the stator voltage and current are required to be transformed to phasor variables with a magnitude and a phase-shift angle. The amplitude is easily found with the maximum of the signal in one period. The third challenge is in the phase angle. For purposes of discovering these angles, a phase lock loop (PLL) is proposed. Many types (analog/continuous, digital/discrete, linear, nonlinear, etc) and plenty of literature is found for the design of PLLs [176], however, we programmed a digital PLL (DPLL) in which the electric frequency can be varied automatically as input. The designed DPLL is shown in Fig. 6. The DPLL consists of several stages: 


\subsubsection{Conditioning}

In this stage, the input signal (in our case the filtered stator current vector $\left[\boldsymbol{i}_{\boldsymbol{s} f}\right]$ ) is transformed to the complex vector notation in $\alpha \beta$ reference frame. Then, the signal is normalized $\left(e^{j \theta}\right)$. See Figure 9.6.

\subsubsection{Phase Error Detector}

This stage is for the discovery of the phase. It is composed of a multiplier block that conducts the product of the conjugate feedback signal $\left(e^{j \widehat{\theta}}\right)$ and the input signal $\left(e^{j \theta}\right)$, where $\hat{\theta}$ is the estimated phase. Thus, the output of this product is $m=e^{j \theta} * e^{-j \widehat{\theta}}=$ $e^{j \theta-j \widehat{\theta}}$. There are two techniques to obtain the phase after the multiplication: (1) using the inverse tangent $\left(\Delta \theta=\tan ^{-1}\left(e^{j \theta-j \widehat{\theta}}\right)\right)$ of the complex signal or (2) using the imaginary component of the complex signal $\left(\Delta \theta=\operatorname{Imag}\left(e^{j \theta-j \widehat{\theta}}\right)=\sin (\theta-\hat{\theta}) \simeq \theta-\hat{\theta}\right)$. We chose the first method.

\subsubsection{Loop-Filter}

A plethora of different types of loop-filters can be implemented. The most common are: (1) designing an analog loop-filter and then converting it into a digital equivalent, following the procedure in [177] and (2) designing a proportional-integral (PI) controller plus a discrete time integrator. The PI controller is designed heuristically, while the integrator is the simple transfer function $1 / s$, where $s$ is the Laplace complex variable.

\subsubsection{Oscillator}

The simplest method to develop a digital oscillator is by introducing the estimated phase $\hat{\theta}$ into an exponential function, as shown in Figure 9.6. 


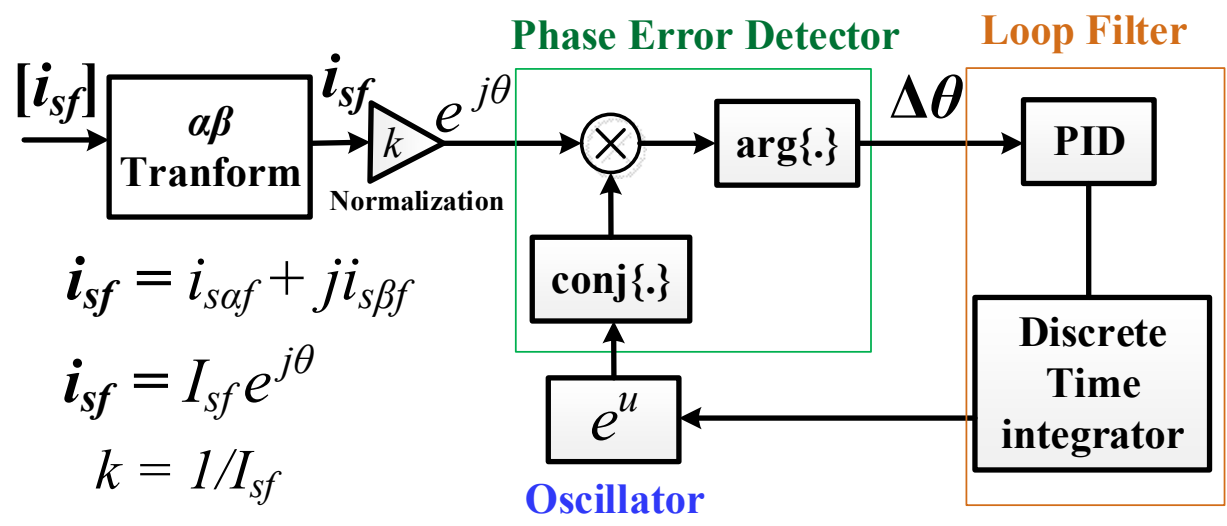

Figure 9.6 Fault Detection Technique (a) Learning Stage (b) Monitoring Stage.

This implementation can be applied to any of the closed-loop vector controllers of Table 9-1, as well as closed-loop scalar controllers. Furthermore, it can be used on any inverterfed IM, in open-loop mode or no control at all, only using PWM and a sinusoidal reference. This technique only requires 3 sensors: two currents and one voltage.

\subsection{Simulation Test-Benches}

Two simulation test-benches (coded and circuit based) are built in MatLab environment to study the transient and steady state regime of any IM with stator ITSC. In these simulations the IM can be started up with any type of drive. It can be used to study gridconnected IM (also named connected directly on-line (DOL)) and/or inverter-fed IM driven by any controller in open and/or closed-loop condition. Both are open simulations (it means that can be modified) for research purposes, however, the coded-based can be cumbersome while the circuit-based is simple to use and hides all mathematical complexity.

The coded test-bench is based on pure MatLab script coding, where the ODE of the faulty IM is solved by a $4^{\text {th }}$ order Runge-Kutta (RK) method. The RK was programmed by the authors to have internal access to the integration and thus provide dynamic changes 
through the simulation. Vector controllers require instantaneous control changes of the voltage source inverter. The programming levels, organization and filing are shown in Figure 9.7.

\section{Main File}

\begin{tabular}{|c|}
\hline $\begin{array}{l}\text { - Initializations and definitions } \\
\text { - } \text { For-loop }\left(\mathrm{T}_{\mathrm{s}}, \mathrm{t}_{\mathrm{i}}, \mathrm{t}_{\mathrm{f}}\right)\end{array}$ \\
\hline $\begin{array}{l}\text { - Measurements at control cycle } \mathrm{k} \\
\text { - DTC coding for calculating input voltage control at contro } \\
\text { cycle k with measurements. } \\
\text { - Call the Solver Function } \\
\text { - Indicate the ODE function to the ODE solver, plus initial } \\
\text { conditions. } \\
\text { - Extract the solution from the solver } \\
\text { - Give the solution to DTC to compute input in the next } \\
\text { instant of control }(\mathrm{k}+1) \text {. } \\
\text { - Return to the beginning }\end{array}$ \\
\hline
\end{tabular}

Solver File Solver: $4^{\text {th }}$
order Runge

Kutta

- Call ODE

ODE File

Faulty IM

ODE

Figure 9.7 Coded Simulation Test-bench programming levels, organization and filing.

The circuit-based test-bench was built in Simulink SimpowerSystems, where the faulty IM is programmed as a block similar to the existing symmetrical ones in the Simulink Library. Among the several ways to approach the modelling of the ODE and its transformation to circuit analysis are: Thevenin equivalent circuit (EC), Norton EC and voltage behind reactance [178]. For simplicity, due to the way the faulty IM ODE was formulated, a Norton EC is selected. The block diagram of the Simulink block is as in Figure 9.8(a). In Figure 9.8(b) the three-phase Norton EC per-phase can be observed. Each phase consists of a current controlled source with a high value resistance in parallel. The voltage of the current controlled sources are fed-back to the Subsystem $\left(v_{s a}, v_{s b}\right.$ and $\left.v_{s c}\right)$. The load torque and the fault severity factor are inputs of the subsystem (they are defined by the user). Inside the subsystem, a $4^{\text {th }}$ order Runge-Kutta with the dynamic equations of 
the faulty IM model are programmed to produce the outputs that will control the current sources plus the electromagnetic torque and speed.

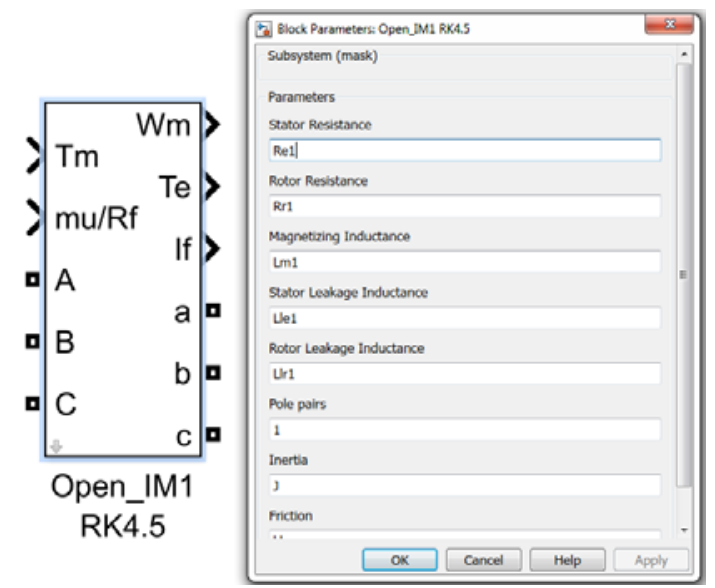

(a)
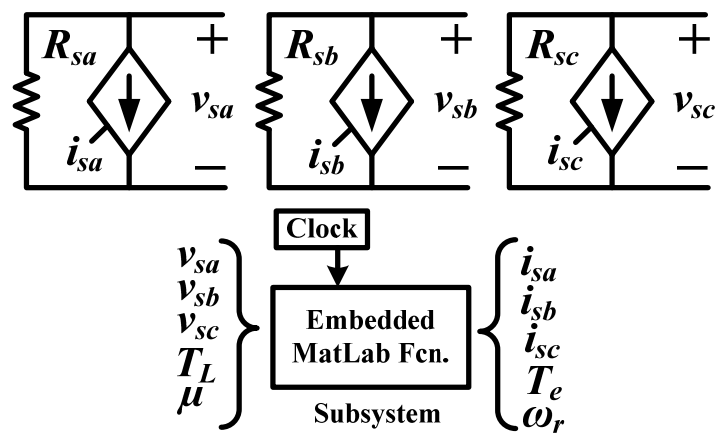

Figure 9.8 (a) Simulink block of the damaged IM (b) Norton EC based Simulation Testbench.

The coded test-bench simulation is the classical way to implement ODEs, not significant contribution is claimed here, howbeit, the circuit test bench software is an original development not available in Simulink or other similar simulation software platforms, to the best of the author's knowledge. The difference of the circuit-based testbench respect to the coded one is that the variables (current, voltage, flux-linkage, torque and speed signals) have units while the in the coded test-bench all the variables are dimensionless data quantities. In this sense, the benefits of the circuit-based test-bench are:

- It can be used to easily test the ITSC phenomena in an IM.

- It can be utilized to test the damaged IM under any type of drive, i.e. pre-existing Simulink IM drives or any other driver in general. 
- It can be effortlessly applied to investigate ITSC in IM with different ratings and parameters.

- The impact on the educative community is that it can be utilized by non-expert practitioners in the area of faulty IM (such as undergraduate or master students). This circuit block hides the complexity of the model and it only requires a user configuration (see Figure 9.8(a)) in a friendly environment.

Two simulation test-benches are built in the MatLab environment to study the transient and steady state regime of any IM with ITSC driven by any controller in open and/or closed-loop condition. The first test bench is based on pure MatLab script coding, where the ordinary differential equations (ODEs) of the faulty IM are solved by a $4^{\text {th }}$ order RungeKutta (RK) method, also programmed by us to have internal access to the integration and to give dynamic changes to the simulation during the processing time. The programming levels, organization and filing are shown in Figure 9.7.

\subsection{Simulation and Experimental Results}

The simulation and experimental test are performed for an 1 HP IM. Table 9-3 shows the nameplate ratings and parameters of the motor. For the purpose of creating the ITSC, 8 taps were placed in the phase " $a$ " [179], [180]. Figure 8.5 shows the experimental test platform. The IM shaft is mechanically coupled to a $250 \mathrm{~W}$ and $42 \mathrm{~V}$ dc permanent magnet (PM) machine. The dc PM machine is acting as a generator with variable load. The sensor board is based on LEM transducers and a dSpace 1104 board is used for the control and condition monitoring. The motor angular speed is measured with a $1000 \mathrm{ppr}$ coupled to the PM machine. The $R_{f}=1 \Omega$ and $N_{s}=510$. Nine cases of ITSC were studied and they are shown in Table 9-4. The first case (c1) is the healthy IM. 
Table 9-3 Parameters and Nameplate Rating for The IM Weg.

\begin{tabular}{|c|c|c|c|c|c|c|c|}
\hline Param. & Value & Param. & Value & Ratin & ig Value $\mathrm{F}$ & Rating & g Value \\
\hline$L_{l s}$ & $32.110^{-3} \mathrm{H}$ & $R_{S}$ & $9.292 \Omega$ & $P_{r}$ & $746 \mathrm{~W}$ & $P F$ & 0.8 \\
\hline$L_{l r}$ & $37.010^{-3} H$ & $R_{r}$ & $7.231 \Omega$ & $V_{r}$ & $460 \mathrm{~V}$ & Poles & 2 \\
\hline$L_{m}$ & $0.895 \mathrm{H}$ & $J_{m}$ & $0.053 \mathrm{Kgm}^{2}$ & $I_{r}$ & $1.47 \mathrm{~A}$ & $n_{r}$ & $1730 \mathrm{rpm}$ \\
\hline
\end{tabular}

Table 9-4 ITSC Test Cases for the Faulty IM

\begin{tabular}{cccc|cccc}
\hline \hline ITSC & \multicolumn{1}{c|}{$\boldsymbol{N}_{\boldsymbol{s a} 2 \boldsymbol{\mu}} \boldsymbol{\mu}(\%)$ Cases } & ITSC & \multicolumn{4}{c}{$\boldsymbol{N}_{\boldsymbol{s a} \boldsymbol{2}} \boldsymbol{\mu}(\%)$} & Cases \\
\hline \hline$U_{5}-a_{1,2}$ & 6 & 1.08 & $\mathrm{c} 2$ & $a_{3}-a_{4}$ & 28 & 5.55 & $\mathrm{c} 6$ \\
$a_{1}-a_{2}$ & 14 & 2.68 & $\mathrm{c} 3$ & $a_{3,2}-a_{4}$ & 42 & 8.25 & $\mathrm{c} 7$ \\
$U_{5}-a_{2}$ & 20 & 3.98 & $\mathrm{c} 4$ & $a_{2,2}-a_{3,2}$ & 47 & 9.31 & $\mathrm{c} 8$ \\
$a_{1}-a_{2,2}$ & 24 & 4.70 & $\mathrm{c} 5$ & $a_{2,2}-a_{3}$ & 61 & 12.01 & $\mathrm{c} 9$
\end{tabular}

\subsubsection{Simulation Results}

The simulations are divided into three parts: (1) Transient Operation of the Faulty IM under DTC, (2) Study of two fault severity factors under DTC and (3) Fault Detection Demonstration.

\subsubsection{Transient Operation of the Faulty IM under DTC}

Two cases of ITSC are shown: case 3 (c3) has a $\mu=2.68 \%$ and case 9 (c9) $\mu=$ $12.01 \%$. The transient (start-up) and steady-state performance are shown. The entire time for the test is $2 \mathrm{sec}$. The simulation integration step time is $10^{-5} \mathrm{sec}$. The simulation comprises the comparison of the estimated and actual $T_{e}$ and $\left|\lambda_{s}\right|$ for the two cases of study. Figure 9.9 presents $\left|\lambda_{s}\right|$ and Figure 9.10 shows $T_{e}$. The estimation problem is depicted in 
Figure 9.9 and Figure 9.10, where the estimated values tracked and achieved the references ( $T_{r e f}$ and $\lambda_{\text {ref }}$ ), while the actual values do not. Case 9 presents large deviations out of the both hysteresis bands. The actual values of case 2 track the references, as the fault severity is small. Also, the double electrical frequency component is observed for c9.

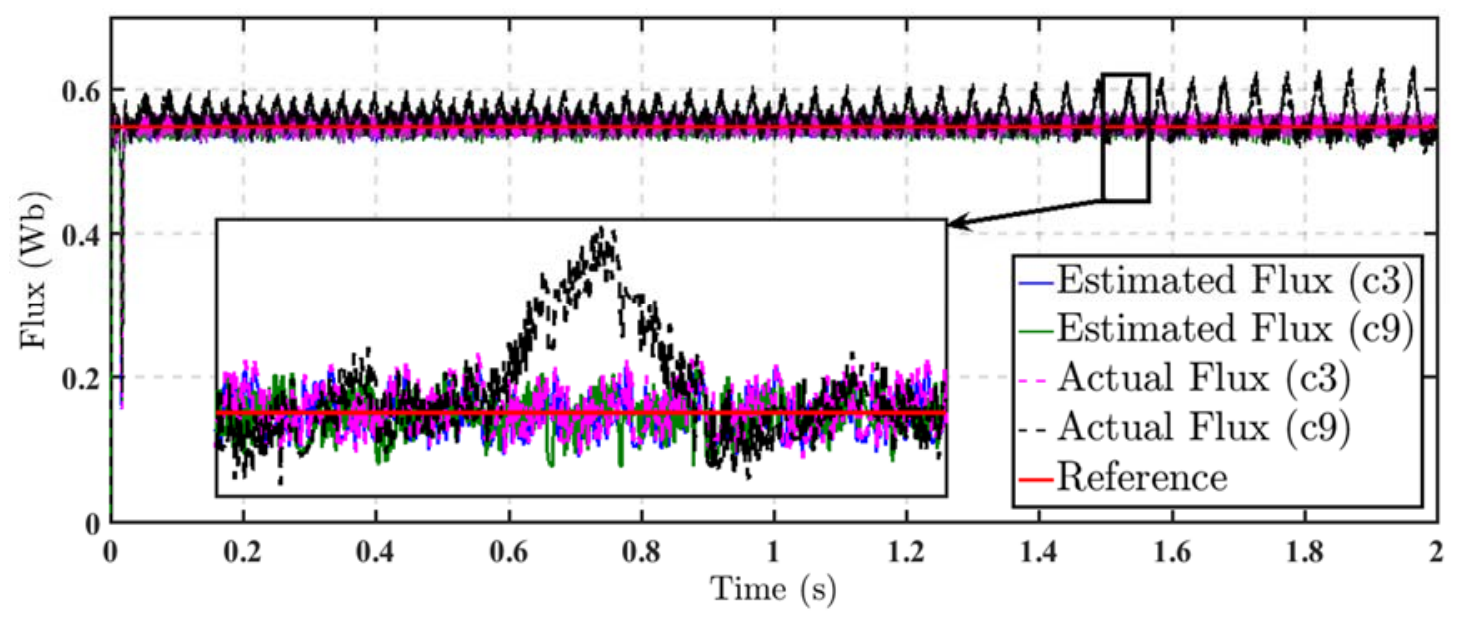

Figure 9.9 Estimated and actual Stator flux amplitude for the two cases of study (case 3 and 9). Simulation Results.

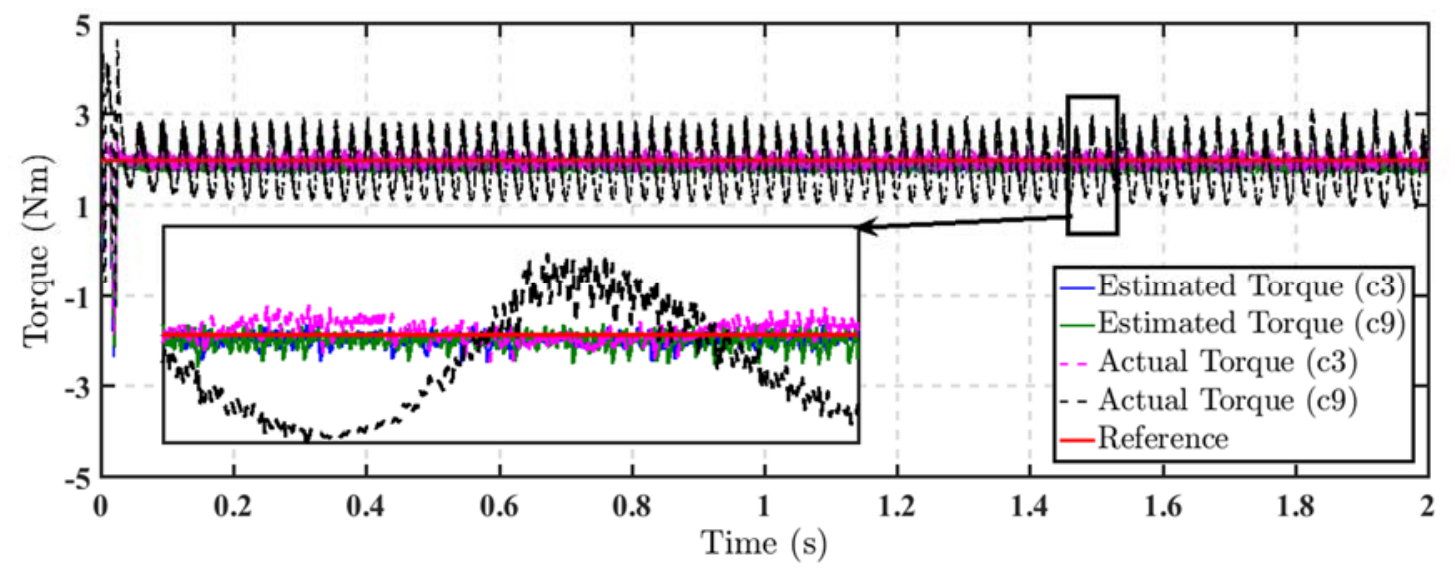

Figure 9.10 Estimated and actual Electromagnetic Torque the two cases of study (case 3 and 9). Simulation Results. 


\subsubsection{Study of two Fault Severity Factors under DTC}

In this section, two fault severity factors (case 3 (c3) with $\mu=2.68 \%$ and case 9 (c9) $\mu=12.01 \%$. .) are examined, prior to the demonstration of the fault detector. For each ITSC, 8 machine operative points are investigated at different torque references (from $T_{\text {ref_i }}=0.6 \mathrm{Nm}$ to $T_{r e f_{-} f}=2 \mathrm{Nm}$ in steps of $\left.0.2 \mathrm{Nm}\right)$. The actual electromagnetic torque and actual flux-linkage (not the estimated ones) for the 8 operative points of the 2 cases are shown in Figure 9.11, Figure 9.12, Figure 9.13 and Figure 9.14. Figure 9.11 shows that the actual electromagnetic torque for case 3 follows the 8 torque references in the same fashion that would be the healthy IM. In Figure 9.12, which shows the same plot as before for the case 9, even when the actual electromagnetic torque follows the torque reference, there is an abnormal torque ripple due to the ITSC (for the 8 torque references), however, it is more evident for the $T_{r e f}=2 \mathrm{Nm}$. From the comparison of the electromagnetic torques, for the two cases on Figure 9.11 and Figure 9.12, it is obvious that for a small $\mu$ the DTC is not strongly affected by the ITSC, and the double frequency component coming from the negative sequence magnetic field is small. This double frequency component is more noticeable for the $T_{r e f}=2 \mathrm{Nm}$, as well. On the other hand, when $\mu$ reaches a level of $12.01 \%$, the fault severity is not considered small anymore (the fault has evolved) and the double torque ripples are significant. However, keep in mind Figure 9.10, which shows that the estimated torque is still close to the reference with not double torque ripple coming from the faulty behavior of the IM. 


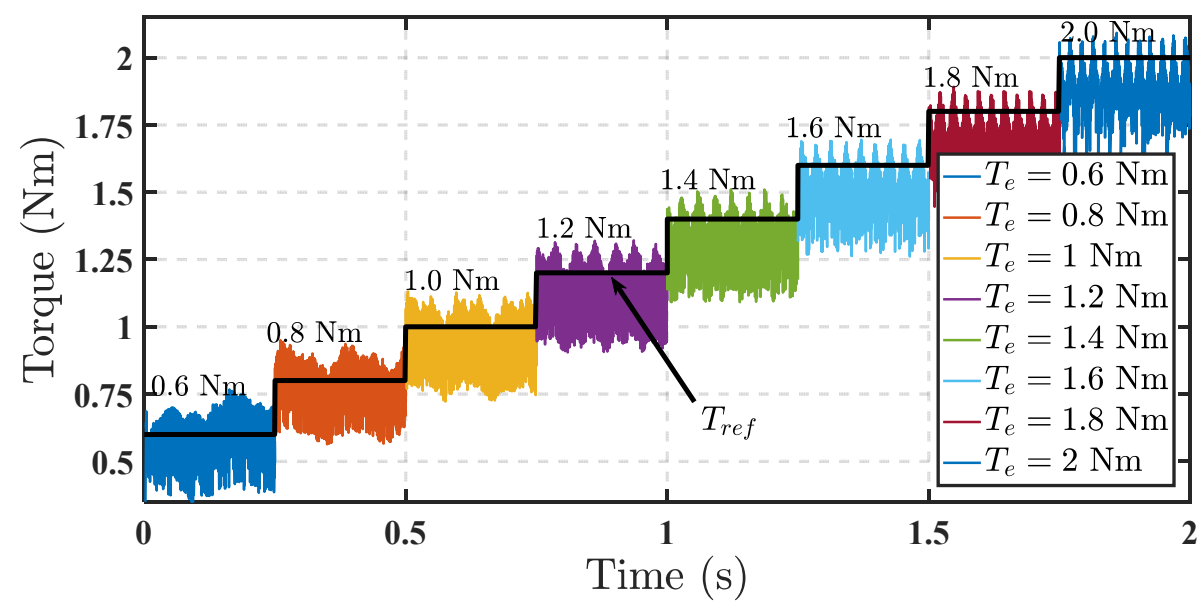

Figure 9.11 Actual Electromagnetic Torque for the 8 machine operative points of the case 3. Simulation Results.

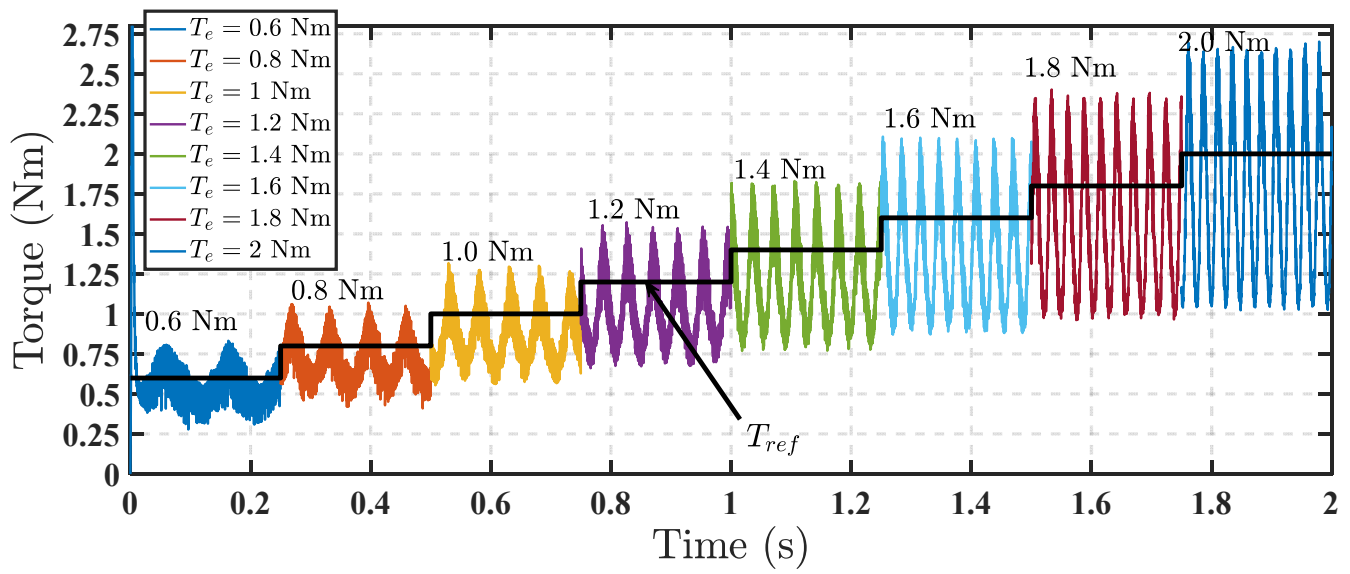

Figure 9.12 Actual Electromagnetic Torque for the 8 machine operative points of the case 9. Simulation Results.

The flux-linkage magnitudes for case 3 is shown in Figure 9.13. Closer study of the fluxlinkages indicates that while the torque reference decreases, the fault effect increases. This detail could not be seen at $2 \mathrm{Nm}$ in the previous analysis (sub-section IX-A-1). The same behavior is noticed in Figure 9.14 for case 9. Nevertheless, case 9 presents bigger disturbances due the larger severity of the fault.

Table 9-5 shows a numerical comparison between the dynamic behaviors of the two cases. The mean absolute error (MAE) is calculated for the torque between the 
instantaneous torque reference $\left(T_{r e f_{-} k}\right)$ and the instantaneous actual electromagnetic torque $T_{e_{-} k}$ and for the flux-linkage between the magnitude of the flux-linkage reference $\left(\lambda_{\text {ref_k } k}\right)$ and magnitude of the flux-linkage $\left(\left|\lambda_{s_{-}}\right|\right)$. As follows, the instantaneous errors are computed as (9.9) and MAE (9.10).

$$
e_{k_{-} T}=\frac{T_{\text {ref_k }}-T_{e_{-} k}}{\text { Rated Torque }} \quad e_{k_{-} \lambda}=\frac{\lambda_{\text {ref_k }}-\left|\lambda_{s_{-} k}\right|}{\lambda_{\text {ref } \_k}}
$$

$$
M A E=\frac{1}{N} \sum_{k=1}^{N}\left|e_{k \_x}\right| \quad x=\{T, \lambda\}
$$

It is observed, from Table 9-5, that the MAE torque values for the case 9 are more than 3 time larger than the ones for the case 3. The MAE flux values are similar for both cases.
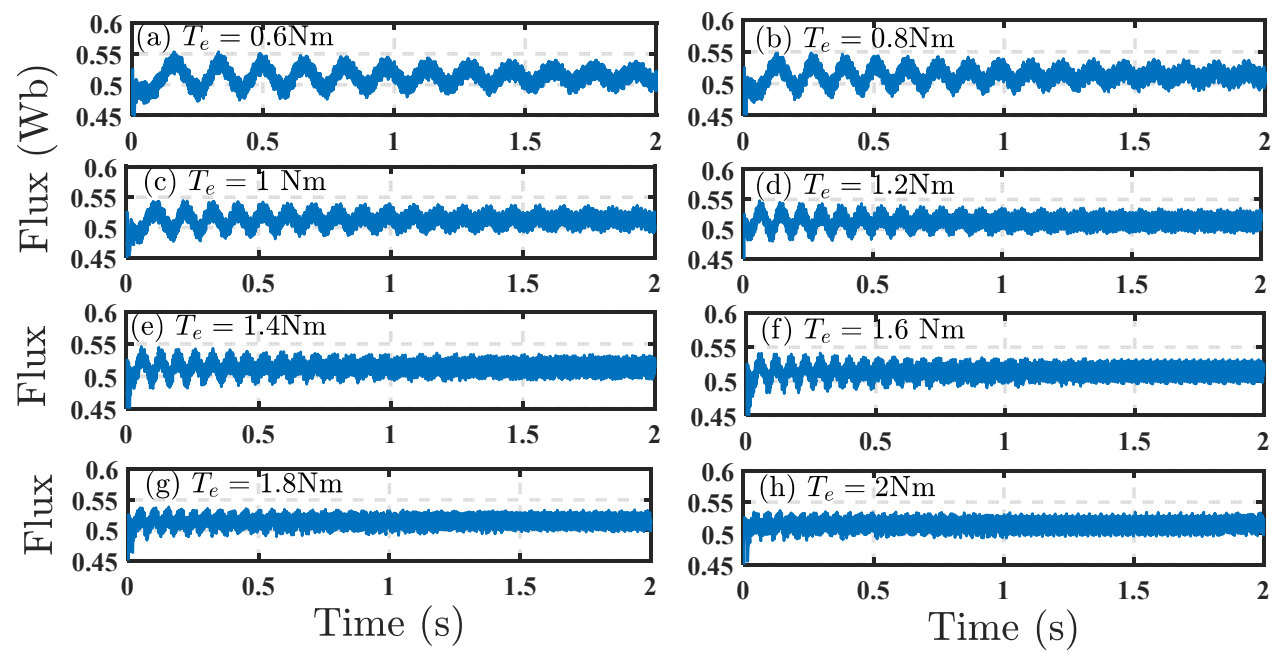

Figure 9.13 Flux-linkages for the 8 machine operative points of the case 3. Simulation Results. 

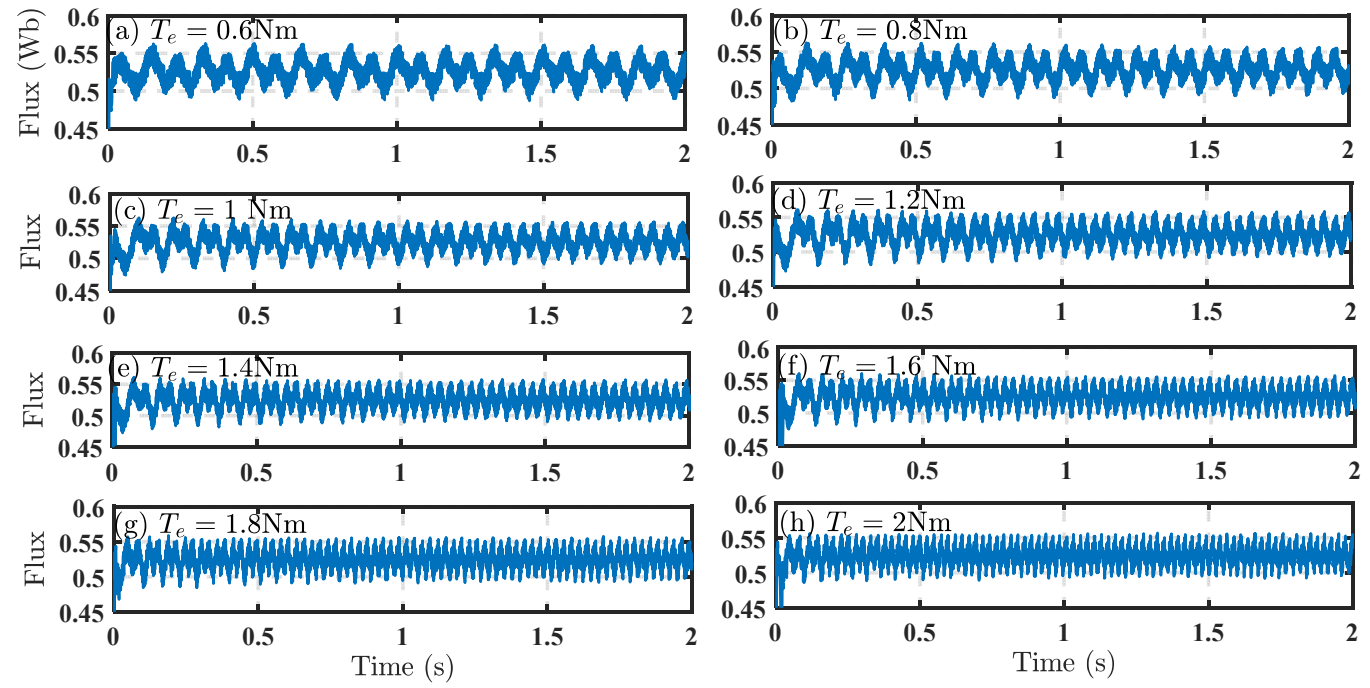

Figure 9.14 Flux-linkages for the 8 machine operative points of the case 9. Simulation Results.

Table 9-5 Actual Torque and Flux MAE for the eight ITSC Test-Cases of Study at the eight Operational Points of the Faulty IM Driven by DTC

\begin{tabular}{|c|c|c|c|c|c|}
\hline \multirow{2}{*}{ MAE } & \multirow{2}{*}{$\mu$} & \multicolumn{2}{c|}{ Case 3 } & \multicolumn{2}{c|}{ Case 9 } \\
\cline { 3 - 7 } & \multicolumn{2}{c|}{0.0286} & \multicolumn{2}{c|}{0.1201} \\
\hline Variables & \multicolumn{3}{c|}{ MAE $T_{e}|\operatorname{MAE}| \lambda_{s} \mid$ MAE $T_{e}$} & MAE $\left|\lambda_{s}\right|$ \\
\hline \multirow{4}{*}{} & 0.6 & 1.73 & 2.59 & 3.11 & 2.41 \\
\cline { 2 - 6 } Operative & 0.8 & 1.75 & 2.28 & 3.33 & 2.49 \\
\cline { 2 - 6 } & 1.0 & 2.11 & 2.08 & 4.43 & 2.53 \\
\cline { 2 - 6 } Point $T_{\text {ref }}$ & 1.2 & 2.44 & 1.87 & 5.64 & 2.44 \\
\cline { 2 - 6 }$(\mathrm{Nm})$ & 1.4 & 2.62 & 1.84 & 6.96 & 2.39 \\
\cline { 2 - 6 } & 1.6 & 2.90 & 1.79 & 8.50 & 2.31 \\
\cline { 2 - 6 } & 1.8 & 3.18 & 1.72 & 10.22 & 2.19 \\
\cline { 2 - 6 } & 2.0 & 3.47 & 1.78 & 12.50 & 2.21 \\
\hline
\end{tabular}

\subsubsection{The Fault Detection Demonstration.}

For the study of the fault detection, 8 cases of ITSC were tested under 8 torque references. Thus, the off-diagonal term of the SC impedance matrix for 64 points are presented in Table 9-6, Figure 9.15 and Figure 9.16. In a similar fashion to the previous simulation results, per each fault severity factor, 8 operational points were investigated for variation of the torque reference from $T_{r e f_{-} i}=0.6 \mathrm{Nm}$ to $T_{r e f_{-} f}=2 \mathrm{Nm}$ in steps of 0.2 Nm. In Table 9-6 and Table 9-7Table 9-7 Off-Diagonal Term of the Impedance Matrix, 
Mechanical Speed And Electrical Frequency for the 64 ITSC Test Cases of the Faulty IM Driven by DTC: From 1.2 to $2 \mathrm{Nm}, 3$ variables are presented for each case: the fault detector $\left(Z_{n p}(\Omega)\right)$ in magnitude and angle, the electrical frequency $\left(f_{e}(\mathrm{~Hz})\right)$ and the rotational mechanical speed $\left(\omega_{r}(\mathrm{rad} / \mathrm{sec})\right.$. From the table, it can be noted that for each torque reference, the electrical frequency and mechanical speed have similar values (same operational point), however, the fault detection increases with $\mu$. Figure 9.15 shows the locus of the off-diagonal impedance component for the 64 cases. Two characteristics can be recognized: (1) the spiral trend feature of the fault detector per fault severity factor in similar pattern as the ones presented for the DOL IM in [35]. Nonetheless, the spiral is not as clear in some cases due to the transient dynamics of the DTC (2) the amplitude of the off-diagonal impedance component separates more from the locus origin as the fault severity factor increases. Observing Figure 9.16, these results indicate that $\left|Z_{n p}\right|$ is affected by the ITSC severity, but not significantly by the operational point of the IM. Additionally, the success of the FD to the ITSC faults can be noticed. In the case of the simulation, the healthy off-diagonal impedance $\left(\left|Z_{n p h}\right|=0\right)$ is zero, as no construction asymmetries were considered. 
Table 9-6 Off-Diagonal Term of the Impedance Matrix, Mechanical Speed And Electrical Frequency for the 64 ITSC Test Cases of the Faulty IM Driven by DTC: From 0.6 to 1.2

\begin{tabular}{|c|c|c|c|c|c|c|c|c|c|c|c|c|}
\hline \multicolumn{13}{|c|}{$\mathrm{Nm}$} \\
\hline $\begin{array}{l}T_{r e f} \\
(\mathrm{Nm})\end{array}$ & \multicolumn{3}{|c|}{0.6} & \multicolumn{3}{|c|}{0.8} & \multicolumn{3}{|c|}{1} & \multicolumn{3}{|c|}{1.2} \\
\hline$\mu$ & $Z_{n p}(\Omega)$ & $\begin{array}{c}f_{e} \\
(\mathrm{~Hz})\end{array}$ & $\begin{array}{c}\omega_{r} \\
(\mathrm{rad} / \mathrm{sec}) \\
\end{array}$ & $Z_{n p}(\Omega)$ & $\begin{array}{c}f_{e} \\
(\mathrm{~Hz})\end{array}$ & $\begin{array}{c}\omega_{r} \\
(\mathrm{rad} / \mathrm{sec}) \\
\end{array}$ & $Z_{n p}(\Omega)$ & \begin{tabular}{|c|}
$f_{e}$ \\
$(\mathrm{~Hz})$ \\
\end{tabular} & $\begin{array}{c}\omega_{r} \\
(\mathrm{rad} / \mathrm{sec}) \\
\end{array}$ & $Z_{n p}(\Omega)$ & \begin{tabular}{|c|}
$f_{e}$ \\
$(\mathrm{~Hz})$ \\
\end{tabular} & $\omega_{r}(\mathrm{rad} / \mathrm{sec})$ \\
\hline 0 & 0 & & 264.8 & 0 & & 357.6 & 0 & & 445.0 & 0 & & 534.5 \\
\hline 0.0108 & $\begin{array}{l}0.034 \angle \\
-1.99\end{array}$ & 6.55 & 286.6 & $\begin{array}{l}0.016 \angle \\
-1.60\end{array}$ & 8.72 & 380.9 & $\begin{array}{l}0.003 \angle \\
-2.05\end{array}$ & 10.9 & 474.6 & $0.010<1.71$ & 13.15 & 570.8 \\
\hline 0.0268 & $\begin{array}{l}0.097 \angle \\
-2.25 \\
\end{array}$ & 6.5 & 284.7 & $\begin{array}{l}0.089 \angle \\
-2.44 \\
\end{array}$ & 8.68 & 379.1 & $\begin{array}{l}0.092 \angle \\
-2.45 \\
\end{array}$ & 10.83 & 472.2 & $\begin{array}{l}0.109 \angle \\
-2.43 \\
\end{array}$ & 13.2 & 575.1 \\
\hline 0.0398 & $\begin{array}{l}0.158 \angle \\
-2.35\end{array}$ & 6.47 & 283.0 & $\begin{array}{l}0.170 \angle \\
-2.58\end{array}$ & 8.6 & 377.0 & $\begin{array}{l}0.221 \angle \\
-2.61\end{array}$ & 10.8 & 469.7 & $\begin{array}{l}0.256 \angle \\
-2.63\end{array}$ & 13.02 & 565.1 \\
\hline 0.0470 & $\begin{array}{l}0.168 \angle \\
-2.47\end{array}$ & 6.42 & 281.7 & $\begin{array}{l}0.170 \angle \\
-2.67\end{array}$ & 8.58 & 375.5 & $\begin{array}{l}0.218 \angle \\
-2.75\end{array}$ & 10.7 & 467.7 & $\begin{array}{l}0.283 \angle \\
-2.70\end{array}$ & 12.96 & 562.7 \\
\hline 0.0555 & $\begin{array}{l}0.367 \angle \\
-2.53 \\
\end{array}$ & 6.38 & 280.0 & $\begin{array}{l}0.334 \angle \\
-2.81 \\
\end{array}$ & 8.52 & 373.6 & $\begin{array}{l}0.378 \angle \\
-2.72 \\
\end{array}$ & 10.75 & 470.8 & $\begin{array}{l}0.397 \angle \\
-2.68 \\
\end{array}$ & 12.96 & 566.3 \\
\hline 0.0825 & $\begin{array}{l}0.557 \angle \\
-2.87\end{array}$ & 6.25 & 275.1 & $\begin{array}{l}0.566 \angle \\
-3.00\end{array}$ & 8.35 & 365.6 & $\begin{array}{l}0.604 \angle \\
-2.90 \\
\end{array}$ & 10.4 & 455.3 & $\begin{array}{l}0.567 \angle \\
-2.82 \\
\end{array}$ & 12.55 & 547.2 \\
\hline 0.0931 & $0.800<2.92$ & 6.18 & 271.5 & $0.893 \angle 3.03$ & 8.25 & 361.9 & $\begin{array}{l}0.874 \angle \\
-3.10 \\
\end{array}$ & 10.3 & 450.6 & $\begin{array}{l}0.925 \angle \\
-3.08 \\
\end{array}$ & 12.41 & 540.6 \\
\hline 0.1201 & $1.068 \angle 3.05$ & 5.98 & 263.4 & $1.163 \angle 2.88$ & 8 & 351.3 & $1.301 \angle 2.88$ & \begin{tabular}{|l|}
9.94 \\
\end{tabular} & 436.6 & $1.253 \angle 2.94$ & 12 & 524.0 \\
\hline
\end{tabular}

Table 9-7 Off-Diagonal Term of the Impedance Matrix, Mechanical Speed And Electrical Frequency for the 64 ITSC Test Cases of the Faulty IM Driven by DTC: From 1.2 to 2

\begin{tabular}{|c|c|c|c|c|c|c|c|c|c|c|c|c|}
\hline \multicolumn{13}{|c|}{$\mathrm{Nm}$} \\
\hline$T_{r e f}$ & \multicolumn{3}{|c|}{1.4} & \multicolumn{3}{|c|}{1.6} & \multicolumn{3}{|c|}{1.8} & \multicolumn{3}{|c|}{2} \\
\hline$\mu$ & $Z_{n p}(\Omega)$ & $\begin{array}{c}f_{e} \\
(\mathrm{~Hz})\end{array}$ & $\begin{array}{c}\omega_{r} \\
(\mathrm{rad} / \mathrm{sec})\end{array}$ & $Z_{n p}(\Omega)$ & \begin{tabular}{|c|}
$f_{e}$ \\
$(\mathrm{~Hz})$
\end{tabular} & $\begin{array}{c}\omega_{r} \\
(\mathrm{rad} / \mathrm{sec})\end{array}$ & $Z_{n p}(\Omega)$ & \begin{tabular}{|c|}
$f_{e}$ \\
$(\mathrm{~Hz})$
\end{tabular} & $\begin{array}{c}\omega_{r} \\
(\mathrm{rad} / \mathrm{sec})\end{array}$ & $Z_{n p}(\Omega)$ & $\begin{array}{c}f_{e} \\
(\mathrm{~Hz})\end{array}$ & $\begin{array}{c}\omega_{r} \\
(\mathrm{rad} / \mathrm{sec})\end{array}$ \\
\hline 0 & 0 & & 616.6 & 0 & & 706.0 & 0 & & 789.9 & 0 & & 889.8 \\
\hline 0.0108 & $0.042 \angle 2.93$ & 15.5 & 668.6 & $\begin{array}{l}0.052 \angle \\
-2.26\end{array}$ & 17.82 & 764.0 & $\begin{array}{l}0.040 \angle \\
-1.34\end{array}$ & 20.25 & 860.4 & $0.047 \angle 0.78$ & 22.72 & 955.6 \\
\hline 0.0268 & $\begin{array}{l}0.113 \angle \\
-2.36 \\
\end{array}$ & 15.55 & 673.9 & $\begin{array}{l}0.122 \angle \\
-2.24 \\
\end{array}$ & 17.9 & 770.9 & $\begin{array}{l}0.114 \angle \\
-2.29 \\
\end{array}$ & 20.3 & 868.5 & $\begin{array}{l}0.109 \angle \\
-2.33 \\
\end{array}$ & 22.84 & 966.0 \\
\hline 0.0398 & $\begin{array}{l}0.247 \angle \\
-2.51 \\
\end{array}$ & 15.3 & 661.6 & $\begin{array}{l}0.242 \angle \\
-2.37 \\
\end{array}$ & 17.62 & 758.1 & $\begin{array}{l}0.212 \angle \\
-2.35 \\
\end{array}$ & 20 & 855.3 & $\begin{array}{l}0.229 \angle \\
-2.46 \\
\end{array}$ & 22.55 & 952.6 \\
\hline 0.0470 & $\begin{array}{l}0.298 \angle \\
-2.17 \\
\end{array}$ & 15.25 & 659.5 & $\begin{array}{l}0.213 \angle \\
-2.42 \\
\end{array}$ & 17.5 & 755.1 & $\begin{array}{l}0.232 \angle \\
-2.46 \\
\end{array}$ & 19.95 & 852.5 & $\begin{array}{l}0.2347 \angle \\
-2.45 \\
\end{array}$ & 22.45 & 950.2 \\
\hline 0.0555 & $\begin{array}{l}0.409 \angle \\
-2.60 \\
\end{array}$ & 15.25 & 663.2 & $\begin{array}{l}0.419 \angle \\
-2.55 \\
\end{array}$ & 17.6 & 760.2 & $\begin{array}{l}0.41 \angle \\
-2.52 \\
\end{array}$ & 20 & 858.1 & $\begin{array}{l}0.406 \angle \\
-2.58 \\
\end{array}$ & 22.5 & 957.3 \\
\hline 0.0825 & $0.503 \angle 3.05$ & 14.75 & 640.3 & $0.656 \angle 2.89$ & 17 & 734.9 & $\begin{array}{l}0.627 \angle \\
-2.69 \\
\end{array}$ & 19.3 & 829.5 & $\begin{array}{l}0.591 \angle \\
-2.77 \\
\end{array}$ & 21.75 & 926.2 \\
\hline 0.0931 & $\begin{array}{l}0.872 \angle \\
-2.87 \\
\end{array}$ & 14.56 & 633.3 & $\begin{array}{l}0.975 \angle \\
-2.81 \\
\end{array}$ & 16.8 & 726.4 & $\begin{array}{l}0.944 \angle \\
-2.86 \\
\end{array}$ & 19.07 & 819.8 & $\begin{array}{l}0.788 \angle \\
-2.97 \\
\end{array}$ & 21.48 & 916.0 \\
\hline 0.1201 & $1.171 \angle 3.14$ & 14.07 & 612.4 & $\begin{array}{l}1.424 \angle \\
-3.11 \\
\end{array}$ & 16.2 & 701.0 & $1.042 \angle 3.12$ & 18.4 & 791.4 & $1.348 \angle 3.136$ & 20.7 & 883.9 \\
\hline
\end{tabular}




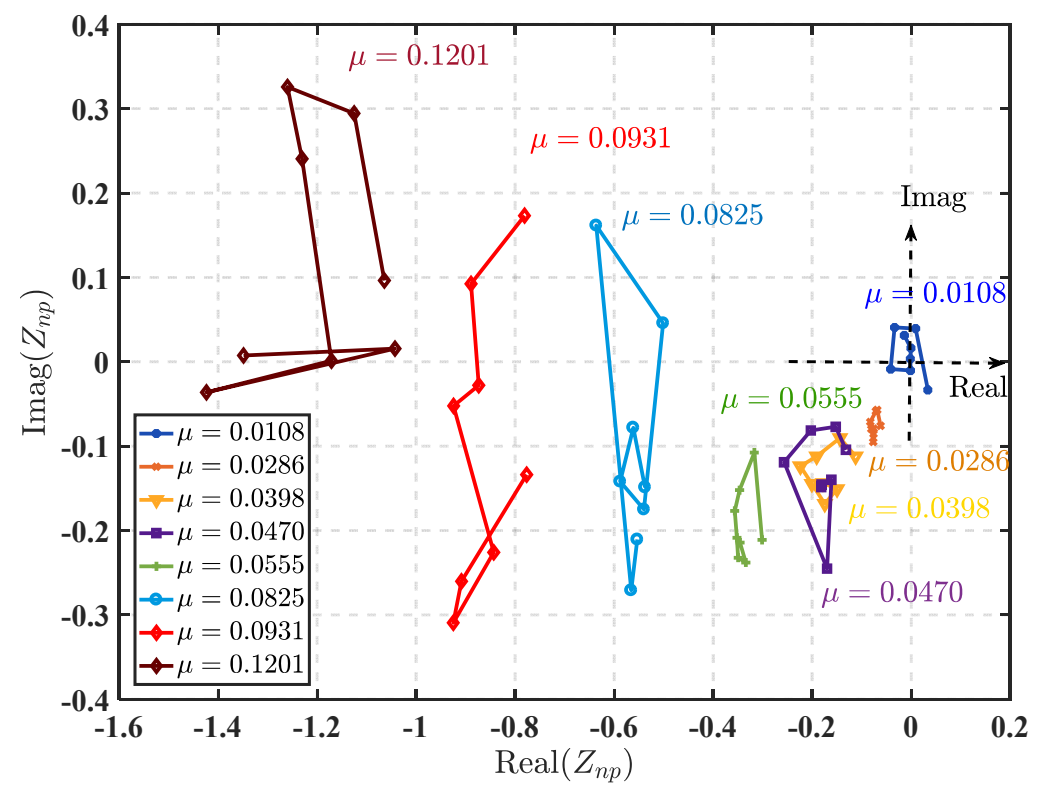

Figure 9.15 Locus of the Off-diagonal Term of the Sequence Component Impedance Matrix for the 64 cases of study. Simulation Results.

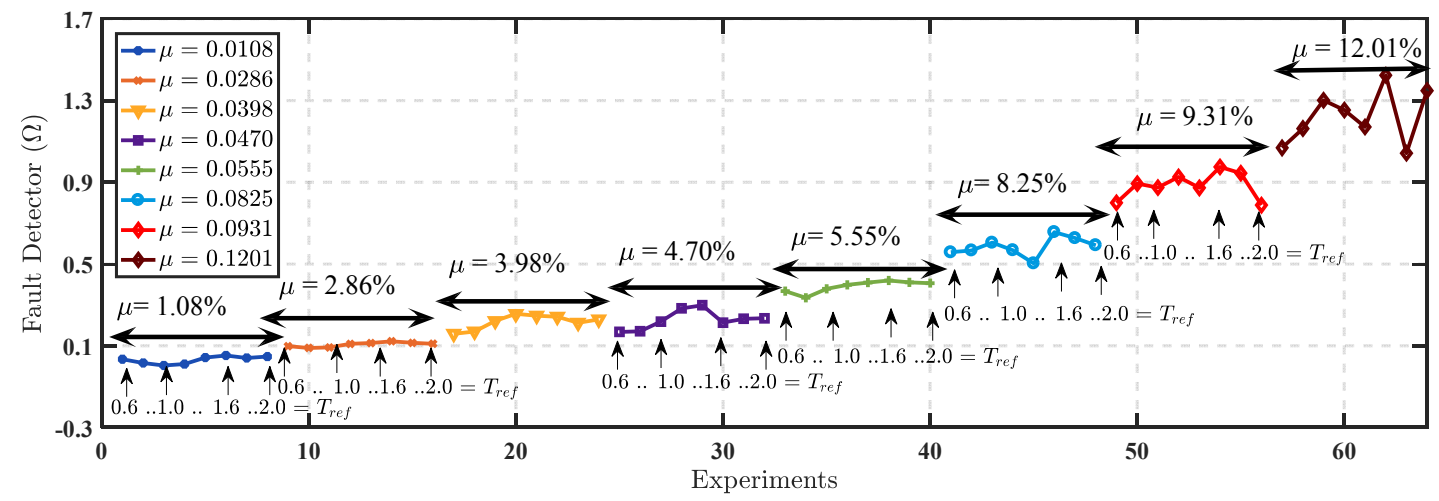

Figure 9.16 Simulation results of the proposed FD technique for the 64 cases of study. Fault detector versus number of experiments.

\subsubsection{Experimental Results}

The experimental results are composed of 2 parts: (1) Study of eight Fault Severity Factors under DTC for one operative condition and (2) Fault Detection Corroboration. For the experimental results, the 8 cases of fault severity factor were tested under one operative point $\left(T_{r e f}=2 \mathrm{Nm}\right)$ as from the simulation results is was demonstrated that the operative 
point (torque reference) does not affect the FD. Furthermore, the torque ripple behavior of the experimental results is larger than the simulation ones, leading to a hiding consequence of the ITSC effects. Typically, there are several reasons for which the estimated torque in the experimental results has higher ripple:

- The torque ripple is being estimated and not measured in the experimental test.

- This variable depends on the shape, geometry and position of the rotor bars in the IM, which are not considered in the simulated model.

- The dc-link voltage in the experimental test presents variations with the switching activities and the operative point of the IM under DTC. In the simulations, the dclink voltage is always constant, as an infinite dc power source is considered.

- Typically, a real IM has small asymmetries inherent to its construction. Some of the asymmetries are static eccentricity, different values of stator resistances perphase, deformation and defects in the stator windings, etc [149].

\subsubsection{Study of eight Fault Severity Factors under DTC for one operative condition}

In Figure 9.17, the estimated electromagnetic torque is depicted for the healthy and 8 ITSC cases of the IM driven by DTC. In the experimental results, the only available torque is the estimated one. The actual torque should be measured with a torque meter or a force meter not accessible in the lab. The increment on the torque ripple can be noted while the fault severity factor increases, however, the differential is not significant due to the nature of the signal (estimated). From the simulations, it was demonstrated that the DTC has no access to the faulty circulating current that creates a large double torque ripple frequency, as shown in Fig. 10 and 12. In [34], [180], the modelling of the faulty IM indicates that the 
electromagnetic torque has two components: (1) the healthy torque and (2) faulty torque term, which is enabled by the circulating current in the faulty part of the winding.

Therefore, the estimated torque and flux will not show the effects of the ITSC as clearly as the actual real values. Notice in Table 9-8, that the MAE for the estimated torque and flux are similar values, where no conclusion can be obtained about the faulty behavior of the IM.

$$
-\mu=0.0470-\mu=0.0555-\mu=0.0825-\mu=0.0931-\mu=0.1201
$$

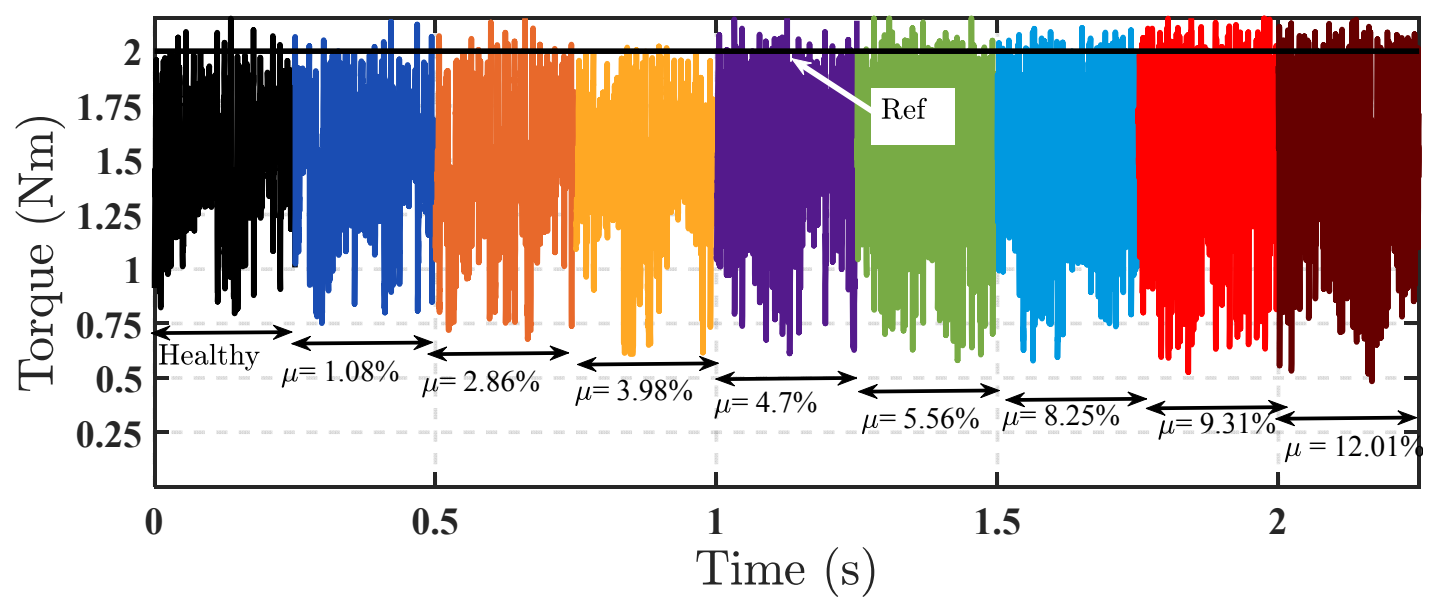

Figure 9.17 Estimated Electromagnetic Torque for the 8 cases of ITSC fault and the healthy IM at $T_{r e f}=2 \mathrm{Nm}$. Experimental results.

Table 9-8 Estimated Torque and Flux MAE for the eight ITSC Test-Cases for the one operational Point $\left(T_{r e f}=2 \mathrm{Nm}\right)$ of the Faulty IM driven by DTC

\begin{tabular}{|c|c|c|}
\hline$\mu$ & $\operatorname{MAE}\left(\widehat{T}_{e}\right)$ & $\mathrm{MAE}\left|\hat{\boldsymbol{\lambda}}_{s}\right|$ \\
\hline 0 & 12.87 & 1.88 \\
\hline 0.0108 & 12.32 & 1.89 \\
\hline 0.0268 & 13.73 & 1.87 \\
\hline 0.0398 & 12.83 & 1.88 \\
\hline 0.0470 & 12.22 & 1.88 \\
\hline 0.0555 & 12.25 & 1.87 \\
\hline 0.0825 & 12.45 & 1.87 \\
\hline 0.0931 & 13.73 & 1.86 \\
\hline 0.1201 & 14.42 & 1.86 \\
\hline
\end{tabular}




\subsubsection{Fault Detection Corroboration}

For the experimental verification, 8 fault cases are studied, as in the simulation part, but only for one operative point of the DTC $T_{r e f}=2 \mathrm{Nm}$, as the simulation demonstrated that the FD technique is not affected by it. In Table 9-9, 3 variables are presented for each case: the fault detector $\left(Z_{n p}(\Omega)\right)$ in magnitude and angle, the electrical frequency $\left(f_{e}(\mathrm{~Hz})\right)$ and the rotational mechanical speed $\left(\omega_{r}(\mathrm{rad} / \mathrm{sec})\right.$. From the table, it can be noted that for each torque reference, the electrical frequency and mechanical speed have similar values (same operational point), however, the fault detection increases with $\mu$.

Table 9-9 Secuence Components and Off-Diagonal Term of the Impedance Matrix for the eight ITSC Test Cases of the Faulty IM driven by DTC at $T_{r e f}=2 \mathrm{Nm}$

\begin{tabular}{|c|c|c|c|}
\hline$\mu$ & $Z_{n p}(\Omega)$ & $f_{e}(\mathrm{~Hz})$ & $\omega_{r}(\mathrm{rad} / \mathrm{sec})$ \\
\hline 0 & $0.1812 \angle 2.7827$ & 78.88 & 1087.5 \\
\hline 0.0108 & $0.3325 \angle 1.2360$ & 78.12 & 1093.3 \\
\hline 0.0268 & $0.5519 \angle 2.5925$ & 77.20 & 1078.0 \\
\hline 0.0398 & $0.6861 \angle-2.5001$ & 78.73 & 1097.0 \\
\hline 0.0470 & $1.9924 \angle 0.7039$ & 73.85 & 1039.4 \\
\hline 0.0555 & $2.3123 \angle-0.3244$ & 73.54 & 1022.6 \\
\hline 0.0825 & $3.4273 \angle 2.5778$ & 74.00 & 1026.0 \\
\hline 0.0931 & $5.7418 \angle 0.4975$ & 69.42 & 967.9 \\
\hline 0.1201 & $11.7956 \angle-.4217$ & 68.81 & 963.6 \\
\hline
\end{tabular}

Comparing simulation and experimental results from Table 9-6 and Table 9-9 at $T_{\text {ref }}=$ $2 \mathrm{Nm}$, the mechanical speeds are correlated, but the electrical frequency is 4 times faster from the experiments than from the simulations. Figure 9.18 shows the locus of the offdiagonal impedance component for the 9 cases. The amplitude of the off-diagonal impedance component separates more from the locus origin as the fault severity factor increases, in the same way as the simulations do. Figure 9.19 shows the fault detector for 
the healthy case and the 8 faults. Notice that the FD increases with the $\mu$. In this case, the healthy off-diagonal impedance $Z_{n p h} \neq 0$, as the IM has construction asymmetries. A comparison between the simulation and experimental results remarks a significant difference in absolute values of the FD but not in the trend of them. It is obvious that the FD has a great sensitivity and it has been able to detect incipient turn faults at least as low as $1.08 \%$ (the minimum $\mu$ tested), which means 6 turns out of 510 .

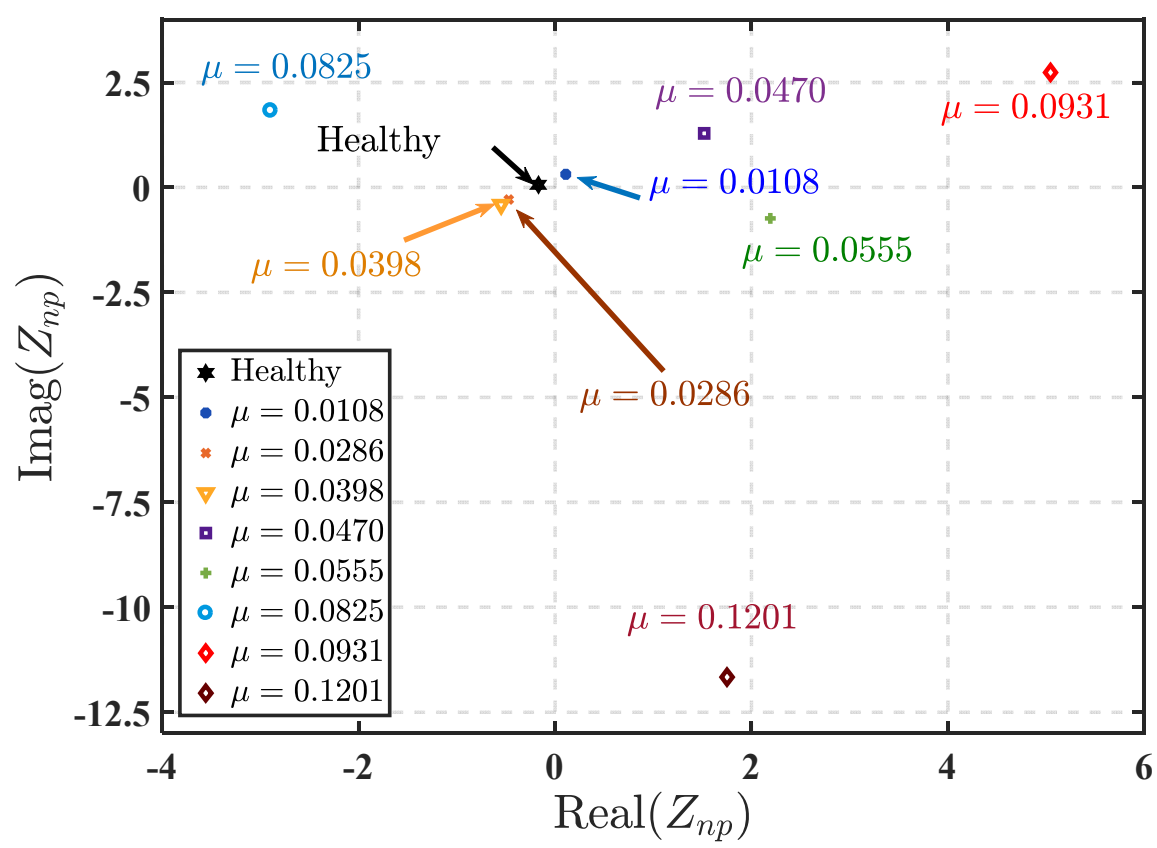

Figure 9.18 Locus of the Off-diagonal Term of the Sequence Component Impedance Matrix for the 64 cases of study. Experimental Results. 


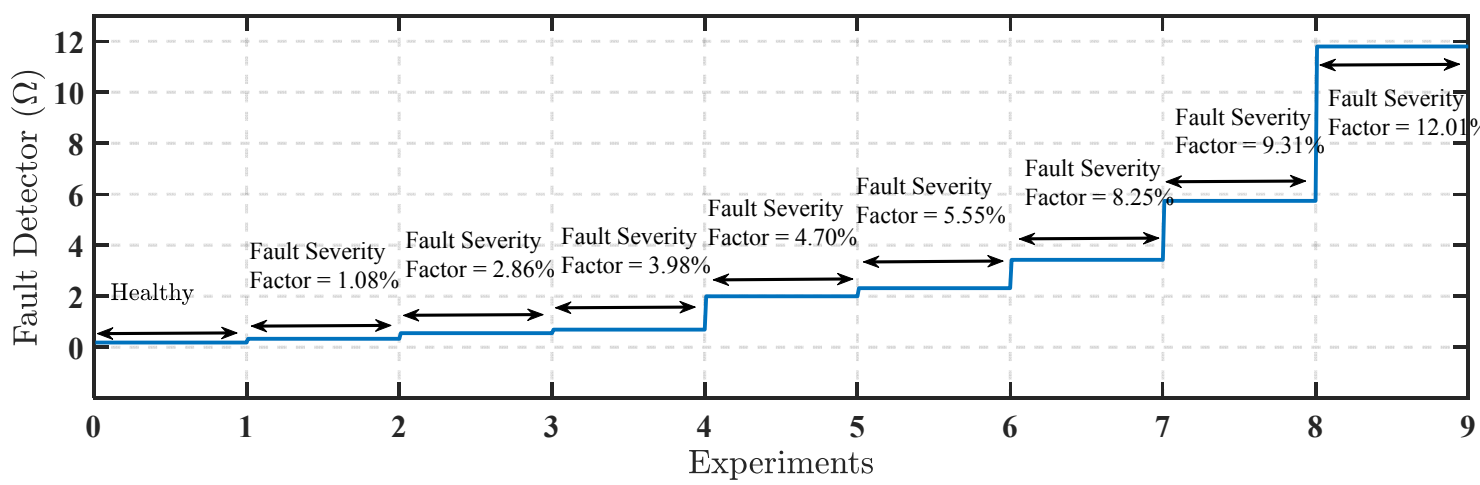

Figure 9.19 Experimental results of the proposed FD technique for the 8 fault test-cases of study and the healthy IM at $T_{r e f}=2 \mathrm{Nm}$. Fault detector versus number of experiments.

\subsection{Conclusion}

An online, not intrusive and robust fault detector of stator turn faults for DTC driven IM is presented. The fault detector is based on the continuous monitoring of the off-diagonal term of the SC impedance matrix. The FD is adapted to suit DTC controlled IM, where many hurdles were overcome for its implementation. The implementation of the FD is explained in detail. Two simulation test-benches were developed for initial testing of the FD. The simulations provide insights on the DTC functioning and the effects of the ITSC on it. Evidence of the signatures hiding phenomena by the closed-loop control were demonstrated, as well as the DTC estimation errors due to the missing terms appearing in the onset of the fault. Finally, experimental test results demonstrate the effectiveness, capabilities and sensitivity of the FD for the DTC driven IM. 


\section{Chapter 10 Condition Monitoring in Power Electronic Devices: Breakdown Mechanism in Gallium Nitride MOSFETs}

\subsection{Introduction}

Although power electronic drives are widespread and are used in a variety of applications, they are prone to several failures. As [12] notes, the two components that are most likely to fail in switch-mode drives are electrolytic filtering capacitors and power semiconductors. Among the latter, the most typical power semiconductor faults are short circuit-and open-circuit faults. Although most of the works in the power converter FD area address ac- $\mathrm{dc}$ or ac-ac converters, an increasing number of recent papers are focused on FD in dc-dc converters.

A detailed review of the recent literature on inverter FD is developed in [181]. Reference [182] notes the characteristics of each of the two main types of switch failures in inverters: while short-circuit fault are typically very destructive and require the adoption of actions to shut down the drive immediately, open-circuit faults do not necessarily lead to system shutdown and can remain undetected for a long time (however, such faults can lead to secondary faults in other converter components that can eventually lead to high repair costs).

Several recent works have addressed the FD of different faults in power electronics components. In this regard, the FD of IGBTs is studied in [183], [184]. In [184], the authors propose a new on-board CM of the aging of solder layers in IGBTs for electric vehicle applications, while [183] proposes an online principal component analysis based algorithm for early fault detection in IGBT switches. The fault detection and degradation of other 
components, such as MOSFETs, is studied in [185]. Despite there is a number of papers describing and analyzing the physics of the failure in power components as MOSFETs or IGBTs, there is still a lack of works proposing the use of the device features (e.g. on-state resistance, threshold voltage ...) for on-line switch CM. This deficiency is partially due to the difficulty in obtaining the required signals that are often small and susceptible to corruption from noise [183].

With the evolution of the semiconductor technology, the trend power electronic converters is turning its view to the new and so-called wide band gap devices such as Silicon Carbide ( $\mathrm{SiC}$ ) or Gallium Nitride $(\mathrm{GaN})$ switching devices. Power GaN transistors have recently demonstrated to be excellent devices for application in power electronics. The high breakdown field and the superior mobility of the 2-dimensional electron gas allow to fabricate transistors with low resistive and switching losses that permit to increase the efficiency of switching mode power converters beyond $99 \%$. GaN-based transistors are currently supposed to be adopted in KW-range power converters; $650 \mathrm{~V}$ transistors are already available on the market, and $1200 \mathrm{~V}$ devices are currently under development.

During operation, GaN power transistors can reach critical conditions, especially in the off-state (with a high $V_{d s}$, in excess of $650 \mathrm{~V}$ ), during hard-switching (where high current and voltage can be simultaneously present), and for high positive gate voltages (in the case of normally-off devices). This chapter reports the most recent results on the gradual and catastrophic degradation of GaN-based power HEMTs. The degradation mechanisms that take place in a GaN power HEMT can be under the following states of the device: off-state, on-state and semi-on state conditions. The phenomenon interested in this chapter is the offstate. Thus, the degradation mechanisms of the HEMT under off-state are [186]: 
1. Off-state (Degradation mode/process)

a. Dynamic-Ron increase: the physical origin can come from:

i. buffer trapping, due to:

1. Ionization of buffer acceptors.

2. Injection of electrons from the substrate.

ii. Or Surface trapping due to injection of electrons from gate to drain, due to poor passivation.

b. Threshold Voltage shift: the physical origin can come from trapping/detrapping of electrons in the gate area.

c. Time-dependent degradation: the physical origin can come from:

i. Generation of source-drain current paths.

ii. Short circuits between gate and channel.

iii. Vertical (drain to substrate) breakdown.

These degradation phenomena appears as a consequence of high $V_{d s}$ stress on the HEMT. Thus, this chapter is dedicated to investigate the voltage breakdown mechanics that will be the commencement stage or the cause of the degradation process.

\subsection{Breakdown Voltage Assessment of GaN HEMT Devices through Physics- Based Modeling}

GaN HEMTs have gained a great deal of attention within the power electronics industry as a result of their capability of operating at higher temperatures, higher switching frequencies, and increased voltage. Theoretical limits lines $\left(r_{o n}-B V\right)$ for lateral HEMT are better than vertical GaN devices as the mobility of the electrons in the two-dimensional electron gas (2DEG) is huge [187]. However, HEMTs are susceptible to surface breakdown 
and are not easily scalable to higher voltages and currents. Thus, there is a necessity to investigate how the BV of the HEMT can be increased. This question can be answered by delving deep into the physics of the HEMT operation. Physics-based models (PBM) can provide us with a specialized set of tools based on the geometry, materials, and physics of HEMT to evaluate mechanisms that can increase the BV [188], [189].

There are several BV mechanisms such as:

- Source-drain breakdown due to short-channel effects, and/or punch-through.

- The presence of relatively high breakdown current components at the gate, which can be either related to the leakage through the Schottky junction, or to surfacerelated conduction.

- Vertical breakdown, which can be due to a poor compensation of the buffer, to the use of a conductive substrate, and can be limited by the adoption of suitable back barrier or heterostructure configurations.

- Impact ionization mechanisms, that may induce a significant increase in drain current due to the generation of electron-hole pairs close to the gate [190].

In the last case, the BV depends on the material critical electric field $\left(E_{\text {crit }}\right)$, or the field needed to initiate the impact ionization causing an avalanche breakdown [191]. Thus, there are 2 ways to avoid the BV: ensuring that the electric field $\left(E_{f}\right)<E_{\text {crit }}$ at the operating point of the HEMT or enlarging the drift distance. However, the drift distance is kept fixed as a constraint. Thus, the only solution available is to redistribute $E_{f}$. From the literature review, there are 3 solutions to improve the BV: varying passivation material, changing the substrate material, and applying a field plate structure. 
Table 10-1 GAN-FET Design Parameters and Cases of Study

\begin{tabular}{|c|c|c|c|c|}
\hline Name & \multicolumn{2}{|c|}{ Value } & Name & Value \\
\hline Substrate & \multicolumn{2}{|c|}{ Thickness $13.98 \mu \mathrm{m}$} & AlGaN Composit & $x=20 \%$ \\
\hline Source/Drain & \multicolumn{2}{|c|}{ Width $1 \mu \mathrm{m}$} & & \\
\hline Gate & \multicolumn{2}{|c|}{ Width $3 \mu \mathrm{m}$} & Case of Study & Fig. 1 - Passiv. - Subst. \\
\hline Active GaN Layer & \multicolumn{2}{|c|}{ Thickness $1 \mu \mathrm{m}$} & Original & Fig. 1(a)-SiO $2-\mathrm{Si}$ \\
\hline $\mathrm{AlGaN}$ & \multicolumn{2}{|c|}{ Thickness $0.02 \mu \mathrm{m}$} & Passivation & Fig. 1 (a)-SiN - Si \\
\hline Doping & Original & Light & GaN Substrate & Fig. 1 (a)-SiN - GaN \\
\hline GaN Layer (Acceptor) & $2 \times 10^{17} \mathrm{~cm}^{-3}$ & $2 \times 10^{14} \mathrm{~cm}^{-3}$ & Light Doping & Fig. 1 (a)-SiN - GaN \\
\hline GaN Layer (Donor) & $2 \times 10^{16} \mathrm{~cm}^{-3}$ & $2 \times 10^{13} \mathrm{~cm}^{-3}$ & Ge Substrate & Fig. 1 (a)-SiN - Ge \\
\hline AlGaN Layer (Donor) & $1 \times 10^{17} \mathrm{~cm}^{-3}$ & $1 \times 10^{14} \mathrm{~cm}^{-3}$ & Field Plate & Fig. 1 (b)-SiN - GaN \\
\hline
\end{tabular}

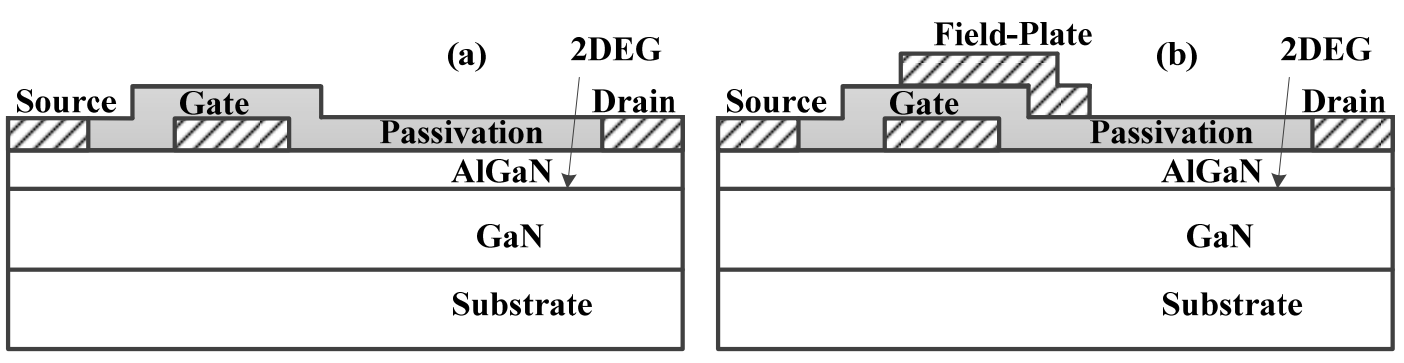

Figure 10.1 Schematic layer structure (a) Base (b) Field Plate.

Different materials and geometric changes have been applied on a single HEMT. The passivation material, substrate, and doping level have been evaluated as well as the impacts of a FP inclusion. For each case, the BV progression and $E_{f}$ distribution is discussed. A comparison of 6 different structures (Table 10-1) is presented. The schematic layers of these cases are shown in Figure 10.1 with the dimensions described in Table 10-1. Table 10-1 indicates the structure for each case of study. For the final case shown in Figure 10.1 (b) [188], a FP is added to reshape the $E_{f}$ distribution in the channel and reduce its peak at the point of interest [192]. 


\subsubsection{Electric Field by FEA of all the Cases}

In this section, a detailed study of all the performed modifications are presented. The strucutres schematics, the true actual dimenstions, its material specfications, the drain current versus the drain-source voltage characteristics and the $2 \mathrm{D}$ electric field in the HEMT are presented.

\subsubsection{Base Structure}

The schematic of the base structure, which has not been drawn at scale is shown in Figure 10.1(a). The specifications of this structure are as follows:

Characteristics of the base case:

- Substrate material: Silicon (Si)

- Passivation material: $\mathrm{SiO} 2$

- No Field plate

- Dimentions: as shown in Table 10-1.

The proportional real dimentions of the base case HEMT are shown in Figure 10.2(a) where it is observe that the biggest region is the substrate. In Figure 10.2(b) the characteristic curve $\left(i_{d}-v_{d s}\right)$ of the HEMT is plotted. From this curve it is noted that the breakdown voltage starts at $366.5 \mathrm{~V}$.

The 2D electric field of the base case of the HEMT is shown in Figure 10.3. In this figure it can be observed the zones where the electric field is more intense. Two zones are of interest: in the vicinity of the gate and drain. However, the intensity on the gate vicinity is superior. A zoom of the electric field near the gate is illustrated at the top of Figure 10.3. In here, the right lower corner of the gate contactor indicates a spike of field. This spike is 
the one responsible for the voltage breakdown mechanism producing a huge gate current that travels to the drain causing the knee point observed in Figure 10.2.
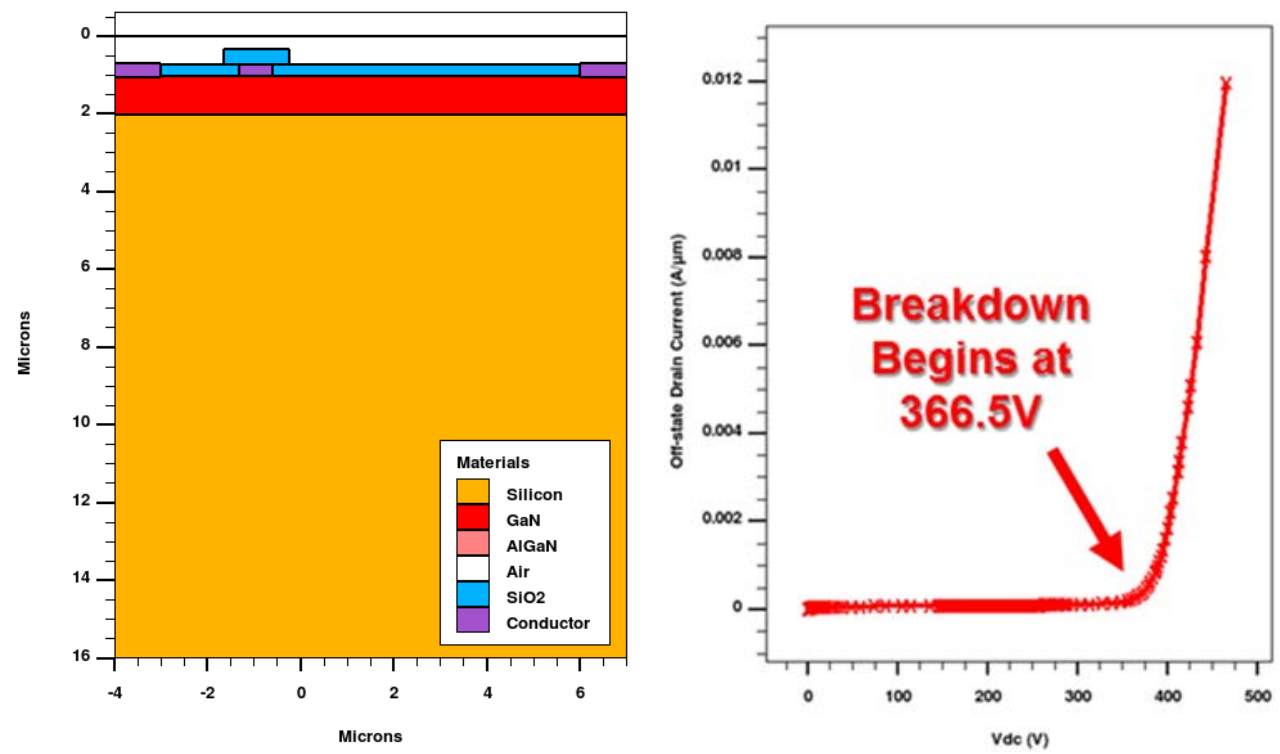

Figure 10.2 (a) True dimentions of the base case HEMT (b) Drain current versus Drainsource voltage characteristic of the base case HEMT.

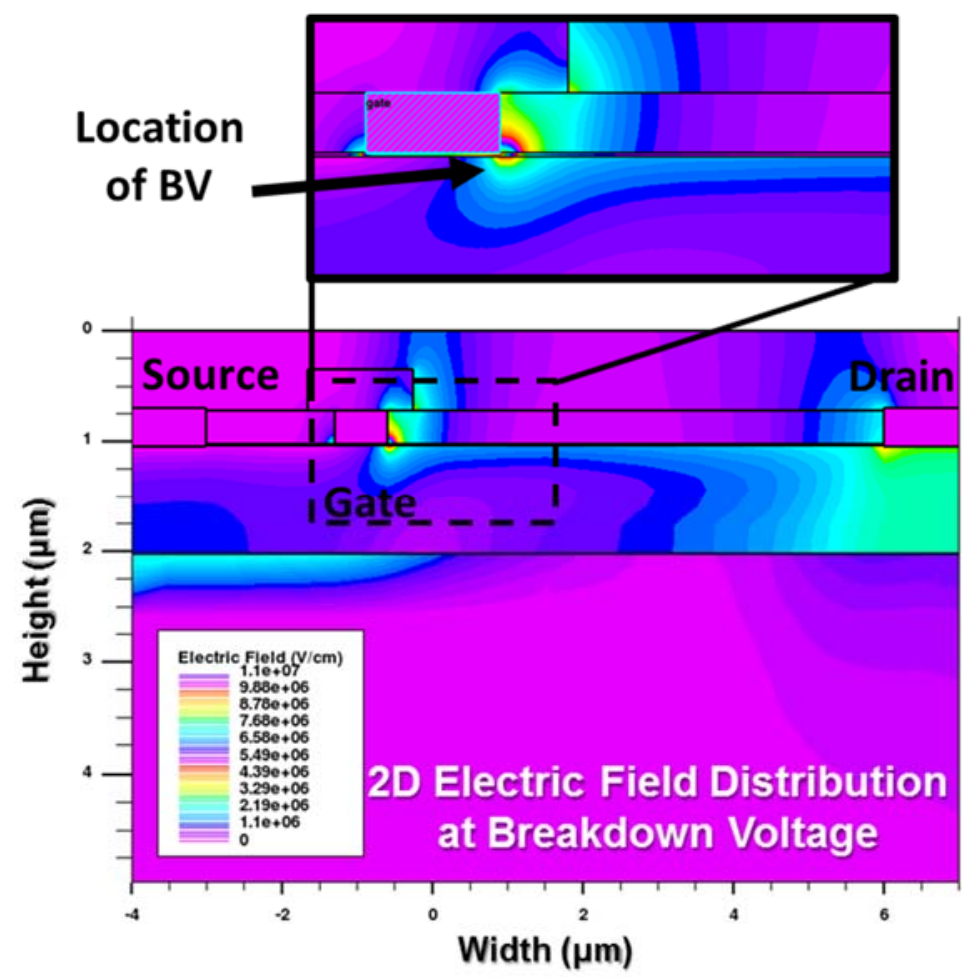

Figure 10.3 Two dimentional electric field forth the base case HEMT. 


\subsubsection{Changing the Passivation Layer}

The schematic of this case is the same as the base structure as in Figure 10.1(a). The specifications of this structure are as follows:

- Same structure than the base

- Substrate material: Silicon (Si)

- Passivation material: SiN

- No Field plate

The proportional real dimentions of this case HEMT are shown in Figure 10.4(a). In Figure 10.4(b) the characteristic curve $\left(i_{d}-v_{d s}\right)$ of the HEMT is plotted. From this curve it is noted that the breakdown voltage starts at $384.3 \mathrm{~V}$.
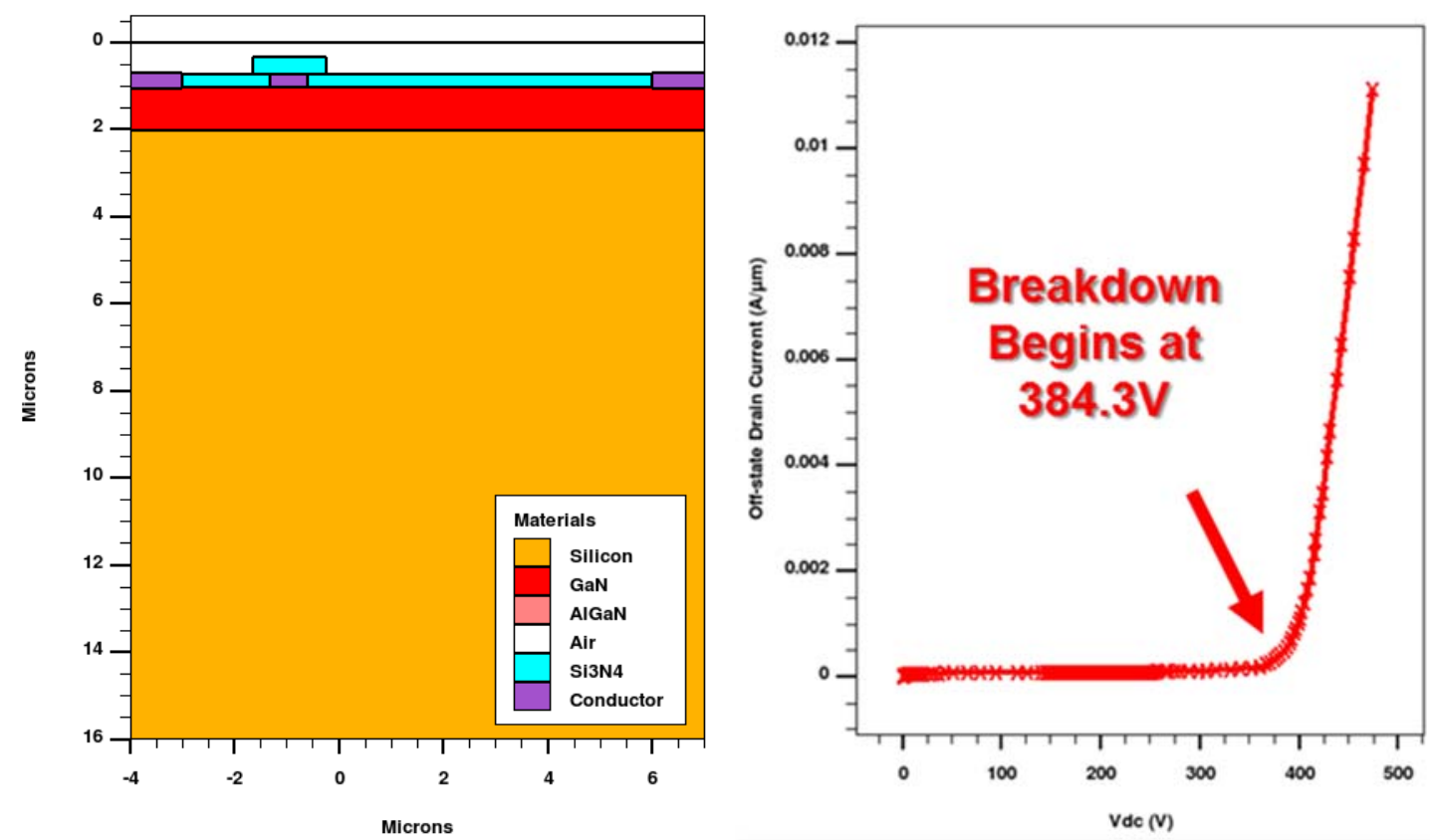

Figure 10.4 (a) True dimentions of the case HEMT with $\mathrm{SiN}$ in the passivation (b) Drain current versus Drain-source voltage characteristic of the case HEMT with SiN in the passivation. 
The 2D electric field of the base case of the HEMT is shown in Figure 10.15. In this figure it can be observed the zones where the electric field is more intense, similar to the base case. The electric field in the vicinity of the gate is more intense. The zoom of the electric field at the top of Figure 10.15 shows that the right lower corner of the gate contactor has a spike. This spike is the one responsible for the voltage breakdown mechanism in the same way as the base case. The difference here is that the SiN, respect with $\mathrm{SiO} 2$, presents a better electric field critical and thus more tolerance to the breakdown mechanism.

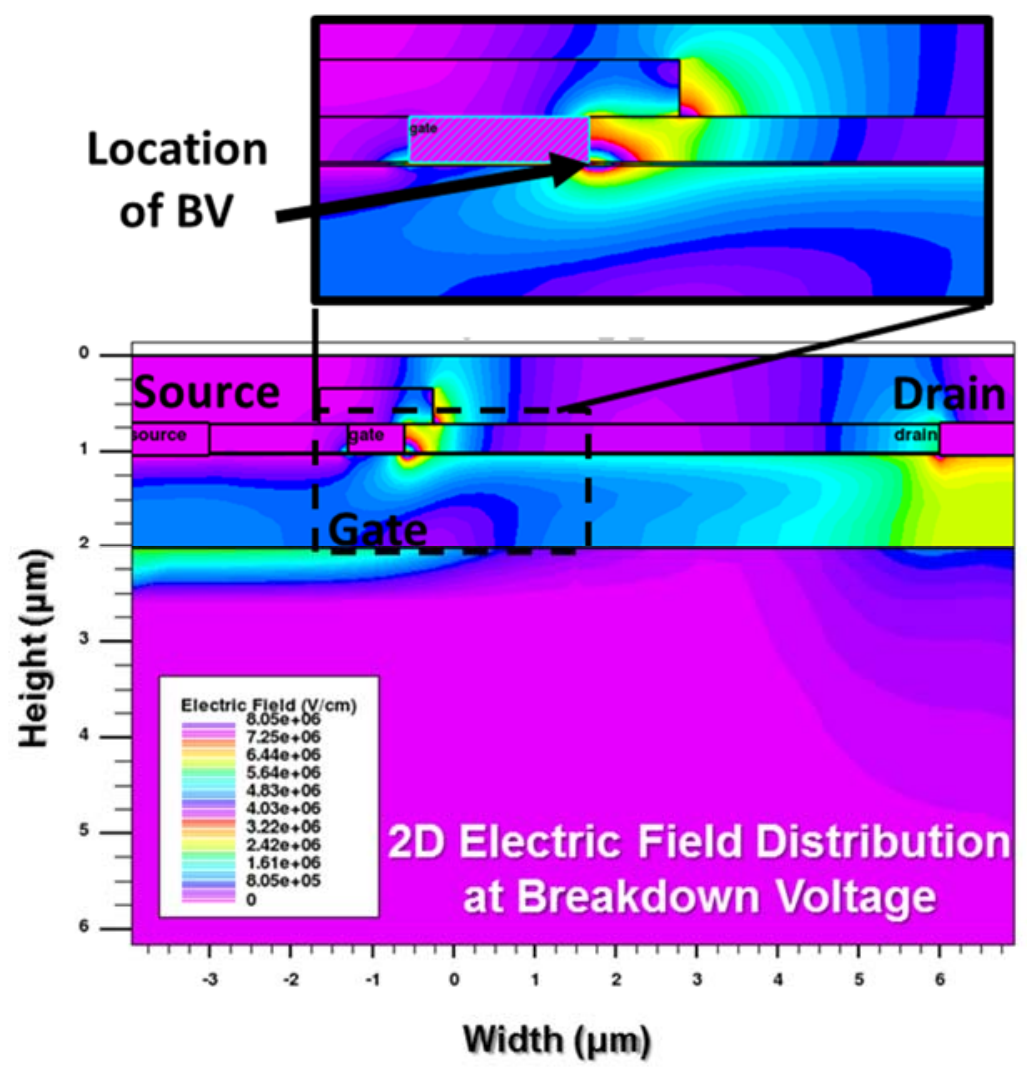

Figure 10.5 Two dimentional electric field forth the case HEMT with $\mathrm{SiN}$ in the passivation. 


\subsubsection{Altering the Doping Profile}

The schematic of this case is the same as the base structure as in Figure 10.1(a). The specifications of this structure are as follows:

- Same structure than the base

- Substrate material: Silicon (Si)

- Passivation material: SiN

- No Field plate

Table 10-2 Doping Parameters for the GaN and AlGaN layers

\begin{tabular}{|l|l|l|}
\hline Name & Value \\
\hline Doping & Original & Light \\
\hline GaN Layer (Acceptor) & $2 \times 10^{17} \mathrm{~cm}^{-3}$ & $2 \times 10^{14} \mathrm{~cm}^{-3}$ \\
\hline GaN Layer (Donor) & $2 \times 10^{16} \mathrm{~cm}^{-3}$ & $2 \times 10^{13} \mathrm{~cm}^{-3}$ \\
\hline AlGaN Layer (Donor) & $1 \times 10^{17} \mathrm{~cm}^{-3}$ & $1 \times 10^{14} \mathrm{~cm}^{-3}$ \\
\hline
\end{tabular}

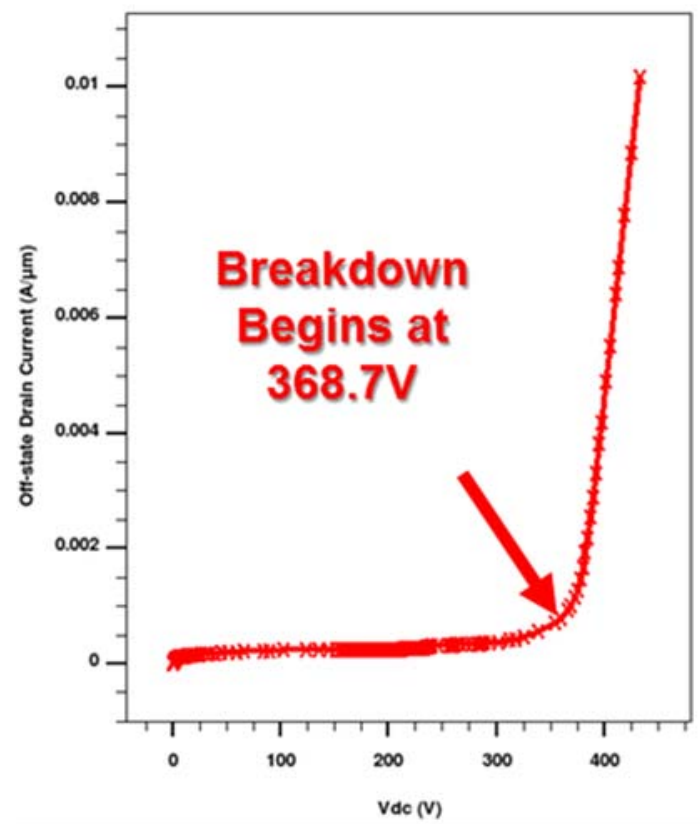

Figure 10.6 Drain current versus Drain-source voltage characteristic of the case HEMT with SiN in the passivation. 
In this subsection, the HEMT GaN and AlGaN layers have been modified. The dopin profile has been increased respect with the previous cases. In Table 10-2 the doping profile is shown. In Figure 10.6 the characteristic curve $\left(i_{d}-v_{d s}\right)$ of the HEMT is plotted. From this curve it is noted that the breakdown voltage starts at $368.7 \mathrm{~V}$. With this study, it has been corroborated that the ideal case is the best one. Thus, the inclusion of impurities is the simulation of the true conditions, however, the desired output in this chapter is the maximum theoretical breakdown voltage possible.

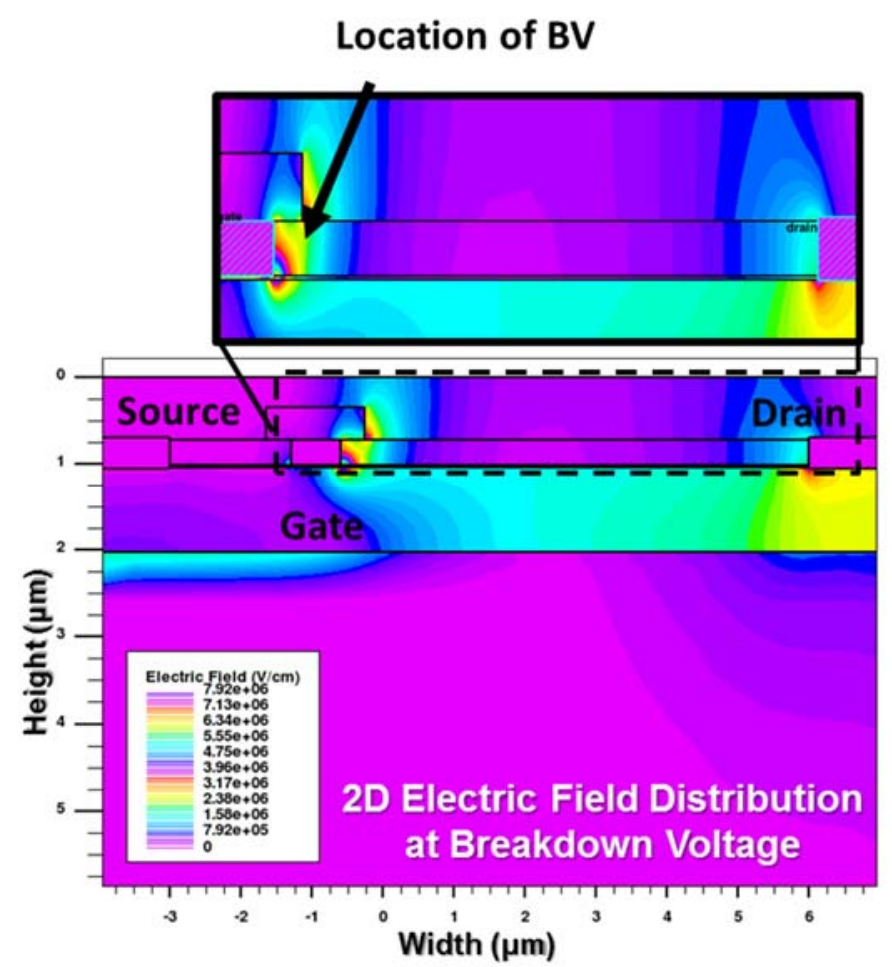

Figure 10.7 Two dimentional electric field forth the case HEMT with $\mathrm{SiN}$ in the passivation.

The $2 \mathrm{D}$ electric field of this case is shown in Figure 10.7. In this figure it can be observed the zones where the electric field is more intense, similar to the previous cases. The electric field in the vicinity of the gate is more intense. The zoom of the electric field 
at the top of Figure 10.7 shows that the right lower corner of the gate contactor has a spike. This spike is the one responsible for the voltage breakdown mechanism in the same way as the previous case. The difference here is that the doping profile reduce the breakdown voltage due to the inclusion of the impurities in the GaN and AlGaN layers, which reduce the mobility on the 2DEG and increase the on-resistance.

\subsubsection{Replacing the Subtrate with Germanium}

The schematic of this case is the same as the base structure as in Figure 10.1(a). The specifications of this structure are as follows:

- Same structure than the base

- Substrate material: Germanium $(\mathrm{Ge})$

- Passivation material: SiN

- No Field plate
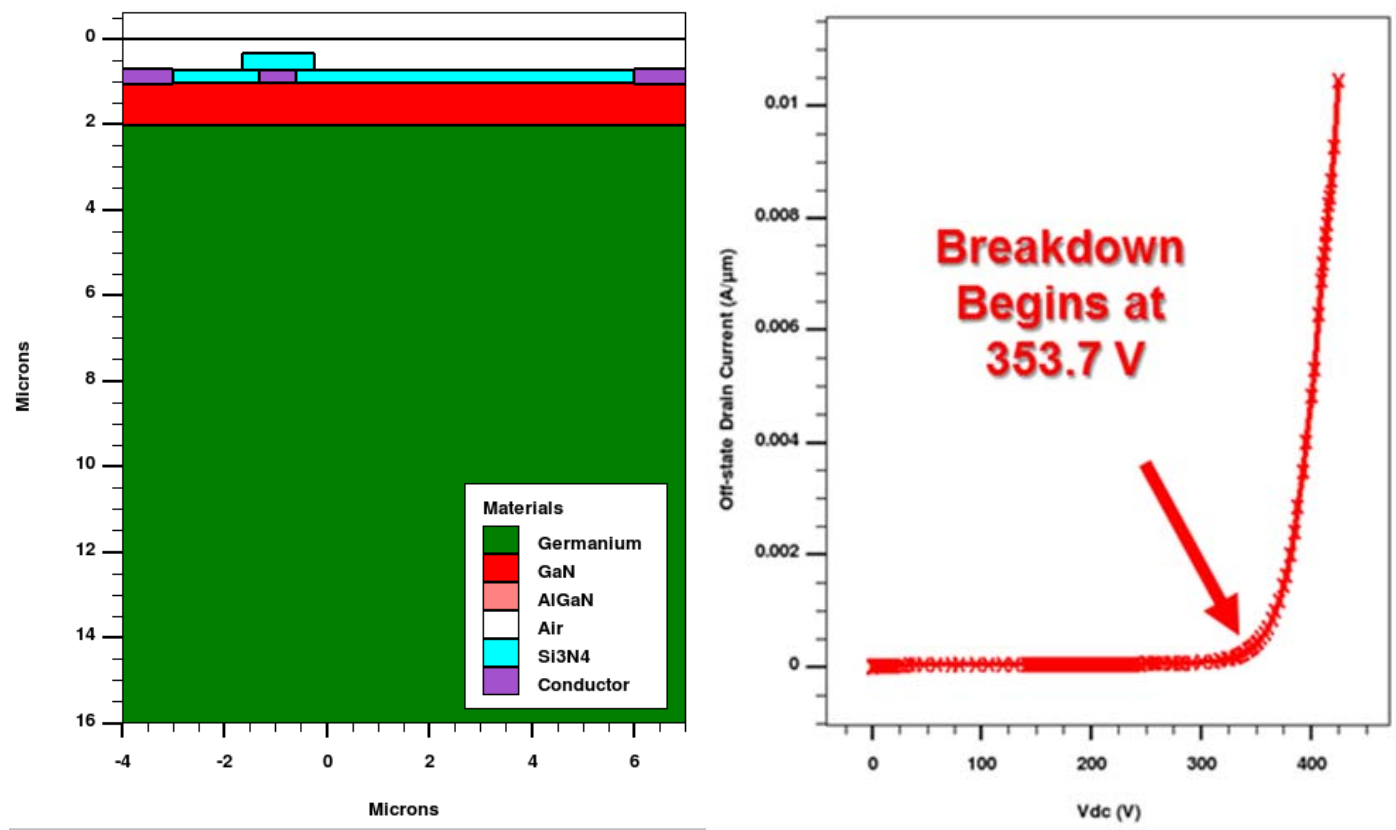

Figure 10.8 (a) True dimentions of the case HEMT with Germaium as substrate (b) Drain current versus Drain-source voltage characteristic of the case HEMT with Germaium as substrate. 
In this subsection, the HEMT substrate have been replaced with Germanium. Figure 10.8(a) shows the geometry of the HEMT while Figure 10.8(b) depicts its characteristic curve $\left(i_{d}-v_{d s}\right)$. From this curve it is noted that the breakdown voltage starts at $353.7 \mathrm{~V}$. It was expected that the breadown voltage decrease as the energy band gap of the Ge is lower than the $\mathrm{Si}$.

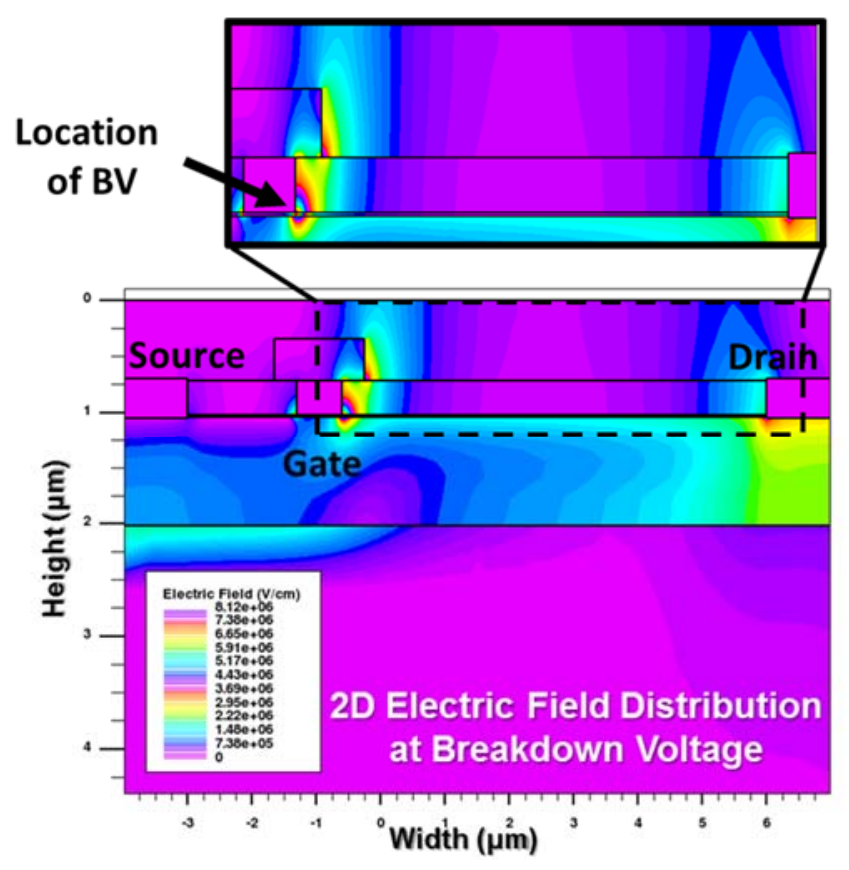

Figure 10.9 Two dimentional electric field forth the case HEMT with Germaium as substrate.

The 2D electric field of this case is shown in Figure 10.9. In the same fashion that the previous cases, this figure shows two zones where the electric field is more intense. The electric field in the vicinity of the gate is more intense. The zoom of the electric field at the top of Figure 10.9 shows that the right lower corner of the gate contactor has a spike. This spike is the one responsible for the voltage breakdown mechanism in the same way as the previous cases. 


\subsubsection{Etching of the Gallium Nitride}

The schematic of this case is the same as the base structure as in Figure 10.1(a). The specifications of this structure are as follows:

- Same structure than the base

- Substrate material: NA

- Passivation material: $\mathrm{SiN}$

- No Field plate
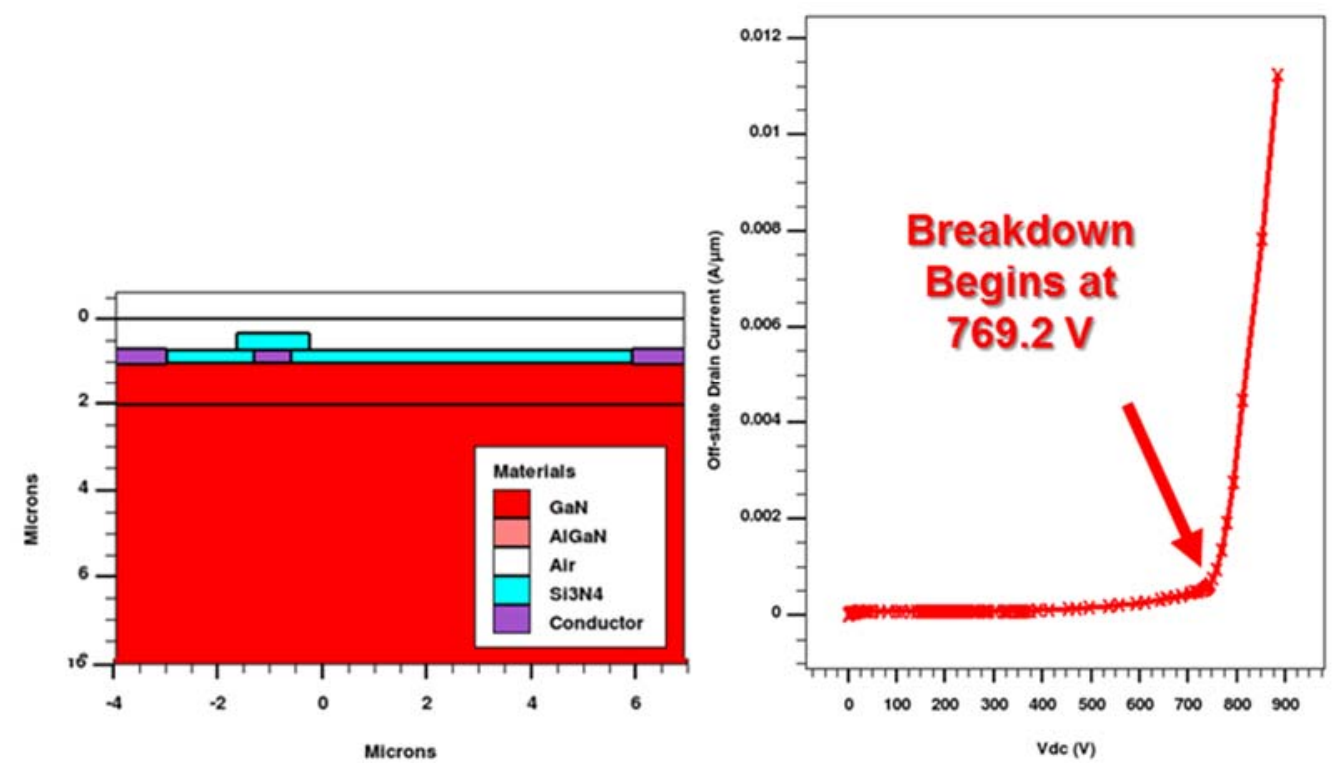

Figure 10.10 (a) True dimentions of the case HEMT with etched substrate (b) Drain current versus Drain-source voltage characteristic of the case HEMT with etched substrate

In this subsection, the HEMT substrate have been etached. Figure 10.10(a) shows the geometry of the HEMT while Figure 10.10(b) depicts its characteristic curve $\left(i_{d}-v_{d s}\right)$. From this curve it is noted that the breakdown voltage starts at $769.2 \mathrm{~V}$. The significant increase of the BV due to substrate removel is explain in [193]. However, a simple 
explanation is that now the substrate is the same GaN, which has a larger energy band gap value.

The 2D electric field of this case is shown in Figure 10.11. In the same fashion that the previous cases, this figure shows two zones where the electric field is more intense. The electric field in the vicinity of the gate is more intense. The zoom of the electric field at the top of Figure 10.11 shows that the right lower corner of the gate contactor has a spike. This spike is the one responsible for the voltage breakdown mechanism in the same way as the previous cases. From this figure it is observed that the intense point is spread.

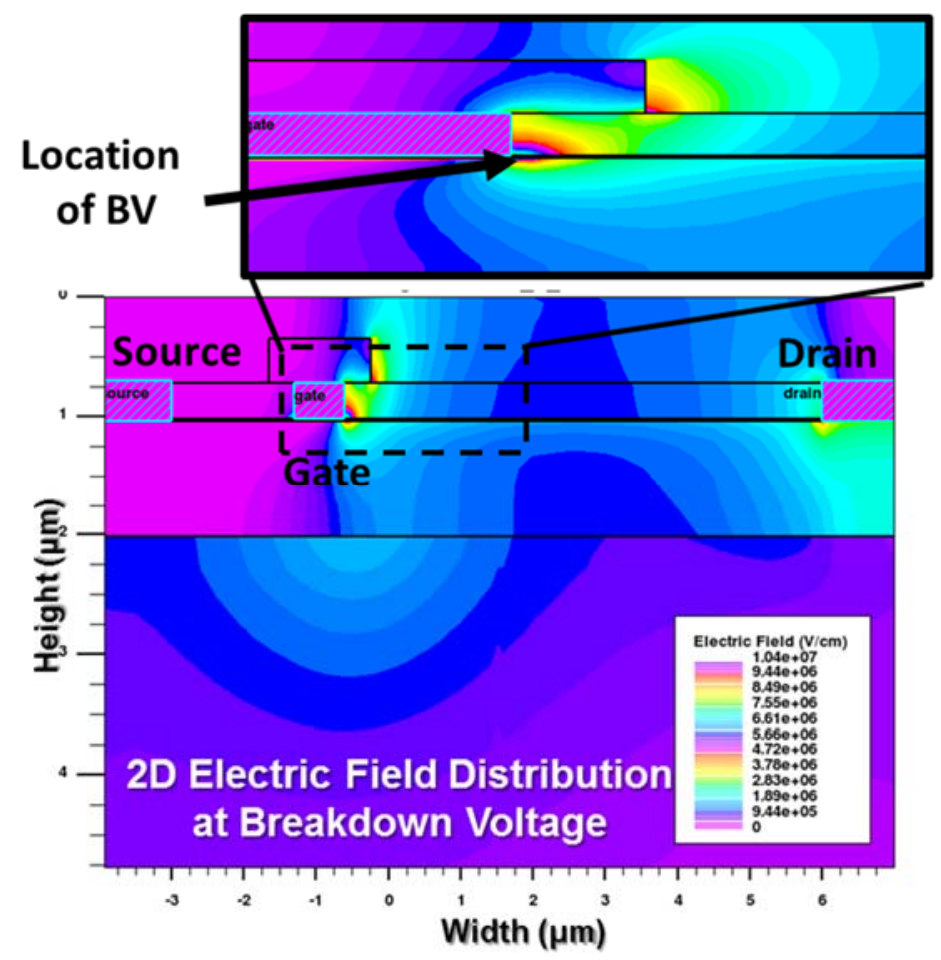

Figure 10.11 Two dimentional electric field forth the case HEMT with etched substrate.

\subsubsection{Applying Field Plate Contact}

The schematic of this case is shown in Figure 10.1(b). The specifications of this structure are as follows: 
- Substrate material: NA

- Passivation material: SiN

- Field plate inclusion
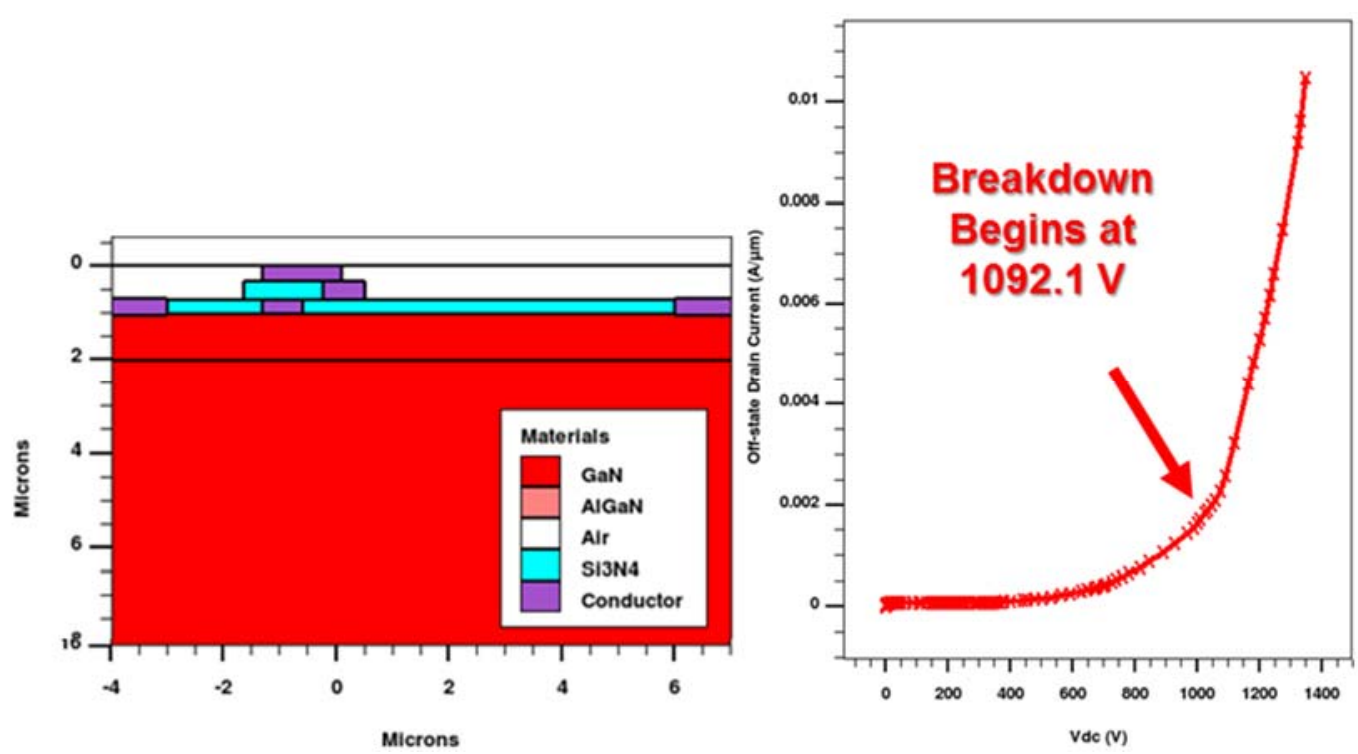

Figure 10.12 (a) True dimentions of the case HEMT with Field plate (b) Drain current versus Drain-source voltage characteristic of the case HEMT with Field plate.

In this subsection, a field plate has been included over the gate of the HEMT. This field plate has been contected to the source voltage (ground). Figure 10.12(a) shows the geometry of the HEMT while Figure 10.12(b) depicts its characteristic curve $\left(i_{d}-v_{d s}\right)$. From this curve it is noted that the breakdown voltage starts at $1092.1 \mathrm{~V}$. The significant increase of the BV due to inclusion of the field plate is well-known as it shift the Electric Peak field in the gate to the field plate that is actually above the passivation layer. The passivation layer has a large voltage breakdown standing larger voltages than the $\mathrm{GaN}$ and AlGaN layers.

The 2D electric field of this case is shown in Figure 10.13. In the different fashion that the previous cases, this figure shows one zone where the electric field is more intense (in 
the vicinity of the gate). The zoom of the electric field at the top of Figure 10.13 shows that the right lower corner of the FP contactor has a spike. This spike is the one responsible for the voltage breakdown mechanism in the same way as the previous cases. Notice that the intense peak at the bottom of the gate has disappeared.

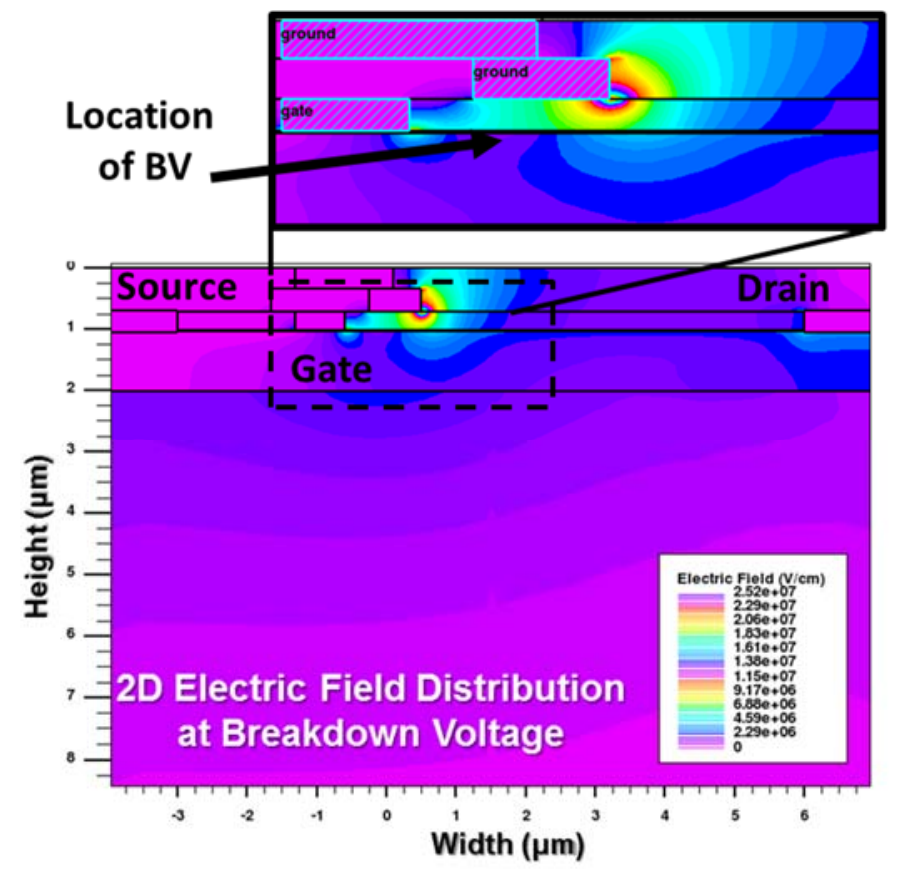

Figure 10.13 Two dimentional electric field forth the case HEMT with Field plate

\subsubsection{Comparative Results Analysis of GaN HEMT}

Results of the BV progression at $v_{g}=0 \mathrm{~V}$ and $E_{f}$ distribution at 2 heights $\left(y_{2}=\right.$ $0.721 \mu \mathrm{m}$ and $\left.y_{4}=1.02 \mu \mathrm{m}\right)$ are shown in Figure 10.14 and Figure 10.15 where the BV is defined as the maximum slope of the I-V curve. Notice that changing the passivation from silicon dioxide $(\mathrm{SiO} 2)$ to silicon nitride ( $\mathrm{SiN}$ ) has a small positive effect on the $\mathrm{BV}$ and $E_{f \max }$ (Figure 10.14(a) and Table 10-3), thus $\mathrm{SiN}$ is kept as the insulator. The next change is the removal of the Si substrate by etching. This results in a huge increase in the BV. This is an expected result as mentioned in [193]. The 4th case is the study of a doping 
variation as stated in Table 10-1 (Light doping). In this case, the variation is not favorable, presenting one of the smallest BV and largest $E_{\text {fmax }}$ (Figure 10.14(a) and Table 10-3). Next, another change of the substrate from Si to germanium $(\mathrm{Ge})$ in the base HEMT also was not positive. Finally, the addition of a FP to the current best case (Si substrate removal) yields the best results as it distributes the $E_{f}$ (Figure 10.15) in a way that does not allow an early BV. Figure 10.14(b) demonstrates that the origin of the BV for the FP case is a drainsource sub-threshold leakage [190]. In Figure 10.15 the $E_{f}$ distribution along the $x$ axis is presented at $y_{2}$ and $y_{4}$ which correspond to the surface of the device and the 2DEG channel, respectively, for all cases of study. Distance $y_{2}$ is the height where the $E_{f \max }$ appears for the FP case and $y_{4}$ is where the $E_{f \max }$ appears for the remaining cases. For a fair comparison, Figure 10.15 is performed at the lowest BV for all structures which occurs in Ge substrate case $(353.7 \mathrm{~V})$. For each case, the drain voltage is as indicated in Table 10-3 which is approximately equal to the Ge BV. Thus, from Figure 10.15 (a) and Table 10-3, it can be noted that as $E_{f \max }$ decreases the $\mathrm{BV}$ increases in all the cases (as expected). However, $E_{f \max }$ is the highest for the FP device which appears to be contradictory. This can be explained noticing that this $E_{f \max }$ is located at $x=0.5 \mu \mathrm{m}$ which is at the end of the FP contact and separated from the 2DEG by the passivation layer (SiN insulator) with very high dielectric strength of $10 \mathrm{MV} / \mathrm{cm}$. From Figure 10.15(b), which corresponds to the cross section along the $2 \mathrm{DEG}$, it is clear that the $E_{f \max }$ of the $\mathrm{FP}$ is the minimum.

\subsubsection{Analysis of the Field Plate Case}

For the final FP case, the $E_{f}$ distribution along the $x$-direction at different heights (at $V_{d s}=B V=1092.1 \mathrm{~V}$ ) is shown in Figure 10.16 and also as a 2D map in Figure 10.17. 
Figure 10.16 shows the key advantage of the FP layout as it redistributes the field and relocates the peak electric field along the $x$ and $y$ plane keeping it below the critical value and hence increasing the BV. The same behavior is more clearly observed in Figure 10.17(a) and (b). It is shown that the highest field strength is present right at the corner of the FP-SiN interface on the drain side of the gate, where a field of $20.7 \mathrm{MV} / \mathrm{cm}$ is experienced.

Table 10-3 BV and Maximum E Field for all Cases at 2 Heights

\begin{tabular}{|l|l|l|l|l|l|l|}
\hline & Original & Passivation & $\begin{array}{l}\text { Light } \\
\text { Doping }\end{array}$ & $\begin{array}{l}\text { Ge } \\
\text { Substrate }\end{array}$ & $\begin{array}{l}\text { GaN } \\
\text { Substrate }\end{array}$ & $\begin{array}{l}\text { Field } \\
\text { Plate }\end{array}$ \\
\hline$E_{\text {fmax } y_{2}}(\mathrm{MV} / \mathrm{cm})$ & 8.02 & 7.07 & 6.83 & 7.23 & 8.08 & 20.7 \\
\hline $\mathrm{BV}(\mathrm{V})$ & 366.5 & 384.3 & 368.7 & 353.7 & 769.2 & 1092.1 \\
\hline$V_{d s}(\mathrm{~V})$ & 354.6 & 351.9 & 354.4 & 353.7 & 354.3 & 350.5 \\
\hline$E_{\text {fmax } y_{4}}(\mathrm{MV} / \mathrm{cm})$ & 7.35 & 6.17 & 6.67 & 7.15 & 4.41 & 4.21 \\
\hline
\end{tabular}
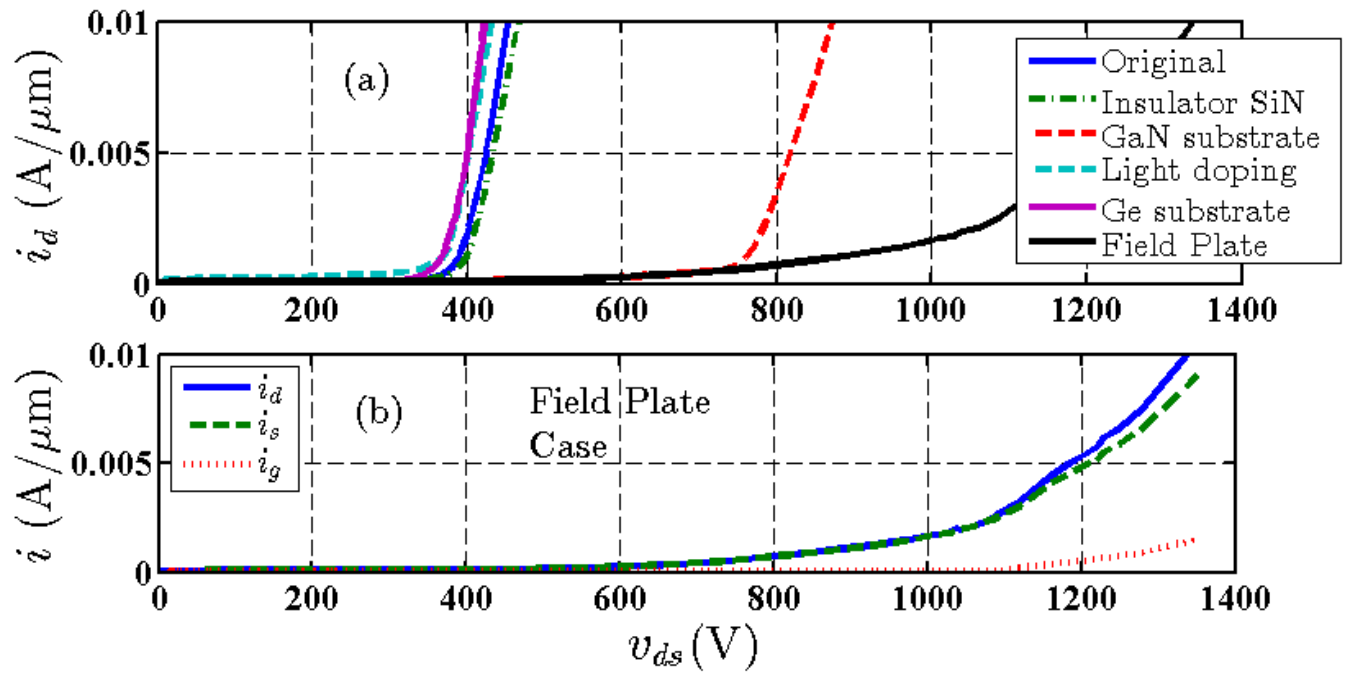

Figure 10.14 (a) BV curves for all cases of study (b) Drain, source and gate currents for the FP case 


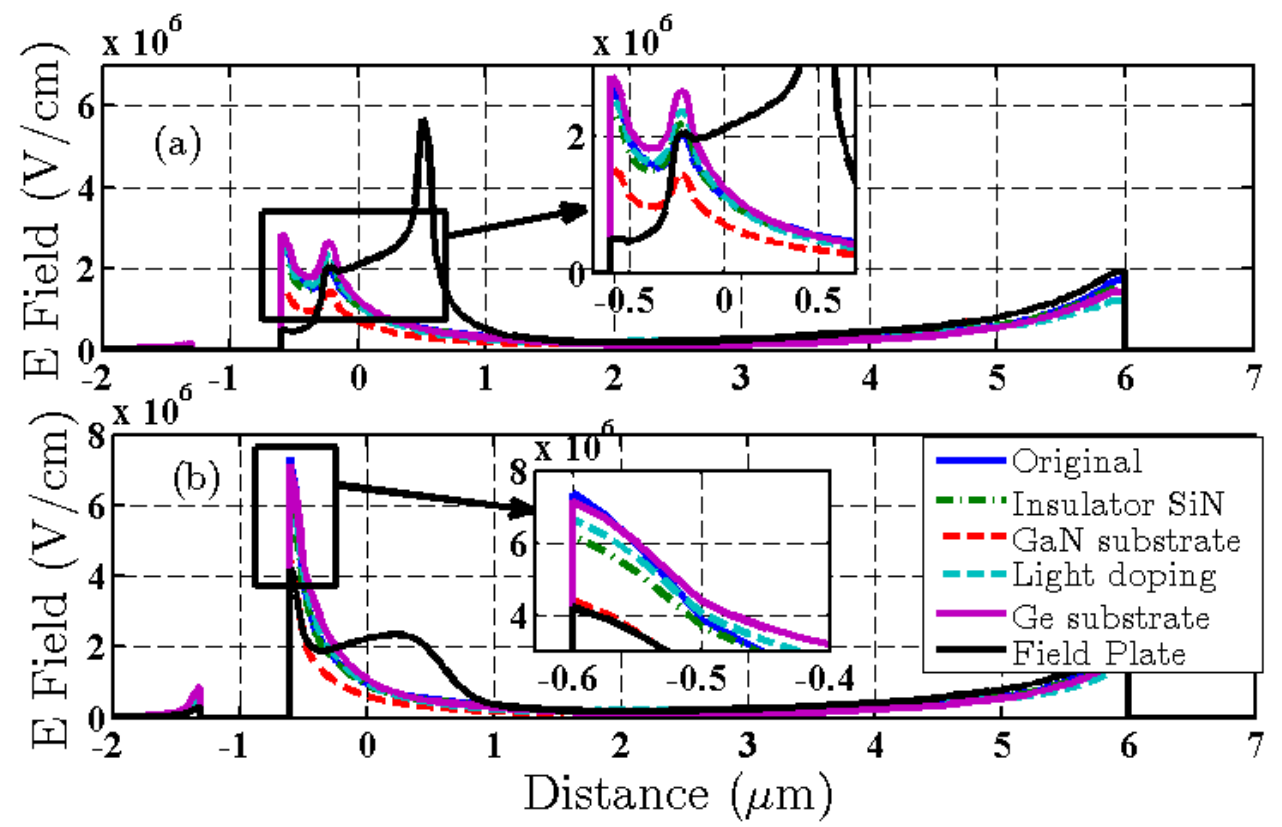

Figure $10.15 \mathrm{E}$ field distribution along $\mathrm{x}$ axis at (a) $\mathrm{y} \_2=0.721 \mu \mathrm{m}$ (b) $\mathrm{y}_{-} 4=1.02 \mu \mathrm{m}$

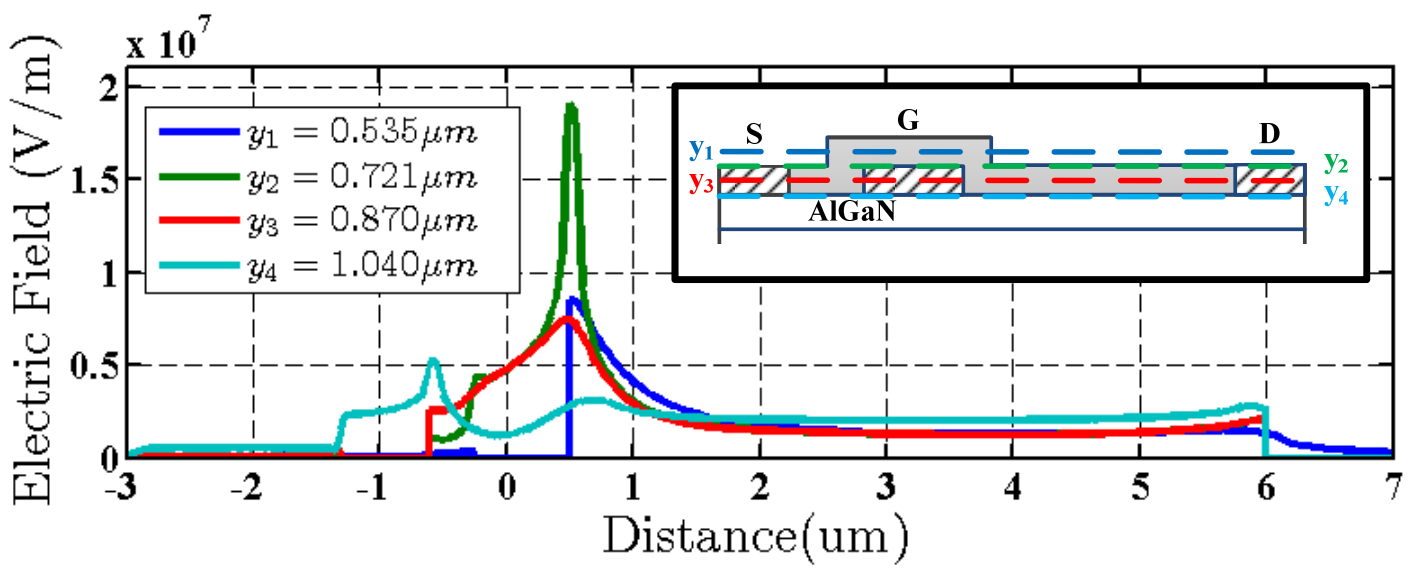

Figure 10.16 E field distribution along the $\mathrm{x}$ axis at $4 \mathrm{y}$ distances for the FP case. 


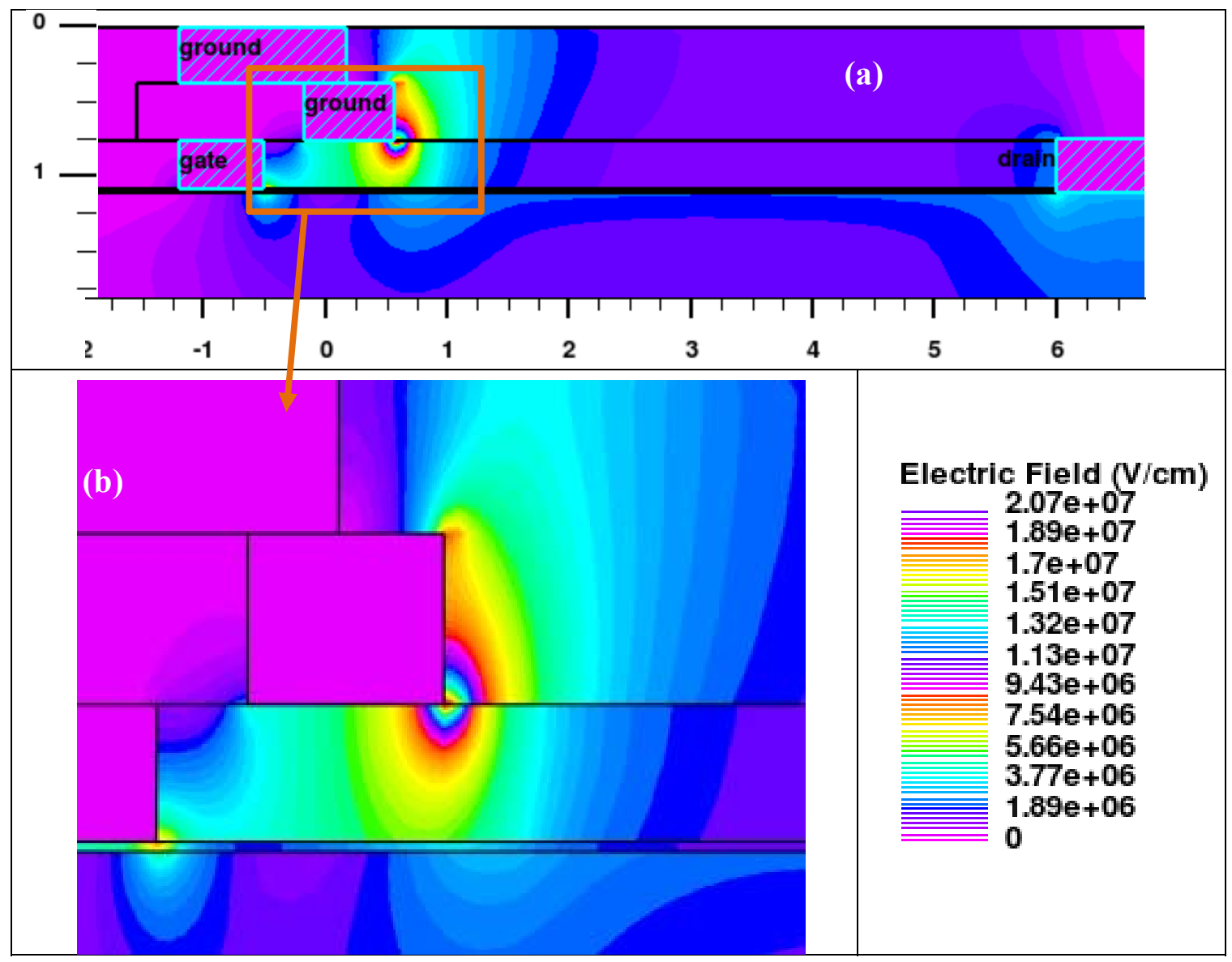

Figure 10.17 2D electric field distribution for the case of the FP.

\subsection{Field-Plate Length Variation on GaN Devices for BV and On-Resistance Characterization}

Single heterojunction field effect transistors (SHFETs) have gathered great attention due to the fact that they can operate higher in temperature, switching frequencies, and voltage, within minimum volumetric dimensions. This miniaturization is enabled by their wide band-gap characteristic, and by higher performance and a lower figure of merit $(F O M=$ $R_{d s(\text { on })} x Q_{G}$ ) [194]. The most famous single heterojunction is the AlGaN/GaN, which renders a two-dimensional electron gas (2DEG) layer [187] with very low on-resistance $\left(R_{d s(o n)}\right)$ as compared to silicon. However, GaN devices have to overcome some hurdles 
before they can completely replace silicon including: cost-reduction in their fabrication, their susceptibility to the surface breakdown, their scalability to higher power and their reliability.

Therefore, there is a necessity to investigate strategies to improve the performance of GaN SHFET by increasing its BV, reducing the FOM and augmenting its reliability. Three solutions have been suggested in literature in an effort to improve the these characteristics and mainly the BV: 1) a variation of the passivation material, 2) changing the substrate material, and 3) the application of a FP on either the source or gate [195]. The purpose of this work is to investigate a FP inclusion on the gate conductor. A variation of the FP length is performed to determine the optimum length, as well as to study its on-resistance.

In this work, a depletion-mode device is used because of its simplicity and the findings for this type of transistor can be extended to the enhancement-mode device without major complications. The dimensions and material properties used are provided in Table 10-4 [196], [197]. The device simulation is performed using commercial Silvaco software for the Finite Element Analysis. The structure schematic is shown in Figure 10.18.

Table 10-4 GAN-FET Design Parameters and Cases of Study

\begin{tabular}{|c|c|c|c|}
\hline Name & Value & Name & Value \\
\hline Source - to - Gate Distance & $1.70 \mu \mathrm{m}$ & Si Substrate Thickness & $2.98 \mu \mathrm{m}$ \\
\hline Gate - to - Drain Distance & $6.60 \mu \mathrm{m}$ & GaN Layer Thickness & $1.00 \mu \mathrm{m}$ \\
\hline Source/Drain Length & $1.00 \mu \mathrm{m}$ & AlGaN Layer Thickness & $0.02 \mu \mathrm{m}$ \\
\hline Gate Width & $3.00 \mu \mathrm{m}$ & AlGaN Composition & $x=20 \%$ \\
\hline
\end{tabular}




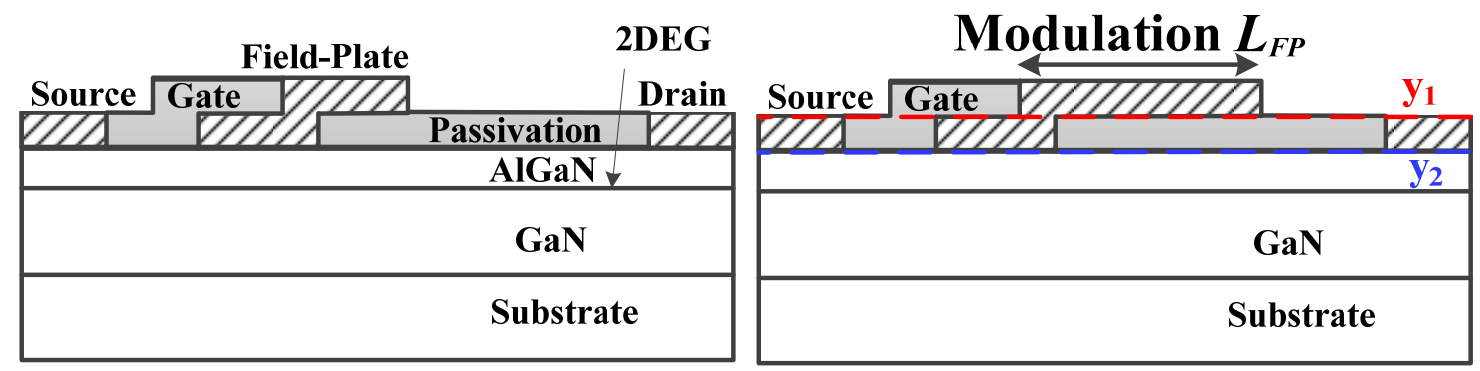

Figure 10.18 Schematic layer structure (a) Gate FP (G-FP) (b) Varying the G-FP.

\subsubsection{Analysis of FP Length on GaN Performance}

The FP is placed directly atop of the gate, stretching toward the drain with a length of $0.6 \mu \mathrm{m}$, beginning from the center of the gate. The length is then increased in $0.5 \mu \mathrm{m}$ steps until it approaches closer to the drain. For each step, the GaN device is swept in off-state $\left(v_{g}=-2 \mathrm{~V}\right)$, where the I-V characteristic curve for each case is shown in Figure 10.19. Figure 10.19 suggests the FP length modulation has only a minor impact on the BV. However, to more clearly demonstrate the progression of the BV versus the FP width, the $\mathrm{BV}$ is calculated by pinpointing the voltage, where the I-V slope is at its maximum. Figure 10.20 reveals that despite the fact all I-V curves appear similar, there are minute differences in where the breakdown occurs. Initially, an increase in the FP length results in an increase of the BV, approaching a maximum value at $3.1 \mu \mathrm{m}$. Following this point, the BV slowly begins to fall, until a drop of $20 \mathrm{~V}$ occurs after the length exceeds $4 \mu \mathrm{m}$. Some fluctuations occur past this point, until a collapse occurs following $5 \mu \mathrm{m}$, as only a small isolation gap remains between the FP and drain contacts after this point.

To gain a better insight into the performance associated with each case, an electric field $\left(E_{f}\right)$ analysis was conducted at two important heights across the length of the structure. $y_{1}$ 
corresponds to the surface of the device $(0.721 \mu \mathrm{m})$ and $y_{2}$ corresponds to the $2 \mathrm{DEG}$ channel $(1.020 \mu \mathrm{m})$ as shown in Figure 10.18(b). For each case, the maximum $E_{f}$ at each height is summarized in Table 10-5. Table 10-5 reveals a desirable range for the device operation, where the FP length is between $1.1 \mu \mathrm{m}$ to $3.1 \mu \mathrm{m}$. The lowest $E_{f}$ peaks are observed in $y_{2}$. This is particularly of interest, because the breakdown typically occurs near or along the 2DEG. As the FP width increases, initially $E_{f \max y_{1}}$ and $E_{f \max y_{2}}$ are inversely correlated until $4.1 \mu \mathrm{m}$, where both peaks rapidly increase. Based on our definition, the best BV occurs with a FP width of $3.1 \mu \mathrm{m}$.

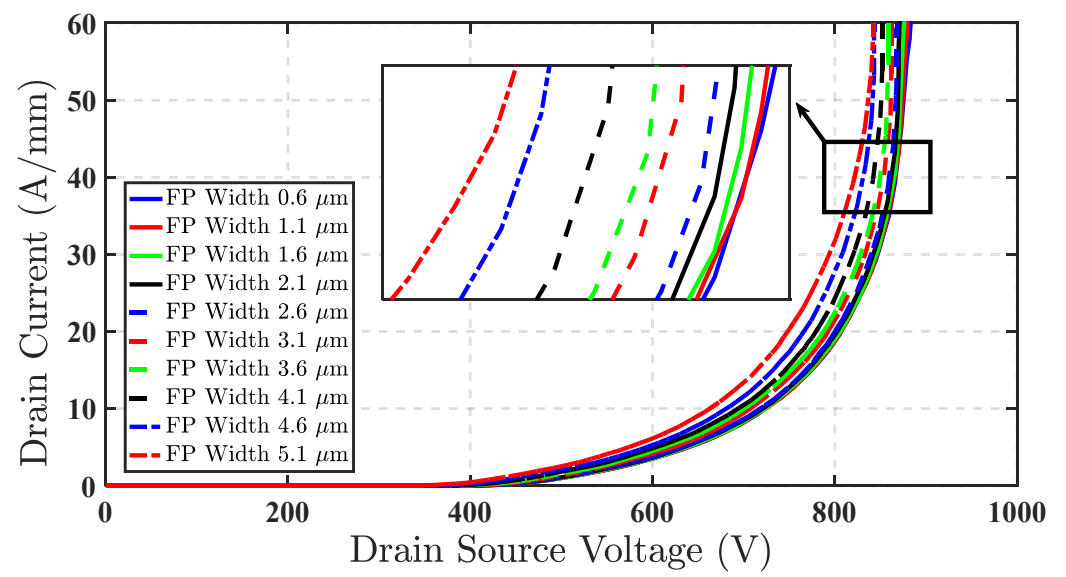

Figure 10.19 Characteristic Curves for all Cases.

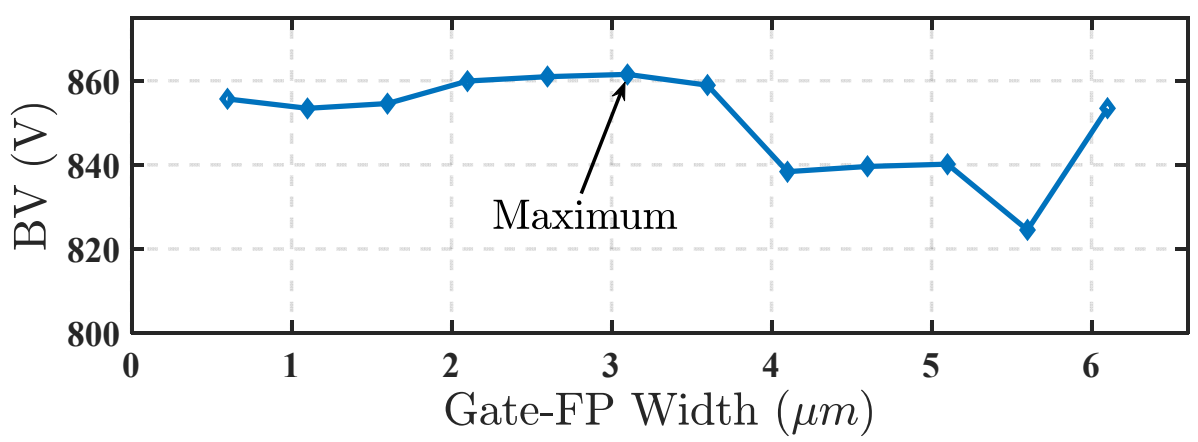

Figure 10.20 Breakdown Voltage versus the Gate-FP Width. 
Table 10-5 BV and Maximum E Field for all Cases at 2 Heights

\begin{tabular}{|c|l|l|l|l|l|l|l|l|l|l|}
\hline $\boldsymbol{L}_{\boldsymbol{F P}}(\boldsymbol{\mu m})$ & $\mathbf{0 . 6}$ & 1.1 & 1.6 & 2.1 & 2.6 & 3.1 & 3.6 & 4.1 & 4.6 & 5.1 \\
\hline $\mathrm{BV}(\mathrm{V})$ & 855.6 & 853.4 & 854.5 & 859.9 & 861.0 & 861.5 & 858.9 & 838.3 & 839.6 & 840.1 \\
\hline$E_{\text {fmax } y_{1}}(\mathrm{MV} / \mathrm{cm})$ & 13.07 & 15.36 & 14.03 & 12.96 & 9.83 & 8.22 & 8.91 & 9.88 & 10.78 & 21.31 \\
\hline$E_{\text {fmax } y_{2}}(\mathrm{MV} / \mathrm{cm})$ & 12.20 & 9.89 & 9.79 & 9.93 & 10.04 & 10.74 & 12.18 & 13.87 & 15.77 & 21.54 \\
\hline$R_{\text {ds (on })}\left(\mu \Omega / \mathrm{cm}^{2}\right)$ & 22.17 & - & - & - & - & 22.34 & - & - & - & 22.33 \\
\hline
\end{tabular}

\subsubsection{Closer Analysis of Three Cases}

A deeper analysis has been conducted for three important cases in particular: the shortest $(0.6 \mu \mathrm{m})$, optimal $(3.1 \mu \mathrm{m})$, and longest $(5.1 \mu \mathrm{m})$ FP width. For these cases, new I-V characteristic curves were generated with the device under on-state $\left(v_{g}=0 \mathrm{~V}\right)$ with a drain voltage of $10 \mathrm{~V}$, where the $R_{d s(o n)}$ of the device was taken. An area factor (AF) was used to generate a normalized resistance based on the area of the device. As can be seen from Table 10-5, all three values produce similar performance. The electric field distribution across each of the three structures is shown in Figure 10.21 and as a 2D map in Figure 10.22. In the $0.6 \mu \mathrm{m}$ case, the FP width is too small, thus the peak is not well-distributed, resulting in concentrations at the edge of the gate facing the drain. Once the FP width increases to $3.1 \mu \mathrm{m}$, the peak field is distributed and a wide isolation to the drain results in the lowest value. For the final case of $5.1 \mu \mathrm{m}$, although the peak is widely distributed, its proximity to the drain results in a higher bias across most of the device as well as significant increases in the peaks along both $y_{1}$ and $y_{2}$. 


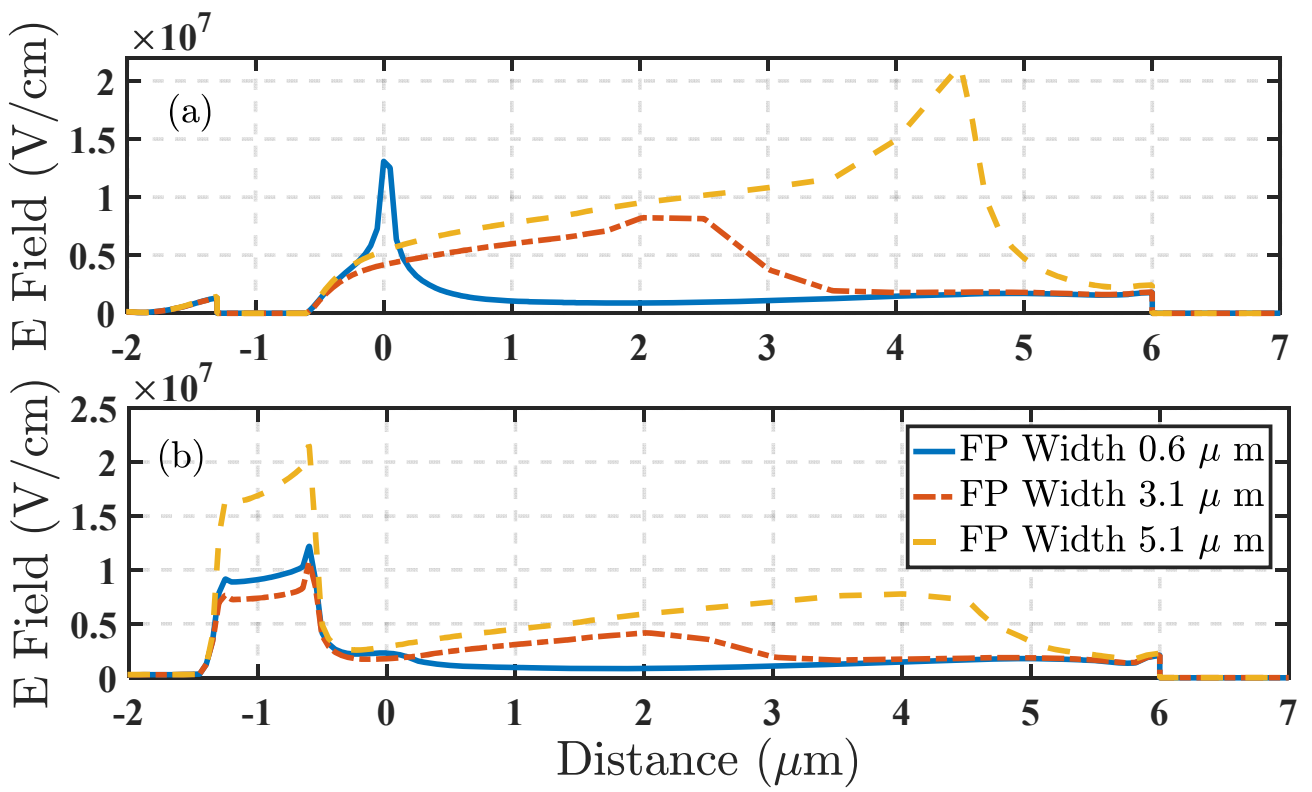

Figure $10.21 E_{f}$ distribution along the $\mathrm{x}$ axis at $2 \mathrm{y}$ distances for the FP case. Simulation performed at $V_{d s}=853 \mathrm{~V}$.

\subsection{Breakdown Voltage Improvement and Analysis of GaN HEMTs through Field Plate Inclusion and Substrate Removal}

Transistors based on $\mathrm{GaN}$ material are extremely promising devices in the area of microwave circuits and power systems because its material properties such as high peak electron velocity, saturation velocity and thermal stability. Theoretical $r_{o n}-B V$ limits for lateral HEMT are better than Vertical GaN $(\mathrm{VGaN})$ devices as the mobility of electrons in the two-dimensional electron gas (2-DEG) is higher than the mobility in the VGaN [187]. Notwithstanding, HEMTs are sensitive to surface breakdown and are not easy to scale to higher power. Therefore, there is a need to study the breakdown voltage (BV) mechanism of the HEMT and investigate strategies to increase it. The BV mechanisms are: sourcedrain breakdown (punch-through), gate-drain breakdown (leakage through the Schottky diode), vertical breakdown (poor compensation of the buffer layer) and impact ionization 
(an electron-hole pair generation close to the gate) [190]. The latter depends on the critical electric field $\left(E_{c r i t}\right)$ of the material, or the field strength required to initiate impact ionization causing an avalanche breakdown [191].

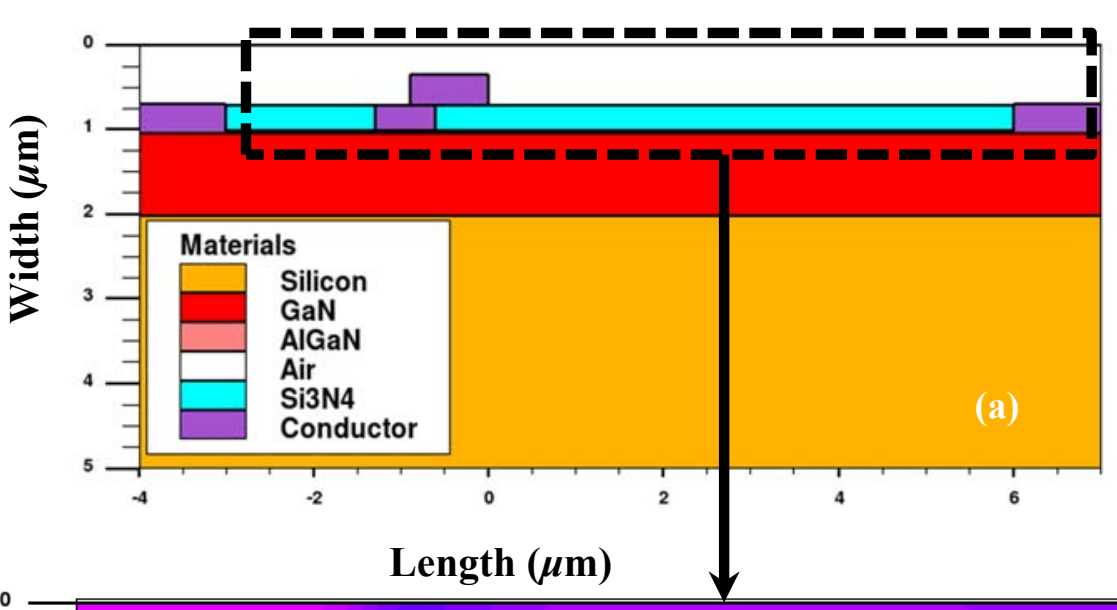

Electric Field (V/cm)
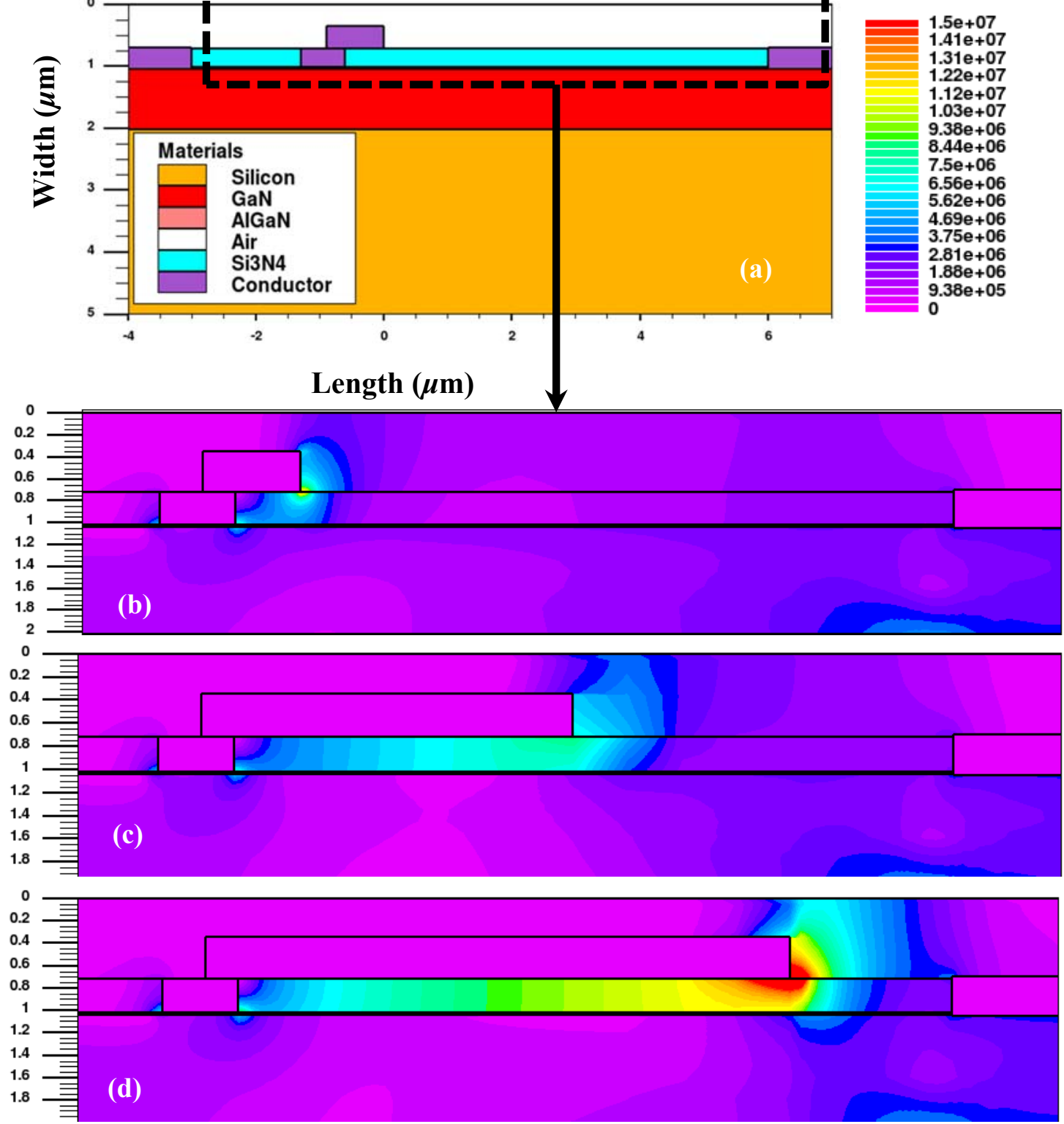

Figure 10.22 2D $E_{f}$ Distribution at $V_{d s}=853 \mathrm{~V}$ (a) schematic (b) shortest $L_{F P}=0.6 \mu \mathrm{m}$ (c) optimal $L_{F P}=3.1 \mu \mathrm{m}(\mathrm{d})$ longest $L_{F P}=5.1 \mu \mathrm{m}$.

There are 2 ways to avoid the BV: ensuring that the electric field is smaller than the material $E_{c r i t}$ at the operating point of the HEMT or enlarging the drift distance $\left(L_{g d}\right)$. The 
drift distance is held constant as a constraint, thus, the only solution available is to redistribute the electric field $\left(E_{f}\right)$. Three solutions have been suggested in literature in an effort to improve the BV: a variation of the passivation material, changing the substrate material, and the application of a field plate (FP) structure on either the source or gate [195].

The use of the FP in GaN-based heterostructure field-effect transistors (HFET) enhances both dc and microwave performance by improving the parameters such as [198]:

- Breakdown voltage

- Current voltage swing

- Linearity

- Efficiency

- Stability

- Reliability And suppressing phenomena, namely:

- surface trap effects

- Current collapse

- Gate leakage

- Dc-to-RF dispersion.

The electric field in the HFET channel peaks at the gate edge, which serves as a vortex for the electric field streamlines. A FP causes another electric field peak at its edge dividing most of the voltage drop between the two peaks and thus reducing the maximum electric field. As shown in [199] this reduction can be further enhanced by using multiple FPs. It is found that significantly higher $\mathrm{BV}$ can be achieved by raising the dielectric constant $\left(\varepsilon_{i}\right)$ 
of the insulator beneath the FP. Also the BV is dependent of the 2-DEG concentration $\left(n_{s}\right)$ [200].

Different BV improvement if found in reference [193]. It proposed the etching of the silicon $(\mathrm{Si})$ substrate which resulted in a significant increase in the BV. Following these two ideas: substrate removal and FP addition, we propose to study the progressive etching of the Si substrate to obtain the optimum Si thickness that results in a higher BV. Subsequently, a FP inclusion investigation is conducted with diverse structures. An initial theoretical study of the FP effects and its modelling is performed continuing with the subsequent research for different FP configurations.

\subsubsection{Field Plate Effects and Modelling}

\subsubsection{Field Plate Effects}

A general structure of the FP is shown in Figure 10.23. The specific geometrical parameters and material variables crucial for the FP analysis are [200]:

- Field plate length $\left(L_{F P}\right)$

- Insulator thickness $\left(t_{i}\right)$

- Gate-drain separation $\left(L_{d g}\right)$

- Channel electron concentration $\left(n_{s}\right)$

- Insulator dielectric constant $\left(\varepsilon_{i}\right)$ 


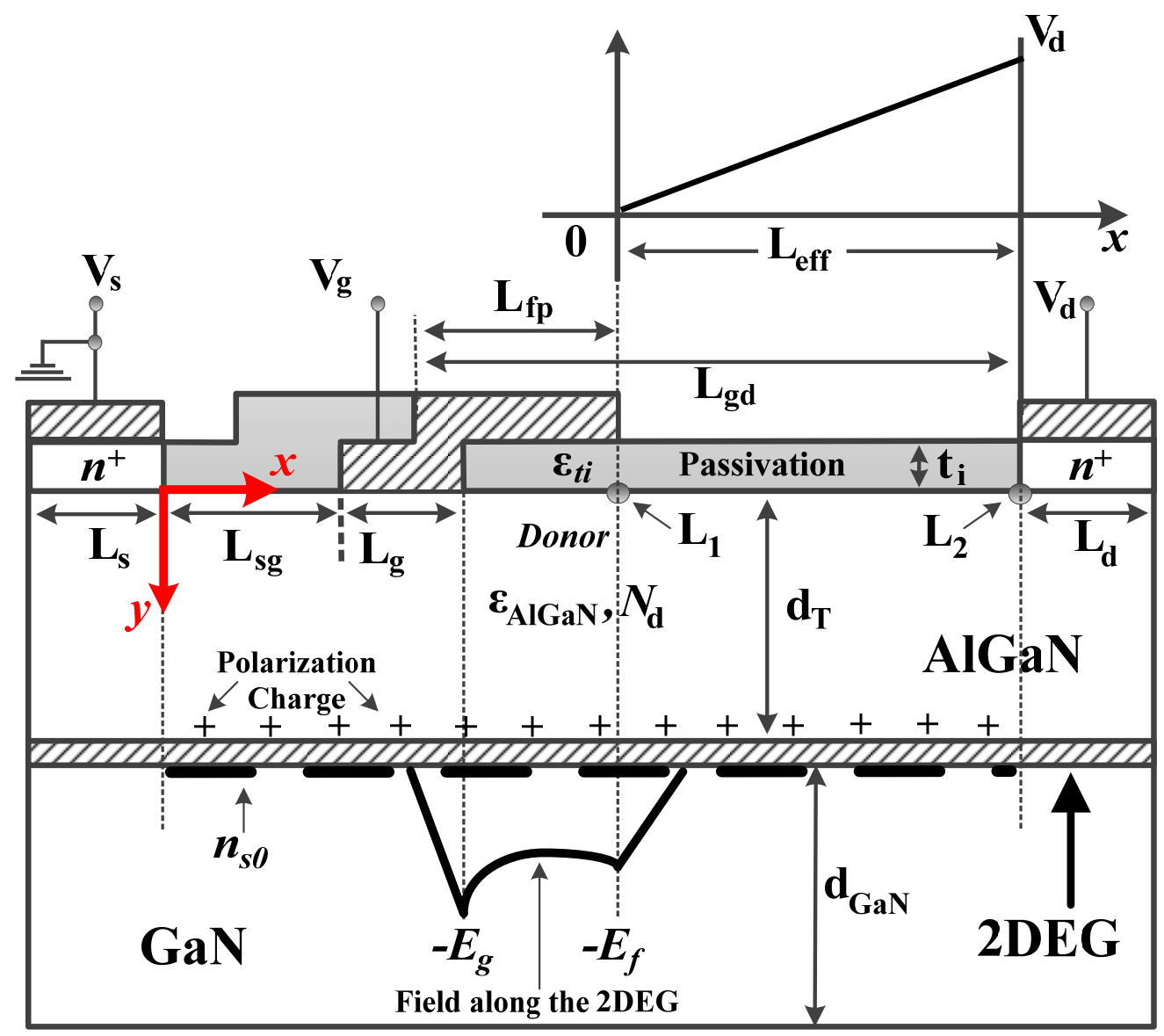

Figure 10.23 Cross-section of the HEMT with a Field Plate (FP-HEMT).

Without a FP the breakdown field distribution along the 2-DEG over $L_{d g}$ is confined over a small distance from the gate edge [200]. So, a high field is reach even for small values of $V_{d}$. Inclusion of FP reduces and spread the field along the 2DEG. It is possible to predict qualitatively several important trends in the behavior of FP-HEMT as a function of these 5 variable aforementioned.

1. Maximum BV is obtained for an optimum $t_{i}$. For large $t_{i}$ the FP effect vanishes and for $t_{i}=0$ the same response is obtained. Optimal $t_{i}$ is in between. 
2. Optimum $t_{i}$ will increase with the increase of $\varepsilon_{i}$ because the electric field is influence by the capacitance action of the FP.

3. Optimum $t_{i}$ reduce with increase of $n_{s}$ on account of the same capacitive action.

4. The BV will decrease with the increase of $n_{s}$.

5. The BV will not increase for increase $L_{F P}$ beyond certain point. This is because the field distribution along the 2-DEG consist on two triangular lobes with peaks near the gate edge and the FP edge (see Fig. 1).

\subsubsection{Theoretical Model of HEMT with Field Plate}

A simple model of the complex charge distribution within the AlGaN donor layer determining $n_{s}$ is proposed in [200]. The components of the charge distribution are as shown in Figure 10.24(a), the polarization dipole charge, $n_{p+}$ and $n_{p-}$, at opposite faces of the donor layer, the insulator-donor layer interface charge, $n_{t i}$, and the ionized unintentional doping change, $n_{d}$, all per $\mathrm{cm}^{2}$. Hence the donor layer charge distribution is equivalent to a single positive sheet change located along the heterojunction (see Figure 10.24(b)). The sheet concentration $n_{f}$ is the algebraic sum of the donor layer charges and so, equals to $n_{s}$. Note that $n_{f}$ is sensitive to charges $n_{t i}$.

A more accurate but complex model of the charge distribution is presented in [201]. In fact, in the reference, it is proposed the electrostatic potential of the total AlGaN layer as:

\begin{tabular}{|l|l|}
\hline$\phi_{\text {AlGaN }}(y)=\left(\frac{P_{\text {SpAlGaN }}-P_{\text {SpGaN }}}{\varepsilon}+2 \xi \gamma_{x x}-\frac{q n_{S}}{\varepsilon}\right)(y)$ & (10.1) \\
\hline
\end{tabular}

where $=\varepsilon_{A l G a N}+\frac{e_{33}{ }^{2}}{C_{33}}, \xi=\frac{e_{31}-e_{33} C_{13}}{\varepsilon C_{33}}, \gamma_{x x}$ is a strain constant defined as $\gamma_{x x}=$ $\frac{a_{G a N}-a_{A l G a N}}{a_{A l G a N}}$ and remaining parameters are summarized in Table 10-6. 


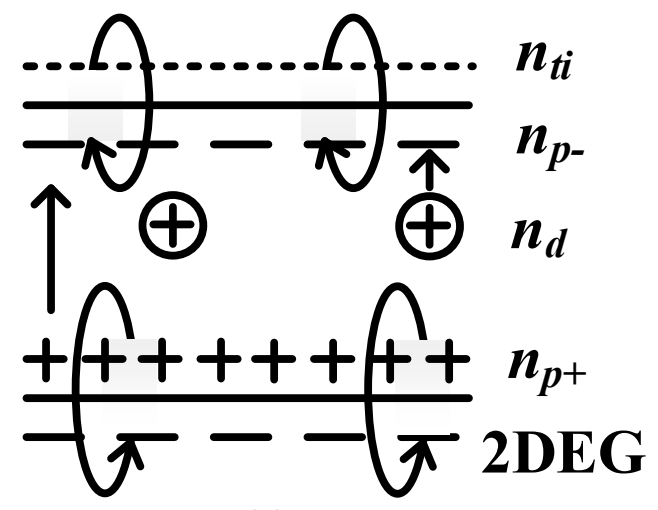

(a)

\section{Insulator layer}

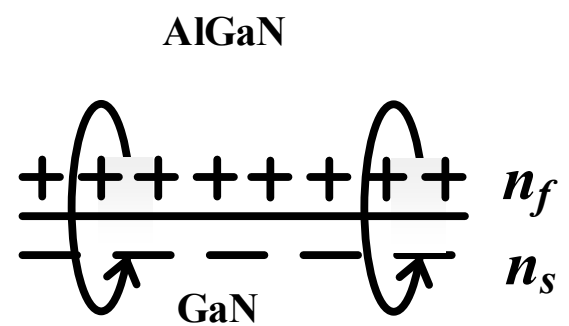

(b)

Figure 10.24 Illustration of the modelling (a) actual complex picture of the charges in the AlGaN layer (b) Simplified model of (a).

Table 10-6 Polarization Parameters for the Electrostatic Potential on AlGaN Devices.

\begin{tabular}{|c|c|}
\hline Variable & Description \\
\hline$P_{\text {spAlGaN }}(m)=-0.052 m-0.029 \mathrm{C} / \mathrm{m}^{2}$ & Spont. pol. AlGaN \\
\hline$P_{\text {spGaN }}=-0.029 \mathrm{C} / \mathrm{m}^{2}$ & Spont. pol. GaN \\
\hline$a_{\text {GaN }}=3.189 \AA$ & Lattice constant \\
\hline$a_{\text {AlGaN }}(m)=-0.77 m+3.189 \AA$ & Lattice constant \\
\hline$e_{31}(m)=-0.11 m-0.49 \mathrm{C} / \mathrm{m}^{2}$ & Piezoelectric cons. \\
\hline$e_{33}(m)=0.73 m+0.73 \mathrm{C} / \mathrm{m}^{2}$ & Piezoelectric cons. \\
\hline$C_{31}(m)=5 m-103 \mathrm{GPa}$ & Elastic constant \\
\hline$C_{33}(m)=-32 m+405 \mathrm{GPa}$ & Elastic constant \\
\hline
\end{tabular}

Now, the Fig. 1 illustrates a cross-section of the HEMT structure with a FP, passivated with $\mathrm{SiN}$ in order to reduce the traps. The field plate is deposited like a second gate electrode above the passivation layer. This study follows [192], however some inconsistences are found which did not let to finish the analytical investigation. Here we establish the formulation of the problem for a future resolution. The origin of the 
coordinates are placed in the surface of the $\mathrm{AlGaN}$ (see Figure 10.23) with a FP length of $L_{1}=L_{F P}$, insulator length of $L_{2}$, the effective length of the insulator layer is then $L_{e f f}=$ $L_{2}-L_{1}$, the applied drain voltage is $V_{d}$, the total thickness of the AlGaN material layer is $d_{T}$ and the thickness of the insulator is $t_{i}$. Assuming a linear distribution of the potential along the insulator, we can write [202]:

$$
\varphi_{f}(x)=\left\{\begin{array}{cc}
0 & x \leq L_{1} \\
\frac{V_{d}\left(x-L_{1}\right)}{L_{e f f}} & L_{1} \leq x \leq L_{2} \\
V_{d} & x \geq L_{2}
\end{array}\right.
$$

where $\varphi_{f}(x)$ represents the potential distribution along the insulator. The potential distribution in the semiconductor AlGaN $\phi(x, y)$ must satisfy Poisson's equation as in (10.3).

$$
\frac{\partial^{2} \phi(x, y)}{\partial x^{2}}+\frac{\partial^{2} \phi(x, y)}{\partial y^{2}}=-\frac{q n_{S}}{\varepsilon_{A l G a N}}
$$

Assuming that the drain region of the structure is complete depleted at the breakdown, the potential function $\phi(x, y)$ can be approximated [192], [202], [203] by the expression as in (10.4) for the 2D distribution as a parabolic approximation.

$$
\phi(x, y)=\phi_{0}(x)+\phi_{1}(x) y+\phi_{2}(x) y^{2}+\phi_{\text {AlGaN }}(y)
$$

where the coefficients $\phi_{0}(x), \phi_{1}(x)$ and $\phi_{2}(x)$ are functions only of $x$. These coefficients are determined by the boundary conditions as follows:

$$
E_{y}(x, 0)=-\left.\frac{\partial \phi(x, y)}{\partial y}\right|_{y=0}=k \frac{\varphi_{f}(x)-\phi_{f}(x)}{t_{i}}
$$


\begin{tabular}{|c|c|}
\hline$\phi\left(x, d_{T}\right)=\phi_{c}(x)$ & (10.6) \\
\hline
\end{tabular}

where $k=\varepsilon_{i} / \varepsilon_{\text {AlGaN }}, \phi_{c}(x)$ is the potential distribution along he two dimensional electron gas channel, $\phi_{f}(x)$ is the potential distribution along the AlGaN surface.

At $y=0, \phi(x, 0)=\phi_{0}(x)=\phi_{f}(x)$ from Figure 10.23. From (10.5) it results:

$$
\phi_{1}(x)=-k \frac{\varphi_{f}(x)-\phi_{f}(x)}{t_{i}}+\frac{\partial \phi_{\text {AlGaN }}}{\partial y}
$$

From (10.6) it is obtained $\phi_{2}(x)$ as:

$$
\phi_{2}(x)=\frac{1}{d_{T}^{2}}\left(\phi_{c}(x)-\phi_{f}(x)-\phi_{1}(x) d_{T}-\phi_{A l G a N}\left(d_{T}\right)\right)
$$

Introducing $\phi_{0}(x), \phi_{1}(x)$ and $\phi_{2}(x)$ back to (10.4) and solving Poisson's equation, it results two ordinary differential equations (ODE) to find the 2D potential distribution.

\subsubsection{Cases of Study and Simulation Results}

Two investigations are proposed in this article: (1) Varying of the thickness of the silicon substrate (2) Field plate inclusion. In general there are two types of FP: source FP (S-FP) and gate FP (G-FP). Two different S-FP are studied as shown in Figure 10.25(b) and (c). The studies performed in this article are based on physics based model (PBM) simulated on Silvaco which can provide us with a specialized set of tools to evaluate mechanisms that can increase the BV [188], [189]. A comparative analysis is carry among all these cases:

- Original (base case) - Figure 10.25 (a)

- Optimal substrate - Figure 10.25 (a)

- FP1 (S-FP) with $\mathrm{SiO}_{2}$ passivation - Figure 10.25 (b) 
- $\quad$ FP1 (S-FP) with SiN passivation - Figure 10.25 (b)

- $\quad$ FP2 (S-FP) with SiN passivation - Figure 10.25 (c)

- $\quad$ FP3 (G-FP) with SiN passivation - Figure 10.25 (d)

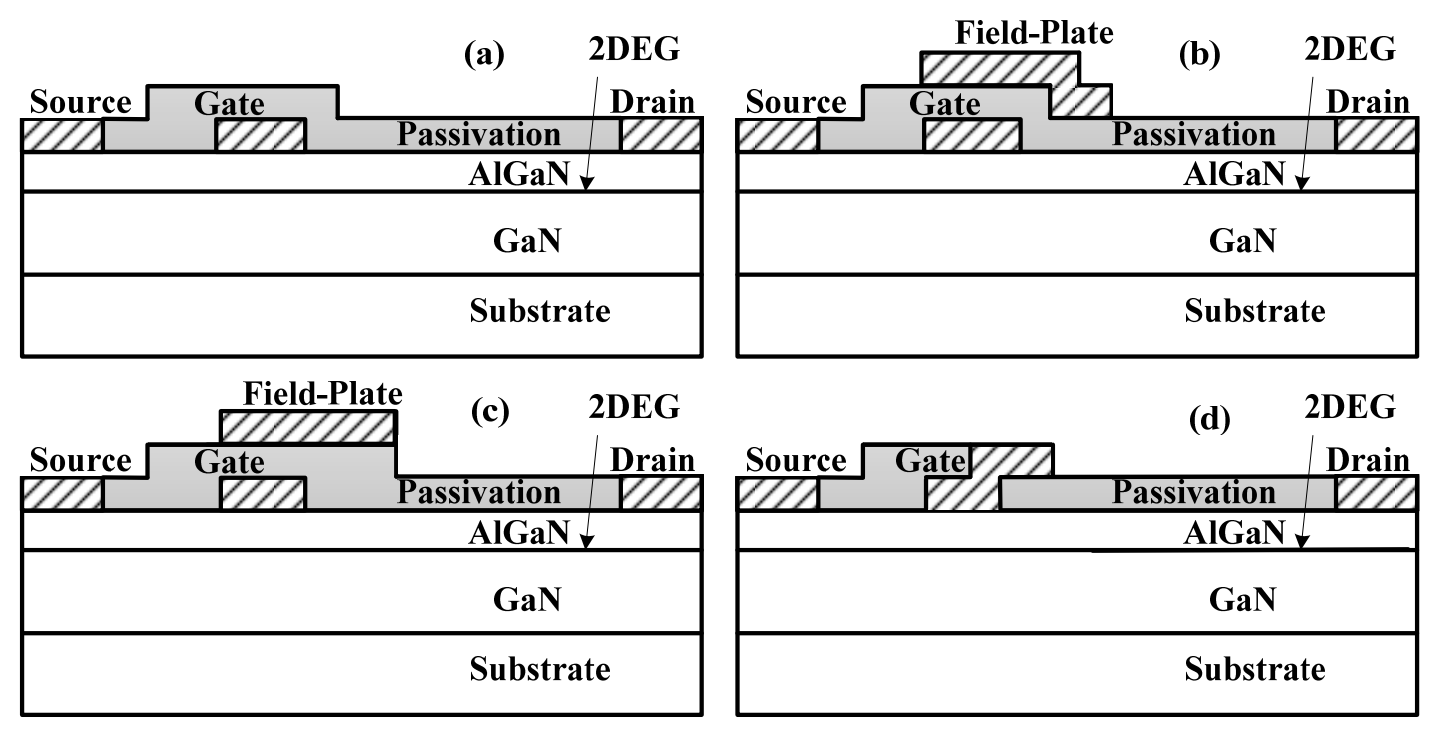

Figure 10.25 Schematic layer structure (a) Base (b) S-FP1 (c) S-FP2 and (d) G-FP3.

\subsubsection{Varying Thickness of the Silicon Substrate}

In the first study, multiple etching depths of the Si substrate are performed in the structure shown in Figure 10.25(a). Dimensions of the HEMT under study are as shown in Table 10-7, where initially a silicon dioxide $\left(\mathrm{SiO}_{2}\right)$ passivation layer is used as well as a $13.98 \mu \mathrm{m}$ Si substrate thickness. This thickness is then reduced in $1 \mu \mathrm{m}$ steps terminating at $0.98 \mu \mathrm{m}$ where the BV progression is shown in Figure 10.26. From this study, the optimum substrate is identified to be is $2.98 \mu \mathrm{m}$ and is used for each of the FP cases. The BV on Figure 10.26 are calculated by developing the I-V characteristic curve for each of the etched cases by finding their maximum slope. The HEMT is swept in off-state $\left(v_{g}=\right.$ $-2 \mathrm{~V})$. 
Table 10-7 GaN-FET Design Parameters and Cases of Study

\begin{tabular}{|c|c|c|c|}
\hline Name & Value & Name & Value \\
\hline $\begin{array}{c}\text { GaNFET } \\
\text { Thickness }\end{array}$ & $\begin{array}{c}\text { Initial: } 16.00 \mu \mathrm{m} \\
\text { Optimized: } 5.00 \mu \mathrm{m}\end{array}$ & Total Width & $11.00 \mu \mathrm{m}$ \\
\hline $\begin{array}{c}\text { Substrate } \\
\text { Thickness }\end{array}$ & $\begin{array}{c}\text { Initial: } 13.98 \mu \mathrm{m} \\
\text { Optimized: } 2.98 \mu \mathrm{m}\end{array}$ & AlGaN Composition & $x=20 \%$ \\
\hline$L_{s}$ & $1.00 \mu \mathrm{m}$ & $L_{g d}$ & $6.90 \mu \mathrm{m}$ \\
\hline$L_{s g}$ & $1.70 \mu \mathrm{m}$ & $L_{e f f}$ & $5.50 \mu \mathrm{m}$ \\
\hline$L_{f p}$ & $1.40 \mu \mathrm{m}$ & $L_{d}$ & $1.00 \mu \mathrm{m}$ \\
\hline$d_{t}$ & $0.02 \mu \mathrm{m}$ & $d_{G a N}$ & $0.98 \mu \mathrm{m}$ \\
\hline$L_{g}$ & $0.70 \mu \mathrm{m}$ & $\varepsilon_{d}$ & 8.82 \\
\hline$N_{d}$ & 0 (ideal) & $L_{1}$ & $0.50 \mu \mathrm{m}$ \\
\hline$L_{2}$ & $6.00 \mu \mathrm{m}$ & $t_{i}$ & $0.30 \mu \mathrm{m}$ \\
\hline Meshing & Initial: 55552 & $\varepsilon_{t i}$ & 7.50 \\
\hline Elements & Optimized: 51520 & & \\
\hline
\end{tabular}

Table 10-7 provides all the geometrical and material parameters for the performed simulations.

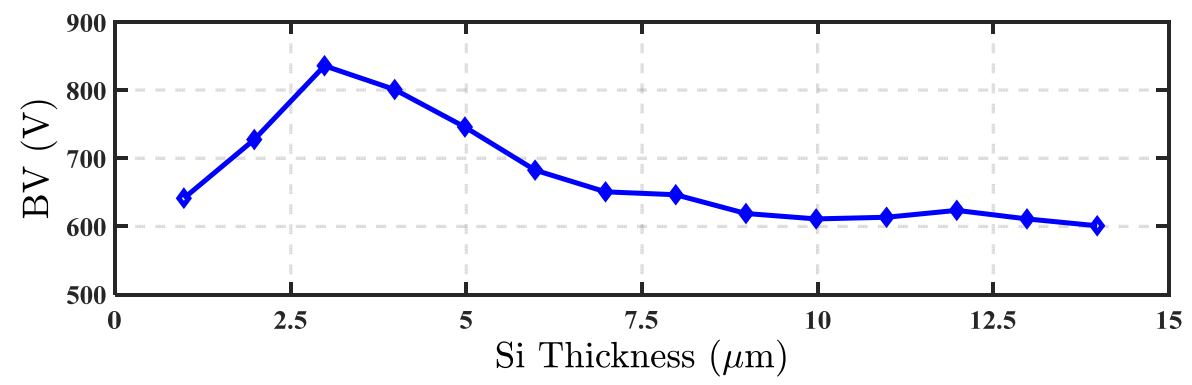

Figure 10.26 BV against the Si substrate thickness for the etching reduction.

\subsubsection{Detailed Study of all the Cases}

In this chapter it is investigated 5 cases of study. The structures of the schematics are shown in Figure 10.25. The details of all the cases are as follows: 


\subsubsection{Base Case}

The schematic of the base structure, which has not been drawn at scale is shown in Figure 10.25(a). The specifications of this structure are as follows:

Characteristics of the base case:

- Substrate material: Silicon (Si)

- Passivation material: $\mathrm{SiO} 2$

- No Field plate

- Dimentions: as shown in Table 10-7.
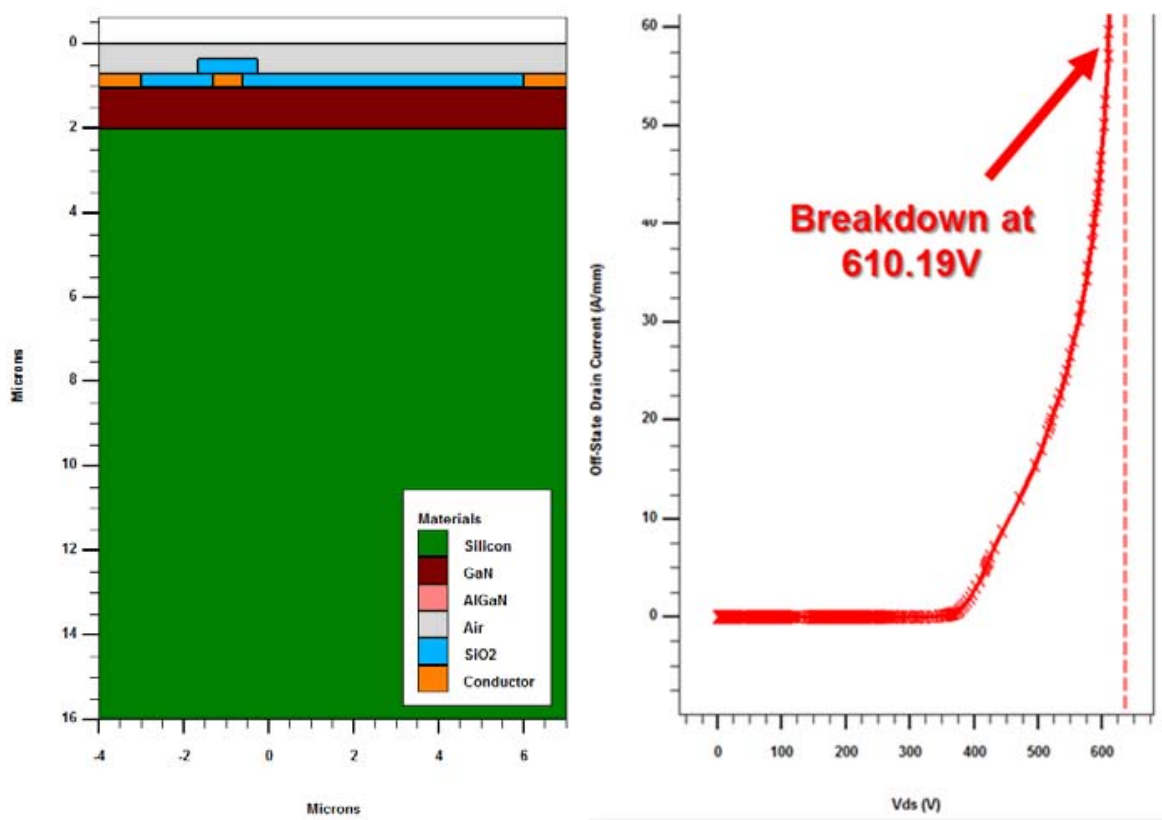

Figure 10.27 (a) True dimentions of the base case HEMT (b) Drain current versus Drainsource voltage characteristic of the base case HEMT.

The proportional real dimentions of the base case HEMT are shown in Figure 10.27(a) where it is observe that the biggest region is the substrate. In Figure 10.27(b) the characteristic curve $\left(i_{d}-v_{d s}\right)$ of the HEMT is plotted. From this curve it is noted that the 
breakdown voltage starts at $610.9 \mathrm{~V}$ in this new definition.

The 2D electric field of the base case of the HEMT is shown in Figure 10.28. In this figure it can be observed the zones where the electric field is more intense. Two zones are of interest: in the vicinity of the gate and drain. However, the intensity on the gate vicinity is superior. A zoom of the electric field near the gate is illustrated at the top of Figure 10.28. In here, the right lower corner of the gate contactor indicates a spike of field. This spike is the one responsible for the voltage breakdown mechanism producing a huge gate current that travels to the drain.

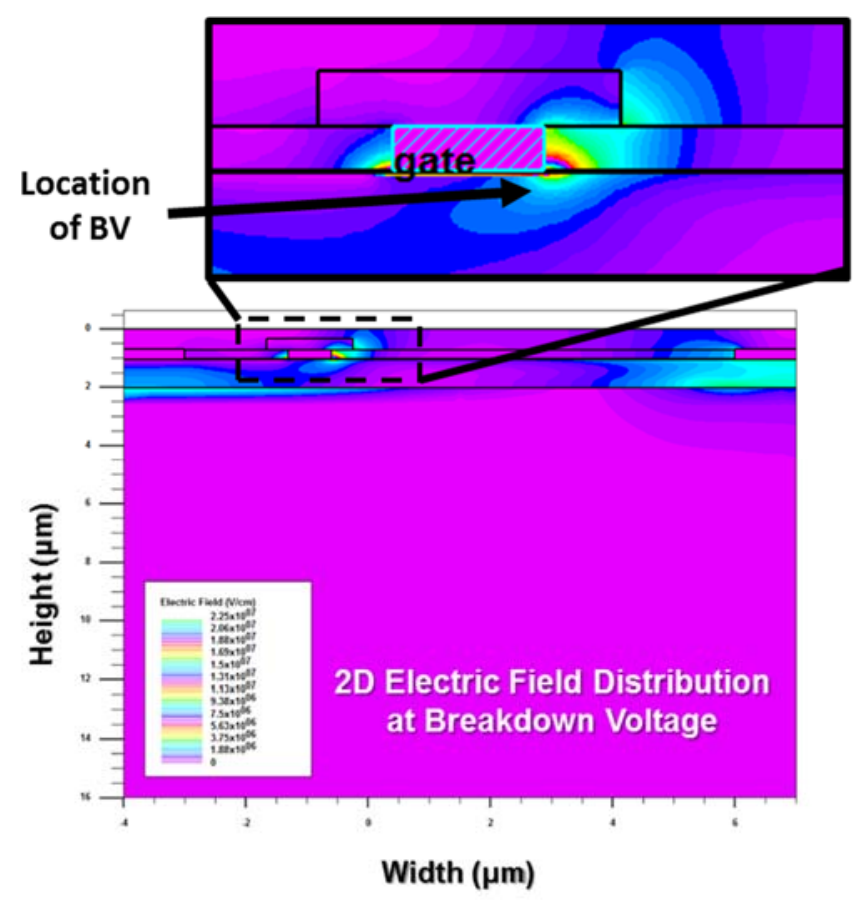

Figure 10.28 Two dimentional electric field forth the base case HEMT.

\subsubsection{Optimum Substrate}

The schematic of the HEMT structure with optimum substrate is the same as the base case on Figure 10.25(a). The specifications of this structure are as follows:

- Substrate material: Silicon (Si) Optimum 
- Passivation material: $\mathrm{SiO} 2$

- No Field plate

- Dimentions: as shown in Table 10-7.
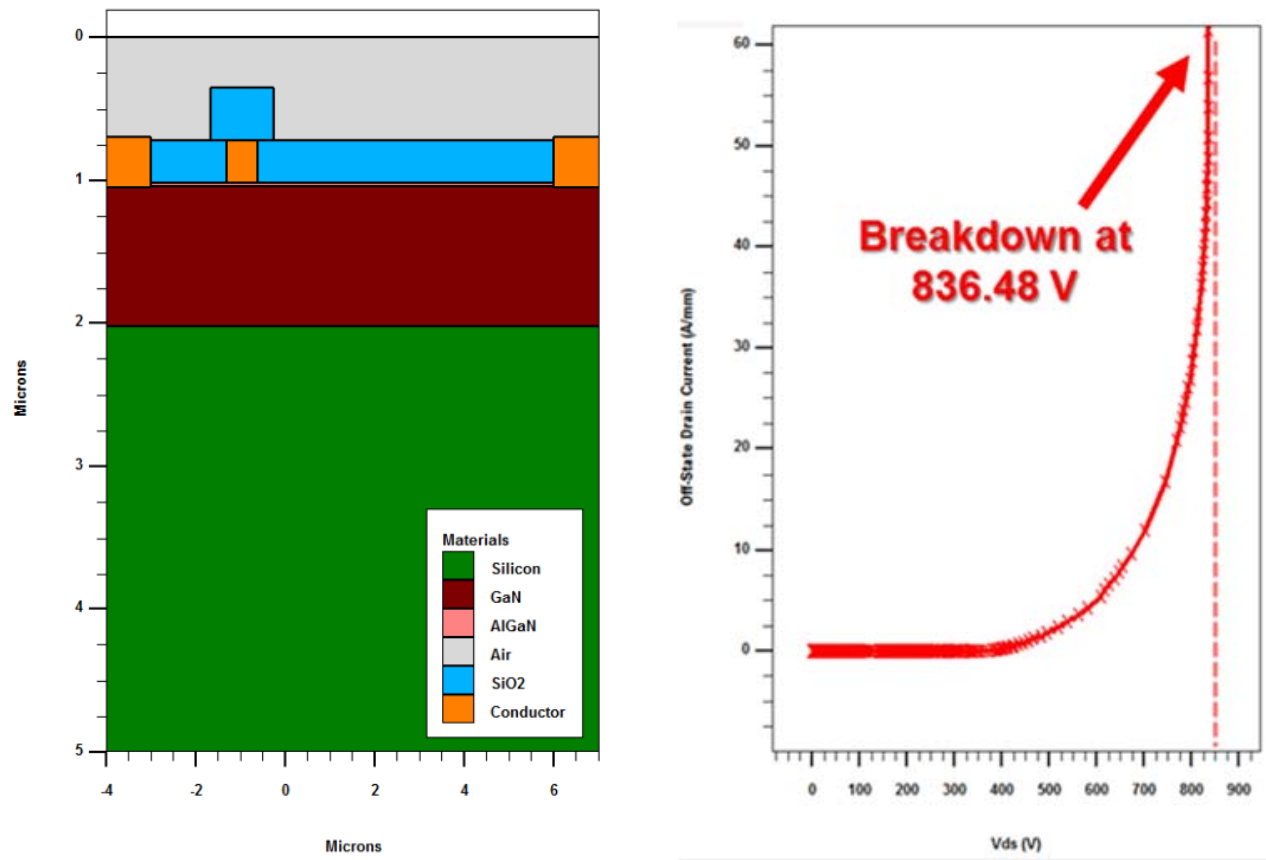

Figure 10.29 (a) True dimentions of the HEMT with optimum substrate (b) Drain current versus Drain-source voltage characteristic of the HEMT optimum substrate.

The proportional real dimentions of this HEMT case are shown in Figure 10.29(a) where it is observe that the the substrate has been etched. In Figure 10.29(b) the characteristic curve $\left(i_{d}-v_{d s}\right)$ of the HEMT is plotted. From this curve it is noted that the breakdown voltage starts at $836.48 \mathrm{~V}$ in this new definition.

The $2 \mathrm{D}$ electric field of the base case of the HEMT is shown in Figure 10.30. In this figure it can be observed two zones where the electric field is more intense. The intensity on the gate's vicinity is superior. A zoom of the electric field near the gate is illustrated at the top of Figure 10.30. In here, the right lower corner of the gate contactor indicates a spike of field. 


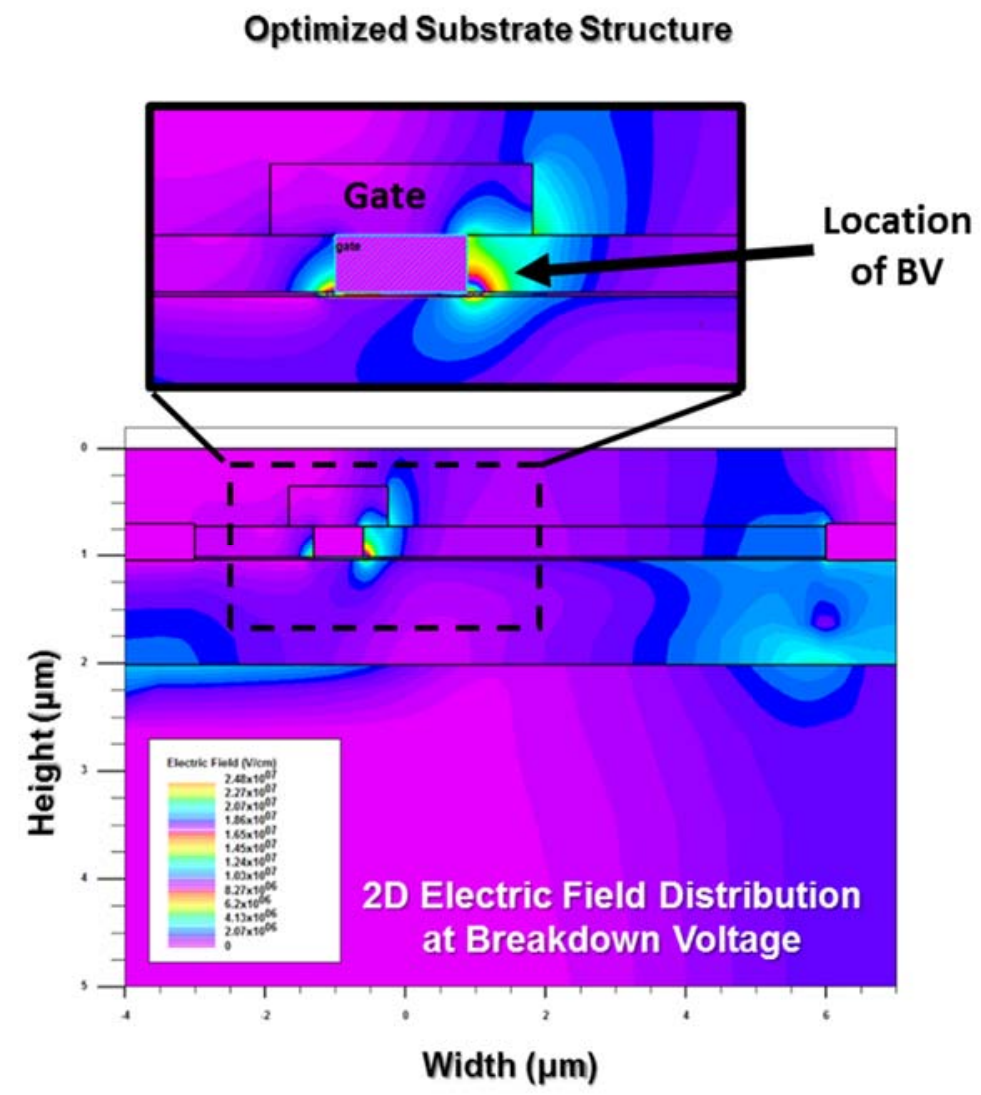

Figure 10.30 Two dimentional electric field forth the HEMT optimum substrate.

\subsubsection{Source Field Plate 1}

The schematic of the HEMT structure with source field plate 1 is shown in Figure 10.25(b). The specifications of this structure are as follows:

- Substrate material: Silicon (Si) Optimum

- Passivation material: $\mathrm{SiO} 2$

- Field plate: Source FP1

- Dimentions: as shown in Table 10-7. 

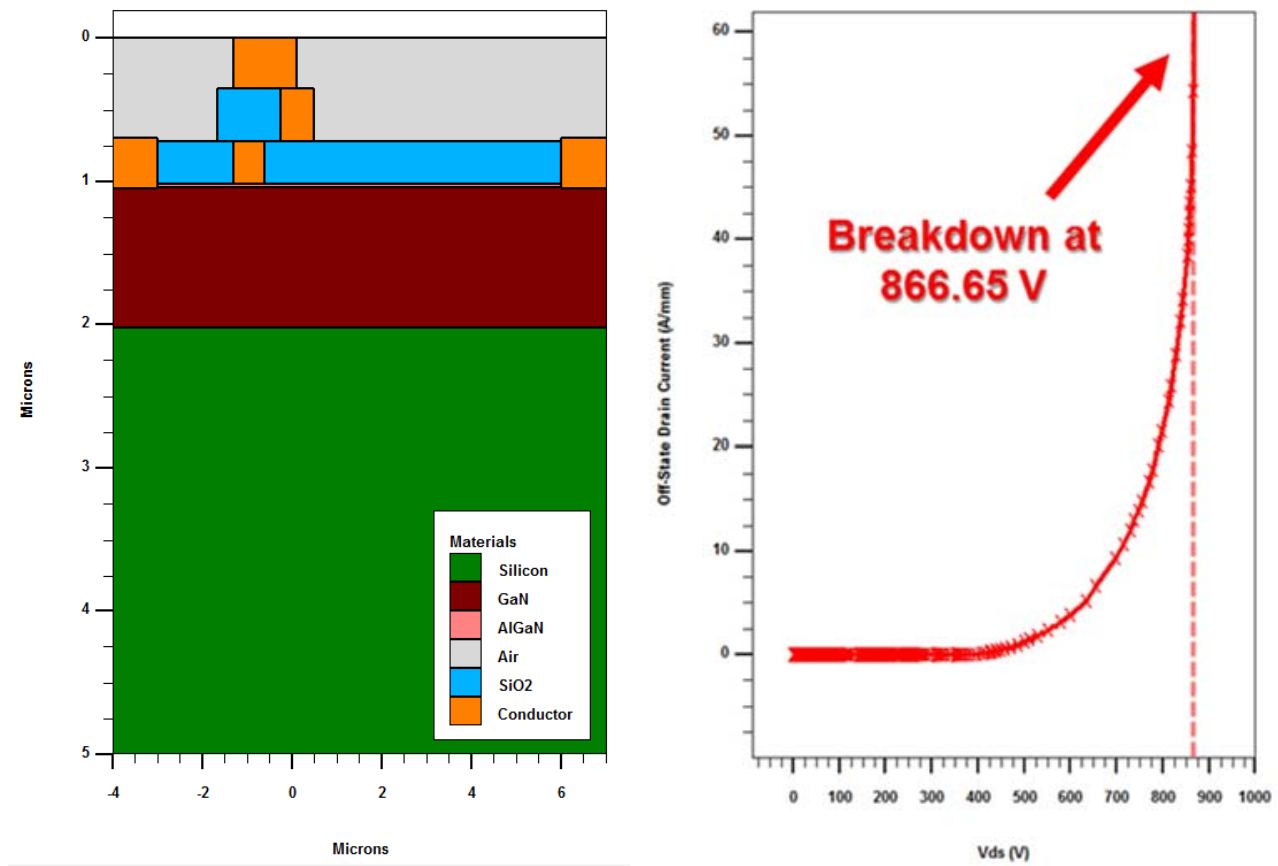

Figure 10.31 (a) True dimentions of the HEMT with Source FP1 (b) Drain current versus Drain-source voltage characteristic of the HEMT Source FP1.

The proportional real dimentions of this HEMT case are shown in Figure 10.31(a) where it is observe that the the substrate has been etched. In Figure 10.31(b) the characteristic curve $\left(i_{d}-v_{d s}\right)$ of the HEMT is plotted. From this curve it is noted that the breakdown voltage starts at $866.65 \mathrm{~V}$.

The 2D electric field of the base case of the HEMT is shown in Figure 10.32. In this figure it can be observed two zones where the electric field is more intense. The intensity on the gate's vicinity is superior. A zoom of the electric field near the gate is illustrated at the top of Figure 10.32. In here, the right lower corner of the field plate contactor indicates a spike of field. In this case, the same situation as explained before in Sub-Section 10.2.1.6. 


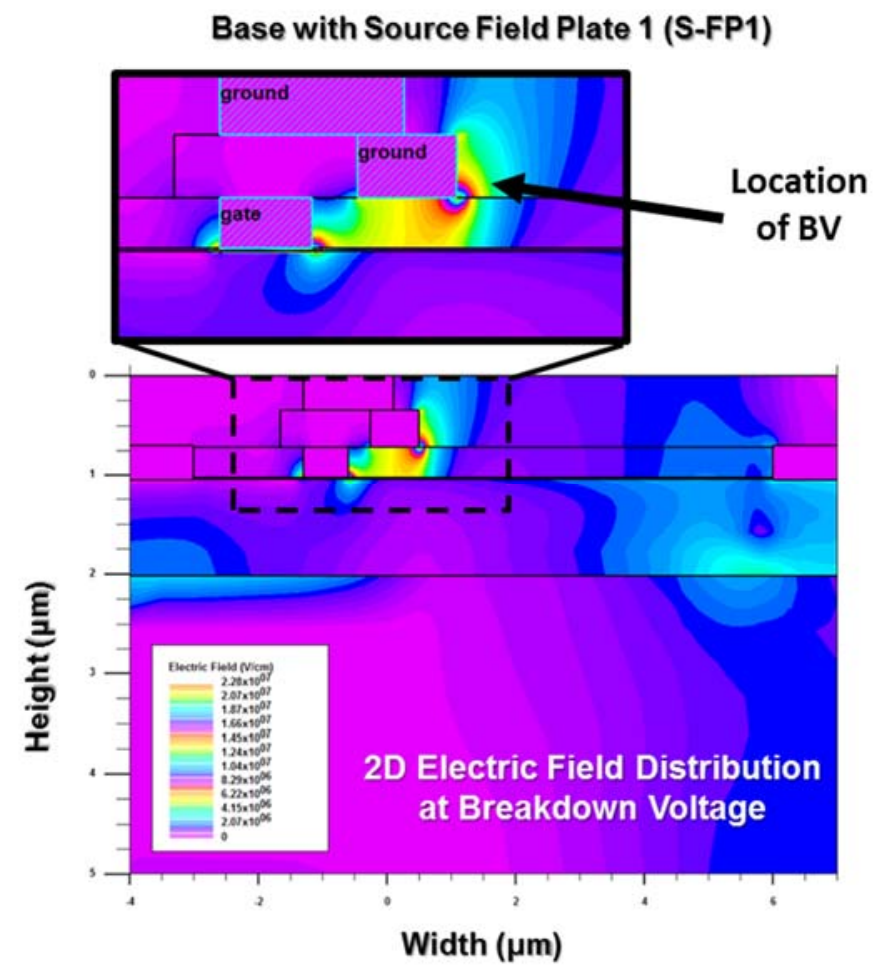

Figure 10.32 Two dimentional electric field forth the HEMT Source FP1.

\subsubsection{Source Field Plate 1 with SiN Insulator}

The schematic of the HEMT structure with source field plate 1 and $\mathrm{SiN}$ insulator is shown in Figure 10.25(b). The specifications of this structure are as follows:

- Substrate material: Silicon (Si) Optimum

- Passivation material: SiN

- Field plate: Source FP1

- Dimentions: as shown in Table 10-7.

The proportional real dimentions of this HEMT case are shown in Figure 10.33(a) where it is observe that the the substrate has been etched. In Figure 10.33(b) the characteristic curve $\left(i_{d}-v_{d s}\right)$ of the HEMT is plotted. From this curve it is noted that the breakdown voltage starts at $880.63 \mathrm{~V}$. 

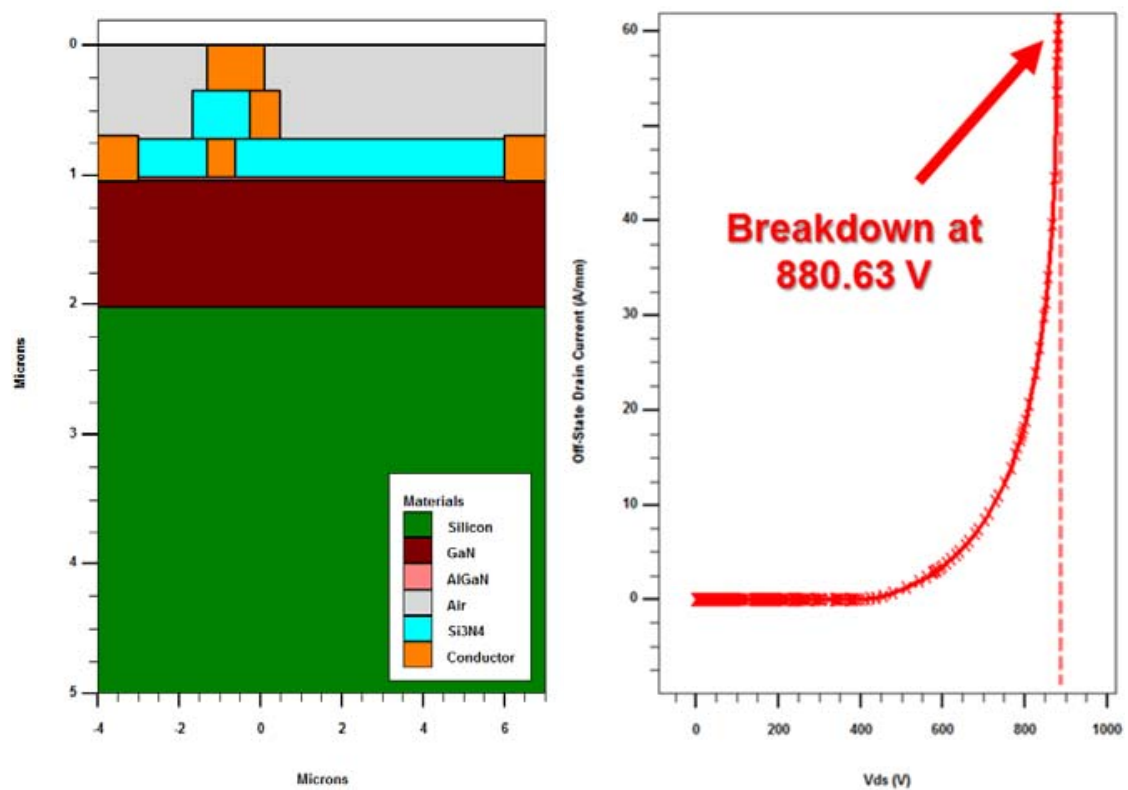

Figure 10.33 (a) True dimentions of the HEMT with Source FP1 and SiN insulator (b) Drain current versus Drain-source voltage characteristic of the HEMT Source FP1 and $\mathrm{SiN}$ insulator.

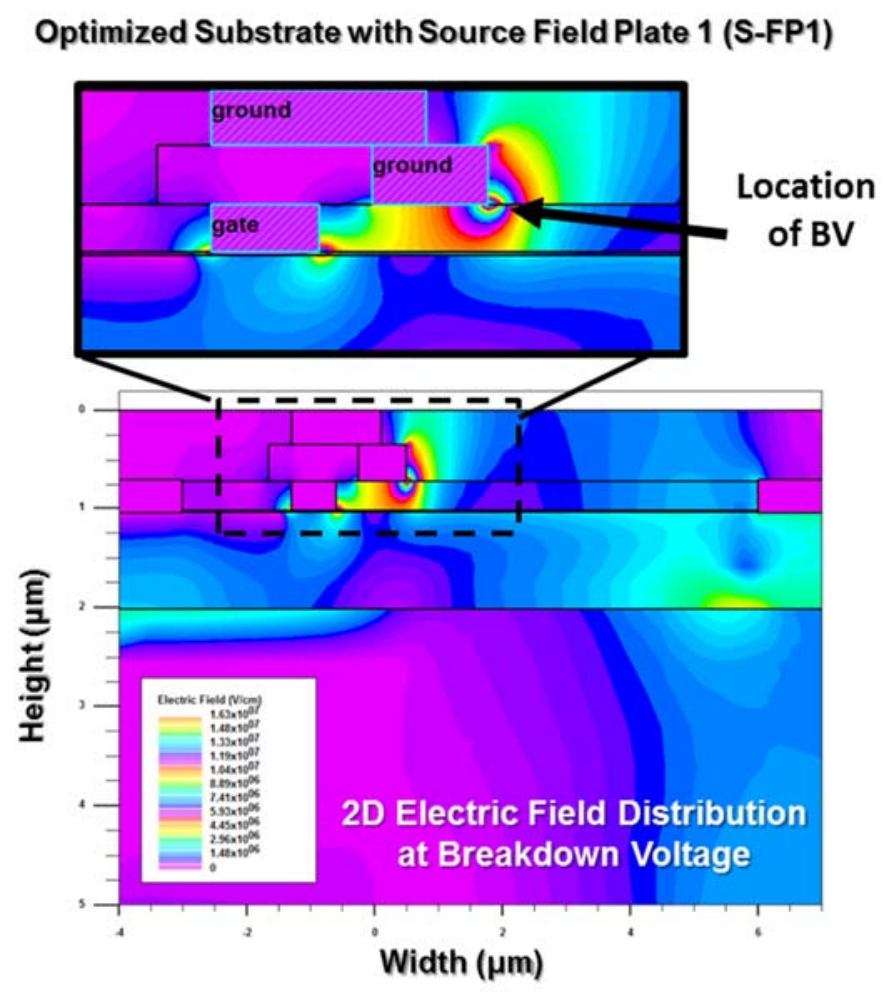

Figure 10.34 Two dimentional electric field forth the HEMT Source FP1 and SiN insulator. 
The 2D electric field of the base case of the HEMT is shown in Figure 10.34. In this figure it can be observed two zones where the electric field is more intense. The intensity on the gate's vicinity is superior. A zoom of the electric field near the gate is illustrated at the top of Figure 10.34. In here, the right lower corner of the field plate contactor indicates a spike of field. In this case, the same situation as explained before in Sub-Section 10.2.1.6.

\subsubsection{Source Field Plate 2 with SiN Insulator}

The schematic of the HEMT structure with source field plate 2 and SiN insulator is shown in Figure 10.25(b). The specifications of this structure are as follows:

- Substrate material: Silicon (Si) Optimum

- Passivation material: $\mathrm{SiN}$

- Field plate: Source FP2

- Dimentions: as shown in Table 10-7.

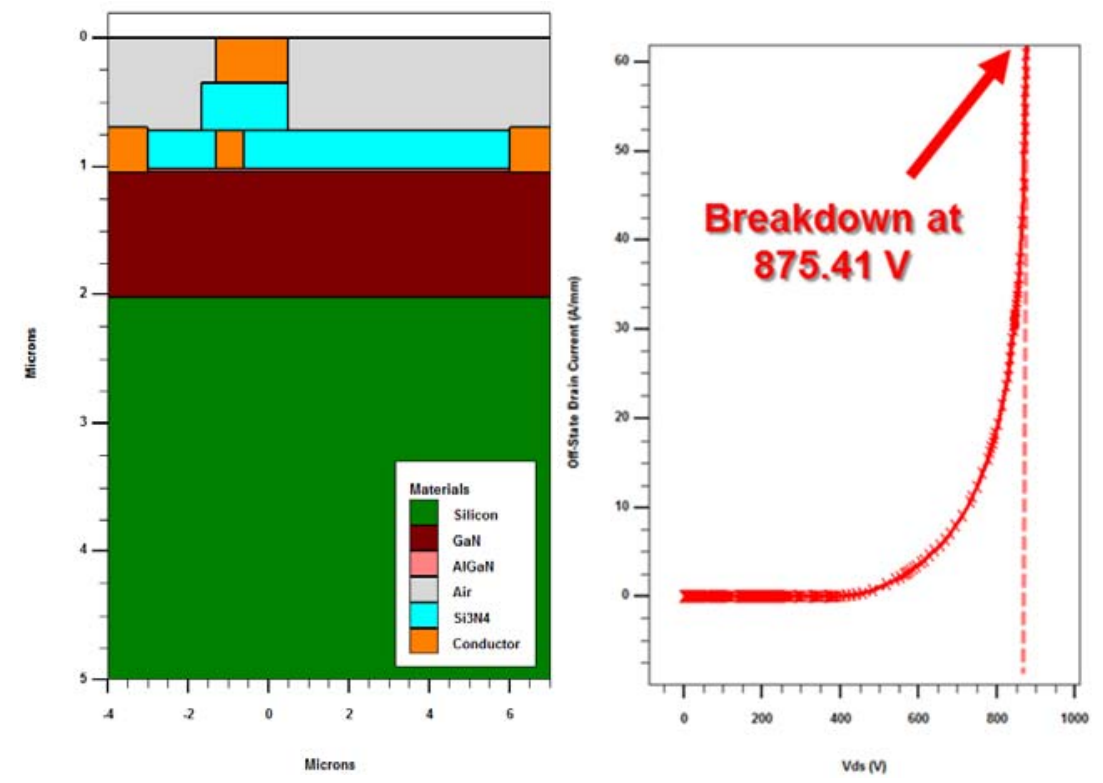

Figure 10.35 (a) True dimentions of the HEMT with S-FP2 and SiN insulator (b) Drain current versus Drain-source voltage characteristic of the HEMT S-FP2 and SiN insulator. 


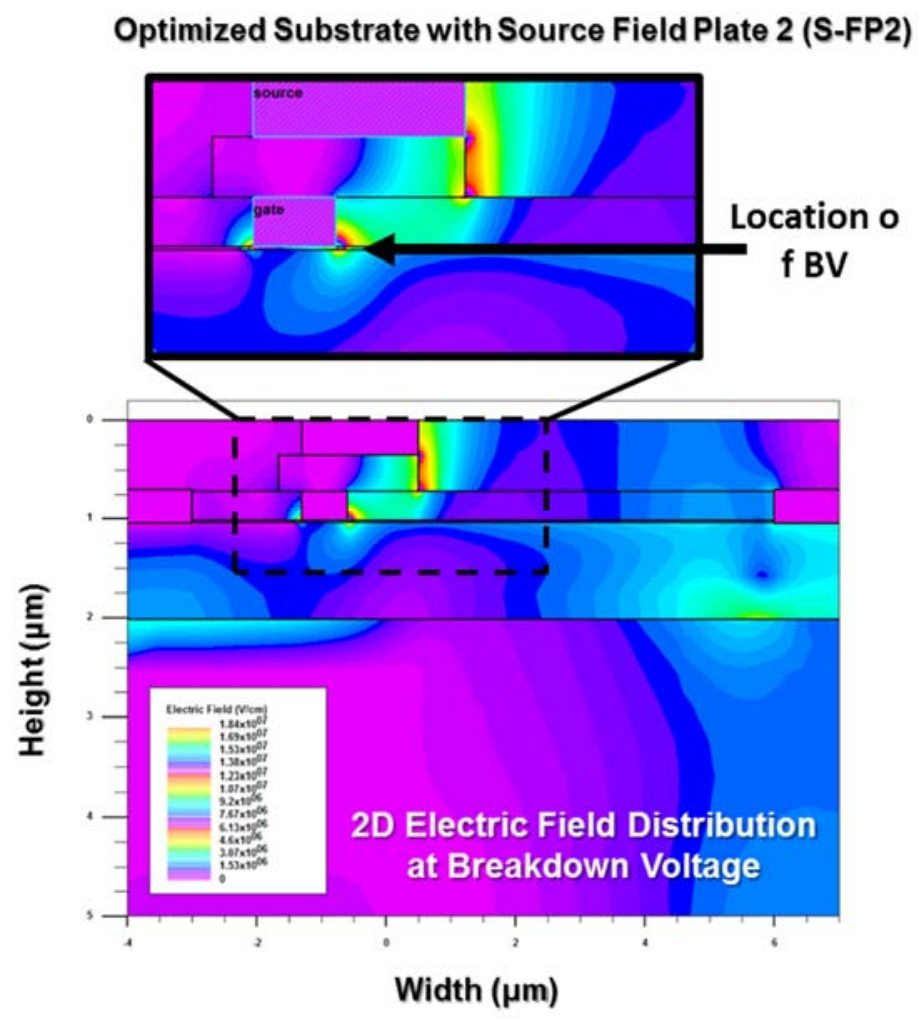

Figure 10.36 Two dimentional electric field forth the HEMT Source FP2 and SiN insulator.

The proportional real dimentions of this HEMT case are shown in Figure 10.35(a) where it is observe that the the substrate has been etched. In Figure 10.35(b) the characteristic curve $\left(i_{d}-v_{d s}\right)$ of the HEMT is plotted. From this curve it is noted that the breakdown voltage starts at $875.41 \mathrm{~V}$.

The 2D electric field of the base case of the HEMT is shown in Figure 10.36. In this figure it can be observed two zones where the electric field is more intense. The intensity on the gate's vicinity is superior. A zoom of the electric field near the gate is illustrated at the top of Figure 10.36. In here, the right lower corner of the field plate contactor indicates a spike of field. In this case, the same situation as explained before in Sub-Section 10.2.1.6. 


\subsubsection{Source Field Plate 3 with SiN Insulator}

The schematic of the HEMT structure with source field plate 3 and SiN insulator is shown in Figure 10.25(b). The specifications of this structure are as follows:

- Substrate material: Silicon (Si) Optimum

- Passivation material: $\mathrm{SiN}$

- $\quad$ Field plate: Source FP3

- Dimentions: as shown in Table 10-7.
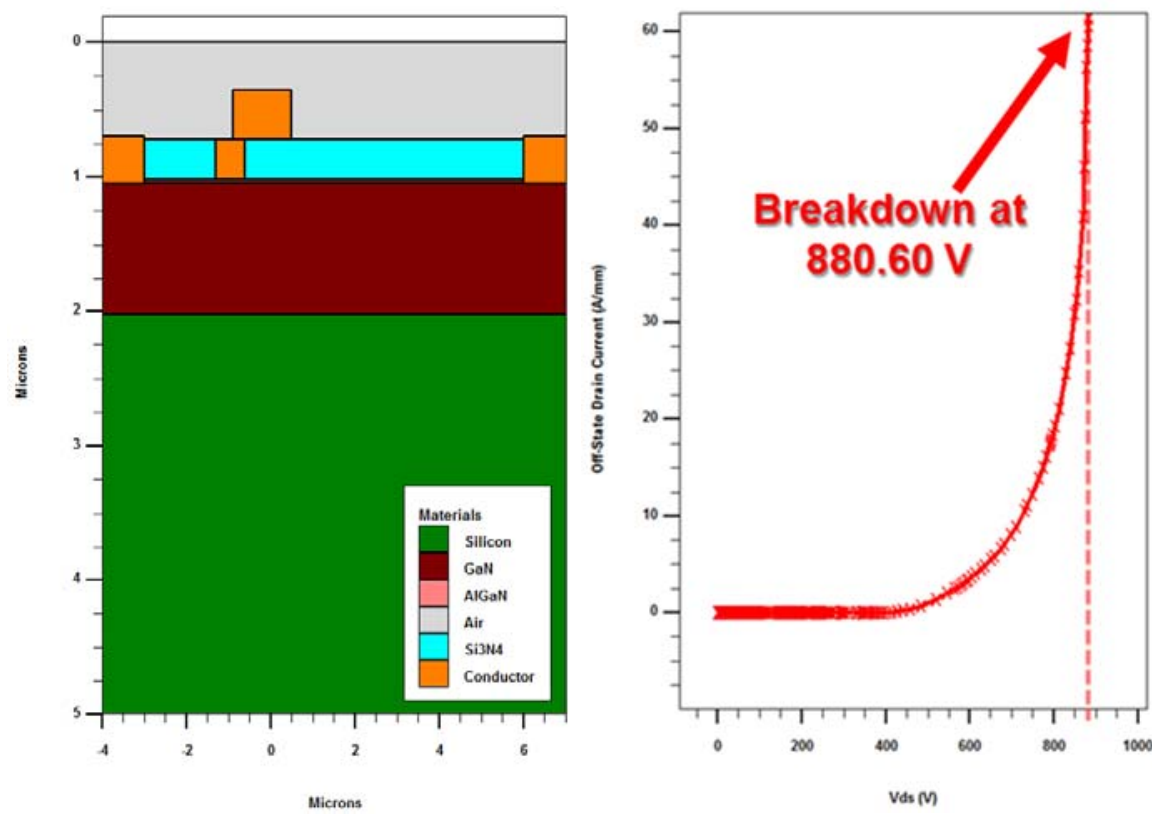

Figure 10.37 (a) True dimentions of the HEMT with Source FP3 and SiN insulator (b) Drain current versus Drain-source voltage characteristic of the HEMT Source FP3 and SiN insulator. 


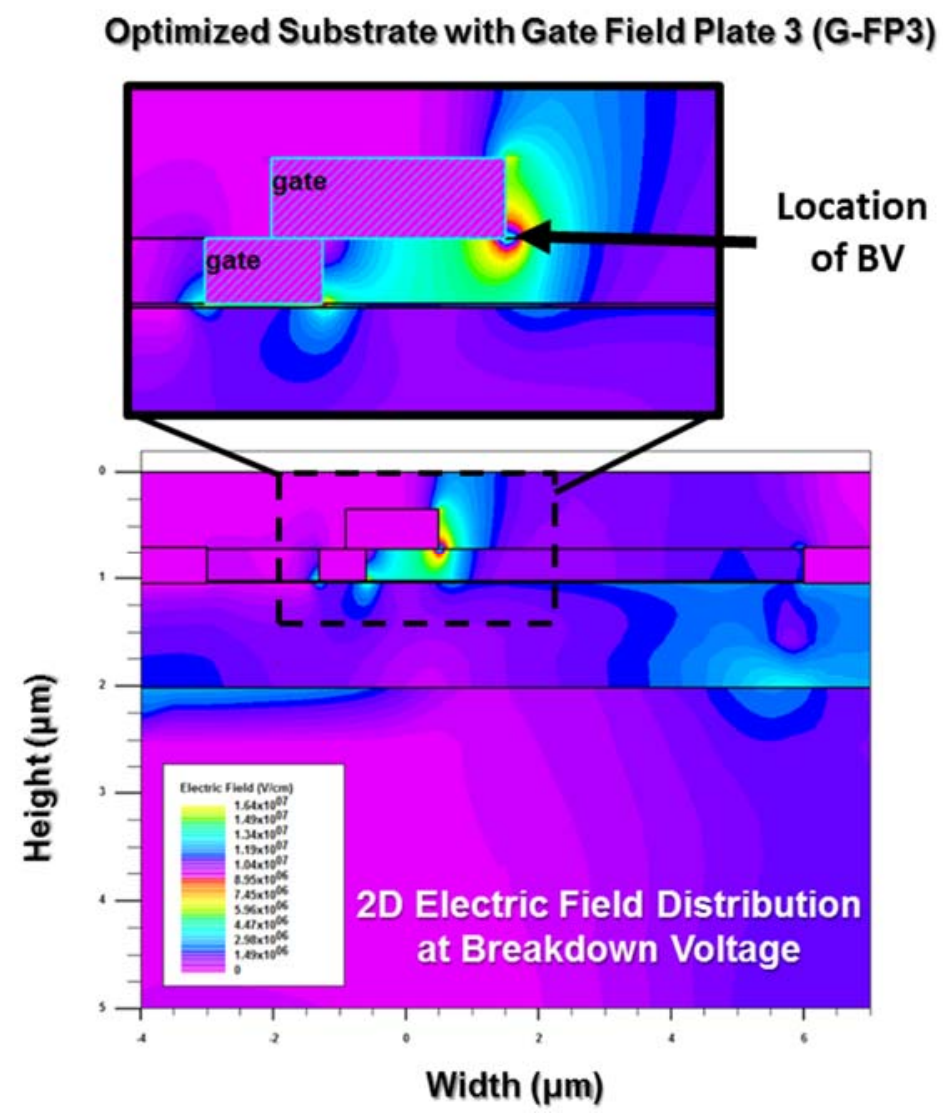

Figure 10.38 Two dimentional electric field forth the HEMT Source FP3 and SiN insulator.

The proportional real dimentions of this HEMT case are shown in Figure 10.37(a) where it is observe that the the substrate has been etched. In Figure 10.37(b) the characteristic curve $\left(i_{d}-v_{d s}\right)$ of the HEMT is plotted. From this curve it is noted that the breakdown voltage starts at $880.60 \mathrm{~V}$.

The 2D electric field of the base case of the HEMT is shown in Figure 10.38. In this figure it can be observed two zones where the electric field is more intense. The intensity on the gate's vicinity is superior. A zoom of the electric field near the gate is illustrated at the top of Figure 10.38. In here, the right lower corner of the field plate contactor indicates a spike of field. In this case, the same situation as explained before in Sub-Section 10.2.1.6. 


\subsubsection{Comparative Results among all Studied Cases}

The structure in Figure 10.25(a) is then modified to evaluate the BV performance with the application of different FP configurations and a modified passivation material. The BV progressions (Figure 10.39(a)) and electric field $\left(E_{f}\right)$ distribution at 4 heights $\left(\mathrm{y}_{1}=\right.$ $0.535 \mu \mathrm{m}, \mathrm{y}_{2}=0.721 \mu \mathrm{m}$ surface of the device, $\mathrm{y}_{3}=0.870 \mu \mathrm{m}$ and $\mathrm{y}_{4}=1.040 \mu \mathrm{m}$ channel) as shown in Figure 10.40 along the $x$ axis have been conducted at $V_{d s}=610 \mathrm{~V}$ which is the smallest BV for the original case (Figure 10.41).

First, substrate etching of the original to the optimal thickness results in a dramatic increase of the BV by $226.3 \mathrm{~V}$. Two S-FP architectures are then tested as modifications to the optimal Si substrate thickness case. A two-part staircase S-FP1 as shown in Figure 10.25(b) results in an immediate $30 \mathrm{~V}$ increase of the $\mathrm{BV}$. The $\mathrm{SiO}_{2}$ passivation layer is then replaced with Silicon Nitride $(\mathrm{SiN})$ resulting in a further increase of $14 \mathrm{~V}$. Next, an alternative single part S-FP architecture (FP2) is evaluated as shown in Figure 10.25(c) keeping $\mathrm{SiN}$ as the passivation layer which results in a slight drop in the BV versus FP1. However, it should be mentioned the BV is only around $5 \mathrm{~V}$ lower with a potential trade-

off in easier fabrication of a single-part structure. A summary of the results are shown in Table III.

Next, a single-part G-FP structure (FP3) is evaluated as depicted in Figure 10.25(d) which results in almost the same performance as FP1. Figure 10.39(b) demonstrates the origin of the BV for the optimal Si substrate thickness case (FP1 with SiN passivation) which points toward drain-source sub-threshold leakage [190]. From Figure 10.41(b) and Table $10-8$, it can be noted that as $E_{f \max }$ decreases the BV increases in all the cases (as 
expected). However, $E_{\text {fmax }}$ is the highest for the FP1-SiN device which appears to be contradictory. This can be explained noticing that this $E_{f \max }$ is located at $x=0.5 \mu \mathrm{m}$, at the end of the S-FP contact and separated from the 2-DEG by the SiN passivation layer with very high dielectric strength of $11 \mathrm{MV} / \mathrm{cm}$.

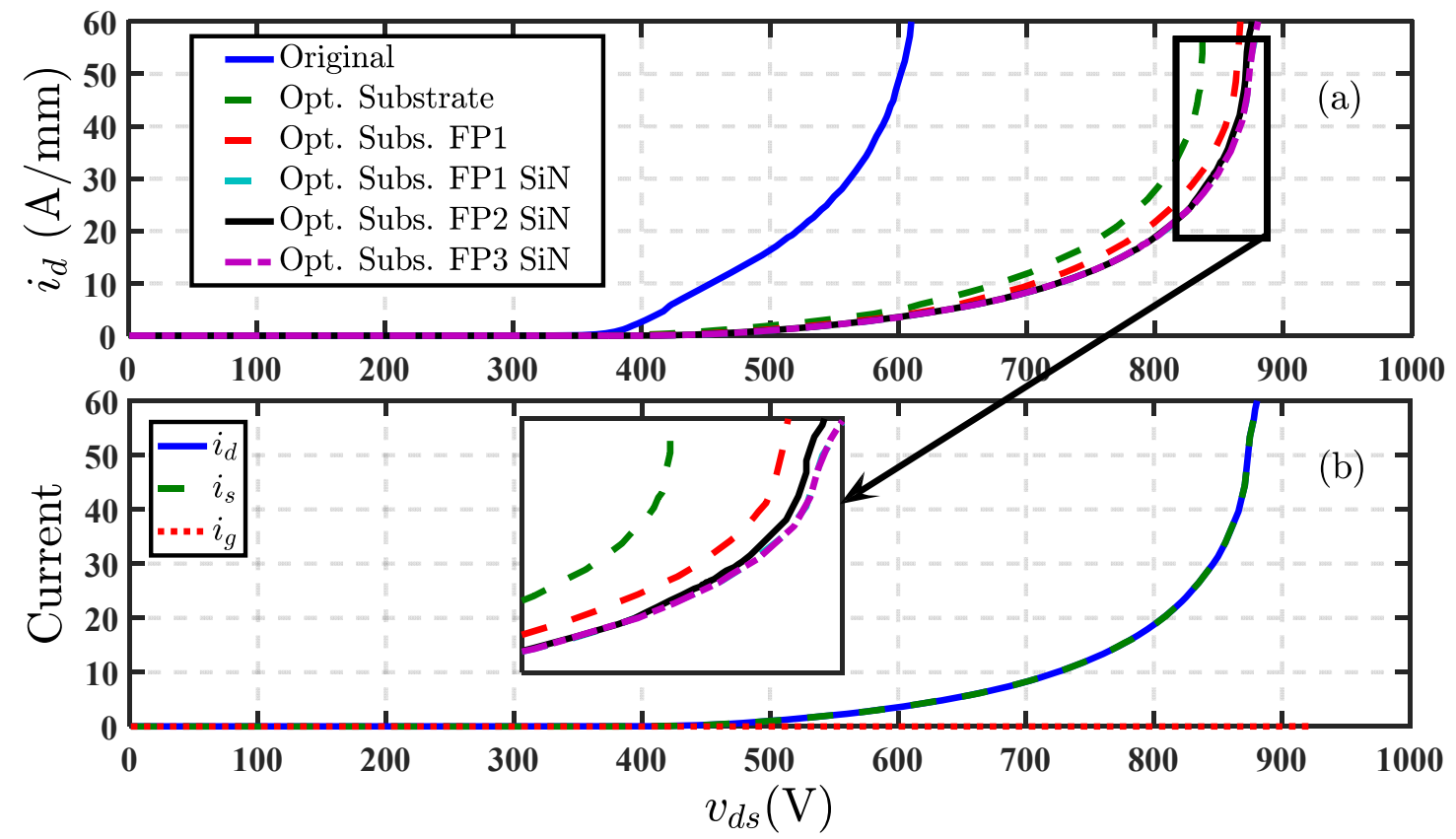

Figure 10.39 (a) BV curves for all cases (b) Drain, source and gate currents for the best Case of Si substrate thickness with FP1 and SiN.

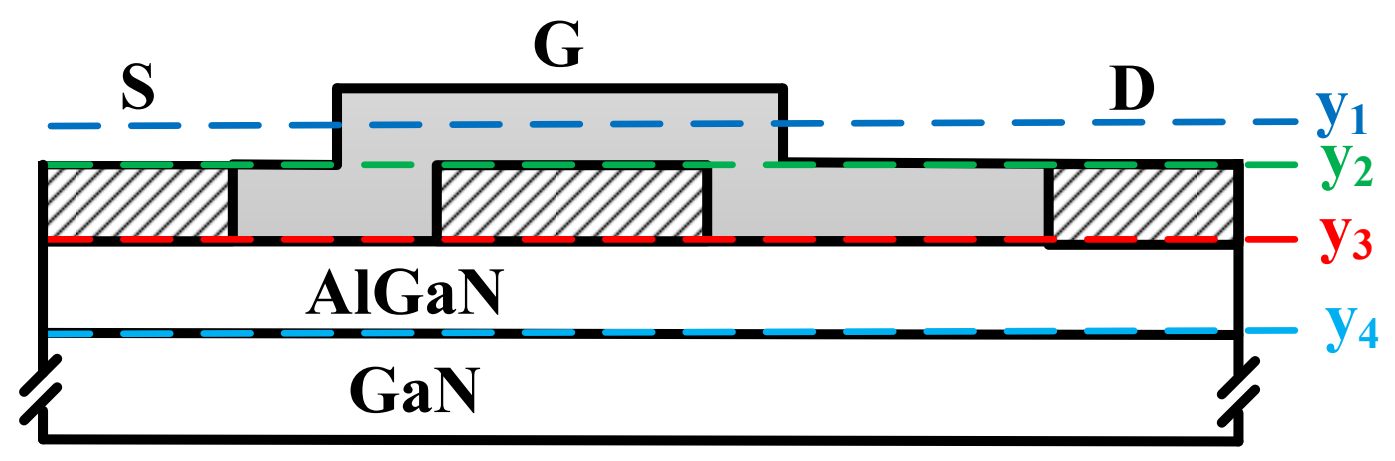

Figure 10.40 Locations of Electric Field Cross Sections. 
Even when the electric field peaks appear to be huge, two points need to be clarify:

1. The electric field peak is not in the AlGaN material if not it is over the insulation layer.

2. The simulation where performed with sharp edges in all the metal conductors. It is well known that sharp edges of the conductors crowd the electric field. Practical implementations required round edges.

3. The simulation were carry out without impurities.

From Figure 10.41(d), which corresponds to the cross section along the 2-DEG $\left(\mathrm{y}_{4}=\right.$ $1.040 \mu \mathrm{m})$, it is clear that the $E_{f \max }$ of the FP1-SiN is the minimum.

\subsubsection{Analysis of the Best Case with Field Plate}

For the best FP case, the electric field distribution along the x-direction at 4 different heights (at BV=880.61 V) is shown in Figure 10.42 and also as a 2D map in Figure 10.43. Figure 10.42 shows the key advantage of the FP layout as it redistributes the field and relocates the peak electric field along the $\mathrm{x}$ and $\mathrm{y}$ plane keeping it below the critical value and hence increasing the BV. The same behavior is more clearly observed in Figure 10.43(c). It is shown that the highest field strength is present right at the corner of the FP$\mathrm{SiN}$ interface on the drain side of the gate at $11.4 \mathrm{MV} / \mathrm{cm}$. 

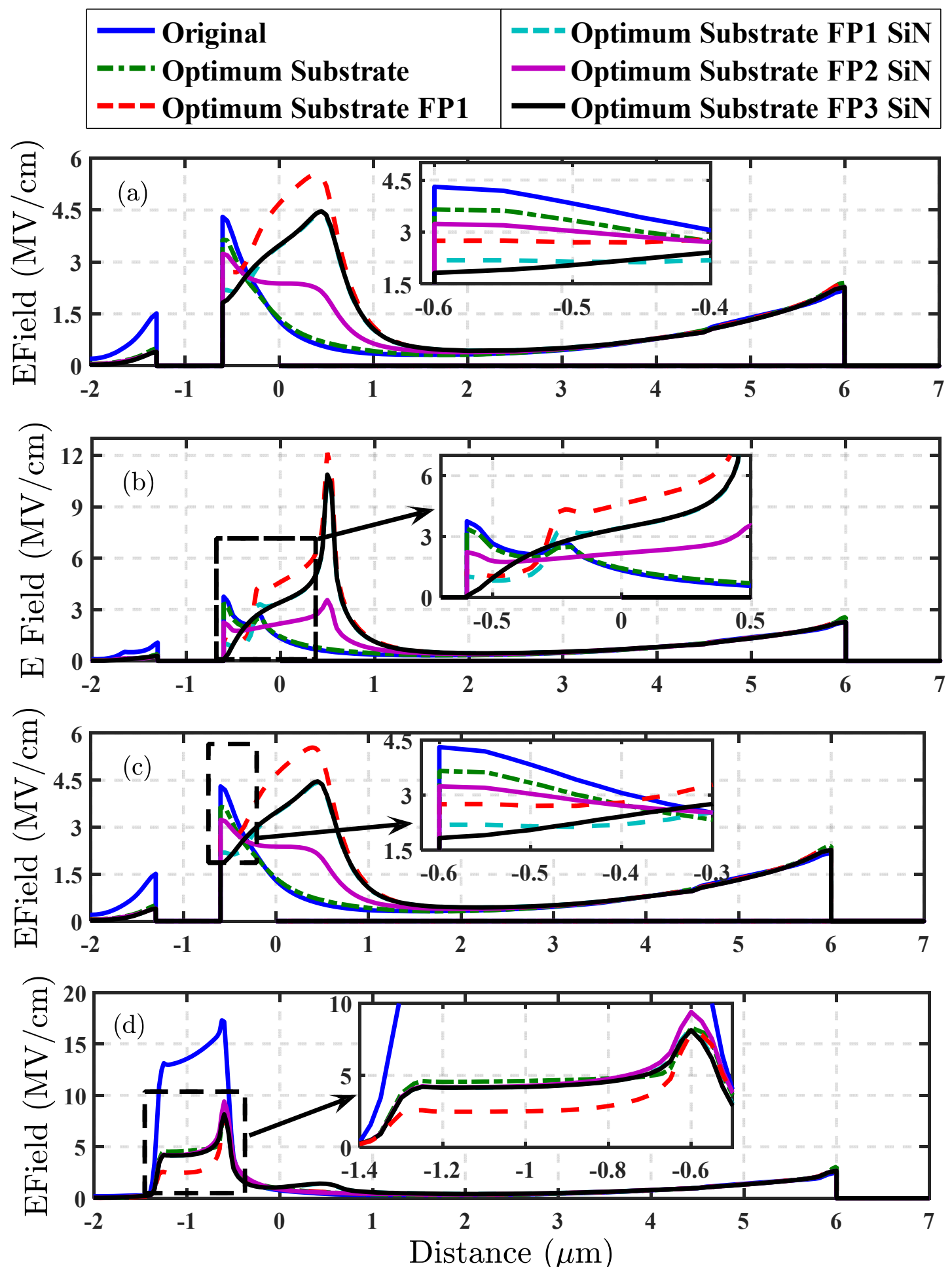

Figure 10.41 Electric field distribution along $x$ axis for all cases (a) $y_{1}=$ $0.535 \mu \mathrm{m}$, (b) $\mathrm{y}_{2}=0.721 \mu \mathrm{m} \quad$ (c) $\mathrm{y}_{3}=0.870 \mu \mathrm{m}$, (d) $\mathrm{y}_{4}=1.040 \mu \mathrm{m}$. 
Table 10-8 GaNFET Design Parameters and Cases of Study

\begin{tabular}{|l|r|r|r|r|r|r|}
\hline & Base & \multicolumn{1}{|c|}{$\begin{array}{l}\text { Optimum } \\
\text { Substrate }\end{array}$} & $\begin{array}{l}\text { Optimum } \\
\text { Substrate } \\
\text { FP1 }\end{array}$ & $\begin{array}{l}\text { Optimum } \\
\text { Substrate } \\
\text { FP1 SiN }\end{array}$ & $\begin{array}{l}\text { Optimum } \\
\text { Substrate } \\
\text { FP2 SiN }\end{array}$ & $\begin{array}{l}\text { Optimum Substrate } \\
\text { FP3 SiN }\end{array}$ \\
\hline $\begin{array}{l}\mathrm{BV} \\
(\mathrm{V})\end{array}$ & 610.19 & 836.48 & 866.65 & 880.63 & 875.41 & 880.60 \\
\hline $\begin{array}{c}E_{f \max y_{1}} \\
(\mathrm{MV} / \mathrm{cm})\end{array}$ & 2.476 & 2.391 & 4.534 & 4.295 & 4.059 & 4.350 \\
\hline $\begin{array}{c}E_{f \max y_{2}} \\
(\mathrm{MV} / \mathrm{cm})\end{array}$ & 3.754 & 3.387 & 12.154 & 10.775 & 3.549 & 10.888 \\
\hline $\begin{array}{c}E_{f \max y_{3}} \\
(\mathrm{MV} / \mathrm{cm})\end{array}$ & 4.302 & 3.653 & 5.544 & 4.421 & 3.234 & 4.463 \\
\hline $\begin{array}{c}E_{f \max y_{4}} \\
(\mathrm{MV} / \mathrm{cm})\end{array}$ & 17.291 & 8.319 & 7.959 & 8.290 & 9.412 & 8.145 \\
\hline
\end{tabular}

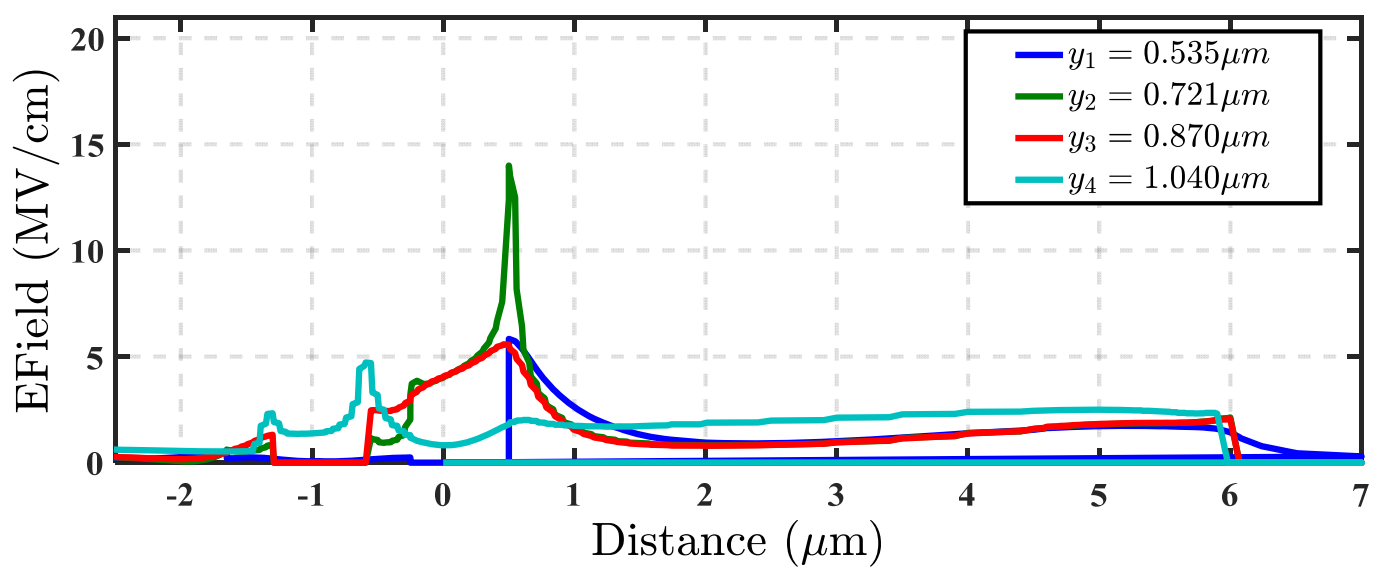

Figure 10.42 E field distribution along the $\mathrm{x}$ axis at $4 \mathrm{y}$ distances for the FP case. 


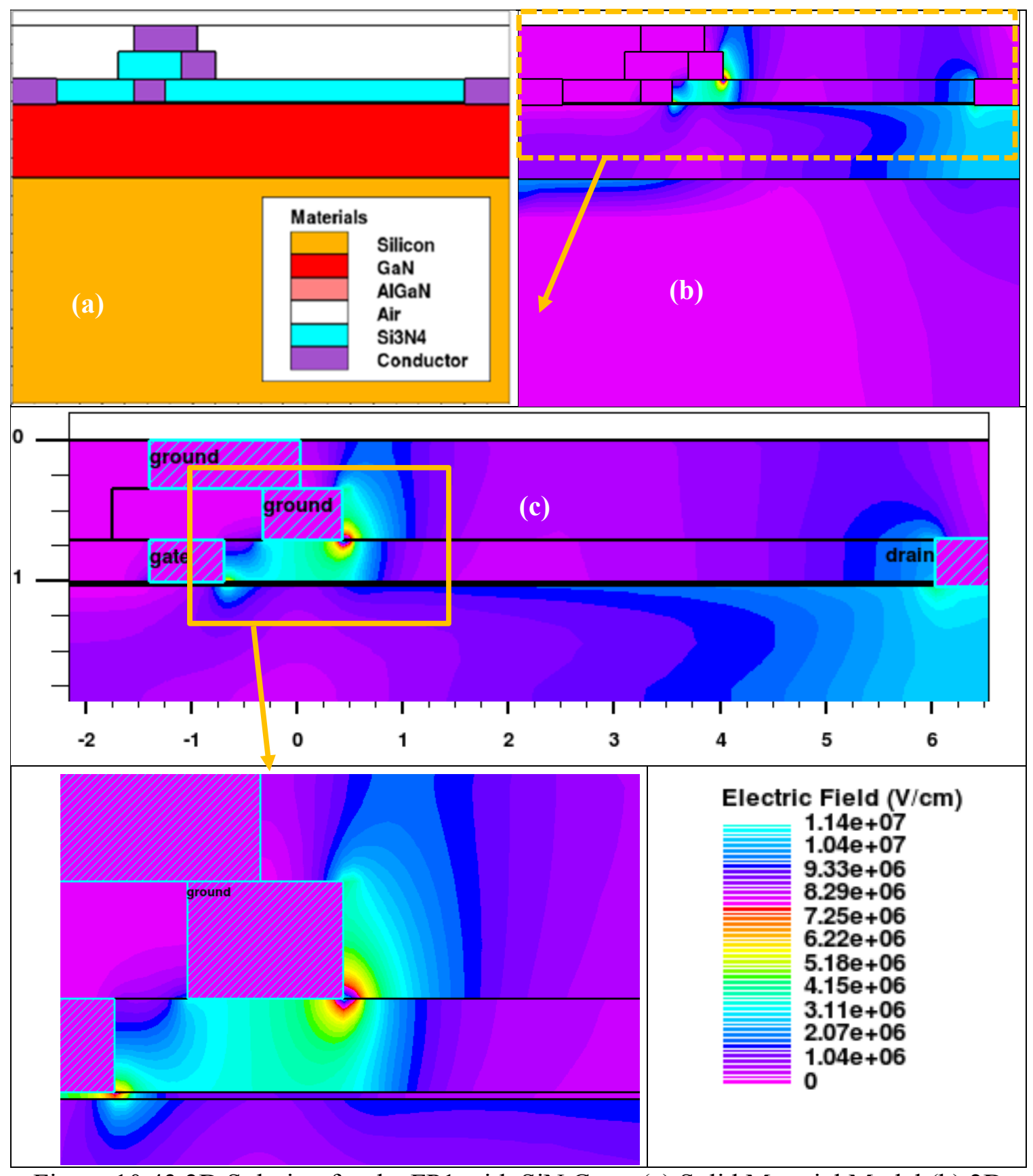

Figure 10.43 2D Solution for the FP1 with SiN Case. (a) Solid Material Model (b) 2D Electric Field Distribution Full View, (c) 2D Electric Field Zoom

\subsection{Conclusion}

In order to increase the $\mathrm{BV}$, it can be concluded that the best way is through a combination of the three well-known techniques: passivation, substrate removal, and a FP 
addition. FP modulation has only a small effect on the BV and $R_{d s(o n)}$, but the $E_{f}$ stress can be reduced with the optimal selection of the FP length.

In this chapter a general theoretical analysis of a heterostructure $(\mathrm{AlGaN})$ with a gate field plate (G-FP) is carry out. A comprehensive comparative study among different field plate configuration was performed. Finite element simulations corroborate the theoretical findings. Furthermore, it is demonstrated that the Si substrate reduction plus the addition of field plate improve the BV in classical HEMTs. Future investigations should focus more in the theoretical solution of the electric field for better insights. Additional FP cases such as multiple FP, dual and double could lead to further increase in the BV mechanism. Future investigation will be performed to explain the physical mechanism behind the optimum substrate removal. 


\section{Chapter 11 Conclusions and Recommendations for Future Research}

\subsection{Conclusions}

From this Chapter 2, it can be concluded that some research topics based on the selection of power electronics, with no order or importance of apparition, are as follows:

- Active regenerative rectifier research: modelling, design and control.

- DC-DC converter research: PCB design for EMI reduction.

- $\quad$ Plug-in battery charger control for electric vehicles.

For the case of the condition monitoring in electrical machines, we have selected the three phase squirrel cage induction machine. Under this machine, the type of failure covered was the inter-turn short-circuit in one phase. The research englobes:

- Modelling.

- Signature discovery for fault detection and identification.

- Developing an online fault detection for inverter-fed IM driven by close loop vector controllers.

A comprehensive study of the ARR was performed in chapter 3, starting with an exhaustive literature review, classification and summarization of the control and design strategies. On top of that, this chapter proposed an improved and general design methodology for the passive components of the ARR. The novelty of the L-filter design strategy lies on the consideration of the modulation technique (centered-SPWM) in a simple manner to reach convergence with the current harmonic analysis in frequency domain. The dc-link capacitor design was based on the dc-link voltage ripple and power balancing. Both design strategies (L-filter and dc-link capacitor) were demonstrated and 
verified by means of simulation and experimental results. All these tests were performed under the ARR being controlled by a deadbeat-type predictive controller (OVC-DCC). Based on non-linear theory, a complete analysis of the OVC-DCC for a typical VSC was developed for the first time in the literature to the best of the author's knowledge. The dynamic performance and accuracy of the proposed controller was tested and corroborated also by means of simulations and experimental results where indices, such as MAE and RMSE, were computed. Two modulation strategies were compared (SPWM and SVM) under 3 criteria: dynamic performance, current THD and losses, finding that SVM performs better. A sensitivity study was performed to test the ARR parameter independency under diverse operating conditions. It was concluded that the OVC-DCC is highly robust to parameter uncertainty of $\pm 50 \%$.

Chapter 4 presented an optimization technique for the PCB design based on the arrangement of the passive components. A software platform was developed to perform the optimization using GA and 3D-FE. The proposed procedure for PCB design was verified by simulation and experimental results. The results show a significant reduction in the radiated electromagnetic field at a susceptible device in a PCB. Even when the optimization procedure was applied only for the sensor's center, the field reduction was extended for a wide region around the sensor. Moreover, the proposed technique not only reduces the fundamental field component, but also all the harmonic contents for the electromagnetic field.

An innovative and simple control strategy based on the integration of a FLC algorithm with minimum and linear MF with a predictive methodology was presented. The advantages of both techniques are exploited to improve the drawbacks and performance of 
the direct torque controller. Simulations and experimental results show an improvement in the control performance of the torque, including ripple and steady state error reduction, and a satisfactory performance at the low-speed region. The proposed control was tested under $\pm 50 \%$ variation of the IM parameters, obtaining similar results and validating its low dependency. Different modulation strategies were studied, and it was found that SVM presents the best performance for the drive application under the proposed controller.

The simplest state-space model for the IM with stator winding inter-turn short-circuit fault on one phase was developed. It was demonstrated that this model can be separated in two modes: "common mode" and "differential mode," representing the healthy and faulty part of the IM. Complex vector and operational EC are derived to obtain the steady state component-sequence EC. Complete fault IM admittance deductions are developed and presented. Simulation for the transient and steady state behavior of the machine under fault condition are performed. Furthermore, SC analysis of the stator current and the torque ripple are studied to evaluate, compare and find usable signatures for FDI of stator windings faults in IM. Finally, torque slip characteristics are analyzed under voltage and frequency variation.

Inter-turn short-circuits in the stator winding of a three-phase IM was fully theoretically analyzed, studied, modelled, simulated, experimentally tested and compared. This work proposes an alternative faulty leakage inductance approach with additional physical meaning compared with the ones presented in the literature. This leakage inductance model has more accurate behavior with respect to the linear one, keeping simplicity of the modelling. The faulty model was completely deduced and verified by comparing simulation with experimental tests under different load conditions, regimes and variation 
of the fault severity factor and faulty resistance. These two variables define the magnitude of the faulty leakage current and thus the damaging of the IM. From the results, it can be concluded that the faulty model is accurate and behaves similarly to the real asymmetric IM. Also, the chapter collects a series of EC that are useful for future FDI techniques, however, they are more utilizable for improving some MRA techniques, such as: off-line impedance signature, parameter estimation and observer-based techniques.

Inter-turn short-circuits in the stator winding of a three-phase squirrel-cage IM driven by a VSI under a DTC was theoretically analyzed, studied, simulated, experimentally tested and compared. This chapter points out the influence of ITSC on the two controlled variables of DTC: electromagnetic torque and stator flux-linkage vector amplitude. From the mathematical models of the asymmetric IM and using the theory of predictive control, a theoretical hypothesis was developed for the DTC reaction to the ITSC fault. Errors in the estimated variables are found throughout the mathematical modelling that explains the DTC compensation and robustness to the ITSC effects. The simulation and experimental results verified the theoretical thesis.

An online, not intrusive and robust fault detector of stator turn faults for DTC driven IM was presented. The fault detector was based on the continuous monitoring of the offdiagonal term of the SC impedance matrix. The FD is adapted to suit DTC controlled IM, where many hurdles were overcome for its implementation. The implementation of the FD was explained in detail. Two simulation test-benches were developed for initial testing of the FD. The simulations provide insights on the DTC functioning and the effects of the ITSC on it. Evidence of the signatures hiding phenomena by the closed-loop control were demonstrated, as well as the DTC estimation errors due to the missing terms appearing in 
the onset of the fault. Finally, experimental test results demonstrate the effectiveness, capabilities and sensitivity of the FD for the DTC driven IM.

In order to increase the $\mathrm{BV}$, it can be concluded that the best way is through a combination of the three well-known techniques: passivation, substrate removal, and a FP addition. FP modulation has only a small effect on the BV and $R_{d s(o n)}$, but the $E_{f}$ stress can be reduced with the optimal selection of the FP length. This is studied in chapter 10 . In this chapter a general theoretical analysis of a heterostructure $(\mathrm{AlGaN})$ with a gate field plate (G-FP) was carried out. A comprehensive comparative study among different field plate configuration was performed. Finite element simulations corroborate the theoretical findings. Furthermore, it was demonstrated that the Si substrate reduction plus the addition of field plate improve the BV in classical HEMTs. Future investigations should focus more in the theoretical solution of the electric field for better insights. Additional FP cases such as multiple FP, dual and double could lead to further increase in the BV mechanism. Future investigation will be performed to explain the physical mechanism behind the optimum substrate removal.

\subsection{Recommendations for Future Research}

This dissertation presented contributions to various aspects of $\mathrm{CM}$ of electric machines, there are several directions in which further research could build upon the results presented in this work. Recommendation for the future research on the modelling of the damaged machine, as well some advices of future research on the detection methods. The need to continue working on the modelling will open the possibilities to improve and enhance the methodologies of fault detection and condition monitoring that are based on model reference approaches, which are the most promising techniques. 
Additionally, some recommendations for the condition monitoring and performance improvement in power electronic converters are presented.

\subsubsection{Recommendations for the Modelling of IM with ITSC}

Based on the definition of the stator leakage inductance of the faulty winding part in the induction machine with stator turn-to-turn fault, the models of the IM can be categorized in three types:

- The model of [34] which considers the stator leakage inductance of the faulty winding part linearly dependent of the fault severity factor.

- The model of [144] which considers the stator leakage inductance of the faulty winding part quadratic dependent of the fault severity factor.

- The model of [179] which considers the stator leakage inductance of the faulty winding part is neither linear nor quadratic fault severity factor and a function indicating the relationship was developed.

Further focus needs to be paid to compare the above-mentioned models in simulations throughout an assessment study. Observe their correlation with the experimental results of a real IM under ITSC for different cases of fault severity factor, fault resistance and loading condition. For the assessment purposes, the three models should be expressed in the same reference frame, possible the best option is to use the multiple reference frame (MRF) or the arbitrary reference frame (ARF), ergo all the others reference frames would be also available with some small mathematical manipulation. Once the models are in the same reference frame, their transformed voltage equations required to be fully developed until the SS form was reached. Thus, the models can be easily simulated by RK ODE integrators. 
Based on the previous results, comparing simplicity and accuracy, there is a need for developing a general model for the IM with stator ITSC. This general model has the capabilities to help the fault detector to locate the phase of the failure. The model will be more cumbersome than the pre-existing ones. Two approaches to develop the general model, that they should be transformed in the ARF, are as follows:

1. Build a model of the IM where each phase of the stator presents an ITSC. Thus, a space vector of the fault severity factor can be used as $[\boldsymbol{\mu}]=\left[\mu_{a}, \mu_{b}, \mu_{c}\right][152]$. The drawback is that the model is obviously more complex, however, when transformed for instance to the stator reference frame $(\alpha \beta)$ then the fault severity factor vector would be $[\boldsymbol{\mu}]=\left[\mu_{\alpha}, \mu_{\beta}\right]$.

2. The models presented in [34], [144], [179] they assume that the fault is going to be in phase $a$. For the case of the stator reference frame, the transformation was concibed respect the $\alpha$ axis, which transform the voltage equations in certain way, which would be different if the fault is in phase $b$ or $c$. It would be interesting to solve the transformation assuming that the ITSC is on phase $b$ and then $c$. It was obvious that the difference among the models will be a rotation of $\frac{2 \pi}{3}$ and $\frac{4 \pi}{3}$, but the specifics of the analytical calculation are necessary to conclude how the model was modified under fault on phase $b$ or $c$. Similar idea was proposed in [8] which use an angle with 3 options, $0, \frac{2 \pi}{3}$ and $\frac{4 \pi}{3}$ to decipher the location of the failure. The drawback in this model is that it does no have a complete physical meaning.

There is a need for further Investigate the bold ITSC, when $R_{f}=0$, and very low fault severity factor. Some research [8], [144] indicates that the faulty circulating current 
increases with the fault severity factor, but subsequently decreases with it. The only manner to verify this hypothesis are throughout numerical analysis: finite element analysis or use of winding functions [125] to obtain the full detailed model of a faulty IM and perform the normal ODE integration. The drawback of these two approaches is the time consumption which reduces the possibility of long time runs in simulation time (tens of seconds) and/or connection with power electronic converter with controllers.

Finally, there is also a need for research and develop models for the following different cases of IM, such as:

$\checkmark$ Phase-to-phase SC

$\checkmark$ Phase-to-core SC

$\checkmark \quad$ ITSC in one of the parallel connected windings (IM with low voltage connection)

$\checkmark$ ITSC in between the parallel connected windings (IM with low voltage connection)

$\checkmark$ Same combinations of faults in the IM connected in delta. There is an approach for this model in [146].

These models should be generalized and transformed to the ARF.

\subsubsection{Recommendations for Online Condition Monitoring of IM}

Chapter 10 proposes and implement a fault detection technique based on the offdiagonal terms of the admittance sequence components matrix. This technique was implemented on an inverter-fed faulty IM driven by DTC. The analyses in this chapter may need to be extended to achieve further deductions of the equivalent circuits and transducer errors or technique sensitivy in a similar way as the reference [35].

New and modern methodologies of fault detection should be developed, compared and assessed for the case when the IM is inverter-fed and drive by the most famous controller 
DTC. Few research has been devoted to this matter and therefore further investigation is required. Among the most promising FD techniques, on DOL IM, the model reference based approach hold the first place. Ergo, these methods must be investigated:

1. Parameter Estimation: this strategy is studied for the DOL IM in [8]. It can be extended for its use in inverter-fed IM. Moreover, the model used in this estimation is a model that lack of physical meaning. Therefore, using the future developed general model the parameter estimation technique can be greatly enhanced. The complication here is in the manipulation of the model to be expressed in only stator quantities such as: stator currents and stator fluxes.

2. Observers: with the SS model of the abnormal IM many observer's techniques can be implemented. The most famous one is the Kalman filter used for the DOL IM. Extension of this technique to inverter-fed IM would be a high contribution to the field of condition monitoring.

Two additional techniques also deserve attention in the case of the faulty IM driven by DTC:

1. Frequency domain analysis has been researched in [156], [162], however, only the third harmonic component in the flux-linkage was investigated. Further analysis is required, for instance the study of the inter-harmonics injected by the DTC, by the fault and by the machine. Their origin discrimination would lead to effective fault detection techniques. This need the study of harmonics that came from IM MMF, slots, rotor bars, skew, etc plus the harmonics from the DTC or the inverter.

2. Power Analysis: the complex power inputted to the IM can be calculated to find the instantaneous active and reactive power. With a power comparative analysis 
between the healthy and faulty IM can be conducted. The input average power and output average power can be computed from the symmetric components equivalent circuits published on [165]. Thus a distribution of the power lost and the reactive power consumption in the ITSC can be identified.

\subsubsection{Recommendations for the Condition Monitoring and Performance Enhancement of Power Electronic Stages}

In Chapter 3, it was studied the design and control of active regenerative rectifier. Some future work in the area is:

1. Investigate the ARR inductor and capacitor design technique for other types of modulations. Specifically, SVM appears to be promising. Implement the L-design strategy based on the current error area for SVM.

2. Improve the capacitor design methodology with a similar concept of voltage area error or energy area error.

3. Study the control of the ARR for other cases that are not only power factor corrector. Adapt and modify OVS-DCC for control of the reactive power.

4. Extend the study the control of the ARR to Active Power Filter for the design and control.

5. Investigate the stability of the ARR based on Lyapunov criteria.

In Chapter 4, it was studied the design a dc-dc converters. The study of the synchronous buck-boost converter for purposes of interconnecting the machine drive to a batterie plus a battery charger stage could be interesting topics of research. In this matters: 
1. Investigate the design and dimensioning of the battery charger compoused by a bidirectional synchronous buck-boost converter and a VSI connected to the grid.

2. Research the control of the previous topology based on power control. For that reason investigate DPC for the VSI. In this stage, study the ancilliary services of the system to the mains.

In Chapter 5, the control of induction machines was investigated. Not too much room was left in this chapter, however, studying alternatives of improving the Fuzzy controller with the weaking field approaches or with some FOC strategies.

For the last chapter, about the study of the breakdown voltage in HEMTs, more investigion is need, even though many research has been devoted. The recommendations in this field are:

1. Study the field plate modelling of the HEMT to find a closed-form formula that indicates how the electric field behavies in the device.

2. Study the modelling of the field plate for different topologies: source FP, gate FP, dual FP, double FP, etc. Compare the analyses and corroborate this findings with FEA.

3. The FEA should be performed for more realistics conditions. For instance, the bordes in this dissertation were perfectly squared. That means 90 degrees of change in the geometry. Typically, experimental HEMTs do not have so sharp edges. They are rounded to avoid peak and electric field concentrations. 


\section{LIST OF REFERENCES}

[1] M. Liserre, A. Dell'Aquila, and F. Blaabjerg, "An overview of three-phase voltage source active rectifiers interfacing the utility," in Power Tech Conference Proceedings, 2003 IEEE Bologna, 2003, vol. 3, p. 8 pp. Vol.3-.

[2] V. Monteiro, J. G. Pinto, B. Exposto, and J. L. Afonso, "Comprehensive comparison of a current-source and a voltage-source converter for three-phase EV fast battery chargers," in 2015 9th International Conference on Compatibility and Power Electronics (CPE), 2015, pp. 173-178.

[3] G. Buja, M. Bertoluzzo, and C. Fontana, "Reactive Power Compensation Capabilities of V2G-Enabled Electric Vehicles," IEEE Trans. Power Electron., vol. 32, no. 12, pp. 9447-9459, Dec. 2017.

[4] M. Kesler, M. C. Kisacikoglu, and L. M. Tolbert, "Vehicle-to-Grid Reactive Power Operation Using Plug-In Electric Vehicle Bidirectional Offboard Charger," IEEE Trans. Ind. Electron., vol. 61, no. 12, pp. 6778-6784, Dec. 2014.

[5] D. R. White, 3: Electromagnetic Interference and Compatibility. Gainesville, VA: Don White Consultants, 1981.

[6] J. W. Kolar, F. Krismer, Y. Lobsiger, J. Muhlethaler, T. Nussbaumer, and J. Minibock, "Extreme efficiency power electronics," in 2012 7th International Conference on Integrated Power Electronics Systems (CIPS), 2012, pp. 1-22.

[7] D. G. Holmes and T. A. Lipo, Pulse Width Modulation for Power Converters: Principles and Practice. John Wiley \& Sons, 2003.

[8] J.-C. Trigeassou, Electrical Machines Diagnosis, 1st ed. USA: John Wiley \& Sons, Inc., 2012.

[9] L. F. S. Alves et al., "SIC power devices in power electronics: An overview," in 2017 Brazilian Power Electronics Conference (COBEP), 2017, pp. 1-8.

[10] A. Lidow, J. Strydom, M. de Rooij, and D. Reusch, GaN Transistors for Efficient Power Conversion. John Wiley \& Sons, 2014.

[11] J. L. Hostetler, P. Alexandrov, X. Li, L. Fursin, and A. Bhalla, "6.5 kV SiC normally-off JFETs \#x2014; Technology status," in 2014 IEEE Workshop on Wide Bandgap Power Devices and Applications, 2014, pp. 143-146.

[12] M. Riera-Guasp, J. A. Antonino-Daviu, and G. A. Capolino, "Advances in Electrical Machine, Power Electronic, and Drive Condition Monitoring and Fault Detection: State of the Art," IEEE Trans. Ind. Electron., vol. 62, no. 3, pp. 1746-1759, Mar. 2015. 
[13] R. W. D. Doncker, "Twenty years of digital signal processing in power electronics and drives," in The 29th Annual Conference of the IEEE Industrial Electronics Society, 2003. IECON '03, 2003, vol. 1, pp. 957-960 vol.1.

[14] P. C. Krause, O. Wasynczuk, S. D. Sudhoff, and S. Pekarek, Analysis of Electric Machinery and Drive Systems. John Wiley \& Sons, 2013.

[15] M. Liwschitz-Garik, Electric Machinery. NY: Van Nostrand, 1946.

[16] H. A. Toliyat and G. B. Kliman, Handbook of Electric Motors. CRC Press, 2004.

[17] Maquinas Electricas, Fifth. Mc Graw Hill, 2003.

[18] J. M. Aller, Máquinas Eléctricas Rotativas, 1st ed. Venezuela: Editorial Equinoccio, 2008.

[19] P. L. Alger, The nature of induction machines. New York: Gordon and Breach, 1965.

[20] P. L. Alger, The nature of polyphase induction machines. New York: Wiley, 1951.

[21] "Report of Large Motor Reliability Survey of Industrial and Commercial Installations, Part I," IEEE Trans. Ind. Appl., vol. IA-21, no. 4, pp. 853-864, Jul. 1985.

[22] A. Bellini, F. Filippetti, C. Tassoni, and G.-A. Capolino, "Advances in Diagnostic Techniques for Induction Machines," IEEE Trans. Ind. Electron., vol. 55, no. 12, pp. 4109-4126, Dec. 2008.

[23] S. Nandi, H. A. Toliyat, and X. Li, "Condition monitoring and fault diagnosis of electrical motors-a review," IEEE Trans. Energy Convers., vol. 20, no. 4, pp. 719 729, Dec. 2005.

[24] P. Zhang, Y. Du, T. G. Habetler, and B. Lu, "A survey of condition monitoring and protection methods for medium voltage induction motors," in IEEE Energy Conversion Congress and Exposition, 2009. ECCE 2009, 2009, pp. 3165-3174.

[25] P. Neti and S. Nandi, "Stator Interturn Fault Detection of Synchronous Machines Using Field Current and Rotor Search-Coil Voltage Signature Analysis," IEEE Trans. Ind. Appl., vol. 45, no. 3, pp. 911-920, May 2009.

[26] J. Yun, K. Lee, K. W. Lee, S. B. Lee, and J. Y. Yoo, "Detection and Classification of Stator Turn Faults and High-Resistance Electrical Connections for Induction Machines," IEEE Trans. Ind. Appl., vol. 45, no. 2, pp. 666-675, Mar. 2009.

[27] S. A. McInerny and Y. Dai, "Basic vibration signal processing for bearing fault detection," IEEE Trans. Educ., vol. 46, no. 1, pp. 149-156, Feb. 2003. 
[28] P. J. Tavner, L. Ran, J. Penman, and H. Sedding, Condition Monitoring of Rotating Electrical Machines, 2nd Revised edition edition. London, U.K: The Institution of Engineering and Technology, 2008.

[29] A. Bellini, F. Filippetti, G. Franceschini, and C. Tassoni, "Closed-loop control impact on the diagnosis of induction motors faults," IEEE Trans. Ind. Appl., vol. 36, no. 5, pp. 1318-1329, Sep. 2000.

[30] P. F. Albrecht, J. C. Appiarius, R. M. McCoy, E. L. Owen, and D. K. Sharma, "Assessment of the Reliability of Motors in Utility Applications - Updated," IEEE Trans. Energy Convers., vol. EC-1, no. 1, pp. 39-46, Mar. 1986.

[31] "Report of Large Motor Reliability Survey of Industrial and Commercial Installations, Part II," IEEE Trans. Ind. Appl., vol. IA-21, no. 4, pp. 865-872, Jul. 1985.

[32] "Report of Large Motor Reliability Survey of Industrial and Commercial Installations: Part 3," IEEE Trans. Ind. Appl., vol. IA-23, no. 1, pp. 153-158, Jan. 1987.

[33] M. Melfi, A. M. J. Sung, S. Bell, and G. L. Skibinski, "Effect of surge voltage risetime on the insulation of low-voltage machines fed by PWM converters," IEEE Trans. Ind. Appl., vol. 34, no. 4, pp. 766-775, Jul. 1998.

[34] R. M. Tallam, T. G. Habetler, and R. G. Harley, "Transient model for induction machines with stator winding turn faults," IEEE Trans. Ind. Appl., vol. 38, no. 3, pp. 632-637, May 2002.

[35] S. B. Lee, R. M. Tallam, and T. G. Habetler, "A robust, on-line turn-fault detection technique for induction machines based on monitoring the sequence component impedance matrix," IEEE Trans. Power Electron., vol. 18, no. 3, pp. 865-872, May 2003.

[36] A. Berzoy, A. A. S. Mohamed, and O. A. Mohammed, "Dynamic space-vector model of induction machines with stator inter-turn short-circuit fault," in IECON 2015 - 41st Annual Conference of the IEEE Industrial Electronics Society, 2015, pp. $003620-003625$.

[37] S. Cheng, "Utilizing the Connected Power Electronic Converter for improved Condition Monitoring of Induction Motors and Claw-Pole Generators," Georgia Institute of Technology, Atlanta, USA, 2012.

[38] A. M. Trzynadlowski, Control of Induction Motors, 1 edition. San Diego: Academic Press, 2000.

[39] P. Vas, Vector control of AC machines. Clarendon Press, 1990. 
[40] D. W. Novotny and T. A. Lipo, Vector Control and Dynamics of AC Drives. Clarendon Press, 1996.

[41] J. A. Santisteban and R. M. Stephan, "Vector control methods for induction machines: an overview," IEEE Trans. Educ., vol. 44, no. 2, pp. 170-175, May 2001.

[42] S. Karmakar, S. Chattopadhyay, M. Mitra, and S. Sengupta, "Induction Motor and Faults," in Induction Motor Fault Diagnosis, Springer, Singapore, 2016, pp. 7-28.

[43] J. M. Aller, "Conversion de Energia Electrica.".

[44] E. Clarke, Circuit Analysis of A-C Power Systems. Wiley, 1943.

[45] G. Escobar, A. M. Stankovic, J. M. Carrasco, E. Galvan, and R. Ortega, "Analysis and design of direct power control (DPC) for a three phase synchronous rectifier via output regulation subspaces," IEEE Trans. Power Electron., vol. 18, no. 3, pp. 823830, May 2003.

[46] J. Ge, Z. Zhao, L. Yuan, T. Lu, and F. He, "Direct Power Control Based on Natural Switching Surface for Three-Phase PWM Rectifiers," IEEE Trans. Power Electron., vol. 30, no. 6, pp. 2918-2922, Jun. 2015.

[47] Y. Zhang and C. Qu, "Direct Power Control of a Pulse Width Modulation Rectifier Using Space Vector Modulation Under Unbalanced Grid Voltages," IEEE Trans. Power Electron., vol. 30, no. 10, pp. 5892-5901, Oct. 2015.

[48] J. A. Restrepo et al., "Direct Power Control of a Dual Converter Operating as a Synchronous Rectifier," IEEE Trans. Power Electron., vol. 26, no. 5, pp. 14101417, May 2011.

[49] Y. Zhang and C. Qu, "Model Predictive Direct Power Control of PWM Rectifiers Under Unbalanced Network Conditions," IEEE Trans. Ind. Electron., vol. 62, no. 7, pp. 4011-4022, Jul. 2015.

[50] S. Kwak and J.-C. Park, "Model-Predictive Direct Power Control With Vector Preselection Technique for Highly Efficient Active Rectifiers," IEEE Trans. Ind. Inform., vol. 11, no. 1, pp. 44-52, Feb. 2015.

[51] J. A. Restrepo, J. M. Aller, J. C. Viola, A. Bueno, and T. G. Habetler, "Optimum Space Vector Computation Technique for Direct Power Control," IEEE Trans. Power Electron., vol. 24, no. 6, pp. 1637-1645, Jun. 2009.

[52] Z. Chen, Y. Luo, Y. Zhu, and G. Shen, "Analysis and design of three-phase rectifier with near-sinusoidal input currents," in Power Electronics and Motion Control Conference, 2009. IPEMC '09. IEEE 6th International, 2009, pp. 1703-1-1703-7. 
[53] Y. Lang, D. Xu, S. R. Hadianamrei, and H. Ma, "A Novel Design Method of LCL Type Utility Interface for Three-Phase Voltage Source Rectifier," in Power Electronics Specialists Conference, 2005. PESC '05. IEEE 36th, 2005, pp. 313-317.

[54] L. A. Moran, J. W. Dixon, and R. R. Wallace, "A three-phase active power filter operating with fixed switching frequency for reactive power and current harmonic compensation," IEEE Trans. Ind. Electron., vol. 42, no. 4, pp. 402-408, Aug. 1995.

[55] Y. Tong, F. Tang, Y. Chen, F. Zhou, and X. Jin, "Design algorithm of grid-side LCL-filter for three-phase voltage source PWM rectifier," in 2008 IEEE Power and Energy Society General Meeting - Conversion and Delivery of Electrical Energy in the 21st Century, 2008, pp. 1-6.

[56] N.-Y. Dai and M.-C. Wong, "Design considerations of coupling inductance for active power filters," in 2011 6th IEEE Conference on Industrial Electronics and Applications (ICIEA), 2011, pp. 1370-1375.

[57] A. Berzoy, A. Elsayed, T. Youssef, and O. A. Mohammed, "Improved design of controlled rectifier for reduced ripple resulting from integration of DC loads to AC systems," in 2014 IEEE PES General Meeting | Conference Exposition, 2014, pp. $1-5$.

[58] Y. Konishi, M. Ishibashi, and M. Nakaoka, "Three-phase current-source softswitching PWM rectifier for high-power applications and its design considerations," in Power Electronics and Variable Speed Drives, 1998. Seventh International Conference on (Conf. Publ. No. 456), 1998, pp. 133-138.

[59] M. Liserre, F. Blaabjerg, and S. Hansen, "Design and control of an LCL-filter-based three-phase active rectifier," IEEE Trans. Ind. Appl., vol. 41, no. 5, pp. 1281-1291, Sep. 2005.

[60] M. Malinowski, M. P. Kazmierkowski, and A. M. Trzynadlowski, "A comparative study of control techniques for PWM rectifiers in AC adjustable speed drives," IEEE Trans. Power Electron., vol. 18, no. 6, pp. 1390-1396, Nov. 2003.

[61] S. Vazquez, J. Rodriguez, M. Rivera, L. G. Franquelo, and M. Norambuena, "Model Predictive Control for Power Converters and Drives: Advances and Trends," IEEE Trans. Ind. Electron., vol. 64, no. 2, pp. 935-947, Feb. 2017.

[62] M. P. Kazmierkowski and L. Malesani, "Current control techniques for three-phase voltage-source PWM converters: a survey," IEEE Trans. Ind. Electron., vol. 45, no. 5, pp. 691-703, Oct. 1998.

[63] P. Cortes, M. P. Kazmierkowski, R. M. Kennel, D. E. Quevedo, and J. Rodriguez, "Predictive Control in Power Electronics and Drives," IEEE Trans. Ind. Electron., vol. 55, no. 12, pp. 4312-4324, Dec. 2008. 
[64] Yongchang, Qin, Zhengxi, and Yingchao, "Comparative study of model predictive current control and voltage oriented control for PWM rectifiers," in 2013 International Conference on Electrical Machines and Systems (ICEMS), 2013, pp. 2207-2212.

[65] A. Berzoy, J. Viola, and J. Restrepo, "Voltage space vector's computation for current control in three phase converters," Rev. Fac. Ing.-Univ. Antioquia, vol. 64, pp. 45-56, 2012.

[66] A. Berzoy, A. A. S. Mohamed, and O. Mohammed, "Design considerations and predictive direct current control of Active Regenerative Rectifiers for harmonic and current ripple reduction," in 2016 IEEE Applied Power Electronics Conference and Exposition (APEC), 2016, pp. 928-935.

[67] Power System Analysis: Short-Circuit Load Flow and Harmonics, Second Edition, 2 edition. Boca Raton: CRC Press, 2011.

[68] M. Bojrup, Advanced Control of Active Filters in a Battery Charger Application. SWEDEN: Department of Industrial Electrical Engineering and Automation (IEA) Lund Institute of Technology (LTH), 1999.

[69] M. A. Hannan, "Effect of DC capacitor size on D-STATCOM voltage regulation performance evaluation," Press Electr. Rev., vol. 88, no. 12, 2012.

[70] J. A. Restrepo et al., "Direct Power Control of a Dual Converter Operating as a Synchronous Rectifier," IEEE Trans. Power Electron., vol. 26, no. 5, pp. 14101417, May 2011.

[71] P. CortÉs, J. RodrÍguez, P. Antoniewicz, and M. Kazmierkowski, "Direct Power Control of an AFE Using Predictive Control," IEEE Trans. Power Electron., vol. 23, no. 5, pp. 2516-2523, Sep. 2008.

[72] T. Noguchi, H. Tomiki, S. Kondo, and I. Takahashi, "Direct power control of PWM converter without power-source voltage sensors," IEEE Trans. Ind. Appl., vol. 34, no. 3, pp. 473-479, May 1998.

[73] Q. Lu, Y. Sun, and S. Mei, Nonlinear Control Systems and Power System Dynamics. Springer Science \& Business Media, 2001.

[74] J. Restrepo, J. M. Aller, A. Bueno, V. Guzman, and M. I. Gimenez, "Generalized Algorithm for Pulse Width Modulation using a Two-Vectors Based Technique," EPE J., vol. 21, no. 2, Jun. 2011.

[75] L. Asiminoaei, P. Rodriguez, and F. Blaabjerg, "Application of Discontinuous PWMModulation in Active Power Filters," IEEE Trans. Power Electron., vol. 23, no. 4, pp. 1692-1706, Jul. 2008. 
[76] F. Luo, D. Boroyevich, P. Mattavelli, and H. Bishnoi, "EMI filter design considering in-circuit impedance mismatching," in 2012 IEEE Energy Conversion Congress and Exposition (ECCE), 2012, pp. 4613-4618.

[77] M. R. Yazdani, H. Farzanehfard, and J. Faiz, "EMI Analysis and Evaluation of an Improved ZCT Flyback Converter," IEEE Trans. Power Electron., vol. 26, no. 8, pp. 2326-2334, Aug. 2011.

[78] A. Bhargava, D. Pommerenke, K. W. Kam, F. Centola, and C. W. Lam, "DC-DC Buck Converter EMI Reduction Using PCB Layout Modification," IEEE Trans. Electromagn. Compat., vol. 53, no. 3, pp. 806-813, Aug. 2011.

[79] H. F. Blanchette and K. Al-Haddad, "Solving EMI-Related Problems for Reliable High-Power Converters Design With Precomputed Electromagnetic Models," IEEE Trans. Power Electron., vol. 25, no. 1, pp. 219-227, Jan. 2010.

[80] A. Berzoy, A. A. S. Mohamed, and O. A. Mohammed, "Reduced Radiated EMI by Optimum Positioning of Inductors on PCB using 3DFEA - GA," Williamsburg, VA, USA, 2015.

[81] K. Zeineddine, A. Uan-Zo-Li, C.-H. Chen, J. Hahn, and D. Han, "A power-efficient method to mitigate the EMI of Switched-Mode Power Supplies," in 2011 IEEE 8th International Conference on Power Electronics and ECCE Asia (ICPE ECCE), 2011, pp. 453-459.

[82] A. Berzoy, A. A. S. Mohamed, and O. A. Mohammed, "Optimizing Power Converter PCB Magnetic Design for Lower EMI Levels," Graz, Austria, 2014.

[83] A. Bhargava et al., "EMI prediction in switched power supplies by full-wave and non-linear circuit co-simulation," in IEEE International Symposium on Electromagnetic Compatibility, 2009. EMC 2009, 2009, pp. 41-46.

[84] B. Stube, B. Schroeder, E. Hoene, and A. Lissner, "A Novel Approach for EMI Design of Power Electronics," in Design, Automation and Test in Europe, 2008. DATE '08, 2008, pp. 170-175.

[85] M. A. Farahat, H. M. B. Metwally, and A. Abd-Elfatah Mohamed, "Optimal choice and design of different topologies of DC-DC converter used in PV systems, at different climatic conditions in Egypt," Renew. Energy, vol. 43, pp. 393-402, Jul. 2012.

[86] A. A. S. Mohamed, A. Berzoy, and O. Mohammed, "Optimized-fuzzy MPPT controller using GA for stand-alone photovoltaic water pumping system," in IECON 2014 - 40th Annual Conference of the IEEE Industrial Electronics Society, 2014, pp. 2213-2218. 
[87] A. A. S. Mohamed, A. Berzoy, and O. Mohammed, "Control parameters optimization for PM DC motor in photovoltaic applications," in 2015 IEEE International Electric Machines Drives Conference (IEMDC), 2015, pp. 17421747.

[88] N. Y. Abed and O. A. Mohammed, "Physics-Based High-Frequency Transformer Modeling by Finite Elements," Magn. IEEE Trans., vol. 46, no. 8, pp. 3249-3252, Aug. 2010.

[89] C. A. Martins and A. S. Carvalho, "Technological trends in induction motor electrical drives," in Power Tech Proceedings, 2001 IEEE Porto, 2001, vol. 2, p. 7 pp. vol.2-.

[90] F. Blaschke, "A new method for the structural decoupling of AC induction machines," Conf Rec IFAC, pp. 1-15, Oct. 1971.

[91] R. Gabriel, W. Leonhard, and C. J. Nordby, "Field-Oriented Control of a Standard AC Motor Using Microprocessors," IEEE Trans. Ind. Appl., vol. IA-16, no. 2, pp. 186-192, Mar. 1980.

[92] P. Vas and M. Alakula, "Field-oriented control of saturated induction machines," IEEE Trans. Energy Convers., vol. 5, no. 1, pp. 218-224, Mar. 1990.

[93] Y. Miloud and A. Draou, "Fuzzy logic speed control of an indirect field-oriented induction machine drive," in The 27th Annual Conference of the IEEE Industrial Electronics Society, 2001. IECON '01, 2001, vol. 3, pp. 2111-2116 vol.3.

[94] W. L. Chan, A. T. P. So, and L. L. Lai, "Evolutionary programming based machine parameters estimation for field oriented control," in Electric Machines and Drives, 1999. International Conference IEMD '99, 1999, pp. 534-536.

[95] J.-H. Kim, J.-W. Choi, and S.-K. Sul, "Novel rotor flux observer using observer characteristic function in complex vector space for field oriented induction motor drives," in Sixteenth Annual IEEE Applied Power Electronics Conference and Exposition, 2001. APEC 2001, 2001, vol. 1, pp. 615-621 vol.1.

[96] M. Depenbrock, "Direct self-control (DSC) of inverter-fed induction machine," IEEE Trans. Power Electron., vol. 3, no. 4, pp. 420-429, Oct. 1988.

[97] I. Takahashi and T. Noguchi, "A New Quick-Response and High-Efficiency Control Strategy of an Induction Motor," IEEE Trans. Ind. Appl., vol. IA-22, no. 5, pp. 820827, Sep. 1986.

[98] T. Noguchi, M. Yamamoto, S. Kondo, and I. Takahashi, "High frequency switching operation of PWM inverter for direct torque control of induction motor," in , 
Conference Record of the 1997 IEEE Industry Applications Conference, 1997. Thirty-Second IAS Annual Meeting, IAS '97, 1997, vol. 1, pp. 775-780 vol.1.

[99] M. P. Kazmierkowski and A. B. Kasprowicz, "Improved direct torque and flux vector control of PWM inverter-fed induction motor drives," IEEE Trans. Ind. Electron., vol. 42, no. 4, pp. 344-350, Aug. 1995.

[100] J.-K. Kang and S.-K. Sul, "Analysis and prediction of inverter switching frequency in direct torque control of induction machine based on hysteresis bands and machine parameters," IEEE Trans. Ind. Electron., vol. 48, no. 3, pp. 545-553, Jun. 2001.

[101] T. G. Habetler, F. Profumo, M. Pastorelli, and L. M. Tolbert, "Direct torque control of induction machines using space vector modulation," IEEE Trans. Ind. Appl., vol. 28, no. 5, pp. 1045-1053, Sep. 1992.

[102] G. Papafotiou, J. Kley, K. G. Papadopoulos, P. Bohren, and M. Morari, "Model Predictive Direct Torque Control-Part II: Implementation and Experimental Evaluation," IEEE Trans. Ind. Electron., vol. 56, no. 6, pp. 1906-1915, Jun. 2009.

[103] R. Vargas, J. Rodriguez, U. Ammann, and P. W. Wheeler, "Predictive Current Control of an Induction Machine Fed by a Matrix Converter With Reactive Power Control," IEEE Trans. Ind. Electron., vol. 55, no. 12, pp. 4362-4371, Dec. 2008.

[104] J. Beerten, J. Verveckken, and J. Driesen, "Predictive Direct Torque Control for Flux and Torque Ripple Reduction," IEEE Trans. Ind. Electron., vol. 57, no. 1, pp. 404412, Jan. 2010.

[105] F. Wang, S. Li, X. Mei, W. Xie, J. Rodriguez, and R. M. Kennel, "Model-Based Predictive Direct Control Strategies for Electrical Drives: An Experimental Evaluation of PTC and PCC Methods," IEEE Trans. Ind. Inform., vol. 11, no. 3, pp. 671-681, Jun. 2015.

[106] J. Rengifo, J. Aller, A. Berzoy, and J. Restrepo, "Predictive DTC algorithm for induction machines using Sliding Horizon Prediction," in 2014 IEEE 5th Latin American Symposium on Circuits and Systems (LASCAS), 2014, pp. 1-4.

[107] E. A. José Sayritupac, J. Rengifo, E. Albanez, J. M. Aller, and J. Restrepo, "Predictive Control Strategy for DFIG Wind Turbines with Maximum Power Point Tracking Using Multilevel Converters," 2nd Workshop Power Electron. Power Qual. Appl., pp. 1-6, Jun. 2015.

[108] L. Djaghdali, S. Belkacem, and F. Naceri, "Reducing of torque and flux ripples in DTC of im based on predictive control," J. Electr. Eng., vol. 16, pp. 247-257, 2016.

[109] D. Casadei, F. Profumo, G. Serra, and A. Tani, "FOC and DTC: two viable schemes for induction motors torque control," IEEE Trans. Power Electron., vol. 17, no. 5, pp. 779-787, Sep. 2002. 
[110] R. Abdelli, D. Rekioua, and T. Rekioua, "Performances improvements and torque ripple minimization for VSI fed induction machine with direct control torque," ISA Trans., vol. 50, no. 2, pp. 213-219, Apr. 2011.

[111] J. C. Viola, J. A. Restrepo, V. M. Guzman, and M. I. Gimenez, "Direct Torque Control of Induction Motors Using a Fuzzy Inference System for Reduced Ripple Torque and Current Limitation," in Power Electronics and Motion Control Conference, 2006. EPE-PEMC 2006. 12th International, 2006, pp. 1161-1166.

[112] M. Ortega, J. Restrepo, J. Viola, M. I. Gimenez, and V. Guzman, "Direct torque control of induction motors using fuzzy logic with current limitation," in 31 st Annual Conference of IEEE Industrial Electronics Society, 2005. IECON 2005, 2005, p. 6 pp.-.

[113] J. A. Restrepo, J. C. Viola, M. Ortega, V. M. Guzman, and M. I. Gimenez, “A fuzzyPWM direct torque control of induction machines for current limitation and reduced torque ripple," in 2005 European Conference on Power Electronics and Applications, 2005, p. 8 pp.-P.8.

[114] S. Gdaim, A. Mtibaa, and M. F. Mimouni, "Design and Experimental Implementation of DTC of an Induction Machine Based on Fuzzy Logic Control on FPGA,” IEEE Trans. Fuzzy Syst., vol. 23, no. 3, pp. 644-655, Jun. 2015.

[115] G. Noriega, J. Restrepo, V. Guzman, M. Gimenez, and J. Aller, "Direct torque control of PMSM using fuzzy logic with PWM," in 2007 42nd International Universities Power Engineering Conference, 2007, pp. 203-209.

[116] G. Noriega, J. Restrepo, A. Bueno, J. M. Aller, M. I. Giménez, and V. Guzmán, "Classic, fuzzy and predictive dtc strategies for the PMSM using the bacterial foraging algorithmas an online parameter estimator," Rev. Fac. Ing. Univ. Antioquia, no. 64, pp. 182-194, Sep. 2012.

[117] R. Ortega, N. Barabanov, G. Escobar, and E. Valderrama, "Direct torque control of induction motors: stability analysis and performance improvement," IEEE Trans. Autom. Control, vol. 46, no. 8, pp. 1209-1222, Aug. 2001.

[118] G. Escobar, A. M. Stankovic, E. Galvan, J. M. Carrasco, and R. Ortega, "A family of switching control strategies for the reduction of torque ripple in DTC," IEEE Trans. Control Syst. Technol., vol. 11, no. 6, pp. 933-939, Nov. 2003.

[119] A. A. S. Mohamed, A. Berzoy, and O. Mohammed, "Design and Hardware Implementation of FL MPPT Control for PV Systems Based on GA and SmallSignal Analysis," IEEE Trans. Sustain. Energy, vol. PP, no. 99, pp. 1-1, 2016.

[120] J. Prieto, M. Jones, F. Barrero, E. Levi, and S. Toral, "Comparative Analysis of Discontinuous and Continuous PWM Techniques in VSI-Fed Five-Phase Induction 
Motor," IEEE Transactions Industrial Electronics, vol. 58, no. 12, pp. 5324-5335, Dec. 2011.

[121] J. W. R. Santana, J. B. Suñe, J. M. A. Castro, A. A. B. Montilla, and J. A. R. Zambrano, "Parameter estimation method for induction machines using instantaneous voltage and current measurements," Rev. Fac. Ing., vol. 0, no. 75, pp. 57-66, May 2015.

[122] J. Rengifo, J. M. Aller, A. Bueno, J. Viola, and J. Restrepo, "Parameter Estimation Method for Induction Machines Using the Instantaneous Impedance During a Dynamic Start-Up," in Andean Region International Conference (ANDESCON), 2012 VI, 2012, pp. 11-14.

[123] X. Chang, V. Cocquempot, and C. Christophe, "A model of asynchronous machines for stator fault detection and isolation," IEEE Trans. Ind. Electron., vol. 50, no. 3, pp. 578-584, Jun. 2003.

[124] S. Williamson and K. Mirzoian, "Analysis of Cage Induction Motors with Stator Winding Faults," IEEE Power Eng. Rev., vol. PER-5, no. 7, pp. 50-51, Jul. 1985.

[125] H. A. Toliyat and T. A. Lipo, "Transient analysis of cage induction machines under stator, rotor bar and end ring faults," IEEE Trans. Energy Convers., vol. 10, no. 2, pp. 241-247, Jun. 1995.

[126] X. Luo, Y. Liao, H. A. Toliyat, A. El-Antably, and T. A. Lipo, "Multiple coupled circuit modeling of induction machines," IEEE Trans. Ind. Appl., vol. 31, no. 2, pp. 311-318, Mar. 1995.

[127] S. Nandi, "A detailed model of induction machines with saturation extendable for fault analysis," IEEE Trans. Ind. Appl., vol. 40, no. 5, pp. 1302-1309, Sep. 2004.

[128] Circuit Analysis of A-C Power Systems... J. Wiley \& sons, Incorporated, 1950.

[129] F. Duan and R. Zivanovic, "A model for induction motor with stator faults," in Universities Power Engineering Conference (AUPEC), 2012 22nd Australasian, 2012, pp. 1-5.

[130] J. M. Aller, "Métodos para el Análisis y Control Dinámico de la Máquina de Inducción." Universidad Simon Bolivar, 1997.

[131] M. Popescu, "Induction motor modelling for vector control purposes," PhD. Thesis, Helsinki University of Technology, Finland, 2000.

[132] G. B. Kliman, W. J. Premerlani, R. A. Koegl, and D. Hoeweler, "A new approach to on-line turn fault detection in AC motors," in , Conference Record of the 1996 IEEE Industry Applications Conference, 1996. Thirty-First IAS Annual Meeting, IAS '96, 1996, vol. 1, pp. 687-693 vol.1. 
[133] A. Bellini, C. Concari, G. Franceschini, E. Lorenzani, and C. Tassoni, "Induction Drives Diagnosis by Signal Injection: Effectiveness and Severity Classification," in 2005 IEEE International Conference on Electric Machines and Drives, 2005, pp. $718-727$.

[134] A. Siddique, G. S. Yadava, and B. Singh, "A review of stator fault monitoring techniques of induction motors," IEEE Trans. Energy Convers., vol. 20, no. 1, pp. 106-114, Mar. 2005.

[135] J. Martinez, F. Philipp, M. Glesner, and A. Arkkio, "An accurate and fast technique for correcting spectral leakage in motor diagnosis," in 2013 9th IEEE International Symposium on Diagnostics for Electric Machines, Power Electronics and Drives (SDEMPED), 2013, pp. 215-220.

[136] O. Imoru, L. Mokate, A. A. Jimoh, and Y. Hamam, "Diagnosis of rotor inter-turn fault of electrical machine at speed using stray flux test method," in AFRICON, 2015, 2015, pp. 1-5.

[137] D. T. Razafimahefa, E. Randrianarisoa, E. J. R. Sambatra, and N. Heraud, "Modeling and faults detection of small power wound rotor induction machine," in 2014 International Conference and Exposition on Electrical and Power Engineering (EPE), 2014, pp. 311-316.

[138] Y. Maouche, A. Boussaid, M. Boucherma, and A. Khezzar, "Modeling and simulation of stator turn faults. Detection based on stator circular current and neutral voltage," in 2013 9th IEEE International Symposium on Diagnostics for Electric Machines, Power Electronics and Drives (SDEMPED), 2013, pp. 263-268.

[139] M. B. K. Bouzid and G. Champenois, "Neural network based method for the automatic detection of the stator faults of the induction motor," in 2013 International Conference on Electrical Engineering and Software Applications (ICEESA), 2013, pp. 1-7.

[140] S. Toma, L. Capocchi, and G. A. Capolino, "Wound-Rotor Induction Generator Inter-Turn Short-Circuits Diagnosis Using a New Digital Neural Network," IEEE Trans. Ind. Electron., vol. 60, no. 9, pp. 4043-4052, Sep. 2013.

[141] A. A. Halacsy, "Leakage Reactance of Transformers [includes discussion]," Trans. Am. Inst. Electr. Eng. Part III Power Appar. Syst., vol. 75, no. 3, Jan. 1956.

[142] I. Hernandez, F. de Leon, and P. Gomez, "Design Formulas for the Leakage Inductance of Toroidal Distribution Transformers," IEEE Trans. Power Deliv., vol. 26, no. 4, pp. 2197-2204, Oct. 2011.

[143] P. Bastard, P. Bertrand, and M. Meunier, "A transformer model for winding fault studies," IEEE Trans. Power Deliv., vol. 9, no. 2, pp. 690-699, Apr. 1994. 
[144] S. M. A. Cruz and A. J. M. Cardoso, "Multiple Reference Frames Theory: A New Method for the Diagnosis of Stator Faults in Three-Phase Induction Motors," IEEE Trans. Energy Convers., vol. 20, no. 3, pp. 611-619, Sep. 2005.

[145] C. H. D. Angelo, G. R. Bossio, S. J. Giaccone, M. I. Valla, J. A. Solsona, and G. O. Garcia, "Online Model-Based Stator-Fault Detection and Identification in Induction Motors," IEEE Trans. Ind. Electron., vol. 56, no. 11, pp. 4671-4680, Nov. 2009.

[146] C. S. Kallesoe, R. Izadi-Zamanabadi, P. Vadstrup, and H. Rasmussen, "ObserverBased Estimation of Stator-Winding Faults in Delta-Connected Induction Motors: A Linear Matrix Inequality Approach," IEEE Trans. Ind. Appl., vol. 43, no. 4, pp. 1022-1031, Jul. 2007.

[147] D. Filipović-Grčić, B. Filipović-Grčić, and K. Capuder, "Modeling of three-phase autotransformer for short-circuit studies," Int. J. Electr. Power Energy Syst., vol. 56, pp. 228-234, Mar. 2014.

[148] Z. Zhao et al., "Measurements and Calculation of Core-Based Curve and Magnetizing Current in DC-Biased Transformers," IEEE Trans. Appl. Supercond., vol. 20, no. 3, pp. 1131-1134, Jun. 2010.

[149] J. Sottile, F. C. Trutt, and J. L. Kohler, "Condition monitoring of stator windings in induction motors. II. Experimental investigation of voltage mismatch detectors," IEEE Trans. Ind. Appl., vol. 38, no. 5, pp. 1454-1459, Sep. 2002.

[150] T. M. Wolbank, K. A. Loparo, and R. Wohrnschimmel, "Inverter statistics for online detection of stator asymmetries in inverter-fed induction motors," IEEE Trans. Ind. Appl., vol. 39, no. 4, pp. 1102-1108, Jul. 2003.

[151] R. M. Tallam, T. G. Habetler, and R. G. Harley, "Stator winding turn-fault detection for closed-loop induction motor drives," IEEE Trans. Ind. Appl., vol. 39, no. 3, pp. 720-724, May 2003.

[152] M. Wolkiewicz, G. Tarchała, T. Orłowska-Kowalska, and C. T. Kowalski, "Online Stator Interturn Short Circuits Monitoring in the DFOC Induction-Motor Drive," IEEE Trans. Ind. Electron., vol. 63, no. 4, pp. 2517-2528, Apr. 2016.

[153] M. Wolkiewicz, G. Tarchała, T. Orlowska-Kowalska, and C. Kowalski, "Stator fault monitoring based on internal signals of vector controlled induction motor drives," in IECON 2016 - 42nd Annual Conference of the IEEE Industrial Electronics Society, 2016, pp. 2651-2656.

[154] M. Wolkiewicz and C. T. Kowalski, "On-line neural network-based stator fault diagnosis system of the converter-fed induction motor drive," in IECON 2013 - 39th Annual Conference of the IEEE Industrial Electronics Society, 2013, pp. 55615566. 
[155] S. Cheng, P. Zhang, and T. G. Habetler, "An Impedance Identification Approach to Sensitive Detection and Location of Stator Turn-to-Turn Faults in a Closed-Loop Multiple-Motor Drive," IEEE Trans. Ind. Electron., vol. 58, no. 5, pp. 1545-1554, May 2011.

[156] S. M. A. Cruz and A. J. M. Cardoso, "Diagnosis of stator inter-turn short circuits in DTC induction motor drives," IEEE Trans. Ind. Appl., vol. 40, no. 5, pp. 1349-1360, Sep. 2004.

[157] S. M. A. Cruz, H. A. Toliyat, and A. J. M. Cardoso, "DSP implementation of the multiple reference frames theory for the diagnosis of stator faults in a DTC induction motor drive," IEEE Trans. Energy Convers., vol. 20, no. 2, pp. 329-335, Jun. 2005.

[158] A. Berzoy, O. Mohammed, and J. Rengifo, "Fuzzy Predictive DTC of induction machines with reduced torque ripple and high performance operation," in 2016 IEEE Applied Power Electronics Conference and Exposition (APEC), 2016, pp. 32003206.

[159] A. Berzoy, A. A. S. Mohamed, and O. Mohammed, "Complex-Vector Model of Inter-Turn Failure in Induction Machines for Fault Detection and Identification," IEEE Trans. Ind. Appl., vol. PP, no. 99, pp. 1-1, 2016.

[160] F. Briz, M. W. Degner, A. Zamarron, and J. M. Guerrero, "Online stator winding fault diagnosis in inverter-fed AC machines using high-frequency signal injection," IEEE Trans. Ind. Appl., vol. 39, no. 4, pp. 1109-1117, Jul. 2003.

[161] P. C. T. Kowalski, R. Wierzbicki, and M. Wolkiewicz, "Stator and Rotor Faults Monitoring of the Inverter-Fed Induction Motor Drive using State Estimators," Automatika, vol. 54, no. 3, pp. 348-355, Jan. 2013.

[162] S. S. Refaat, H. Abu-Rub, and A. Iqbal, "ANN-based system for inter-turn stator winding fault tolerant DTC for induction motor drives," in 2015 17th European Conference on Power Electronics and Applications (EPE'15 ECCE-Europe), 2015, pp. 1-7.

[163] F. Briz, M. W. Degner, A. B. Diez, and J. M. Guerrero, "Online diagnostics in inverter-fed induction machines using high-frequency signal injection," IEEE Trans. Ind. Appl., vol. 40, no. 4, pp. 1153-1161, Jul. 2004.

[164] A. Berzoy, J. Rengifo, and O. Mohammed, "Fuzzy Predictive DTC of Induction Machines with Reduced Torque Ripple and High Performance Operation," IEEE Trans. Power Electron., vol. PP, no. 99, pp. 1-1, 2017.

[165] A. Berzoy, A. A. S. Mohamed, and O. A. Mohammed, "Stator winding inter-turn fault in induction machines: Complex-vector transient and steady-state modelling," in 2017 IEEE Inter. Electric Machines and Drives Conf. (IEMDC), 2017, pp. 1-7. 
[166] S.-K. Chung, "A phase tracking system for three phase utility interface inverters," IEEE Trans. Power Electron., vol. 15, no. 3, pp. 431-438, May 2000.

[167] J.-Z. Yang and C.-W. Liu, "A precise calculation of power system frequency," IEEE Trans. Power Deliv., vol. 16, no. 3, pp. 361-366, Jul. 2001.

[168] I. Kamwa and R. Grondin, "Fast adaptive schemes for tracking voltage phasor and local frequency in power transmission and distribution systems," IEEE Trans. Power Deliv., vol. 7, no. 2, pp. 789-795, Apr. 1992.

[169] A. K. Pradhan, A. Routray, and A. Basak, "Power system frequency estimation using least mean square technique," IEEE Trans. Power Deliv., vol. 20, no. 3, pp. 18121816, Jul. 2005.

[170] V. V. Terzija, M. B. Djuric, and B. D. Kovacevic, "Voltage phasor and local system frequency estimation using Newton type algorithm," IEEE Trans. Power Deliv., vol. 9, no. 3, pp. 1368-1374, Jul. 1994.

[171] T. Lin, M. Tsuji, and E. Yamada, "A wavelet approach to real time estimation of power system frequency," in SICE 2001. Proceedings of the 40th SICE Annual Conference. International Session Papers (IEEE Cat. No.01TH8603), 2001, pp. 5865.

[172] L. L. Lai, W. L. Chan, C. T. Tse, and A. T. P. So, "Real-time frequency and harmonic evaluation using artificial neural networks," IEEE Trans. Power Deliv., vol. 14, no. 1, pp. 52-59, Jan. 1999.

[173] S. A. Souza, M. Oleskovicz, D. V. Coury, T. V. Silva, A. C. B. Delbem, and E. V. Simoes, "FPGA implementation of Genetic Algorithms for frequency estimation in power systems," in 2008 IEEE Power and Energy Society General Meeting Conversion and Delivery of Electrical Energy in the 21st Century, 2008, pp. 1-6.

[174] J. Szafran and W. Rebizant, "Power system frequency estimation," Transm. Distrib. IEE Proc. - Gener., vol. 145, no. 5, pp. 578-582, Sep. 1998.

[175] C. L. Fortescue, "Method of symmetrical co-ordinates applied to the solution of polyphase networks," Proc. Am. Inst. Electr. Eng., vol. 37, no. 6, pp. 629-716, Jun. 1918.

[176] M. Rice, Digital Communications: A Discrete-Time Approach, 1 edition. Upper Saddle River, N.J: Pearson, 2008.

[177] W. L. Brogan, Modern Control Theory, 3 edition. Englewood Cliffs, N.J: Pearson, 1990.

[178] L. Wang and J. Jatskevich, "A voltage-behind-reactance induction machine model for the EMTP-Type solution," in 2009 IEEE PES General Meeting, 2009, pp. 1-1. 
[179] A. Berzoy, A. A. S. Mohamed, and O. Mohammed, "Complex-Vector Model of Interturn Failure in Induction Machines for Fault Detection and Identification," IEEE Trans. Ind. Appl., vol. 53, no. 3, pp. 2667-2678, May 2017.

[180] A. Berzoy, A. A. S. Mohamed, and O. Mohammed, "Impact of Inter-Turn ShortCircuit Location on Induction Machines Parameters through FE Computations," IEEE Trans. Magn., vol. PP, no. 99, pp. 1-1, 2017.

[181] J. O. Estima, N. M. A. Freire, and A. J. M. Cardoso, "Recent advances in fault diagnosis by Park's vector approach," in 2013 IEEE Workshop on Electrical Machines Design, Control and Diagnosis (WEMDCD), 2013, pp. 279-288.

[182] J. O. Estima and A. J. M. Cardoso, “A New Algorithm for Real-Time Multiple OpenCircuit Fault Diagnosis in Voltage-Fed PWM Motor Drives by the Reference Current Errors," IEEE Trans. Ind. Electron., vol. 60, no. 8, pp. 3496-3505, Aug. 2013.

[183] J. M. Anderson, R. W. Cox, and P. O'Connor, "Online algorithm for early stage fault detection in IGBT switches," in 2013 9th IEEE International Symposium on Diagnostics for Electric Machines, Power Electronics and Drives (SDEMPED), 2013, pp. 1-8.

[184] B. Ji, V. Pickert, W. P. Cao, and L. Xing, "Onboard condition monitoring of solder fatigue in IGBT power modules," in 2013 9th IEEE International Symposium on Diagnostics for Electric Machines, Power Electronics and Drives (SDEMPED), 2013, pp. 9-15.

[185] I. Vaalasranta, J. Pippola, and L. Frisk, "Power MOSFET failure and degradation mechanisms in flyback topology under high temperature and high humidity conditions," in 2013 9th IEEE International Symposium on Diagnostics for Electric Machines, Power Electronics and Drives (SDEMPED), 2013, pp. 16-22.

[186] M. Meneghini et al., "Reliability and failure analysis in power GaN-HEMTs: An overview," in 2017 IEEE International Reliability Physics Symposium (IRPS), 2017, p. 3B-2.1-3B-2.8.

[187] K. Shenai, "Switching Megawatts with Power Transistors," Electrochem. Soc. Interface, vol. 22, no. 1, pp. 47-53, Jan. 2013.

[188] S. Vitanov et al., "Physics-Based Modeling of GaN HEMTs," IEEE Trans. Electron Devices, vol. 59, no. 3, pp. 685-693, Mar. 2012.

[189] F. Li, Q. H. Liu, and D. P. Klemer, "Numerical Simulation of high electron mobility transistors based on the spectral element Method," Appl. Comput. Electromagn. Soc. J., vol. 31, no. 10, pp. 1144-1150, 2016. 
[190] G. Meneghesso, M. Meneghini, and E. Zanoni, "Breakdown mechanisms in AlGaN/GaN HEMTs: An overview," Jpn. J. Appl. Phys., vol. 53, no. 10, p. 100211, Sep. 2014.

[191] "Wiley: GaN Transistors for Efficient Power Conversion, 2nd Edition - Alex Lidow, Johan Strydom, Michael de Rooij, et al." [Online]. Available: http://www.wiley.com/WileyCDA/WileyTitle/productCd-1118844769.html. [Accessed: 19-Oct-2016].

[192] M. Kaddeche, A. Telia, and A. Soltani, "Study of field plate effects onAlGaN/GaN HEMTs," in 2009 International Conference on Microelectronics - ICM, 2009, pp. $362-365$.

[193] D. Visalli et al., "Experimental and simulation study of breakdown voltage enhancement of AlGaN/GaN heterostructures by Si substrate removal," Appl. Phys. Lett., vol. 97, no. 11, p. 113501, Sep. 2010.

[194] Andrew Binder and Jiann-Shiun Yuan, "Optimization of an Enhancement-mode $\mathrm{AlGaN} / \mathrm{GaN} / \mathrm{AlGaN}$ DHFET towards a High Breakdown Voltage and Low Figure of Merit," presented at the Workshop on Wide Bandgap Power Devices and Applications, New Mexico, USA, 2017.

[195] W. Saito et al., "Field-Plate Structure Dependence of Current Collapse Phenomena in High-Voltage GaN-HEMTs," IEEE Electron Device Lett., vol. 31, no. 7, pp. 659661, Jul. 2010.

[196] Alberto Berzoy, Christopher R. Lashway, and Osama Mohammed, "Breakdown Voltage Improvement and Analysis of GaN HEMTs through Field Plate Inclusion and Substrate Removal," presented at the Workshop on Wide Bandgap Power Devices and Applications, New Mexico, USA, 2017.

[197] C. R. Lashway, A. Berzoy, N. Elsayad, and O. Mohammed, "Breakdown voltage assessment of GaN HEMT devices through physics-based modeling," in 2017 International Applied Computational Electromagnetics Society Symposium - Italy (ACES), 2017, pp. 1-2.

[198] S. Karmalkar, M. S. Shur, G. Simin, and M. A. Khan, "Field-plate engineering for HFETs," IEEE Trans. Electron Devices, vol. 52, no. 12, pp. 2534-2540, Dec. 2005.

[199] H. Xing, Y. Dora, A. Chini, S. Heikman, S. Keller, and U. K. Mishra, "High breakdown voltage AlGaN-GaN HEMTs achieved by multiple field plates," IEEE Electron Device Lett., vol. 25, no. 4, pp. 161-163, Apr. 2004. 
[200] S. Karmalkar and U. K. Mishra, "Enhancement of breakdown voltage in AlGaN/GaN high electron mobility transistors using a field plate," IEEE Trans. Electron Devices, vol. 48, no. 8, pp. 1515-1521, Aug. 2001.

[201] B. Jogai, J. D. Albrecht, and E. Pan, "Effect of electromechanical coupling on the strain in $\mathrm{AlGaN} / \mathrm{GaN}$ heterojunction field effect transistors," J. Appl. Phys., vol. 94, no. 6, pp. 3984-3989, Sep. 2003.

[202] S. K. Chung, "Analytic model for field-plate-edge breakdown of planar devices terminated with field plate and semiresistive layer," IEE Proc. - Sci. Meas. Technol., vol. 151, no. 1, pp. 21-24, Jan. 2004.

[203] K. K. Young, "Short-channel effect in fully depleted SOI MOSFETs," IEEE Trans. Electron Devices, vol. 36, no. 2, pp. 399-402, Feb. 1989. 
VITA

\section{ALBERTO BERZOY}

27 August 1979

1997-2003

2003-2008

2013-2017

2008-2018

Jan - Aug 2017

2018 - Present
Born, Caracas, Venezuela

B.Sc. Simón Bolívar University, Venezuela

M.Sc. Simón Bolívar University, Venezuela

Teaching Assistant, Florida International University

Associate Prof., Simon Bolivar University, Venezuela

Dissertation Year Fellowship, FIU, USA

Hardware Power Electronic Engineer at Sonnen Batterie Inc

\section{SELECTED PUBLICATION AND PRESENTATIONS}

1. A. Berzoy, and O. A. Mohammed and Jose Restrepo "Analysis of the Impact of Stator Inter-Turn Short-Circuit Faults on Induction Machines driven by Direct Torque Control" in Trans. Energy Conversion. Revision R1.

2. A Berzoy, H Eldeeb, O. Mohammed, "Online Fault Detection of Stator Winding Faults in IM driven by DTC using the Off-diagonal term of the Symmetrical Component Impedance Matrix," in IEEE Trans. Power Electron. Submitted

4. A. Berzoy, J. Rengifo and O. Mohammed, "Fuzzy Predictive DTC of Induction Machines With Reduced Torque Ripple and High-Performance Operation," in IEEE Trans. on Power Electronics, vol. 33, no. 3, pp. 2580-2587, March 2018.

5. A. Berzoy, A. A. S. Mohamed and O. Mohammed, "Impact of Inter-Turn ShortCircuit Location on Induction Machines Parameters Through FE Computations," in IEEE Transactions on Magnetics, vol. 53, no. 6, pp. 1-4, June 2017.

6. A. Berzoy, A. A. S. Mohamed and O. Mohammed, "Complex-Vector Model of Interturn Failure in Induction Machines for Fault Detection and Identification," in IEEE Trans. on Industry App., vol. 53, no. 3, pp. 2667-2678, May-June 2017.

7. A. Berzoy, A. a. S. Mohamed, and O. Mohammed, "Optimizing power converter PCB design for lower EMI," COMPEL - Int. J. Comput. Math. Electr. Electron. Eng., vol. 34, no. 5, pp. 1364-1380, Sep. 2015.

8. Alberto Berzoy, Hassan Eldeeb, O. Mohammed, “Online Fault Detection of Stator Winding Faults in IM driven by DTC using the Off-diagonal term of the 
Symmetrical Component Impedance Matrix," in 2018 IEEE Applied Power Electronics Conference and Exposition (APEC), 2018.

9. Christopher Lasway, Alberto Berzoy, O. Mohammed, "Field-Plate Length Variation on GaN Devices for BV and On-Resistance Characterization" IEEE ACES, 2018.

10. Christopher R. Lashway, Alberto Berzoy, Nour Elsayad and O. Mohammed, "Breakdown Voltage Assessment of GaN HEMT Devices through Physics-Based Modeling," IEEE ACES, 2017.

11. Alberto Berzoy, A. A. S. Mohamed, O. A. Mohammed, "Stator Winding Inter-turn Fault in Induction Machines: Complex-Vector Transient and Steady-State Modelling," IEEE IEMDC, 2017

12. A. Berzoy, C. R. Lashway, H. Moradisizkoohi and O. A. Mohammed "Breakdown Voltage Improvement and Analysis of GaN HEMTs through Field Plate Inclusion and Substrate Removal" Wide Bandgap Power Devices and Applications 2017. New Mexico. USA

13. Alberto Berzoy, O. Mohammed, and J. Rengifo, "Fuzzy Predictive DTC of induction machines with reduced torque ripple and high performance operation," in 2016 IEEE Applied Power Electronics Conference and Exposition (APEC), 2016, pp. 3200-3206.

14. A. Berzoy, A. A. S. Mohamed, and O. Mohammed, "Design considerations and predictive direct current control of Active Regenerative Rectifiers for harmonic and current ripple reduction," in 2016 IEEE Applied Power Electronics Conference and Exposition (APEC), 2016, pp. 928-935.

15. A. Berzoy, A. A. S. Mohamed, and O. A. Mohammed, "Inter-turn short-circuit fault model for magnetically coupled circuits: A general study," in 2016 IEEE Industry Applications Society Annual Meeting, 2016, pp. 1-7.

16. A. Berzoy, A. A. S. Mohamed, and O. A. Mohammed, "Dynamic space-vector model of induction machines with stator inter-turn short-circuit fault," in IECON 2015 - 41st Annual Conference of the IEEE Industrial Electronics Society, 2015, pp. $003620-003625$.

\section{AWARDS}

- Wipda 2017 Travel Award

"Breakdown Voltage Improvement and Analysis of GaN HEMTs through Field Plate Inclusion and Substrate Removal" Authors: A. Berzoy, C. R. Lashway, H. Moradisizkoohi and O. A. Mohammed

- APEC 2016 Best Paper Presentation Award and Travel Award 2016 "Design Considerations and PDCC of Active Regenerative Rectifiers for Harmonic 
and Current Ripple Reduction" Authors: A. Berzoy, A. Mohamed and O. A. Mohammed

- IAS 2016 Travel Award

"Inter-Tum Short-Circuit Fault Model for Magnetically Coupled Circuits: A General Study” Authors: A. Berzoy, A. Mohamed and O. A. Mohammed

- IECON 2015 Best Paper Presentation Award

"Dynamic modeling of Induction Machine with stator inter-turn short circuit". Authors: A. Berzoy, A. Mohamed and O. A. Mohammed

- IECON 2014 Best Paper Conference 2014 "Optimized-Fuzzy MPPT Controller Using GA for Stand-alone Photovoltaic Water Pumping System" Authors: A. Mohamed, A. Berzoy and O. A. Mohammed

- GPSC FIU Travel Awards 2014-2015-2016-2017 GPSC is Graduate and Professional Student Committee at FIU. I obtained 4 consecutively years this award as support for travel to present conference papers.

\section{ORGANIZATION AND MEMBERSHIPS}

- IEEE Student Member

2013-Present

- IEEE-FIU IAS Student Branch Chapter Chair

2016-Present

- IEEE PES Secretariat Professional Branch Chapter

2016-Present 